GEORG-AUGUST-UNIVERSITÄT GÖTTINGEN

\title{
Partikelbildung bei der Alkenozonolyse und ihre Kopplung an die Radikalchemie
}

\author{
vorgelegt von \\ Claudia Keunecke \\ aus Bad Gandersheim
}

Göttingen 2012 



\title{
Partikelbildung bei der Alkenozonolyse und ihre Kopplung an die Radikalchemie
}

\author{
Dissertation zur Erlangung des \\ mathematisch-naturwissenschaftlichen Doktorgrades \\ „Doctor rerum naturalium“ \\ der \\ Georg-August-Universität Göttingen
}

vorgelegt von

Claudia Keunecke

aus Bad Gandersheim

Prüfer:

Dr. T. Zeuch

Prof. Dr. M.A. Suhm

angefertigt im

Institut für Physikalische Chemie

der Georg-August-Universität zu Göttingen

2012 

Referent: Dr. T. Zeuch

Korreferent: Prof. Dr. M. A. Suhm

Tag der mündlichen Prüfung: 11.05.2012 
Meine von mir verfasste Dissertation ist kein Plagiat, und den Vorwurf weise ich mit allem Nachdruck von mir.

(Karl Theodor Maria Nikolaus Johann Jacob Philipp Franz Joseph Sylvester Freiherr von und zu Guttenberg, 2011) 


\section{Danksagung}

Viele Menschen haben mich in meinem Promotionsvorhaben unterstützt, bei denen ich mich an dieser Stelle bedanken möchte.

Zuerst geht dieser Dank natürlich an die offensichtlichen Personen: Dr. Thomas Zeuch und Prof. Dr. Martin Suhm, die Betreuung bzw. das Korreferat dieser Arbeit übernommen haben. Dr. Zeuch zeichnete sich dabei besonders durch sein Engagement sowie seine Begeisterungsfähigkeit für Messergebnisse und Interpretationsversuche aller Art aus, was das Arbeiten zu einem Erlebnis (der guten Art, um Missverständnisse an dieser Stelle zu vermeiden) machte. Prof. Suhm war bei experimentellen und theoretischen Problemen stets bereit, seine Expertise auf dem Gebiet der IR-Spektrokopie, der Molekulardynamik und zahlreichen anderen Aspekten zur Verfügung zu stellen.

Auch Herrn Prof. Hoyermann danke ich für viele Gespräche (manchmal auch mit wissenschaftlichem Thema) in den letzten Jahren, die Farbe in den Alltag gebracht haben.

Eine große Hilfe im Laboralltag war neben Michael Schlote und Clemens Heymann auf jeden Fall Werner Noack, der jederzeit bereit war, mir bei praktischen Problemen zur Seite zu stehen und mich auch politik- und nachrichtentechnisch auf dem neuesten Stand hielt. Auch bei den Werkstätten, stellvertretend für ihre Mitarbeiter seien die Leiter Volker Meyer, Andreas Knorr und Hans-Joachim Schlette genannt, konnte ich mich immer auf die zeitnahe Erfüllung meiner Wünsche verlassen.

Unterstützung fand ich im letzten Jahr bei experimentellen Arbeiten auch durch einige Bachelorstudenten, denen ich für ihr hohes Engagement und ihr Interesse an dem manchmal nervenraubenden Feld der Aerosolbildung danken möchte. Dies sind Philip Carlsson (zyklische Alkene), Christoph Dierking (ungesättigte Alkohole), Bastian Krüger (lineare Alkene), Benjamin Schröder (ungesättigte Carbonylverbindungen) und Janina Dege $\left(\mathrm{SO}_{2}\right.$-Simulationen).

Wenn der Blondinenfaktor zuschlug, fand ich bei Computerproblemen Unterstützung bei Norbert Neisen und Dr. Rainer Oswald, die sich nicht von meiner Unkenntnis (und teilweise auch Beratungsresistenz) abschrecken ließen und damit meist größere Katastrophen abwenden konnten.

Im Laufe des letzten Jahres hat sich außerdem eine Kooperation mit einer 
Arbeitsgruppe aus Mainz ergeben, die es sich zur Aufgabe gemacht hat, das produzierte Aerosol zu analysieren. Für den hohen Arbeitsaufwand und die insgesamt nette Zusammenarbeit danke ich daher dem zuständigen Doktoranden Martin Beck wie auch seinem Arbeitsgruppenleiter Prof. Thorsten Hoffmann.

Meine Arbeitsgruppe hat sich in den mittlerweile über vier Jahren meiner Mitgliedschaft bis auf die permanenten Mitarbeiter einmal komplett ausgetauscht. Jeder Zusammensetzung war jedoch das gute Arbeitsklima gemein, so dass sich auch Treffen außerhalb des Institutes zu spaßigen Aktivitäten entwickelten. Besonders hervorheben möchte ich hier die Mitglieder, die diese Arbeit korrekturgelesen haben: Jonas Altnöder, Sascha Abraham und Julia Zischang. Mit Petra Lawecki werde ich wohl immer die Woche bei der H-Bond Tagung in Verbindung bringen, in der wir den Conference Desk gerockt haben. Dr. Jan Lennard Wolf danke ich für die Einarbeitung in die verwendeten Methoden.

Das unterhaltsamste Trio seit Tick, Trick und Track hat in den letzten Monaten viel zu meiner Aufheiterung beigetragen. Mein Dank geht deswegen auch an João Oliveira, Jonas Feldt und ganz besonders Dr. Johannes Dieterich. Johannes danke ich zusätzlich noch für die Zeit, die er in das Korrekturlesen dieser Arbeit, das Gucken schlechter Filme, die Soleiproduktion sowie die Lösung von Computer- und Theoriefragen investiert hat.

Weiterhin möchte ich Herrn Prof. Botschwina für jahrelange gute Nachbarschaft, nette Gespräche in Mensen und Cafeterien sowie sein stetes Interesse an meiner Arbeit (und Person) danken.

Der gesamten Institutsbelegschaft, ganz besonders Dr. Markus Hold, danke ich für die gute Arbeitsatmosphäre und Hilfsbereitschaft, die das Arbeiten sehr angenehm machte.

Die wichtigsten Personen in meinem Leben sollen am Ende genannt werden: meine Familie, die mir - jeder für sich und alle zusammen - jeden Tag aufs neue beweisen, dass Blut doch dicker ist als Wasser. Neben meinen Eltern, die mir das Studium ermöglicht haben, haben auch mein Bruder Tobias und meine Großmutter stets hinter mir gestanden und meine Ziele geteilt - DANKE! : )

Widmen möchte ich diese Arbeit meiner Oma Gretchen, die leider in der Zeit der Anfertigung verstorben ist. 


\section{Inhaltsverzeichnis}

1. Einleitung 1

2. Theorie 13

2.1. Spektroskopische Grundlagen ... . . . . . . . . . . . . 13

2.1.1. Welle-Korpuskel-Dualismus . . . . . . . . . . . . . . 14

2.1.2. Schwinqunqsspektroskopie . . . . . . . . . . . . . . 15

2.1.3. FTIR-Spektroskopie ... . . . . . . . . . . . . . . 18

2.1.4. LAMBERT-BeERsches Gesetz . . . . . . . . . . . . . 20

2.2. Die Ozonolvse . . . . . . . . . . . . . . . . . . 22

2.2.1. Der allgemeine Ozonolysemechanismus nach CRIEGEE . 22

2.2.2. Die Foldaechemie . . . . . . . . . . . . . . . . . . 24

2.2.2.1. Unimolekulare Foldereaktionen . . . . . . . 24

2.2.2.2. Bimolekulare Folgereaktionen . . . . . . . . 27

2.2.2.3. Die Bedeutung von Sauerstoff als Reaktionspartner in bimolekularen Reaktionen . . . . . . . 29

2.3. Spezifische Reaktionsverläufe für die untersuchten Substanzen . 33

2.3.1. Reine Kohlenwasserstoffverbindungen . . . . . . . . . . 34

2.3.2. Voroxidierte Edukte . . . . . . . . . . . . . . . . . 36

2.4. Die Rolle von Radikalfängern . . . . . . . . . . . . . . . . . 42

2.4.1. Cvclohexan ... . . . . . . . . . . . . . . . . 43

2.4.2. Butanolisomere . . . . . . . . . . . . . . . . . . . 46

2.4.3. Wasserstoffperoxid . . . . . . . . . . . . . . . . 47

2.5. Die Kinetik von Ozonolvsereaktionen . . . . . . . . . . . . . . 47

2.6. Struktur-Reaktivitäts-Zusammenhang: SAR . . . . . . . . . . 51

2.7. Nukleationstheorien . . . . . . . . . . . . . . . . . 52

2.8. Der Einfluss von Schwefeldioxid $\left(\mathrm{SO}_{2}\right)$ auf die Ozonolysereaktion 54

2.8.1. Oxidation und Kinetik . . . . . . . . . . . . . . . 54

2.8.2. Auswirkungen von $\mathrm{SO}_{2}$ auf den Ozonolysemechanismus 56

3. Versuchsaufbau und experimentelle Methoden 59

3.1. Der experimentelle Aufbau . . . . . . . . . . . . . . 59

3.1.1. Ozonerzeuquna . . . . . . . . . . . . . . . . . 59

3.1.2. FTIR-Spektrometer. . . . . . . . . . . . . . . . 60

3.1.3. SMPS Partikelgrößenverteilungsanalysator . . . . . . . 61 
3.1.4. Die Aerosolzelle . . . . . . . . . . . . . . . . . . . . . 67

3.1.5. Die Vormischkammern . . . . . . . . . . . . . . . . . . 69

3.2. Die Durchführung verschiedener Experimentvarianten . . . . . . 70

3.2.1. Allqemeines Vorgehen . . . . . . . . . . . . . . . . . . 71

3.2.2. Partikelbildungsmessungen . . . . . . . . . . . . . . . 74

3.2.3. Kinetikmessungen . . . . . . . . . . . . . . . . . . . . 74

3.2.4. Produktbildunqsmessungen . . . . . . . . . . . . . . 76

3.2.5. Radikalfängerexperimente . . . . . . . . . . . . . . . . 76

4. Kalibrierung und Konzentrationsbestimmung 79

4.1. Kohlenwasserstoffverbindungen . . . . . . . . . . . . . 79

4.1.1. Carbonsäuren . . . . . . . . . . . . . . . . . . 79

4.1.2. Alkene, Alkohole, Aldehyd und Keton . . . . . . . . . . . 81

4.2. Ozon . . . . . . . . . . . . . . . . . . . . . . . 84

4.2.1. Methoden der Ozonkonzentrationsbestimmung . . . . . . 84

4.2.1.1. IR-Messungen . . . . . . . . . . . . . . . . 85

4.2.1.2. UV-Optik ....... . . . . . . . . . 87

4.2.1.3. Der Ozonanalysator . . . . . . . . . . . . . . 90

4.2.2. Kreuzkalibrierung: UV-Optik vs. Analysator und UV-Optik vs. IR-Messung . . . . . . . . . . . . . . . . . . 93

4.3. Additiva . . . . . . . . . . . . . . . . 95

4.3.1. $\mathrm{SO}_{3} \ldots \ldots \ldots \ldots \ldots$

4.3.2. $\mathrm{H}_{2} \mathrm{O} \ldots \ldots \ldots \ldots$

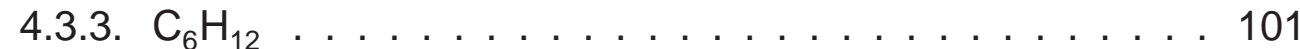

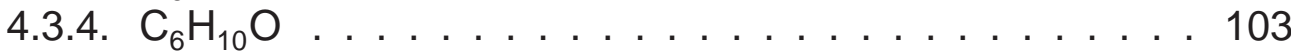

5. Auswertungsmethoden 105

5.1. Bestimmuna der Ozonkonzentration . . . . . . . . . . . 105

5.2. Infrarotspektren . . . . . . . . . . . . . . . . . 107

5.2.1. Kinetikmessungen . . . . . . . . . . . . . . . . 108

5.2.2. Produktbildungsstudien . . . . . . . . . . . . . . . . 109

5.3. Partikelbildunasmessungen . . . . . . . . . . . . . . . . . 111

5.4. Quantenchemische Rechnungen . . . . . . . . . . . . 112

6. Erqebnisse 119

6.1. Kinetikstudien . . . . . . . . . . . . . . . . . . . . 119

6.1.1. $\mathrm{C}_{6}-$ Kohlenwasserstoffe . . . . . . . . . . . . . . . 121

6.1.2. Voroxidierte Verbindungen . . . . . . . . . . . . . . . 124

6.1.2.1. Alkohole . . . . . . . . . . . . . . . . . . . . . 124

6.1.2.2. Carbonvlverbindungen . . . . . . . . . 125

6.1.2.3. Carbonsäuren . . . . . . . . . . . . . 126 
6.2. Produktbildungsstudien - Qualitative Auswertung . . . . . . . . . 130

6.2.1. $\mathrm{C}_{6}$-Kohlenwasserstoffe. . . . . . . . . . . . . . . . . . 131

6.2.1.1. Methylcvclopenten . . . . . . . . . . . . . . . . 131

6.2.1.2. 1-Hexen und 2-Methyl-1-penten . . . . . . . . . 136

6.2.2. Voroxidierte Verbindungen ... . . . . . . . . . . . . . . . 140

6.2.2.1. Ungesättiate Alkohole . . . . . . . . . . . . . . . 140

6.2.2.2. Carbonvlverbindungen . . . . . . . . . . . . . 143

6.2.2.3. Carbonsäuren . . . . . . . . . . . . . . . . . 149

6.2.3. Ketenbilduna - Zusammenfassuna . . . . . . . . . . . . . 153

6.2.4. Produktstudien unter $\mathrm{SO}_{2}$-Beteiligung . . . . . . . . . . 159

6.2.4.1. Endozyklische Alkene . . . . . . . . . . . . . . 160

6.2.4.2. Exozyklische Alkene . . . . . . . . . . . . . . . . 166

6.3. Produktstudien - Quantitative Auswertung . . . . . . . . . . . . . 169

6.3.1. OH-Ausbeute . . . . . . . . . . . . . . . . . . . . . 169

6.3.2. CO-Ausbeute . . . . . . . . . . . . . . . . . . . 172

6.3.2.1. C6-Kohlenwasserstoffe . . . . . . . . . . . . . 172

6.3.2.2. Voroxidierte Verbindungen . . . . . . . . . . . . 174

6.4. Partikelbildunasstudien ... . . . . . . . . . . . . . 175

6.4.1. $\mathrm{C}_{6}$-Kohlenwasserstoffe. ............... 177

6.4.1.1. Einfluss des Gesamtdruckes und des Badgases 177

6.4.1.2. Einfluss von Radikalfängern . . . . . . . . . . . 183

6.4.2. Voroxidierte Verbindungen . . . . . . . . . . . . . . . 188

6.4.2.1. Alkohole . . . . . . . . . . . . . . . . . 188

6.4.2.2. Fazit: Partikelbildung durch die Ozonolyse ungesättigter Alkohole .. . . . . . . . . . . . . . . . 194

6.4.2.3. Carbonylverbindungen . . . . . . . . . . . 196

6.4.2.4. Fazit: Partikelbildung durch die Ozonolyse ungesättigter Carbonylverbindungen . . . . . . . . 200

6.4.2.5. Carbonsäuren . . . . . . . . . . . . . . . 202

6.4.2.6. Untersuchung der Bedeutung sekundärer Reaktionen . . . . . . . . . . . . . . . . 207

6.4.2.7. Fazit: Partikelbildung durch die Ozonolyse ungesättigter Carbonsäuren . . . . . . . . . . . . 214

6.4.3. Fazit: Partikelbilduna aus der Alkenozonolyse . . . . . . . 216

6.5. Experimente unter Schwefeldioxidzugabe . . . . . . . . . . . . . 219

6.5.1. Cyclohexen . . . . . . . . . . . . . . . . . . . 222

6.5.2. Methylcyclopenten . . . . . . . . . . . . . . . . . . 226

6.5.3. Methylcyclohexen . . . . . . . . . . . . . . . . . . . . 227

6.5.4. $\alpha$-Pinen . . . . . . . . . . . . . . . . . . . . . . . . . . . . 228

6.5.5. Methylencyclohexan . . . . . . . . . . . . . . . . 231

6.5.6. $\beta$-Pinen . . . . . . . . . . . . . . . . . . . . . . 232

6.5.7. Auswirkungen des Gesamtdruckes in $\mathrm{SO}_{2}$-Anwesenheit . 233 
6.5.8. Einfluss durch Wasserzudabe . . . . . . . . . . . . . . . 242

6.6. Abschätzunq der Clusterstabilitäten . . . . . . . . . . . . . . 244

6.7. Partikelphasenanalyse . . . . . . . . . . . . . . . . . . . . . 249

6.7.1. Methode. . . . . . . . . . . . . . . . . . . . . 249

6.7.2. Analvseergebnisse . . . . . . . . . . . . . . . . 250

6.7.2.1. $\alpha$-Pinen . . . . . . . . . . . . . . . . 250

6.7.2.2. Butensäure . . . . . . . . . . . . . . . . . 252

7. Diskussion 257

7.1. Zentrale Rolle der $\mathrm{OH} / \mathrm{HO}_{2}$-Chemie? - Auswirkungen der Verwendung verschiedener Badgase . . . . . . . . . . . . . 257

7.1.1. Kinetikstudien . . . . . . . . . . . . . . . . . . 257

7.1.2. Produktbildungsstudien - Abhängigkeit von Gesamtdruck und Badgas . . . . . . . . . . . . . . . . . . . 262

7.2. Partikelbildungsmessungen . . . . . . . . . . . . . . 270

7.2.1. Beeinflussung der Intermediatchemie - Auswirkungen des Badgaswechsels . . . . . . . . . . . . . 272

7.2.2. Beeinflussung der Intermediatchemie durch die Struktur des Eduktes . . . . . . . . . . . . . . . 279 7.2.2.1. Auswirkungen der Vorfunktionalisierung der Edukte . . . . . . . . . . . . . . 279

7.2.2.2. Auswirkungen der Druckabhängigkeit - Die Rolle der CRIEGEE-Intermediate . . . . . . . . . . . 285

7.3. $\mathrm{SO}_{2}$-Experimente - Neue Ansatzpunkte durch Betrachtung des druckabhängigen Verhaltens? . . . . . . . . . . . . . . . . . 291

\begin{tabular}{ll}
\hline 8. Zusammenfassung 297 & 297
\end{tabular}

9. Ausblick 305

A. Anhana I

A.1. Verwendete Substanzen . . . . . . . . . . . . . . . I I

A.2. Experimentelle Bedinqungen . . . . . . . . . . . . . II

A.2.1. Kinetikmessungen . . . . . . . . . . . . . . . . . II

A.2.2. Produktbilduna ... . . . . . . . . . . . . . . . III

A.2.3. Partikelbildunasexperimente .............. IV

A.3. Geschwindiakeitskoeffizienten für die Ozonolvsereaktion . . . . . V V

A.3.1. Ergebnisse der einzelnen Kinetikmessungen . . . . . . . VIII

B. Kohlenstoffmonoxidausbeuten der einzelnen Experimente XI

C. Reinspektren der verwendeten Substanzen (Auswahl) XIII 
D. Eraänzende Abbildungen zu Produktbildungsstudien XV

D.1. 1-Hexen . . . . . . . . . . . . . . . . XV

D.2. 2-Methyl-1-penten . . . . . . . . . . . . . . . XVII

D.3. 3-Buten-1-0l . . . . . . . . . . . . . . . . . . XVIII

D.4. 1-Penten-3-0 . . . . . . . . . . . . . . . . . . . . . . XX

D.5. 4-Penten-1-0l . . . . . . . . . . . . . . . . . . . . . XXII

D.6. 1-Penten-3-on . . . . . . . . . . . . . . . . . . . . . XXIV

D.7. 4-Penten-1-al . . . . . . . . . . . . . . . . . . . . . . XXVI

D.8. Methacrvlsäure . . . . . . . . . . . . . . . XXVIII

D.9. Butensäure . . . . . . . . . . . . . . . XXX

E. Erqänzende Abbildunqen zur Partikelbildung - Butensäureozonolyse unter Zugabe verschiedener Additiva

XXXIII

F. Ergänzende Werte zu Clusterstabilitäten unter $\mathrm{SO}_{2}$-Beteiligung XXXV

$\begin{array}{ll}\text { G. Ergänzung zur UV-Analytik XXXVII } & \text { X }\end{array}$

H. Ergänzende Reaktionsmechanismen XXXIX

I. Potenzielle Folgereaktionen voroxidierter Edukte XLIII

I.1. Abfanareaktionen des CRIEGEE-Intermediates und gebildete Produkte als Gegenstand zukünftiger Untersuchungen . . . . . . XLIII

Literaturverzeichnis LXXI 



\section{Einleitung}

Das Verständnis klimaverändernder Prozesse ist eine der zentralen wissenschaftlichen Herausforderungen der Gegenwart, jedoch sind viele Aspekte dieses Themas noch unverstanden und mit großen Unsicherheiten behaftet.

Der Kohlenstoffkreislauf beinhaltet beispielsweise unter anderem die Aufnahme und Freisetzung gasförmiger Spezies, wie Kohlenstoffdioxid und Methan, bis hin zu biogenen volatilen und semivolatilen organischen Verbindungen. Während erstere ihren Einfluss vor allem auf das Strahlungsbudget der Erde ausüben, spielen letztere eine direkte Rolle in photochemischen Kreisläufen der Atmosphäre und bilden damit einen Teil des globalen Kohlenstoffzyklus. Durch die photochemische Oxidation dieser Spezies werden unter anderem troposphärisches Ozon und Sekundäres Organisches Aerosol (SOA) gebildet, wobei die Emission dieser Spezies in allen Arten von Ökosystemen, von jahrhundertealten Wäldern über Grasland bis hin zu städtischen Gegenden, stattfindet. Die Raten sind dabei abhängig vom Typ des emittierenden Systems (Ackerland, Wald, Grasland o.a.), der Gesundheit der Vegetation, Jahreszeit, Temperatur, Intensität der Sonneneinstrahlung und einigen anderen Umgebungsfaktoren. Gleichzeitig sind die Ergebnisse von Feldstudien für eine Abschätzung der emittierten Mengen stark mit Unsicherheiten behaftet, die ihren Ursprung unter anderem in der Messmethode bzw. des in den Untersuchungen gewählten Bezugspunktes haben (,leaf scale vs. tower based methods on canopy scale vs. landscape scale with baloon profiling or aircraft methods") [1].

Von neuen, die Luftqualität betreffenden gesetzlichen Regelungen über Maximalgrenzen von Ozon- und Feinstaubgehalt (z.B. US Ambient Air Quality Standards oder deutsche Feinstaubplaketten, die den Zugang zu verschiedenen Innenstädten regeln [2]) motiviert, wird die Untersuchung biogener volatiler organischer Substanzen (BVOC) immer weiter vorangetrieben und auf neue Ebenen gebracht. Dazu gehört die Identifikation neuer biogen emittierter Sub- 
stanzen, Messungen ihrer Emissions- und Reaktionsraten auf regionaler oder globaler Ebene, die Bestimmung der dabei entstehenden Reaktionsprodukte und der jeweiligen Ausbeuten.

Nach wie vor bleibt noch immer der atmosphärische Oxidationsmechanismus zu klären, insbesondere für Komponenten, deren Oxidation zu schwerflüchtigen und kondensationsfähigen Produkten führt. Die Charakterisierung des Aerosols bezüglich der entstehenden Ausbeuten und ihres molekularen Aufbaus ist eine Herausforderung für die aktuelle Forschung in diesem Gebiet [1].

Nachdem nachteilige Auswirkungen von aggressiven Spurengasen sowie Feinstaubpartikeln auf die menschliche Gesundheit entdeckt wurden, kam es zur Festlegung bestimmter primärer (für den Schutz der menschlichen Gesundheit) und sekundärer Grenzwerte (z.B. Schutz vor schlechter Sicht, Gesundheit von Tieren und Pflanzen) mit dem Ziel, bis zum Jahr 2020 mehr als 230000 vorzeitige Todesfälle zu verhindern. Diese Regelungen umfassen neben Ozon auch Kohlenstoffmonoxid, Bleistäube, Stickstoffdioxid und Schwefeldioxid, wobei verschiedene Maximalwerte je nach Expositionsdauer (Stunden- vs. Jahresmittel) definiert sind. In Bezug auf die Feinstaubbelastung (Particulate Matter - $P M)$ sind zwei Standards definiert, die sich auf den Größenbereich des Partikeldurchmessers beziehen. Damit wird der unterschiedlichen Art der Gefährdung Rechnung getragen, die aus der jeweiligen Partikelgröße resultiert [2]. Ein weiteres Problem ergibt sich jedoch aus der Tatsache, dass die Feinstaubbelastung massebasiert gemessen wird und Grenzwerte festgelegt sind, obwohl Studien darauf hinweisen, dass eher die Anzahl und Größe der Partikel, also eher die Oberfläche als die Masse, für die nachteiligen gesundheitlichen Folgen entscheidend sind [3].

Die Belastung durch partikuläre Materie, welche den Oberbegriff für extrem kleine, feste Partikel ebenso wie für flüssige Tröpfchen bildet, ist vor allem in einem Größenbereich unter $10 \mu \mathrm{m}$ problematisch, da diese die natürlichen Barrieren in Nase, Rachen und Bronchien überwinden und daher bis in die Lunge gelangen, wo sie vor allem bei Personen mit geschwächtem Immunsystem, wie älteren Menschen, Kindern oder Erwachsenen mit Vorerkrankungen wie Asthma, negative gesundheitliche Folgen haben können. Dabei kann es zu akuten Erkrankungen, wie entzündlichen Reaktionen oder Herz- und Lungenkrankheiten, wie auch zu Langzeitschäden, etwa einer dauerhaft reduzierten Lungen- 
funktion, chronischer Bronchitis und damit zu den bereits erwähnten vorzeitigen Todesfällen kommen.

Partikel unter $10 \mu \mathrm{m}$ werden noch einmal in zwei Unterklassen aufgeteilt, denen spezifische Gefährdungen zugeschrieben werden: Partikel mit einem aerodynamischen Durchmesser zwischen 2.5-10 $\mu \mathrm{m}$ (inhalable coarse particles) und Partikel einer Größe von unter $2.5 \mu \mathrm{m}$ (fine particles). Erstere werden vor allem durch Industrievorgänge, wie Abschleifprozesse oder das Aufwirbeln von Staub durch Straßenverkehr oder Wind freigesetzt. Fine particles sind so klein, dass sie bezüglich ihrer Oberflächenbeschaffenheit lediglich per Elektronenmikroskop nachgewiesen werden können. Als direkte Quellen kommen dabei alle Arten von Verbrennungsprozessen, einschließlich der in Automotoren, Energiekraftwerken, privater Holzverbrennung und Waldbränden sowie Brandrodungen in Frage. Es existieren für $\mathrm{PM}_{2.5}$-Partikel auch einige indirekte Quellen, da bei bestimmten Arten von Industrieanlagen sowie den genannten Verbrennungsprozessen Gase (z.B. $\mathrm{NO}_{x}, \mathrm{CO}, \mathrm{SO}_{2}$ ) emittiert werden, die in der Atmosphäre weiterreagieren und auf diese Weise über sekundäre Reaktionswege zur Partikelbildung führen können [2].

Seit mehr als 50 Jahren ist der Zusammenhang zwischen atmosphärischen Oxidationsmitteln [4], der Emmission von Kohlenwasserstoffverbindungen und der aus der Reaktion beider Partner resultierenden Partikelbildung bekannt und Gegenstand intensiver Forschung. Im Herbst 2011 lassen sich mit Google unter den Stichworten „particle formation, atmosphere“ über 3.44 Millionen Einträge im Internet finden, davon sind immerhin noch 661000 als wissenschaftliche Artikel gekennzeichnet. Wird diese Suche zusätzlich um "isoprene“, das atmoshärisch relevante und in großer Menge (600 Tg/Jahr [5]) emittierte Terpen, eingegrenzt, finden sich 24800 Treffer, von denen es sich bei 18600 um Beiträge aus wissenschaftlichen Zeitschriften handelt1.

Dieses Beispiel soll lediglich zeigen, wie intensiv gegenwärtig auf dem Gebiet der atmosphärischen Partikelbildung geforscht wird, allerdings auch die Problematik verdeutlichen. Aktuell konzentriert sich die Hauptrichtung in der Forschung auf atmosphärische Prozesse und versucht daher, diese Bedingungen möglichst genau zu imitieren, ein Bereich ist dabei die Ozonolyse von Koh-

\footnotetext{
${ }^{1}$ Eingesehen am 6. November 2011.
} 
lenwasserstoffen. Für diese Experimente werden riesige Forschungsreaktoren in Dimensionen eines Einfamilienhauses konstruiert und in Betrieb genommen. Beispiele dafür sind etwa die AIDA-Smog Chamber am KIT in Karlsruhe mit einem Volumen von $78 \mathrm{~m}^{3}$ oder auch die auf europäischer Ebene von $\mathrm{Zu}$ sammenschlüssen mehrerer Partnern im Rahmen des EUROCHAMP-Projekts konstruierte $200 \mathrm{~m}^{3}$ fassende EUPHORE- oder die sogar $270 \mathrm{~m}^{3}$ große $S A$ PHIR-Reaktionskammer2.

Ein Problem ließ sich allerdings trotz mit hohem experimentellen Aufwand betriebener Arbeit auf diesem Gebiet noch nicht lösen - niemand kann bisher den Prozess der ozonolyse- oder anderweitig induzierten Partikelneubildung geschlossen erklären, niemand konnte eine allgemeingültige Theorie aufstellen, die auf sämtliche, oder zumindest die wichtigsten, in der Atmosphäre vorkommenden Substanzen und Substanzklassen anwendbar ist. Stattdessen werden immer neue Hypothesen aufgestellt, die den Befund eines bestimmten Experimentes unter bestimmten Bedingungen mit einer bestimmten organischen Verbindung erklären sollen. Allerdings krankt dieses Vorgehen daran, dass durch die Simulation der sehr geringen atmosphärischen Konzentrationen an den Grenzen der Nachweisbarkeit von Edukten und Produkten gearbeitet werden muss. Hier können schon geringe prozentuale Abweichungen zu großen Diskrepanzen in den Ergebnissen führen, die aufgrund des oft singulären Charakters der Messungen nicht als eine zufällige Streuung der Anfangsbedingungen interpretiert werden. Auch die "Compound $X$ “ [6] ist ein beliebter Kandidat für die bisher noch offene Position der nukleationsinitiierenden Substanz, die eventuell für JEDE partikelbildende Reaktion verantwortlich sein kann (oder sein soll). Das zugrunde liegende Problem ist daher die Unkenntnis des genauen Mechanismus, der den entscheidenden Schritt zur Nukleation darstellt. Auch was "Compound $X$ " ist (oder ob sie überhaupt existiert) ist unbekannt, auch wenn einige Arbeitsgruppen die Stoffgruppe der Amine aufgrund von Feld- und Labormessungen für einen vielversprechenden Kandidaten halten [6].

Die Folge dieser Unkenntnis wirkt sich jedoch stark auf die Vorhersagegenauigkeit von Klimamodellen und damit der globalen Erwärmung aus. Partikel kön-

\footnotetext{
${ }^{2}$ Unter den genannten Namen lassen sich ebenfalls im Internet detaillierte Beschreibungen abrufen, daher wird hier auf eine Aufzählung der Webseiten verzichtet.
} 
nen das Klima auf verschiedene Arten beeinflussen. Sie können beispielsweise direkte Wirkungen haben, wie die durchmesserabhängige Absorption bzw. Streuung von Infrarotstrahlung, die von der Sonne auf die Erde trifft und zu einer Erwärmung oder, bei Verringerung der auftreffenden Strahlungsmenge durch abschirmende Prozesse, Abkühlung führen kann [7]. Problematisch ist der erste Effekt auch, wenn sich größere (Ruß-) Partikel auf Schnee- und Eisflächen niederschlagen, die absorbierte Wärmeenergie an diese abgeben und damit zu einer erhöhten Schmelze führen [8]. Allerdings überwiegen vermutlich die indirekten Effekte der Partikel, welchen eher abkühlende Effekte zugeschrieben werden. Aerosole mit einem Durchmesser oberhalb von 100 nm können als Wolkenkondensationskeime (cloud condensation nuclei - $C C N$ ) dienen. In einer übersättigten Wasserdampfumgebung induzieren sie die Kondensation der Wassermoleküle auf innen, wodurch die Wolkenbildung gefördert wird. Gleichzeitig beeinflussen sie auch die Eigenschaften der so gebildeten Wolken, indem sie durch das Angebot von vielen Kondensationskeimen die Größe der einzelnen Wassertröpfchen verringern, das Abregnen verzögern und damit die Lebensdauer der Wolken erhöhen [7].

Durch natürliche Nukleationsereignisse wird sowohl direkt wie auch indirekt Einfluss auf Wetter und Klima ausgeübt. Die Unsicherheit in den Vorhersagen von Nukleationsereignissen, ihrer Häufigkeit, Stärke und Lokalisierung hat daher direkte Auswirkung auf die aus diesen Daten erarbeiteten klimarelevanten Simulationen, z.B. die Wolkenbildung und das Albedo der Erde betreffend, wie Abbildung 1.1]zeigt.

Ein weiterer Nachteil der genannten Smog Chamber-Experimente ist die apparativ aufwendige und zeitaufwendige experimentelle Durchführung. Aufgrund der riesigen Volumina dauert die Durchführung eines Nukleationsexperimentes mit den nötigen Evakuierungs-, Befüllungs- und Durchmischungszyklen einen ganzen Tag oder länger, weshalb die erhaltenen experimentellen Ergebnisse sehr ausführlich bearbeitet und interpretiert werden müssen. Allerdings besitzen sie durch die nur schwer mögliche Reproduzierbarkeit der Bedingungen stets eher singulären Charakter, da eine Wiederholung der jeweiligen Experimente sehr aufwendig wäre und daher keine statistische Betrachtung der Messergebnisse möglich ist. Gleichzeitig macht dies eine Reproduktion der Daten nicht einfacher, da vor allem die Durchmischung der Reaktanden mit Propellern 


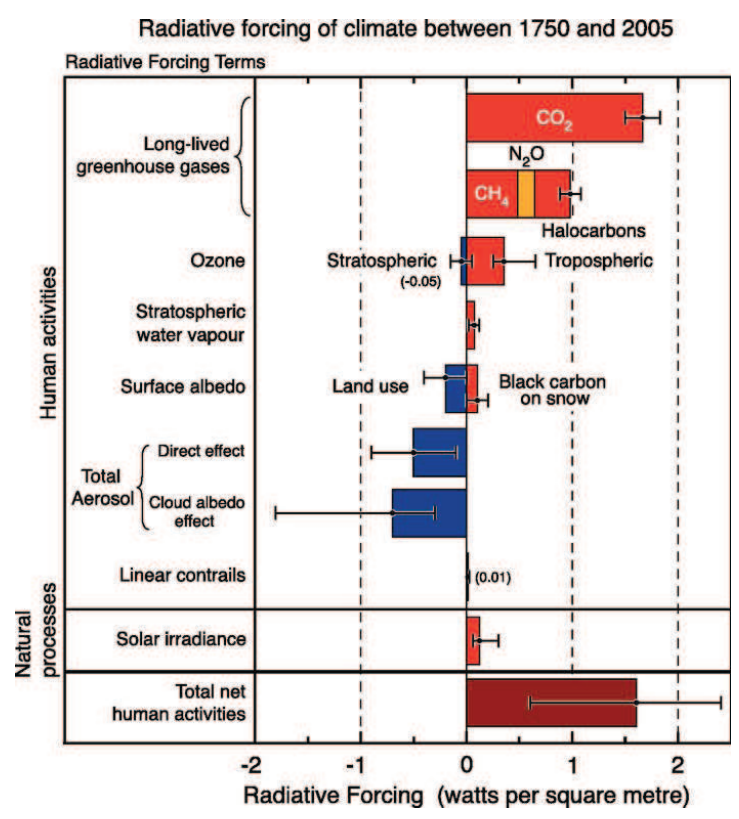

Abb. 1.1.: Verschiedene Effekte auf den vom IPCC eingeführten Strahlungsantrieb (radiative forcing) und ihre Unsicherheiten, entnommen aus dem aktuellen Bericht des Weltklimarates (IPCC - Intergovernmental Panel on Climate Change) [7].

oder ähnlichem nur bedingt kontrolliert erfolgen kann.

Die atmosphärische Partikelbildung kann durch verschiedene Prozesse verursacht werden, von denen ein Teil die atmosphärische Oxidation von biogen oder anthropogen emittierten ungesättigten Kohlenwasserstoffverbindungen darstellt. Die Hauptvertreter dieser oxidativen Substanzen sind neben Hydroxyl$(\mathrm{HO})$ und Nitrat-Radikalen $\left(\mathrm{NO}_{3}\right)$ auch das Spurengas Ozon $\left(\mathrm{O}_{3}\right)$. Durch Oxidation der organischen Verbindungen wird über meist mehrere Zwischenschritte die Flüchtigkeit der Produkte so weit herabgesetzt, dass ab einem bestimmten Punkt die Nukleation einsetzt [9]. Neben diesen, auf organische Komponenten zurückführbaren Vorgängen der Aerosolbildung sind auch solche unter ausschließlicher Beteiligung anorganischer Verbindungen bekannt. Vertreter sind beispielsweise die binäre (Schwefelsäure / Wasser [10]) oder ternäre (Schwefelsäure / Wasser / Ammoniak [10]) Nukleation. Auch eine ioneninduzierte Partikelbildung [10] oder eine durch lodoxide und Halogenverbindungen verursachte Aerosolbildung [11] konnte bereits nachgewiesen werden. Zusätzlich wird auch über die Möglichkeit einer Partikelbildung aus der Reaktion von organischen 
Verbindungen und Schwefelsäure diskutiert [12].

Neuere Arbeiten erörtern teilweise sehr kontrovers, welche Rolle dem organischen und dem anorganischen Anteil der Partikelbildung für die Keimproduktion, die die Partikelanzahl in einem betrachteten Volumen determiniert, und dem Masseaufbau, als Maß für die Partikelgröße, zukommen. DoNAHUE et al. [13] kommen in ihren neuesten Arbeiten zu dem Schluss, dass Schwefelsäure und aus oder mit inr gebildete Cluster sich zwar positiv auf die Aerosolbildung auswirken können, der Hauptteil des Partikelwachstums jedoch auf Kondensationsprozesse mit organischen Verbindungen, etwa oxidierten Ozonolyseprodukten, zurückzuführen ist.

Der in der hier vorgelegten Arbeit verfolgte Ansatz ist in Kenntnis der Problematik bei Experimenten in Rauchkammern ein anderer. Er schließt aus den Vorgängen in der Gasphase, charakterisiert durch die Kinetik und Produktbildung, auf die Entwicklung der Partikelphase. Statt eine Simulation der realen Atmosphäre anzustreben, werden hier ausdrücklich als solche zu verstehende Modellexperimente durchgeführt, in denen nur in einigen Fällen atmosphärenrelevante Konzentrationen oder Substanzen verwendet werden. Die unter vergleichsweise hohen Anfangskonzentrationen erhaltenen Ergebnisse sollen einen Teil zur Grundlagenforschung beitragen, um möglicherweise die entscheidenden, zur Partikelbildung führenden Vorgänge der Ozonolysereaktion zu identifizieren.

Der Untersuchung der Ozonolysereaktion wurde der Vorzug gegenüber anderen möglichen zuvor beschriebenen Varianten gegeben, da sie im Vergleich mit anderen nukleationsinduzierenden Prozessen mit recht einfachen Mitteln unter kontrollierten Bedingungen durchführbar ist (vgl. Abschnitt 3 ) und Ozon außerdem das in der höchsten Konzentration vorliegende der zuvor genannten Spurengase ist [14].

Der Vorteil in der eingesetzten, lediglich $64 \mathrm{~L}$ fassenden Reaktionszelle liegt aufgrund ihrer Konstruktion in der Möglichkeit, die Reaktionsbedingungen sehr präzise kontrollieren zu können. Dies betrifft vor allem die Expansionsbedingungen, die Durchmischung der Reaktanden und die Druckvariation - Parameter, die sich als kritische Punkte innerhalb des Nukleationsprozesses herausgestellt haben, wie sich aus Versuchen mit einer Vorgängerapparatur ergab [15, 16]. 
Oxidierte Reaktanden: Variation von Oxidationsgrad und Abstand der Funktionalitäten

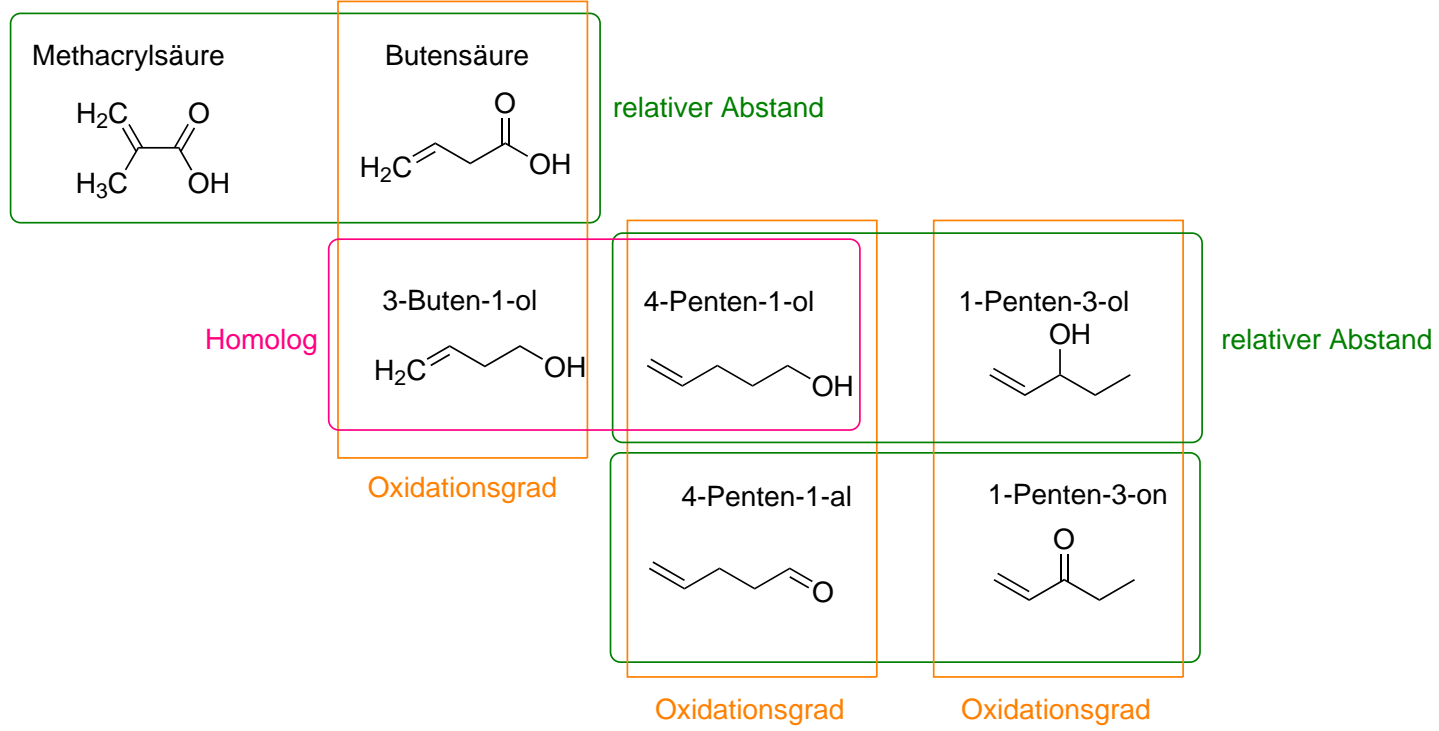

Abb. 1.2.: Oxidierte Komponenten und die betrachteten Abhängigkeiten im Reaktionsverhalten.

Um die genannten kritischen Punkte identifizieren und in ihrer Wirkung charakterisieren zu können, bildet die gezielte Variation verschiedener Parameter den Kernpunkt dieser Arbeit. Zum einen gehört dazu die Auswahl der verwendeten Edukte. Diese umfasst ...

- die Verwendung von Substanzen mit verschiedenartigen funktionellen Gruppen (ungesättigte Carbonsäuren, Carbonylverbindungen, Alkohole), um die Auswirkungen des Oxidationsgrades einer zusätzlichen funktionellen Gruppe im Alken zu analysieren (Abb. 1.2, senkrecht).

- die Variation des relativen Abstandes zwischen den funktionellen Gruppen, um die Auswirkungen auf das Partikelbildungsvermögen und die gebildeten Gasphasenprodukte zu betrachten (Abb. 1.2, waagerecht).

- die Verwendung reiner Kohlenwasserstoffverbindungen 3 unter Betrachtung der Auswirkungen auf Partikelausbeuten und Reaktionsprodukte, um durch die Veränderung des reaktiven Zentrums zur Aufklärung verschie-

${ }^{3}$ Dieser Begriff wird im Folgenden für die nicht voroxidierten Komponenten verwendet, um sie von den sauerstoffhaltigen Edukten abzugrenzen. 
Reine Kohlenwasserstoffe: Variation des reaktiven Zentrums

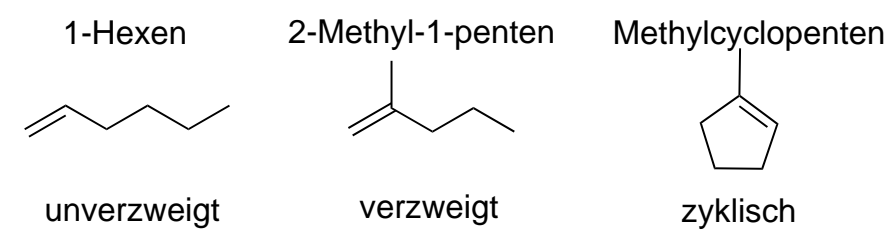

Abb. 1.3.: Reine Kohlenwasserstoffverbindungen und Darstellung der untersuchten Abhängigkeiten.

dener Reaktionskanäle und Verzweigungen innerhalb des Mechanismus zu gelangen (Abb. 1.3).

Zum anderen wird die Reaktionsumgebung von außen beeinflusst. Dies geschieht anhand ...

- der Durchführung der Experimente bei verschiedenen Gesamtdrücken, da die Wahrscheinlichkeit der Stabilisierung energiereicher Zwischenprodukte mit zunehmendem Druck und der daraus resultierenden steigenden Häufigkeit desaktivierender Stöße mit Badgasmolekülen wächst. Es wird i.d.R. der Druckbereich von 30 mbar bis 1000 mbar untersucht, die Variation beträgt also mehr als einen Faktor 30.

- der Verwendung verschiedener Badgaszusammensetzungen, da durch Variation des Sauerstoffgehaltes die Beschreitung bestimmter Reaktionskanäle bevorzugt oder unterdrückt werden kann (s. Abb. 1.4).

Eine entscheidende Weiche im Partikelbildungsprozess stellt nach Untersuchungen zahlreicher Arbeitsgruppen [12, 17.-21] der Vinylhydroperoxidkanal4 dar, über den die in der Ozonolyse entstehenden Intermediate zum Teil zerfallen können. Auf den Zerfall über diesen Reaktionskanal kann, wie in Abb. 1.4 schematisch dargestellt, über Variation des Druckes (Beeinflussung der Intermediatstabilität) und die Wahl der Eduktstruktur oder des Badgases Einfluss (Beeinflussung der Peroxy- / Alkoxychemie) genommen werden. Während die Vorgänge zwischen der Bildung des CRIEGEE-Intermediates in der Ozonolysereaktion und des Eintritts in die Peroxy- / Alkoxychemie recht gut

\footnotetext{
${ }^{4}$ Die hier angesprochenen Prozesse werden ab Kapitel2.2.2.1 ausführlich beschrieben.
} 


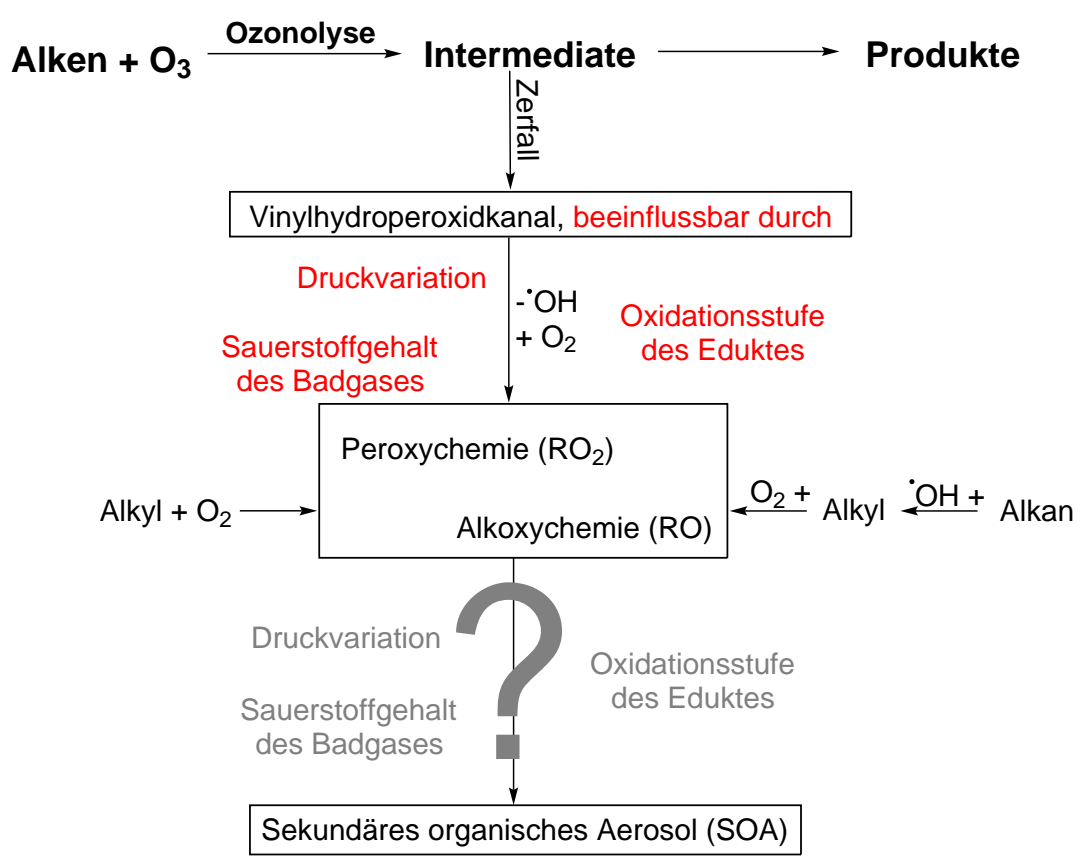

Abb. 1.4.: Einflussmöglichkeiten auf (möglicherweise) entscheidende Schritte im Partikelbildungsprozess.

verstanden sind, bleiben in den Schritten von diesem Punkt bis zur Bildung von sekundärem organischen Aerosol noch einige offene Fragen zu klären. Dies soll durch gezielte Beeinflussung der intermediären Chemie erreicht werden. Dieser Reaktionspfad besitzt wegen des natürlichen Vorkommens von $\mathrm{OH}$ Radikalen auch Bedeutung für die atmosphärischen Vorgänge.

Schließlich werden auch einige atmosphärenrelevante Partikelbildungsexperimente durchgeführt, um zu überprüfen, ob die aus den Modellexperimenten gezogenen Schlüsse auch für biogene Verbindungen anwendbar sind. Bei den in Abbildung $1.5 \mathrm{im}$ oberen Teil gezeigten Verbindungen handelt es sich um häufig in Untersuchungen zur Ozonolyse als Modellsubstanzen verwendete Strukturanaloga zu den unten dargestellten biogen emittierten Terpenen $\alpha$ - und $\beta$-Pinen, da sie das gleiche Strukturmotiv am reaktiven Zentrum zeigen.

Mit diesen Substanzen werden vor allem die Auswirkungen von $\mathrm{SO}_{2}{ }^{-}$ Zugaben und ihrer Variation auf Partikel- und Produktbildung während der Ozonolyse untersucht. Dieses Thema wird schon länger in verschiedenen Arbeitsgruppen bearbeitet. Wegen des hohen Nukleationsvermögens in während der 
Biogen emittierte Terpene und zugehörige Modellsubstanzen

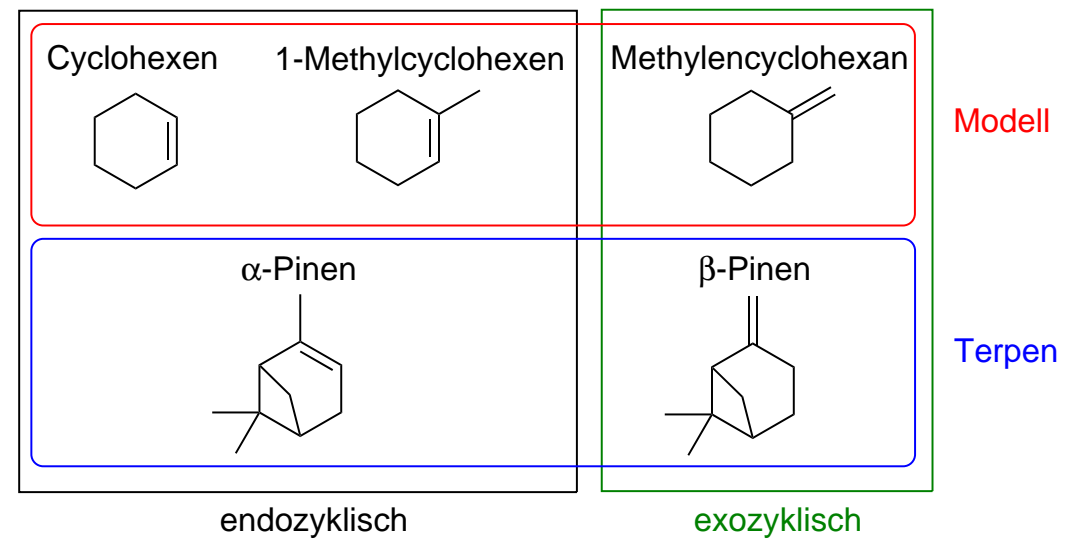

Abb. 1.5.: Reine, biogen emittierte Kohlenwasserstoffverbindungen und zugehörige Modellsubstanzen.

Ozonolyse aus Schwefeldioxid entstehender Schwefelsäure und ihres atmosphärischen Vorkommens (bekannt z.B. aus Verbrennung fossiler Brennstoffe und dem daraus folgenden Auftreten sauren Regens) wurde dieser Säure z.T. schon die Rolle der "Compound $X$ " zugeschrieben, was allerdings in letzter Zeit zunehmend bezweifelt wird [22].

Wegen des hohen Interesses am Schwefelsäureeinfluss wird daher durch Variation der zugegebenen $\mathrm{SO}_{2}$-Partialdrücke, als Unterthema der schon genannten Badgasvariation, untersucht, ob die (anorganische) Schwefelsäurebildung oder die (organische) Nukleation durch die Alken-Ozon-Reaktion die Partikelausbeuten dominiert. Zudem werden auch hier Produktstudien durchgeführt, um festzustellen, ob exo- und endozyklische Alkene unterschiedlich auf $\mathrm{SO}_{2}$-Anwesenheit reagieren. Von besonderem Interesse ist auch in diesem Themenkomplex die Auswirkung des Gesamtdruckes.

In den folgenden Abschnitten werden zunächst die theoretischen Grundlagen zu den Untersuchungen dargestellt (Kapitel 2), die sich mit verschiedenen Aspekten der Alkenozonolyse beschäftigen. Kapitel 3 behandelt im Anschluss daran den experimentellen Aufbau und beschreibt das Vorgehen bei den verschiedenen Experimentvarianten. Aufgrund der Diversität der verwendeten organischen Edukte und den daraus folgenden unterschiedlichen Eigenschaften 
wird in Kapitel 4 der Konzentrationsbestimmung der einzelnen Verbindungen ein eigener Abschnitt gewidmet, bevor in Kapitel[5] eine Beschreibung der Auswertungsmethoden erfolgt. Das Kernstück der hier vorliegenden Arbeit bildet Kapitel 6, in dem die Darstellung der Ergebnisse der verschiedenen Experimente zu Kinetik, Produkt- und Partikelbildung erfolgt. Um für den Leser trotz des großen Umfanges und der Verschiedenartigkeit der untersuchten Edukte und Aspekte dieser Daten ein möglichest hohes Maß an Übersichtlichkeit zu erhalten, erfolgen Teile der Interpretation sowie des Vergleichs mit Literaturstudien bereits am Ende der jeweiligen Auswertungskapitel. Eine globale Zusammenführung erfolgt anschließend in der Diskussion in Kapitel 7, bevor mit Zusammenfassung (Kap. 8) und Ausblick (Kap. 9) die Arbeit beendet wird. 


\section{Theorie}

\subsection{Spektroskopische Grundlagen}

Spektroskopie ist abgeleitet von lat. spectrum für Bild, Erscheinung und bezeichnet die Lehre von Erzeugung, Beobachtung, Registrierung, Ausmessung sowie Deutung von Spektren. Ein Spektrum ist dabei die Bezeichnung für jede Anordnung von Dingen oder Eigenschaften nach ihrer Größe. Die Darstellung von elektromagnetischer Strahlung kann z.B. in Abhängigkeit von deren Wellenlänge, Schwingungsfrequenz oder Energie erfolgen ( [23], S. 4231).

Die Spektroskopie ist ein experimentelles Verfahren, das anhand der Aufnahme und Auswertung eines Spektums („Farbzerlegung“) von Lichtquellen untersucht, wie Materie und elektromagnetische Strahlung miteinander wechselwirken. Die Deutung der Spektren und ihrer Feinstruktur erfolgt mit Mitteln der Quantenmechanik und der theoretischen Chemie. Durch Absorption, Emission oder Streuung elektromagnetischer Strahlung an Atomen, Molekülen, Ionen und Radikalen kann die Charakterisierung dieser Verbindungen erfolgen. So kann die Konstitution einzelner Spezies ermittelt, ihr Auftreten nachgewiesen und ihre Eigenschaften bestimmt werden ( [23], ebd.). Die Methode ist grundsätzlich unabhängig davon einsetzbar, ob die Atome und Moleküle in der Gasphase, flüssig, als Festkörper oder an einem Festkörper adsorbiert vorliegen ([24], S. 1).

In den im Rahmen dieser Arbeit durchgeführten Experimenten wurde die FTIR-Spektroskopie benutzt, um die Konzentrationen der Reaktanden und die erhaltenen Produkte sowie deren Beschaffenheit zu charakterisieren. Die Grundlagen dieser Methode werden im Folgenden beschrieben 1 .

${ }^{1}$ Eine ausführliche Einführung in die Thematik bieten z.B. BANWELL und MCCASH [25] sowie HOLLAS [24]. Die folgenden Ausführungen orientieren sich hauptsächlich an diesen. 


\subsubsection{Welle-Korpuskel-Dualismus}

Das Spektrum elektromagnetischer Strahlung erstreckt sich über einen Bereich von Radiowellen $\left(\lambda=10^{3} \mathrm{~m}\right)$ bis zur Gammastrahlung $\left(\lambda=10^{-12} \mathrm{~m}\right)$ (u.a. [24], S. 1). Das für den Menschen sichtbare Licht umfasst lediglich den Wellenlängenbereich zwischen $380 \mathrm{~nm}$ und $780 \mathrm{~nm}$ ( [26], S. 2449). $\lambda$ [m] bezeichnet dabei die Wellenlänge, die der Abstand zweier benachbarter Wellenorte gleicher Schwingungsphase ist. Sie berechnet sich aus dem Quotienten von Lichtgeschwindigkeit $c$ (ca. 2,998 $\cdot 10^{8} \mathrm{~m} / \mathrm{s}$ im Vakuum) und Schwingungsfrequenz $\nu$ $\left[\mathrm{Hz}=\mathrm{s}^{-1}\right]$ ( [24], S. 37):

$$
\lambda=\frac{c}{\nu}
$$

Eine für die Spektroskopie wichtige Größe ist weiterhin die Wellenzahl $\tilde{\nu}$ $\left[\mathrm{cm}^{-1}\right]$, die als Kehrwert der Wellenlänge die Anzahl von Schwingungen pro einer bestimmten Länge (üblicherweise Zentimeter) angibt. Sie ist proportional zur Energie einer Welle.

$$
\tilde{\nu}=\frac{1}{\lambda}
$$

Die Definitionen der klassischen Physik behandeln das Licht ausschließlich als Welle. Je kürzer jedoch die Wellenlänge der Strahlung durch einen zunehmenden Impuls wird (de Broglie-Wellenlänge), desto mehr ähneln die Eigenschaften der Wellenzüge denen eines bewegten Teilchens (Welle-KorpuskelDualismus). Die Gesetzmäßigkeiten, die das Licht ausschließlich als Welle betrachten, waren mit wachsenden technischen Möglichkeiten Anfang des 20. Jahrhunderts schließlich nicht mit experimentellen Befunden in Einklang zu bringen? 2 .

Dieser Widerspruch wurde mit der Postulierung der Strahlungsgleichung am Schwarzen Strahler durch MAX PLANCK aufgelöst, nach der die Energie auf diskrete Mengen (Quanten) beschränkt wird. Die Energie eines Ozillators der

\footnotetext{
${ }^{2} \mathrm{Vgl}$. etwa die ausbleibende UV-Katastrophe, die aus dem RAYLEIGH-JEANS-Gesetz folgen würde ( [27], S. 338f.).
} 
Frequenz $\nu$ kann unter Verwendung der Fundamentalkonstanten $h=6.62608$. $10^{-34} \mathrm{Js}$ (PLANCK'sches Wirkungsquantum) berechnet werden:

$$
E_{\text {Photon }}=h \cdot \nu
$$

Auch von Albert EINSTEIn wurde das Licht als Korpuskel, Photonen genannt, interpretiert. Die Definition der Lichtintensität $\left[\frac{\mathrm{J}}{\mathrm{m}^{2} \cdot \mathrm{s}}\right]$ ergibt sich aus der Gesamtenergie $E$ (Produkt aus Anzahl der Photonen und Energie der einzelnen Photonen), die bei Bestrahlung mit Licht pro Zeiteinheit $t$ auf eine Fläche $A_{F}$ fällli , in Verbindung mit Gl. 2.3. [ [28], S. 5).

$$
\begin{aligned}
I & =\frac{E}{A_{\mathrm{F}} \cdot t} \\
& =\frac{n_{\text {Photonen }} \cdot h \cdot \nu_{\text {Photon }}}{A_{\mathrm{F}} \cdot t}
\end{aligned}
$$

\subsubsection{Schwingungsspektroskopie}

Wie bereits der Begriff der elektromagnetischen Strahlung nahelegt, enthält diese Art Strahlung einen elektrischen $(\vec{E})$ und einen magnetischen Anteil $(\vec{H})$, die beiden Felder stehen orthogonal zueinander. Elektromagnetische Strahlung wird durch die periodische Änderung eines Dipolmoments erzeugt, wobei die Frequenz der des oszillierenden Dipols entspricht.

Wie beschrieben, benutzt die Schwingungsspektroskopie im IR-Bereich die Eigenschaft von Materie, mit elektromagnetischer Strahlung zu wechselwirken, indem sie diese beispielsweise absorbiert. Dazu müssen verschiedene Voraussetzungen erfüllt sein. Zunächst ist es notwendig, dass sich ein Dipolmoment ausbilden kann. Licht kann lediglich absorbiert werden, wenn es mit einem in gleicher Frequenz oszillierenden Dipol in Wechselwirkung tritt, dessen Energieniveaus nach PLANCK ebenfalls gequantelt sind (s. Gl. 2.6, nach [24], S. 4f.).

\footnotetext{
${ }^{3} A$ für die Fläche wird mit dem Index $F$ versehen, um eine Verwechslung mit der Absorbanz A zu vermeiden.
} 


$$
E=n \cdot h \cdot \nu \text { mit } n=0,1,2, \ldots
$$

Nur in diesem Fall kann ein Photon seine gesamte Energie als Quant an den Oszillator abgeben und diesen in einen energetisch angeregten Zustand überführen. Durch thermische Relaxation, d.h. Stöße mit Nachbarmolekülen, bei denen Überschussenergie in Form von kinetischer Energie übertragen wird, kann das Molekül wieder in seinen Grundzustand zurückkehren. Dieser Effekt ist ebenfalls durch spontane Emission möglich, bei der Strahlung diffus in alle Raumrichtungen ausgesendet wird ( [25], S. 13ff.).

Beispielsweise im Falle eines zweiatomigen heteronuklearen Moleküls kann sich ein Dipol ausbilden, sofern sich die Elektronegativitäten der Atome genug unterscheiden. Eine durch Schwingung verursachte periodische Änderung des Dipolmoments kann beispielsweise durch IR-Strahlung angeregt werden. Die Bindungsstärke eines Moleküls beschreibt in diesem Fall gleichzeitig die Stärke der Anziehungskräfte zwischen den beiden entgegengesetzten Ladungen eines Dipols. In der klassischen Physik wird die Molekülschwingung beschrieben durch die Theorie des harmonischen Oszillators. Diese wird unter der Annahme abgeleitet, dass die Vibrationsbewegung dem HoOKE'schen Gesetz (GI. 2.7) gehorcht. Dieses sagt aus, dass die Rückstellkraft $F$ proportional zur Auslenkung vom Gleichgewichtsabstand $\left(r-r_{\mathrm{e}}\right)$ ist, $k$ ist dabei eine (molekülspezifische) Proportionalitätskonstante (Kraftkonstante $k>0$ ).

$$
F=-k \cdot\left(r-r_{e}\right)
$$

Die Potentialkurve wird durch eine Parabel dargestellt und die Quantelung der Schwingungsenergie nach PLANCK liefert durch Lösung der SchrödingerGleichung für den harmonischen Oszillator äquidistante Energieniveaus nach folgender Gleichung:

$$
E_{\mathrm{v}}=h \cdot \nu_{0} \cdot\left(v+\frac{1}{2}\right)
$$


$v$ steht in dieser Gleichung für die Schwingungsquantenzahl, die ganzzahlige Werte ab Null annehmen kann, $\nu_{0}[\mathrm{~Hz}]$ für die Eigenschwingungsfrequenz des Oszillators ( [24], S. 23).

Nach dieser Interpretation der Schwingung ist es unmöglich, dass ein Molekül dissoziiert, da die potentielle Energie für große Abstände ins Unendliche läuft. Da in der Realität der Bindungsbruch bei zu großer Auslenkung jedoch stattfindet, kann die Gesetzmäßigkeit von HOOKE nur bei geringen Auslenkungen vom Gleichgewichtsabstand als gültig angesehen werden.

Die dem tatsächlichen Verhalten eines Moleküls eher entsprechende Beschreibung liefert das Bild des anharmonischen Oszillators. Da die Federkonstante $k$ bei der Dissoziation (d.h. großen Abständen $r$ ) in zwei neutrale und sich daher nicht gegenseitig beeinflussende Atome Null wird, kann $r$ ohne weitere Änderung der potenziellen Energie unendlich groß werden. Daher muss die Potentialkurve bei $r \rightarrow \infty$ gegen einen Grenzwert laufen, diese Asymptote entspricht der Dissoziationsenergie $D_{\mathrm{e}}$. In der Nähe der Dissoziationsgrenze strebt $k$ gegen Null, die Bindung wird schwächer und die Kurve verläuft im Vergleich zur Potentialkurve des harmonischen Oszillators flacher. Bei einem Abstand der Kerne, der kleiner als der Gleichgewichtsabstand ist, verläuft die Kurve des anharmonischen Oszillators steiler, weil die Abstoßung der positiv geladenen Atomkerne eines Moleküls oder die negativ geladenen inneren Schalen eine weitere Annäherung verhindern. Das Morse-Potential $V(x)$ (Gl. 2.9. nach [24], S. 134) ist ein Beispiel für den Verlauf der Potentialkurve eines anharmonischen Oszillators, der jedoch für kleine Molekülabstände keine realen Ergebnisse liefert. Aus inm folgt weiterhin, dass die gequantelten Energieniveaus nicht äquidistant sind, sondern mit zunehmender Quantenzahl $v$ in der Nähe der Dissoziationsenergie zusammenrücken.

$$
V(x)=D_{\mathrm{e}} \cdot[1-\exp (-a x)]^{2}
$$

$x=r-r_{\mathrm{e}}$ steht dabei für die Auslenkung, $a$ und $D_{\mathrm{e}}$ sind charakteristische Konstanten für einen bestimmten elektronischen Zustand eines Moleküls.

Während für den harmonischen Oszillator (ohne Berücksichtigung der elektrischen Anharmonizität) lediglich Schwingungsanregungen nach der Auswahl- 
regel $\Delta v= \pm 1$ erlaubt sind, sind für den anharmonischen Oszillator auch Übergänge mit $\Delta v= \pm 2, \pm 3 \ldots$ möglich, welche als Obertonschwingungen bezeichnet werden.

\subsubsection{FTIR-Spektroskopie}

Allgemein kann die Lage eines Moleküls aus $N$ Atomen im Raum durch drei Raumkoordinaten pro Atom beschrieben werden, es besitzt somit $3 N$ Freiheitsgrade. Da für die Translations- und Rotationsbewegung jeweils 3 Freiheitsgrade für jede Raumachse abgezogen werden müssen, bleiben 3N-6 Freiheitsgrade für die Schwingungsbewegung bestehen. Sofern ein betrachtetes Molekül linear ist und bei der Rotation um die Bindungsachse nahezu kein Trägheitsmoment besitzt, entfällt dieser Freiheitsgrad und es sind 3N-5 Grundschwingungen möglich ( [25], S. 94). Schwingungen entlang einer Bindungsachse werden als Valenz- oder Streckschwingungen bezeichnet, bei Deformationsoder Knickschwingungen handelt es sich um Schwingungen, bei denen sich der Bindungswinkel ändert. In beiden Fällen handelt es sich um so genannte Normalschwingungsmoden, bei denen alle Atome des bewegten Moleküls in derselben Frequenz schwingen und gleichzeitig ihre Ruhelage durchlaufen ( [25], S.95f.).

Mit wachsender Atomzahl nimmt auch die Zahl der möglichen Schwingungen zu, weshalb hochmolekulare Verbindungen komplexe Spektren aufweisen, die in festgelegten Bereichen als Gesamtbild für bestimmte Strukturen spezifisch sind (Fingerprintbereich, bei C-C-Gerüstschwingungen z.B. im Bereich von $700-1400 \mathrm{~cm}^{-1}$ ). Funktionelle Gruppen zeigen unabhängig von der Gesamtstruktur einer bestimmten Substanz stets eine oder mehrere charakteristische Banden im selben Frequenzbereich, die so genannten charakteristischen Gruppenfrequenzen ( [25], S. 110ff.). Somit ist es möglich, Rückschlüsse auf die Struktur einer unbekannten Substanz zu ziehen und in Verbindung mit weiteren analytischen Verfahren wie der NMR-Spektroskopie oder Massenspektrometrie eine Strukturaufklärung zu erreichen.

Die Absorptionsspektroskopie weist in der Praxis den Mangel auf, dass durch die Aufspaltung in die einzelnen Frequenzen an einem Gitter der Lichtstrahl nur in abgeschwächter Form bei Probe bzw. Detektor angelangt. Zudem muss 
durch sukzessive Verstellung des Gitters oder Bewegung des Detektors jede Wellenlänge einzeln durchgescannt werden, was die Aufnahme eines Spektrums zeitlich ausdehnt. Es bietet also Vorteile, wenn gleichzeitig die gesamte Lichtintensität aller sich überlagernder Wellen auf eine Probe trifft und auch detektiert werden kann. Die von der Strahlungsquelle ausgesendete Strahlung besteht aus vielen sich überlagernden Wellenlängen, die von der Kreisfrequenz $\omega_{1}$ und Zeit $t$ abhängt. Die allgemeine Darstellung einer Schwingung $S_{1}$ in Form einer Cosinusfunktion ergibt sich dabei als $S_{1}=A_{1} \cdot \cos \left(\omega_{1} \cdot t\right)$, wobei $A_{1}$ die maximale Amplitude ist. Da die verschiedenen Frequenzen des Lichts damit jedoch auch gleichzeitig verschiedene Schwingungszustände beeinflussen können, ist das sich ergebende Gesamtbild in einem Spektrum nicht sofort eindeutig einer bestimmten Anregung zuzuordnen. Abhilfe bietet die so genannte FourierTransformations-Spektroskopie, die auch in der vorliegenden Arbeit angewandt wird. Diese Methode ermöglicht, die einzelnen Intensitäten und Frequenzen einer Schwingung zu ermitteln. Zu den genaueren mathematischen Zusammenhängen sei auf die Arbeiten von NOTHDURFT [29] und dort zitierten Arbeiten sowie BANWELL [25], S. 26ff., und HolLAS [24], S. 45ff., verwiesen.

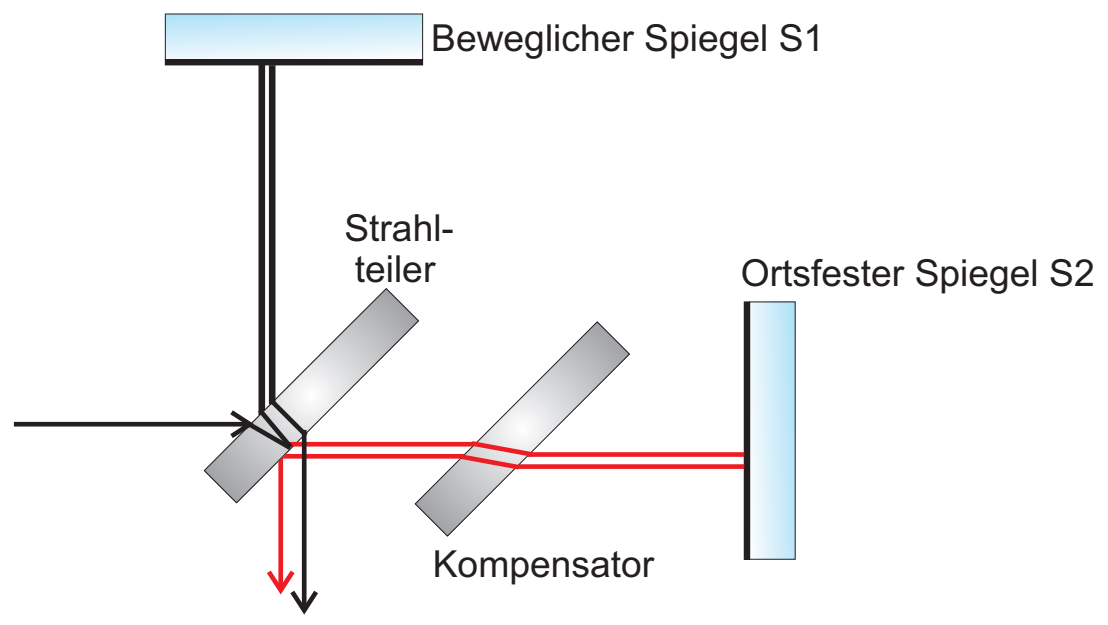

Abb. 2.1.: Schematischer Aufbau eines MICHELSON-Interferometers, nach [27], S. 512.

Während es für die Spektroskopie in niederfrequenten Bereichen unter $100 \mathrm{GHz}$ Detektoren mit kurzen Reaktionszeiten gibt, so dass Spektren in Abhängigkeit von der Zeit aufgenommen werden können, bietet sich im Bereich von IR- und UV / VIS-Strahlung $(\nu>600 \mathrm{GHz})$ eher eine Auftragung gegen 
die Frequenz an. Dazu wird ein Michelson-Interferometer wie in Abb. 2.1 verwendet, durch welches das Spektrum direkt in der Längendomäne gemessen werden kann ( [24], S. 49f.).

Das Interferometer besteht aus einem festen (S2) und einem beweglichen Spiegel (S1) ebenso wie einem Strahlteiler aus einem geeigneten lichtdurchlässigen Material, das so beschichtet ist, dass die Hälfte der einfallenden Strahlung reflektiert wird ( [25], S. 123f.). Trifft ein monochromatischer Strahl auf den Strahlteiler, wird ein Teil durch inn auf den beweglichen Spiegel und von dort aus zurück durch den Strahlteiler auf den Detektor gelenkt. Der zweite Teilstrahl wird auf den festen Spiegel geworfen und anschließend wiederum auf den Strahlteiler, wo er erneut geteilt wird. Ein Teil wird dabei auf den Detektor abgebildet. Erreichen die Strahlen den Detektor, haben sie zwei verschiedene optische Wege mit der Differenz $\Delta s$ zurückgelegt, die von der Stellung des beweglichen Spiegels abhängig ist. Ist diese Differenz exakt Null oder ein ganzzahliges Vielfaches der Wellenlänge $(\Delta s=n \cdot \lambda, n=0,1,2 .$.$) , kommt es zu$ konstruktiver Interferenz, d.h. die Amplitudenmaxima werden addiert. Von destruktiver Interferenz wird gesprochen, wenn sich die Teilstrahlen auslöschen. Sie liegt vor bei $\Delta s=(2 n+1) \cdot \frac{\lambda}{2}$ ( [30], S. 2772). Ein im Aufbau enthaltener Kompensator stellt dabei sicher, dass die Lichtstrahlen den gleichen Weg durch das Material des Strahlteilers zurücklegen.

Nach Fouriertransformation des Interferogramms aus der Längen- in die Wellenzahldomäne resultiert ein Spektrum, in dem die Intensitäten in Abhängigkeit der Frequenz aufgetragen sind.

\subsubsection{LAMBERT-BEERsches Gesetz}

In der Praxis können weder Emission der Strahlungsquelle noch Empfindlichkeit des Detektors als absolut konstant angenommen werden. Die von einer in den Strahlengang eingebrachten Probe herbeigeführte Absorption wird daher durch den Vergleich des Intensitätsspektrums I mit dem Intensitätsspektrum $I_{0}$ des reinen Signalwegs bestimmt. Durch Bildung des Quotienten wird ein Transmissionsspektrum erhalten, wobei sich die Transmission $T$ in die dekadische Absorbanz A umrechnen lässt. 


$$
\begin{aligned}
T & :=\frac{l}{I_{0}} \\
A & :=-\lg (T)
\end{aligned}
$$

Über den experimentellen Befund, dass die Intensitätsänderung $d /$ eines Lichtstrahls beim Durchgang durch eine Probe proportional zur Konzentration $c$ selbiger, der Intensität / der Lichtquelle sowie der Änderung der vom Lichtstrahl durch die Probe zurückgelegten Weglänge $\mathrm{d} L$ ist, ergibt sich nach Umformung das Lambert-BeERSche GeSeTZ (GI. 2.14, [27], S. 514f.).

$$
\begin{aligned}
\mathrm{d} l & =-\kappa \cdot c \cdot l \cdot \mathrm{d} L \\
\epsilon & :=\frac{\kappa}{\ln (10)} \\
\Rightarrow A & =\epsilon \cdot c \cdot l
\end{aligned}
$$

In diesen Gleichungen stellen sowohl $\kappa$ als auch $\epsilon$ stoffspezifische Konstanten dar. Im ersten Fall handelt es sich um den natürlichen molaren Absorptionskoeffizienten $\left[\frac{\mathrm{m}^{2}}{\mathrm{~mol}}\right]$, letzterer ist der dekadische molare Absorptionskoeffizient $\left[\frac{\mathrm{m}^{2}}{\mathrm{~mol}}\right]$. In Gl. 2.14] wird ein linearer Zusammenhang zwischen Absorbanz und Konzentration hergestellt, durch Messung der Absorbanz kann also die Konzentration in einer Probe bestimmt werden. Da in praktischen Messungen das Signal in einem Spektrum eine endliche Linienbreite besitzt, ist es erforderlich, die Absorbanz über den Wellenzahlbereich der vollständigen Bande zu ermitteln und zu integrieren. Dabei bleibt die Linearität des LAMBERT-BEERschen Gesetzes erhalten und der Einfluss unsystematischer Fehler verringert. Es wird die integrale Absorbanz $\tilde{A}$ und der integrale Absorptionskoeffizient $\mathcal{A}$ erhalten $\left[\frac{\mathrm{m}^{2}}{\mathrm{~mol}}\right]$, wofür sich folgender Zusammenhang ergibt ( [27], S. 514):

$$
\tilde{A}:=\mathcal{A} \cdot c \cdot l
$$




\subsection{Die Ozonolyse}

In den folgenden Abschnitten wird zunächst der allgemeine Reaktionsablauf bei der Ozonolyse beschrieben. Anschließend wird näher auf die Folgechemie in der Gasphase eingegangen, bevor schließlich die speziellen literaturbekannten Erkenntnisse zum jeweiligen Reaktionsmechanismus und wichtiger Einflussgrößen bezüglich der in dieser Arbeit untersuchten Verbindungen vorgestellt werden.

\subsubsection{Der allgemeine Ozonolysemechanismus nach Criegee}

Bereits seit kurz nach der Entdeckung des Ozons im 19. Jahrhundert durch CHRISTIAN FRIEDRICH SCHÖNBEIN [31] wurde kontrovers über die Art, wie dieses mit Kohlenwasserstoffverbindungen reagiert, diskutiert. 1975 hat RuDOLF CRIEGEE den damaligen Kenntnisstand für die Reaktionsfolge bei der Ozonolysereaktion in flüssiger Phase vorgestellt [32], der seitdem zunehmend detaillierter untersucht und weiterentwickelt wurde [21,33]. Diese unter der Bezeichnung CRIEGEE-MEChANISMUS bekannte Reaktionsfolge (Abb. 2.2) ist mit einigen Einschränkungen auch auf die Gasphasenreaktion übertragbar.

In Lösung wie auch der Gasphase wird in Reaktionsfolge (I.) das Ozonmolekül zunächst an die Doppelbindung der ungesättigten Kohlenwasserstoffverbindung addiert (konzertierte [2+3]-Cycloaddition), woraus das sogenannte Primärozonid $(P O Z)$ resultiert. Dieses zerfält aufgrund seiner hohen Überschussenergie von 200-250 kJ/mol (chemische Aktivierung) [33] durch vor allem in der Gasphase nur unzureichend erfolgende Stoßstabilisierung zunächst unter Cycloreversion in eine Carbonylverbindung sowie ein Carbonyloxid. Letzteres wird in der Literatur häufig als CRIEGEE-Intermediat $(C l)$ bezeichnet. Dabei kann das $\mathrm{Cl}$ in verschiedenen Konformationen entstehen: der syn- und der anti-Konformation, je nachdem, ob die $\mathrm{O}-\mathrm{O}$-Bindung des $\mathrm{Cl}$ in die gleiche oder entgegengesetzte Richtung zum Rest $R_{1}-R_{4}$ mit der höchsten Priorität zeigt (vgl. Abb. 2.3). Die höchste Priorität ergibt sich in der Regel aus dem höheren Molekulargewicht eines der Substituenten, da es sich bei den Resten meist um reine Kohlenwasserstoffketten handelt. 


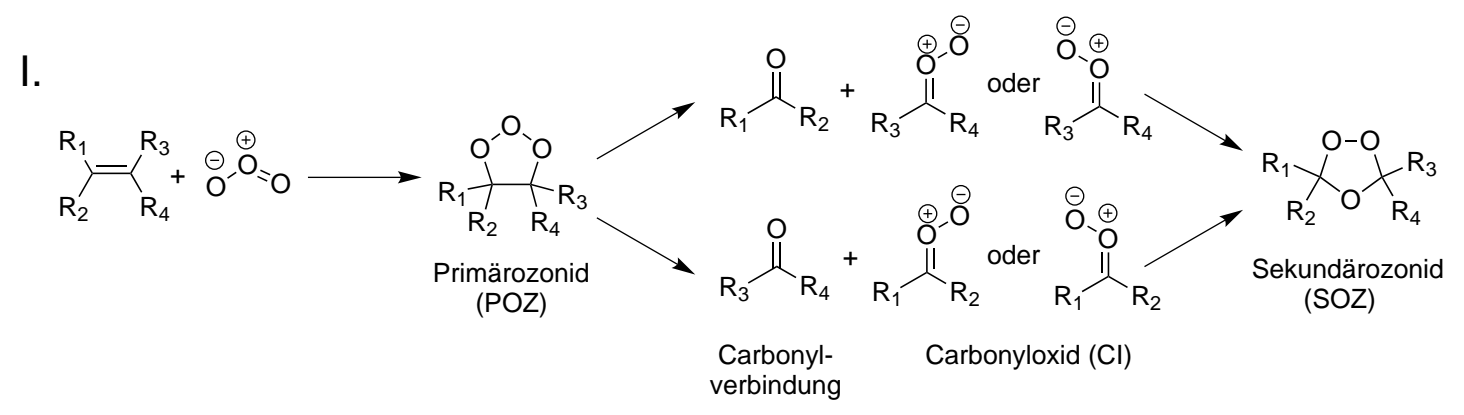

II.

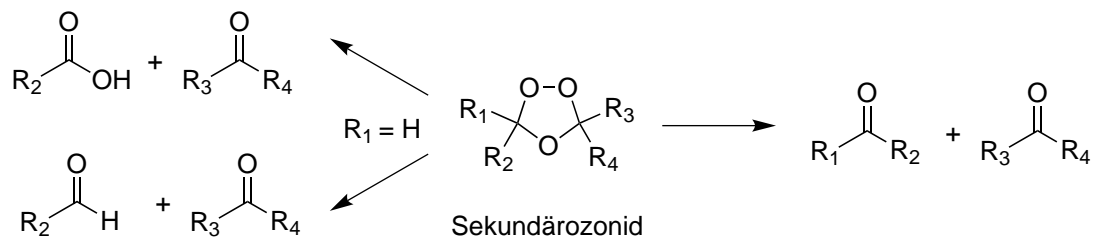

$$
\begin{aligned}
& \text { (SOZ) }
\end{aligned}
$$

Abb. 2.2.: Der CRIEGEE-Mechanismus, nach [32].

Im Unterschied zur flüssigen Phase wird das CRIEGEE-Intermediat in der Gasphasenreaktion meist nicht als Zwitterion, sondern als Diradikal dargestellt, wie in Abbildung 2.3 gezeigt. In der Realität muss wohl von einer Art Mischcharakter ausgegangen werden, wie UHEREK festgestellt hat [34]. Das CI besitzt noch immer eine so hohe Überschussenergie, dass in der Gasphase durch eine ineffektive Energieabführung aufgrund des fehlenden Lösungsmittelkäfigs die Rekombination von Carbonylverbindung und $\mathrm{Cl}$ zum Sekundärozonid nicht mehr quantitativ erfolgen kann - dies ermöglicht eine Vielzahl von Nebenreaktionen, die, oft über radikalische Zwischenstufen, zu einer großen Zahl sekundärer Reaktionsfolgen führen.

Auch nach dem Zerfall des Primärozonids und der daraus resultierenden Verteilung der Überschussenergie auf Translations-, Rotations- und Vibrationsmoden sind die Bruchstücke noch energetisch angeregt, diese schwingungsangeregten Verbindungen sind also ebenfalls chemisch aktiviert [33, 35].

Nur bei ausreichend langer Lebensdauer und hohen Konzentrationen in der Gasphase reagieren das stabilisierte $\mathrm{Cl}(S C l)$ und die typischerweise stoßstabilisierbare Carbonylverbindung (Keton oder Aldehyd) anschließend in einem weiteren Reaktionsschritt zum Sekundärozonid (SOZ), welches schließlich in die Produkte zerfällt (Teil II. in Abb. 2.2). Abhängig von der Substitution an 
der Doppelbindung sowie den Reaktionsbedingungen können hier Carbonyl, Säure- und andere Funktionalitäten in der Verbindung entstehen. Liegt das CRIEGEE-Intermediat ausreichend lange im Reaktionsgemisch vor, ist die bimolekulare Reaktion mit der Carbonylverbindung zum Sekundärozonid jedoch nur eine der Möglichkeiten. Auch Reaktionen mit anderen Substanzen der Reaktionsmischung sind neben den stets favorisierten, da auf kürzeren Zeitskalen ablaufenden, unimolekularen Zerfallsreaktionen und Isomerisierungen des $\mathrm{CI}$ denkbar [18, 33, 36].

\subsubsection{Die Folgechemie}

\subsubsection{Unimolekulare Folgereaktionen}

Die aus dem Primärozonid entstehenden CRIEGEE-Intermediate können in drei Gruppen eingeteilt werden, innerhalb derer sich bestimmte Merkmale der jeweiligen Folgechemie charakterisieren lassen. Neben dem unsubstituierten $\mathrm{Cl}$ (auch $\mathrm{C}_{1}$-CRIEGEE-Intermediat genannt) existieren mono- und disubstituierte Intermediate, wobei die letzten beiden in syn- oder anti-Konformation auftreten können (vgl. Abb. 2.3).

Substitutionsgrad:

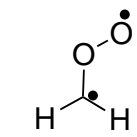

unsubstituiert

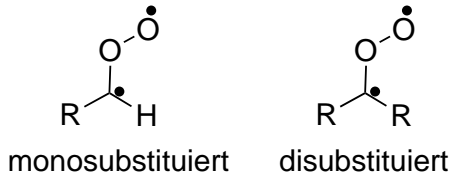

Konformation: Priorität $\mathrm{R}_{\mathrm{x}}>\mathrm{R}_{\mathrm{y}}$
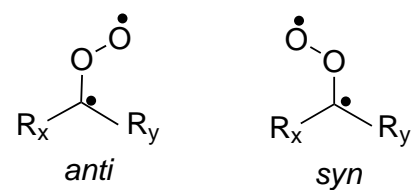

Abb. 2.3.: Mögliche Strukturen der CRIEGEE-Intermediate in Gasphasenreaktionen.

Streng gilt diese Benennung zwar nur für monosubstituierte Verbindungen, allerdings kann sie zur besseren Unterscheidung auch auf Verbindungen mit unterschiedlichen Resten unterschiedlicher Priorität angewandt werden. Da die C-O-Bindung aufgrund ihres partiellen Doppelbindungscharakters nicht frei drehbar ist, ist die Unterscheidung der beiden Konformere und ihrer spezifischen Reaktionskanäle sinnvoll [33]. An welchem der beiden an der Doppel- 
bindung beteiligten Kohlenstoffatome das $\mathrm{Cl}$ gebildet wird, ist von der Alkenstruktur determiniert. Nach Untersuchungen von RICKARD et al. [37] wird das CRIEGEE-Intermediat bevorzugt an dialkylsubstituierten Kohlenstoffatomen gebildet.

Das unsubstituierte CRIEGEE-Intermediat besitzt nur ein Kohlenstoffatom. Aus ihm kann nach Dioxiranbildung heiße, d.h. schwingungsangeregte Ameisensäure sowie, wiederum in einem druckabhängigen Kanalverhältnis, kleinere Bruchstücke, wie Kohlenstoffmonoxid, Kohlenstoffdioxid sowie HCO-, OH- und $\mathrm{H}$-Radikale, entstehen (Abb. 2.4). Dies ist beispielsweise für die Ethenozonolyse bei NEEB et al. beschrieben [38]. Wie in Tabelle 2.1 anhand verschiedener Studien gezeigt, können sich die experimentell bestimmten Ausbeuten der einzelnen Zerfallsprodukte stark unterscheiden. Anhand der Fragmente wird üblicherweise auf die Verteilung auf die verschiedenen Reaktionskanäle des $\mathrm{C}_{1}$-Cl-Zerfalls und indirekt auch auf die Ausbeute für das gebildete $\mathrm{Cl}$ rückgeschlossen. Diese Differenzen problematisieren daher die Interpretation der Bedeutung der CRIEGEE-Intermediate im Ozonolysemechanismus und somit in der Partikelbildung.

\begin{tabular}{|c|c|c|c|}
\hline Zerfallsreaktion & Atkinson [39] & Neeb [38] & Alam [40] \\
\hline $\mathrm{H}_{2} \mathrm{COO} \longrightarrow \mathrm{CO}_{2}+2 \mathrm{H}$ & 0.06 & \multirow[b]{2}{*}{0.23} & 0.05 \\
\hline $\mathrm{H}_{2} \mathrm{COO} \longrightarrow \mathrm{CO}_{2}+\mathrm{H}_{2}$ & 0.13 & & 0.24 \\
\hline $\mathrm{H}_{2} \mathrm{COO} \longrightarrow \mathrm{CO}+\mathrm{H}_{2} \mathrm{O}$ & 0.44 & \multirow[t]{2}{*}{\} 0.23} & 0.17 \\
\hline $\begin{array}{c}\mathrm{H}_{2} \mathrm{COO} \longrightarrow \mathrm{HCO}+\mathrm{OH} \\
\mathrm{H}_{2} \mathrm{COO} \longrightarrow \mathrm{HCOOH}\end{array}$ & $\frac{0}{0}$ & & $\frac{0.11}{\text { nicht best }}$ \\
\hline $\mathrm{H}_{2} \mathrm{COO}+\mathrm{M} \longrightarrow \mathrm{H}_{2} \mathrm{COO}$ & 0.37 & 0.50 & 0.54 \\
\hline
\end{tabular}

Tab. 2.1.: Ausbeuten der Zerfallsreaktionen des $\mathrm{C}_{1}-\mathrm{Cl}$.

Das monosubstituierte anti-Konformer zeigt ein ähnliches Verhalten wie das unsubstituierte CRIEGEE-Intermediat (Abbildung 2.4), da beide aufgrund der Struktur keine direkte $\mathrm{H}$-Radikalabstraktion und Bildung eines $\mathrm{OH}$-Radikals erlaubt. Auch bei dem $\mathrm{C}_{1}$-Cl wird zunächst aus einem Dioxiran die später weiter zerfallende schwingungsangeregte Säure gebildet, jedoch konkurriert dieser unimolekulare Zerfall aufgrund der höheren Stabilisierungswahrscheinlichkeit mit bimolekularen Reaktionen.

Dem syn-konformierten sowie dem disubstituierten CRIEGEE-Intermediat 


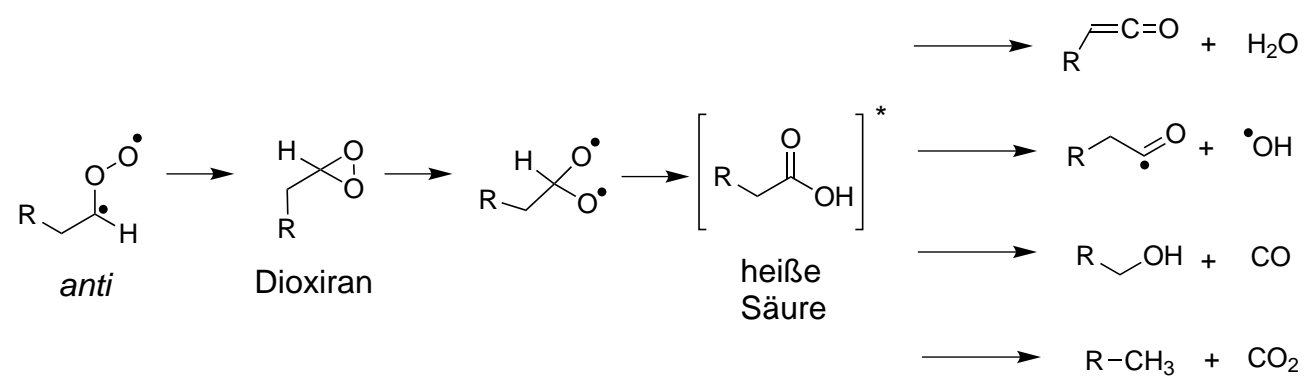

Abb. 2.4.: Zerfall der schwingungsangeregten Ameisensäure aus einem anti$\mathrm{Cl}$, nach JOHNSON und MARSTON [33].

ist die Weiterreaktion über den Vinylhydroperoxidkanal möglich, da beide ein $\mathrm{H}$-Atom in $\beta$-Position zur Carbonyloxidgruppe besitzen, was eine 1,4-HUmlagerung zum Vinylhydroperoxid und anschließenden Zerfall in ein $\mathrm{OH}$ - und ein Vinoxylradikal zur Folge hat (Abb. 2.5).

Die verschiedenen spezifischen Folgereaktionen bieten die Möglichkeit, auch die Anteile gebildeter syn- und anti-Konformere zu bestimmen. Da erstere über den Hydroperoxidkanal unter Bildung eines $\mathrm{OH}$-Radikals zerfallen, kann über den Anteil der gebildeten Radikale auf die Wahrscheinlichkeit der Bildung des syn-Cl rückgeschlossen werden. Dies geschieht häufig über den Einsatz von Radikalfängern wie Cyclohexan und anschließender Quantifizierung der in der Reaktion $\mathrm{C}_{6} \mathrm{H}_{12}+\mathrm{OH}$ gebildeten Produkte (vgl. Abb. 2.19 in Abschnitt 2.4.1). Eine Isomerisierung zwischen syn- und anti-Konformation wird aufgrund der hohen Barriere von ca. $120 \mathrm{~kJ} / \mathrm{mol}$ als eher unwahrscheinlich angesehen [33].

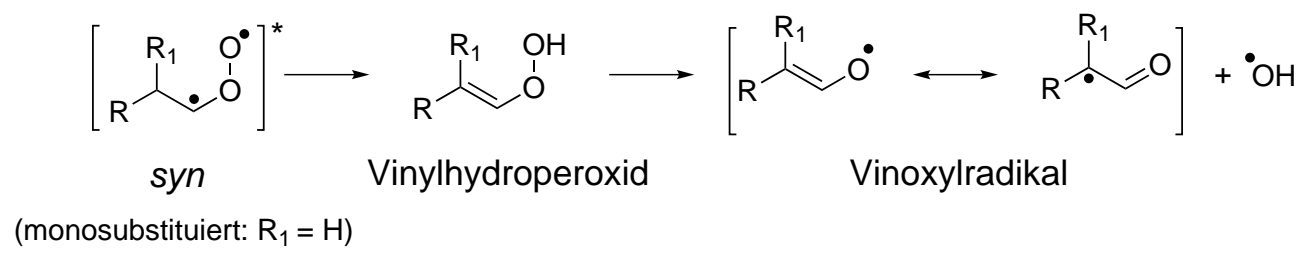

Abb. 2.5.: Zerfall eines syn-Cl, nach JOHNSON et al. [33].

Ein Spezialfall des disubstituierten CRIEGEE-Intermediates ist das zyklische, das mit seiner Struktur ebenfalls zur 1,4-H-Umlagerung fähig ist. Die Weiterreaktion unter $\mathrm{CO}_{2}$-Abspaltung ist gegenüber der über den Vinylhydroperoxidkanal nur von untergeordneter Bedeutung [33]. 


\subsubsection{Bimolekulare Folgereaktionen}

Falls das CRIEGEE-Intermediat seine Überschussenergie effektiv durch Stöße abgeben kann, steht dieses nun stabilisierte $\mathrm{Cl}(S C l)$ aufgrund seiner längeren Lebensdauer neben der weiterhin möglichen unimolekularen Chemie auch als Reaktionspartner für bimolekulare Folgereaktionen zur Verfügung. Zumindest das $\mathrm{C}_{1}-\mathrm{Cl}$ konnte bereits in der Gasphase nachgewiesen werden [41]. Der Anteil an $\mathrm{SCl}$ ist erhöht, falls der Hydroperoxidkanal blockiert ist, da der unimolekulare Zerfall über diesen Kanal wesentlich schneller als die meisten bimolekularen Reaktionen verläuft [42]. Das wiederum hat zur Folge, dass lediglich eine Konkurrenzsituation zwischen langsameren unimolekularen und möglichen bimolekularen Reaktionen besteht, was letztere auch bei geringeren Partialdrücken wahrscheinlicher werden lässt.

\begin{tabular}{|c|c|}
\hline Alken & $\boldsymbol{Y}_{\text {Scl }}$ [\%] \\
\hline \hline Cyclohexen & 3.2 \\
Methylcyclohexen & 10.4 \\
$\alpha$-Pinen & 12.5 \\
\hline Methylencyclohexan & 21.6 \\
$\beta$-Pinen & 24.9 \\
\hline
\end{tabular}

Tab. 2.2.: Ausbeuten des stabilisierten CRIEGEE-Intermediates (SCI) für verschiedene zyklische Alkene. Sämtliche Werte stammen von PAULSON et al. [43].

Mehrere Faktoren üben dabei einen Einfluss auf die Stabilisierung der angeregten Intermediate und damit die SCl-Ausbeute aus. Beispielsweise ist dieser Anteil für exozyklische Reaktanden höher als bei endozyklischen, da die Ozonolyse von Substanzen mit Doppelbindungen außerhalb des Ringes zur Bildung von zwei Molekülen führt (z.B. $\mathrm{CO}_{2}$-Abspaltung) [43,44]. Dadurch wird die Überschussenergie zum einen als Translationsenergie auf die beiden Produktverbindungen verteilt, zum anderen bietet die Beibehaltung der Ringstruktur eine große Anzahl von Schwingungsmoden, die eine Engergieumverteilung und -speicherung ermöglichen [45]. Auch die Molekülgröße hat Einfluss auf die SCIAusbeute, da mit der Anzahl der Kohlenstoffatome auch die Anzahl verfügbarer Schwingungsmoden steigt, so dass die im Molekül enthaltene Überschussenergie mit höherer Effizienz in nichtreaktive Moden verteilt werden kann [44]. Diese 
Auswirkungen auf die Stabilisierbarkeit lassen sich an den in Tabelle 2.2 gegebenen Werten ablesen [43].

Der entscheidende Parameter für die Stabilisierung von CRIEGEEIntermediaten ist der Gesamtdruck, da er sich direkt über die steigende Wahrscheinlichkeit der stattfindenden energieabführenden Stöße auf die Effektivität der Stoßstabilisierung auswirkt [46, 47].

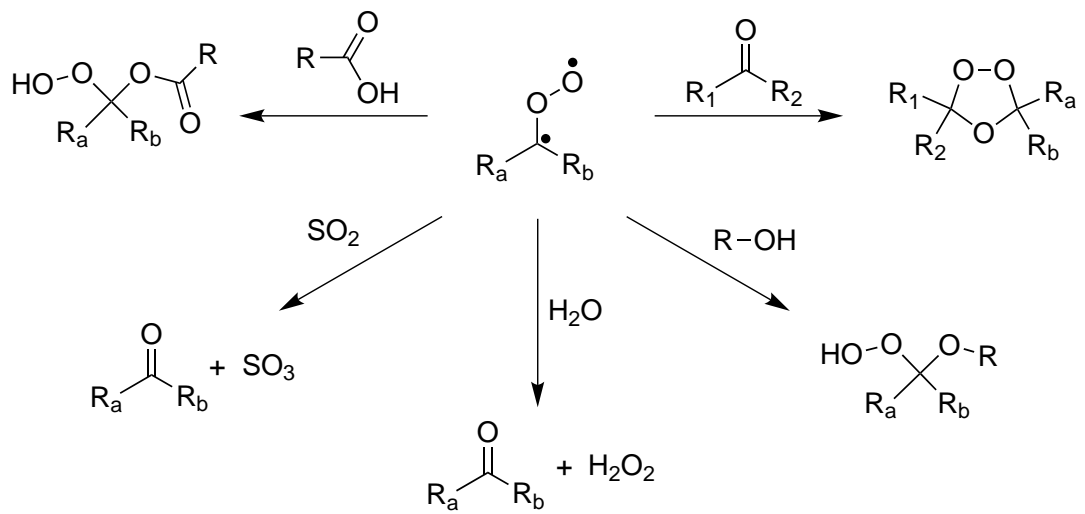

Abb. 2.6.: Mögliche bimolekulare Reaktionen des CRIEGEE-Intermediats, nach [18, 33, 48-51].

In der natürlichen Atmosphäre ist Wasser der wichtigste Reaktionspartner für das stabilisierte CRIEGEE-Intermediat, da es durch seine hohe Konzentration den geringen Geschwindigkeitskoeffizienten dieser Reaktion kompensiert [18, 33, 52] (s. Abb. 2.6). Schneller läuft die Reaktion dagegen mit oxidierten Verbindungen wie Säuren [18], Alkoholen [52], Schwefeldioxid [33, 46] sowie Carbonylverbindungen [18] ab, wobei der letzte Vorgang die Reaktion zum Sekundärozonid darstellt.

SADEZKY et al. [36,53] postulieren zudem, dass aus der Selbstreaktion der $\mathrm{Cl}$ langkettige Oligomere gebildet werden, bis diese Kettenreaktion durch $\mathrm{HO}_{2}$ Radikale unterbrochen wird; beispielsweise für das $\mathrm{C}_{1}-\mathrm{Cl}$ sowie die Ozonolyse von trans-3-Hexen (vgl. Abb. 2.7) konnte dies bereits nachgewiesen werden.

Wie hier gezeigt, sind dabei der Kettenstart durch die Reaktion des Alkens mit einem $\mathrm{OH}$-Radikal, wie es in der Ozonolysereaktion entsteht und die Anlagerung eines Sauerstoffmoleküls nötig, bevor die kettenverlängernden Reaktionen der $\mathrm{Cl}$ stattfinden können. Daher wird im folgenden Abschnitt kurz auf die Bedeutung von Sauerstoff in der Folgechemie der Ozonolysereaktion ein- 


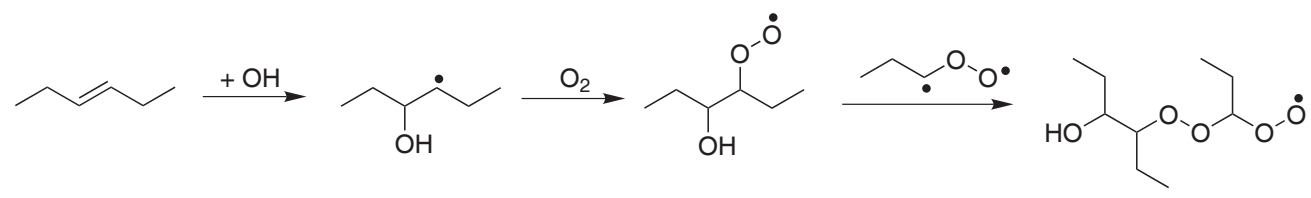

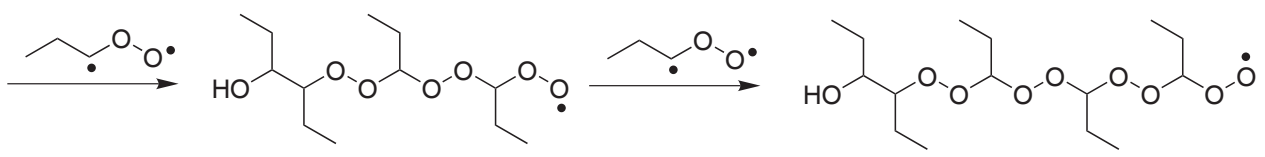

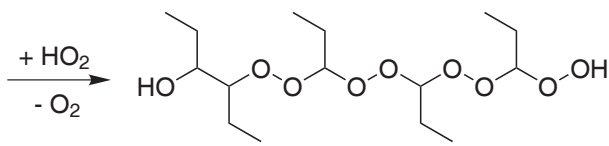

Abb. 2.7.: Postulierte Oligomerisierungsreaktion bei der trans-3-HexenOzonolyse, nach SADEZKY et al. [36].

gegangen.

\subsubsection{Die Bedeutung von Sauerstoff als Reaktionspartner in bimolekularen Reaktionen}

Wie schon in Abbildung 2.5 im vorangegangenen Abschnitt gezeigt, zerfällt das syn-konformierte CRIEGEE-Intermediat über den Vinylhydroperoxidkanal, im Verlauf der Reaktionsfolge bildet sich nach $\mathrm{OH}$-Radikalabspaltung ein mesomeriestabilisierbares Vinoxylradikal. Dieses kann in verschiedenen Kanälen weiter zerfallen bzw. reagieren, abhängig von Faktoren wie z.B. der Art des verwendeten Badgases. In Abbildung 2.8 ist dargestellt, wie sich Sauerstoffanwesenheit auf den Reaktionsverlauf auswirkt.

Das Vinoxylradikal (Teil I.) kann entweder mit Sauerstoff zu einem Peroxyradikal (im Folgenden auch als ", $\mathrm{RO}_{2}$ " abgekürzt) reagieren, das mit einem weiteren dieser Art unter Sauerstoffabspaltung ein stabilisiertes Alkoxyradikal („RO“) bildet. Dieses kann, ebenso wie das Peroxyradikal selbst, möglicherweise als „nukleationsinduzierendes Molekül“ (NIM) wirken [18] und in der Folge entweder zerfallen oder isomerisieren.

Die Bedeutung der genannten Verbindungen als Partikelvorläufer ist abhängig von ihrer Stabilisierung bzw. Isomerisierung, da hier größere Moleküle vorhanden bleiben als bei auftretenden Zerfallsreaktionen. Analog zur mit dem Gesamtdruck steigenden Wahrscheinlichkeit der Cl-Stabilisierung [47] nimmt 
I. Bildung von Alkoxy- und Peroxyradikalen:

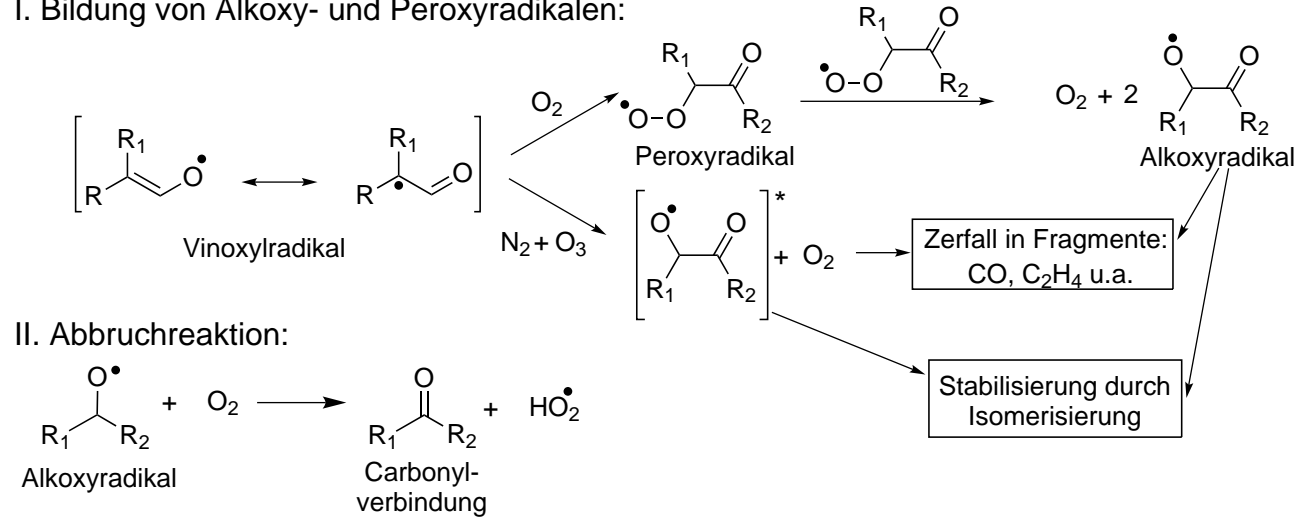

Abb. 2.8.: Postulierte Folgechemie des Vinylhydroperoxidradikals nach HOYERMANN et al. sowie WOLF [20,48].

auch in der Alkoxy- / Peroxychemie die Bedeutung der Isomerisierung gegenüber dem Zerfall bei höheren Reaktionsdrücken zu, wie HOYERMANN et al. [20] für die Reaktion zyklischer Kohlenwasserstoffverbindungen mit Sauerstoffatomen und die daraus entstehenden zyklischen Alkoxyradikale zeigen konnten. In der Dissertation von WOLF [48] wird zudem die These aufgestellt, dass schon Alkoxyradikale mit einem kleinerem $\mathrm{C}_{6}$-Grundkörper aus der Ozonolysereaktion bei atmosphärischen Bedingungen stabilisiert werden können, da sie durch ihren Ursprung aus der Alken $+\mathrm{O}_{3}$-Reaktion eine geringere Überschussenergie besitzen sollten als Produkte, die aus der Reaktion von Sauerstoffatomen stammen.

In Teil II. der Abbildung 2.8 wird die mögliche Abbruchreaktion mit Sauerstoff gezeigt, bei der das Alkoxyradikal zu einer Carbonylverbindung und einem $\mathrm{HO}_{2}{ }^{-}$ Radikal reagiert. Als Abbruchreaktion wird sie in diesem Zusammenhang wegen ihrer tendenziell hemmenden Auswirkung auf die Bildung von Partikelkeimen durch die Entstehung von Produkten mit niedrigerem Molekulargewicht bezeichnet. Zudem können die entstehenden $\mathrm{HO}_{2}$-Radikale möglicherweise ablaufende Oligomerisierungsreaktionen (vgl. Abb. 2.7) unterbinden. Die in beiden Fällen I. und II. mögliche Fragmentierung der sauerstoffhaltigen Radikale kann zu verschiedenen, durch Infrarotspektroskopie nachweisbaren Gasphasenprodukten, wie $\mathrm{CO}$, Ethen und verschiedenen Ketenderivaten führen, die als Maß für den Zerfall über den Hydroperoxidkanal und allgemein das Stabilisierungsvermögen des Systems durch verschiedene experimentelle Bedingungen 
genutzt werden (vgl. Kap. 6.2).

Radikalinduzierte Reaktionsschritte bei der $\beta$-Pinen-Ozonolyse in Sauerstoffanwesenheit

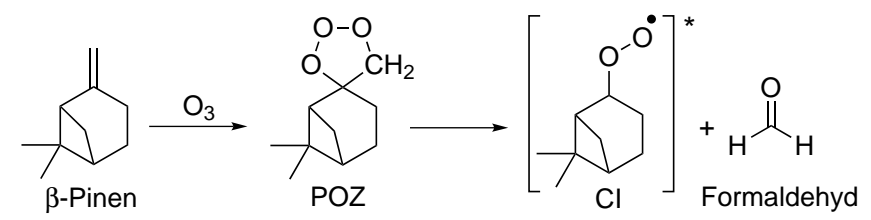

$\downarrow \begin{aligned} & -\mathrm{OH} \\ & +\mathrm{O}_{2}\end{aligned}$

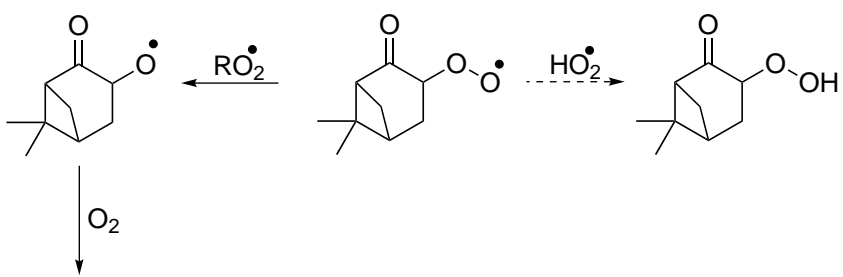

$\underbrace{\mathrm{O}-\mathrm{OH}}_{=\mathrm{O}}$
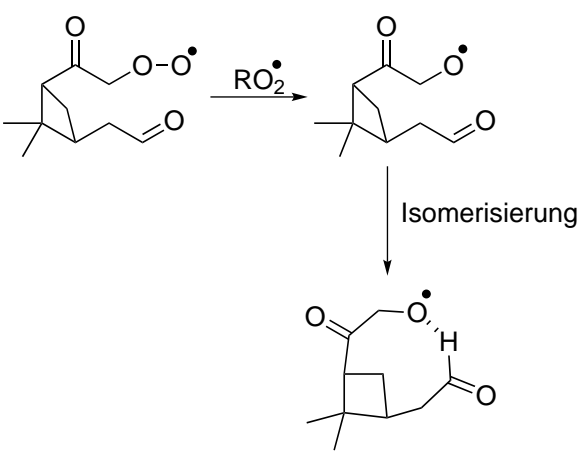

$\mathrm{O}_{2}$<smiles></smiles><smiles>CC(C)(C)C(CC(=O)O[Na])C(=O)O</smiles><smiles>CCCCCCCCCCC(C)(C)C(CC(=O)O)C(=O)O</smiles>

Abb. 2.9.: Einfluss auf gebildete Produkte durch Radikale bei der $\beta$ Pinenozonolyse nach DOCHERTY et al. [54]. 
In verschiedenen Studien wurde festgestellt, dass sich das Verhältnis von $\mathrm{HO}_{2}$ - und Peroxyradikalen auf die Partikelbildung auswirken kann. Der Effekt ist jedoch abhängig von der Alkengrundstruktur des eingesetzten Eduktes. Während eine Erhöhung des Verhältnisses von $\mathrm{HO}_{2} \mathrm{zu} \mathrm{RO}_{2}$ bei der Ozonolyse von endozyklischen Verbindungen wie Cyclohexen [55] oder $\alpha$-Pinen [19] für eine Zunahme der Aerosolausbeute sorgt, wurde beim exozyklischen $\beta$-Pinen ein Rückgang beobachtet [54].

Für diesen Befund werden Acylperoxyradikale (Strukturmotiv $\mathrm{R}-(\mathrm{C}=\mathrm{O})-\mathrm{O}-\mathrm{O})$ verantwortlich gemacht, denen in ihrem Verhälthis $\mathrm{zu}$ $\mathrm{HO}_{2}$-Radikalen eine Bedeutung für die Nukleation zugeschrieben wird. Die Interpretation von KEYWOOD et al. [55] bzw. DOCHERTY et al. [54] besagt, dass bei direkter Bildung dieser Strukturen durch ein niedriges $\mathrm{OH}$ / PeroxyradikalVerhältnis weniger Aerosol gebildet wird, während für Alkene, aus denen die Acylperoxyverbindungen erst nach mehreren Zwischenschritten entstehen, ein höheres Verhältnis für die Unterdrückung nötig ist. Dieser Effekt wird durch den Mechanismus in Abbildung 2.9 erklärt, in dem die Bildung schwerflüchtiger, zur Nukleation fähiger Verbindungen erst nach mehreren Reaktionsschritten erfolgt. Da die Reaktionen von $\mathrm{HO}_{2}$-Radikalen mit entstehenden Peroxyverbindungen zunächst zu Substanzen mit geringerer Flüchtigkeit führen, kann somit eine hohe $\mathrm{HO}_{2}$-Konzentration zur Unterdrückung der Aerosolbildung führen.

Dieser Reaktionsverlauf ist allerdings nur für relativ große Alkene als bedeutsam anzusehen. Für kleine Kohlenstoffgerüste kann nicht von einer genügenden Reduktion des Dampfdrucks durch Ozonolyse und Bildung von Alkoxyund Peroxyverbindungen für die effektive Bildung von Partikeln oder ihren Vorläufern ausgegangen werden. Hier ist die Bildung von Oligomerstrukturen nach dem Vorbild von SADEZKY et al. [36, 53], gezeigt in Abb 2.7, wahrscheinlicher, wobei die Erhöhung der $\mathrm{HO}_{2}$-Konzentration sich aufgrund ihrer kettenabbrechenden Funktion negativ auf das Partikelbildungsvermögen des betrachteten Systems auswirken sollte.

Die $\mathrm{HO}_{2}$-Konzentration in der Reaktionsmischung sollte höher sein, wenn Stickstoff statt Synthetischer Luft als Badgas verwendet wird, da zum einen die Wahrscheinlichkeit höher ist, dass bei Bildung von $\mathrm{OH}-$ Radikalen während der Ozonolyse die in Tabelle2.3 (Kap. 2.5) genannte Reaktion $\mathrm{OH}+\mathrm{O}_{3} \longrightarrow \mathrm{HO}_{2}+\mathrm{O}_{2}$ abläuft. Der Abbau der $\mathrm{HO}_{2}$-Radikale durch Ozon besitzt dabei nicht nur einen 
langsameren Geschwindigkeitskoeffizienten, sondern sollte auch wegen der geringeren $\mathrm{HO}_{2}$ - gegenüber der $\mathrm{OH}$-Konzentration in wesentlich geringerem Maße ablaufen. Entscheidend sollte jedoch sein, dass zum anderen in Sauerstoffabwesenheit während der Ozonolysereaktion Peroxyradikale $\left(\mathrm{RO}_{2}\right)$ in geringerem Maße gebildet werden. Dies hat zur Folge, dass der Abbau von $\mathrm{HO}_{2}$ Radikalen nach dem folgenden Schema ( [56], S. $81 \mathrm{ff}$.) nicht mehr ablaufen kann.

$$
\begin{aligned}
& \mathrm{RO}_{2}+\mathrm{HO}_{2} \rightarrow \mathrm{ROOH}+\mathrm{O}_{2} \\
& \mathrm{RO}_{2}+\mathrm{HO}_{2} \rightarrow \mathrm{RO}-\mathrm{H}+\mathrm{H}_{2} \mathrm{O}+\mathrm{O}_{2} \\
& \mathrm{RO}_{2}+\mathrm{HO}_{2} \longrightarrow \mathrm{RO}-\mathrm{X}+\mathrm{HOX}+\mathrm{O}_{2} \\
& \mathrm{RO}_{2}+\mathrm{HO}_{2} \longrightarrow \mathrm{ROH}+\mathrm{O}_{3}
\end{aligned}
$$

\subsection{Spezifische Reaktionsverläufe für die untersuchten Substanzen}

Nach der Darstellung der allgemeinen Abläufe und möglichen Reaktionskanälen bei der Ozonolyse im vorangegangenen Abschnitt sollen nun die speziellen Reaktionen und aus innen folgenden Zwischen- und Endprodukte vorgestellt werden. Vor allem die entstehenden CRIEGEE-Intermediate und die aus innen gebildete spezifischen Folgeprodukte wurden in mehreren Arbeiten anderer Arbeitsgruppen (z.B. [21, 36, 53, 57]) als entscheidend für die Produkt- und Partikelbildung durch die Ozonolysereaktion benannt. Vor allem die Ozonolyseprodukte, denen aus strukturellen Gründen nicht der Zerfall über den Vinylhydroperoxidkanal offensteht, können über verschiedene Prozesse zur partikulären Materie beitragen. Sie können unter bestimmten Umständen stabilisiert werden und ihr Dampfdruck durch sekundäre Reaktionen soweit reduziert werden, dass sekundäres organisches Aerosol (SOA) entsteht [21], welches dann in Partikelbildungsmessungen nachweisbar ist. 


\subsubsection{Reine Kohlenwasserstoffverbindungen}

Zunächst können in Abbildung 2.10 die möglichen Strukturen der CRIEGEEIntermediate für einige der untersuchten reinen Kohlenwasserstoffverbindungen betrachtet werden. Hier wird zu jeder der verwendeten Grundstrukturen ein Beispiel gegeben, da die Reaktion für stukturell ähnliche Substanzen (z.B.Methylcyclohexen vs. Methylcyclopenten) analog verläuft. Dabei fällt auf, dass lediglich in der Ozonolyse von 1-Hexen und 2-Methyl-1-penten $\mathrm{C}_{1}$-Cls gebildet werden, die die nach dem Vorschlag von SADETZKY et al. [36] eine Oligomerisierungsreaktion eingehen können.
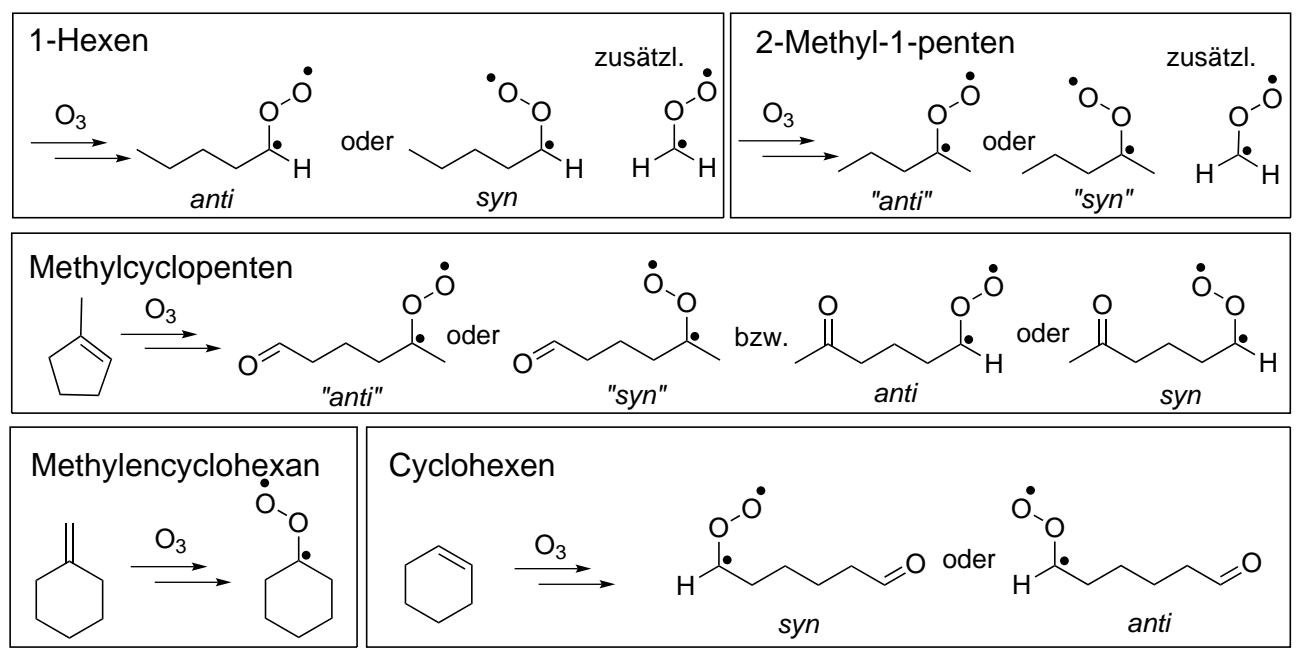

Abb. 2.10.: Mögliche Strukturen der CRIEGEE-Intermediate für die verwendeten Grundstrukturen der reinen Kohlenwasserstoffverbindungen.

Weiterhin ist zu beachten, dass bei den zyklischen Ausgangsverbindungen nur ein $\mathrm{Cl}$ gebildet wird, weshalb durch das Fehlen einer Translationsmode eine höhere Überschussenergie aus der Ozonanlagerung im Molekül verbleibt und die $\mathrm{Cl}$ des endozyklischen Methylcyclopentens und Cyclohexens schlechter zu stabilisieren sein sollten (vgl. Tabelle 2.2, S. 27). Günstiger für die Verteilung dieser Energie ist dagegen die Beibehaltung der zyklischen Struktur im Falle des exozyklischen Methylencyclohexans [44].

Beispielhaft ist in Abbildung 2.11 dargestellt, wie der Zerfall des Primärozonids zu den verschiedenen Carbonyl- und Carbonyloxidverbindungen führt und wie letztere unimolekular weiterreagieren. Die dargestellten Reaktionen sind 


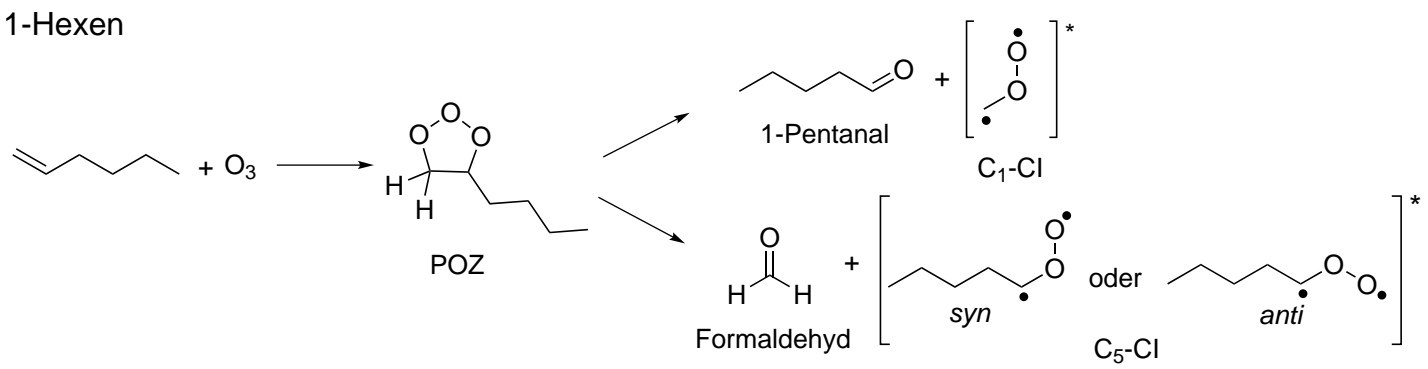

Unimolekulare Folgereaktionen:

Zerfall des syn-Cl über den Hydroperoxidkanal

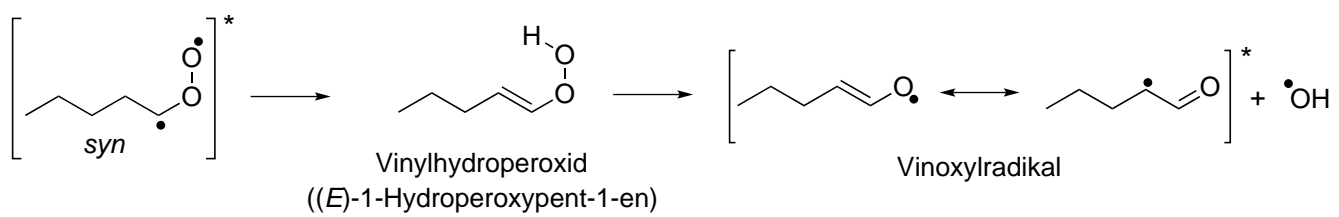

Zerfall von $\mathrm{C}_{1}-\mathrm{Cl}(\mathrm{R}=\mathrm{H})$ und anti- $\mathrm{Cl}\left(\mathrm{R}=\mathrm{C}_{4} \mathrm{H}_{9}\right)$

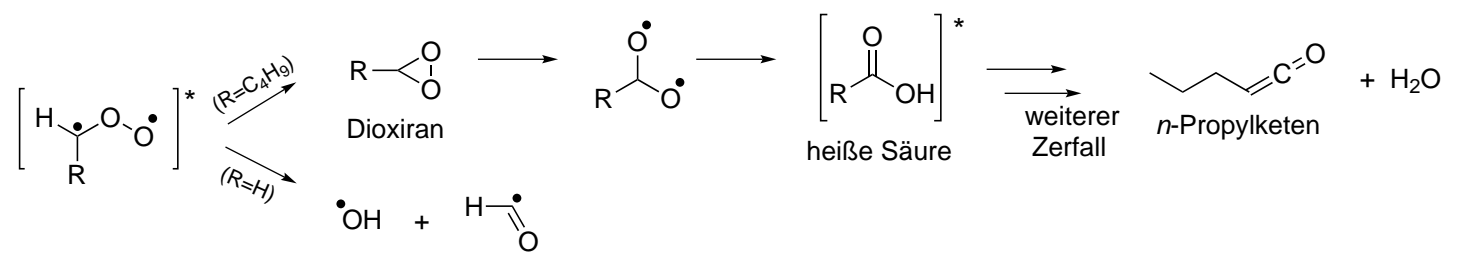

Abb. 2.11.: Unimolekulare Folgechemie des 1-Hexens, adaptiert nach WOLF [48].

dabei lediglich als explizites Beispiel für die im vorherigen Abschnitt in den Abbildungen 2.4 und 2.5 allgemein gezeigten unimolekularen Folgereaktionen der Intermediate zu sehen.

Wie in Abschnitt 2.2.2.1 bereits beschrieben, erfolgt die Bildung der Cls nicht statistisch, sondern bevorzugt an dem Kohlenstoffatom der Doppelbindung mit der höheren Alkylsubstituierung [37]. Für 1-Hexen, das nur un- und einfachsubstituierte Intermediate bilden kann, ergibt sich nach Untersuchungen von RICKARD et al. [37] beispielsweise ein Verhältnis von 50:50 für $\mathrm{C}_{1}$ - und $\mathrm{C}_{5}-\mathrm{Cl}$, für 2-Methyl-1-buten mit der Möglichkeit zur Bildung eines disubstituierten $\mathrm{Cl}$ liegt das Verhältnis von $\mathrm{C}_{1}-\mathrm{zu} \mathrm{C}_{4}$-Cl dagegen schon bei 28:72. Aus diesen Verhältnissen ergeben sich folglich auch verschieden ausgeprägte Ausbeuten der entstehenden Folgeprodukte, die in der Folge auf die ablaufenden Prozesse bei der Partikelbildung Einfluss ausüben. Eine weitere Unterscheidung zwischen 
syn- und anti-Konformeren ist zudem beispielsweise für 1-Hexen anhand der zusätzlichen Information, dass mit einer Ausbeute von $32 \%$ das $\mathrm{OH}$-Radikal gebildet wird $\left(Y_{\mathrm{OH}}=0.32\right)$, möglich. Dies wird als Maß für den unimolekularen Zerfall des syn-Cl gesehen, da es unter $\mathrm{OH}$-Radikalbildung über den Vinylhydroperoxidkanal weiter reagiert. Zwar wird aufgrund fehlender Gegenbeweise meist davon ausgegangen, das das syn / anti-Verhältnis 1:1 beträgt [33, 42], allerdings ist für einzelne Substanzen bereits gezeigt worden, dass diese Annahme nicht immer gerechtfertigt ist [45].

\subsubsection{Voroxidierte Edukte}

Nach Untersuchungen zur Ethenozonlyse von NEEB et al. [57] haben stabilisierte CRIEGEE-Intermediate (Abb. 2.12) neben einer unimolekularen Folgechemie (vgl. Abb. 2.13) die Möglichkeit, mit Hydroxylverbindungen bimolekulare Reaktionen einzugehen.

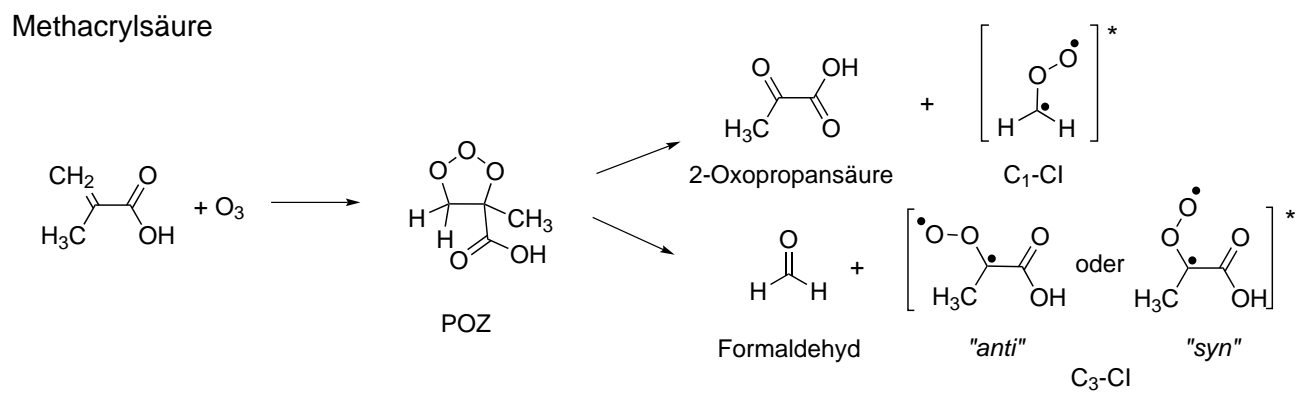

Abb. 2.12.: Initialer Schritt der Methacrylsäureozonolyse.

Dies ist nicht nur bei der Verwendung von speziell eingesetzten Fängermolekülen, wie Ameisensäure, Essigsäure und Methanol [57] bzw. Formaldehyd [58], von Bedeutung. Vielmehr ergeben sich im Zusammenhang der im Rahmen der vorliegenden Arbeit durchgeführten Experimente vor allem interessante Möglichkeiten für Reaktionen der gebildeten $\mathrm{Cl}$ mit den Eduktmolekülen der verwendeten voroxidierten Verbindungen. In einer weiteren Arbeit von NEEB et al. [59] wird diese Reaktionsmöglichkeit explizit für Acryl- und die in dieser Arbeit verwendete Methacrylsäure angegeben. Die Untersuchungen zur Bestimmung der Reaktionsgeschwindigkeitskoeffizienten haben dabei gezeigt, dass die verwendeten ungesättigten Carbonsäureedukte signifikant langsamer 
Unimolekulare Folgereaktionen:

Zerfall des disubstituierten $\mathrm{Cl}$ über den Hydroperoxidkanal

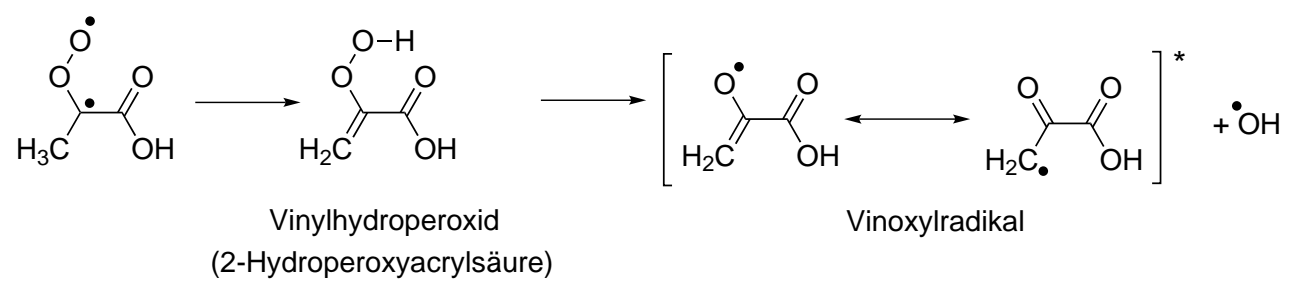

Zerfall des $\mathrm{C}_{1}-\mathrm{Cl}$<smiles>CCCOCCC</smiles>

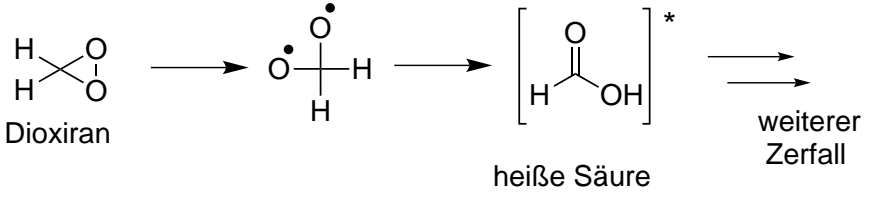

$\mathrm{OH}+\mathrm{H} \underset{\mathrm{O}}{\stackrel{0}{O}}$

Abb. 2.13.: Unimolekulare Folgechemie der Methacrylsäureozonolyse.

verbraucht werden, sobald der Reaktionsmischung Ameisensäure zugesetzt wurde. Da aufgrund der Verwendung von Cyclohexan in großem Überschuss4 $\mathrm{OH}$-Radikale als zusätzliche Reaktionspartner ausgeschlossen werden konnten, folgern die Autoren, dass der schnellere Abbau der Säuremoleküle auf bimolekulare Reaktionen mit den stabilisierten $\mathrm{Cl}$ zurückzuführen sei. Ein vergleichbarer Effekt konnte bei der Untersuchung analoger Aldehyd- und Ketonstrukturen (Methacrolein und Methylvinylketon) nicht festgestellt werden.

HATAKEYAMA et al. [58] postulieren dagegen eine Abfangreaktion der $\mathrm{Cl}$ durch Carbonylverbindungen. Sie schließen sich dem ursprünglich von NIKI et al. [60] gemachten Vorschlag zur $\mathrm{Cl}+$ Aldehydreaktion an, der in Abb. 2.14] gezeigt ist, machen allerdings keine Angaben zur Effizienz dieser Reaktion im Vergleich zu der mit anderen Verbindungen.

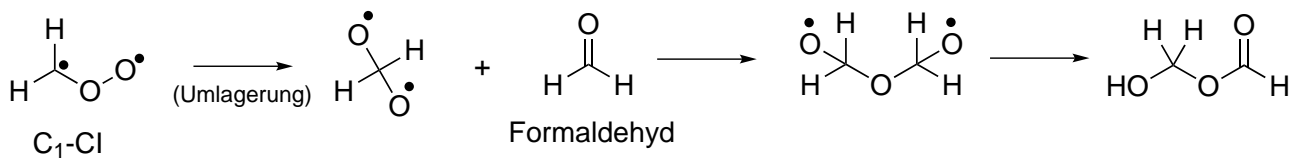

Abb. 2.14.: Vorschlag zur $\mathrm{C}_{1}-\mathrm{Cl}+$ Aldehyd-Reaktion nach HATAKEYAMA et al. [58].

\footnotetext{
${ }^{4}$ Etwa 60fach im Vergleich zum umgesetzten Alken, um > $90 \%$ OH-Radikale abzufangen.
} 
Untersuchungen von LEE und KAMENS [52] kamen zu dem Ergebnis, dass die Effektivität von SCl-Fängern in der Reihenfolge Wasser < Acetaldehyd < Methanol < Ameisensäure zunimmt, also organische Hydroxylguppenträger mit möglichst hohem Oxidationsgrad für diese Aufgabe am besten geeignet sind 5

Vorschlag nach Neeb (1999)

Bimolekulare Folgereaktion der stabilisierten $\mathrm{Cl}$ (am Beispiel Wasser, Ameisensäure und des verwendeten Edukts, weitere Reaktionen analog zu Abb. 2.7):<smiles>O=COCOO</smiles>
Hydroperoxymethylformiat<smiles>CC(OO)[C@@H](C)OOCC(=O)O</smiles><smiles>C=C(C)C(=O)OC(C)C(=O)OO</smiles>

Hydroperoxymethylmethacrylat

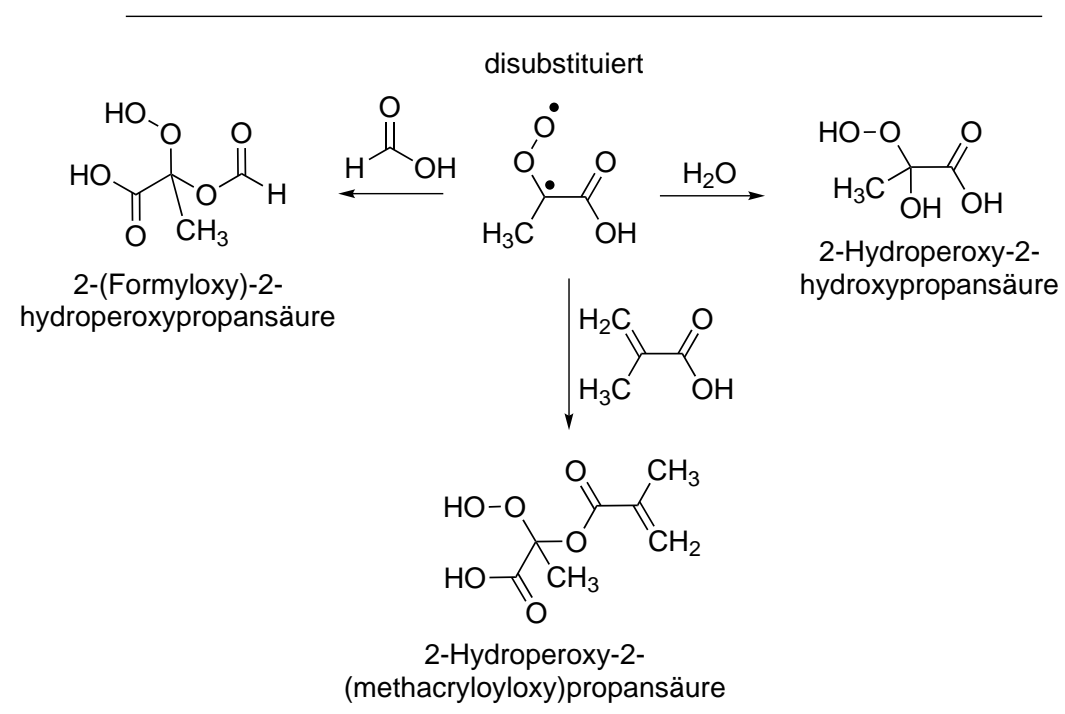

Abb. 2.15.: Bimolekulare Folgechemie der stabilisierten $\mathrm{Cl}$ der Methacrylsäure, nach einem Vorschlag von NEEB et al. [59].

${ }^{5}$ Auf diese Punkte wird im weiteren Verlauf der Arbeit (Kap. 6, Kap. 7) detaillierter eingegangen. 
Abbildung 2.15zeigt diese postulierte Reaktion für die CRIEGEE-Intermediate mit verschiedenen Reaktionspartnern, die aus der Ozonolyse von Methacrylsäure entstehen können. Zum einen ist als atmosphärisch relevanter, da in hoher Konzentration vorhandener, Reaktand Wasser dargestellt.

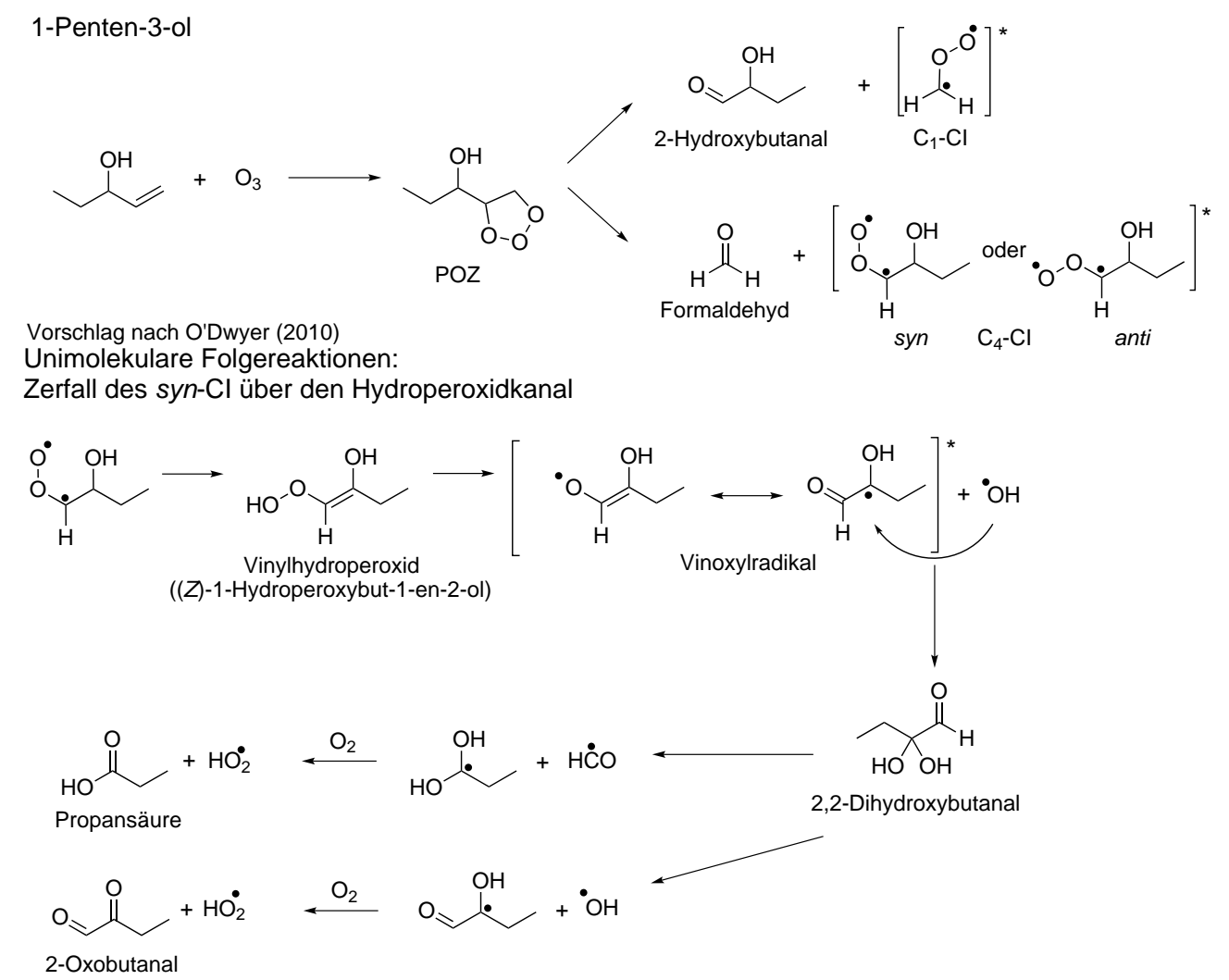

Direkter Zerfall

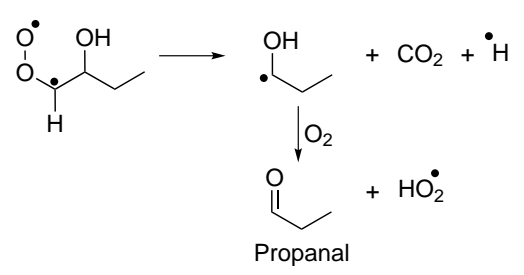

Abb. 2.16.: Ozonolysemechanismus des 1-Penten-3-ol mit Folgereaktionen nach einem Vorschlag von O'DWYER et al. [61].

Zum anderen wurden Ameisensäure und Methacrylsäure gewählt, da diese Substanzen für die Laborexperimente wegen erhöhter Konzentrationen von 
größerer Bedeutung sind. Während erstere aus der Ozonolyse und ihren Folgereaktionen entstehen kann und somit als Reaktionspartner zur Verfügung steht, ist letztere als Edukt aufgrund ihres hohen Partialdrucks in der Reaktionsmischung der wahrscheinlichste Reaktionspartner. In diesen Reaktionen entstehen innerhalb weniger Reaktionsschritte hochoxidierte Hydroperoxyverbindungen, die durch die Vielzahl an Sauerstoffatomen ein hohes Molekulargewicht und damit meist auch einen geringen Dampfdruck aufweisen. Aus diesem Grund könnten sie durchaus zur partikulären Materie beitragen.

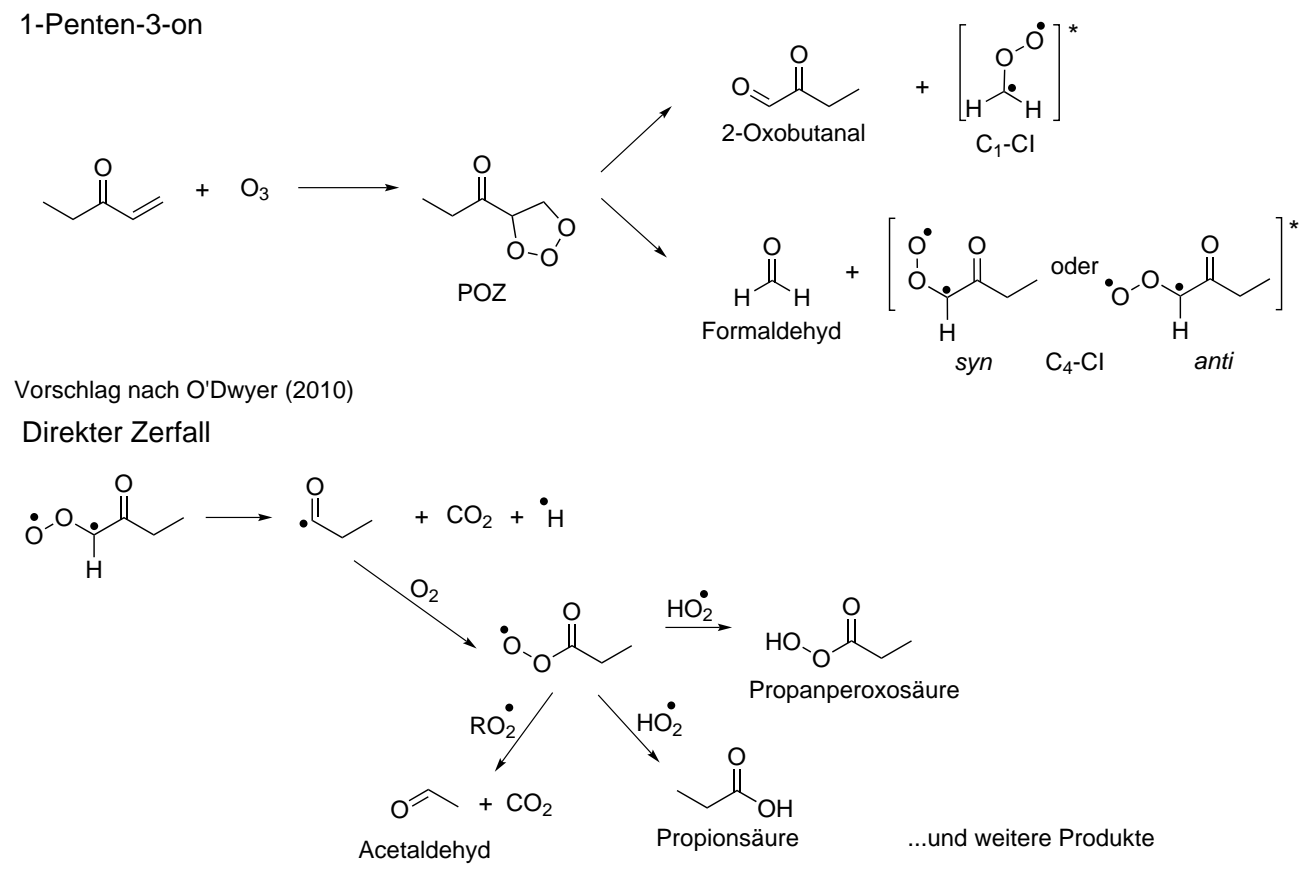

Abb. 2.17.: Ozonolysemechanismus des 1-Penten-3-on mit Folgeraktionen nach einem Vorschlag von O'DWYER et al. [61].

Auch für einige der untersuchten Alkohole und Carbonylverbindungen lassen sich Mechanismusvorschläge in der Literatur finden. Diese Verbindungen sind aufgrund ihres natürlichen Vorkommens von Interesse und rücken neben den biogen emittierten Terpenen, wie Isopren, $\alpha$ - und $\beta$-Pinen, immer weiter in den Fokus wissenschaftlicher Untersuchungen zu atmosphärischen Vorgängen. Von den untersuchten Verbindungen sind 1-Penten-3-ol und das analoge Keton bereits als biogen emittierte VOCs bekannt, sie werden, wie weitere Pentenole und Pentenale, vor allem durch grünblättrige Pflanzen bei Stress 
durch Verletzungen oder Trocknung abgegeben [62] oder entstehen durch die atmosphärische Oxidation von Alkenen [63]. Auch 3-Buten-1-ol kommt in der natürlichen Atmosphäre vor, allerdings wird es nicht nur biogen, sondern auch anthropogen in Polymersyntheseprozessen der Plastikindustrie freigesetzt [64].

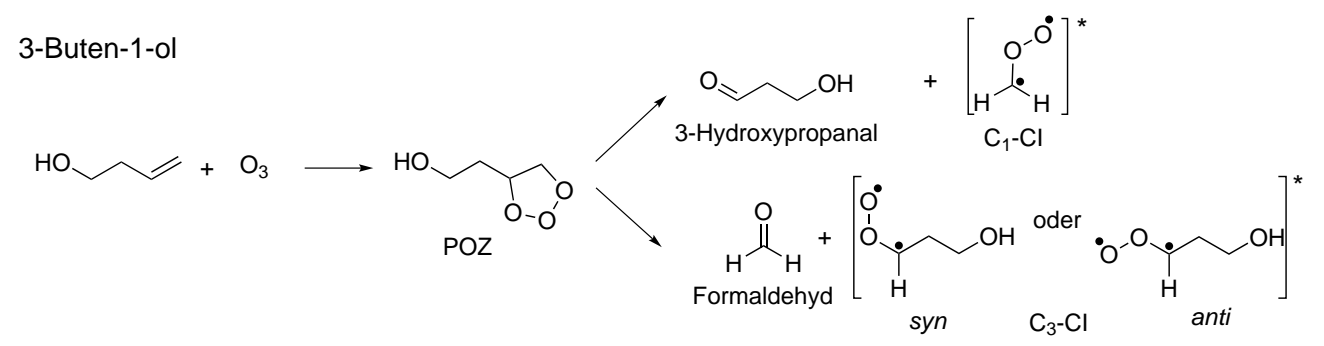

Vorschlag nach Grosjean (1993)

Unimolekulare Folgereaktionen: Zerfall des $\mathrm{C}_{3}-\mathrm{Cl}$ (u.a. Hydroperoxidkanal)

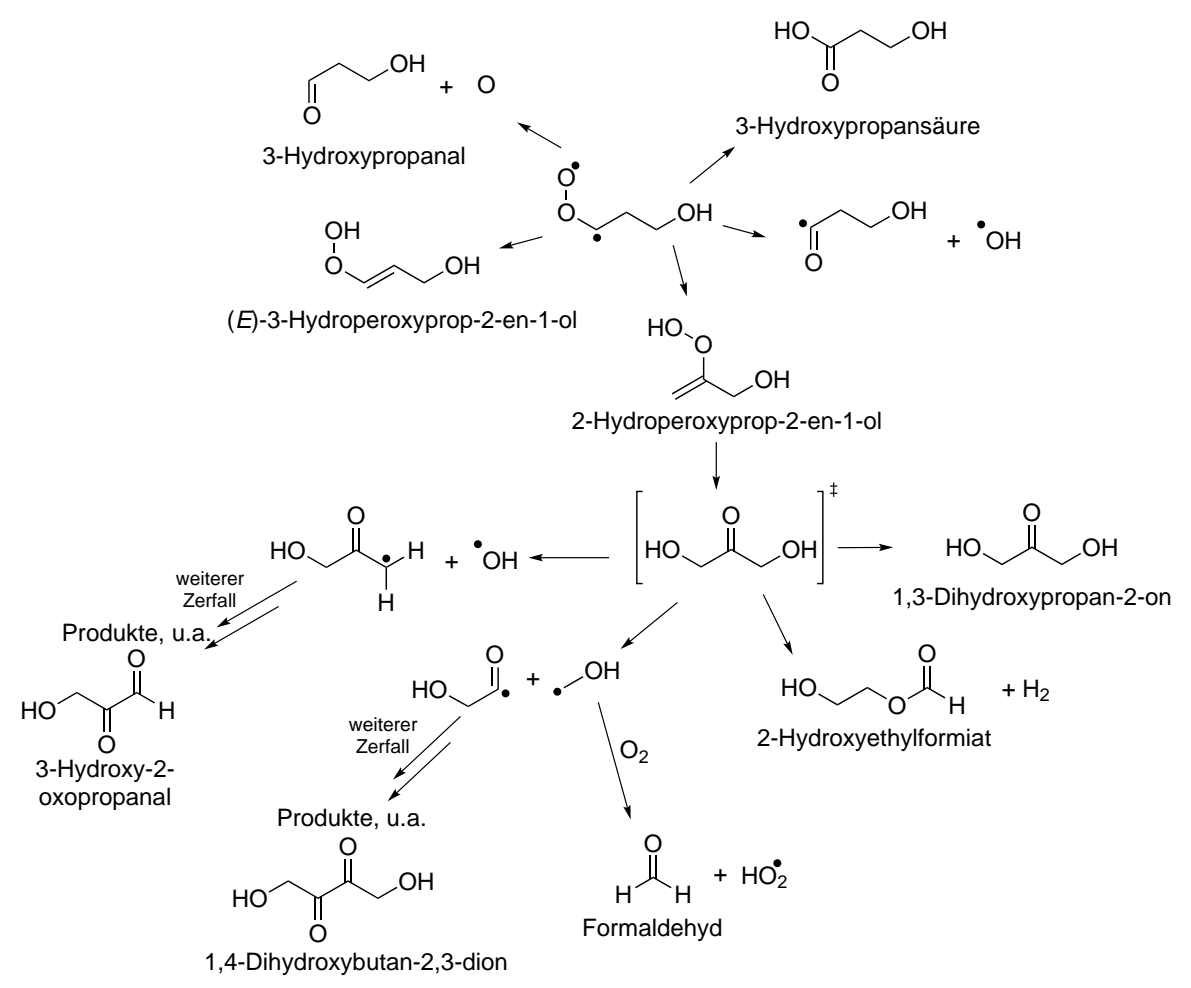

Abb. 2.18.: Ozonolysemechanismus des 3-Buten-1-ol mit unimolekularen Folgeraktionen nach einem Vorschlag von GROSJEAN et al. [65].

O'DWYER et al. [61] schlagen für 1-Penten-3-ol und 1-Penten-3-on Mechanismen vor, deren Folgereaktionen nach Bildung der $\mathrm{Cl}$ ausschließlich unimolekulare Zerfälle bzw. Reaktionen mit Sauerstoffmolekülen des Badgases beinhalten. Dieses untermauern sie durch den FTIR-spektroskopischen 
und gaschromatographisch-massenspektrometrischen Nachweis der stabilen Produkte. Im Fall von 1-Penten-3-ol sind dies neben Formaldehyd auch 2Hydroxybutanal und Propanal (Abb. 2.16). Aus der Ozonolyse von 1-Penten3-on resultieren nach diesen Untersuchungen die Verbindungen Formaldehyd und 2-Oxobutanal (Abb. 2.17).

Für 4-Penten-1-ol und den entsprechenden Aldehyd sind bisher (u.U. da sie bisher nicht in Feldmessungen nachgewiesen wurden) keine Literatur bezüglich des Ozonlysereaktionsverhaltens vorhanden. Für diese werden jeweils nur die ersten Schritte bis zur Bildung der CRIEGEE-Intermediate gezeigt (Abb. 1.5 und (1.6), die als gesichert gelten können. Die weiteren potenziellen Reaktionsschritte sind der Vollständigkeit halber an gleicher Stelle in Anhang $\mathbb{H}$ zu finden.

3-Buten-1-ol wurde bereits als Modellsubstanz zusammen mit und für das biogen emittierte cis-3-Hexen-1-ol von GrosJEAN et al. [65] untersucht. Auch hier haben die Autoren neben dem chromatographischen Produktnachweis einen Vorschlag für die Reaktionswege erarbeitet, der in Abbildung 2.18 gezeigt ist.

\subsection{Die Rolle von Radikalfängern}

Im Rahmen einiger in dieser Arbeit vorgestellten Experimente wurden verschiedene Radikalfänger in die reaktiven Mischungen zugegeben, um die Rolle der während der Ozonolysereaktion gebildeten radikalischen Produkte im Partikelund Produktbildungsprozess zu untersuchen. Die mechanistischen Grundlagen zu diesen Untersuchungen finden sich in den folgenden Abschnitten, geordnet nach der Art des Fängermoleküls.

Durch den Einsatz dieser Substanzen sollen bestimmte radikalische Intermediate in einer Reaktionsfolge abgefangen und somit die sonst aus innen folgenden Reaktionskanäle blockiert werden. Auf diese Weise sollen Experimente in Bezug auf die betrachtete und eigentlich interessierende Hauptreaktion, hier die Ozonolysereaktion ungesättigter Kohlenwasserstoffverbindungen, aussagekräftiger werden, da sie nur noch in geringerem Maße von Nebenreaktionen beeinflusst werden sollte. Auch sollte eine Vereinfachung des Mechanismus folgen, da Nebenkanäle auf diese Weise in der Betrachtung vernachläs- 
sigt werden können. Allerdings ist nicht zu unterschätzen, dass auch die Radikal + Radikalfänger-Reaktion potenziell zu reaktiven und daher für den weiteren Reaktionsverlauf zu beachtenden Produkten führen kann und der Einsatz von eigentlich bestimmte Kanäle blockierenden Substanzen die Interpretation eines beobachteten Effektes deshalb nicht immer vereinfachen muss.

Generell kann eine Vielzahl verschiedener Verbindungen dafür eingesetzt werden, entstehende Radikale in einer reaktiven Mischung abzufangen. Neben gesättigten Kohlenwasserstoffverbindungen oder Alkoholen, wie das im Verlauf der nächsten Kapitel vorgestellte Cyclohexan sowie verschiedene Butanolisomere, ist beispielsweise auch die Verwendung von Kohlenstoffmonoxid möglich, wie in einigen Studien, z.B. denen von O'DWYER et al. oder GUTBROD et al. [61, 66], vorgestellt wurde. Von der Verwendung dieses Stoffes wurde jedoch Abstand genommen, da er nicht nur schon in geringen Konzentrationen hochgiftig ist, sondern auch in den durchgeführten Kinetik- und Produktstudien die Auswertung gebildeter CO-Ausbeuten behindert hätte, die einen wertvollen Hinweis auf Zerfallskanäle innerhalb des Mechanismus liefern können. Auch Wasserstoffperoxid $\left(\mathrm{H}_{2} \mathrm{O}_{2}\right)$ kann zum Abfangen von $\mathrm{OH}$-Radikalen eingesetzt werden [19].

\subsubsection{Cyclohexan}

Die am häufigsten in wissenschaftlichen Arbeiten als Radikalfänger eingesetzte Verbindung ist das Cyclohexan (CHA; s. z.B. [37,40,67,-69]), wobei von der Bildung von Folgeprodukten ausgegangen wird, die keinen Einfluss auf den weiteren Reaktionsverlauf und aufgenommene Messwerte ausüben. Das Hauptprodukt der $\mathrm{C}_{6} \mathrm{H}_{12}+\mathrm{OH}$-Reaktion in Synthetischer Luft ist Cyclohexanon (im Weiteren als $\mathrm{CHON}$ abgekürzt) mit einer Ausbeute von $Y_{\mathrm{CHON}}=0.5$. Die Konzentration dieser Substanz wird daher, gemeinsam mit Cyclohexanol als weiterem Nebenprodukt, oft als Marker für die während der Reaktion gebildeten $\mathrm{OH}$-Radikale benutzt. Wie Abbildung 2.19 zeigt, wird neben dem Hauptprodukt jedoch eine Vielzahl weiterer Intermediate und Zerfallsprodukte gebildet.

Nach einem C-C-Bindungsbruch in $\beta$-Position bezüglich der Radikalfunktion liegt das zuvor zyklische Intermediat linear mit jeweils einer radikalischen und einer aldehydischen Endgruppe vor. Erstere kann unter Sauerstoffanlagerung 
und weiteren Reaktionen mit Peroxyverbindungen oder weiteren Sauerstoffmolekülen in wenigen Reaktionsschritten zur Bildung hochoxidierter Verbindungen führen. Diese weisen mehrere alkoholische oder aldehydische Strukturmotive, aber keine die Instabilität oder Reaktivität erhöhenden Radikalfunktionen mehr auf. So können sie als Endprodukte mit hohen Molekulargewichten und dadurch bedingter reduzierter Flüchtigkeit im Vergleich zur Ausgangsverbindung durchaus in Partikelbildungsexperimenten zur detektierbaren Ausbeute an partikulärer Materie beitragen. Diese Überlegungen werden in den später dargestellten Ergebnissen zur Partikelbildung des 2-Methyl-1-pentens (Kap. 6.4.1.2) von Bedeutung sein. 


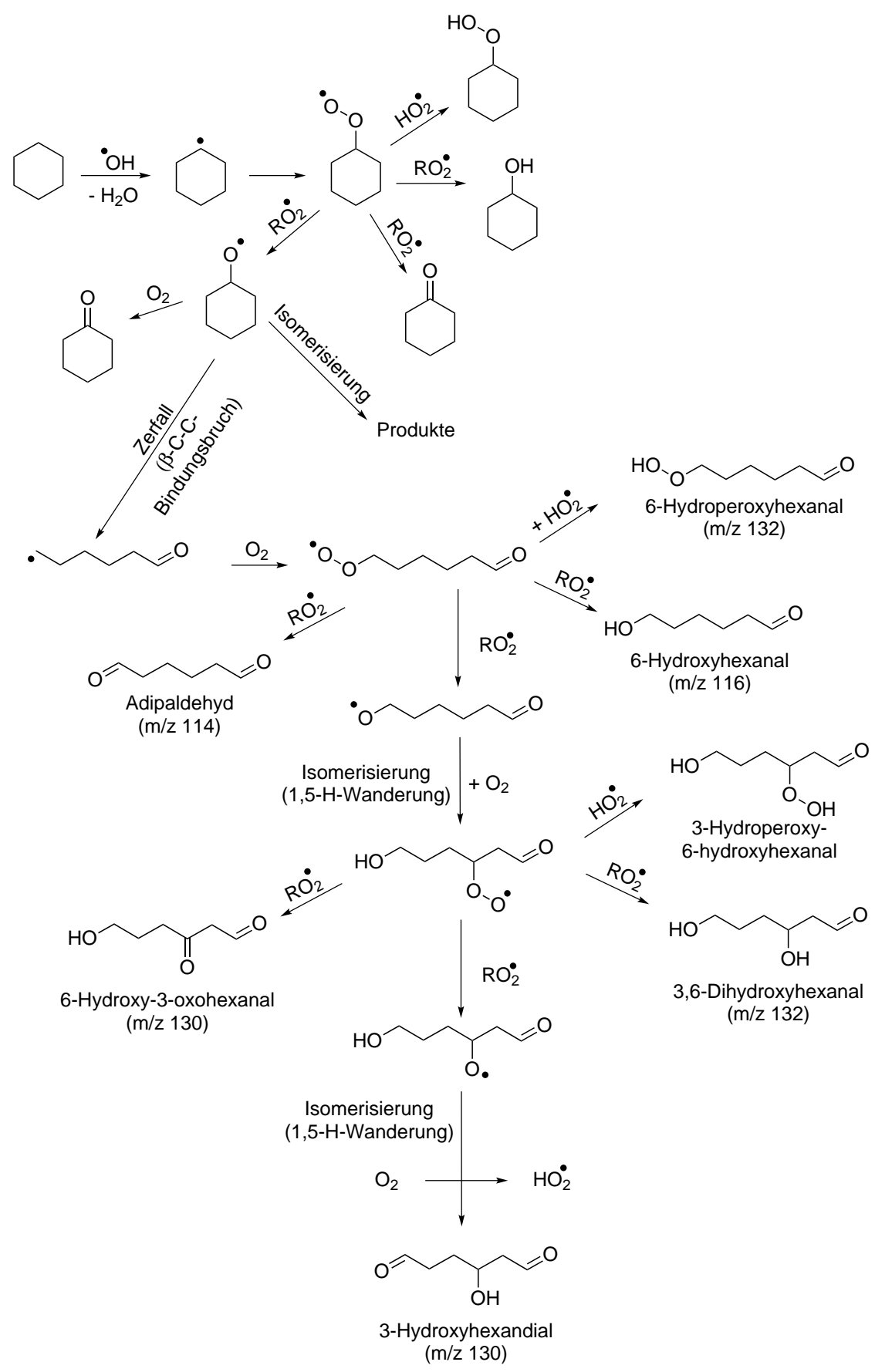

Abb. 2.19.: Folgechemie der $\mathrm{C}_{6} \mathrm{H}_{12}+\mathrm{OH}-$ Reaktion, nach ALAM et al. [40]. 


\subsubsection{Butanolisomere}

Auch verschiedene Butanolverbindungen können zum Abfangen von $\mathrm{OH}-$ Radikalen benutzt werden. Neben tert-Butanol werden auch 1-Butanol (1BuOH, [70]) und 2-Butanol (2BuOH, [55]) eingesetzt, die letzten beiden wurden auch in einigen der später vorgestellten Experimenten verwendet.

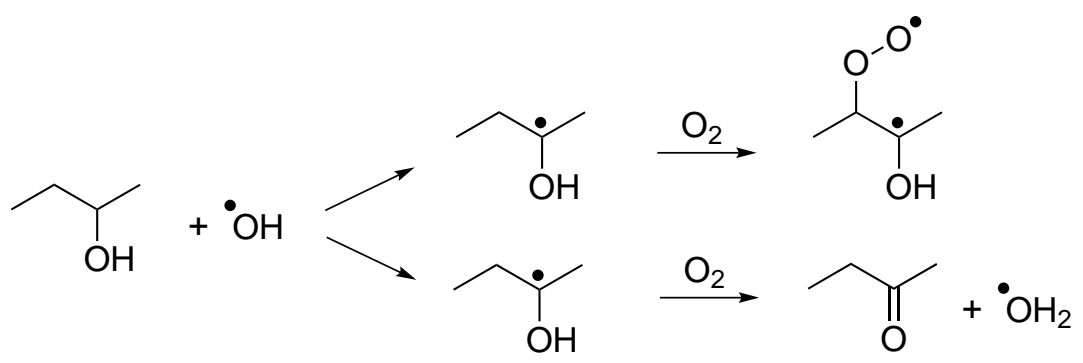

Abb. 2.20.: Folgechemie der 2-Butanol + OH-Reaktion, entnommen aus KEYWOOD et al. [55].

Im Vergleich zur Abfangreaktion durch Cyclohexan entstehen hier Endprodukte mit einem geringeren Molekulargewicht, also auch mit einem höheren Dampfdruck. Daher können diese schwerer kondensieren und sollten bei Partikelmessungen nicht zur nachgewiesenen kondensierbaren Materie beitragen. Bei den besagten Produkten handelt es sich vor allem um das höher oxidierte Butanon. In einer Nebenreaktion werden, wie auch beim Cyclohexan, $\mathrm{HO}_{2}{ }^{-}$ Radikale gebildet, wie in dem von KEYWOOD [55] präsentierten Mechanismus (vgl. Abbildung 2.20) gezeigt ist.

Bei der Verwendung von Alkoholen als Fängermoleküle können diese allerdings noch an anderer Stelle in den Mechanismus eingreifen. Unter anderem ist nach Untersuchungen von BoNN et al. [18] auch die Reaktion von Alkoholverbindungen mit CRIEGEE-Intermediaten möglich, wie sie ebenfalls von TOBIAS und ZIEMANN [71] in der Ozonolyse von 1-Tetradecen beobachtet wurde.

Die Beeinflussung der Messergebnisse durch beide Reaktionen und daraus folgende Nebenprodukte sollte nicht von vornherein ausgeschlossen werden. 


\subsubsection{Wasserstoffperoxid}

In Kenntnis der für Alkohole als OH-Fänger geschilderten Problematik sprachen sich HenRY und Donahue [19] für die Verwendung von Wasserstoffperoxid $\left(\mathrm{H}_{2} \mathrm{O}_{2}\right)$ aus. Am Beispiel der $\alpha$-Pinenozonolyse konnten sie zeigen, dass der Einsatz dieser Substanz, bei dem die Abfangreaktion lediglich $\mathrm{HO}_{2}$-Radikale produziert, die Aerosolausbeute im Vergleich zu Experimenten in Butanolanwesenheit erhöht. Dies lässt sich auf das erhöhte $\mathrm{HO}_{2} / \mathrm{RO}_{2}$-Verhältnis zurückführen, da sich unter vermehrter $\mathrm{HO}_{2}$-Anwesenheit weniger flüchtige und multifunktionale Hydroperoxide zu bilden scheinen als unter $\mathrm{RO}_{2}$-reichen $\mathrm{Be}$ dingungen. Wie bereits in Abschnitt 2.2.2.3 angedeutet, wirkt sich die Veränderung des Radikalpools recht empfindlich auf die Aerosolbildung aus. Daher ist auch die Verwendung von $\mathrm{H}_{2} \mathrm{O}_{2}$ nicht unproblematisch, da es etwa unter Einstrahlung von UV-Licht in OH-Radikale zerfallen kann, was dem Zweck seines Einsatzes entgegensteht.

\subsection{Die Kinetik von Ozonolysereaktionen}

Die Ozonolyse erfolgt nach einer Reaktion zweiter Ordnung, da die Addition der Ozonmoleküle an die Doppelbindung der Kohlenwasserstoffverbindung praktisch irreversibel erfolgt. Es liegt eine Elementarreaktion vor [72]. Eine Reaktion zweiter Ordnung ist von den Konzentrationen beider Reaktanden abhängig, es gilt folglich für die Reaktionsgeschwindigkeit der Reaktion [73]:

$$
\begin{aligned}
& \mathrm{O}_{3}+\text { Alken } \stackrel{k(T)}{\longrightarrow} \text { Produkte } \\
& v=k(T) \cdot c\left(\mathrm{O}_{3}\right) \cdot c \text { (Alken) }
\end{aligned}
$$

Allgemein ergibt sich die Reaktionsgleichung 2.16 unter Beachtung der stöchiometrischen Faktoren der Edukte und unter Beibehaltung des Geschwindigkeitsgesetzes 2.17 zu [48]:

$$
\nu\left(\mathrm{O}_{3}\right) \cdot \mathrm{O}_{3}+\nu(\text { Alken }) \cdot \text { Alken } \stackrel{k(T)}{\longrightarrow} \text { Produkte }
$$


Folgende Größen werden in den Gleichungen verwendet:

- $k(T)$ : temperaturabhängiger Geschwindigkeitskoeffizient der betrachteten Reaktion

- $v=\frac{1}{\nu_{i}} \cdot \frac{\mathrm{d} a}{\mathrm{~d} t}$ : Reaktionsgeschwindigkeit in festem Volumen $V$ und homogener Phase

- $c_{\mathrm{i}}$ : zeitabhängige Konzentration der Komponente $i$

$\cdot t$ : Zeit

- $\nu_{\mathrm{i}}$ : stöchiometrischer Koeffizient (für Edukte definitionsgemäß negativ, für Produkte positiv).

Die in Gl.2.18genannten Produkte umfassen dabei verschiedene Verbindungen und Verbindungsklassen. Neben den resultierenden KohlenwasserstoffSauerstoffverbindungen verschiedener Zusammensetzungen bildet sich bei der Reaktion in der Gasphase auch eine Vielzahl verschiedener Radikale6, die in den Ablauf der Reaktion eingreifen und zusätzlich zum Verbrauch der Edukte beitragen. Um nun den Geschwindigkeitskoeffizienten für die eigentlich betrachtete Alken-Ozon-Reaktion bestimmen zu können, muss der aus der Abnahme der Edukte experimentell ermittelte Gesamtgeschwindigkeitskoeffizient um die radikalischen Nebenreaktionen, durch die die Edukte wie auch entstehende (Zwischen-) Produkte simultan abgebaut werden können, korrigiert werden. Die wichtigsten Nebenreaktionskanäle werden durch die Bildung von $\mathrm{OH}$ - und $\mathrm{HO}_{2}$-Radikalen ermöglicht. Allerdings werden, wie im CRIEGEEMechanismus (vgl. Kap. 2.2) dargestellt, auch weitere Radikale, wenn auch in geringeren Anteilen, gebildet. Dabei sind die Reaktionsgeschwindigkeiten unter Radikalbeteiligung oft um ein Vielfaches höher als die eigentlich betrachtete Ozonolysereaktion, wie in Tab. 2.3 beispielhaft für das biogen emittierte $\alpha$-Pinen und verschiedene relevante Radikalreaktionen gezeigt wird.

In den Kinetikexperimenten ist der Einsatz von Radikal- und Cl-Fängern [59] ebenfalls vielfach üblich, um die weiterführenden Reaktionskanäle zu blockie-

${ }^{6} \operatorname{Im}$ Gegensatz zu der ursprünglichen von CRIEGEE betrachteten Reaktion in der flüssigen Phase, in der die Produkte meist zwitterionischer Natur sind, vgl. Kap. 2.2. 


\begin{tabular}{|c|c|c|}
\hline Reaktion & $\boldsymbol{k}_{\mathbf{2 9 8}} \mathrm{K}\left[\frac{\mathbf{c m}^{3}}{\text { molecules }^{3}}\right]$ & Referenz \\
\hline$\alpha-$ Pinen $+\mathrm{O}_{3} \longrightarrow$ Produkte & $(8.3 \pm 1.3) \cdot 10^{-17}$ & ATKINSON [74] \\
\hline$\alpha-$ Pinen $+\mathrm{OH} \longrightarrow$ Produkte & $(5.3 \pm 1.4) \cdot 10^{-11}$ & ATKINSON [75] \\
\hline $\mathrm{OH}+\mathrm{O}_{3} \longrightarrow \mathrm{HO}_{2}+\mathrm{O}_{2}$ & $(7.3 \pm 1.4) \cdot 10^{-14}$ & ATKINSON [76] \\
$\mathrm{HO}_{2}+\mathrm{O}_{3} \longrightarrow \mathrm{HO}+2 \mathrm{O}_{2}$ & $(2.0 \pm 1.6) \cdot 10^{-15}$ & ATKINSON [76] \\
$\mathrm{H}+\mathrm{O}_{3} \longrightarrow \mathrm{OH}+\mathrm{O}_{2}$ & $(2.8 \pm 1.6) \cdot 10^{-11}$ & ATKINSON [77] \\
\hline $\mathrm{H}+\mathrm{O}_{2}+\mathrm{M} \longrightarrow \mathrm{HO}_{2}+\mathrm{M}$ & $(5.4 \pm 1.3) \cdot 10^{-32} \cdot \mathrm{c}\left(\mathrm{N}_{2}\right)$ & ATKINSON [76] \\
\hline $\mathrm{OH}+\mathrm{Cyclohexan} \longrightarrow$ Produkte & $(7.3 \pm 1.4) \cdot 10^{-11}$ & ATKINSON [78] \\
$\mathrm{OH}+1$ - Butanol $\longrightarrow$ Produkte & $(8.5 \pm 0.4) \cdot 10^{-12}$ & ATKINSON [75] \\
$\mathrm{OH}+2$ - Butanol $\longrightarrow$ Produkte & $(8.7 \pm 1.4) \cdot 10^{-12}$ & ATKINSON [75] \\
\hline
\end{tabular}

Tab. 2.3.: Exemplarischer Vergleich der Geschwindigkeitskoeffizienten für die Ozonolyse- und relevante Radikalreaktionen.

ren und den Geschwindigkeitskoeffizienten ohne Beeinflussung durch Nebenreaktionen zu bestimmen.

Durch diese Nebenreaktionen kann der zusätzliche Verbrauch der Edukte durch verschiedene sekundär gebildete Radikale für die betrachteten Edukte bei experimenteller Bestimmung zu stöchiometrischen Koeffizienten von $\nu_{\mathrm{i}} \neq-$ 1 führen, trotz einer Reaktion zweiter Ordnung mit einem nominellen Reaktandenverhältnis Alken zu Ozon von 1: 1. Zur Quantifizierung dieses Einflusses wird die Größe des relativen Alkenverbrauchs als Quotient aus Alken- und Ozonverbrauch definiert:

$$
\alpha:=-\nu_{\text {Alken }}=\frac{\Delta p(\text { Alken })}{\Delta p\left(\mathrm{O}_{3}\right)}
$$

Im Falle des bevorzugten Verbrauchs der Alkene durch OH-Radikale würde der relative Verbrauch Werte von $\alpha>1$ annehmen, falls $\alpha<1$ bestimmt wird, ist von einem erhöhten Umsatz des Ozons, z.B. durch $\mathrm{HO}_{2}$, auszugehen (vgl. Reaktionsgeschwindigkeiten in Tabelle 2.3/7.

Bei der folgenden Betrachtung nach einem Vorschlag von NEEB et al. [35] wird die Reaktion von $\mathrm{HO}_{2}$-Radikalen mit Ozon vernachlässigt. Es wird statt-

${ }^{7}$ Ozon wird trotz eines höheren Geschwindigkeitskoeffizienten nicht überwiegend von $\mathrm{OH}$ Radikalen abgebaut, da letztere bevorzugt mit dem vorliegenden Alken reagieren. 
dessen davon ausgegangen, dass diese Radikale ausschließlich mit sich selbst reagieren, daher kann der stöchiometrische Koeffizient des Ozons angenommen werden als $\nu\left(\mathrm{O}_{3}\right)=-1$ [48] und es folgt $\alpha:=-\nu$ (Alken).

Der relative Verbrauch $\alpha$ kann experimentell bestimmt werden, indem der Verbrauch von Alken und Ozon gegeneinander aufgetragen werden (s. Abschnitt 5.2.1) und als Ergebnis der Wert der Steigung, $\alpha$, anschließend in die Geschwindikeitsgleichung eingesetzt wird.

Weiterhin wird die Reaktionsvariable $x$ definiert ( [73], S. 167).

$$
x(t):=\frac{1}{\nu_{\mathrm{i}} \cdot V} \int_{t=0}^{t} \mathrm{~d} n_{i}
$$

Mit dieser können die folgenden Stoffmengenbilanzen aufgestellt werden:

$$
\begin{aligned}
c\left(\mathrm{O}_{3}\right) & =c_{0}\left(\mathrm{O}_{3}\right)-x \\
c(\text { Alken }) & =c_{0}(\text { Alken })-\alpha \cdot x \\
& =c_{0}(\text { Alken })-\alpha \cdot\left(c_{0}\left(\mathrm{O}_{3}\right)-c\left(\mathrm{O}_{3}\right)\right) \\
& =\gamma+\alpha \cdot c\left(\mathrm{O}_{3}\right)
\end{aligned}
$$

Innerhalb der vorangegangenen Gleichungen wurde dabei substituiert:

$$
\gamma=c_{0}(\text { Alken })-\alpha \cdot c\left(\mathrm{O}_{3}\right)
$$

Die allgemeine Lösung der sich aus dem Geschwindigkeitsgesetz 2.17]ergebenden Differentialgleichung nach Trennung der Variablen und Partialbruchzerlegung [73] wird in GI. 2.26 angegeben.

$$
\frac{1}{\nu(\text { Alken }) c_{0}\left(\mathrm{O}_{3}\right)-\nu\left(\mathrm{O}_{3}\right) c_{0}(\text { Alken })} \cdot \ln \left(\frac{c_{0}\left(\mathrm{O}_{3}\right)\left(c_{0}(\text { Alken })+\nu(\text { Alken }) x\right)}{c_{0}(\text { Alken })\left(c_{0}\left(\mathrm{O}_{3}\right)+\nu\left(\mathrm{O}_{3}\right) x\right)}\right)=k(T) \cdot t
$$

Unter Berücksichtigung der Stoffmengenbilanzen 2.21-2.24 folgt daraus die explizite Lösung, die auch zur späteren Auswertung der Kinetikmessungen herangezogen wird (s. Kapitel 5.2.1). 


$$
\underbrace{\frac{1}{\gamma} \cdot \ln \left(\frac{c_{0}\left(\mathrm{O}_{3}\right)\left(\gamma+\alpha \cdot c\left(\mathrm{O}_{3}\right)\right)}{c_{0}(\text { Alken }) \cdot c\left(\mathrm{O}_{3}\right)}\right)}_{\text {Lösung „L“ }}=k(T) \cdot t
$$

Durch dieses Vorgehen ist es gleichzeitig möglich, die Ausbeute an $\mathrm{OH}$ Radikalen $\left(Y_{\mathrm{OH}}\right)$ zu bestimmen, welche abhängig vom verwendeten Alken ist. Dazu wird näherungsweise davon ausgegangen, dass die Kohlenwasserstoffverbindung neben Ozon ausschließlich von $\mathrm{OH}$-Radikalen verbraucht wird und gleichzeitig auch die Radikale exklusiv mit den Kohlenwasserstoffen reagieren. Unter diesen Annahmen kann die $\mathrm{OH}$-Ausbeute nach der Gleichung 2.28 aus dem experimentell bestimmten Wert von $\alpha$ berechnet werden (vgl. Abschnitt 6.3.1).

$$
Y_{\mathrm{OH}}=\alpha-1
$$

\subsection{Struktur-Reaktivitäts-Zusammenhang: $S A R$}

In der Theorie der SAR (Structure Activity Relationship) wird die Bedeutung der chemischen Struktur für das (z.B. kinetische) Reaktionsverhalten beschrieben. Auf diese Weise gelingt es, Geschwindigkeitskoeffizienten für Verbindungen durch theoretische Betrachtungen zu erhalten, die den zuvor praktisch gemessenen möglichst genau entsprechen sollen [79]. So kann die anschließende ausschließlich theoretisch erfolgende Bestimmung von Reaktionsgeschwindigkeiten für neue oder nur schlecht praktisch zu vermessende Substanzen Ergebnisse in einem realistischen Größenbereich liefern, wie die Werte in den genannten Arbeiten von MCGILLEN et al. [79, 80] für offenkettige und heteroatomige (u.a. voroxidierte) Alkene zeigen.

Generell wird durch Alkylsubstituenten an der Doppelbindung des Alkens die Reaktionsgeschwindigkeit erhöht, was auf den zunehmenden positiven induktiven Effekt zurückzuführen ist. Gleiches gilt, wenn im Edukt eine Ringspannung enthalten ist, wie im Fünfring des Methylcyclopentens [81].

Auf Grundlage dieses Prinzips ist es möglich, strukturell analoge Verbindungen als Vergleichssubstanzen bei der Untersuchung des Verhaltens während 
der Ozonolysereaktion heranzuziehen, für den Fall, dass für die Verbindung selbst noch keine experimentell bestimmten Literaturwerte verfügbar sind.

Konkret wurde diese Möglichkeit für Methylcyclopenten genutzt, für das mit 1Methylcyclohexen eine intensiv untersuchte Modellsubstanz existiert [48], wobei beim ersteren jedoch, die höhere Ringspannung des Moleküls zu beachten ist. Auch für 2-Methyl-1-penten wurde mit 2-Methyl-1-buten [37] ein Strukturanalogon gefunden.

Weitere Vergleiche werden an an den jeweiligen Stellen in der Auswertung bzw. Diskussion (Kap. 6 bzw. 7) der Experimente gezogen und benannt.

\subsection{Nukleationstheorien}

In diesem Abschnitt sollen die verschiedenen Ansätze zur Interpretation von Nukleationsprozessen vorgestellt werden, um eine Einordnung der im Rahmen dieser Arbeit betrachteten Phänomene vornehmen zu können.

Üblicherweise wird zwischen homogener, heterogener und reaktionskontrollierter Nukleation unterschieden.

- Homogene Nukleation: Hier findet die Partikelbildung, das heißt die Bildung flüssiger (oder fester) Teilchen, aus einer Phase statt. Dies geschieht, wenn z.B. im Falle eines Einkomponentensystems der Substanz „A“ in einer übersättigten Gasphase (Sättigung $S>1$ ) der temperaturabhängige Sättigungsdampfdruck $\left(p_{A}^{0}(T)\right)$ durch den Partialdruck der betrachteten Komponente $A\left(p_{A}\right)$ überschritten wird und folgt Gleichung 2.29 .

$$
S:=\frac{p_{\mathrm{A}}}{p_{\mathrm{A}}^{0}(T)}
$$

Bei diesen Bedingungen müssen sich dann ausreichend viele Moleküle zu einer gemeinsamen Struktur zusammenfinden und stabilisiert werden, um letztendlich einen Partikel zu bilden. Dies ist jedoch aufgrund der sphärischen Form flüssiger Partikel und der Gültigkeit der KELVINGleichung ( [73], S. 421) relativ unwahrscheinlich, da nach dieser nicht nur 
der Sättigungsdampfdruck über gekrümmten Oberflächen größer als über ebenen ist, sondern mit wachsendem Tröpfchenradius auch noch weiter zunimmt. Das bedeutet, dass die Moleküle eines Clusters mit steigender Clustergröße mit wachsender Wahrscheinlichkeit abdampfen, während sie sich mit sinkender anlagern. Dies macht die Bildung stabiler Partikel im messbaren Größenbereich in großer Zahl durch rein homogene Nukleation solange eher unwahrscheinlich, bis ein Grenzdurchmesser erreicht ist, ab dem der Partikel aufgrund des Vorliegens eines metastabilen Zustands stabilisiert wird [10].

- Heterogene Nukleation: Die heterogene Nukleation unterscheidet sich von der vorher beschriebenen durch die Verfügbarkeit von Kondensationskeimen. Hier können ionische Moleküle (z. B. $\mathrm{NH}_{3}, \mathrm{H}_{2} \mathrm{SO}_{4}, \mathrm{HCl}$ ) oder andere stabile Oberflächen, wie etwa schon vorhandene Cluster als „Katalysator" für das Einfangen der Moleküle in der Gasphase dienen. Die entstehenden Partikel sind schon mit kleineren Durchmessern stabil, da etwa bei ionischen Keimen elektrostatische Kräfte wirken. Diese verringern die Verdampfungsrate aus den Partikeln, wodurch auch eine langsamere Aufkondensation eine Durchmesserzunahme ermöglicht und die Nukleation auch einsetzen kann, ohne dass die Gasphase dazu stark übersättigt sein muss [10].

- Reaktionskontrollierte Nukleation: Als reaktionskontrollierte Nukleation wird ein Vorgang bezeichnet, bei dem die Reaktionen leichtflüchtiger Substanzen mit hohem Dampfdruck zu schwerflüchtigen, kondensierbaren Produkten führen. Die Produkte, meist hochoxidierte Verbindungen mit dementsprechend geringem Dampfdruck, die als NIM (nukleationsinduzierende Moleküle) bezeichnet werden, können dann als Partikelkeime wirken. Hier handelt es sich also um eine Unterklasse der homogenen Nukleation, da im Vorfeld eine chemische Reaktion nötig ist, während die beiden erstgenannten Mechanismen auch durch rein physikalische Vorgänge bestimmt werden.

Neben den in Abschnitt 2.2.2.2 genannten Alkoxy- und Peroxyverbindungen kommen bei Betrachtung einer Ozonolysereaktion auch aus Carbonylverbindung und CRIEGEE-Intermediat gebildete Sekundärozonide 
[82] oder die CRIEGGE-Intermediate selbst [48] als Kandidaten für NIM in Frage, ebenso wie die aus $\mathrm{Cl}$ gebildeten Oligomere [36].

\subsection{Der Einfluss von Schwefeldioxid $\left(\mathrm{SO}_{2}\right)$ auf die Ozonolysereaktion}

Wie in Abbildung 2.6 zu sehen ist, kann unter anderem das CRIEGEEIntermediat mit Schwefeldioxid bimolekulare Reaktionen eingehen. $\mathrm{SO}_{2}$ aus biogenen (z.B. vulkanische Gase) und anthropogenen (z.B. Verbrennung fossiler Brennstoffe) Quellen stellt einen Teil der atmosphärischen Spurengase dar, daher soll in diesem Abschnitt der Stand der Forschung zu seinem Einfluss auf die Nukleation zusammengefasst werden.

\subsubsection{Oxidation und Kinetik}

$\mathrm{SO}_{2}$ kann durch atmosphärische Spurengase wie Ozon, $\mathrm{OH}$-Radikale oder CRIEGEE-Intermediate (GI. 2.32) oxidiert werden. Folgende Reaktionen sind in der Literatur bekannt, für die meisten davon wurden Geschwindigkeitskoeffizienten bestimmt.

$$
\begin{array}{rll}
\mathrm{SO}_{2}+\mathrm{O}_{3} & \stackrel{k_{1}}{\rightarrow} & \mathrm{SO}_{3}+\mathrm{O}_{2} \\
\mathrm{SO}_{2}+\mathrm{HO}_{2} & \stackrel{k_{2}}{\rightarrow} & \mathrm{SO}_{3}+\mathrm{OH} \\
\mathrm{SO}_{2}+\mathrm{Cl} & \stackrel{k_{3}}{\rightarrow} & \mathrm{SO}_{3}+\text { Keton / Aldehyd } \\
\mathrm{SO}_{2}+\mathrm{OH} & \stackrel{k_{4}}{\rightarrow} & \mathrm{HOSO}_{2} \\
\mathrm{HOSO}_{2}+\mathrm{O}_{2} & \stackrel{k_{5}}{\rightarrow} & \mathrm{SO}_{3}+\mathrm{HO}_{2} \\
\mathrm{SO}_{3}+\mathrm{H}_{2} \mathrm{O} & \stackrel{k_{6}}{\rightarrow} & \mathrm{H}_{2} \mathrm{SO}_{4} \\
\mathrm{HOSO}_{2}+\mathrm{O}_{2}+\mathrm{M} & \stackrel{k_{7}}{\rightarrow} & \mathrm{HSO}_{5}+\mathrm{M}
\end{array}
$$

Neben der Oxidation zu Schwefelsäure durch Folgereaktionen mit OH-Radikalen und Wasser (Reaktion 2.33-2.35, STOCKWELL-CALVERTMechanismus [87]), die mit den höchsten Geschwindigkeitskoeffizienten die be- 


\begin{tabular}{|c|c|c|}
\hline Reaktion & $\boldsymbol{k}\left[\frac{\mathbf{c m}}{\mathbf{m}} \mathbf{m}\right.$.lecules & Referenz \\
\hline \hline $\mathrm{SO}_{2}+\mathrm{O}_{3}$ & $1.89 \cdot 10^{-22}$ & DEMORE [83] \\
\hline $\mathrm{SO}_{2}+\mathrm{HO}_{2}$ & $2 \cdot 10^{-17}$ & BURROWS [84] \\
\hline $\mathrm{SO}_{2}+\mathrm{Cl}$ & $4 \cdot 10^{-15}$ & JOHNSON [85] \\
\hline $\mathrm{SO}_{2}+\mathrm{OH}$ & $1.3 \cdot 10^{-12}$ & ATKINSON [76] \\
\hline $\mathrm{HOSO}_{2}+\mathrm{O}_{2}$ & $4.3 \cdot 10^{-13}$ & ATKINSON [76] \\
\hline $\mathrm{SO}_{3}+\mathrm{H}_{2} \mathrm{O}$ & $9 \cdot 10^{-13}$ & REINER [86] \\
\hline $\mathrm{HOSO}_{2}+\mathrm{O}_{2}+\mathrm{M}$ & nicht bekannt & - \\
\hline
\end{tabular}

Tab. 2.4.: Geschwindigkeitskoeffizienten der in Gl. 2.30 bis 2.36 gezeigten Reaktionen.

deutendsten sind, kann $\mathrm{SO}_{2}$ auch direkt mit Ozon reagieren und dabei Partikel bilden. Diese Reaktion läuft jedoch bis zu zehn Größenordnungen langsamer als die übrigen ab. Die Radikalkonzentrationen können daher innerhalb der Reaktionsmischung durch die Ozonolysereaktion nicht in dem Maße angepasst werden, um diese Differenz auszugleichen, wie für Ethen [88] und Isopren [67] bereits festgestellt wurde. Daher ist sie in Anwesenheit von $\mathrm{OH}$-Radikalen, die z.B. während der Ozonolyse von Alkenen gebildet werden, nicht konkurrenzfähig und der in Blindproben ohne Alkenzugabe beobachtete Effekt auf die Parti-

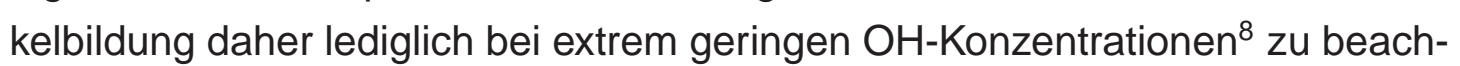
ten.

Wenn die CRIEGEE-Intermediate aus der Ozonolysereaktion ausreichend stabilisiert werden, können sie bimolekulare Reaktionen mit $\mathrm{SO}_{2}$ eingehen, aus denen das höher oxidierte $\mathrm{SO}_{3}$ sowie eine Carbonylverbindung entstehen. Allerdings sind die Angaben der Kenngrößen dieser Reaktionen mit einer großen Unsicherheit behaftet [33], da allein schon die Angaben des Anteils an entstehendem stabilisiertem $\left(\mathrm{C}_{1^{-}}\right) \mathrm{Cl}$ zwischen den einzelnen Untersuchungen stark schwanken. Wie in Tabelle 2.1 (auf Seite 25) exemplarisch gezeigt wurde, schwanken die Vorhersagen zwischen $Y_{\mathrm{C}_{1}}=0.37$ [39] und $Y_{\mathrm{C}_{1}}=0.54$ [40]. Diese Unsicherheit in den Anfangskonzentrationen wirkt sich bei Annahme einer

${ }^{8}$ Dies wäre beispielsweise bei Ozonolyseexperimenten mit hohem Ozonüberschuss und nur geringer Alkenanfangskonzentration der Fall. In durchgeführten Blindproben unter Zugabe von ausschließlich Wasser zur $\mathrm{SO}_{2} / \mathrm{O}_{3}$-Mischung wurden zwar auswertbare Verteilungen beobachtet, diese lagen jedoch bezüglich Anzahl und Masse weit unter der normalen Streubreite der Messwerte. 
Reaktion zweiter Ordnung (analog zur Ozonolyse, vgl. Abschnitt 2.5) direkt auf die Verlässlichkeit der Aussagen zu Reaktionsgeschwindigkeit und Geschwindigkeitskoeffizient der betrachteten Reaktion (s. Gl. 2.17) aus.

Weiterhin bestehen Unterschiede im Reaktionsverhalten der verschiedenen $\mathrm{Cl}$-Konformationen, da theoretische Studien gezeigt haben, dass antisubstituierte $\mathrm{Cl}$ um bis zu fünf Größenordnungen schneller reagieren sollen als ihre syn-Pendants oder disubstituierte $\mathrm{Cl}[33,89,90]$. In einer Studie postulierten LAAKSONEN et al. 2008, dass das in Reaktion 2.36 gebildete $\mathrm{HSO}_{5}$ aufgrund seines höheren Molekulargewichtes ein effektiverer Kandidat für die Rolle eines NIM als Schwefelsäure sein kann [91]. Allerdings wird diese Verbindung nach Aussage der Autoren lediglich oberhalb eines Gesamtdruckes von 80 mbar gebildet und in einer neueren Arbeit von 2010 konnte die Bedeutung der Substanz für die Partikelbildung nicht bestätigt werden [92].

\subsubsection{Auswirkungen von $\mathrm{SO}_{2}$ auf den Ozonolysemechanismus}

Wenn die Ozonolyse von Alkenen in Anwesenheit von Schwefeldioxid abläuft, müssen die Mechanismen der $\mathrm{SO}_{2}+\mathrm{O}_{3}$ - und Alken $+\mathrm{O}_{3}$-Reaktion zusammengeführt werden. Neben der Oxidation durch $\mathrm{OH}$-Radikale werden auch Reaktionen mit weiteren Ozonolyseprodukten, wie organischen Peroxiden, als bedeutsam angenommen [93].

Durch die Eigenschaften des Schwefeldioxids und aus inm entstehenden Folgeprodukten wird das Einsetzen von Nukleation nicht ausschließlich von der Flüchtigkeit der gebildeten Produkte bestimmt. $\mathrm{Da} \mathrm{H}_{2} \mathrm{SO}_{4}$ als Säure wirkt, können sich innerhalb der Cluster Wasserstoffbrückenbindungen ausbilden, so dass die Keime bei kleinerer Molekülanzahl stabiler sein können als durch (homogene) organische Nukleation gebildete. Weiterhin können auf diesen Kondensationskeimen durch Säure-Base-Reaktionen schwer- und mittelflüchtige Ozonolyseprodukte gebunden werden und ein „Einfangen“ organischer Ozonolyseprodukte durch die sauren Eigenschaften der Keime zusätzlich katalysiert werden. Auch hier können stabilisierende zwischenmolekulare Wechselwirkungen für eine effektivere Nukleation sorgen. Beispielsweise wird vermutet, dass 
durch die katalytische Wirkung an den sauren Partikeloberflächen eine Reaktion entstehender Aldehyde zu schwerflüchtigeren Verbindungen wie Carbonsäuren oder eine Oligomerbildung stattfindet [94], was bisher jedoch noch nicht im Detail aufgeklärt werden konnte. Dieser Vorgang würde eine höhere Partikelausbeute bezüglich der detektierbaren Masse bedeuten, allerdings weniger Auswirkungen auf die Anzahl haben. Einen solchen Vorgang, bei dem mittelflüchtige Carbonylverbindungen aus der Gas- in die Partikelphase übergehen, sollte sich anhand der Gasphasenanalyse mittels IR-Spektroskopie nachweisen lassen - die Carbonylbande sollte in Differenzspektren in $\mathrm{SO}_{2}$-Anwesenheit abnehmeng. GARLAND et al. schlagen einen in Abbildung 2.21 gezeigten Mechanismus für diese säurekatalysierte Aldehydreaktion vor [95].

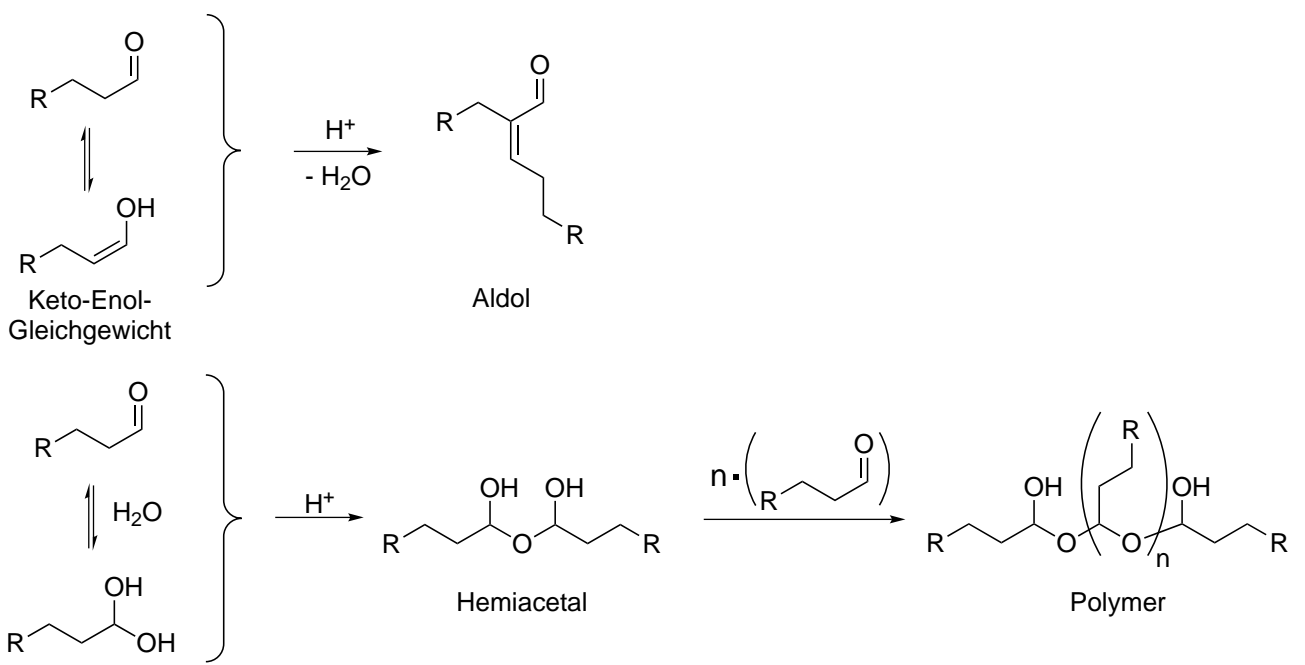

Abb. 2.21.: Die säurekatalysierte Reaktion der Aldehyde, entnommen aus GARLAND et al. [95].

Auch ein Mechanismus, in dem das stabilisierte $\mathrm{Cl}$ das Schwefeldioxid direkt oxidiert ist in der Literatur zu finden, wobei das $\mathrm{SCl}$ durch $\mathrm{SO}_{2}$ im Reaktionsverlauf zu einer Carbonylverbindung reduziert wird [46, 58, 96] (s. Abb. 2.22).

Zusätzlich bleibt eine direkte Reaktion des $\mathrm{SO}_{2}$ mit aus der Ozonolyse entstehenden Intermediaten und Produkten eine denkbare Möglichkeit. BONN et al. berichten in einer neueren Arbeit von der Bildung von Organosulfaten aus der Reaktion $\mathrm{SO}_{2}+\mathrm{SCl}$ und vermuten, dass auch diese Verbindungen als NIM

\footnotetext{
${ }^{9}$ Das experimentelle Vorgehen wird in Kap. 5.2.2 beschrieben.
} 


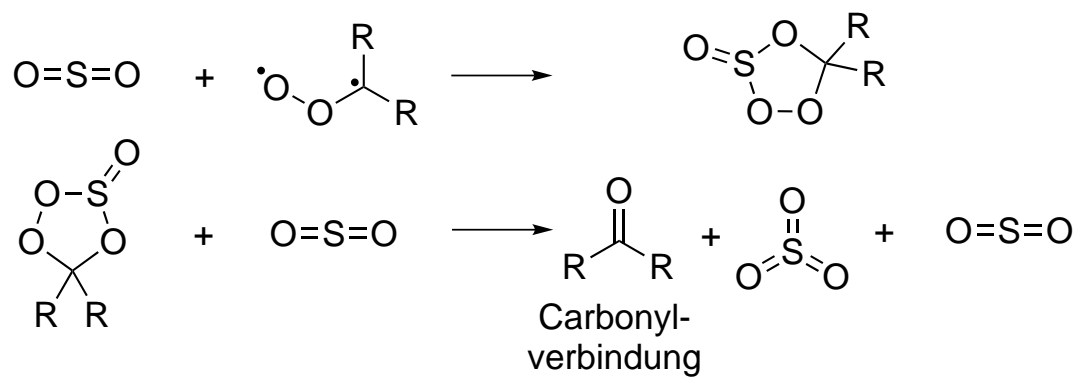

Abb. 2.22.: Reaktion eines stabilisierten CRIEGEE-Intermediates mit $\mathrm{SO}_{2}$, nach HATAKEYAMA et al. [46, 58, 96].

wirken könnten, genauso wie Produkte aus Schwefelsäure und Sekundärozonid. Diese Reaktionswege werden allerdings hauptsächlich für große Alkene an Bedeutung gewinnen, z.B. Sesquiterpenen, bei denen durch bessere Möglichkeiten zur Energieumverteilung im Molekül ein höherer Anteil der CRIEGEEIntermediate stabilisiert werden kann [12]. Aufgrund ihrer niedrigen Dampfdrücke sind jedoch auch Cluster aus organischen Säuren in Verbindung mit Schwefelsäure aussichtsreiche Quellen für Nukleationskeime [22]. 


\section{Versuchsaufbau und experimentelle Methoden}

Im Rahmen der Doktorarbeit von Lennard Wolf [48] wurde die bestehende und im Laufe dieses Kapitels beschriebene Apparatur aus Photolysezelle (PZ), Ozonisator, FTIR-Spektrometer sowie SMPS-Aufbau um eine weitere Messzelle, im Folgenden Aerosolzelle (AZ) genannt, mit Vormischkammern (VMK) ergänzt. In diesem Kapitel werden die einzelnen Teile des Gesamtaufbaus in inrer Funktionsweise beschrieben. Da große Teile schon in meiner 2008 angefertigten Staatsexamensarbeit [15] verwendet und beschrieben wurden, wird sich das folgende Kapitel an diesen Ausführungen orientieren. Dies trifft auch für die experimentellen Abläufe zu, die sich in großen Teilen kaum verändert haben. In den Unterkapiteln folgt die Beschreibung der Hauptbestandteile der Apparatur in einer Reihenfolge, die dem Ablauf während der Experimente entspricht.

\subsection{Der experimentelle Aufbau}

\subsubsection{Ozonerzeugung}

In einem Laborozonisator der Firma Sander (Laborozonisator 301.7) wird durch stille elektrische Entladung zwischen Glas / Aluminium-Elektroden bei einer Spannung von $U=7 \mathrm{kV}$ reiner Sauerstoff $\left(\mathrm{O}_{2} 4.5\right.$, Fa. Air Liquide) in Ozon umgewandelt. Dieses wird auf Silikakugeln in zwei hintereinander geschalteten Kühlfallen, die mit einer Trockeneis / Silikonölmischung auf ca. -40 bis $-50^{\circ} \mathrm{C}$ heruntergekühlt werden, adsorbiert. In der Regel wird der Ozonisator ca. 25 bis 30 Minuten bei 200 Iph (liter per hour bezogen auf $20^{\circ} \mathrm{C}, 1$ bar absolut) 
Sauerstofffluss unter Kühlwasserdurchfluss betrieben, um eine für den Messtag ausreichende Ozonmenge bereitzustellen. Nach Beendigung des Ozonolysevorganges folgt nach einem zehnminütigen Spülvorgang zur Entfernung von Ozonresten aus dem Gerät die Evakuierung und Entfernung des Restsauerstoffs aus dem System. Anschließend lässt sich das Ozon durch Spülen mit Helium (He 4.6, Fa. Air Liquide) wiederum in die Gasphase bringen und kann auf diese Weise über Schlauchverbindungen in die dafür vorgesehene Vormischkammer (s. Abschnitt 3.1.5) eingebracht werden.

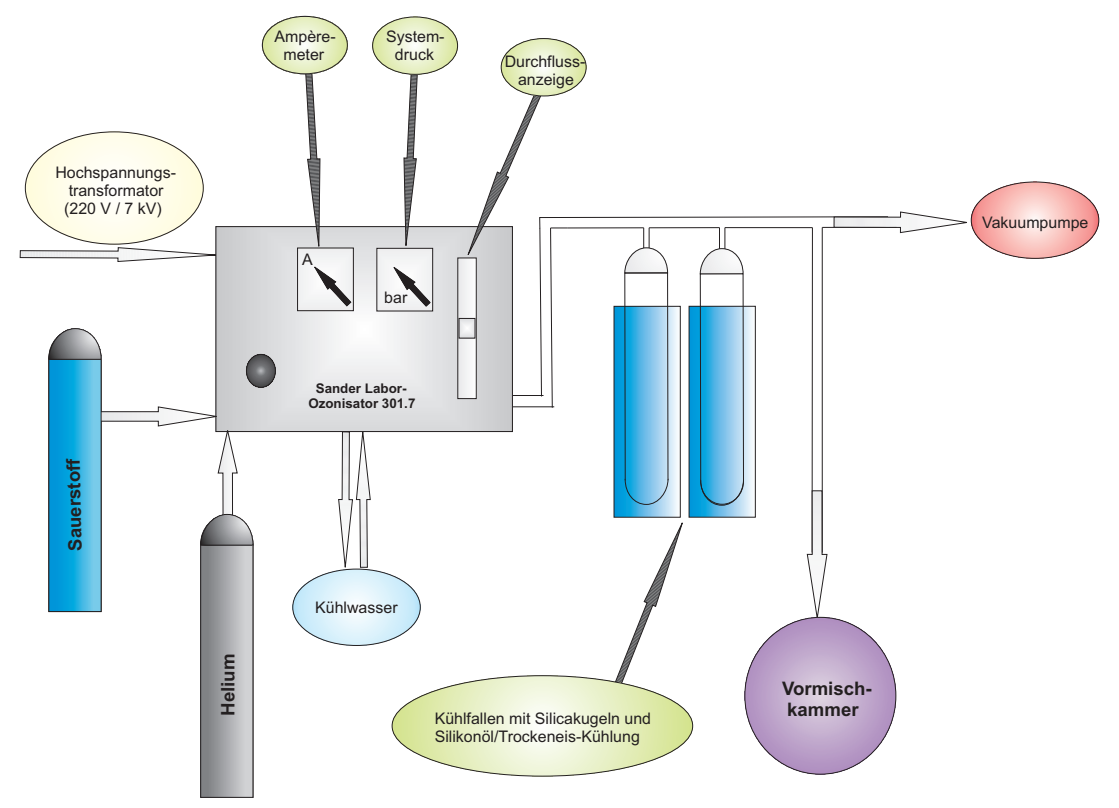

Abb. 3.1.: Schematischer Aufbau des Laborozonisators.

\subsubsection{FTIR-Spektrometer}

Die Aufnahme der IR-Spektren erfolgt über ein IR-Spektrometer (IFS 66, Fa. Bruker). Dieses besteht aus der Infrarotquelle, dem Michelson-Interferometer, Probenraum und Detektor. Die Emission der Infrarotstrahlung erfolgt durch einen Globarstift aus Siliciumcarbid im Bereich von 400 bis $6000 \mathrm{~cm}^{-1}$ ( [29], S. 18). Die Lichtquelle sendet einen Parallelstrahl zum Michelson-Interferometer. Es stehen zwei externe, mit flüssigem Stickstoff gekühlten MCT-Detektoren zur Verfügung $\left(\mathrm{Hg} / \mathrm{Cd} / \mathrm{Te}\right.$, Typ FTIR-16-1.00 mit $1.00 \mathrm{~mm}^{2}$ aktiver Detektorfläche, Wellenzahlbereich 500-5000 $\mathrm{cm}^{-1}$; außerdem Typ MCT-13-2.00 mit $4.00 \mathrm{~mm}^{2}$ 
aktiver Detektorfläche, Messbereich von 570 bis $6000 \mathrm{~cm}^{-1}$, beide Fa. BFI Optilas). Dabei wurde der erstgenannte bei Messungen in der für die Experimente nicht verwendeten Photolysezelle, der andere für Experimente in der Aerosolzelle verwendet. Die Datenaufnahme und Kommunikation mit dem Spektrometer erfolgt mit der Messsoftware OPUS 3.04. Diese Software ermöglicht auch die weiterführende Bearbeitung und Auswertung der Spektren an einem Rechner unter OS/2. Diese Arbeitsschritte bestehen vor allem aus Division und Subtraktion von Spektren, Umwandlung erhaltener Transmissions- in Absorbanzspektren sowie der Integration charakteristischer Banden. Erhaltene Spektren können anschließend als ASCII-Dateien ausgegeben und mit Grafikprogrammen (hier: ORIGIN 8G) weiterverarbeitet werden.

Die Spektrenaufnahme erfolgte unter folgenden Standardeinstellungen:

- Auflösung: $\Delta \tilde{\nu}=1.0 \mathrm{~cm}^{-1}$

- Blende: $5 \mathrm{~mm}$ (PZ) bzw. $3 \mathrm{~mm}(\mathrm{AZ})$

- Messbereich: $600-5000 \mathrm{~cm}^{-1}$

Abhängig vom zu beobachtenden Experiment wurden unterschiedlich viele Interferogramme zu einem Spektrum gemittelt. Bei schnell ablaufenden Reaktionen sind das in der Regel 72 Interferogramme in 30 Sekunden, bei langsameren Reaktionsabläufen, Kalibriermessungen oder gering konzentrierten Reaktionsmischungen in den Partikelbildungsexperimenten wurden 200 Scans in ca. 85 Sekunden durchgeführt und gemittelt.

\subsubsection{SMPS Partikelgrößenverteilungsanalysator}

Die Anzahlgrößenverteilung gebildeter Partikel lässt sich direkt mit dem an die Apparatur angeschlossenen Scanning Mobility Particle Sizer (SMPS, Model 3936, Fa. TSI) bestimmen. Das SMPS stellt eine der genauesten Messmethoden zur Bestimmung von Partikelgrößenverteilungen im Nanometer- und niedrigen Mikrometerbereich dar, ist allerdings ungeeignet für flüchtige Substanzen ( [97], S. 24). Es besteht aus den zwei Hauptkomponenten

- Elektrostatischer Klassierer (Model 3080, Fa. TSI) 
- Kondensationspartikelzähler (Condensation Particle Counter, CPC 3022A, Fa. TS/).

Zur Größenselektion der Aerosole steht ein Differentieller Mobilitätsanalysator (Differential Mobility Analyser, DMA 3081L/3085N, Fa. TSI) zur Verfügung, wobei die lange Trennsäule (DMA 3081L, kurz LDMA) mit einem theoretischen Messbereich von 13-833 nm in der Regel für die Partikelbildungsexperimente, die kurze (DMA 3085N, NDMA genannt, theoretischer Messbereich 4.4 $168 \mathrm{~nm}$ ) dagegen für Experimente mit sehr kleinen erwarteten Partikeldurchmessern, wie etwa Blindproben oder in seltenen Fällen Partikelbildungsexperimente mit sehr kleinen Anfangskonzentrationen, verwendet wurden.

In der Praxis engt sich dieser theoretische Messbereich jedoch abhängig von den Einstellungen für Flussraten sowie Scanzeiten ein, so dass standardmäBig in einem Messbereich von 21.3-777.4 nm (LDMA) bzw. 5.62-205.4 nm (NDMA) gearbeitet wurde. Diese Einschränkung war akzeptabel, da sich die Nachweisgrenzen in der Regel außerhalb der auftretenden Partikelgrößenverteilungen befanden. Dabei betrugen interner Umhüllungsfluss (sheath air bzw. Mantelluft) $2.0 \mathrm{lpm}$, der Probenfluss durch das Verdünnungsverhältnis $10: 1$ $0.2 \mathrm{lpm}$ - das Gerät lief also im low flow mode. Zur Vermeidung experimenteller Artefakte wurden die Scanzeiten, also die Zeit, in denen der CPC die Spannung von theoretisch maximal $U=10-10000 \mathrm{~V}$ und wieder zurück durchläuft, auf $75 \mathrm{~s}$ (up scan time) bzw. $45 \mathrm{~s}$ (down scan time) festgelegt, was in einem praktisch erreichbaren Spannungsbereich von 10 - $9865 \mathrm{~V}$ für den LDMA sowie 11 - $9646 \vee$ für den NDMA und den oben genannten Messbreichen resultierte. Dle Aufnahmezeit für eine Partikelgrößenverteilung betrug also insgesamt 2 min.

Der standardmäßig gewählte 120 s-Takt (75 s up scan-, 45 s down scan time), der die Einengung des theoretischen auf den praktisch möglichen Messbereich bedingte, sorgte einerseits für eine möglichst vollständig erfasste GröBenverteilung und hielt andererseits die Verfälschung der integrierten Gesamtmasse durch die bezüglich des Partikeldurchmessers kubisch gewichteten Schwankungen in der Partikelanzahl minimal. Bei zu kurzen Scanzeiten können experimentelle Artefakte durch Partikelreste vorheriger Messungen auftreten, die im System verbleiben und fälschlicherweise als weitere Verteilung 
aufgenommen und gezählt werden.

Das SMPS (Abb. 3.2) arbeitet ausschließlich bei Atmosphärendruck und Raumtemperatur ( [97], S. 25). Daher wird bei Reaktionen, die bei variablen Drücken unterhalb von 1 bar ablaufen, nach Abwarten der für die jeweilige Substanz nötigen Reaktionszeit zunächst die Zelle mit dem verwendeten Badgas auf einen Druck von $p_{\text {ges }}=1.0 \pm 0.05$ bar gebracht. Nach Befüllen der Zelle mit Badgas auf den aktuell herrschenden Atmosphärendruck wird eine Verbindung zum SMPS geöffnet, während gleichzeitig Stickstoff kontrolliert über einen Flussregler nachströmt ( FC 260, Fa. Tylan, 2 SLM He, wobei SLM für standard liter per minute steht). Als Standardeinstellungen in den hier vorgestellten Experimenten wurde der Zellenregler mit $20.0 \pm 2.0 \%$ (Ist-Wert) betrieben.

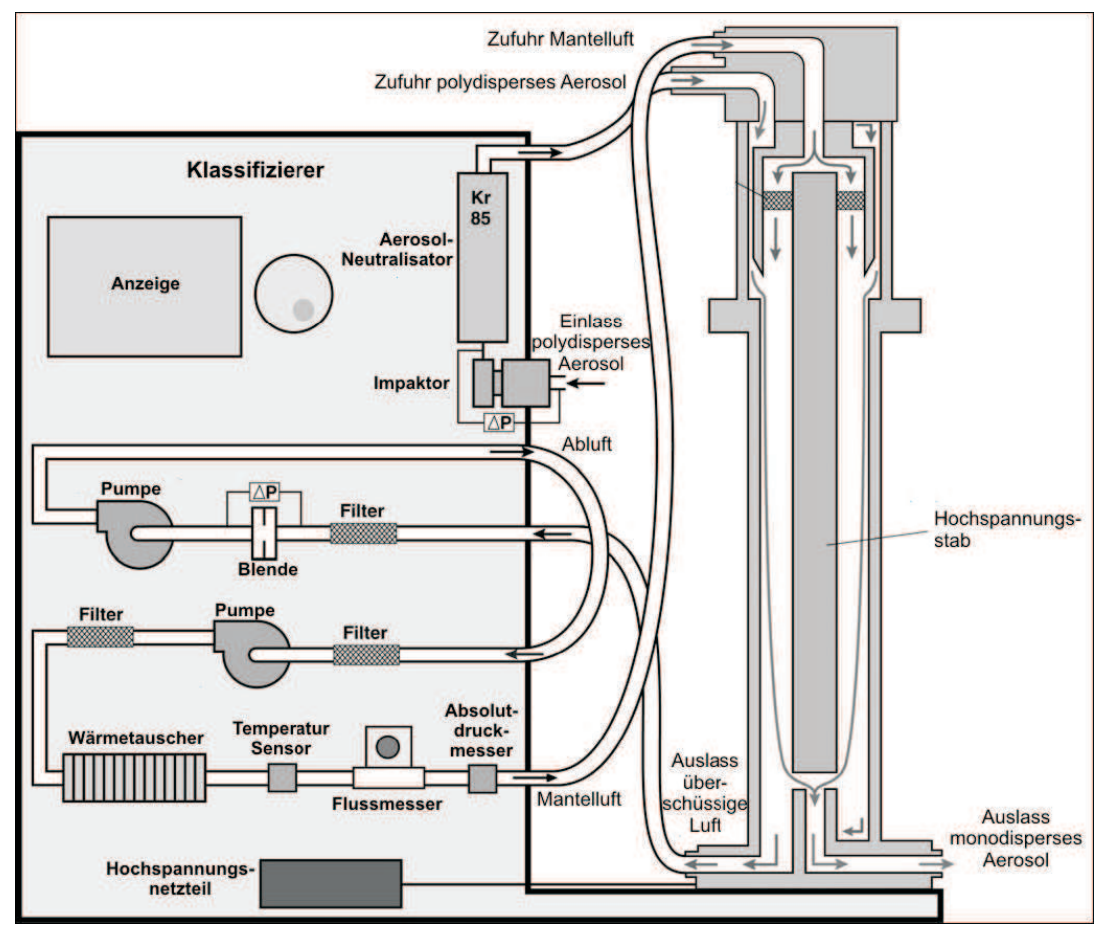

Abb. 3.2.: Schematischer Aufbau des Klassierers 3080L der Firma TSI (Diagramm @TSI Incorporated [98]), nach BRESCH [99], S. 60.

Durch den Einlass gelangt eine polydisperse Partikelprobe, die Durchmesser verschiedener Größenordnungen aufweist, oder monodisperse Nanopartikel, deren Durchmesser innerhalb einer bestimmten Größenordnung liegen, in das Gerät und werden durch eine Düse beschleunigt. Vor und hinter dieser Düse herrscht eine Druckdifferenz, da die im Klassierer integrierte Pumpe 
kontinuierlich arbeitet und so für einen konstanten Gasfluss der Probe sorgt. Dabei werden übergroße Partikel aus dem Luftstrom im Impaktor (Abb. 3.3) mit einem Innendurchmesser von $0.0457 \mathrm{~cm}$ abgetrennt, indem die Trägheit gröBerer Partikel ausgenutzt wird und ausschließlich Aerosol mit Durchmessern unterhalb eines vom Gerät messbaren Maximaldurchmessers mit dem Strom weitergetragen werden. Dies geschieht nach Passage der beschleunigenden Düse durch die Umlenkung des Gasstroms um $90^{\circ}$ mittels der Imp aktorplatte. Dieser Umlenkung können die größeren Partikel wegen ihrer erhöhten Trägheit nicht folgen, wodurch eine fraktionierte Absetzung erreicht werden kann.

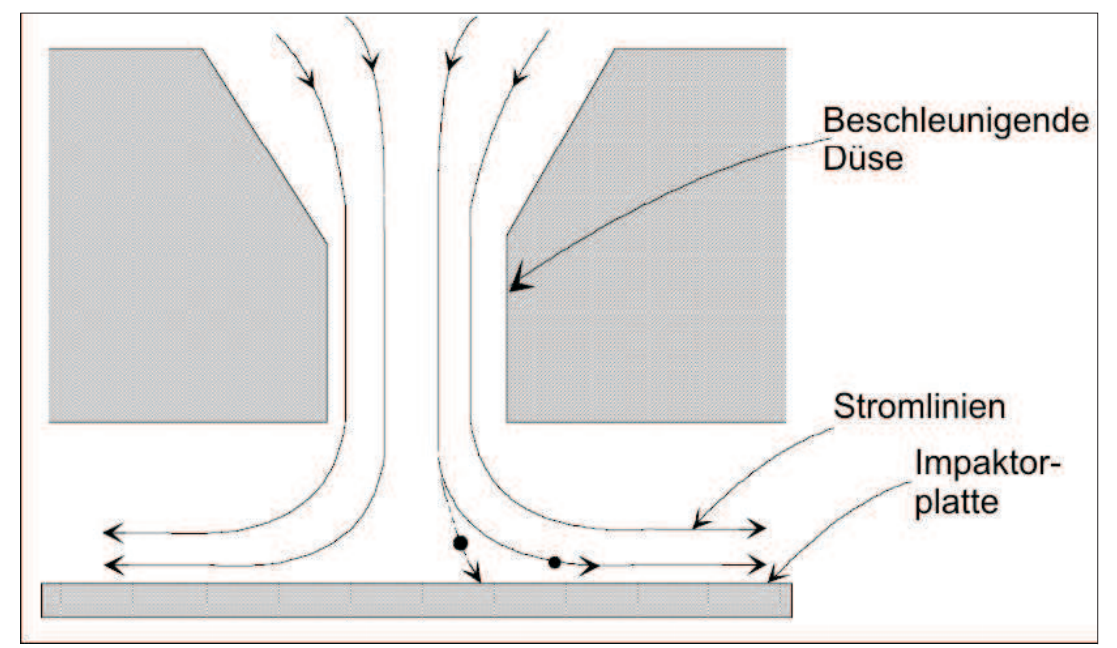

Abb. 3.3.: Funktionsweise des Impaktors, nach BRESCH [99], S. 61.

Nach Durchfluss des Impaktors wird der bipolare Partikelstrom zunächst durch einen ebensolchen Ladungsneutralisator mit ${ }^{85} \mathrm{Kr}$-Strahlungsquelle, einem $\beta$-Strahler, geleitet. Hierbei wird das Aerosol-Trägergas ionisiert, wobei sich als primäre lonisationsprodukte positiv geladene lonen und freie Elektronen bilden. Letztere können durch Gasmoleküle mit hoher Elektronenaffinität, wie zum Beispiel Sauerstoff, aufgenommen werden und zur Bildung negativ geladener lonen führen. Weitere Moleküle, wie z. B. Wasser, können sich an diese zunächst positiv und negativ geladenen lonen anlagern und ionische Cluster ausbilden. Wenn Mobilität und Masse der positiv und negativ geladenen ionischen Cluster vergleichbar sind, sollten sich die Ladungen neutralisieren 
( [100], S. 867 f.)1].

Für das Ladungsniveau der Partikel stellt sich nach genügend langer Zeit sowohl für negative als auch für positive Ladungen ein definiertes Gleichgewicht ein, das in seiner Form dem Boltzmannschen Ladungsgleichgewicht ähnelt und in einen definierten und berechenbaren Anteil positiver bzw. negativer Partikel separiert werden kann, wenn ein elektrisches Feld angelegt wird. Näheres dazu findet sich in der Arbeit von BRESCH ( [99], S. 62).

Nach vollendeter Einstellung eines Ladungsgleichgewichts gelangt der Partikelstrom schließlich in den DMA (Trennsäule, rechts in Abb. 3.2). Dieser besteht aus einem Spannung führenden Stab, der innerhalb eines geschlossenen Gehäuses platziert ist. Dessen elektrisches Feld ist durchstimmbar und an ihm wird der Probenfluss (Sample Flow) im Verhältnis 1: 10 durch den Umhüllungsfluss (Sheath Flow) verdünnt in einer definierten Abwärtsbewegung vorbeigeführt. Dem Gehäuse werden die Partikel entsprechend Abbildung 3.2 von oben in einem definierten Abstand zum Spannung führenden Stab zugeführt. Durch die auf die Partikel wirkende Schwerkraft sowie den Gasfluss durch den Sheath Flow bewegen sich diese von oben nach unten am Hochspannungsstab vorbei, wobei positiv geladene Teilchen abhängig von Ladung und Durchmesser sowie Viskosität des verwendeten Badgases und der mittleren freien Weglänge der Partikel zu diesem Stab hin beschleunigt werden?2.

Im Anschluss an die Selektion nach elektrischer Mobilität werden nur gröBenselektierte Partikel eines bestimmten Bereichs an den CPC (Abb. 3.4) weitergeleitet, der zum Nachweis der Nanopartikel dient.

Potenziell wäre die Detektion von Partikelproben durch ein Transmissionselektronenmikroskop (TEM) möglich, wozu die Proben allerdings abgeschieden werden müssen, was eine in situ-Untersuchung zeitabhängiger Effekte ausschließt. Auch der Nachweis, bei dem geladene Partikel auf eine Anode gelenkt und der Strom mit einem Pikoamperemeter gemessen wird, wäre möglich. Dieser ist aber störanfällig auf Umgebungsluft und detektiert lediglich elektrisch geladene Teilchen ( [99], S. 66 f.). Eine

\footnotetext{
${ }^{1}$ Genaueres zur Theorie der bipolaren Ladungsverteilung ist in der Arbeit von WIEDENSOHLER beschrieben ([101], S. 387-389).

${ }^{2}$ Zur theoretischen Berechnung der elektrischen Mobilität der Partikel, der vom DMA selektierten Partikel in Abhängigkeit von Durchmesser und Ablenkspannung sei ebenfalls auf BRESCH verwiesen ( [99], S. 64-66).
} 


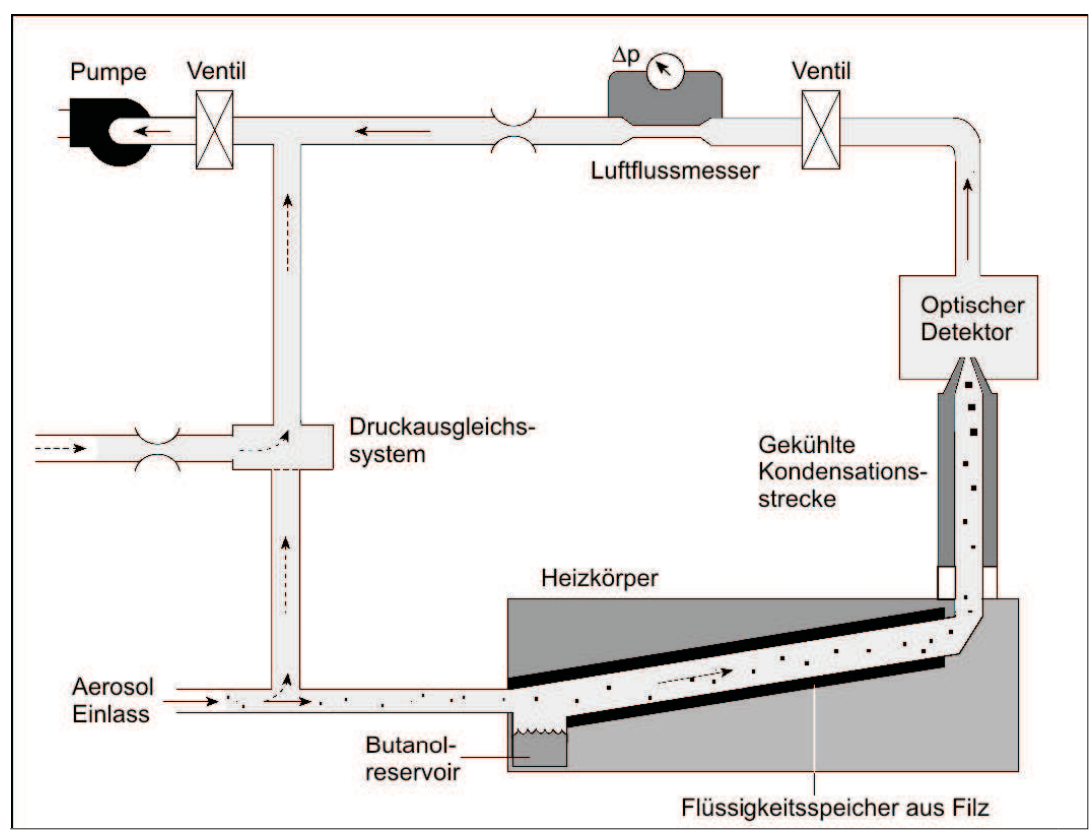

Abb. 3.4.: Schematischer Aufbau des 3022A CPC der Firma TSI (Diagramm (CTSI Incorporated [102]), nach BRESCH [99], S. 67.

dritte Möglichkeit, die in dem hier vorgestellten Experiment auch realisiert wird, besteht in der Detektion der Partikel über Lichtstreuung. Da mit dieser Methode jedoch Nanopartikel mit einem Durchmesser von weniger als $1 \mu \mathrm{m}$ nur schlecht erfasst und die Partikelgrößenverteilung nur unzureichend charakterisiert würde, werden die Partikel im so genannten Sättiger (saturator) bei $35^{\circ} \mathrm{C}$ einer mit $n$-Butanol gesättigten Atmosphäre ausgesetzt. In dieser Umgebung wirken die Partikel als Kondensationskeime, es setzt sich Flüssigkeit auf innen ab, wobei sie auf $\mu \mathrm{m}-\mathrm{Größe}$ anwachsen, wenn sie nach dem Sättiger durch einen Kühler (condensator) mit einer Temperatur von $10^{\circ} \mathrm{C}$ geleitet werden, in dem die dann übersättigte Butanolatmosphäre größenunabhängig auf den Partikeln kondensiert. Das CPC auf Butanolbasis ist eher für Nanopartikel mit einem Durchmesser im Bereich von 10-500 nm geeignet ( [99], S. 67). Die Partikel können so schließlich durch Laserstreuung detektiert und gezählt werden, wobei dieser Bereich des CPC auf $36^{\circ} \mathrm{C}$ beheizt ist, damit sich kein Butanol auf den Optikbauteilen niederschlagen kann3. Das Ergebnis ist

${ }^{3}$ Zur genaueren Funktionsweise und theoretischen Grundlagen s. ebenfalls BRESCH [99], S. $67 \mathrm{f}$. 
eine Zuordnung zwischen Partikelgröße und -anzahlkonzentration, die sich in den meisten betrachteten Fällen bei ausreichend hohen Partikelanzahlen als Lognormalverteilung darstellt.

Die Steuerung des SMPS erfolgte über einen Computer unter Windows XP mit dem Programm AIM (Aerosol Instrument Manager). Die Ausgabe besteht aus einer tabellarischen Auflistung und liefert die Partikelanzahlgrößen- und Flächenverteilung (unter der Annahme, dass die Partikel sphärisch sind) sowie eine Volumen- bzw. Massenverteilung (unter der Annahme einer homogenen Dichte der Partikel von $\rho_{\text {Partikel }}=1.2 \mathrm{~g} \cdot \mathrm{cm}^{-3}$ ). Je nach Auswahl einer Messgröße kann ein Spektrum angezeigt werden, wobei eine Statistiktabelle Integralwerte der Verteilungen enthält, womit z.B. die Partikelgesamtzahl und andere Kenngröße der Verteilung, wie Mediane und geometrische Standardabweichungen, geliefert werden.

\subsubsection{Die Aerosolzelle}

Beim Reaktor handelt es sich um eine doppelwandige temperierbare Edelstahlkugel, die innere und äußere Schale wurde jeweils aus zwei Halbkugelschalen (VA-Edelstahl) von $4 \mathrm{~mm}$ bzw. $5 \mathrm{~mm}$ Dicke verschweißt, wobei die Innendurchmesser $48.5 \mathrm{~cm}$ bzw. $54.3 \mathrm{~cm}$ betragen. Der Raum zwischen den Kugelschalen kann mittels Swagelok-Einlässen $(\varnothing 10 \mathrm{~mm})$ an der unteren Hälfte sowie Auslässen ( $\varnothing 14 \mathrm{~mm}$ ) an der oberen Hälfte der Außenschale mit WasserGlykol-Mischungen gefüllt und über einen Kryostaten (Typ WK 500, Fa. Lauda, effektiver Temperaturbereich $10-30^{\circ} \mathrm{C}$ ) temperiert werden. An der Unterseite der Zelle befindet sich ein $30 \mathrm{~cm}$ ISO-K-Flansch mit O-Ring-Vitondichtung, der mit jeweils zwei DN-16-KF- und DN-25-KF-Flanschen versehen ist. Mittels zweier Durchgangshebelventile kann die Zelle mit der angeschlossenen Drehschieberpumpe (D16BCS, Fa. Leybold, Endvakuum etwa 0.03 mbar, Leckrate $<3.10^{-4} \mathrm{mbar} \mathrm{L} \mathrm{s}^{-1}$ ) verbunden werden. In einem der beiden Kleinflansche ist ein $\mathrm{Ni}$-CrNi-Thermoelement installiert, der andere ist mit zwei Druckmessköpfen für den Hochdruck- bzw. Niederdruckbereich versehen (1000 mbar bzw. 10 mbar Baratron, Fa. MKS). An der Oberseite der Zelle befindet sich ein $16 \mathrm{~cm}$ ISOK-Flansch mit jeweils drei integrierten DN-16-KF- und DN-25-KF-Flanschen. Über zwei Eckventile können die zu untersuchende Substanz über eine Ro- 
tulexverbindung aus Glaskolben bzw. "vom Normalfall abweichende" Badgase (wie reiner Sauerstoff, Schwefelhexafluorid, Helium) direkt in die Zelle eingefüllt werden. Ein Hebeleckventil (DN-16-KF) dient zur Befüllung der Zelle mit Stickstoff als Badgas, eines der größeren (DN-25-KF) hat die gleiche Funktion bei der Verwendung von Synthetischer Luft. Zwei weitere große Hebeleckventile zu beiden Seiten des Badgaseinlasses für Synthetische Luft dienen dazu, die Inhalte der Vormischkammern möglichst schnell und unter gründlicher Durchmischung in die Reaktionszelle zu expandieren.
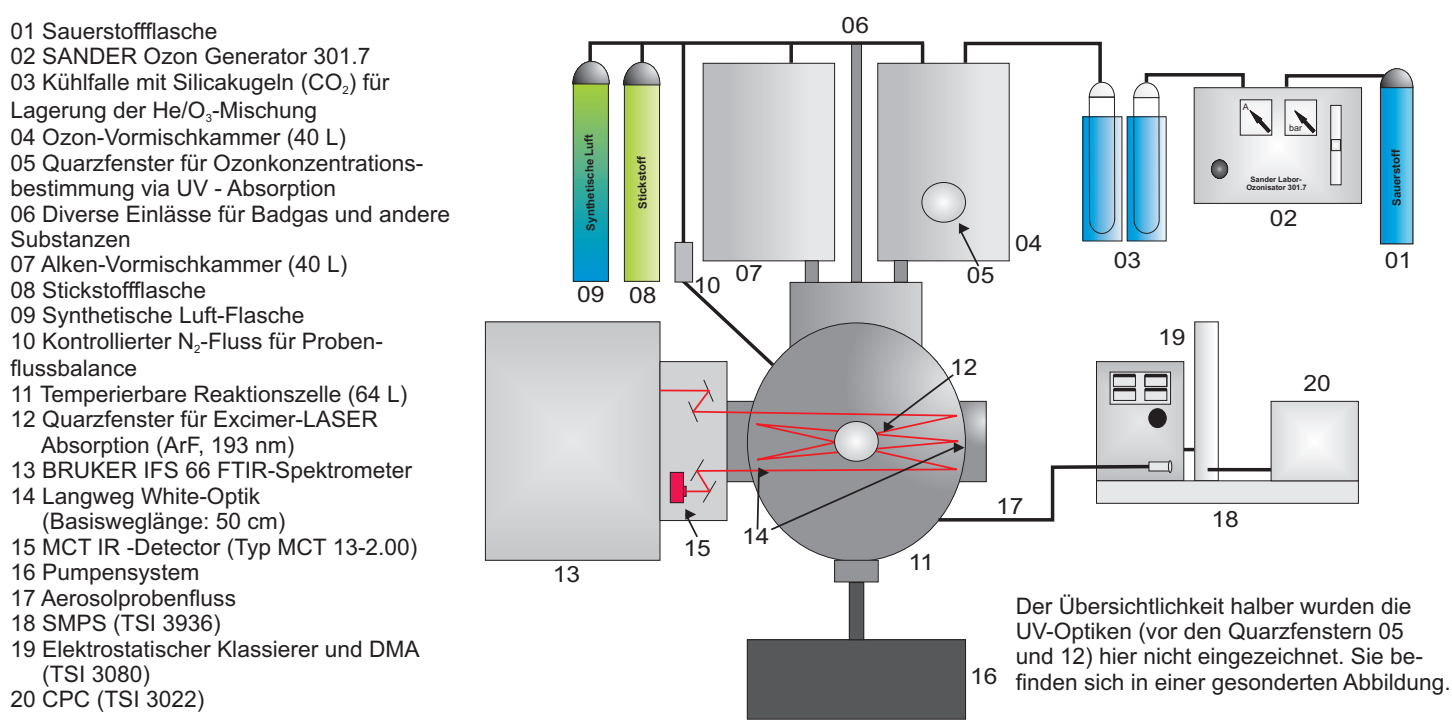

Abb. 3.5.: Schematischer Aufbau der gesamten Apparatur, modifiziert nach WOLF [48], S. 47.

Auf halber Höhe der Reaktionszelle befinden sich parallel zur Orientierung der Vormischkammern zwei gegenüberliegende $8 \mathrm{~cm}$ ISO-K-Flansche, in die Quarzfenster ( $\varnothing 50 \mathrm{~mm}, \varnothing_{\text {effektiv }} 40 \mathrm{~mm}, F a$. Linos) eingebaut sind. Durch diese kann die Strahlung einer Hg-Dampflampe zur Ozonkonzentrationsbestimmung eingekoppelt werden 4 . Auf gleicher Höhe befinden sich orthogonal zwei ISOK-Flansche $(16 \mathrm{~cm})$, auf denen die von außen durch Schrauben justierbaren Spiegel für die WHITE-Optik installiert sind. Im $45^{\circ}$-Winkel zwischen diesen Flanschen sind vier DN-16-KF-Flansche an der Zelle angebracht. Zwei einander gegenüberliegende sind mit Hebeleckventilen ausgestattet und dienen der

${ }^{4} \mathrm{Da}$ ein identischer Aufbau zur Ozonkonzentrationsbestimmung an der OzonVormischkammer angebracht ist, wird im Abschnitt4.2.1 näher auf diesen eigegangen. 
Probennahme über eine Verbindung zum SMPS bzw. dem durch einen Flusszähler (FC 260, Fa. Tylan, 2 SLM He) definierten Nachströmen eines Stickstofflusses zum Druckausgleich für die entnommene Gasmenge. Dabei ist die Verbindung der Zelle zum SMPS über einen möglichst kurzen Gummischlauch hergestellt, was einen schnellen Start der Aerosolmessung sowie eine Verringerung der Depositionsverluste auf dem Weg ermöglicht. Die restlichen zwei bisher ungenutzten Kleinflansche können z.B. für den Anschluss eines Hygrometers oder eines kommerziellen Ozonanalysators genutzt werden.

Die gesamte Zelle ist inklusive aller Flansche mit einer Schichtdicke von etwa $35 \mu \mathrm{m}$ tefloniert (TempCoat 1505, Fa. Impreglon) worden, um Wandverluste des Aerosols zu minimieren sowie die Adsorption von Reaktanden und anschließende unkontrollierte Nachreaktion zu verhindern. Das gesamt Kugelvolumen beträgt $64.0 \pm 0.5 \mathrm{~L}$.

\subsubsection{Die Vormischkammern}

Die Reaktion zwischen ungesättigten Kohlenwasserstoffverbindungen und Ozon läuft auf kurzen Zeitskalen ab. Aus diesem Grund ist es wichtig, die Edukte schnell und unter Gewährleistung einer zügigen Durchmischung im Reaktionsbehälter zusammenzuführen, um lokale Konzentrationsgradienten $\sqrt{5}$ möglichst gering zu halten. Zu diesem Zwecke besitzt die Apparatur zwei Vormischkammern $\left(V_{\mathrm{VMK}}=40.5 \pm 0.3 \mathrm{~L}\right.$ ), in denen die Reaktanden bereitgestellt und durch Öffnen der Verbindungen schnell und unter effektiver Durchmischung in die Reaktionszelle eingebracht werden können. Durch die Einstellung eines bestimmten Vordrucks in den Vormischkammern kann gleichzeitig der Gesamtdruck während der Reaktion innerhalb der Zelle festgelegt werden. Eine definierte Befüllung der Aerosolzelle ist unter der Berücksichtigung des Volumenverhältnisses der Vormischkammern zur Aerosolzelle (vgl. Gl. 4.3 in Abschnitt 4.2.1.2) durch Expansion innerhalb von $4 \mathrm{~s}$ in einem Druckbereich von ca. 10 - 460 mbar möglich. Bei Experimenten oberhalb dieses Druckes musste

${ }^{5}$ Das Hauptmerkmal der hier beschriebenen Einfüllmethode stellt die rasche Befüllung der Zelle dar, wodurch auch die Reaktionszeit unter einem großen Druckgradienten minimiert wird - dies ist besonders unter dem Gesichtspunkt der gesamtdruckabhängigen Partikelausbeuten, die im Ergebnisteil beschrieben werden, von großem Vorteil. 
die Zelle dann zusätzlich mit dem entsprechenden Badgas direkt aus der Flasche befüllt werden, was bei einem Solldruck von 1 bar maximal $20 \mathrm{~s}$ dauert. Eine der Vormischkammern ist wie der Reaktionsbehälter teflonbeschichtet und mit $8 \mathrm{~cm}$ ISO-K-Flanschen mit Quarzfestern zur Ozonkonzentrationsbestimmung via UV-Optik und Hg-Dampflampe ausgestattet (diese wird in Abschnitt 4.2.1.2 beschrieben). An der Oberseite beider Kammern befinden sich DN-25KF-Flansche, an denen durch Verzweigungsrohre Anschlussmöglichkeiten für Bauteile wie elektronische Druckmessköpfe (Alkenvorkammer: 10 mbar Baratron, Ozonvorkammer: 100 mbar Baratron, beide Fa. MKS), Federmanometer für 1 bar Gesamtdruck sowie eine Nachströmmöglichkeit für Stickstoff über den Flusszähler bei Betrieb des Ozonanalysators bestehen. An der Unterseite jeder Kammer befinden sich zwei DN-16-KF-Flansche. Über die daran installierten Eckventile können die jeweiligen Badgase bis zu einem zugelassenen Maximaldruck von 1 bar eingefüllt werden. Über eine weitere KleinflanschEckventil-Verbindung kann das Ozon / Helium-Gemisch in die Ozon-VMK bzw. über eine Kleinflansch-Rotulex-Verbindung leichtflüchtige ungesättigete Kohlenwasserstoffverbindungen oder andere Additiva (Radikalfänger) direkt aus den Glaskolben in die Alken-VMK eingegeben werden, wobei die eingegebenen Konzentrationen volumetrisch über den Druckanstieg bestimmt werden. Beide Vormischkammern sind über Wellblechschläuche (DN-25-KF-Flansch) mit der Aerosolzelle verbunden, so dass eine Expansion aus den Vorkammern sowohl simultan aus beiden Vorratsbehältern oder einzeln in beliebiger Reihenfolge erfolgen kann. Seitlich sind die Kammern über identische FlanschWellblechschlauch-Verbindungen an das Pumpsystem angeschlossen, so dass alle Teile der Gesamtapparatur unabhängig voneinander evakuiert werden können.

\subsection{Die Durchführung verschiedener Experimentvarianten}

In diesem Abschnitt wird zunächst das allgemeine Vorgehen bei einem Experiment geschildert, anschließend folgt die Beschreibung der einzelnen Varianten. 


\subsubsection{Allgemeines Vorgehen}

Zu Beginn jedes Messtages wurde zunächst, wie in Abschnitt 3.1.1 beschrieben, Ozon hergestellt und der Aufbau für 10 Minuten passiviert. Dazu wurde in Ozon-Vormischkammer und Aerosolzelle etwa 70 mbar Ozon / HeliumGemisch eingegeben (entspricht einem Ozonpartialdruck von etwa 150$200 \mathrm{~Pa}$ ), um eventuell vorhandene Substanzreste vorheriger Messungen oxidativ zu entfernen. Dieser Vorgang wurde bei der Verwendung von voroxidierten Kohlenwasserstoffverbindungen auch zwischen den Partikelbildungsversuchen wiederholt, da diese Verbindungen durch ihre reduzierte Flüchtigkeit potenziell schwieriger über reine Spülvorgänge aus den System zu entfernen waren und reproduzierbare Anfangsbedingungen geschaffen werden sollten. Zwischen Produkt- bzw. Kinetikmessungen oder bei Verwendung von nicht voroxidierten Kohlenwasserstoffverbindungen wurde nicht passiviert, da bei ersteren lediglich IR-Spektren aufgenommen wurden und letztere durch Spülen mit Badgas und Abpumpen ausreichend gut aus dem System zu entfernen waren.

Generell stand neben der Ozonvormischkammer auch ein analoger Behälter zur Verfügung, in dem die verwendeten organischen Edukte für die Versuche aus einem Glaskolben über Kleinflansch-Rotulexverbindungen vorgelegt werden konnte (Alken-VMK). Diese Möglichkeit wurde allerdings lediglich bei Messungen mit nicht voroxidierten Komponenten genutzt, da nur von diesen aufgrund des hohen Dampfdruckes problemlos ausreichend große Substanzmengen eingefüllt werden konnten 6 . Auf Grund des niedrigen Dampfdrucks der verwendeten voroxidierten Komponenten wurden diese nicht in die Vormischkammern, sondern direkt in die Aerosolzelle eingebracht. Dazu wurde zunächst ein Glaskolben mit der Substanz befüllt, dieser an die Zelle angeschlossen und die Atmosphäre über der Flüssigkeit abgepumpt. So konnte bis zum Beginn der Messungen innerhalb des Kolbens wieder genug Substanz in

\footnotetext{
${ }^{6}$ Aufgrund des Volumenverhälnisses zwischen Vorkammer und Aerosolzelle konnte höchstens das 0.26-fache der in der VMK eingefüllten Substanz in die AZ übergehen (vgl. GI,4.3), weshalb fast das Vierfache der später im Experiment gewünschten Substanzmenge vorgelegt werden musste. Dies wäre bei Substanzen mit geringer Flüchtigkeit (z.B. Carbonsäuren) nur mit extrem langen Befüllzeiten und damit verbundenen Unsicherheiten durch Leckrate o.ä. zu erreichen. Außerdem ist die Alkenvormischkammer nicht mit einer Teflonbeschichtung versehen, was vor allem bei der Verwendung von Carbonsäuren die Entfernung aus dem System schwieriger gestaltet.
} 
die Gasphase übergehen und den Sättigungsdampfdruck erreichen, um auch mit den schwerflüchtigeren Carbonsäuren höhere Konzentrationen in der Reaktionszelle zu erzielen, wenn dies gewünscht war. Ein weiterer Vorteil dieses Vorgehens ist der geringere Substanzverbrauch als beim Einfüllen über die Vormischkammer. Die Verunreinigung der Dampfphase im Kolben durch eventuell eingedrungene Raumluft ist grundsätzlich als so gering anzusehen, dass der Druckanstieg in der Zelle durch Einlass aus dem Kolben mit dem Anfangspartialdruck der Substanz in der Reaktionsmischung gleichgesetzt werden kann.

Nachdem die Reaktionszelle mehrfach mit dem gewählten Badgas gespült und wieder evakuiert worden ist, werden mit dem Infrarotspektrometer Leerkanalspektren für den späteren Reaktionsdruck aufgenommen $\left(p_{\text {ges }}=10\right.$ 1000 mbar, immer 200 Scans gemittelt). Während der Aufnahme wird die Ozonvormischkammer mit der gewünschten Konzentration und dem für die Reaktion nötigen Gesamtdruck präpariert, die Alkenvormischkammer wird nach eventuellem Befüllen mit dem verwendeten organischen Edukt mit Badgas auf den gleichen Gesamtdruck aufgefüllt. Nach erneuter Evakuierung der Aerosolzelle bis auf den Enddruck (ca. $p_{\text {ges }}=0.05$ mbar) wird der gewünschte Eduktpartialdruck (falls die Vormischkammer nicht verwendet wird) direkt in die Zelle eingegeben und der Inhalt beider Vormischkammern (in diesem Fall ist die Alken-VMK lediglich mit Badgas befüllt, um gleiches Durchmischungsverhalten und schnelle Befüllung der AZ zu gewährleisten) in die Aerosolzelle expandiert, womit der Startzeitpunkt $t_{\text {Start }}=0 \mathrm{~s}$ der Reaktion festgelegt ist.

Als letzter Schritt wird die Zelle umgehend, falls nötig bei einem gewünschten Reaktionsdruck von $p_{\text {ges }}>460$ mbar, mit Badgas auf den gewünschten Gesamtdruck aufgefüllt und die FTIR-Messung gestartet. Die genauen Konzentrationen der ungesättigten Kohlenwasserstoffverbindungen in der Reaktionsmischung werden durch Vergleich der ersten FTIR-Messung mit Spektren von im Vorfeld durchgeführten Kalibriermessungen und den sich daraus ergebenden Konzentrationsumrechnungen (s. Kap. 4) bestimmt.

Die substanz- und experimentabhängigen Einfüllschemata lassen sich Tabelle 3.1 entnehmen. Bei Experimenten, in denen außer Ozon und untersuchtem Kohlenwasserstoff weitere Additiva verwendet wurden, wurden die Substanzen so eingefüllt, dass es nicht zu einer verfühten Reaktion einzelner Komponenten kommen konnte. 


\begin{tabular}{|c|c|c|c|}
\hline $\begin{array}{c}\text { EXPERIMENTFORM } \\
\text { (verwendete Substanzen) }\end{array}$ & $\begin{array}{l}\text { Aerosol- } \\
\text { zelle }\end{array}$ & Alken-VMK & Ozon-VMK \\
\hline $\begin{array}{l}\text { PARTIKELBILDUNG f }\left(p_{\text {ges }}\right) \\
\text { (voroxidierte Alkene, } \\
\text { 1HEX, 2M1P, MCPE) }\end{array}$ & Alken & $\begin{array}{l}\text { Badgas (optional } \\
+ \text { Radikalfänger) }\end{array}$ & Ozon \\
\hline $\begin{array}{c}\text { PARTIKELBILDUNG } \mathbf{f}\left(\boldsymbol{p}_{\text {ges }}\right) \\
\text { (Pinene, } \mathrm{cHEX}, \mathrm{MCHa} \\
\mathrm{MCHe})\end{array}$ & $-^{*}$ & $\begin{array}{l}\text { Badgas (optional } \\
\text { + Radikalfänger) }\end{array}$ & Ozon \\
\hline $\begin{array}{l}\text { PARTIKELBILDUNG } f\left(p_{\mathrm{O}_{2}}\right) \\
\text { (voroxidierte Alkene) }\end{array}$ & $\mathrm{O}_{2}$, Alken & Badgas & Ozon \\
\hline $\begin{array}{c}\text { PARTIKELBILDUNG } \mathbf{f}\left(p_{\mathrm{SO}_{2}}\right) \\
\text { (Pinene, cHEX, MCHa, } \\
\text { MCHe, MCPE) }\end{array}$ & $\mathrm{H}_{2} \mathrm{O}, \mathrm{SO}_{2}$ & Alken & Ozon \\
\hline $\begin{array}{c}\text { KINETIK-/ } \\
\text { PRODUKTSTUDIEN } \\
\text { (voroxidierte Alkene, } \\
\text { 1HEX, 2M1P, MCPE) }\end{array}$ & Alken & $\begin{array}{l}\text { Badgas (optional } \\
+ \text { Radikalfänger) }\end{array}$ & Ozon \\
\hline $\begin{array}{l}\text { PRODUKTSTUDIEN } \mathbf{f}\left(p_{\mathrm{SO}_{2}}\right) \\
\text { (cHEX, } \alpha \text {-Pinen) }\end{array}$ & $\mathrm{SO}_{2}$ & Alken & Ozon \\
\hline
\end{tabular}

Tab. 3.1.: Einfüllschema der Edukte, abhängig von ungesättigter Kohlenwasserstoffverbindung und Experimentvariante.

*Bei Radikalfängerexperimenten wurde das Alken in der Aerosolzelle vorgelegt. 


\subsubsection{Partikelbildungsmessungen}

Nach der Expansion aus den Vormischkammern und ggf. Einstellen des gewünschten Reaktionsdrucks wurde die IR-Messung nach $t_{\text {Reaktion }}=20 \mathrm{~s}$ gestartet. Durch das Absorbanzspektrum dieser ersten FTIR-Messung erfolgte die grobe Überprüfung der in der Aerosolzelle befindlichen Startkonzentrationen, um Befüllungsfehler auszuschließen. Gegen Ende der auf Basis der Geschwindigkeitskoeffizienten ermittelten Reaktionszeit wurde ein weiteres IR-Spektrum aufgenommen, um zu überprüfen, ob das Ozon vollständig umgesetzt worden war. Anschließend wurde die Zelle, wie bei der Durchführung von Niederdruckexperimenten nötig, auf $p_{\text {ges }}=1000$ mbar aufgefüllt und die Verbindung zum Nachströmen des $\mathrm{N}_{2}$ geöffnet. Anschließend wurde die Verbindung zum SMPS geöffnet, die Messung gestartet und über 6 - 8 Samples verfolgt. Nach Ende der Messung wurden die Verbindungen wieder geschlossen und die Zelle abgepumpt. Danach folgte ein Spülzyklus (bei Messungen mit voroxidierte Alkenen zusätzlich mit zehnminütiger Passivierungsphase) und Evakuierung bis auf den Enddruck, bevor das nächste Leerkanalspektrum für die Folgemessung aufgenommen wurde.

Im Rahmen dieser Arbeit standen zwei verschiedene DMA-Modelle zur Verfügung, der LDMA ( $L=$ Long), der für den theoretischen Messbereich von 13 $833 \mathrm{~nm}$ optimiert ist (praktisch: $21.3-777.4 \mathrm{~nm}$ ) sowie ein NDMA ( $N=$ Nano), dessen theoretischer Messbereich 4.4 - 168 nm (praktisch: 5.62 - 205.4 nm, vgl. Abschnitt 3.1.3) umfasst. Dabei wurde der LDMA grundsätzlich für Partikelbildungsexperimente eingesetzt, der NDMA vor allem für Blindproben und teilweise für Partikelbildungsexperimente, bei denen Verteilungen mit geringen Medianen von vornherein erwartet oder nach Einsatz des LDMA, z.B. wegen abgeschnittener Verteilungen im Bereich kleiner Mediane, für nötig befunden wurde.

\subsubsection{Kinetikmessungen}

Die Vorbereitung dieser Experimente entspricht der der Partikelbildungsexperimente (s. Tabelle 3.1). Bei Kinetikmessungen werden beide Vormischkammern jedoch grundsätzlich auf 1000 mbar Vordruck aufgefüllt und der Inhalt in die Ae- 
rosolzelle expandiert, so dass die Experimente bei einem Reaktionsdruck von $p_{\text {ges }} \approx 460 \pm 10$ mbar ablaufen. Dieser Druck entspricht dem durch reine Expansion aus den Vormischkammern maximal erreichbaren Enddruck und stellt einen Kompromiss dar. Zum einen ist er ausreichend hoch, um die Ergebnisse mit denen anderer Arbeitsgruppen zu vergleichen, die meist bei Atmosphärendruck arbeiten, zum anderen muss der Druck nach der Expansion nicht manuell durch Badgaszugabe erhöht werden. Dies hätte nicht nur einen potenziellen Unsicherheitsfaktor bedeutet, sondern auch den Beginn der ersten IR-Messung verzögert 7 , was vor allem bei schnell reagierenden Kohlenwasserstoffverbindungen und hohen Anfangskonzentrationen problematisch wäre.

Die Anfangskonzentrationen wurden sowohl absolut wie auch im relativen Verhältnis zueinander variiert, um zufällige Ausreißer besser identifizieren zu können, bevor die in den einzelnen Messungen erhaltenen Geschwindigkeitskoeffizienten zu einem Gesamtergebnis gemittelt wurden.

Die erste IR-Messung in den später beschriebenen Experimenten wurde nach $t_{\text {Reaktion }}=2 \mathrm{~s}$ gestartet. Zunächst wurden zehn Spektren à 72 Scans über eine Zeitdauer von je 30 Sekunden aufgenommen. Anschließend folgten mit Beginn der 6. und 8. Reaktionsminute noch einmal zwei Aufnahmen mit jeweils 200 Scans in $85 \mathrm{~s}$. Von diesem Schema wurde lediglich bei der Substanz Methylcyclopenten abgewichen, da diese Substanz sehr schnell mit Ozon reagiert und bei dem beschriebenen Vorgehen schon nach Aufnahme des ersten Spektrums so weit abreagiert ist, dass eine Auswertung nicht mehr sinnvoll möglich ist. Daher wurde bei dieser Verbindung neben dem üblichen Verfahren mit 72-Scan-Spektren bei $t_{\text {Reaktion }}=2 \mathrm{~s}$ (hiervon jedoch nur vier, da die Reaktion danach vollständig abgelaufen war) auch Messungen von vier Spektren mit jeweilis lediglich 37 Scans durchgeführt, die dann in nur $20 \mathrm{~s}$ aufgenommen werden können, allerdings auch ein schlechteres Signal-zu-Rausch-Verhältnis aufweisen.

\footnotetext{
${ }^{7}$ Das Auffüllen der Zelle von 460 mbar auf 1000 mbar dauert bei einem Vordruck von 3 bar am Reduzierventil der Badgasflasche etwa 15 Sekunden.
} 


\subsubsection{Produktbildungsmessungen}

Auch bei Produktstudien wurden die Edukte wie in Tabelle 3.1 zusammengefasst für das Experiment bereitgestellt. Hier wurden verglichen mit Partikelmessungen deutlich höhere, jedoch stets konstante Ausgangskonzentrationen, verwendet, damit auch die Produkte in höheren Konzentrationen gebildet wurden. Dadurch traten in den IR-Spektren höhere Absorbanzsignale der gebildeten Spezies auf und das Signal-zu-Rausch-Verhältnis verbesserte sich, was die Identifikation bestimmter Linien vereinfachte. Um sowohl Zwischen- als auch Endprodukte zu detektieren, wurden nicht nur am Ende der geschätzten Reaktionszeit (meist nach 10 - 12 Minuten), sondern bereits ab der sechsten Messminute alle zwei Minuten 200-Scan-Spekten aufgenommen. Die hohe Zahl an Scans, über die gemittelt wurde, sorgte zusätzlich für verhältnismäßig rauscharme Spektren.

Allgemein wurden Produktspektren unter variablen Bedingungen aufgenommen, die anhand der Partikelmessungen als vielversprechend hinsichtlich potenziell unterschiedlicher bevorzugter Reaktionspfade und damit der Bildung unterschiedlicher Endprodukte identifiziert wurden. Da mit der Variation des Gesamtdruckes während der Reaktion sowie, bei einigen untersuchten Substanzen, der An- und Abwesenheit von $\mathrm{O}_{2}$ bei ansonsten konstanten Eduktkonzentrationen verschiedene Partikelausbeuten erhalten wurden, wurden auch Produktstudien im Hoch- bzw. Niederdruckregime und in verschiedenen Badgasen (Synthetische Luft bzw. reiner Stickstoff) durchgeführt, um anhand der IR-Absorbanzen die bei einem auftretenden oder ausbleibenden Nuklationsereignis gebildeten Gasphasenprodukte zu identifizieren und so möglicherweise auf entscheidende Verzweigungen im ablaufenden Reaktionsmechanismus rückschließen zu können.

\subsubsection{Radikalfängerexperimente}

Beim Einsatz von Substanzen, die die in der Ozonolysereaktion (vgl. Abschnitt 2.2 und 2.4) intermediär entstehenden $\mathrm{OH}$-Radikale abfangen sollen, wurden diese in die Vormischkammern eingegeben und das jeweils verwendete Alken in der Aerosolzelle vorgelegt (s. Tabelle 3.1). Um den meist um mehrere 
Größenordnungen über der Ozonolysereaktion liegenden Geschwindigkeitskoeffizienten für Reaktionen von OH-Radikalen mit Alken Rechnung zu tragen (Tabelle 2.3), wurden die Anfangskonzentrationen der Fängermoleküle so eingestellt, dass ein merklicher Anteil der Radikale bevorzugt mit dem Fänger und nicht dem Alken reagieren sollte.

Teilweise wurde auch der Partialdruck der Fängersubstanz variiert, um aus möglichen Unterschieden in den Messergebnissen bei verschiedenen Anteilen abgefangener Radikale aus der reaktiven Mischung auf die Bedeutung der radikalischen Nebenreaktionen innerhalb des Gesamtmechanismus rückschließen zu können.

Grundsätzlich wurde der in der Aerosolzelle während der Reaktion vorliegende Partialdruck der Fängersubstanzen volumetrisch über Druckanstieg in der Vormischkammer und anschließender Berücksichtigung des Volumenverhältnisses (GI.4.3) bestimmt. Eine Konzentrationsbestimmung per IR-Messung war wegen der extrem hohen verwendeten Konzentrationen und teilweise nichtlinearem Verhalten der Signale, von denen sich einige zusätzlich in der Sättigung befinden, nicht genau genug möglich.

Die experimentellen Bedingungen befinden sich in den vorangegangenen Abschnitten, weil die Radikalfängerexperimente jeweils Variationen der Partikel- (Abschnitt 3.2.2), Kinetik- (Abschnitt 3.2.3) und Produktmessungen (Abschnitt 3.2.4) darstellen. 


\section{Kalibrierung und Konzentrationsbestimmung}

In diesem Kapitel wird das Vorgehen bei der Kalibrierung der verwendeten Substanzen zur späteren Konzentrationsbestimmung in den Hauptexperimenten beschrieben. Diese werden dabei der Übersicht halber nach ihrer Funktion im Reaktionsablauf (Reaktand, Additiv) und innerhalb dessen weiter nach ihrem Hauptstrukturmotiv (Oxidationsgrad, relativer Abstand funktionaler Gruppen usw.) aufgeteilt.

\subsection{Kohlenwasserstoffverbindungen}

Generell konnte die Konzentrationsbestimmung der ungesättigten Kohlenwasserstoffverbindungen über volumetrische Eingabe in die Reaktionszelle in Verbindung mit Infrarotspektroskopie erfolgen. Allerdings ließ sich diese Art der Bestimmung nicht problemlos auf die Carbonsäuren anwenden, wie in den folgenden Abschnitten beschrieben wird.

\subsubsection{Carbonsäuren}

Da die Carbonsäuren Acrylsäure und Methacrylsäure schon in meiner 2008 angefertigten Staatsexamensarbeit [15] im Mittelpunkt der Untersuchungen standen, waren die Probleme bei der Konzentrationsbestimmung dieser Substanzen mittels Infrarotspektroskopie bekannt.

Das Hauptproblem ließ sich in der Bildung von Carbonsäuredimeren lokalisieren, die partialdruck- und temperaturabhängig erfolgte. Normalerweise ist es sinnvoll, einen für die jeweilige Substanz charakteristischen überlagerungsfrei- 
en Peak zu wählen, der zum einen eine möglichst hohe Intensität besitzt, um die sich aus dem Signal-zu-Rausch- (S/R) Verhältnis ergebenden Unsicherheiten zu minimieren und zum anderen möglichst schmal ist. Eine geringe FWHM (full width at half maximum) minimiert Unsicherheiten, die auf potenziellen Grundlinienabweichungen vom waagerechten Idealfall basieren und verringert außerdem die Wahrscheinlichkeit, dass die Bande später durch entstehende Produkte überlagert wird. Weiterhin sollte die gewählte Bande möglichst nicht im Bereich der Wasser- oder Kohlenstoffdioxidsignale liegen, da diese Banden durch stark nichtlineares Verhalten bei Konzentrations- und Druckänderungen oft nicht vollständig aus aufgenommenen Spektren herausgerechnet werden können und bei geringen Konzentrationen der interessierenden Substanz für eine Fehlbestimmung sorgen können (vgl. z.B. $\mathrm{SO}_{2}$, Abschnitt 4.3.1). Für den experimentellen Ablauf erwies sich als problematisch, dass aus der volumetrisch in die Zelle eingegebenen Menge nicht linear auf die per IR-Messung bestimmten Konzentration geschlossen werden konnte. Die Auswertung von im IR-Spektrum auftretenden Peaks ist grundsätzlich auf zwei verschiedene Arten möglich: über die Auswertung der integralen Absorbanz oder der Peakhöhen (verhältnisse).

Bei der Betrachtung der integralen Absorbanz wird über den ausgewählten Peak integriert und das Integral als Maß für die weitere Auswertung genutzt. Bei Auswertung über die reinen Peakhöhen, die sich anbietet, wenn kein über die gesamte Peakbreite freistehendes Signal zur Integration vorhanden ist, kann das relative Höhenverhältnis zwischen zwei charakteristischen Punkten (vorzugsweise zueinander benachbarte Maxima und Minima) als Bezugsmaß dienen. Im Idealfall sollte die Absorbanz gemäß dem LAMBERT-BEERschen Gesetz bei einer Auftragung gegen die eingefüllte Konzentration linear von dieser abhängen (GI.4.5). Allerdings ergaben die Kalibriermessungen für die genannten Carbonsäuren weder einen linearen Zusammenhang noch durchlief eine lineare Annährerung der gemessenen Absorbanzwerte den Ursprung (nicht gezeigt). Auch konnte kein anderer mathematischer Zusammenhang, wie Polynome verschiedener Grade, den Verlauf geschlossen erklären. Als weiterer Effekt trat in der Anfangsphase der Reaktion eine Änderung der relativen Intensitäten der Monomer- (Wellenzahlbereich ca. 3650-3550 $\mathrm{cm}^{-1}$ ) hin zu den Dimerpeaksignalen (ca. $3500-2500 \mathrm{~cm}^{-1}$ ) auf, die wohl auf den Expansionsvorgang zu- 
rückzuführen ist, wo sich nach starken Druckgradienten und daraus folgenden Temperaturschwankungen im Verlauf der ersten zwei Folgeminuten ein Gleichgewicht erst neu einstellen musste. Da in der Literatur diese Probleme bekannt sind und sich trotz intensiver Bemühungen 11 keine korrekte Korrelation über den gesamten gewünschten Konzentrationsbereich ergab, wurde die Konzentration der Carbonsäuren bei den in dieser Arbeit präsentierten Experimenten nach dem Vorbild von NEEB et al. [59] lediglich volumetrisch bestimmt, ohne auf die tendenzielle Unterschätzung der tatsächlich vorliegenden Konzentration durch Dimerisierung der Säuremoleküle Rücksicht zu nehmen. Die Experimente zur Untersuchung der Partikelbildung wurden bei einem konstanten, über Druckanstieg bestimmten Partialdruck von $p_{\text {Säure }}=5 \mathrm{~Pa}$ durchgeführt, wobei diese jeweils zu Beginn der Reaktion anhand eines IR-Spektrums relativ zu einem Referenzspektrum überprüft wurde. Im Fall von Kinetik- und Produktbildungsstudien wurden direkt im Anschluss der Reaktion Referenzspektren mit gleichen Substanzkonzentrationen zu den gleichen Zeitpunkten nach der Expansion aufgenommen, um im Reaktionsspektrum verbleibene Edukte möglicht umfassend aus den Spektren herausrechnen zu können.

\subsubsection{Alkene, Alkohole, Aldehyd und Keton}

Die Kalibrierungen können für Substanzen, die sich in ihrer Signalintensität linear mit der Konzentration verhalten, immer nach dem gleichen Schema ablaufen. Es werden zunächst Leerkanalspektren (Intensität $I_{0}$ ) bei Niederdruckbedingungen ( $p_{\text {ges }} \approx 30$ mbar) und im Hochdruckregime ( $p_{\text {ges }} \approx 1000$ mbar) aufgenommen. Nach Abpumpen des Badgases auf den Enddruck wurde die Leckrate (Druckanstieg in der Zelle bei geschlossener Verbindung zur Pumpe innerhalb einer bestimmten Zeitspanne) bestimmt. Anschließend werden nacheinander verschiedene Partialdrücke der zu kalibrierenden Substanz in die Zelle eingegeben, diese zunächst auf einen Gesamtdruck von 30 mbar und anschließend auf 1000 mbar mit Badgas aufgefült, wobei bei jedem Druckregime ein IR-Spektrum aufgenommen wird (Intensität $l$ ). So sollten eventuell vorhan-

\footnotetext{
${ }^{1}$ Es wurden theoretische Berechnungen der relativen Intensitäten auf $B 3 L Y P$ 6-311+G(d,p)Niveau mit dem Programm GAUSSIAN 03 [103] durchgeführt und anschließend eine Umrechnung der postulierten Dimer- in eine Monomerkonzentration getestet, ohne jedoch eine ausreichende Genauigkeit der Konzentrationsbestimmung zu erreichen.
} 
dene druckabhängige Veränderungen der Absorbanzsignale entdeckt und ihnen bei der späteren Bestimmung des Kalibrierkoeffizienten Rechnung getragen werden. In den nach Gleichung 4.1 aus aufgenommenden Einkanalspektren berechneten Absorbanzspektren werden nun eine oder mehrere überlagerungsfreie Signale ausgewählt und zwischen zwei Grenzpunkten integriert. Die Banden sollten dabei möglichst nicht im Bereich der Kohlenstoffdioxid- oder Wassersignale liegen, damit die Rotationsbanden dieser beiden schlecht oder gar nicht aus den Spektren abzuziehenden „Hintergrundsubstanzen“ durch additives Verhalten der Signale die Integrationswerte nicht verfälschen2. Die gemessenen integralen Absorbanzen werden im nächsten Schritt gegen die tatsächlich, das heißt um die Leckrate korrigierte, eingefüllte Substanzmenge aufgetragen.

Der Auftragung der Kalibriergeraden lag dabei eine Abhängigkeit basierend auf dem LAMBERT-BEERschen Gesetz (GI. 4.5, 4.1) zugrunde:

$$
A=\lg \frac{l_{0}}{l}=\epsilon_{\lambda} \cdot c \cdot d
$$

Da sowohl der Absorptionskoeffizient $\epsilon_{\lambda}$ der Substanz als auch die durchstrahlte Schichtdicke $d$ während der Experimente konstant sind, ist die Absorbanz direkt von der eingefüllten Konzentration $c$ abhängig. Aus einer Auftragung der integralen Absorbanz gegen den jeweilig eingefüllten Partialdruck der Substanz und der sich bei linearem Verlauf ergebenden konstanten Steigung (entspricht dem Kalibrierkoeffizienten $\kappa$ ) kann die Substanzkonzentration daher bei anschließenden Messungen aus der Absorbanz berechnet werden:

$$
\begin{aligned}
A & =\kappa \cdot C \\
c & =\frac{A}{\kappa}
\end{aligned}
$$

\footnotetext{
${ }^{2} \mathrm{Im}$ verwendeten Programm OPUS stehen mehrere Integrationsmethoden zur Wahl. Bei den in dieser Arbeit durchgeführten Messungen wurde immer nach Methode „ $B$ “ vorgegangen. Bei dieser werden die äußeren Grenzen des Integrals vorgegeben, die Berechnung der Fläche des Peaks erfolgt dann bis zu einer Geraden, die zwischen den Grenzen des Integrals gezogen wird. Der Vorteil dieser Methode ist, dass eine vorherige Korrektur der Grundlinienhöhe auf einen Absorbanzwert von Null, wie er bei schlechter werdendem Detektorsignal durch Erwärmung über den Tag auftritt, überflüssig wird.
} 
Für die betrachteten Integrationsbereiche ergaben sich dabei folgende Werte für die Kalibrierkoeffizienten:

\begin{tabular}{|c|c|c|}
\hline Substanz & Integrationsgrenzen $\left[\mathbf{c m}^{-1}\right]$ & $\begin{array}{c}\text { Koeffizient } \boldsymbol{\kappa} \\
{\left[\mathbf{c m}^{-1} \cdot \mathbf{P a}^{-1}\right]}\end{array}$ \\
\hline \hline Ozon & $1073-970$ & 1.797 \\
\hline 3-Buten-1-ol & $3125-3045$ & 2.066 \\
& $3045-2805$ & 0.242 \\
\hline 1-Penten-3-ol & $3130-3050$ & 0.0215 \\
& $3050-2800$ & 0.2643 \\
\hline 1-Penten-3-on & $1233-1145$ & 0.3551 \\
\hline 4-Penten-1-al & $960-870$ & 0.5394 \\
\hline 1-Hexen & $958.8-861.8$ & 0.543 \\
\hline 2-Methyl-1-penten & $933-845$ & 0.557 \\
\hline Methylcyclopenten & $3100-3020$ & 0.2145 \\
\hline Cyclohexan & $2990-2820$ & 4.40 \\
\hline$\alpha$-Pinen & $807-768$ & 0.195 \\
& $3030-2805$ & 8.326 \\
\hline$\beta$-Pinen & $910-835$ & 0.594 \\
& $3030-2830$ & 7.031 \\
\hline 1-Methylcyclohexen & $3030-2820$ & 5.578 \\
\hline Methylencyclohexan & $925-825$ & 0.669 \\
\hline
\end{tabular}

Tab. 4.1.: Angabe der experimentell für den verwendeten Versuchsaufbau ermittelten Kalibrierkoeffizienten. Die Werte für Ozon, $\alpha$-, $\beta$-Pinen, Methylencyclohexan und 1-Methylcyclohexen wurden von WOLF [48] übernommen. 


\subsection{Ozon}

\subsubsection{Methoden der Ozonkonzentrationsbestimmung}

Eine verlässliche Bestimmung der in der Reaktionszelle vorliegenden Ozonkonzentration ist problematisch. Die Ozonkonzentrationsmessung konnte während der Experimentdurchführung nur über IR-Spektren erfolgen, da eine Auswertung der UV-Aufzeichnungen erst nach Ende des Messtages möglich war (s. dazu Abschnitt 4.2.1.2). Allerdings war dadurch nur eine grobe Abschätzung der enthaltenen Ozonmenge zu erreichen, da die charakteristische $\nu_{3^{-}}$ Schwingung zwischen 1080 - $980 \mathrm{~cm}^{-1}$ nicht immer einen Rückschluss auf den wahren Konzentrationswert zuließ. Aus diesen Gründen wurde bei der Durchführung von Partikelmessungen zwar immer ein IR-Spektrum des Reaktionsgemisches aufgenommen, dieses diente allerdings lediglich der groben Überprüfung der verwendeten Reaktandenkonzentrationen. Bei Kinetik- und Produktbildungsmessungen hatte die Ozonkonzentrationsbestimmung über FTIR dagegen eine größere Bedeutung zur Bestimmung der Restkonzentration in der Reaktionsmischung, um ggf. Eduktpeaks aus Produktspektren herausrechnen zu können. Zu diesem Zweck lagen Kalibrierspektren bei verschiedenen Konzentrationen und Gesamtdrücken vor [48], mit denen die Korrekturen vorgenommen werden konnten.

Für eine ausreichende Genauigkeit der Werte musste außerdem eine Methode benutzt werden, mit der die Ozonkonzentration schon vor der Eingabe in die Reaktionszelle bestimmt werden konnte. Diese Notwendigkeit ergab sich aus den Experimenten an der Vorgängerapparatur (s. z.B. [15]), an der die einzige Möglichkeit der Konzentrationsbestimmung die IR-Messung mit einer recht großen Unsicherheit darstellte. Da das Ozon / Helium-Gemisch keinen konstanten Ozonanteil aufwies, musste dieses Gemisch immer zu Beginn des Experiments in die Zelle gegeben, ein IR-Spektrum zur Überprüfung aufgenommen und anschließend die verwendete Kohlenwasserstoffverbindung bei Niederdruckbedingungen zugefügt werden, um danach die Zelle auf den gewünschten Reaktionsdruck zu bringen. Problematisch waren dabei die nicht komplett wiederholbaren Abläufe, da beispielsweise die Einfüllzeit des Alkens von der gewünschten Sollkonzentration abhing. So fand die Reaktion ab Beginn 
der Alkenzugabe über verschieden lange Zeiten bei Niederdruckbedingungen und bei großen lokalen Konzentrationsgradienten statt, bevor die Reaktion nach Badgaszugabe im Hochdruckregime abgeschlossen wurde.

Da diese Einfüllprozedur relativ schlecht reproduzierbare Ergebnisse lieferte, wurde der Aufbau mit den beiden Vormischkammern entwickelt [48], in denen die Edukte in den gewünschten und im Vorfeld der Reaktion überpüfbaren Konzentrationen vorgelegt (UV-Absorption für Ozon bzw. volumetrische Bestimmung für Kohlenwasserstoffverbindungen) und unter definierten und reproduzierbaren Bedingungen erst in der Aerosolzelle zusammengeführt werden. Auch das Problem lokaler Konzentrationsunterschiede konnte auf diese Weise gelöst werden, da beide Reaktanden-Badgasmischungen durch den Expansionsvorgang schnell und gründlich durchmischt werden.

Zur Bestimmung der genauen Ozonanfangskonzentration wurde standardmäßig auf die nachträgliche Auswertung der aufgenommenen UV-Daten zurückgegriffen. Zur weiteren Absicherung wurde eine Kreuzkalibrierung mit einem kommerziell erhältlichen Ozonanalysator durchgeführt (Abschnitt 4.2.2). So wurde sichergestellt, dass zum einen die Umrechnung des Spannungsabfalls der UV-Optik-Dioden in eine Ozonkonzentration (Analysator vs. UV-Optik) und zum anderen das Expansionsverhältnis der VMK und AZ zur Berechnung der Ozonkonzentration in der reaktiven Mischung (UV-Optik vs. IR-Messung) korrekt erfolgten. Für letzteres wurde kurzzeitig eine weitere UV-Optik direkt an der Aerosolzelle angebracht.

Zunächst werden in den nächsten Abschnitten die generell in den vorgestellten Experimenten verwendeten Konzentrationsbestimmungsmethoden vorgestellt, bevor diese im Anschluss im Hinblick auf die erhaltenen Ergebnisse und ihrer Übereinstimmung in einer Kreuzkalibrierung verglichen werden (Abschnitt 4.2.2.

\subsubsection{IR-Messungen}

Die oben beschriebene Problematik der Ozonkonzentrationsbestimmung über IR-Spektroskopie ergibt sich, da einerseits die Signale des Ozons durch Schwingungen der verwendeten Kohlenwasserstoffverbindungen oder entstehender Produkte überlagert sein können und anderseits die Absorption nicht- 
lineare Abhängigkeiten von Druck- und Konzentrationsänderungen zeigte. Die Abhängigkeit der Bandenform vom vorherrschenden Gesamtdruck ist in Abbildung $4.1 \mathrm{a}$ anhand der asymmetrischen Streckschwingung $\left(\nu_{3}\right.$-Bande als intensivstes Signal im Spektrum) dargestellt. Es ist zu erkennen, dass sich die Intensität der Bande bei Druckzunahme in einigen Bereichen (ca. 1060$1045 \mathrm{~cm}^{-1}$ und 1035-1010 $\mathrm{cm}^{-1}$ ) erhöht, in anderen nahezu konstant bleibt (1035-1040 $\left.\mathrm{cm}^{-1}\right)$ und es im rechten Zweig bei Druckänderungen auch zu einer Veränderung der Bandenform in P- und R-Zweig kommt.

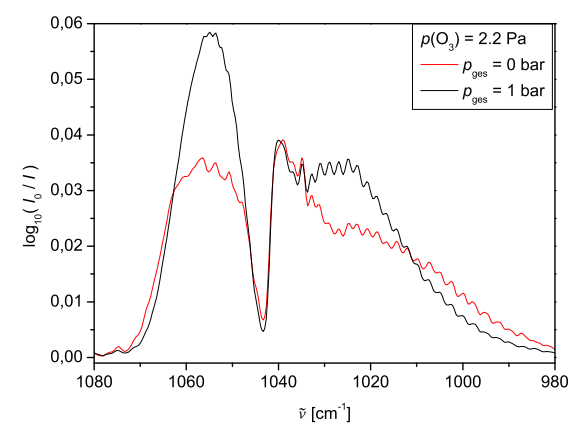

(a) IR-Spektrum des Ozons (hohe Ozonkonzentration) bei verschiedenen Gesamtdrücken.

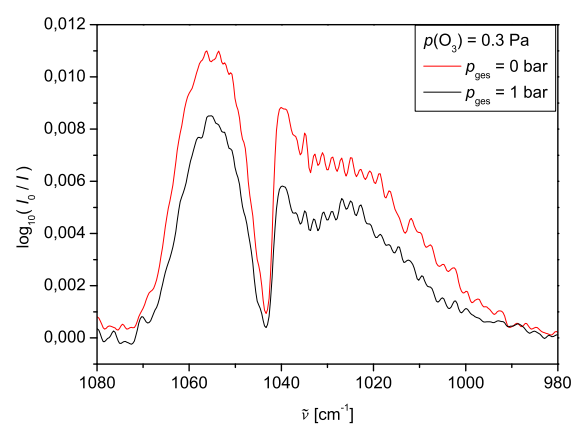

(b) IR-Spektrum des Ozons (geringe Ozonkonzentration) bei verschiedenen Gesamtdrücken.

Abb. 4.1.: IR-Spektrum des Ozons - Darstellung der konzentrationsabhängigen Bandenform bei Änderung des Gesamtdruckes, entnommen aus [48].

Das nichtlineare Verhalten im Bezug auf die Bandenform bei verschiedenen Konzentrationen zeigt sich darin, dass sich die Absorbanz bei einem geringeren Ozonpartialdruck über den gesamten Wellenzahlbereich relativ gleichmäßig bei Druckerhöhung vergrößert (vgl. Abb 4.1b).

Dieser Effekt ist wahrscheinlich durch Druckverbreiterung bei höheren Gesamtdrücken sowie Auflösungsartefakte, ähnlich wie bei den Rotationsbanden des Kohlenstoffmonoxids, zu erklären [48]. Zusätzlich ist auch eine Adsorption von Ozon sowohl auf den Goldspiegeln der WHITE-Optik als auch auf den Quarzfenstern der AZ in Lichtweg möglich, die jedoch hauptsächlich im Niederdruckbereich auftritt. Zumindest das Problem der Spiegel- und Fensterbelegung sollte im Hochdruckbereich durch die Badgaszugabe und das damit verbundene „Freispülen“ der Optikbauteile nicht zu große Auswirkungen ha- 
ben. Die Vermutung der Fenster- / Spiegelbelegung durch Ozon schien auch beim Einsatz der UV-Optik zu greifen, da sich die Lichtintensität nach Ozoneinlass bei der Badgaszugabe noch einmal um bis zu $2 \%$ erhöhte, (vgl. 4.2.1.2) was nominell einer sich verringernden Ozonkonzentration entspräche, zu der es aber durch das abgeschlossene Gefäß mit durch die aufgebrachte Teflonbeschichtung inerten Reaktorwänden nicht kommen konnte. Insgesamt ließ sich beim Vergleich der IR-Spektren beobachten, dass sich die Konzentration bei höheren Gesamtdrücken wesentlich sicherer bestimmen ließ als im Niederdruckbereich. Dies könnte sich eventuell aber auf zufällig unterschiedliche Ozonkonzentrationen in der Heliummischung zurückführen lassen, die unterschiedliche Einfüllmengen bedingen und sich daher auch unterschiedlich auf die Spiegelbelegung auswirken können ( [48], S. 57f.).

\subsubsection{UV-Optik}

Das Herzstück der UV-Optik ist eine Hg-Dampflampe. Für die Messungen zu den voroxidierten Substanzen wurde UV-AUFBAU 1 verwendet, für Anschlussmessungen zu reinen Kohlenwasserstoffen wurde dieser ersetzt durch UVAUFBAU 2 (s. Abb. 4.2), der neben einer höheren UVC-Strahlungsleistung von $20 \mathrm{~W}$ den Vorteil besonders konstanter Strahlungsleistungen über einen längeren Zeitraum besitzt. Dies ist durch den Einsatz der neuen UV-Strahlungsquelle möglich. Weiterhin wurden Quarzlinsen von der UV-Lampe aus gesehen vor und hinter der Vormischkammer bzw. Aerosolzelle zur Fokussierung der durch die Quarzfenster transmittierten Strahlung platziert. Die Aufbauten besitzen die im Folgenden angegebenen Kenngrößen.

- UV-Aufbau 1:

Strahlungsquelle: TUV PL-L 36 W/4P 1CT, Fa. Philips, UVCStrahlungsleistung $10.9 \mathrm{~W}$.

Linsen: Fa. Linos, $\varnothing_{\mathrm{L} 1}=50 \mathrm{~mm}, f_{\mathrm{L} 1}=100 \mathrm{~mm} ; \varnothing_{\mathrm{L} 2}=18 \mathrm{~mm}$, $f_{\mathrm{L} 2}=30 \mathrm{~mm}$.

- uV-Aufbau 2:

Strahlungsquelle: UV-Strahlungsquelle NNI 60/35 XL, Fa. UVConsulting Peschl, UVC-Strahlungsleistung $20 \mathrm{~W}$. 
Linsen: Fa. qioptiq, $\varnothing_{\mathrm{L} 1}=50 \mathrm{~mm}, f_{\mathrm{L} 1}=100 \mathrm{~mm} ; \varnothing_{\mathrm{L} 2}=31.5 \mathrm{~mm}$, $f_{\mathrm{L} 2}=40 \mathrm{~mm}$.

Die Registrierung der Strahlungsleistung erfolgt über zwei Photodioden (JEC1C, Fa. Laser Components) mit einer aktiven Detektorfläche von $0.96 \mathrm{~cm}^{2}$. Beide Lichtquellen emittieren die Strahlung sehr schmalbandig um ihre Hauptlinie bei $254 \mathrm{~nm}$ (Halbwertsbreite full width at half maximum, $F W H M \approx 5 \mathrm{~nm}$ ), zusätzlich sind die Photodioden mit einem Highcut-Filter ( $275 \mathrm{~nm}$ ) ausgestattet, damit die Nebenlinien oberhalb von $280 \mathrm{~nm}$ nicht zu der gemessenen Leistung beitragen. Da die Ausgabespannung $U$ der Dioden proportional zur auf die Dioden treffende Strahlungsintensität $/$ ist, kann letztere durch Digitalvoltmeter (VC960, Fa. Voltcraft) ausgelesen und auf die Intensitätswerte rückgeschlossen werden. Mit den Voltmetern war eine Datenaufnahme und -speicherung möglich, so dass die Geräte nachträglich mit einem Computer ausgelesen und die Daten mit Hilfe einer vorbereiteten EXCEL-Tabellenvorlage ausgewertet werden können. Dabei wurde im Zweikanalbetrieb gearbeitet, die Referenzspannung $I_{0}$ also über die Referenzdiode auf Seiten der UV-Lampe zeitgleich zur Signalspannung I nach Durchgang durch das Probengefäß aufgezeichnet. Dieser Betrieb hat sich als weniger fehleranfällig als der Einkanalbetrieb mit abwechselnder Messung der Leer- und Substanzmessung erwiesen, da zumindest die erste verwendete UV-Lampe in ihrer Leistung nicht ausreichend konstant über typische Messdauern von 10-15 min emittierte [48].

Neben Leistungsschwankungen der Lampe stellte auch die Temperaturabhängigkeit der registrierten Spannung ein Problem dar [48]. Diese Unsicherheit konnte allerdings durch die Installation einer Klimaanlage reduziert werden. Die Bestimmung der Ozonkonzentration erfolgte nach folgendem Ablauf:

- Spülen der VMK mit Badgas.

- Ablesen der Leerspannung $U_{0}$ am Signalvoltmeter und Berechnung der Sollspannung beim Einfüllen der gewünschten $\mathrm{O}_{3}$-Menge mit einer vorbereiteten EXCEL-Tabelle.

- Einfüllen des $\mathrm{O}_{3} / \mathrm{He}$-Gemisches bis leicht unter den Spannungs-Sollwert (d.h., etwas höhere Ozonkonzentration als gewünscht). 


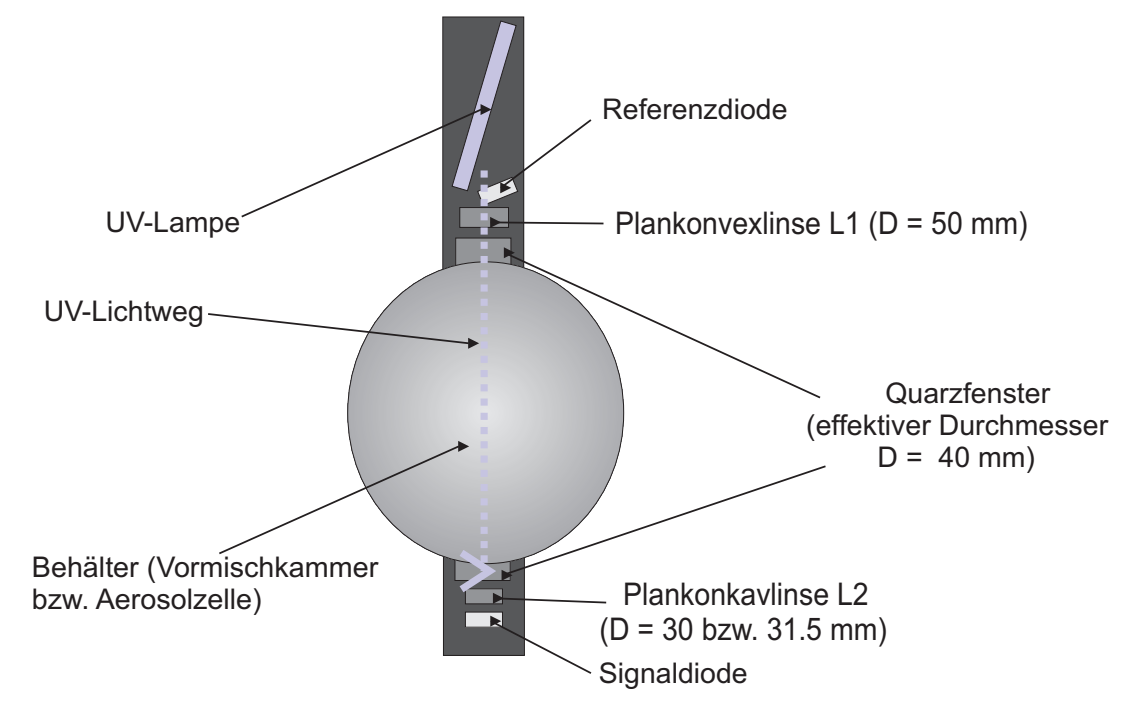

Abb. 4.2.: Schematischer Darstellung der UV-AufBAUTEN 1 bzw. 2 an Aerosolzelle bzw. Ozon-Vormischkammer zur Ozonkonzentrationsbestimmung.

- Einfüllen des Badgases (wobei die angezeigte Spannung durch Freispülen der mit Ozon belegten Quarzfenster wiederum leicht anstieg).

- Gegebenenfalls Abpumpen des Gemisches und wieder Auffüllen mit Badgas auf den Solldruck, bis die gewünschte Signalspannung erreicht ist, und über das bekannte Verdünnungsverhältnis zwischen VMK und $A Z$ die später vorliegende Ozonkonzentration in der Reaktionsmischung bestimmen.

- Die VMK für mindestens fünf Minuten mit der eingefüllten Ozonmenge belassen, um die UV-Daten lang genug für die anschließende Auswertung aufzunehmen.

- Nach der Expansion sollte die UV-Aufnahme an der VMK mit der verbleibenen Ozonmenge wiederum für mind. fünf Minuten laufen, bevor die Kammer komplett abgepumpt und die nächste Messung vorbereitet werden kann.

Dieser Ablauf wurde auch für die im Abschnitt4.2.2 durchgeführte Kalibrierung beibehalten. 
Die Bestimmung der Ozonkonzentration erfolgte dabei unter Nutzung des Zusammenhanges 3

$$
\begin{aligned}
\Delta n\left(\mathrm{O}_{3}\right) & =V_{\mathrm{VMK}} \cdot\left(c_{\text {Anfang }}-c_{\text {Ende }}\right) \\
\frac{\left(c_{\text {Anfang }}-c_{\text {Ende }}\right)}{c_{\text {Ende }}} & =\frac{V_{\mathrm{AZ}}}{V_{\mathrm{VMK}}}
\end{aligned}
$$

\subsubsection{Der Ozonanalysator}

Der Ozonanalysator (Model $\mathrm{O}_{3} 41 \mathrm{M}-\mathrm{LCD}, \mathrm{Fa}$. ansynco) dient der direkten und kontinuierlichen Ozonkonzentrationsbestimmung in einem bestimmten Volumen, wobei der Messbereich von 0.001-10 ppm reicht [104]. Das Messprinzip basiert (wie im Experiment mit der UV-Optik direkt genutzt) auf der UVAbsorption des Ozons bei $254 \mathrm{~nm}$ und dem LAMBERT-BEERschen-Gesetz (Gl. 4.5), wobei diese Methode selektiv und linear in dem angegebenen Messbereich wirkt.

$$
T:=\frac{l}{I_{0}}
$$

Durch Teflonschlauchverbindungen wird das Gerät an der Rückseite mit dem Probenvolumen verbunden, wobei ein internes Pumpsystem für den nötigen Gastransport und Durchfluss sorgt. Die Messwerte können nach Einstellung des gewünschten Messbereiches direkt am Display abgelesen werden. Neben Ozon können mit diesem Gerät potenziell auch $\mathrm{NO}_{x}(x=1,2)$ nachgewiesen werden. Druck- und Temperatursensoren am Gerät sorgen für eine automatische Korrektur der Messwerte auf Normalbedingungen $\left(T_{0}=273 \mathrm{~K}\right.$, $p_{0}=1013 \mathrm{hPa}$ ). Generell werden Messungen nach dem Einstrahlprinzip aufgenommen, d.h. zur Vermeidung von Abgleichfehlern finden Messungen der Intensitäten $I_{0}$ (Referenzmessung) und I (Probenmessung) in der gleichen Küvette statt. Zu jeder Messung von I wird eine Referenzmessung durchgeführt, um von eventuellen Schwankungen der UV-Intensität über die Zeit unabhängig zu sein. Bei stromlosem Magnetventil passiert das Probengas zunächst den Ozonscrubber, der selektiv und quantitativ das enthaltene Ozon abbaut. Die

${ }^{3}$ Die Bestimmung der Alkenkonzentration bei Eingabe über die Vormischkammern erfolgt wegen gleicher Volumenverhältnisse analog. 
Messküvette wird ca. $6 \mathrm{~s}$ gespült, anschließend wird innerhalb von $1 \mathrm{~s}$ der Wert $I_{0}$ für die Transmission gemessen (Referenzmessung), wobei die genaue Dauer der Messung vom Referenzdetektor vorgegeben wird, der zeitgleich die Intensität der UV-Lampe beobachtet. Anschließend werden die Ausgangssignale über A/D-Wandler in eine Frequenz (Pulsfolge) umgewandelt und integriert. Die A/D-Wandler arbeiten bis zu einer maximalen Wandlungsfrequenz von $1.6 \mathrm{MHz}$ bei $10 \mathrm{~V}$ Spannungspegel vom Elektrometerverstärker. In der Regel wird eine Spannung von 6-8 $\mathrm{V}$ angelegt, so dass eine Wandlerrate von 0.9-1.2 $\mathrm{MHz}$ resultiert. Im folgenden Schritt wird die Probe durch das interne Magnetventil in die Messküvette geleitet, die wiederum $6 \mathrm{~s}$ gespült wird. Dort findet eine Messung von I nach dem gleichen Schema statt, die genaue Dauer der Messung wird also wieder vom Erreichen eines bestimmten Referenzdetektorsignals bedingt. Abschließend werden Gasdruck und -temperatur erfasst und die Ozonkonzentration wie folgt berechnet [104]:

$$
\left[\mathrm{O}_{3}\right] / \mathrm{ppm}=\frac{10^{6}}{\epsilon \cdot l} \cdot \ln \frac{I_{0}}{l} \quad\left(\text { bei } p_{0} \text { und } T_{0}\right)
$$

Mit Berücksichtigung der Temperatur- und Druckkorrektur auf Grundlage des idealen Gasgesetzes $p V=n R T$ folgt schließlich

$$
\left[\mathrm{O}_{3}\right] / \mathrm{ppm}=\frac{10^{6}}{\epsilon \cdot L} \cdot \ln \frac{I_{0}}{l} \cdot \frac{p_{0}}{p} \cdot \frac{T}{T_{0}}
$$

- $\epsilon=$ Extinktionskoeffizient des Ozons bei $\lambda=253.7 \mathrm{~nm}$.

- $L$ = optische Weglänge der Messküvette, hier $70.8 \mathrm{~cm}$.

- $I_{0}=$ Intensität (UV-Energie) der ozonfreien Referenzprobe nach Durchgang durch den Ozonfilter.

- $I$ = Intensität nach Durchgang der ozonhaltigen Probe.

- $p=$ aktueller Druck in der Messzelle in [atm].

- $T=$ aktuelle Temperatur in der Messzelle in $[\mathrm{K}]$. 
Mit dem vom Gerät angenommenen Extinktionskoeffizienten von $\epsilon=308 \mathrm{~atm}^{-1} \cdot \mathrm{cm}^{-1}$ [105] und einer optischen Weglänge von $L$ von $70.8 \mathrm{~cm}$ [104] ergibt sich ein gerätspezifischer Kalibrierfaktor $K$ von

$$
K=\epsilon \cdot L=21800 \mathrm{~atm}^{-1}
$$

Jedem Messwert geht eine gleitende Mittelung von drei Messungen à 15$20 \mathrm{~s}$ voraus, aus deren Mittelwerten wiederum gleitende Mittelwerte erzeugt wurden. Daher konnte der erste Konzentrationswert nach etwa $60 \mathrm{~s}$ abgelesen werden. Eine Messung dauert ca. 15-20 Sekunden (programmierbare Integrationszeit $t_{95}$, über die der Messwert bestimmt wurde = kleineste Einstellzeit: $60 \mathrm{~s}$, da gleitend über drei Messwerte integriert wird), wobei jedoch erst nach zwei bis drei Minuten mit verlässlichen Werten zu rechnen war, was durch Restluft im System bedingt wurde. Nach dieser Anlaufzeit wurden für etwa drei bis vier Minuten sinnvolle und konstante Messwerte angezeigt, bevor die Konzentrationen durch Verdünnungseffekte, bedingt durch nachströmendes Gas zum Druckausgleich, stetig sank. Da das Gerät lediglich bei Atmosphärendruck arbeitet, wurde der normalerweise für das Nachströmen bei SMPS-Einsatz verwendete Flussregler ( $F C$ 260, Fa. Tylan, 2 SLM He, betrieben mit 2 bar $\mathrm{N}_{2}$-Vordruck) an die Vormischkammer angeschlossen, um das vom Analysator abgesaugte Volumen auszugleichen. Da trotz der Maximaleinstellung (100\%) nicht der gesamte Fluss von $90 \mathrm{~L} / \mathrm{h}$ Luft über die Dauer der Messung kompensiert werden konnte, wurde zusätzlich gelegentlich manuell Badgas direkt über die Ventile nachgegeben. Aufgrund der zunehmenden Verdünnung der Ozonmischung in der benötigten Messzeit (mit Anlaufzeit insgesamt ca. 5 min, macht bei einem Fluss von $90 \mathrm{~L} / \mathrm{h}$ einen Gasaustausch von ca. $7.5 \mathrm{~L}$, was bei einem Gesamtvolumen von $40.5 \mathrm{~L}$ einem Anteil von fast $20 \%$ entspricht) und der Festlegung auf Atmosphärendruck im Probengefäß war der Ozonanalysator nicht für den standardmäßigen Einsatz bei den laufenden Experimenten geeignet. Zum einen würde die Ozonkonzentration tendenziell zu gering ausfallen, zum anderen sollten Experimente bei verschiedenen Gesamtdrücken durchgeführt werden, die unterschiedliche Vordrücke in den VMK unterhalb von 1 bar nötig machen. Daher wurde der Analysator lediglich dafür benutzt, die installierte UV- 
Optik zur Ozonkonzentrationsbestimmung auf ihre Richtigkeit und Genauigkeit hin zu überprüfen.

\subsubsection{Kreuzkalibrierung: UV-Optik vs. Analysator und UV-Optik vs. IR-Messung}

Nachdem die UV-Optik installiert worden war, mit der die in der VMK und unter Voraussetzung des Verdünnungsverhältnisses $f$ (nach Gl.4.3: bei der Expansion aus beiden VMK simultan $f=0.26$, bei Expansion aus nur einer VMK $f=0.35$ ) auch die in der Aerosolzelle enthaltene Ozonmenge bestimmt werden konnte, sollte die Richtigkeit dieser Annahmen überprüft werden. Dies sollte verhindern, dass sich eventuell systematisch fehlerhaft bestimmte Ozonkonzentrationen auf fehlerbehaftete Experimenten und spätere Fehlschlüsse bei der Auswertung und Interpretation auswirken. Bei der Kreuzkalibrierung wurden alle zur Verfügung stehenden Methoden zur Ozonkonzentrationsbestimmung simultan eingesetzt, um die Ergebnisse später miteinander zu vergleichen.

Zunächst wurde zusätzlich zu der standardmäßig an der OzonVormischkammer installierten UV-Optik der Ozonanalysator an die Vormischkammer angeschlossen und eine weitere UV-Optik an den Quarzfenstern der Aerosolzelle angebracht, die nach dem gleichen Prinzip wie die an der VMK aufgebaut ist und funktioniert.

Erst erfolgte die Überprüfung der Ozonkonzentrationsmessung innerhalb der Vormischkammer. Dazu wurde wie gewohnt die Leerspannung $I_{0}$ abgelesen und durch Zugabe der Ozon / Helium-Mischung die gewünschte Sollspannung I eingestellt, die der jeweiligen Ozonkonzentration entsprach. Anschließend wurde die Vormischkammer mit Badgas auf einen Gesamtdruck von 1 bar aufgefüllt, der Ozonanalysator gestartet und nach der oben beschriebenen Anlaufzeit die Konzentrationswerte abgelesen. Danach wurde der Analysator wieder von der Vormischkammer getrennt und ihr Inhalt in die Aerosolzelle expandiert. Dort wurde der ankommende Inhalt nicht nur IR-spektroskopisch, sondern auch durch die zweite UV-Optik vermessen.

Insgesamt ergab sich eine zufriedenstellende Übereinstimmung bei der Be- 
stimmung der Ozonkonzentrationen mit verschiedenen Techniken in einem Bereich von höchstens $\pm 10 \%$, wie Tabelle 4.2 zeigt. Dabei ist zu beachten, dass die mit IR-Spektroskopie bestimmte Ozonkonzentration nicht mit der gleichen Genauigkeit angegeben werden kann wie die übrigen Messwerte. Dies liegt an den oben genannten Problemen, vor allem an den Auflösungsartefakten, die aus der benutzten Auflösung von $1 \mathrm{~cm}^{-1}$ resultiert. Der Ozonanalysator wurde in einem weiteren Schritt der Kalibrierung auch direkt an die Aerosolzelle angeschlossen, wobei sich ähnlich gute Übereinstimmungen mit der Bestimmung über die UV-Messung in der Vormischkammer sowie UV- und IR-Messung in der Zelle ergaben (hier nicht gezeigt).

\begin{tabular}{|c|c|c|c|c|}
\hline $\begin{array}{c}x\left(\mathrm{O}_{3}, \mathrm{VMK}(\mathrm{UV}),\right. \\
\text { Soll }) / \mathrm{ppm}\end{array}$ & $\begin{array}{c}x\left(\mathrm{O}_{3}, \mathrm{VMK}(\mathrm{Analy}-\right. \\
\text { sator }) / \mathrm{ppm}\end{array}$ & $\begin{array}{c}x\left(\mathrm{O}_{3}, \mathrm{AZ}(\mathrm{Ex}-\right. \\
\text { pansion })) / \mathrm{ppm}\end{array}$ & $\begin{array}{c}x\left(\mathrm{O}_{3}, \mathrm{AZ}(\mathrm{UV}),\right. \\
\text { Ist }) / \mathrm{ppm}\end{array}$ & $\begin{array}{c}x\left(\mathrm{O}_{3}, \mathrm{AZ}\right. \\
(\mathrm{IR})) / \mathrm{ppm}\end{array}$ \\
\hline \hline $4.13(1.07)$ & aus $(-)$ & 1.07 & 0.81 & $\approx 1$ \\
\hline $4.18(1.07)$ & $4.2(1.09)$ & 1.09 & 1.09 & $\approx 1$ \\
\hline $8.03(2.09)$ & $9.1(2.37)$ & 2.09 & 2.04 & $\approx 2$ \\
\hline $7.33(1.91)$ & $7.4(1.92)$ & 1.90 & 1.97 & $\approx 2$ \\
\hline $11.45(2.98)$ & $12.3(3.20)$ & 2.98 & 2.93 & $\approx 3$ \\
\hline
\end{tabular}

Tab. 4.2.: Vergleich der bestimmten Ozonkonzentrationen $x$ mit Hilfe verschiedener Messmethoden. Zum Vergleich der Werte aus VMK und AZ müssen erstere Werte mit dem Faktor für das Volumenverhältnis $(f=0.26)$ multipliziert werden. Die Ergebnisse sind in Klammern angegeben.

Mit den Ergebnissen der Kreuzkalibrierung konnte davon ausgegangen werden, dass die standardmäßige Bestimmung der Ozonkonzentration über die UV-Optik (Sollkonzentration mit Hilfe der abgelesenen Werte einstellen ergibt später richtige Istkonzentration für das Experiment mit nachträglicher Auswertung) eine ausreichende Genauigkeit und Absicherung für den weiteren Einsatz besitzt.

Der kommerziell erhältliche Ozonanalysator eignet sich aus verschiedenen Gründen nicht für den standardmäßigen Einsatz bei den Aerosolexperimenten. Zum einen ist der Betrieb des Gerätes nur bei Atmosphärendruck in der Vormischkammer möglich. Dies ist allerdings für Untersuchungen der druckabhängigen Partikelbildung nicht praktikabel, da so zwangsläufig die erreichbaren Minimaldrücke in der Zelle limitiert wären. Aus den Expansionsverhältnis- 
sen würde sich ein Enddruck von ca. 460 mbar (bei der Expansion aus beiden Vorkammern) bzw. ca. 350 mbar (bei alleiniger Nutzung der Ozonkammer) ergeben. Da die meisten Kohlenwasserstoffverbindungen jedoch, wie in Kapitel 6 dargestellt, oft erst deutlich unterhalb von 200 mbar Reaktionsdruck auf die veränderten Bedingungen reagieren, wären diese Enddrücke deutlich zu hoch für eine umfassende Untersuchung zum Mechanismus der Partikelformation. Zum anderen zeigt die Betrachtung der höheren Konzentrationswerte, dass die Abweichungen im Bereich der Nachweisgrenze des Analysators $\left(x_{O_{3}}=10 \mathrm{ppm}\right)$ zwischen den Werten des Analysators und der übrigen Methoden deutlich gröBer werden. Dies würde den verwendbaren Konzentrationsbereich bei Betrieb an der VMK stark einschränken.

Die Annahme der festen Expansionsverhältnisse und daraus resultierenden Ozonkonzentrationen in der Aerosolzelle musste beibehalten werden, da der Betrieb des Ozonanalysators an der Aerosolzelle problematisch wäre. Weil das Ozon erst gleichzeitig mit oder kurz nach der Kohlenwasserstoffverbindung eingegeben wird und dort abreagiert, könnten keine verlässlichen Konzentrationswerte erhalten werden, würde eventuell das Gerät durch die von Ozon verschiedenen Edukte geschädigt werden und außerdem noch die aerosolhaltige Reaktionsmischung unter Umständen so verdünnt werden, dass die anschließende Aerosolmessung, die das eigentliche Kernthema der hier durchgeführten Untersuchungen darstellt, weniger zuverlässige Werte liefern würde.

Auch die UV-Optik ließ sich für Ozonolyseversuche nicht direkt an der Zelle einsetzen. Dies liegt an der Bildung von Nanopartikeln während der Reaktion, an denen die UV-Strahlung gestreut wird (MIE-Streuung) [106], wodurch die gemessene Intensität $I$ als Maß für die Extinktion verfälscht werden kann.

\subsection{Additiva}

\subsection{1. $\mathrm{SO}_{2}$}

Im Rahmen dieser Arbeit wurden Experimente durchgeführt, die den Einfluss von $\mathrm{SO}_{2}$ auf die Partikelbildung verschiedener biogener Substanzen und Modellverbindungen untersuchen sollten. Um dabei annähernd atmosphärische 
Bedingungen zu simulieren, mussten dazu so geringe Konzentrationen eingesetzt werden, dass diese nicht mehr durch IR-Messungen nachweisbar waren und nicht direkt aus einem Vorratsgefäß, sondern nur nach Vorverdünnung mit Trägergas volumetrisch eingefüllt werden konnten. Außerdem sollte auf diese Art, von sehr geringen Konzentrationen ausgehend, eine mögliche Grenzkonzentration identifiziert werden, ab der Schwefeldioxid einen Einfluss im Partikelbildungsmechanismus auszuüben beginnt.

Zunächst wurde eine direkte Kalibrierung vorgenommen. Dazu wurden Leerkanalspektren bei verschiedenen Gesamtdrücken des Badgases (ca. 30 mbar, ca. 100 mbar, ca. 500 mbar und ca. 1000 mbar; jeweils in Synthetischer Luft und über 200 Scans gemittelt) aufgenommen. Nach Abpumpen auf den Enddruck und Bestimmung der Leckrate wurde volumetrisch eine definierte Menge Schwefeldioxid in die Zelle eingefüllt und anschließend unter sukzessivem Auffüllen mit Badgas bei den genannten Drücken die Kalibrierspektren gemessen. So konnte bei der Auswertung der Spektren im Anschluss die Druckabhängigkeit losgelöst von möglichen Konzentrationsunterschieden durch den Einfüllvorgang betrachtet werden (vgl. Abbildung 4.3). Integriert wurde jeweils über die Bande der asymmetrischen (1405-1302 $\left.\mathrm{cm}^{-1}\right)$ sowie die der symmetrischen Streckschwingung (1240-1059 $\left.\mathrm{cm}^{-1}\right)$ [107].

Der Auftragung der integralen Absorbanz gegen den $\mathrm{SO}_{2}$-Partialdruck, der Kalibriergeraden, lag dabei die Abhängigkeit basierend auf dem LAMBERTBEERschen Gesetz (GI. 4.5, 4.1) sowie 4.2 zugrunde. Für die beiden betrachteten Integrationsbereiche ergaben sich dabei folgende Werte aus der Auftragung 4.4

$$
\begin{aligned}
& \text { - } \kappa_{1}=2.880 \pm 0.025 \quad\left(1405-1302 \mathrm{~cm}^{-1}\right) \\
& \text { - } \kappa_{2}=0.3739 \pm 0.0039\left(1240-1059 \mathrm{~cm}^{-1}\right)
\end{aligned}
$$

Wie in Abbildung $4.3 \mathrm{zu}$ sehen ist, weist die symmetrische Streckschwingung deutlich höhere integrale Absorbanzen auf als die schwächer absorbierende asymmetrische Streckschwingung, erstere würde auch einen Nachweis bei recht niedrigen Konzentrationen ermöglichen. Allerdings ist die symmetrische auch wesentlich stärker druckabhängig (vgl. Abb. 4.3 und 4.5) als die 


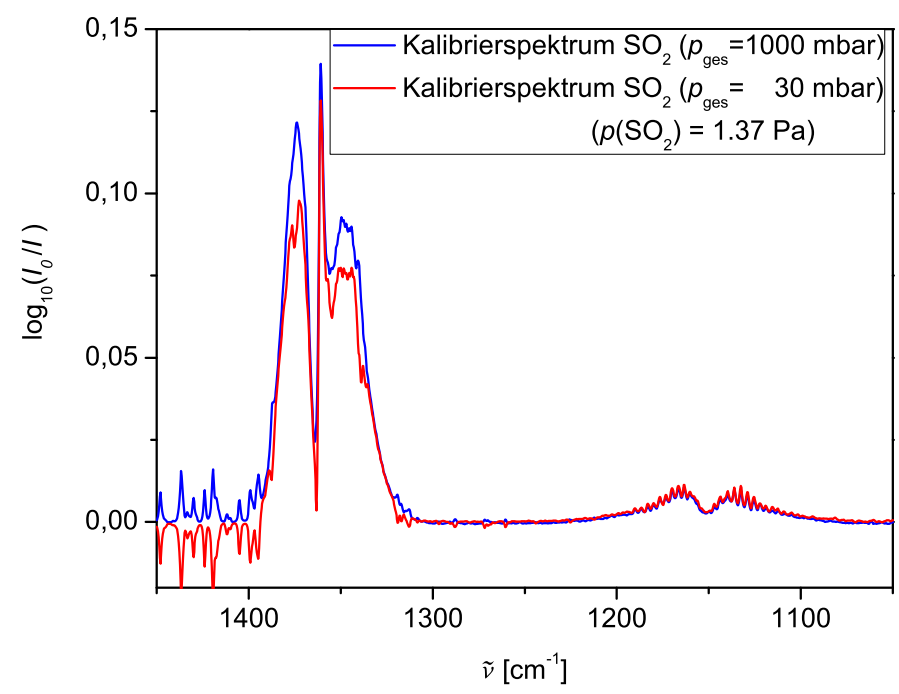

Abb. 4.3.: Absorbanzspektren des $\mathrm{SO}_{2}$ bei identischen Partialdrücken und verschiedenen Gesamtdrücken.

asymmetrische Streckschwingung, was jedoch vor allem bei höheren Partialdrücken (oberhalb von $p_{\mathrm{SO}_{2}}=2 \mathrm{~Pa}$ ) oder niedrigen Zelldrücken, gerade im später in den Partikelbildungsexperimenten interessierenden Bereich von unter $p_{\text {ges }}=500$ mbar, zum Tragen kommt.

Damit die im Experiment benötigten geringen Konzentrationen erreicht werden konnten, wurde für die eigentlichen Messungen eine niedrig konzentrierte $\mathrm{SO}_{2} / \mathrm{N}_{2}$-Mischung in einem 2 L-Glaskolben vorbereitet, aus dem wiederum geringe Mengen in die Aerosolzelle eingefüllt wurden. Der Glaskolben wurde dazu zunächst an einer Glaslinie mit Drehschieberpumpe vorbereitet. Die Linie ist mit zwei Druckmessköpfen für einen Druckbereich bis 10 bzw. 1000 mbar sowie Rotulex-Anschlüssen ausgerüstet, über die Badgasflasche, $\mathrm{SO}_{2}$-Flasche und Glaskolben miteinander verbunden sind und letzterer befüllt werden kann. Zunächst wurde der Kolben evakuiert und mehrmals mit Stickstoff gespült. Anschließend wurden verschiedene Partialdrücke Schwefeldioxid in den Kolben gegeben und mit Stickstoff ( $\geq 99.9990 \%$, Fa. Air Liquide) auf 1000 mbar aufgefüllt. Aus diesem Kolben wurde anschließend in die Aerosolzelle expandiert und die mit IR detektierte Menge mit der aus den Volumenverhältnissen zwischen Kolben und Aerosolzelle erwarteten Sollkonzentration im Reaktor verglichen. 

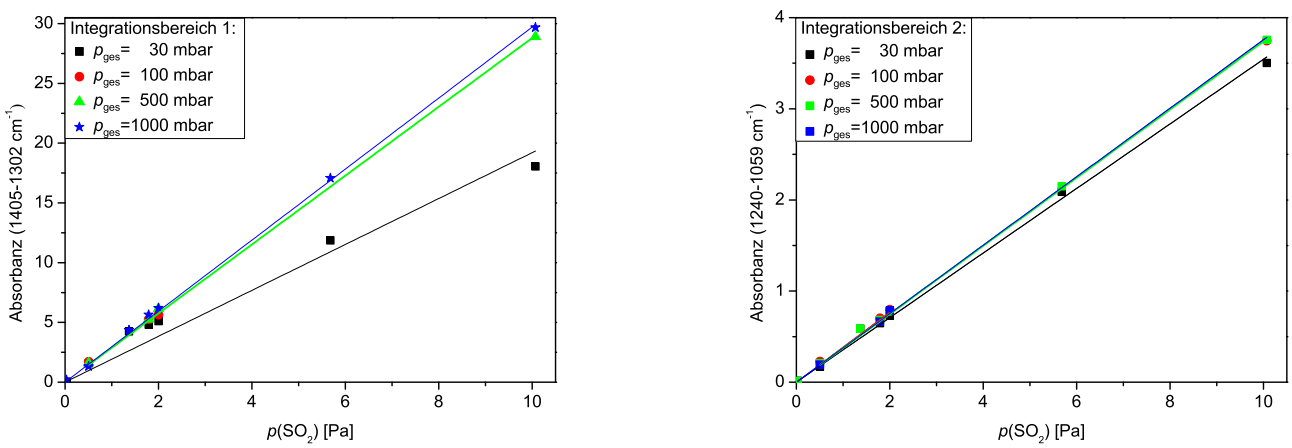

Abb. 4.4.: Auftragungen der Absorbanz gegen den Partialdruck von $\mathrm{SO}_{2}$ für den Integrationsbereich $1405-1302 \mathrm{~cm}^{-1}$ und den Integrationsbereich $1240-1059 \mathrm{~cm}^{-1}$, es ergeben sich Korrelationskoeffizienten > $99 \%$ bei $p_{\text {ges }} \geq 500$ mbar.

Dabei wurden sowohl Tests durchgeführt, bei denen aus Kolben mit niedriger Vorkonzentration ein großer Druckanstieg realisiert wurde, als auch solche, bei denen aus einem Kolben, der mit einem hohen $\mathrm{SO}_{2}$-Partialdruck befüllt war, nur geringe Mengen in die Aerosolzelle eingelassen wurden. Dadurch sollten Fehler durch unterschiedliches Strömungsverhalten bei schnellem oder langsamen Einfüllvorgang vermieden und die Richtigkeit der in die spätere Konzentrationsberechnung eingehenden Verdünnungsverhältnisse überprüft werden.

Die Berechnung der in der Aerosolzelle vorliegenden $\mathrm{SO}_{2}$-Konzentration über die durchgeführte Kalibrierung und Gleichung 4.2 ergab durchschnittlich eine Abweichung von ca. $10 \%$ von der aufgrund des Volumenverhältnisses erwarteten. Die Differenz lässt sich auf Unsicherheiten bei der $\mathrm{SO}_{2}$-Eingabe in den Glaskolben zurückführen, da sich die Genauigkeit der Druckmessköpfe lediglich auf $10^{-2}$ mbar beschränkte und durch Auf- und Abrunden der zweiten Nachkommastelle der Schwefeldioxidpartialdruck lediglich auf $\pm 0.01 \mathrm{mbar}$ genau einfüllen ließ. Aus diesem Grund wurde die tatsächlich vorliegende $\mathrm{SO}_{2}$ Konzentration jeder Kolbenfüllung überprüft. Zunächst wurde grundsätzlich ein $\mathrm{SO}_{2}$-Partialdruck von 0.5 mbar in den Glaskolben eingefüllt und mit Stickstoff auf 1000 mbar aufgefüllt. Von dieser Mischung wurden anschließend so viel in die Aerosolzelle expandiert, dass ein Druckanstieg von 20 mbar stattfand und rechnerisch so viel $\mathrm{SO}_{2}$ in der Zelle vorliegen sollte (ca. $1 \mathrm{~Pa}$ ), dass es ohne 

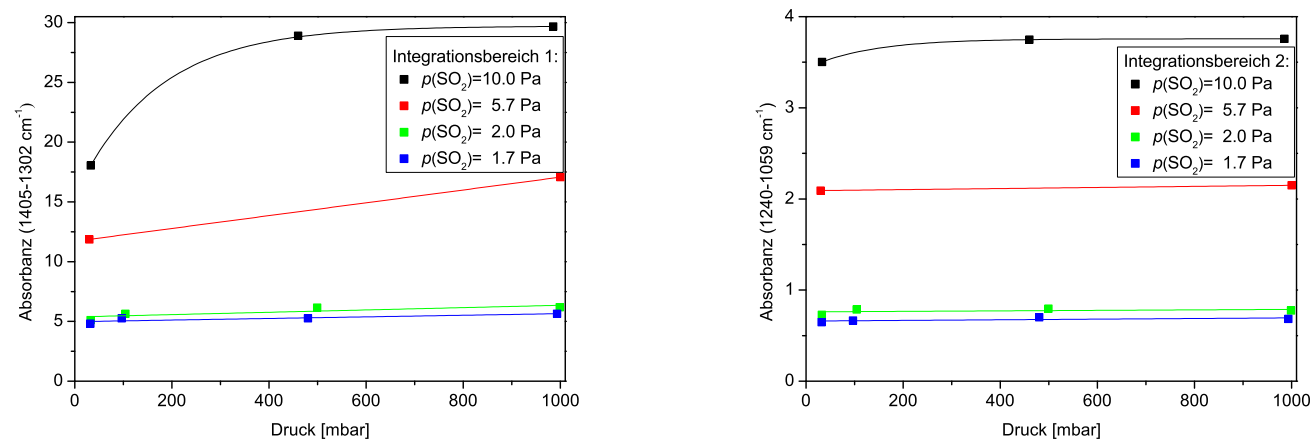

Abb. 4.5.: Auftragungen der Absorbanz gegen den Zelldruck für den Integrationsbereich 1405-1302 $\mathrm{cm}^{-1}$ und den Integrationsbereich 1240$1059 \mathrm{~cm}^{-1}$.

konzentrationsinduzierte Nichtlinearitäten spektroskopisch nachweisbar ist. Anschließend wurde die Aerosolzelle mit Synthetischer Luft auf 500 mbar aufgefüllt, da die Absorbanz bei diesem Druck ausreichend linear von der Konzentration abhängig ist. Anschließend wurde aus der gemessenen Absorbanz der tatsächlich vorliegende Schwefeldioxidgehalt der Mischung bestimmt und daraus die einzufüllenden Vormischungsmengen für bestimmte $\mathrm{SO}_{2}$-Konzentrationen in der Zelle berechnet.

\subsection{2. $\mathrm{H}_{2} \mathrm{O}$}

Bei einigen der $\mathrm{SO}_{2}$-Zugabeexperimenten wurde der Reaktionsmischung Wasser als weiteres Additiv zugesetzt, um die Bildung von Schwefelsäure aus Schwefeldioxid zu beschleunigen (vgl. Abschnitt 2.8). Eine Kalibrierung, bei der sich aus den Absorbanzen auf genaue Konzentrationen innerhalb der Reaktionszelle rückschließen ließ, war für Wasser nicht möglich. Einerseits verhalten sich seine Banden höchst empfindlich gegenüber Konzentrations- und Druckänderungen, andererseits verhalten diese Abhängigkeiten nichtlinear. So hätte eine genaue Kalibrierung einen sehr hohen Aufwand bedeutet. Der Hauptgrund gegen eine pascalgenaue Wasserkalibrierung war jedoch die Tatsache, dass der Strahlengang des Spektrometers zum Teil durch die Raumluft verlief und trotz konstantem Spülen des Spektrometers mit trockener Luft Schwankun- 
gen der Luftfeuchtigkeit im Labor (durch Öffnen der Tür o.ä.) sehr deutlich im Spektrum zu sehen sind, ohne sich jedoch genau quantifizieren zu lassen. Da kein Hygrometer zur Messung der in der Zelle herrschenden Luftfeuchtigkeit zur Verfügung stand, wurde die Menge des in den $\mathrm{SO}_{2}$-Experimenten teilweise eingesetzten Wassers (demineralisiertes Wasser, Hausleitung) lediglich volumetrisch bestimmt und anhand des Volumenanteils und unter Annahme der Gültigkeit des idealen Gasgesetzes die vorliegende Luftfeuchtigkeit nach Gl. 4.9 abgeschätzt.

$$
\begin{array}{rr}
p_{\text {Sätt., } \mathrm{H}_{2} \mathrm{O}} \cdot V_{\text {Kolben }}= & p_{\mathrm{AZ}} \cdot V_{\mathrm{AZ}} \\
\Leftrightarrow p_{\mathrm{AZ}}= & \frac{p_{\text {Sätt., } \mathrm{H}_{2} \mathrm{O}} \cdot V_{\text {Kolben }}}{V_{\mathrm{AZ}}}
\end{array}
$$

Mit den bekannten Volumina des Glaskolbens für Wasser ( $\left.V_{\text {Kolben }}=500 \mathrm{~mL}\right)$, der Reaktionszelle $\left(V_{\mathrm{Az}}=64.5 \mathrm{~L}\right)$ und des Sättigungsdampfdrucks von Wasser bei der herrschenden Raum- und Zellentemperatur von ${ }^{2} 2^{\circ} \mathrm{C}$ ( $p_{\mathrm{Sätt} . \mathrm{H}_{2} \mathrm{O}, 22 \mathrm{C}}=26.447$ mbar, entnommen aus [108]) ließ sich zunächst abschätzen, dass mit diesem Vorgehen ein Druck von rechnerisch

$$
p_{\mathrm{AZ}}=\frac{23.4 \mathrm{mbar} \cdot 0.5 \mathrm{~L}}{64.5 \mathrm{~L}} \approx 0.205 \mathrm{mbar}
$$

erreicht werden könnte. Allerdings berücksichtigt diese Rechnung noch nicht den Druckgradienten zwischen Wasserkolben und evakuierter Reaktionszelle. Da sich die Siedetemperatur von Wasser bei Druckhalbierung um ca. $10^{\circ} \mathrm{C}$ verringert [108], begann das im Kolben befindliche Wasser direkt bei Öffnung der Verbindung zwischen Kolben und Zelle zu sieden, wodurch leicht Druckanstiege von mehr als 5 mbar in der Aerosolzelle realisierbar waren.

Allgemein lässt sich die relative Luftfeuchte $R H$ bei Annahme einer Umgebungstemperatur von ${ }^{\circ 2} \mathrm{C}$ basierend auf Literaturwerten [108] berechnen über

$$
R H=\frac{p_{\mathrm{H}_{2} \mathrm{O}, \text { Einlass }}}{p_{\text {Sätt. }, \mathrm{H}_{2} \mathrm{O}, 22 \mathrm{C}}} \cdot 100 \%
$$

Damit ergibt sich bei den durchgeführten Experimenten bei den gewählten Wasserpartialdrücken jeweils folgende relative Luftfeuchte: 


\begin{tabular}{|c|c|c|c|c|}
\hline $\begin{array}{c}\text { Eingefüllter } \mathrm{H}_{2} \mathrm{O}-\text {-Partialdruck } \\
\left(p_{\left.\mathrm{H}_{2} \mathrm{O}, \text { Einlass }\right)}\right) \text { [mbar] }\end{array}$ & $3.0 \cdot 10^{-3}$ & 0.1 & 1.0 & 7.0 \\
\hline $\ln \mathrm{AZ}$ vorliegende $R H$ & $0.011 \%$ & $0.38 \%$ & $3.8 \%$ & $26 \%$ \\
\hline
\end{tabular}

Tab. 4.3.: Bestimmung der relativen Luftfeuchte in der Aerosolzelle.

Der mit 3.0.10-3 mbar sehr gering erscheinende Partialdruck entspricht dem Wert eines „trockenen“ Experimentes ohne weitere Wasserzugabe, da laut Herstellerangaben maximal ein Anteil von 3 ppm Wasser in der Synthetischen Luft enthalten sind. Durch Undichtigkeiten im Bereich der Ozonzuführung über Schlauchverbindungen in die Vormischkammer könnte dieser Wert noch etwas höher ausfallen und eventuell um 5 ppm liegen (zur Begründung s. Kap. 6.5.8).

\subsection{3. $\mathrm{C}_{6} \mathrm{H}_{12}$}

Cyclohexan reagiert in seinen Absorbanzsignalen sehr empfindlich auf Konzentrations- und Druckänderungen. Daher war hier eine Kalibrierung nach dem Vorbild der übrigen verwendeten reinen Kohlenwasserstoffverbindungen mit der Aufnahme von Spektren bei verschiedenen Einfüllmengen und anschließender Auftragung der integrierten Absorbanz ausgewählter Signale gegen die Partialdrücke nicht zwangsläufig aussagekräftig. Auch die Bestimmung der in reaktiven Mischungen vorliegenden Partialdrücken aus Vergleich und Faktorenbildung mit vorliegenden Kalibrierspektren konnte nur sehr begrenzt erfolgen. Zudem ergeben sich Schwierigkeiten aus den hohen Substanzkonzentrationen bei Radikalfängerexperimenten, da sich die Signale zum Teil in der Sättigung befanden, wie in Abbildung 4.6 beispielhaft gezeigt wird. Die Verwendung so hoher Partialdrücke war jedoch nicht zu vermeiden, da durch das Cyclohexan ein signifikanter Teil der während der Reaktion entstehenden $\mathrm{OH}$-Radikale abgefangen werden sollte, um die aus ihnen folgenden Reaktionsschritte zu behindern. Wenn die Reaktionsgeschwindigkeitskoeffizienten der Kohlenwasserstoffverbindungen mit $\mathrm{OH}$-Radikalen, z.B. $\alpha-$ Pinen $+\mathrm{OH} \longrightarrow$ Produkte, in einer ähnlichen Größenordung wie $\mathrm{OH}+$ $\mathrm{C}_{6} \mathrm{H}_{12} \longrightarrow$ Produkte liegen (vgl. Tabelle 2.3 in Kapitel 2.5), ist es ausreichend, über die Bereitstellung einer etwas höheren Cyclohexankonzentration die Re- 
aktionsgeschwindigkeit der Radikalfängerreaktion zu erhöhen und damit zu begünstigen 4 . Allerdings ist die $\mathrm{H}$-Abstraktionsreaktion an kleineren Alkenen durch $\mathrm{OH}$-Radikale meist deutlich schneller als die Ozonolyse der olefinischen Doppelbindung. Als Beispiel kann hier Cyclohexen mit Geschwindigkeitskeffizi-

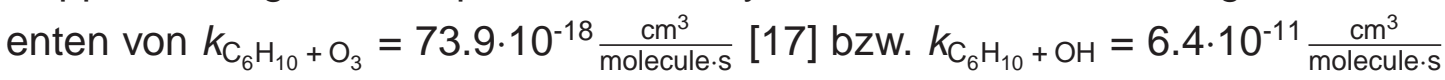
[109] dienen, bei dem die Radikalreaktion um mehrere Größenordnungen schneller verläuft. Dementsprechend müsste, um die Radikale effektiv und vollständig abzufangen, eine um mindestens den gleichen Faktor im Vergleich zur Alkenkonzentration erhöhter Cyclohexanpartialdruck zur Reaktionsmischung zugegeben werden.

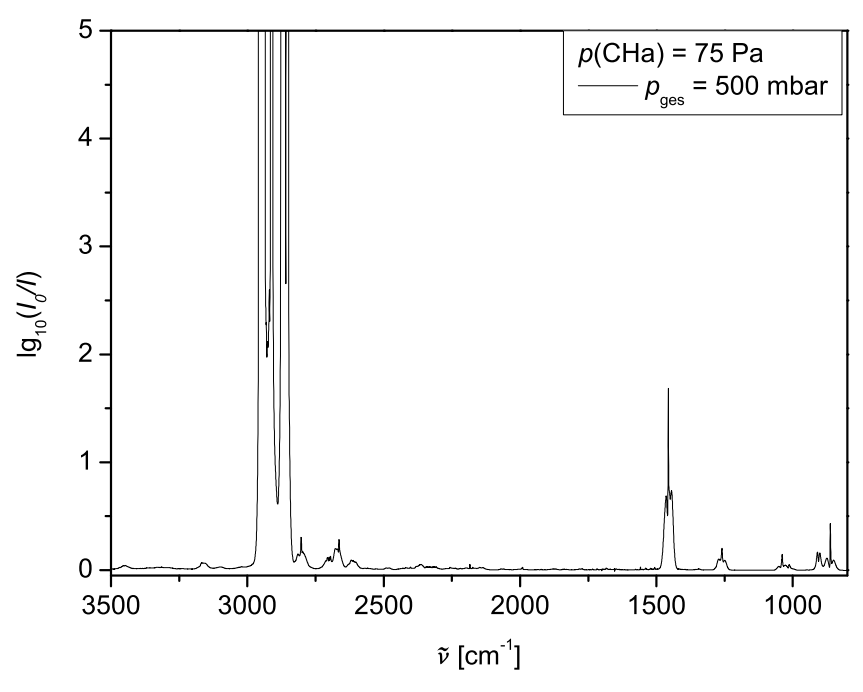

Abb. 4.6.: Reinspektrum des Cyclohexans.

Stattdessen wurden lediglich vereinzelte Reinspektren des Cyclohexans aufgenommen, die in ihren Partialdrücken denen der Reaktionsspektren entsprachen, um die Signale aus Kinetik- oder Produktspektren möglichst vollständig herausrechnen zu können. Dies gelang jedoch nicht immer umfassend, da die oben genannten Abhängigkeiten von Gesamt- und Partialdruck auch in den relativen Verhältnissen einzelner Signale zueinander sichtbar wurden.

${ }^{4}$ Die Reaktion $\mathrm{OH}+\mathrm{O}_{3} \longrightarrow \mathrm{HO}_{2}+\mathrm{O}_{2}$ verläuft wesentlich, d.h. ca. drei Größenordnungen, langsamer und ist daher vernachlässigbar. 


\subsection{4. $\mathrm{C}_{6} \mathrm{H}_{10} \mathrm{O}$}

Cyclohexanon wurde als Additiv lediglich in wenigen Partikeltestmessungen mit der Substanz 2-Methyl-1-penten verwendet. Da es bei der Verwendung des Radikalfängers Cyclohexan zu unerwarteten Ergebnissen kam (s. Kap. 6.4.1.2), sollte durch Zugabe von $\mathrm{C}_{6} \mathrm{H}_{10} \mathrm{O}$ überprüft werden, ob sich die Zunahme an partikulärer Materie durch Folgeprodukte des Radikalfängers, als Hauptprodukt entsteht Cyclohexanon, erklären ließ (vgl. Kap 2.4.1] und [40]). In diesen Experimenten wurde Cyclohexanon in die Vormischkammer eingegeben; die Konzentrationen wurden zwischen einer Menge, die in der $\mathrm{C}_{6} \mathrm{H}_{12}+\mathrm{OH}$-Reaktion realistisch entstehen kann und einem deutlich höheren Partialdruck variiert, um reine Konzentrationseffekte ausschließen zu können. Da diese Tests jedoch keine Erklärung für die beobachteten Abweichungen lieferten, wurden diese Experimente nicht ausführlich weiterverfolgt. Eine Kalibrierung war wegen nicht durchgeführter IR-spektroskopischer Charakterisierung der Produktbildung und Reaktionsgeschwindigkeitskoeffizienten obsolet. 


\section{Auswertungsmethoden}

\subsection{Bestimmung der Ozonkonzentration}

Wie in Abschnitt 4.2.1.2 beschrieben, wird der Anfangspartialdruck des Ozons im Reaktionsgemisch über eine UV-Optik und die nachträgliche Auswertung der aufgenommenen Referenz- und Signalspannungswerte bestimmt. Da die Lampenleistung über den Messtag nicht ideal konstant bleibt und auch nicht gleichbleibend von Signal- und Referenzdiode registriert wird, muss eine Korrelation zwischen der Leerspannung dieser beiden Messwerten (d.h. ohne Ozoneingabe) hergestellt werden. Diese ist nötig, um die Messwerte mit Ozonzugabe anschließend korrekt auswerten zu können, ohne dass Leistungsschwankungen fälschlicherweise als Konzentrationsänderungen angenommen werden.
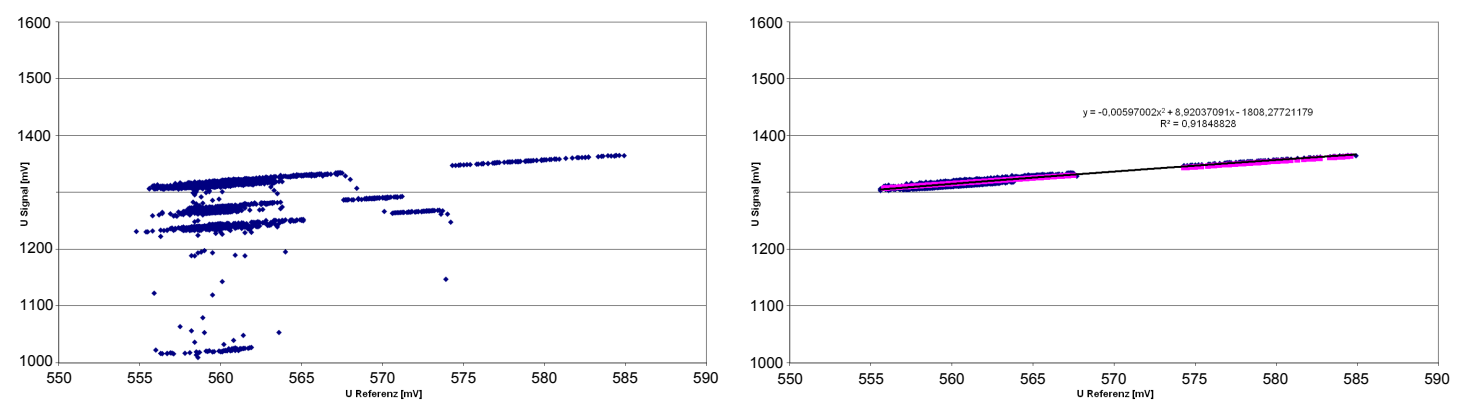

Abb. 5.1.: Korrelation der Signal- und Referenzspannung vor (links) und nach (rechts) der Korrektur um die Ozonmesswerte.

Daher wurden die ausgelesenen Spannungswerte, wie in Abbildung 5.1 gezeigt, in einem vorbereiteten EXCEL-Sheet zunächst um die Substanzmesswerte reduziert und anschließend eine polynomische Anpassung (schwarze Kurve) durch die verbliebenen Leerspannungswerte (restliche blaue Punkte) 
vorgenommen. Die magentafarbene Linie stellt dabei lediglich eine Hilfsgerade zur Separierung der Leer- und Substanzmesswerte dar.

Nach Korrektur aller Messwerte um die polynomische Ausgleichsfunktion werden diese gegen die Messzeit aufgetragen, wobei für jede Expansion, also jede einzelne durchgeführte Ozonolysereaktion, ein Peak mit zwei Plateaus erhalten wird (Abb. 5.2).

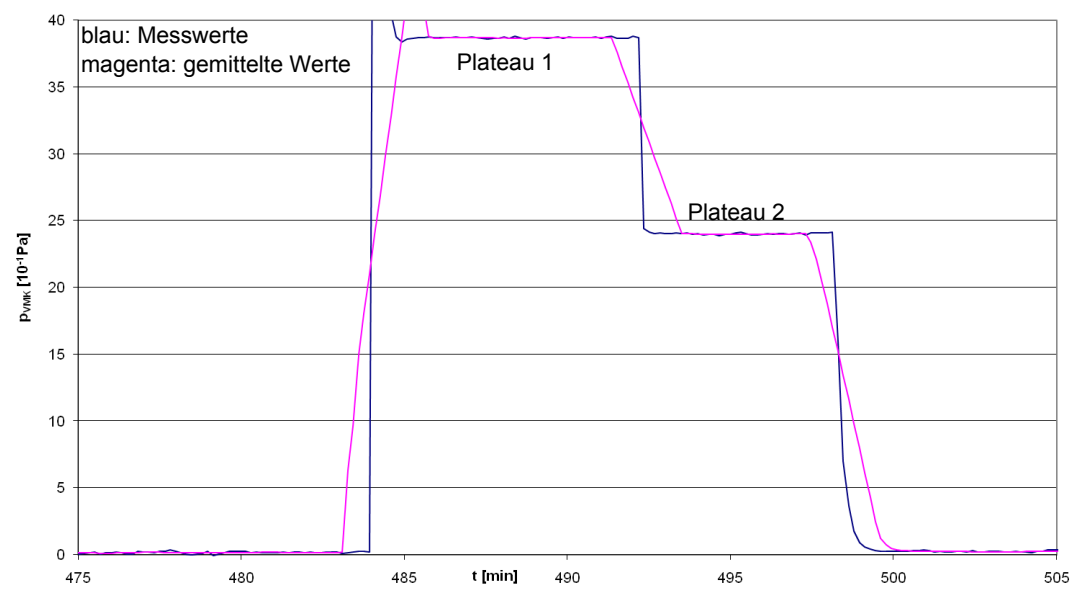

Abb. 5.2.: Ausschnitt der Auftragung des Ozonpartialdrucks in der Vormischkammer, aufgetragen gegen die Aufnahmezeit des Voltmeters.

Das erste Plateau entspricht stets dem Ozonpartialdruck der Vormischkammer vor $\left(p_{0, \mathrm{VMK}}\right)$, das zweite dem nach der Expansion in die Aerosolzelle $\left(p_{\text {Ende,VMK }}\right)$. Der in der Reaktionsmischung enthaltenen Anfangspartialdruck $p_{0}\left(\mathrm{O}_{3}\right)$ ergibt sich somit aus der Differenz der beiden Plateaus multipliziert mit einem Faktor für das Volumenverhältnis der Ozonvormischkammer zur Aerosolzelle $\left(\frac{V_{\text {MMK }}}{V_{\text {AZ }}}\right)$, wie in GI. 5.1]beschrieben.

$$
p_{0}\left(\mathrm{O}_{3}\right)=\frac{V_{\mathrm{VMK}}}{V_{\mathrm{AZ}}} \cdot\left(p_{0, \mathrm{VMK}}-p_{\text {Ende, } \mathrm{VMK}}\right)
$$




\subsection{Infrarotspektren}

Generell wird der Verbrauch der Edukte wie auch die Zunahme der Produkte $(\Delta)$ aus der Differenz der Anfangspartialdrücke $\left(p_{0}\right)$ zu dem am Ende der betrachteten Reaktionszeit vorliegenden Partialdruck $(p)$ bestimmt.

$$
\Delta=p_{0}-p
$$

Zunächst werden die aufgenommenen Reaktionsspektren, die aufgrund der gewählten Anfangskonzentrationen mit einem deutlichen Alkenüberschuss Reste der Kohlenwasserstoffverbindung und teilweise auch des Ozons enthalten, eduktkorrigiert. Dazu wird über einen Vergleich der Bandenintensitäten der betrachteten Verbindung in Reaktions- und einem unter ähnlichen Bedingungen aufgenommenen Reinspektrum (v.a. bezüglich Gesamt- und Partialdruck um z.B. bei Substanzen mit Rotationsaufspaltung eine Fehlbestimmung zu verhindern) der Faktor zwischen diesen bestimmt. Anschließend wird das Produkt aus diesem Faktor und dem Reinspektrum von dem Reaktionsspektrum abgezogen, um ein eduktkorrigiertes Spektrum zu erhalten, in dem die auftretenden Signale nur noch den Produktverbindungen zuzuordnen sind. Diese Berechnung (Faktor aus Intensitätsverhältnis multipliziert mit Referenzkonzentration) erlaubt gleichzeititg die Bestimmung der vorliegenden Restkonzentration der Edukte und damit nach Gl. 5.2 die Berechnung des Substanzverbauchs.

Durch den Einkanalbetrieb des Spektrometers und der daraus folgenden zeitliche Verzögerung zwischen Leerkanal- und Substanzspektrum kann es sowohl zu Schwankungen der Grundlinie (durch eine stetige Erwärmung des stickstoffgekühlten Detektors und daraus folgendem schlechter werdendem Signal) als auch der Signale von Wasser und $\mathrm{CO}_{2}$ (da der Strahlengang durch die Laborluft verläuft) kommen. Beide Probleme lassen sich oft nicht durch bloße Division zur Erstellung eines Absorbanzspektrums (GI. [5.3) beheben. Daher sollte nach der Eduktkorrektur vor der Auswertung der Produktsignale bei Bedarf zunächst die Korrektur der Grundlinie und der nicht aussagekräftigen Hintergrundsubstanzen erfolgen. Für ersteres steht in OPUS eine Korrekturfunktion zur Verfügung, die nach Markierung einiger Punkte auf der „krummen“ Grundlinie diese glät- 
tet, ohne die Signale zu verformen, zweiteres lässt sich durch Subtraktion von Reinspektren in der gleichen Weise wie die Eduktkorrektur durchführen.

$$
A=\lg \frac{I_{0}}{l}
$$

\subsubsection{Kinetikmessungen}

Im Falle von Kinetikmessungen wird durch die Eduktkorrektur lediglich die korrekte Bestimmung der Faktoren und daraus folgend die Abnahme des Eduktpartialdrucks überprüft. Anschließend wird die weiterführende Auswertung mit einem vorbereiteten EXCEL-Skript vorgenommen.

Durch Eingabe der berechneten Faktoren zu jedem Reaktionszeitpunkt und der möglichst genau zu bestimmenden Anfangskonzentrationen von Kohlenwasserstoffverbindung und Ozon ergibt sich die verbliebene Eduktkonzentration, die Differenzen beider ergeben die Umsätze der Edukte. Diese werden gegeneinander aufgetragen (vgl. Abb. 5.3, links), um aus der Steigung den exerimentell bestimmten Koeffizienten $\alpha$ zu berechnen.
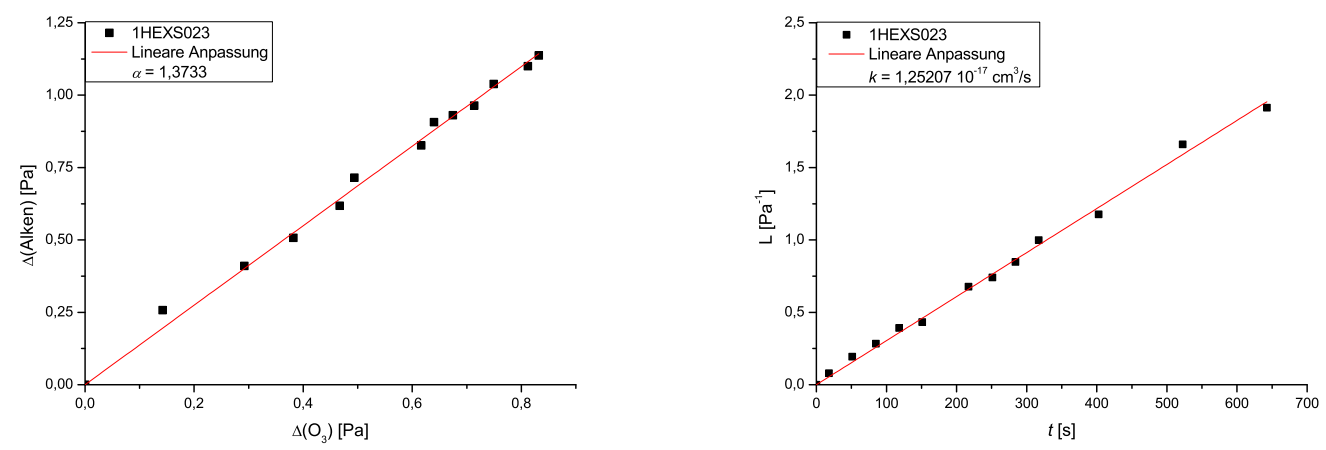

Abb. 5.3.: Bestimmung des relativen Verbrauchs $\alpha$ (links) und Auftragung der Lösung des Zeitgesetzes nach Gl. 2.27 gegen die Reaktionszeit (rechts).

Während die Ozonolyse als Reaktion erster Ordnung theoretisch zu stöchiometrischen Koeffizienten von $\nu_{\mathrm{i}}=1$ und damit auch zu einer Steigung von $\alpha=1$ führen sollte, können während der Reaktion gebildete Radikale dieses Verhält- 
nis durch den zusätzlichen Verbrauch eines oder beider Edukte beeinflussen. $\alpha \neq 1$ gibt daher den zusätzlichen Verbrauch eines Edukts durch in der Ozonolysereaktion gebildete Radikale an. Es ergäbe sich $\alpha<1$ bei Radikalreaktionen, die zusätzlich Ozon verbrauchen, was allerdings unter den gegebenen Bedingungen nicht auftritt. Bei $\alpha>1$ wird hauptsächlich der Kohlenwasserstoff durch Radikale abgebaut. Nach Bestimmung von $\alpha$ wird mit Hilfe dieses Wertes mit Gleichung 2.27 S. 51]der Geschwindigkeitskoeffizient $k(T)$ bestimmt. Dazu wird in einer Auftragung die linke Seite der Lösungsfunktion (,L“) gegen die Reaktionszeit aufgetragen, wodurch sich der Geschwindigkeitskoeffizient aus der Steigung ergibt. Um den erhaltenen Wert zu überprüfen, wird auch die rechte Seite der expliziten Lösung $\left(, k(T) \cdot t^{\prime \prime}\right)$ gegen die Zeit aufgetragen, bei korrekter Bestimmung von $k(T)$ können beide Ausgleichsgeraden zur Deckung gebracht werden (vgl. Abb. 5.3, rechts). Dies stellt gleichzeitig auch sicher, dass die Annahme von ledigleich $\mathrm{OH}$-Radikal induzierten Nebenreaktionen und damit einer $\mathrm{OH}$-Ausbeute von $Y_{\mathrm{OH}}=\alpha-1$ (vgl. Gl. 2.28) in Kap. 2.5) plausibel ist.

\subsubsection{Produktbildungsstudien}

Produktstudien werden, abgesehen von den deutlich höheren eingesetzten Eduktkonzentrationen, analog zu Kinetikmessungen durchgeführt und in Bezug auf die IR-Spektren auch ähnlich ausgewertet. Nach Grundlinien-, Wasserund $\mathrm{CO}_{2}$-Korrektur werden auch hier die ausgewählten Absorbanzspektren (Leerkanal- und Substanzspektrum jeweils über 200 Scans gemittelt) zunächst um die verbleibenden Edukte korrigiert und damit gleichzeitig der Verbauch des jeweiligen Reaktanden bestimmt. Dieser wird zur späteren Bestimmung der jerweiligen Produktausbeuten benötigt, da diese immer auf Verbauch eines der Edukte, in den hier vorgestellten Studien meist Ozon, normiert werden:

$$
Y_{\text {Produkt }}=\frac{\Delta p_{\text {Produkt }}}{\Delta p_{\text {Edukt }}}
$$

Generell werden Produktspektren stets sowohl in Synthetischer Luft als auch in Stickstoff und darin jeweils unter Nieder- und Hochdruckbedingungen (meist 30 bzw. 1000 mbar; Ausnahmen werden bei der Darstellung der jeweiligen Er- 
gebnisse gekennzeichnet) aufgenommen, da diese Einstellungen den Randbereichen der Bedingungen innerhalb der Partikelbildungsuntersuchungen entsprechen.

Bei der Bestimmung der verschiedenen Produktausbeuten wird analog zur Eduktkorrektur vorgegangen, d.h. anhand vorher aufgenommener Reinspektren der betrachteten Verbindungen mit bekannter Konzentration wird nach Vergleich der Peakhöhen ein Faktor bestimmt, mit dem das entsprechende Signal aus dem Produktspektrum subtrahiert werden kann. Aus dem Faktor, multipliziert mit bekannter Referenzkonzentration, ergibt sich so die Ausbeute des gebildeten Produktes.

Standardmäßig wurden die Produktspektren auf die Signale der folgenden Verbindungen in den genannten Wellenzahlbereichen hin überprüft. Die Wahl gerade dieser Signale ergab sich aus charakteristischen Banden, wie etwa spitze Q-Zweige.

- Kohlenstoffmonoxid $\left(2225-2025 \mathrm{~cm}^{-1}\right)$

- Formaldehyd $\left(1745 \mathrm{~cm}^{-1}\right)$

- Keten $\left(2200-2100 \mathrm{~cm}^{-1}\right)$

- Ethen $\left(950 \mathrm{~cm}^{-1}\right)$

- Ameisensäure $\left(1106 \mathrm{~cm}^{-1}\right)$

Zusätzlich ist von Interesse, inwiefern die unter verschiedenen experimentellen Bedingungen gebildete Menge dieser Verbindungen durch unterschiedliche Druck- oder Badgasumgebungen beeinflusst werden kann. Dies kann Aufschluss darüber geben, welche Reaktionskanäle bevorzugt oder behindert werden. Zu diesem Zweck werden Differenzen der verschiedenen Produktspektren gebildet, beispielsweise jeweils zwischen Hoch- und Niederdruckspektren im gleichen Badgas oder zwischen Spektren in Synthetischer Luft und Stickstoff bei gleichem Druck. Bei der Differenzbildung müssen allerdings die leicht unterschiedlichen Eduktumsätze der Edukte während der einzelnen Reaktionen beachtet werden und die Spektren daher auf einen bestimmten Umsatz eines der Edukte normiert werden. 


\subsection{Partikelbildungsmessungen}

Bei der Untersuchung von Partikelausbeuten wird häufig die logarithmische Normalverteilung (Lognormalverteilung) zur Beschreibung herangezogen, da die in einem statistischen Prozess natürlich entstehenden Aerosole durch diese Funktion meist hinreichend genau beschrieben werden können [10].

Im Allgemeinen können die gemessenen Partikelverteilungen direkt ausgewertet werden. Dazu werden aus den pro Experiment jeweils 6-8 aufgenommenen Verteilungen die drei mit der höchsten Gesamtanzahl herangezogen. Die einzelnen Kenngrößen dieser drei Verteilungen werden untereinander gemittelt und als Ergebnis in Abhängigkeit der Variablen (z.B. Gesamtdruck, Ozon-, Alken- oder Sauerstoffpartialdruck) aufgetragen. Die betrachteten Kenngrößen der Verteilung sind dabei die Gesamt- bzw. Maximalanzahl $N \pm \Delta N\left[\# \mathrm{~cm}^{-3}\right]$, der Median $d_{N} \pm \Delta d_{N}[\mathrm{~nm}]$, die Gesamtmasse $M \pm \Delta M\left[\mu \mathrm{g} \mathrm{m}^{-3}\right]$ sowie die geometrische Standardabweichung $\sigma_{\mathrm{g}} \pm \Delta \sigma_{\mathrm{g}}$.

Vor allem für die in dieser Arbeiten vorgestellten Ergebnisse des Cyclohexens (cHEX) konnte der Ansatz der Lognormalverteilung ausgenutzt werden, da diese Verbindung zum einen relativ wenige Partikelkeime bildet, wodurch die Häufigkeitsverteilung keiner perfekten Lognormalfunktion entspricht. Zum anderen folgt aus der geringen Keimbildung ein großer Durchmesser der einzelnen Partikel durch Aufkondensation schwerflüchtiger Produkte, wodurch ein signifikanter Anteil der Verteilung außerhalb der gerätespezifischen Messgrenzen liegt.

Um eine vollständige Verteilung zu erhalten, wird daher an eine Durchschnittsverteilung aus mehreren Samples der zugehörigen Messung eine Lognormalverteilung angepasst, deren Kenngrößen anschließend als Endergebnis des Partikelbildungsexperimentes ausgewertet werden. Damit die erhaltene Simulation möglichst genau gerät, wird die Funktion in ORIGIN an die durchschnittliche Verteilung von vier Samples einer Messung angeglichen, wobei die Verteilung (Gl.5.5) durch die Partikelanzahl $N$, die geometrische Standardabweichung $\sigma_{g}$ und den mittleren Partikeldurchmesser $\frac{d_{p}}{d_{N}}$ parametrisiert ist. Der Divisor 64 folgt dabei aus der Messsoftware, da 64 Messkanäle pro $10 \mathrm{~nm}$ Einheit des Medians zur Verfügung stehen. 


$$
\mathrm{d} N=\frac{\ln (10)}{64} \frac{N}{\sqrt{2 \pi} \cdot \ln \left(\sigma_{\mathrm{g}}\right)} \cdot \exp \left(-\frac{1}{2}\left(\frac{\ln \left(\frac{d_{\mathrm{p}}}{d_{\mathrm{N}}}\right)}{\ln \left(\sigma_{\mathrm{g}}\right)}\right)^{2}\right)
$$

Mit Hilfe dieser Formel wird ausgehend von den gemessenen Werten der Kenngrößen die Lognormalverteilung an die durchschnittliche Anzahlverteilung angepasst, wobei ein möglichst geringer Wert für $\sigma_{\mathrm{g}} z \mathrm{z}$ verwenden ist, damit die simulierte Kurve nicht zu breit gerät. Dieses würde vor allem einen großen Einfluss auf die Masse ausüben und den Wert durch Beiträge von Partikeln mit großen Durchmessern eventuell daher zu stark überschätzen, um noch aussagekräftig zu sein. Bei der Anpassung dienen vor allem die Flanken der gemessenen Verteilung als Orientierungspunkte, da die Spitze oft „abgeschnitten“ ist, daher keiner logarithmischen Normalfunktion mehr entspricht und keine Aussagekraft mehr hat. Für die Partikel wurde eine größenunabhängige durchschnittliche Dichte von $\rho_{\mathrm{p}}=1.2 \mathrm{~g} \cdot \mathrm{cm}^{-3}$ sowie eine sphärische Form angenommen, um die Massenverteilung nach Gleichung 5.6 zu erhalten. $V\left(d_{p}\right)=\pi \frac{d_{p}^{3}}{6}$ bezeichnet dabei das Partikelvolumen.

$$
\mathrm{d} m\left(d_{\mathrm{p}}\right)=\rho_{\mathrm{p}} \cdot V\left(d_{\mathrm{p}}\right) \cdot \mathrm{d} N\left(d_{\mathrm{p}}\right)
$$

In Abbildung 5.4 sind die Ausgangsverteilung sowie die beiden simulierten Kurven beispielhaft für eine Messung des Cyclohexens dargestellt.

\subsection{Quantenchemische Rechnungen}

Für einige potenziell entstehende Ozonolyseprodukte sowie Clusterverbindungen aus diesen Substanzen mit $\mathrm{SO}_{2}$ oder $\mathrm{H}_{2} \mathrm{SO}_{4}$ wurden quantenchemische Rechnungen vorgenommen. Dazu wurden jeweils Strukturoptimierungen und anschließende Frequenzrechnungen zur Vorhersage von IR-Spektren durchgeführt.

Für berechnete IR-Spektren zu Produkten aus der Reaktion der Carbonylund Alkoholverbindungen sowie für 1-Hexen, 2-Methyl-1-penten und Methylcyclopenten wurde dazu das Programm GAUSSIAN 03 [110] mit dem Dichtefunktional B3LYP und dem Basissatz $6-311+G(d, p)$ verwendet. Für die Produkte 


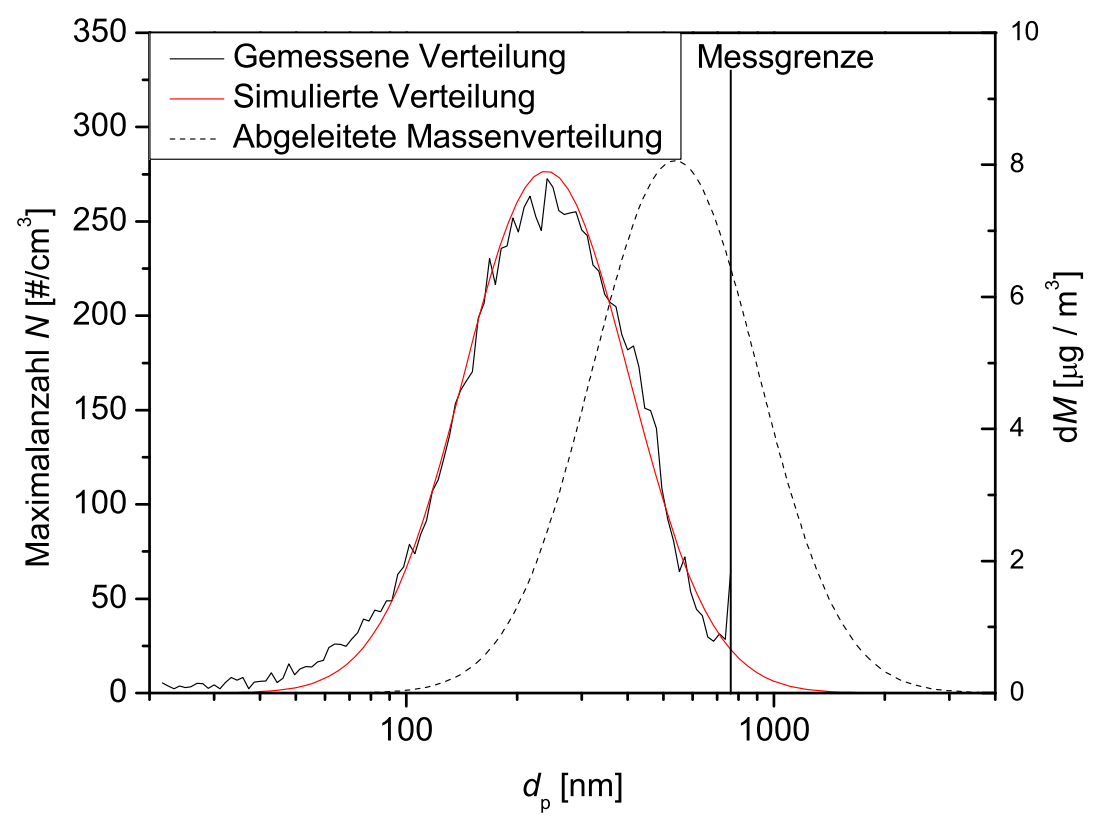

Abb. 5.4.: Simulation einer Partikelverteilung.

aus Carbonsäuren, der atmosphärisch relevanten Verbindung $\alpha$-Pinen und der Modellverbindung Cyclohexen (vgl. z.B. die Darstellung der Mechanismen in Abb. 2.15 sowie Anhang 1.2 und die nachgewiesenen Produkte von JOHNSON et al. [33]) wurde mit GAUSSIAN O9 REVISION A.02 [111] bei gleicher Methode und Basissatz gearbeitet.

In den Abbildungen [5.5 und 5.6 sind die behandelten Strukturen gezeigt. Hier handelt es sich um mittel- oder schwerflüchtige direkte Produkte der Ozonolysereaktion oder potenziell entstehende Produkte unter Beteiligung der CRIEGEE-Intermediate der verwendeten Edukte. Bei letzteren wird jeweils unter dem Substanznamen kenntlich gemacht, welches der Intermediate bimolekular mit weiteren Molekülen reagiert hat.

Die Erstellung der Molekülstrukturen erfolgt in GAUSSVIEW 4.1.2, nach der Frequenzrechnung werden die berechneten Linienspektren einer Gaußverbreiterung unterzogen, wobei eine Halbwertsbreite $(H W H M)$ von $8 \mathrm{~cm}^{-1}$ zugrundegelegt wird. Die erhaltenen simulierten Infrarotspektren werden schließlich mit experimentell gemessenen Spektren verglichen, um Signale den potenziellen Produkten zuordnen zu können. Für diesen Vergleich sollten jedoch zuvor die unter harmonischen Näherungen berechneten Spektren bezüglich ihrer Wel- 
lenzahl verschoben werden, um den real auftretenden Abweichungen in der Signallage durch anharmonische Effekte, wie etwa durch Eigenschaften des anharmonischen MORSE-Potentials ( [73], S. 616 f.) oder anders induzierten Bandendeformationen, Rechnung zu tragen. Zusätzlich ist zu beachten, dass sich diese Einflüsse mit steigender Wellenzahl verstärken, so dass teilweise die Wahl verschiedener Korrekturfaktoren in Bereichen der Gerüst- bzw. CHValenzschwingungen sinnvoll ist.

In der Literatur wird beispielsweise der Verschiebungsfaktor 0.9614 für den Basissatz $(6-31 G(d))$ angegeben [112]. Da in den gezeigten Simulationen jedoch mit einem größeren Basissatz gearbeitet wurde, sollte die Verschiebung tendenziell geringer ausfallen. Für Keten (Kapitel 6.2.1.2) wurde die Frequenzverschiebung testweise durch den Vergleich eines charakteristischen Signals des bekannten Gasphasenprodukts mit dem entsprechenden Peak im simulierten Spektrum erhalten und dieser ermittelte Verschiebungsfaktor anschlieBend auf weitere Simulationen für das strukturverwandte $n$-Propylketen (Kap. 6.2.1.2 angewandt. Ansonsten wurde der Wert abgeschätzt, die jeweils für die Infrarotspektren verwendeten Skalierungsfaktoren lagen mindestens bei 0.96 [112, 113] und werden an den jeweiligen Stellen in der Auswertung angegeben. Generell ist in der Literatur eine große Anzahl spezifischer, auf die in den Rechnungen verwendeten Basissätze und Moleküle zugeschnittene, Skalierungsfaktoren zu finden. Eine recht aktuelle Zusammenstellung findet sich in ALECU et al. [114] und der darin zitierten Literatur.

Als Ergänzung für die Experimente mit Schwefeldioxid wurden weiterhin Rechnungen zur Abschätzung von Clusterstabilitäten angefertigt, da diese Cluster als mögliche NIM und im Allgemeinen bedeutsam für die atmosphärische Partikelbildung angesehen werden (vgl. Kap. 2.8 und darin zitierte Literatur). Es wurden Cluster aus organischen Ozonolyseprodukten (GAUSSIAN 03 [110]) bzw. Wasser (GAUSSIAN 09 [111]) mit Schwefelsäure gerechnet, dabei wurde zunächst ebenfalls die Methode $B 3 L Y P / 6-311+G(d, p)$ verwendet. Anschließend wurden die Berechnungen der in ihrer Atomzahl geringeren $\mathrm{H}_{2} \mathrm{O} / \mathrm{H}_{2} \mathrm{SO}_{4}$-Cluster mit dem größeren Basissatz 6-311++ $\mathrm{G}(3 \mathrm{df}$,3dp) wiederholt, um die Aussagekraft der mit kleinerem Basissatz erhaltenen numerischen 
Ergebnisse zu prüfen 11. Weiterhin wurde für sämtliche Strukturen harmonische Schwingungsfrequenzen bestimmt, um Aussagen über die Art des jeweils vorliegenden Extrempunktes treffen zu können.

Um die Stabilität der Aggregate zu erhalten, wurden die Bildungsenthalpien bei $T=0 \mathrm{~K}$ für Oligomere und die beteiligten Monomere ermittelt. Der Vergleich der erhaltenen Werte liefert nach dem HESS'SCHEN SATZ [73] die Bindungsbzw. Dissoziationsenergie $D_{0}$ nach Gl. 5.7] die Subtraktion nullpunktskorrigierter freier GiBBS-Energien der Monomere von denen der Oligomere bei Normalbedingungen ( $T=298.150 \mathrm{~K}, p=1013.25 \mathrm{mbar}$ ) ergibt die GIBBS-Energie $\Delta_{\mathrm{R}} G$ der Clusterbildung (Gl. 5.8).

$$
\begin{gathered}
D_{0}=-\left(\Delta_{\mathrm{f}} H(\text { Oligomer }, 0 \mathrm{~K})-\sum \Delta_{\mathrm{f}} H(\text { Monomere }, 0 \mathrm{~K})\right) \\
\Delta_{\mathrm{R}} G=\Delta_{\mathrm{f}} G(\text { Oligomer, } 298.150 \mathrm{~K})-\sum \Delta_{\mathrm{f}} G(\text { Monomere, } 298.150 \mathrm{~K})
\end{gathered}
$$

Aus den durchgeführten Rechnungen ergeben sich Energien in der Einheit Hartree [Eh Molekül ${ }^{-1}$ ], die Umrechnung in [ $\mathrm{J} \mathrm{mol}^{-1}$ ] erfolgt über die Gleichung 5.9 mit den aufgezählten Konstanten nach WEDLER [73].

$$
\mathrm{Eh}=\hbar^{2} / m_{\mathrm{e}} \mathrm{a}_{0}^{2}
$$

- $\hbar=1.0545887 \cdot 10^{-34} \mathrm{~J} \mathrm{~s}$ (Planck'sches Wirkungsquantum)

- $m_{\mathrm{e}}=9.10939 \cdot 10^{-31} \mathrm{~kg}$ (Masse des Elektrons)

- $a_{0}=5.2917706 \cdot 10^{-11} \mathrm{~m}$ (Bohr'scher Radius)

- $N_{\mathrm{A}}=6.02214 \cdot 10^{23} \mathrm{~mol}^{-1}$ (Avogadrozahl)

In Kapitel 6.6 werden die berechneten Molekülstrukturen und zugehörigen Energien gezeigt, erstere wurden mit dem XYZVIEWER v0.970 exportiert.

\footnotetext{
${ }^{1}$ Die unterschiedlichen verwendeten Programmversionen von Gaussian ergeben sich aus der Tatsache, dass die neuere Version zu Beginn der Arbeit noch nicht verfügbar war und aufwendigere Rechnungen am Rechenzentrum durchgeführt wurden, wo die ältere Version zugänglicher war. Um beide Programmversionen auf ihre Vergleichbarkeit zu prüfen, wurde für die Substanz Glutaraldehyd als Testfall Optimierung und Frequenzrechnung in beiden Versionen durchgeführt, wobei sich kein signifikanter Unterschied der Bildungsenthalpie ergab.
} 
Die Auswahl der an den Oligomeren beteiligten organischen Verbindungen richtete sich nach ihrer Identifikation als Hauptprodukte der jeweiligen Ozonolysereaktion, wie die nachfolgende Auflistung zeigt. Dabei wurden jeweils die (potenziell schwerflüchtigsten) Carbonyl- und Säureverbindungen herangezogen.

\begin{tabular}{|c|c|c|c|}
\hline \multirow{2}{*}{ Edukt } & Produkt & $\begin{array}{c}\text { bekannte } \\
\text { Ausbeute [\%] }\end{array}$ & Referenz \\
\hline \hline \multirow{2}{*}{$\alpha$-Pinen } & Pinonaldehyd & 17.5 & JOHNSON [33] \\
\cline { 2 - 4 } & Pininsäure & - & JOHNSON [33] \\
\hline \multirow{2}{*}{$\beta$-Pinen } & Pininsäure & - & JENKIN [115] \\
\cline { 2 - 4 } & Norpinsäure & - & JENKIN [115] \\
\hline \multirow{2}{*}{ Cyclohexen } & Pentanal & 17 & KALBERER [116] \\
\cline { 2 - 4 } & 4-Oxobutansäure & 7 & KALBERER [116] \\
\hline
\end{tabular}

Tab. 5.1.: Hauptprodukte der Ozonolysereaktion verschiedener ungesättigter Verbindungen, die als Teil der Oligomere berücksichtigt wurden. 
Gaussian 03 B3LYP $(6-311+G(d, p))$

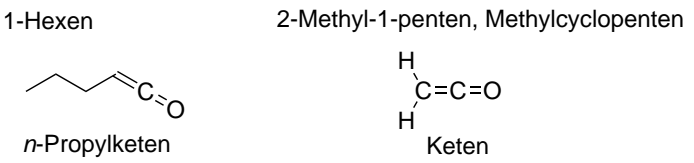

3-Buten-1-ol<smiles>OCCC(CO)OOCOO</smiles>

2-((Hydroperoxymethyl)peroxy)butan-1,4-diol $\left(\mathrm{C}_{3}-\mathrm{Cl}+\mathrm{O}_{2}+\mathrm{C}_{1}-\mathrm{Cl}\right)$

1-Penten-3-ol<smiles>CCC(O)C(CO)OOCOO</smiles>

2-((Hydroperoxymethyl)peroxy)pentan-1,3-diol $\left(1 \mathrm{P} 3 \mathrm{~L}+\mathrm{OH}+\mathrm{O}_{2}+\mathrm{C}_{1}-\mathrm{Cl}\right)$

1-Penten-3-on<smiles>CCC(=O)OOCOO</smiles>

Hydroperoxymethylpropanperoxoat

$\left(\mathrm{C}_{1}-\mathrm{Cl}+\right.$ Propionylperoxylradikal)

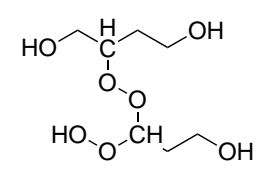

2-((1-Hydroperoxy-3-hydroxypropyl)peroxy)butan-1,4-dio $\left(\mathrm{C}_{3}-\mathrm{Cl}+\mathrm{O}_{2}+\mathrm{C}_{3}-\mathrm{Cl}\right)$

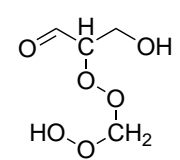

2-((Hydroperoxymethyl)peroxy)-3-hydroxypropanal $\left(\mathrm{C}_{3}-\mathrm{Cl}+\mathrm{O}_{2}+\mathrm{C}_{1}-\mathrm{Cl}\right)$

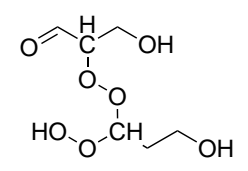

2-((1-Hydroperoxy-3hydroxypropyl)peroxy)-

3-hydroxypropanal $\left(\mathrm{C}_{3}-\mathrm{Cl}+\mathrm{O}_{2}+\mathrm{C}_{3}-\mathrm{Cl}\right)$

$$
\begin{array}{r}
\text { oxobutylpropaneperoxoa } \\
\left(\mathrm{C}_{4}-\mathrm{Cl}+\right.\text { Propionyl- } \\
\text { peroxylradikal })
\end{array}
$$

Abb. 5.5.: Strukturen, für die mit Gaussian 03 Frequenzrechnungen angefertigt wurden. 
Gaussian 09 B3LYP $(6-311+G(d, p))$

(Nach Vorschlag von Neeb, 1999)

Methacrylsäure
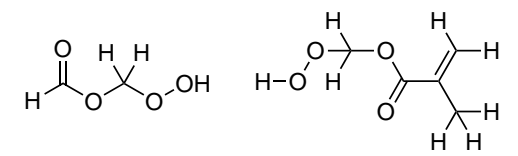

Hydroperoxymethyl-

$$
\text { formiat }
$$

Hydroperoxy-

$\left(\mathrm{C}_{1}-\mathrm{Cl}+\right.$ Ameisensäure $)$

methylmethacrylat

$\left(\mathrm{C}_{1}-\mathrm{Cl}+\mathrm{MACS}\right)$
Cyclohexen

Glutaraldehyd

$\alpha-P i n e n$

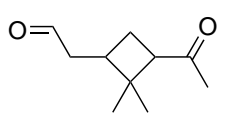

Pinonaldehyd
Butensäure<smiles>O=C(O)CC(O)OO</smiles><smiles>O=CCC(=O)O</smiles>

3-Hydroperoxy-3- Malonsäure 3-Oxopropansäure hydroxypropansäure

(aus bimolekularen Folgereaktionen des $\mathrm{C}_{3}-\mathrm{Cl}$ mit Wasser)<smiles>C1CCC2OOC(C1)O2</smiles>

CHEX-SOZ<smiles>O=CCCC(=O)OO</smiles>

Peroxy-4-oxobutansäure

Abb. 5.6.: Strukturen, für die mit Gaussian 09 Frequenzrechnungen angefertigt wurden. 


\section{Ergebnisse}

\subsection{Kinetikstudien}

In den folgenden Abschnitten werden die Ergebnisse der durchgeführten Kinetikstudien geordnet nach Substanzklassen der Edukte vorgestellt. Hauptsächlich wurden diese in Synthetischer Luft als Badgas durchgeführt, da dies sowohl den atmosphärischen Bedingungen als auch den Laborbedingungen vieler Arbeitsgruppen entspricht. Für die meisten Verbindungen wurden keine Messungen in Stickstoff durchgeführt, da in der Literatur standardmäßig eher Synthetische Luft verwendet wird. Lediglich für die Carbonsäuren und 1-Penten-3-ol fanden auch Experimente in Stickstoffatmosphäre statt, da sich in den Partikelbildungsmessungen zu den Carbonsäuren erstmals interessante Aspekte bei der Verwendung verschiedener Badgase gezeigt hatten (s. Kap. 6.4.2.5). Die Untersuchung des Alkohols diente als Vergleich dieses Verhaltens für verschiedene Oxidationsgrade.

In der Literatur sind meist Studien bei Atmosphärendruck zu finden, allerdings wurden die in dieser Arbeit ermittelten Größen bei $p_{\text {ges }}=460 \pm 10 \mathrm{mbar}$ durchgeführt, da dies der maximal allein durch Expansion aus den Vorkammern erzielbare Druck war, mit dem Vorteil, dass die IR-Messung ohne weitere Verzögerungen durch Auffüllen mit Badgas gestartet werden konnte. Auf die Ergebnisse sollte dies keinen Einfluss haben, wie die Ergebnisse von WOLF [48] für vergleichende Kinetikstudien bei 500 und 1000 mbar gezeigt haben. Auch wurden, anders als in einigen Studien anderer Arbeitsgruppen, keine Radikalfänger verwendet (vgl. z.B. [59, 117] und andere). Die Interpretation der erhaltenen Werte muss daher immer unter Berücksichtigung der Abweichungen in den experimentellen Voraussetzungen erfolgen.

Die folgenden Werte für die Geschwindigkeitskoeffizienten wurden durch Auf- 
tragungen der Lösungsfunktion 2.27 Kap. 2.5, gegen die Reaktionszeit erhalten. Die sich daraus ergebende Einheit ist $\left[\mathrm{Pa} \cdot \mathrm{s}^{-1}\right]$, welche durch folgende Gleichung unter Voraussetzung der Gültigkeit des Idealen Gasgesetzes ( [73] S. 18) in die gängigere $\left[\mathrm{cm}^{3} \mathrm{~s}^{-1}\right]$ umgerechnet werden kann ( $p$ : Druck [Pa], $V$ : Volumen $\left[\mathrm{m}^{3}\right], n$ : Stoffmenge [mol], $N=n \cdot N_{\mathrm{A}}$ : Teilchenanzahl [1], $R=8.31451 \mathrm{~J} \cdot \mathrm{mol}^{-1} \cdot \mathrm{K}^{-1}$ : allgemeine Gaskonstante, $T$ : Temperatur $[\mathrm{K}]$, $N_{\mathrm{A}}=6.02214 \cdot 10^{23} \mathrm{~mol}^{-1}$ : Avogadrozahl, $c=\frac{N}{V}$ Konzentration $\left[\mathrm{cm}^{-3}\right.$ ], Konstanten entnommen aus [73] Umschlagsseite):

$$
\begin{aligned}
p & =\frac{n}{V} \cdot R T=c \cdot \frac{R T}{N_{\mathrm{A}}} \\
c & =\frac{p N_{\mathrm{A}}}{R T}: \\
\frac{c}{\mathrm{~m}^{-3}} & =\frac{p}{\mathrm{~Pa}} \cdot \frac{6.02214 \cdot 10^{23} \mathrm{~mol}^{-1}}{8.31451 \mathrm{~J} \cdot \mathrm{mol}^{-1} \cdot \mathrm{K}^{-1} \cdot 298 \mathrm{~K}} \approx 2.43 \cdot 10^{20} \frac{p}{\mathrm{~Pa}} \\
\frac{c}{\mathrm{~cm}^{-3}} & \approx 2.43 \cdot 10^{14} \frac{\mathrm{p}}{\mathrm{Pa}} \mathrm{bzw} . \\
(\mathrm{Pa} \cdot \mathrm{s})^{-1} & \approx 4.1105 \cdot 10^{-15} \mathrm{~cm}^{3} \mathrm{~s}^{-1}
\end{aligned}
$$

Es werden an dieser Stelle aufgrund der großen Datenmenge für jede Substanz lediglich die Auftragungen der Lösungsfunktion unter Angabe eines Durchschnittswertes für Geschwindigkeitskonstante $(\bar{k})$ und relativen Umsatz $(\bar{\alpha})$ über alle Messwerte unter Angabe eines Fehlers und eines Literaturwertes gezeigt. Die Bestimmung dieser Größen erfolgte nach den in Kapitel 2.5 genannten Gleichungen (Gl. 2.27 bzw. Gl. 2.19) für jede Einzelmessung, bevor aus allen Werten ein Mittelwert gebildet wurde. Der Fehler wurde meist als Größtfehler des Mittelwertes bestimmt, lediglich für 1-Hexen und 2-Methyl1-penten wurde er als statistischer Fehler mit einem Konfidenzintervall von $95.5 \%$ bestimmt, der dazu verwendete Student'sche Faktor wurde dem Fehlerrechnungsskript des Instituts entnommen [118]. Die Einbeziehung des Student'schen Faktors sollte bei den recht nahe beieinander liegenden Einzelwerten der genannten Substanzen eine Unterschätzung der Streuung verhindern.

Der Übersichtlichkeit halber ist erst im Anhang in Tabelle A.6 eine Aufstellung der Einzelwerte unter Angabe der für die IR-Messungen verwendeten Experimentnummer zu finden, die Farben der Experimentbezeichnungen entspre- 
chen denen in den Auftragungen dieses Kapitels. Eine Zusammenstellung der genauen experimentellen Bedingungen einer größeren Zahl an Vergleichsstudien verschiedener Arbeitsgruppen findet sich tabellarisch ebenfalls im Anhang (A.3). Tabelle A.2 in Anhang A.2.1 beinhaltet eine detaillierte Zusammenstellung der experimentellen Bedingungen der im Rahmen dieser Arbeit durchgeführten Messungen.

\subsection{1. $C_{6}$-Kohlenwasserstoffe}

In Abbildung 6.1 sind die Auftragungen zu den Kinetikmessungen von 1-Hexen (1HEX) und 2-Methyl-1-penten (2M1P) gezeigt. Es ergeben sich folgende Werte als Gesamtergebnisse aus den durchgeführten Messungen:

\begin{tabular}{|c|c|c|c|c|c|}
\hline Edukt & $\boldsymbol{k}\left[10^{-17} \frac{\mathrm{cm}^{3}}{\text { molecule.s }}\right]$ & $\boldsymbol{\alpha}_{\exp }$ & $\boldsymbol{K}_{\text {Lit }}\left[10^{-17} \frac{\mathrm{cm}^{3}}{\text { molecule } \mathrm{s}}\right]$ & Fänger & Referenz \\
\hline 1HEX & $1.16 \pm 0.15$ & 1.4 & 0.94 & $\mathrm{CHA}$ & [117] \\
\hline 2M1P & $1.20 \pm 0.06$ & 1.7 & 1.31 & $\mathrm{CHA}$ & [65] \\
\hline \multirow[t]{2}{*}{ MCPE } & \multirow[t]{2}{*}{51} & \multirow[t]{2}{*}{2.3} & 67.0 & ohne & [72] \\
\hline & & & 83.2 & $\begin{array}{l}\mathrm{CHA}, \\
2 \mathrm{BuOH}\end{array}$ & [81] \\
\hline
\end{tabular}

Tab. 6.1.: Ergebnisse der Kinetikauswertungen für reine $\mathrm{C}_{6}$ Kohlenwasserstoffverbindungen. Bei den in den Referenzen verwendeten Fängersubstanzen handelt es sich um CHA: Cyclohexan, 2BuOH: 2-Butanol.

Wie anhand der tabellarischen Zusammenstellung der Werte erkennbar, liegen Mess- und Literaturwerte der offenkettigen Verbindungen recht nah beieinander, zudem liegen auch theoretisch vorhergesagte Werte von MCGILLEN et al. [79] in dieser Größenordnung (vgl. Tabelle A.3). Auch die relative Abfolge der Geschwindigkeitskoeffizienten der drei Alkene ist plausibel, da das 2-Methyl-1penten aufgrund eines höheren Substitutionsgrades und folglich stärkerem $+l$ Effekt schneller reagieren sollte als das lineare 1-Hexen. Für Methylcyclopenten ist aufgrund der Ringspannung mit dem höchsten Wert der drei betrachteten Alkene zu rechnen, zusätzlich ist durch die Ringstruktur auch auf beiden Seiten der Doppelbindung eine Kohlenwasserstoffrestsubstitution zu finden. Auf eine Fehlerangabe wurde für die letzte Verbindung verzichtet, da die aufgrund der 

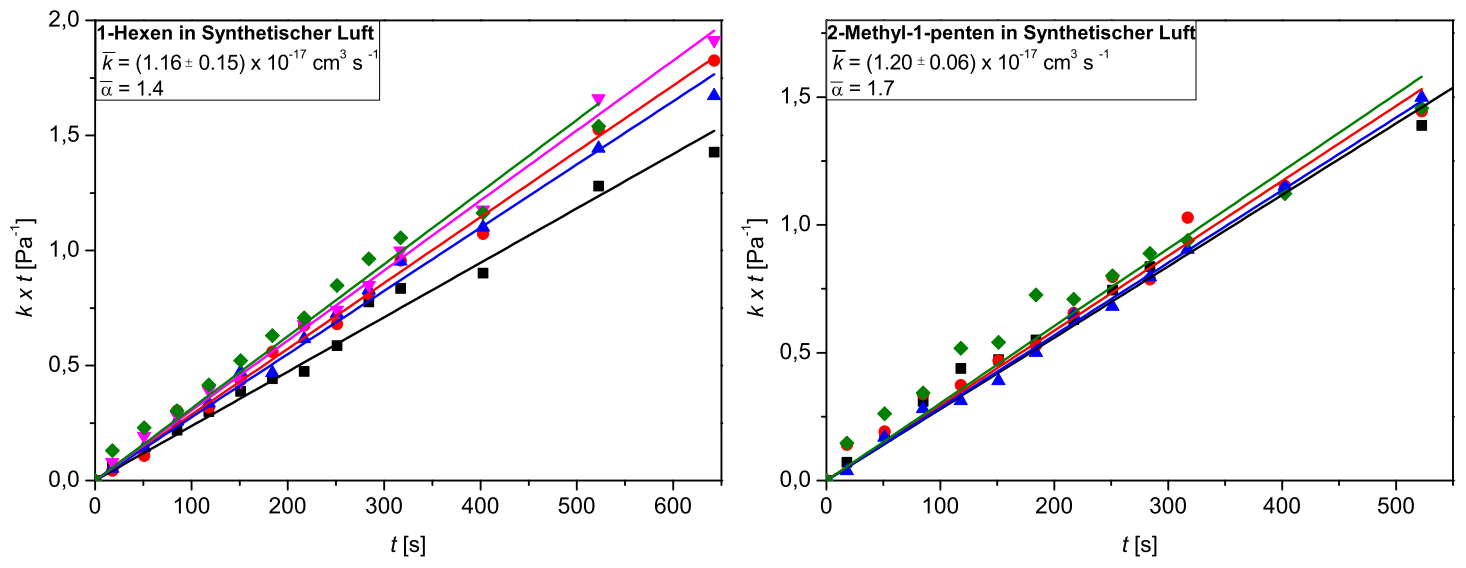

Abb. 6.1.: Darstellung der Ergebnisse der Kinetikmessungen für 1-Hexen (links) und 2-Methyl-1-penten (rechts). Alle Experimente wurden in Synthetischer Luft bei $p_{\text {ges }}=460 \pm 10$ mbar und $T=295 \pm 2 \mathrm{~K}$ durchgeführt.

Abb. 6.2: Darstellung der Ergebnisse der Kinetikmessungen für Methylcyclopenten. Alle Experimente wurden in Synthetischer Luft bei $p_{\text {ges }}=460 \pm 10$ mbar und $T=295 \pm 2 \mathrm{~K}$ durchgeführt.

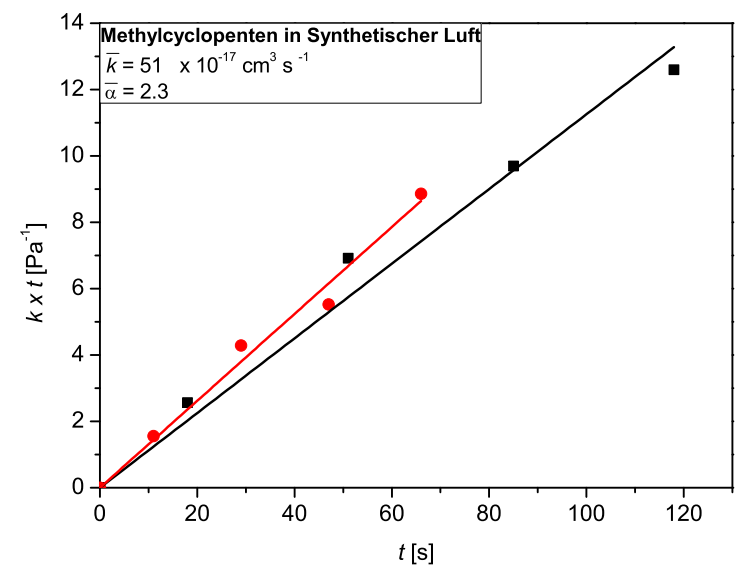

hohen Reaktionsgeschwindigkeit schwierige Handhabung eine genauere Charakterisierung sehr aufwendig gemacht hätte.

Die für die beiden offenkettigen Alkene experimentell sowohl nach oben wie unten abweichenden Werte könnten sich durch den systematischen Fehler einer nicht berücksichtigten $\mathrm{HO}_{2}$-Chemie erklären lassen.

Im Fall der zyklischen Verbindung lässt sich die relativ große Abweichung zu den Literaturwerten durch die sehr schnell ablaufende Reaktion erklären. Durch die hohe Reaktionsgeschwindigkeit mussten die Anfangskonzentrationen sehr niedrig gewählt werden, um die Reaktion zu verlangsamen, damit der Verbrauch der Edukte überhaupt beobachtet werden konnte und die Reaktion nicht schon während der ersten IR-Messung vollständig abgelaufen war. 
Dadurch konnten nicht nur weniger Messzeitpunkte pro Kinetikmessung für die linearen Anpassungen zur Bestimmung des Geschwindigkeitskoeffizienten herangezogen werden, sondern es verkompliziert sich auch die Konzentrationsbestimmung der Edukte 1 , was zu einer größeren Unsicherheit der bestimmten Restkonzentrationen und damit der sich ergebenden Steigung in den Kinetikauftragungen führt. Letztendlich kann über die Güte des erhaltenen Zahlenwertes keine gesicherte Aussage getroffen werden, da die verfügbaren und daher beide in der Tabelle 6.1 gezeigten Literaturwerte untereinander ebenfalls recht stark voneinander abweichen. Auch hier ist die Übereinstimmung unter Berücksichtigung der verschiedenen verwendeten Versuchsaufbauten insgesamt zufriedenstellend.

Ebenfalls ist in Tabelle 6.1 ersichtlich, dass der relative Verbrauch $\alpha$ für alle Substanzen stets größer als 1, was auf einen zusätzlichen Verbrauch des Alkens durch $\mathrm{OH}$-Radikale zurückzuführen ist [48]. Zwischen den Experimenten wurden Alken- und Ozonanfangspartialdrücke variiert, wobei der relative Verbrauch in Experimenten mit Ozonüberschuss geringer ausfiel als bei Alkenüberschuss. Dies ist auf Nebenreaktionen des Ozons, sowohl mit $\mathrm{OH}$ als auch mit $\mathrm{HO}_{2}$-Radikalen zurückzuführen. Wie in Tabelle 2.3 in Kapitel 2.5 bereits erwähnt, läuft die Reaktion der $\mathrm{OH}$-Radikale mit Ozon um einige Größenordnungen langsamer ab als die mit den meisten Alkenen, so dass vor allem $\mathrm{HO}_{2}$-Radikale für den zusätzlichen Ozonverbrauch verantwortlich sein sollten, da diese Reaktion bei hoher Ozonkonzentration gegenüber der $\mathrm{HO}_{2}+$ Alken-Reaktion bevorzugt abläuft. Da in der vorliegenden Auswertung durch die Bestimmung von $\alpha$ jedoch nur Nebenreaktionen unter Beteiligung von $\mathrm{OH}$-Radikalen berücksichtigt und korrigiert werden, sind die stark durch $\mathrm{HO}_{2}$ Reaktionen beeinflussten Messergebnisse möglicherweise nur bedingt verlässlich. Für die Bestimmung von $\alpha$ und daraus folgend der $\mathrm{OH}$-Radikalausbeute (s. Kap. 6.3.1) wurden daher nur Experimente unter Alkenüberschussbedingungen herangezogen, während alle Messungen zur Bestimmung des Geschwindigkeitskoeffizienten genutzt wurden.

${ }^{1}$ Für die sichere Identifikation eines der Edukte beim gegebenen Signal-zu-Rausch-Verhältnis ist erfahrungsgemäß ein Partialdruck von etwa 0.02 Pa nötig, vgl. auch [48], S. 77. 


\subsubsection{Voroxidierte Verbindungen}

Für die im Folgenden beschriebenen Kinetikmessungen können nicht in allen Fällen Literaturwerte zum Vergleich herangezogen werden, da einige der Verbindungen erstmals im Zusammenhang mit der Ozonolyse untersucht wurden. Die Ergebnisse für die voroxidierten Verbindungen werden nach steigendem Oxidationsgrad geordnet dargestellt.

\subsubsection{Alkohole}

\begin{tabular}{|c|c|c|c|c|c|}
\hline Edukt & $\boldsymbol{k}\left[10^{-17} \frac{\mathrm{cm}^{3}}{\mathrm{molecule}^{-s}}\right]$ & $\boldsymbol{\alpha}_{\exp }$ & $\boldsymbol{k}_{\text {Lit }}\left[10^{-17} \frac{\mathrm{cm}^{3}}{\mathrm{molecule} \cdot \mathrm{s}}\right]$ & Fänger & Referenz \\
\hline \hline 4P1L & $1.18 \pm 0.45$ & 1.5 & - & - & \\
\hline 1P3L & $2.15 \pm 0.32$ & $1.3(\mathrm{SL})$ & 1.64 & CO & {$[61]$} \\
& $2.90 \pm 0.02$ & $0.9\left(\mathrm{~N}_{2}\right)$ & - & - & \\
\hline 3B1L & $0.864 \pm 0.102$ & 1.4 & 0.489 & CHA & {$[65]$} \\
\hline
\end{tabular}

Tab. 6.2.: Ergebnisse der Kinetikauswertungen für die ungesättigten Alkohole.
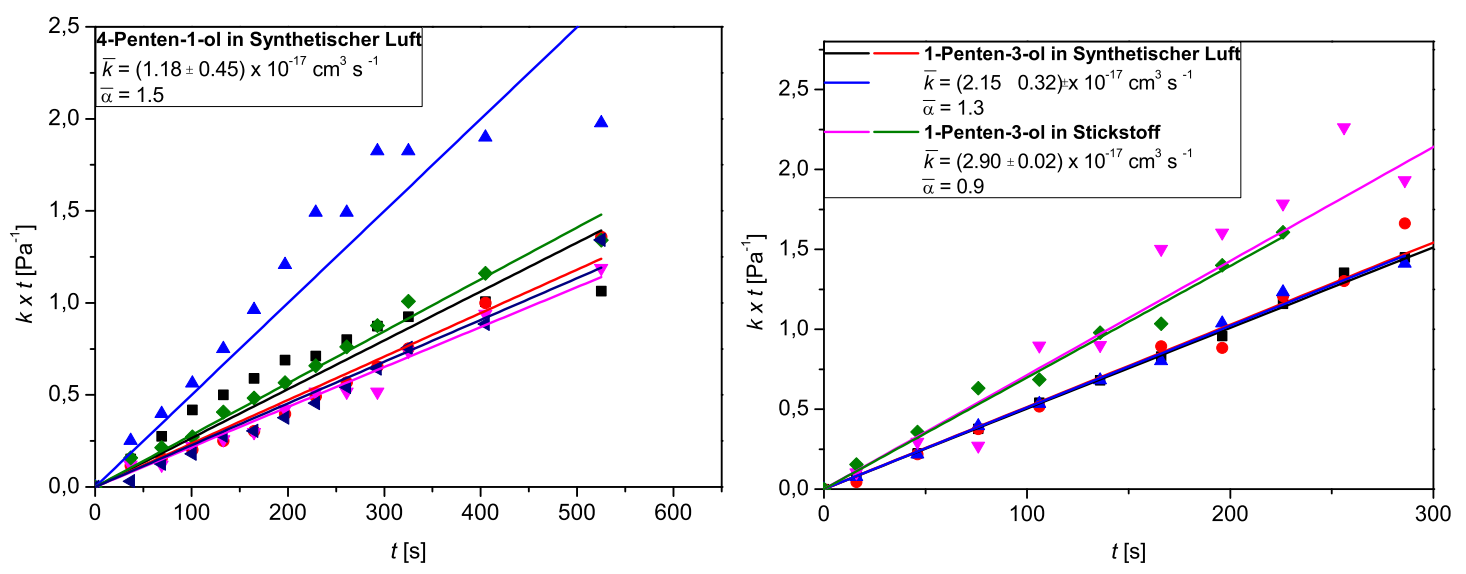

Abb. 6.3.: Darstellung der Ergebnisse der Kinetikmessungen für 4-Penten-1ol (links) und 1-Penten-3-ol (rechts). Alle Experimente wurden bei $p_{\text {ges }}=460 \pm 10$ mbar und $T=295 \pm 2 \mathrm{~K}$ durchgeführt. Für 1-Penten3-ol wurden Messungen sowohl in Stickstoff als auch in Synthetischer Luft durchgeführt.

Auch bei diesen Ergebnissen lässt sich eine relativ gute Übereinstimmung mit den Literaturwerten konstatieren, wenn diese existieren. Lediglich für 3- 


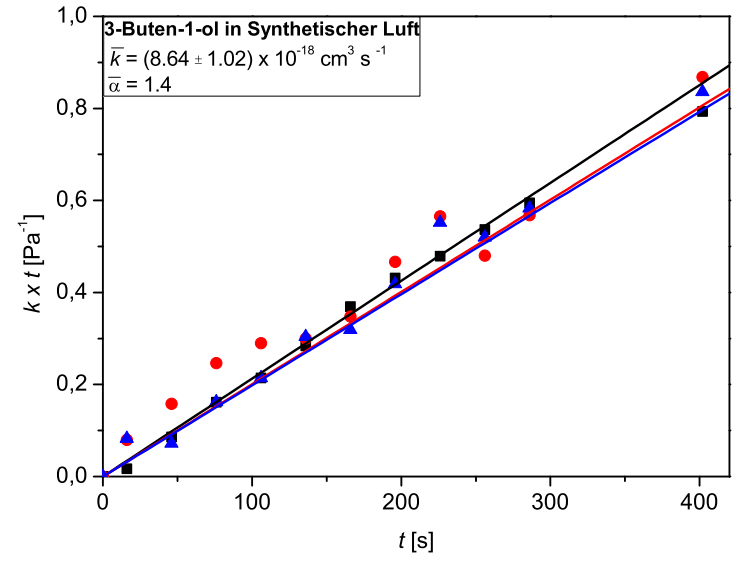

Abb. 6.4: Darstellung der Ergebnisse der Kinetikmessungen für 3-Buten-1-ol. Alle Experimente wurden bei $p_{\text {ges }}=460 \pm 10$ mbar und $T=295 \pm 2 \mathrm{~K}$ in Synthetischer Luft durchgeführt.

Buten-1-ol weicht der Wert um etwa einen Faktor 2 ab. Außerdem ist am Beispiel der beiden Messungen des 1-Penten-3-ol zu sehen, dass die Reaktion in Stickstoff als Badgas beschleunigt zu laufen scheint. Dies ließe sich, wie im vergangenen Abschnitt bei Experimenten mit Ozonüberschuss, durch eine veränderte intermediäre Chemie mit höherer Beeinflussung durch $\mathrm{HO}_{2}$-Radikale in unbekanntem Ausmaß und damit anderer auftretender systematischer Fehler erklären. Generell scheint auch die relative Abfolge der Geschwindigkeitskoeffizienten sinnvoll, da die Reaktionsgeschwindigkeit mit steigender Kettenlänge zunimmt.

\subsubsection{Carbonylverbindungen}

Für die untersuchten Carbonylverbindungen wurde zuvor lediglich die Ozonolysereaktion von 1-Penten-3-on untersucht, für 4-Pentenal war kein Literaturwert verfügbar. Auch hier lässt sich eine gute Übereinstimmung des experimentellen mit dem Literaturwert von GROSJEAN et al. [65] experimentell und von MCGILLEN et al. [80] über die SAR-Theorie theoretisch vorhergesagten (s. Tabelle A.3 im Anhang) feststellen. Lediglich der Wert von O'DWYER et al. [61] weicht mit $k=(11.7 \pm 1.5) \cdot 10^{-18} \frac{\mathrm{cm}^{3}}{\text { molecule.s }}$ (s. Tab. A.3) etwa um einen Faktor zwei ab.

Allerdings ist hier zu bemerken, dass die beiden erstgenannten Literaturwerte und der experimentell bestimmte Wert im Rahmen der Messgenauigkeit und der zu erwartenden Abweichungen durch die (Nicht-) Verwendung von Radikalfängern sehr gut übereinstimmen. In der Arbeit von O'DWYER et al. dagegen wurden mit zehnfachem Alkenüberschuss recht extreme Reaktionsbedin- 


\begin{tabular}{|c|c|c|c|c|c|}
\hline Edukt & $\boldsymbol{k}\left[10^{-18} \frac{\mathrm{cm}^{3}}{\text { molecule.s }}\right]$ & $\boldsymbol{\alpha}_{\text {exp }}$ & $\boldsymbol{k}_{\text {Lit }}\left[10^{-18} \frac{\mathrm{cm}^{3}}{\text { molecule.s }}\right]$ & Fänger & Referenz \\
\hline \hline 4PAL & $7.84 \pm 0.22$ & 1.2 & - & - & \\
\hline 1P3N & $6.79 \pm 0.46$ & 1.4 & 6.04 & CHA & {$[69]$} \\
\hline
\end{tabular}

Tab. 6.3.: Ergebnisse der Kinetikauswertungen für die ungesättigten Carbonylverbindungen.
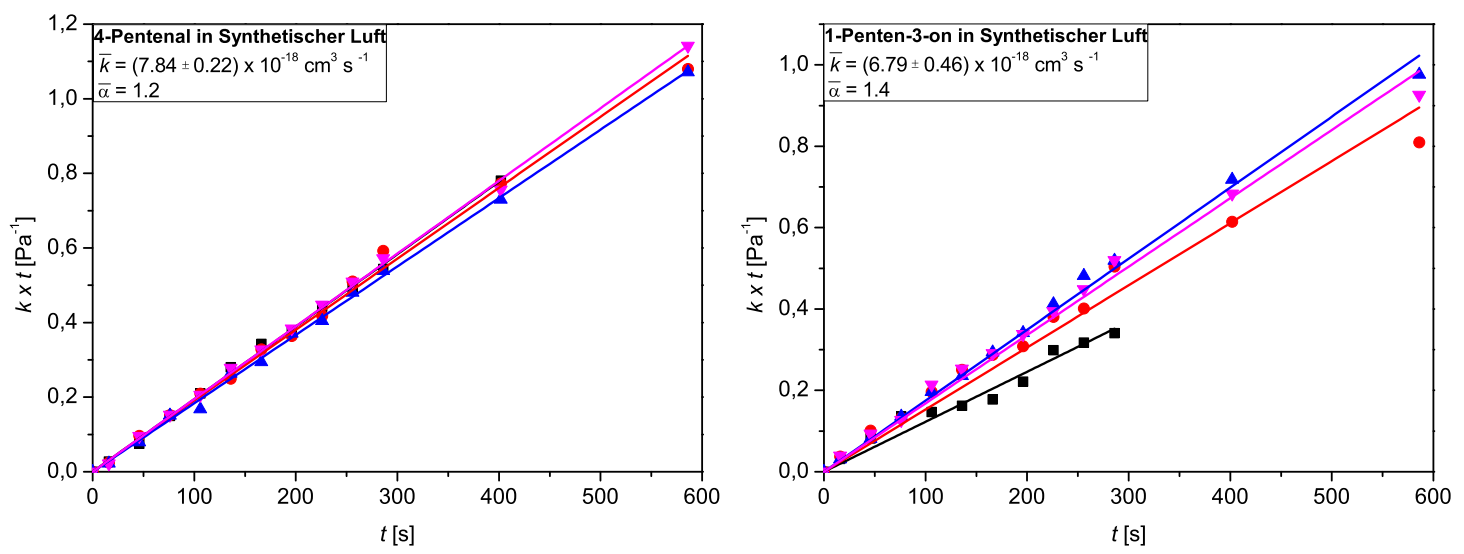

Abb. 6.5.: Darstellung der Ergebnisse der Kinetikmessungen für 4-Pentenal (links) und 1-Penten-3-on (rechts). Alle Experimente wurden in Synthetischer Luft bei $p_{\text {ges }}=460 \pm 10 \mathrm{mbar}$ und $T=295 \pm 2 \mathrm{~K}$ durchgeführt.

gungen gewählt und zusätzlich lediglich die Ozonkonzentration als Reaktionsvariable betrachtet. Durch eine fehlende Korrektur des Radikaleinflusses und der daraus folgenden erhöhten Umsätze durch $\mathrm{OH}$-Radikale (v.a. Alken, aber auch Ozon) und $\mathrm{HO}_{2}$ (v.a. Ozon), wird folglich die Geschwindigkeitskonstante bei Voraussetzung einer Reaktion pseudoerster Ordnung ( $k_{\text {uni }}^{\prime}=k_{\text {bim }}$. [Alken]) durch Abbau des Ozons zu groß bestimmt, was wiederum den Wert des bimolekularen Geschwindigkeitskoeffizienten $k_{\text {bim }}$ durch eine Begünstigung des Ozonabbaus durch $\mathrm{HO}_{2}$ nach oben verfälscht.

\subsubsection{Carbonsäuren}

Nur die Methacrylsäure wurde bereits vor einigen Jahren von NEEB et al. [59] untersucht, daher ist für die in diesem Abschnitt betrachteten Substanzen lediglich für diese Säure in Synthetischer Luft und unter Zugabe von Cyclohexan als Radikal- und Ameisensäure (HFO) als Cl-Fänger ein Vergleichswert 
vorhanden. In einer weiteren Arbeit von MULLA et al. [119] wurden die gleichen Additiva zum Abfangen der während der Reaktion entstehenden Radikale und CRIEGEE-Intermediate zugegeben, was die Vergleichbarkeit mit den experimentell erhaltenen Ergebnissen weiter erschwert. Für einen sinnvollen Vergleich werden daher zusätzlich die analogen Alkene 2-Methyl-propen (2MPR) und 1-Buten (1BEN) herangezogen.

\begin{tabular}{|c|c|c|c|c|c|}
\hline Edukt & $\boldsymbol{k}\left[10^{-18} \frac{\mathrm{cm}^{3}}{\text { molecule.s }}\right]$ & $\alpha_{\text {exp }}$ & $\boldsymbol{K}_{\text {Lit }}\left[10^{-18} \frac{\mathrm{cm}^{3}}{\text { molecule } \mathrm{s}}\right]$ & Fänger & Referenz \\
\hline \multirow[t]{3}{*}{ MACS } & 3.99 (UV 1) & $2.7\left(\mathrm{~N}_{2}\right)$ & & & \\
\hline & 3.74 (UV 1) & $2.4(\mathrm{SL})$ & $4.1 \pm 0.4$ & $\begin{array}{l}\text { CHA, } \\
\text { HFO }\end{array}$ & [59] \\
\hline & & & $2.25 \pm 0.74$ & $\begin{array}{l}\text { CHA, } \\
\text { HFO }\end{array}$ & [119] \\
\hline$\overline{2} \overline{\mathrm{MP}} \overline{\mathrm{R}}$ & & & $11.1 \pm 1.2$ & -1 & [120] \\
\hline BENS & $\begin{array}{l}6.24 \pm 0.31 \\
419+0.13\end{array}$ & $1.4\left(\mathrm{~N}_{2}\right)$ & & - & (UV 2) \\
\hline$\overline{1 \mathrm{~B}} \overline{\mathrm{EN}}$ & & & $9.64 \pm 0.87$ & - & [120] \\
\hline
\end{tabular}

Tab. 6.4.: Ergebnisse der Kinetikauswertungen für die ungesättigten Carbonsäuren. Bei experimentell ermittelten Werten wird angegeben, welcher Aufbau zur Ozonbestimmung (vgl. Abschnitt4.2.1.2) verwendet wurde.

In den Ergebnissen für Methacrylsäure (Abb. 6.6) zeigen sich gute Übereinstimmungen mit dem Literaturvergleichswert von NEEB [59]. Abweichungen lassen sich auf die allgemeinen Probleme bei der Konzentrationsbestimmung der Carbonsäuren zurückführen. Da sich für diese Substanzen ein dampfdruckund temperaturabhängiges Monomer-Dimer-Gleichgewicht einstellt, können die Abweichungen in den Reaktionsbedingungen durchaus zu einer Differenz in den Konzentrationsbestimmungen führen. Weiterhin wird von den Autoren betont, dass sie um diese Schwierigkeit der tendenziell unterschätzten Konzentrationen durch volumetrische Konzentrationsbestimmung wissen, allerdings trotzdem ohne weitere Korrekturen um gebildete Säuredimere gearbeitet wurde. In den in dieser Arbeit präsentierten durchgeführten Experimenten wurde ebenso verfahren und als zusätzliche Schwierigkeit das sich kurz nach der Expansion verändernde relative Verhältnis zwischen Monomer- und Dimerbande im IRSpektrum ausgemacht, die eine Bestimmung der Restkonzentration ebenfalls 
Abb. 6.6: Darstellung der Ergebnisse der Kinetikmessungen für Methacrylsäure. Es wurden Experimente sowohl in Synthetischer Luft als auch in Stickstoff bei $p_{\text {ges }}=460 \pm 10$ mbar und $T=295 \pm 2 \mathrm{~K}$ durchgeführt.

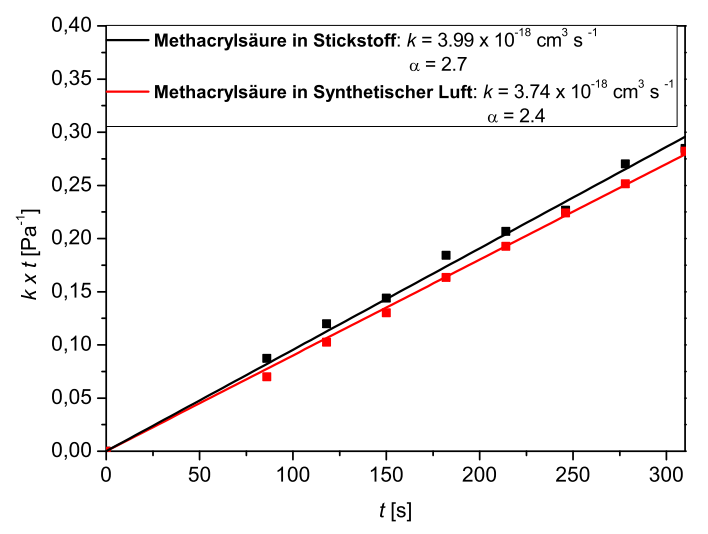

erschwert. Daher - und weil nur jeweils eine der durchgeführten Messungen in jedem Badgas einen realistischen, d.h. weitgehend linearen, Verlauf zeigte wurden für die Messwerte in diesem Fall auch keine Fehler angegeben.

Die Ergebnisse der Butensäurekinetik in Abb. 6.7 zeigen eine zufriedenstellende lineare Abhängigkeit und eine geringe Abweichung der einzelnen Messreihen untereinander. Die Kinetik dieser Carbonsäure wurde ausführlicher betrachtet und die Messungen in größerem zeitlichen Abstand durchgeführt, so dass beide in Kap. 4.2.1.2 beschriebenen UV-Optiken zur Ozonbestimmung zum Einsatz kamen. Die gezeigten Ergebnisse wurden mit UV-AUFBAU 2 erhalten, in Tabelle A.6 (Anhang, $\mathbb{\text { XX }}$ ) ist für beide verwendeten Badgase jeweils zum Vergleich ein Messwert unter Verwendung von UV-AUFBAU 1 angegeben. Die tendenziell geringer ausfallenden Werte bei UV-AUFBAU 1 lassen sich vermutlich durch die geringere Strahlungsleistung der verwendeten UV-Lampe, damit verbunden einer geringeren Nachweisempfindlichkeit und einer potenziell zu hoch bestimmten Anfangskonzentration, erklären. Sie sind an dieser Stelle lediglich zur Veranschaulichung der Bedeutung einer sorgfältigen Bestimmung der Anfangskonzentrationen bei dieser Art von Messungen angegeben.

Auffällig ist für Methacrylsäure der Badgasvergleich, da im Gegensatz zu den Messungen für den ungesättigten Alkohol 1-Penten-3-ol, wo in Synthetischer Luft ein höherer Wert für $\alpha$ erhalten wurde, der relative Verbrauch für die Säuren in Stickstoff höher auszufallen scheint als in Synthetischer Luft? Allerdings konnten für Methacrylsäure nur jeweils ein Experiment pro Badgas

\footnotetext{
${ }^{2}$ Eine genauere Betrachtung der relativen Verbräuche bei der Cearbonsäureozonolyse und ein Interpreationsversuch findet sich in Abschnitt 6.4.2.6
} 

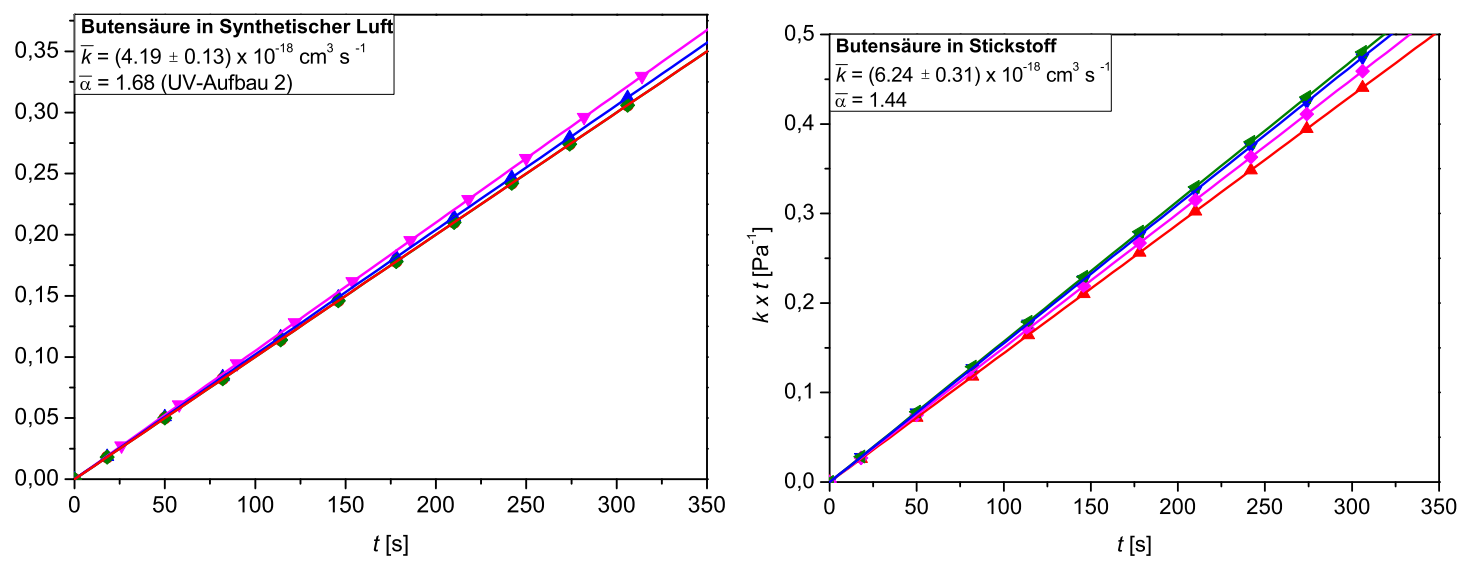

Abb. 6.7.: Darstellung der Ergebnisse der Kinetikmessungen für Butensäure in Synthetischer Luft (links) und Stickstoff (rechts) bei $p_{\text {ges }}=460 \pm 10$ mbar und $T=295 \pm 2 \mathrm{~K}$.

sinnvoll ausgewertet werden, was die Aussagekraft in Frage stellt. Butensäure, für die eine größere Zahl an Messungen vorliegt, zeigt das gleiche Verhalten wie 1-Penten-3-ol. Für eine gesicherte Aussage ist an dieser Stelle jedoch die Datenmenge mit nur einem Alkenol und zwei Carbonsäuren zu gering. Auch kann ein Einfluss der Schwierigkeiten in der Konzentrationsbestimmung durch Dimerisierungseffekte, die nur für die Säuren und nicht für die geringer oxidierten Verbindungen auftraten, nicht ausgeschlossen werden.

Allerdings sind die Beobachtungen für 1-Penten-3-ol zum Badgaseffekt auf den Geschwindigkeitskoeffizienten auch für die beiden untersuchten Säuren weiterhin gültig, da die Werte in Stickstoff höher ausfallen, wie bereits in Untersuchungen von JAPAR et al. [121] festgestellt wurde.

Generell scheinen die vergleichbaren Ergebnisse in Synthetischer Luft mit dem alten UV-Aufbau für die Geschwindigkeitskoeffizienten auch bei Vergleich mit den analogen Alkenen sinnvoll, da das lineare 1-Buten etwas langsamer reagiert als das 2-Methylpropen, ebenso wie die Butensäure langsamer als Methacrylsäure mit Ozon reagiert. 


\subsection{Produktbildungsstudien - Qualitative Auswertung}

Wie in Kapitel 5 beschrieben, wurde die Bildung von Kohlenstoffmonoxid, Ethen, Keten (-derivaten), Formaldehyd und Ameisensäure als charakteristische Produkte in den Ozonolyseexperimenten betrachtet. Diese sollen Hinweise auf den Mechanismus und die Aufteilung auf verschiedene Reaktions- und Zerfallskanäle und ihre Abhängigkeiten liefern. Da die schwerflüchtigsten Produkte aufgrund ihres Überganges in die Partikelphase in der Gasphase nicht mehr nachweisbar sein sollten, kann die Betrachtung dieser kleineren Verbindungen indirekt Aufschluss über die intermediäre Chemie geben.

Außerdem wurde versucht, weitere in den Produktspektren auftretende Peaks durch Vergleich mit zuvor aufgenommenen Reinspektren der betreffenden Substanzen (falls kommerziell verfügbar) oder theoretisch durch Frequenzrechnungen erhaltene IR-Spektren bestimmten Verbindungen zuzuordnen. Dies ist in den folgenden Abschnitten, geordnet nach aufsteigendem Oxidationsgrad, dargestellt. Sämtliche in diesem und dem betreffenden Kapitel des Anhanges (D) gezeigten Produktspektren sind durch Subtraktion entsprechender Reinspektren möglichst vollständig um die verwendeten Edukte und bei Bedarf zusätzlich um Wasser- und Kohlenstoffdioxidsignale bereinigt worden. Am Ende des Kapitels findet sich schließlich eine Zusammenfassung der Ergebnisse zur Beeinflussung des Reaktionsverhaltens biogen relevanter Terpene und Modellsubstanzen bei Schwefeldioxidzugabe. Eine Auswahl an Reinspektren einiger verwendeter Edukte findet sich im Anhang (C).

Zusätzlich wurde für Kohlenstoffmonoxid und $\mathrm{OH}$-Radikale eine quantitative Auswertung durchgeführt, die auf Grundlage der Kinetikmessungen erhalten wurden. Die Zusammenstellung dieser ausgewählten quantitativen Ergebnisse findet sich im anschließenden Unterkapitel.

Zunächst wird in diesem Kapitel eine qualitative Auswertung der auftretenden Produkte und ihrer relativen Ausbeuten unter verschiedenen Reaktionsbedingungen vorgenommen. Wegen der recht großen Anzahl untersuchter Edukte wird lediglich für Methylcyclopenten eine ausführliche Beschreibung mit Darstellung aller Spektren durchgeführt, da sich bei diesem alle oben genannten 
Produkte beobachten ließen. Für die übrigen ungesättigten Reaktanden wird in der Regel eine tabellarische Übersicht der nachgewiesenen Standardprodukte und ergänzend ein Überblick substanzspezifischer Besonderheiten und Produkte präsentiert, die der ersten Gruppe zugehörigen Produktspektren sind im Anhang zu finden (Kap. D).

Die genauen Reaktionsbedingungen sind in Tabelle A.3 in Abschnitt A.2.2 aufgelistet, im vorliegenden Kapitel werden lediglich die wichtigsten Zahlenwerte zur Orientierung genannt.

\subsection{1. $\mathrm{C}_{6}$-Kohlenwasserstoffe}

Es wurden Reaktionsspektren bei Hochdruck- (1000 mbar) und Niederdruckbedingungen (ca. 30 mbar für $1 \mathrm{HEX}$ und 2M1P, ca. 50 mbar für MCPE) nach einer zuvor festgelegten Reaktionszeit, bei der die Umsetzung der Edukte abgeschlossen war (1HEX, 2M1P: $t_{R}=345 \mathrm{~s}$; MCPE: $t_{R}=223 \mathrm{~s}$ ), aufgenommen. Für die offenkettigen Verbindungen wurden Anfangskonzentrationen von jeweils $5.0 \mathrm{~Pa}$, für das zyklische Methylcyclopenten von $3.0 \mathrm{~Pa}$, gewählt und stets mit ca 1.0 Pa Ozon zur Reaktion gebracht. Dabei wurden sowohl Messungen in Synthetischer Luft als auch in Stickstoff durchgeführt.

\subsubsection{Methylcyclopenten}

In der Ozonolyse von Methylcyclopenten konnten alle charakteristischen Zerfallsprodukte beobachtet werden. Daher wird für diese Substanz etwas ausführlicher auf die Betrachtung der Spektren eingegangen. Neben den Reaktionsspektren werden exemplarisch auch Differenzspektren zwischen den verschiedenen Druckbedingungen gezeigt.

Für die weiteren behandelten Substanzen werden anschließend jeweils nur noch die aussagekräftigsten Auftragungen präsentiert.

Zunächst kann bei Betrachtung der Reaktionsspektren bei Hochdruckbedingungen in Abbildung 6.8a exemplarisch festgestellt werden, dass unabhängig vom verwendeten Badgas größtenteils die gleichen Verbindungen im Produktgemisch enthalten sind, da die gleichen Signale auftauchen, wenn auch in unterschiedlichen Intensitäten. Bei Betrachtung der Differenzspektren beider 


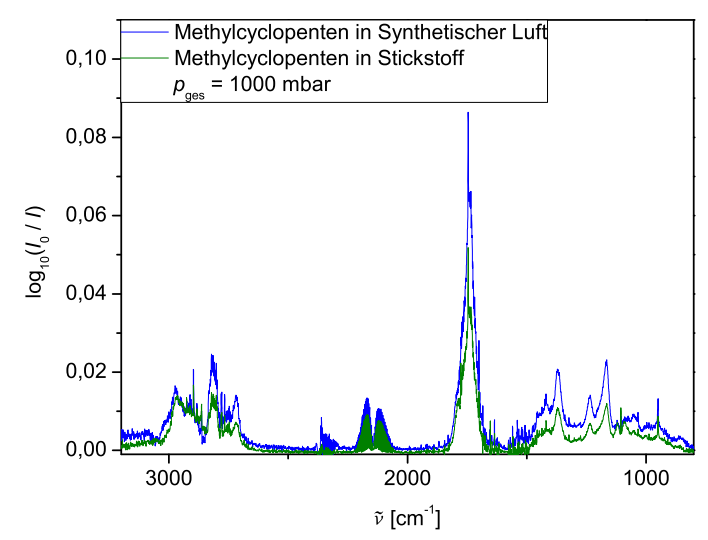

(a) Gesamtreaktionsspektrum des Methylcyclopentens in Synthetischer Luft und Stickstoff bei einem Gesamtdruck von jeweils 1000 mbar.

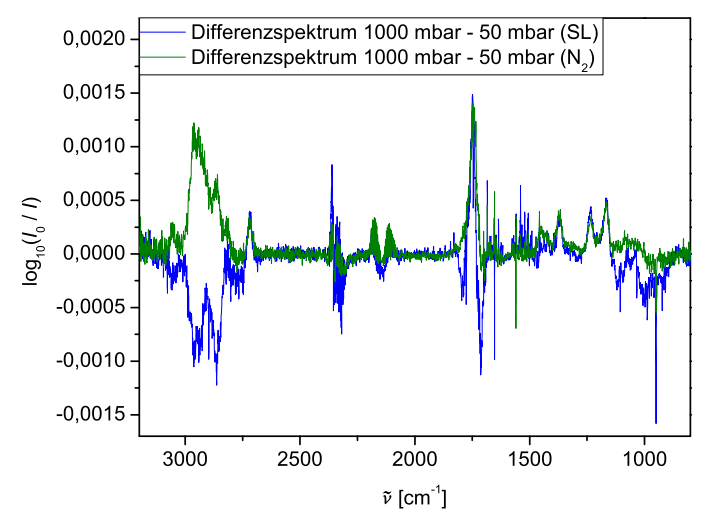

(b) Differenzspektren zwischen Hoch- und Niederdruckmessungen des Methylcyclopentens in Synthetischer Luft und Stickstoff als Badgas.

Abb. 6.8.: Gesamtansicht der Reaktionsspektren bei Hochdruckbedingungen sowie der Differenzspektren unter verschiedenen Druckregimes für Methylcyclopenten.

Badgase bei verschiedenen Gesamtdrücken (Abb. 6.8D) werden die ersten Unterschiede deutlich, die auf die Sauerstoffan- und -abwesenheit zurückzuführen sind. Auf Differenzen im C-H-Streckschwingungsbereich zwischen 3100$2800 \mathrm{~cm}^{-1}$ und einem Signal bei $2710 \mathrm{~cm}^{-1}$ (vermutlich eine aldehydische C$\mathrm{H}$-Schwingung) wird aus Mangel an Vergleichsprodukten zur Zuordnung im weiteren nicht weiter eingegangen, eventuell gehört es zum Profil der Formaldehydabsorbanz. Außerdem können auch Unterschiede bei den Signalen für die unter Abschnitt 5.2.2 genannten betrachteten Produkte festgestellt werden, die in den folgenden Abbildungen gezeigt werden.

Der spitze Q-Zweig der Ameisensäure bei $1106 \mathrm{~cm}^{-1}$ ist in Abb. 6.9alam deutlichsten im Reaktionsspektrum unter Niederdruckbedingungen und in Sauerstoffanwesenheit zu erkennen. Unter den übrigen drei Reaktionsbedingungen (Niederdruck und Hochdruck in Stickstoff, Hochdruck in Synthetischer Luft) hat das Signal eine geringere, fast identische Intensität. Ameisensäure kann generell auf verschiedene Arten innerhalb der Ozonolysereaktion gebildet werden. Sowohl zu einem geringen Teil aus dem Zerfall des $\mathrm{C}_{1}$-CRIEGEE-Intermediates (vgl. Theorieteil und [38]), als auch potenziell aus dem des großen $\mathrm{Cl}$ über Dioxiranbildung und Zerfall zu heißer Ameisensäure, die anschließend stoßsta- 


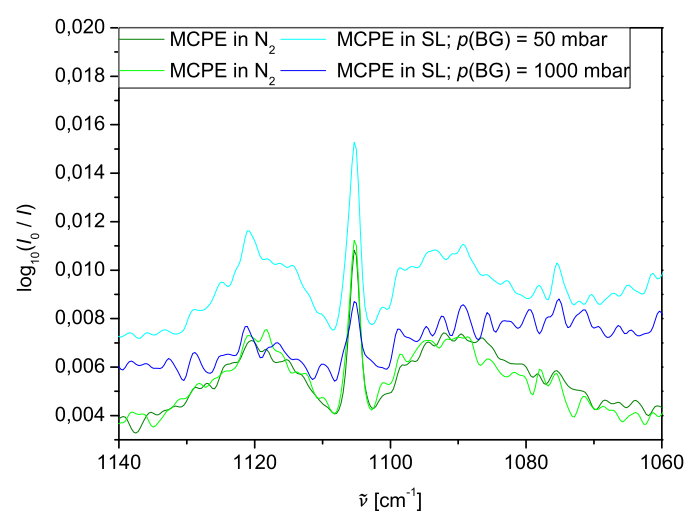

(a) Ameisensäureabsorbanz, Reaktionsspektren.

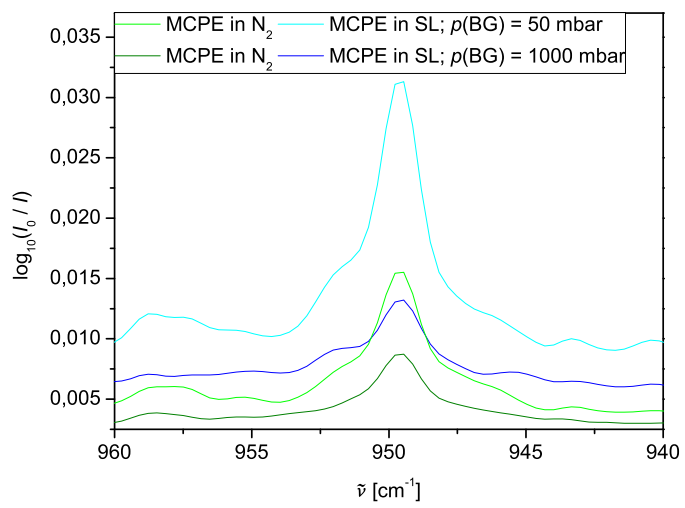

(c) Ethenabsorbanz, Reaktionsspektren.

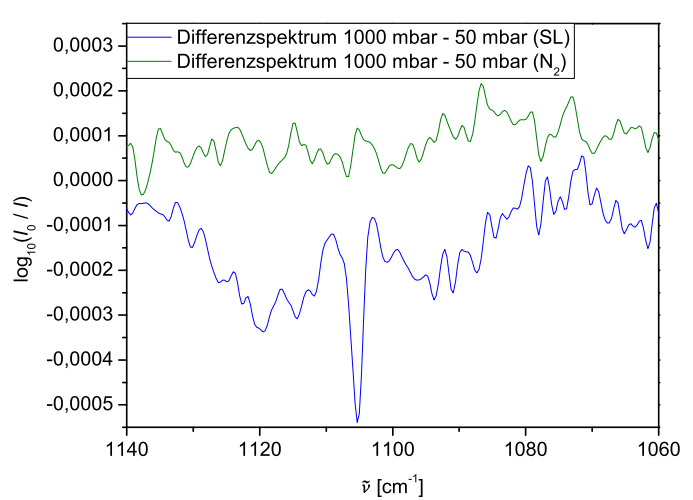

(b) Ameisensäureabsorbanz, Druckabhängigkeit.

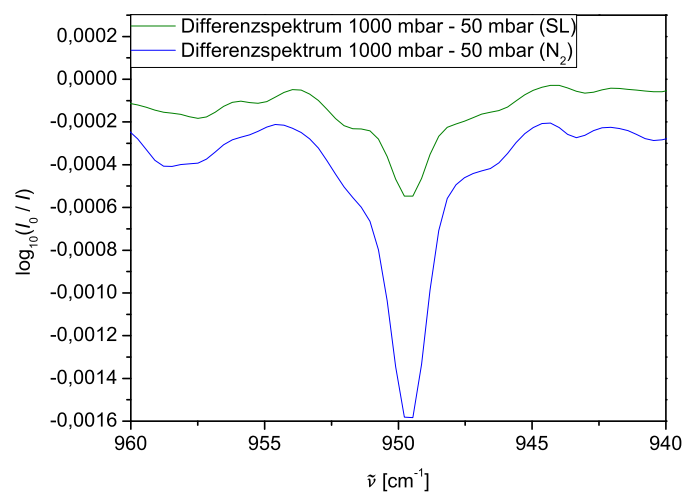

(d) Ethenabsorbanz, Druckabhängigkeit.

Abb. 6.9.: Detailbereiche der Reaktions- und Differenzspektren bei verschiedenen Druckbedingungen für Methylcyclopenten (Ameisensäure, Ethen).

bilisiert werden kann oder weiter zerfällt (vgl. z.B. Abb. 2.11) ist die Produktion möglich. Da während der Ozonolyse von Methylcyclopenten jedoch kein $\mathrm{C}_{1}-\mathrm{Cl}$ aus dem Zerfall des Primärozonids entstehen kann (s. Abb. 2.10), ist der zweite Reaktionsweg entscheidend. Ein solcher Mechanismus wurde von ASCHMANN et al. bereits für Cyclohexen vorgeschlagen [122].

Die Bildung aus den CRIEGEE-Intermediaten würde zum Auftreten einer gewissen Druckabhängigkeit in der Ameisensäureausbeute führen, da der Zerfall der energiereichen Intermediate mit sinkendem Gesamtdruck immer wahrscheinlicher wird. Diese Druckabhängigkeit ist auch vorhanden, wie die Differenzbildung zwischen Hoch- und Niederdruckmessung in Abbildung 6.9b]zeigt, 
allerdings deutet der große Unterschied in der Intensität der Signale zwischen den beiden Badgasen darauf hin, dass die unterschiedliche Ausbeute nicht ausschließlich der Druckabhängigkeit geschuldet ist. Vielmehr scheint neben den Niederdruckbedingungen auch Sauerstoff nötig für eine besonders hohe Ausbeute zu sein, so dass vermutlich sekundäre Prozesse der Alkoxy- / Peroxychemie (vgl. KROLL et al. [21]) bestimmter intermediär gebildeter Verbindungen unter Sauerstoffbeteiligung zur Ameisensäureausbeute beitragen.

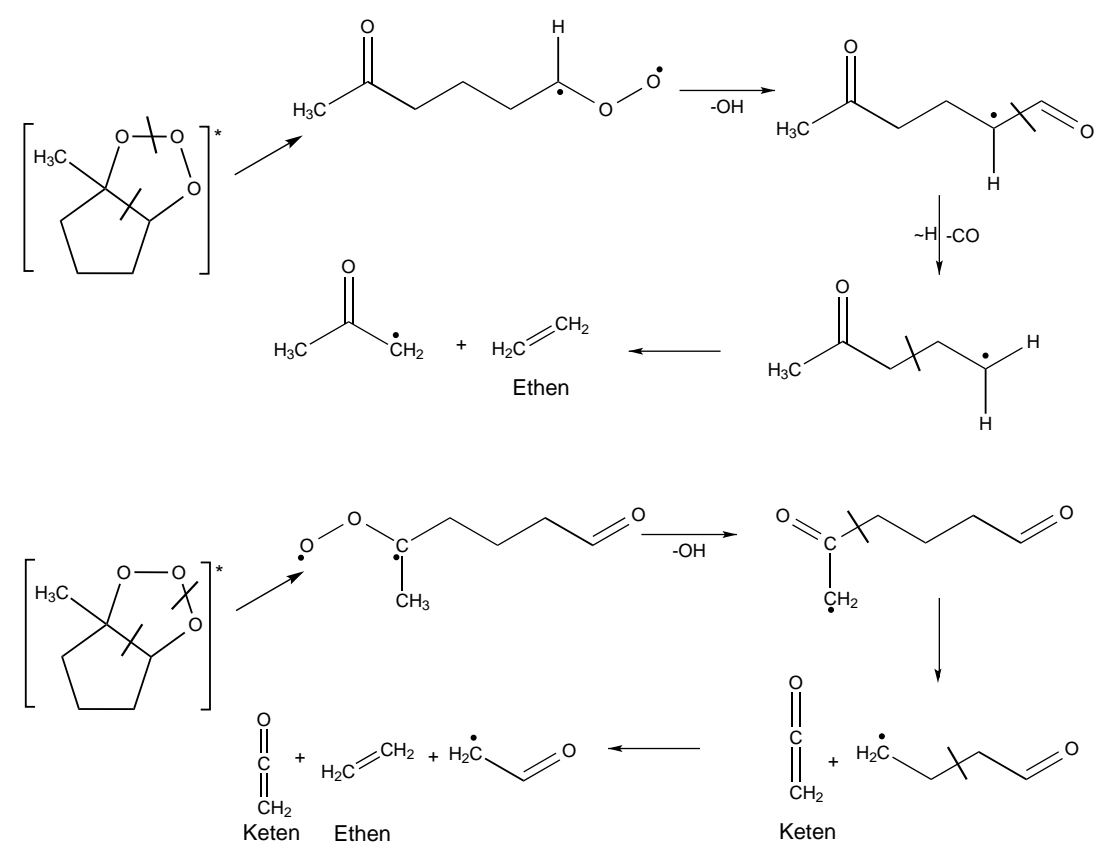

Abb. 6.10.: Postulierter Bildungsweg für Ethen und Keten aus der Methylcyclopentenozonolyse, analog zum Vorschlag aus WOLF et al. [17] für Methylcyclohexen.

Auch Ethen ist im Spektrum zu erkennen, hier kann ein Peak bei $949 \mathrm{~cm}^{-1}$ beobachtet werden (Abb. 6.9C). Es ist die gleiche Tendenz wie bei der Bildung von Ameisensäure zu beobachten, da auch hier in Synthetischer Luft unter Niederdruckbedingungen im Vergleich zu Atmosphärendruck eine deutlich größere Ausbeute als unter vergleichbaren Bedingungen in Stickstoff resultiert (Abb. 6.9d). Diese Beobachtungen gelten ebenfalls für Keten bei $2200-2100 \mathrm{~cm}^{-1}$, mit einem scharfen Q-Zweig bei $2150 \mathrm{~cm}^{-1}$ [123] (Abb. 6.11a und 6.11b). Die negativen Intensitäten dieser zentralen Banden weisen auf eine erhöhte Bildung im Niederdruckbereich und im Vergleich der präsentierten Differenzspektren eine 
höhere Ausbeute unter Sauerstoffanwesenheit hin.

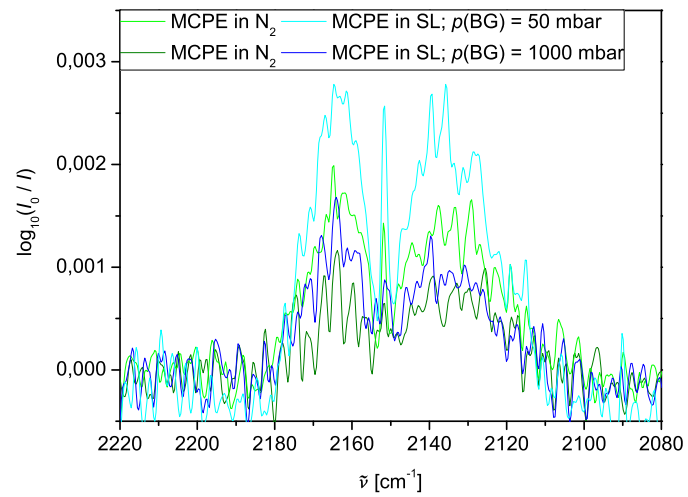

(a) Ketenabsorbanz, Reaktionsspektren. Sämtliche Spektren wurden CO-bereinigt.

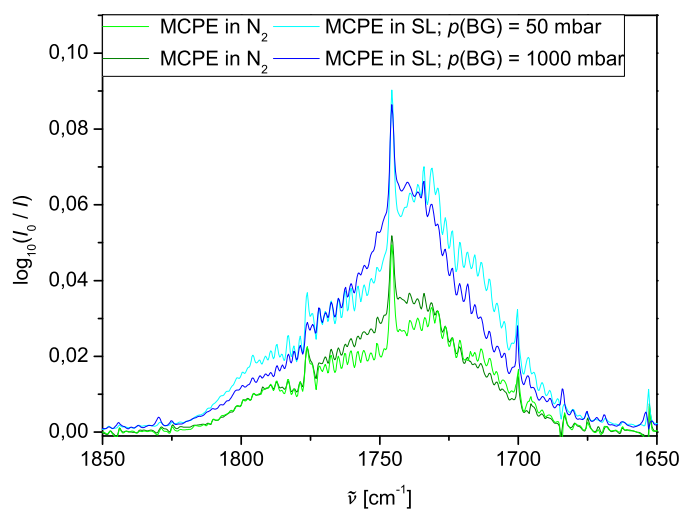

(c) Carbonylabsorbanz, Reaktionsspektren.

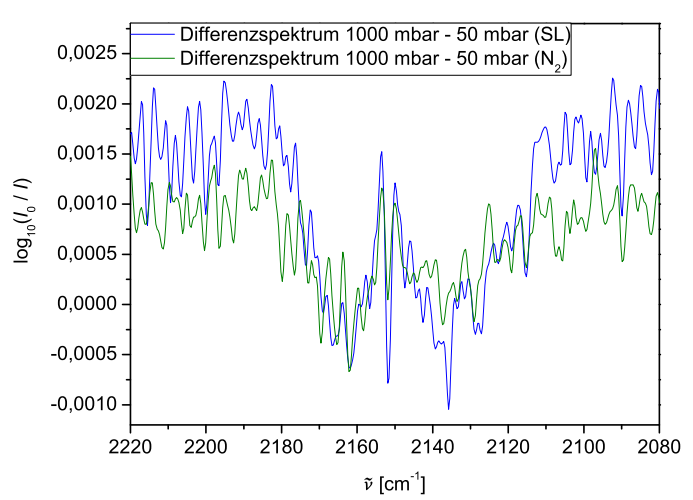

(b) Ketenabsorbanz, Druckabhängigkeit. Sämtliche Spektren wurden CO-bereinigt.

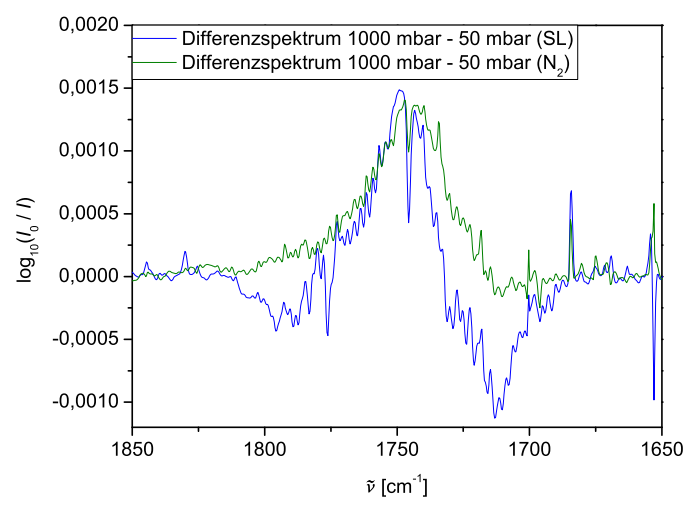

(d) Carbonylabsorbanz, Druckabhängigkeit.

Abb. 6.11.: Detailbereiche der Reaktions- und Differenzspektren bei verschiedenen Druckbedingungen für Methylcyclopenten (Keten, Formaldehyd).

Die druckabhängige Bildung von Ethen und Keten kann, vergleichbar mit der Bildung von Ameisensäure, mit einer Stoßstabilisierung von radikalischen Zwischenprodukten erklärt werden, die bei geringeren Drücken zu den genannten Verbindungen fragmentieren. Ein Vorschlag für diesen Reaktionsweg ist bei Wolf et al. [17] für Methylcyclohexen und $\alpha$-Pinen (vgl. Abb. 2.8 in Kap. 2.2.2.3) zu finden. Dieser Mechanismus würde auch die unterschiedlichen Ausbeuten bei Betrachtung der Differenzspektren erklären, da Sauerstoff als Reaktionspartner des Vinoxylradikals beteiligt ist. Methylcylopenten besitzt ebenso wie die von WOLF bearbeiteten Substanzen eine endozyklische Grundstruktur, 
weshalb die Gültigkeit des Mechanismus angenommen werden kann. Weiterhin wurde ein Vorschlag von WOLF et al. für die Bildung von Ethen und Keten aus dem Primärozonids des Methylcyclohexens erarbeitet (Abb. 6.10), der für das ebenfalls endozyklische Methylcyclopenten zutreffen könnte.

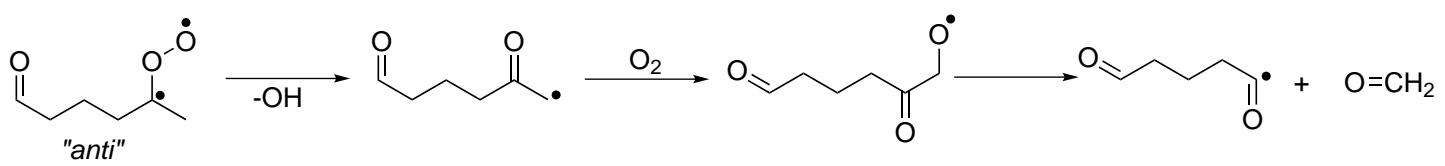

Abb. 6.12.: Postulierter Bildungsweg für Formaldehyd aus der Methylcyclopentenozonolyse, adaptiert nach TUAZON et al. [124].

Im Carbonylbereich zeigt sich unter anderem Formaldehyd (Q-Zweig bei $\left.1745 \mathrm{~cm}^{-1}\right)$. Hier scheint in Abb. 6.11C die Ausbeute zunächst eher in Stickstoff als Badgas erhöht. Bei Betrachtung der Druckabhängigkeit lässt sich in Abb. 6.11d eine stärkere relative Intensitätsänderung durch Druckveränderung in Sauerstoffanwesenheit beobachten. Wie Ameisensäure kann auch Formaldehyd nicht direkt aus dem POZ-Zerfall resultieren, er könnte auch hier durch sekundäre Prozesse unter Sauerstoffbeteiligung für den Produktaufbau erklärt werden. Prinzipiell könnte allerdings auch der Cl-Zerfall zur Formaldehydbildung führen, ohne dass Sauerstoff direkt beteiligt sein müsste.

TUAZON et al. schlagen für die Ozonolyse von 2,3-Dimethyl-2-buten eine mögliche sekundäre Quelle für Formaldehyd vor. Hier wird aus dem CRIEGEEIntermediat zunächst ein Alkoxyradikal gebildet, welches im weiteren Verlauf unter Reaktion mit Sauerstoff zerfällt. Ein entsprechendes, auf die Methylcyclopentenozonolyse angewandtes Schema ist in Abbildung 6.12 gezeigt. Diese Reaktion würde durch die Abhängigkeit von der Sauerstoffkonzentration nicht nur die erhöhte Bildung in Synthetischer Luft erklären, sondern auch die Druckabhängigkeit, da der Alkoxy- / Peroxyzerfall in der chemischen Aktivierung des Moleküls begründet ist, der unter Niederdruckbedingungen oder durch Anlagerung eines weiteren Sauerstoffmoleküls beschleunigt erfolgt.

\subsubsection{1-Hexen und 2-Methyl-1-penten}

In Tabelle 6.5 wird eine Übersicht der beobachteten Produkte gegeben. In dieser Aufstellung, wie auch allen nachfolgenden dieser Art, steht die Anzahl der 
„+"-Zeichen lediglich für die relativen Bandenintensitäten in den vier verschiedenen Reaktionsbedingungen (Synthetische Luft oder Stickstoff, Hoch- oder Niederdruckmessung) zueinander und treffen keine Aussage über einen Vergleich der Ausbeuten unter den verschiedenen Edukten. „" steht für ein nicht nachweisbares Signal. Mit einem Fragezeichen werden sehr schwache, nicht sicher zu identifizierende Banden gekennzeichnet.

\begin{tabular}{|c|c|c|c|c|c|c|}
\hline Alken & Produkt & $\mathrm{SL}, \mathrm{HD}$ & $\mathrm{SL}, \mathrm{ND}$ & $\mathrm{N}_{2}, \mathrm{HD}$ & $\mathrm{N}_{2}, \mathrm{ND}$ & Abb. \\
\hline 1HEX & $\mathrm{H}_{2} \mathrm{CO}$ & +++ & ++ & + & ++ & D.1c \\
& $\mathrm{HCOOH}$ & + & +++ & + & ++ & D.1d] \\
& Propylketen & + & ++ & + & ++ & D.1a \\
& $\mathrm{C}_{2} \mathrm{H}_{4}$ & 0 & $+(?)$ & 0 & + & D.1b \\
\hline \multirow{7}{*}{$2 \mathrm{M} 1 \mathrm{P}$} & $\mathrm{H}_{2} \mathrm{CO}$ & ++ & ++ & ++ & ++ & D.2d] \\
& $\mathrm{HCOOH}$ & ++ & +++ & ++ & +++ & D.2b \\
& Keten & 0 & + & 0 & + & D.2C \\
\hline
\end{tabular}

Tab. 6.5.: Nachgewiesene Produkte für 1-Hexen und 2-Methyl-1-penten.

Die Verwendung verschiedener Badgase hat keine Auswirkungen auf die Produktzusammensetzungen unter Hochdruckbedingungen, wie die Abbildungen $\mathrm{D} .1 \mathrm{a}$ und $\mathrm{D} .2 \mathrm{a}$ zeigen. Die Spektren zeigen über den gesamten Wellenzahlbereich die gleichen Absorbanzen, lediglich die relativen Intensitäten unterscheiden sich etwas. Es ist beispielsweise erkennbar, dass Formaldehyd (scharfer Q-Zweig bei $1745 \mathrm{~cm}^{-1}$ ) in beiden Umgebungen in gleichen Maße gebildet wird und auch die rotationsaufgespaltene CO-Bande $\left(2250-2050 \mathrm{~cm}^{-1}\right)$ in beiden Spektren nicht signifikant voneinander abweicht. Die Sauerstoffan- oder -abwesenheit scheint sich also nicht entscheidend auf die Art der Produkte oder ihre Ausbeuten auszuwirken.

Dagegen ist fast überall eine (unterschiedlich stark) ausgeprägte Druckabhängigkeit der ausgewerteten Produkte zu beobachten, die einzige Ausnahme ist Formaldehyd in der 2-Methyl-1-pentenozonolyse.

Formaldehyd kann z.B. aus dem Zerfall des Primärozonids gebildet werden. Im Gegensatz zu anderen Intermediaten kann die Lebensdauer dieser Verbindung kaum über den Gesamtdruck beeinflusst werden, weshalb die Formaldehydausbeute im IR-Spektrum druckunabhängig erscheinen sollte, solange nicht andere Reaktionskanäle ebenfalls zur Bildung dieser Substanz beitragen. Aller- 
dings ist in beiden Fällen auch der Beitrag anderer, die Bande überlagernder Carbonylverbindungen zur Signalstärke nicht auszuschließen. Beim Vergleich der Ausbeuten aus der 1-Hexen- und der 2-Methyl-1-pentenozonolyse in Abb. D.2e fällt auf, dass sich bei Normierung auf gleiche Ozonumsätze bei der letztgenannten Verbindung deutlich mehr Formaldehyd bildet. Da Formaldehyd aus dem Zerfall des Primärozonids in gleichem Maße wie das disubstituierte CRIEGEE-Intermediat gebildet wird (vgl. den Mechanismus für 1-Hexen in Abbildung 2.11), kann das Formaldehyd als Nachweis für einen höheren Anteil gebildeter disubstituierter $\mathrm{Cl}$ für 2-Methyl-1-penten als für 1-Hexen genutzt werden, wie in Kapitel 2.3.1 anhand der Vergleichssubstanz 2-Methyl-1-buten bereits postuliert wurde 3 .

Ameisensäure $\left(1106 \mathrm{~cm}^{-1}\right)$ kann sowohl zu einem geringen Teil aus dem Zerfall des $C_{1}$-CRIEGEE-Intermediates [38] als auch potenziell laut ASCHMANN et al. [122] aus dem Zerfall des großen $\mathrm{Cl}$ über eine schwingungsangeregte Säure entstehen. Die Produktion aus den CRIEGEE-Intermediaten würde aufgrund der möglichen Stoßstabilisierung bei höheren Drücken bei der Differenzbildung zu einer höheren Ausbeute führen und stimmt mit den Beobachtungen für 1Hexen überein.

Wie die Spektren in Abbildung D.2b zeigen, ist im Falle des 2-Methyl-1penten für Ameisensäure kein signifikanter Einfluss des Druckes oder des Badgases auf die Bildung zu erkennen. Es ist lediglich erkennbar, dass unter Niederdruckbedingungen etwas mehr Ameisensäure gebildet wird, wobei sich dort wiederum in Sauerstoffanwesenheit eine etwas höhere Intensität zeigt. Diese Unterschiede sind jedoch so gering, dass sie auch von leicht differierenden Umsätzen in den einzelnen Experimenten verursacht werden könnten.

Abbildung D.1a zeigt bei $2031 \mathrm{~cm}^{-1}$ sowohl in Synthetischer Luft als auch in Stickstoff bei einem Gesamtdruck von etwa 30 mbar Signale, die in den Hochdruckspektren nicht auftauchen. Der Vergleich mit einem quantenchemisch berechneten und um den empirischen Verschiebungsfaktor 0.9658 korrigiertent 4 IR-Spektrum von $n$-Propylketen, wie es während der Ozonolyse aus 1-Hexen

\footnotetext{
${ }^{3}$ Zur Wiederholung: 1-Hexen: $\mathrm{C}_{1}: \mathrm{C}_{5}=50: 50 ; 2$-Methyl-1-buten: $\mathrm{C}_{1}: \mathrm{C}_{4}=28: 72$ [37].

${ }^{4}$ Dieser Faktor ergibt sich aus dem Vergleich eines berechneten und gemessenen Ketenspektrums und wird aufgrund der strukturellen Ähnlichkeit auch auf das $n$-Propylketen angewandt.
} 


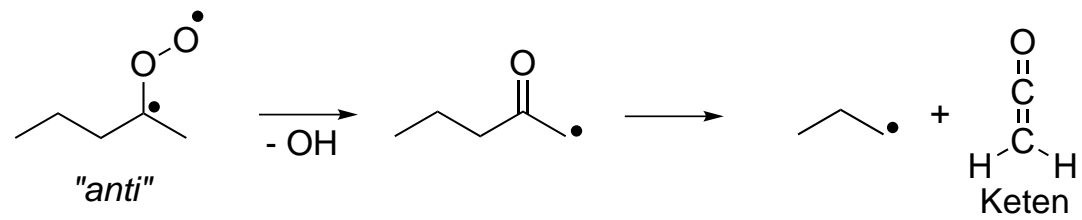

Abb. 6.13.: Möglicher Bildungsweg für Keten aus der 2-Methyl-1pentenozonolyse in Analogie zu einem von WOLF vorgeschlagenen Mechanismus [17].

entstehen kann, legt die Vermutung nahe, dass diese Verbindung für die auftretende Bande verantwortlich ist. Ein möglicher Bildungsweg verläuft über das anti-Cl, wobei der Zerfall der schwingungsangeregten Säure zu $n$-Propylketen als Produkt führt (s. Abbildung 2.11 in den theoretischen Betrachtungen oder 6.20a in Abschnitt 6.2.3).

Die übrigen betrachteten Produkte sind aufgrund ihrer geringen Intensität kaum zu erkennen. Bei $949 \mathrm{~cm}^{-1}$ zeigt sich in Abbildung D.1b unter den jeweiligen Niederdruckbedingungen ein Signal geringer Intensität, wobei es in Stickstoff etwas ausgeprägter erscheint. Hier kann die Bildung von Ethen angenommen werden, da es bei dieser Wellenzahl einen scharfen Q-Zweig zeigt. Allerdings kann es aufgrund der geringen Intensität im Vergleich zum gegebenen S/R-Verhältnis nicht sicher nachgewiesen werden.

In Abbildung D.2C zeigt sich unter Niederdruckbedingungen in Synthetischer Luft als Badgas eine Bande schwacher Intensität im Bereich der Ketenabsorption. Allerdings lässt sich auch durch Vergleich des Spektrums mit einem theoretisch berechneten für Keten (Korrekturfaktor 0.9658) und einem Vergleichsspektrum aus der Methylcyclopentenozonolyse aufgrund der geringen Intensität keine gesicherte Aussage treffen. Mechanistisch ist jedoch die Entstehung von Keten wahrscheinlich, da es in Analogie zu einem von WOLF [17] für 1Methylcyclohexen als druckabhängig vorgeschlagenen Mechanismus gebildet werden könnte, wie Abb. 6.13zeigt.

Die geringe oder nicht auftretende Ethenausbeute für diese beiden Edukte im Gegensatz zu dem in der Methylcyclopentenozonolyse deutlich erkennbaren Peak lässt sich mit der geringeren chemischen Aktivierung der Intermediate der ersten beiden Substanzen erklären, was zu einer geringeren Fragmentation führt. Der höhere Wert der zyklischen Verbindung ist auf die Ringspannung des 
Fünfringes zurückzuführen, die sich bereits in den Kinetikstudien auswirkte.

\subsubsection{Voroxidierte Verbindungen}

Für die sauerstoffhaltigen Reaktanden wurde ein Partialdruck von etwa 5.0 Pa vorgelegt und mit ca. 1.0 Pa Ozon zur Reaktion gebracht. Es wurden jeweils Spektren unter Niederdruckbedingungen (4-Penten-1-ol: 20 und 50 mbar, für alle anderen 30 mbar) und Hochdruckbedingungen (4-Penten-1-ol: 500 mbar, für alle anderen 1000 mbar) in Stickstoff und Synthetischer Luft aufgenommen, die im Folgenden gezeigt werden. Die Reaktionszeiten wurden, abhängig von den Geschwindigkeitskoeffizienten, so gewählt, dass die Umsetzung abgeschlossen war:

- 3-Buten-1-ol (3B1L), 1-Penten-3-ol (1P3L): $702 \mathrm{~s}$

- 4-Penten-1-ol (4P1L): $462 \mathrm{~s}$

- 1-Penten-3-on (1P3N), 4-Pentenal (4PAL): $582 \mathrm{~s}$

- Methacrylsäure (MACS), Butensäure (BENS): 1200 s

Für die voroxidierten Verbindungen werden zusätzlich zu den Differenzspektren zur Veranschaulichung der Druckabhängigkeit solche zwischen den verschiedenen Badgasen gezeigt, da ein besonderes Augenmerk bei den später präsentierten Nukleationsereignissen auf der Abhängigkeit vom verwendeten Badgas lag.

\subsubsection{Ungesättigte Alkohole}

Wie in den jeweiligen Abbildungen (D.3a, D.6a, D.9a) erkennbar, ändert sich die Produktzusammensetzung bei Atmosphärendruck nicht wesentlich durch die Verwendung verschiedener Badgase, die Intensitätsunterschiede weisen lediglich auf die leicht reduzierte Bildung einiger Verbindungen in Sauerstoffabwesenheit hin. Einige Unterschiede lassen sich dagegen in Abb. D.3blzwischen den verschiedenen Druckregimes im Bereich der Gerüst-, Carbonyl- und $\mathrm{C}-\mathrm{H}$ Schwingungen erkennen, auf die im Folgenden näher eingegangen wird. 


\begin{tabular}{|c|c|c|c|c|c|c|}
\hline Alken & Produkt & SL,HD & SL,ND & $\mathrm{N}_{2}, \mathrm{HD}$ & $\mathrm{N}_{2}, \mathrm{ND}$ & Abb. \\
\hline 3B1L & $\mathrm{HCOOH}$ & + & ++ & ++ & +++ & $\mathrm{D} .5 \mathrm{~b}$ \\
& $\mathrm{C}_{2} \mathrm{H}_{4}$ & + & ++ & + & ++ & $\mathrm{D} .5 \mathrm{~b}$ \\
& $\mathrm{H}_{2} \mathrm{CO}$ & ++ & ++ & ++ & +++ & $\mathrm{D} .5 \mathrm{c}$ \\
& $\mathrm{Keten}_{1}$ & + & ++ & + & ++ & $\mathrm{D} .5 \mathrm{~d}$ \\
\hline 1P3L & $\mathrm{H}_{2} \mathrm{CO}$ & + & ++ & + & +++ & $\mathrm{D} .8 \mathrm{~d}$ \\
& $\mathrm{HCOOH}$ & + & ++ & + & +++ & $\mathrm{D} .8 \mathrm{~b}$ \\
& $\mathrm{CO}$ & + & +++ & + & ++ & $0 . \mathrm{A}$. \\
\hline \multirow{6}{*}{ 4P1L } & $\mathrm{H}_{2} \mathrm{CO}$ & ++ & ++ & ++ & ++ & $\mathrm{D} .11 \mathrm{C}$ \\
& $\mathrm{HCOOH}$ & 0 & ++ & + & +++ & $\mathrm{D} .11 \mathrm{~b}$ \\
& $\mathrm{C}_{2} \mathrm{H}_{4}$ & 0 & ++ & 0 & ++ & $\mathrm{D} .11 \mathrm{~b}$ \\
& $\mathrm{CO}$ & + & +++ & + & ++ & $\mathrm{D} .11 \mathrm{~d}$ \\
\hline
\end{tabular}

Tab. 6.6.: Nachgewiesene Produkte für 3-Buten-1-ol, 1-Penten-3-ol und 1Penten-4-ol.

Zunächst wird in Tabelle 6.6 deutlich, dass Ameisensäure wie zuvor vermehrt unter den jeweiligen Niederdruckbedingungen gebildet wird, wobei jedoch stets unter sauerstofffreien Bedingungen eine höhere Intensität beobachtet wurde als in Synthetischer Luft. Dies könnte mit einer ameisensäurebildenden Isomerisierung, etwa einen H-Shift, erklärt werden, zu der es in Sauerstoffanwesenheit nicht kommt, weil das betreffende Intermediat in diesem Fall recht schnell mit $\mathrm{O}_{2}$ reagiert. Weiterhin ist für alle Alkenole eine breite Bande im Bereich zwischen 1100-1050 cm-1 , der verstärkt unter Hochdruckbedingungen und in Synthetischer Luft auftritt, zu erkennen (Abb. D.5b, D.8b, D.11b). Sie konnte bisher keiner bestimmten Struktur zugeordnet werden, allerdings könnte es sich hier um ein Artefakt aus der Differenzbildung des P-Q-R-Motivs der Ameisensäure handeln. Da Ameisensäure ebenfalls Signale im höheren Wellenzahlbereich (ca. 1776 und 3300-2800 $\mathrm{cm}^{-1}$ ) zeigt und die relativen Intensitätsänderungen im Gerüst- und der freien $\mathrm{O}-\mathrm{H}$-Schwingung um $2850 \mathrm{~cm}^{-1}$ übereinstimmen, scheint die Annahme, dass auch die Signale in diesem hohen Wellenzahlbereich sowie die im $\mathrm{C}=\mathrm{O}$-Carbonylbereich z.T. durch Ameisensäure erklärt werden können, gerechtfertigt.

Ethen $\left(949 \mathrm{~cm}^{-1}\right)$ wird in Ozonolyse des 3-Buten-1-ol und 4-Penten-1-ol gebil$\operatorname{det}(A b b . \mathrm{D} .5 \mathrm{~b}$, Abb. D.11b), hier ist die Bildung nicht badgas- sondern lediglich druckabhängig, wie die Signale zeigen. 
Formaldehyd zeigt für 3-Buten-1-ol und 1-Penten-3-ol in Stickstoff unter Niederdruckbedingungen (Abb. D.5C, D.8d) die stärkste Bande, während die Bildung für 4-Penten-1-ol (Bild D.11C) druck- und badgasunabhängig erfolgt.

In Abbildung $\mathrm{D.5d}$ ist der Bereich der CO-Absorbanz gezeigt. Wie hier an der deutlich von der Waagerechten abweichenden Grundlinie zu erkennen ist, entsteht bei der Ozonolyse von 3-Buten-1-ol Keten. Die CO-korrigierte Bande ist in Abschnitt 6.2.3 in Abbildung 6.18 vergleichend zur entsprechenden Bande der Butensäure gezeigt. Allerdings ist schon in den nicht um CO korrigierten Spektren zu erkennen, dass sowohl $\mathrm{CO}$ als auch Keten in den Niederdruckexperimenten vermehrt gebildet werden, wobei die Signale in Sauerstoffanwesenheit tendenziell intensiver beobachtet werden. Da 4-Penten-1-ol im Bereich der CO-Signale keine Anzeichen für Ketenbildung zeigt, wird an dieser Stelle in Abb. $\mathrm{D} .11 \mathrm{~d}$ einmal beispielhaft die rotationsaufgelöste CO-Bande der Reaktionsspektren dargestellt, die später bei allen Substanzen für die quantitative CO-Ausbeute genutzt wurde (Kap. 6.3.2). Diese wurden jeweils so verschoben, dass die Spektren der verschiedenen Badgase bei identischem Gesamtdruck übereinanderliegen. Die Ausbeuten unterscheiden sich unter Hochdruckbedingungen nicht signifikant. Auch bei einem Gesamtdruck von 50 mbar unterscheiden sich die Banden nicht stark, allerdings scheint in Synthetischer Luft etwas mehr $\mathrm{CO}$ zu entstehen. Eine deutliche Differenz ist dagegen erst bei einem Gesamtdruck von 20 mbar zu erkennen. Hier ist unter Sauerstoffanwesenheit eine deutlich höhere Ausbeute zu beobachten 5 , somit kann auf einen druckabhängigen Zerfall eines Intermediats geschlossen werden, der in Sauerstoffanwesenheit, eventuell durch die Destabilisierung eines bestimmten Intermediates durch Anlagerung von $\mathrm{O}_{2}$, unterhalb von $p_{\text {ges }}=50$ mbar verstärkt auftritt.

Die Differenzspektren zwischen Reaktionen in Synthetischer Luft und Stickstoff bei ansonsten identischen Bedingungen (Abb. D.4, D.7, D.10) zeigen Unterschiede in ähnlichen Wellenzahlbereichen wie die zwischen verschiedenen Druckbedingungen. Auch die Ausprägung der Differenzen und ihre Tendenzen sind vergleichbar. Die Beeinflussung des Produktgemisches ist unter Niederdruckbedingungen meist stärker ausgeprägt, Sauerstoff scheint also unter Bedingungen, bei denen die Ozonolyseprodukte länger als instabile Interme-

${ }^{5}$ Die genauen Zahlenwerte finden sich in Tabelle B.2. 
diate vorliegen, einen höheren Einfluss auszuüben. Allerdings ist zu bedenken, dass sich Änderungen auch durch eine unterschiedliche Gasphasen-zuPartikelphasen Aufteilung der Produkte ergeben könnten.

\subsubsection{Carbonylverbindungen}

Die Auftragungen der Reaktionsspektren unter Hochdruckbedingungen (Abb. D.12a, D.15a) zeigen bei beiden Carbonylverbindungen im Gerüstschwingungsbereich eine stärkere Abhängigkeit vom verwendeten Badgas. Auch eine Druckabhängigkeit der Produktausbeuten ist vor allem im Bereich unterhalb von $1200 \mathrm{~cm}^{-1}$ gut zu erkennen (Abb. 6.14).

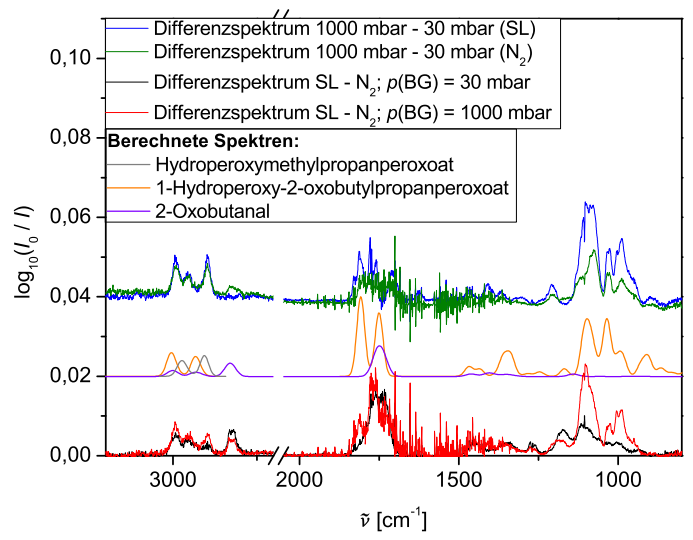

(a) Vergleich der Differenzspektren für die 1Penten-3-onozonolyse mit theoretisch berechneten IR-Spektren.

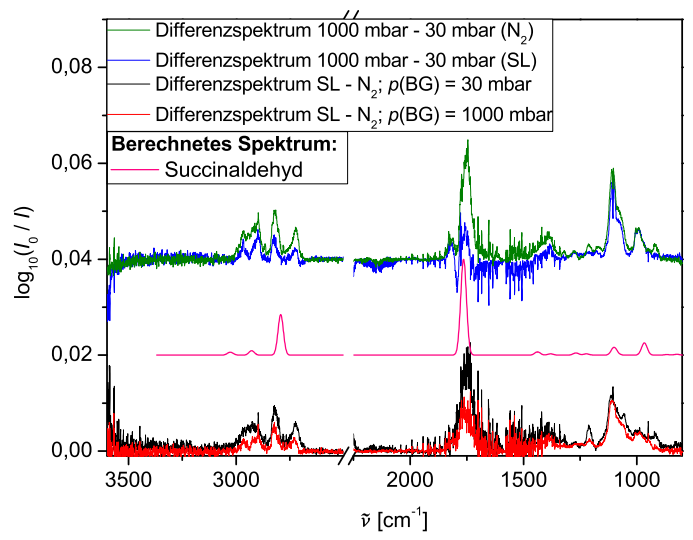

(b) Vergleich der Differenzspektren des 4Penten-1-als mit theoretisch berechneten IR-Spektren.

Abb. 6.14.: Bildung der möglichen Peroxyprodukte, Vergleich der Differenzspektren mit Theoriepektren $(B 3 L Y P / 6-311+G(d, p))$.

Zusätzlich findet sich im Falle des 1-Penten-3-ons im Stickstoffdifferenzspektrum ein nicht in Synthetischer Luft beobachtetes Signal bei $2840-2790 \mathrm{~cm}^{-1}$, das unter Hochdruckbedingungen stärker ausgeprägt ist und zu einer aldehydischen $\mathrm{C}-\mathrm{H}$-Schwingung passen könnte. Bei Betrachtung der Differenzspektren verschiedener Badgase in Abb. 6.14fällt außerdem in den Spektren beider Edukte auf, dass eine Druckänderung einen ähnlichen Effekt wie die Änderung der Reaktionsumgebung durch einen Badgaswechsel von Synthetischer Luft zu Stickstoff hat, da sich die Signalposition und Bandenform in allen Differenzspektren ähnelt. Daraus kann geschlossen werden, dass eine Druckreduktion 
einen ähnlich destabilisierenden Effekt auf die gebildeten Intermediate ausübt wie die Änderung des Sauerstoffpartialdrucks in der Reaktionsmischung. Die Reaktion von $\mathrm{O}_{2}$ mit bestimmten Intermediaten scheint daher wichtig für den Aufbau des Produktgemisches in der Gasphase zu sein.

Denkbar wäre, dass bei hohen Gesamtdrücken die Intermediate durch Stoßstabilisierung ausreichend lange Lebensdauern besitzen, um mit dem auch in Stickstoffatmosphäre vorliegenden Restsauerstoff (abgeschätzt etwa $0.5 \mathrm{~Pa}$ ) verzögert zu den gleichen Produkten zu reagieren, die in Synthetischer Luft auch ohne vorherige Stoßstabilisierung durch die wesentlich höhere $\mathrm{O}_{2}$-Konzentration und daraus folgende schnellere Reaktionsgeschwindigkeit resultieren. Eine andere Erklärung bietet wieder eine veränderte Gasphasenzu-Partikelphasen-Aufteilung der Produkte, die Einfluss auf die Bandenintensitäten nehmen könnte.

Der Vergleich mit theoretisch berechneten IR-Spektren (Abb. 6.14) verschiedener sauerstoffhaltiger Produkte zeigt eine große Übereinstimmung mit den erhaltenen Differenzspektren. Neben 2-Oxobutanal (violett), das innerhalb der Ozonolysereaktion des 1-Penten-3-ons aus dem Zerfall des Primärozonids als stabiles Produkt entsteht, wurden ebenfalls zwei Verbindungen unter Einbeziehung intermediär entstehender Radikale in Betracht gezogen. Zum einen handelt es sich um das Hydroperoxymethylpropanperoxoat, das aus der Reaktion eines Propionylperoxyl-Radikals mit einem $\mathrm{C}_{1}-\mathrm{Cl}$ entsteht und in grau aufgetragen wurde. Zum anderen wurde mit der Struktur des 1Hydroperoxy-2-oxobutylpropanperoxoat, das Produkt der analogen Reaktion des Propionylperoxyl-Radikals mit einem $\mathrm{C}_{4}-\mathrm{Cl}$, berechnet. Die Bildung der beiden Peroxyverbindungen ist Untersuchungen von $\mathrm{Hou}$ et al. [125] entnommen und in Abb. 6.15a) beschrieben. Im Falle des 4-Pentenal zeigen sich einige Übereinstimmungen der gemessenen Spektren mit einem theoretisch für das aus dem POZ-Zerfall stammenden Succinaldehyd (1,4-Butandial, $\mathrm{C}_{4} \mathrm{H}_{5} \mathrm{O}_{2}$ ) bestimmten, wie beispielsweise die aldehydische $\mathrm{CH}$-Schwingung bei $2820 \mathrm{~cm}^{-1}$, Signale im CH-Bereich (3040-2670 $\left.\mathrm{cm}^{-1}\right)$, im Carbonylbereich $\left(1764 \mathrm{~cm}^{-1}\right)$ und im Gerüstbereich (1100 bzw. $\left.963 \mathrm{~cm}^{-1}\right)$. Alle berechneten Spektren wurden um den Faktor 0.963 (Valenzbereich) bzw. 0.976 (Carbonyl- und Gerüstbereich) verschoben.

Durch die große Ähnlichkeit der Spektren und die Unsicherheit durch em- 


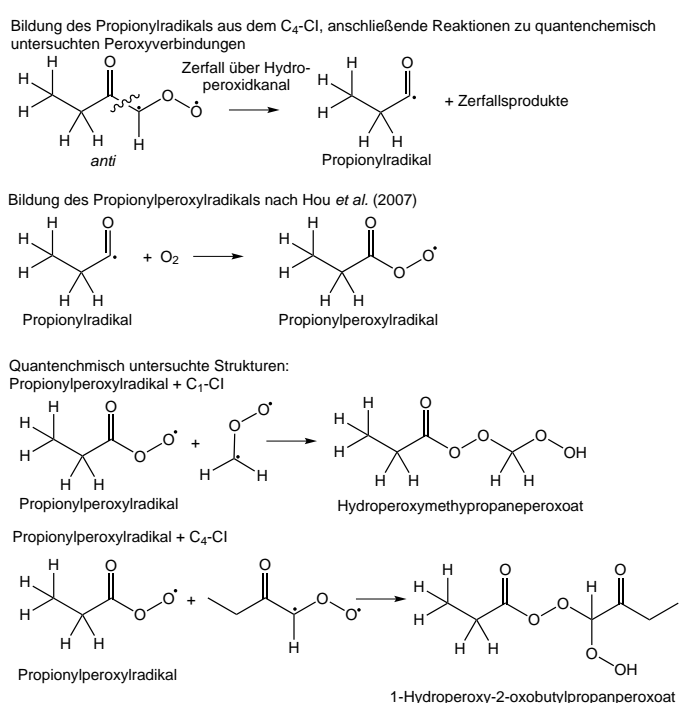

(a) Bildung der quantenchemisch untersuchten Peroxystrukturen nach $\mathrm{Hou}$ et al. [125].

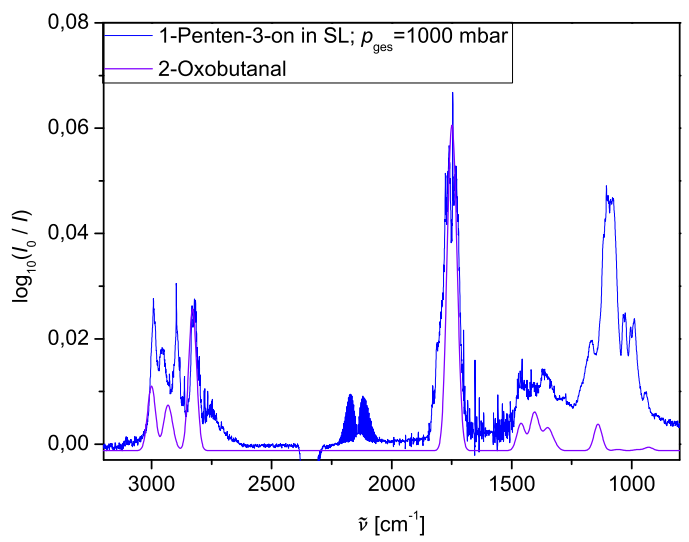

(b) Vergleich des Hochdruckreaktionsspektrums von 1-Penten-3-on in Synthetischer Luft und quantenchemisch berechnetem Spektrum von 2-Oxobutanal.

Abb. 6.15.: Bildung der möglichen Peroxyprodukte, Vergleich des Reaktionsspektrums mit 2-Oxobutanal $(B 3 L Y P / 6-311+G(d, p))$.

pirisch gewählte Verschiebungsfaktoren kann allerdings nicht mit Sicherheit bestimmt werden, ob und welche der möglicherweise aus Radikalreaktionen entstehende Verbindungen im Reaktionsgemisch vorliegt. Die Bildung von 2Oxobutanal kann dagegen als gesichert angesehen werden, sie wurde zuvor schon in anderen Untersuchungen beobachtet [61] und die Übereinstimmungen des theoretischen Spektrums in Abb. 6.15b mit dem Hochdruckreaktionsspektrum in Synthetischer Luft ist sehr hoch (Banden bei 1455, 1412, 1357, $\left.1169 \mathrm{~cm}^{-1}\right)$. Zusätzlich kann durch Betrachtung der Druckdifferenzspektren festgestellt werden, dass das Signal bei $2840-2790 \mathrm{~cm}^{-1}$ im Synthetische LuftSpektrum nicht mehr auftaucht, hier kann also auf eine druckunanhängige Bildung und damit den Zerfall des (im untersuchten Bereich druckunabhängig zerfallenden) Primärozonids als alleinige Quelle geschlossen werden. In Stickstoff ist das Signal unter Hochdruckbedingungen stärker ausgeprägt, was möglicherweise auf die Bildung eines weiteren Aldehyds hinweist, welcher im gleichen Bereich absorbiert und in Stickstoff vermehrt gebildet wird. Eine andere Möglichkeit ist, dass in Stickstoff unter Hochdruckbedingungen weitere Kanäle 
zur Bildung von 2-Oxobutanal führen, die unter Niederdruckbedingungen oder in Sauerstoffanwesenheit nicht beschritten werden können. Auch die Bildung des Succinaldehyds ist als wahrscheinlich anzusehen.

\begin{tabular}{|c|c|c|c|c|c|c|}
\hline Alken & Produkt & $\mathrm{SL}, \mathrm{HD}$ & $\mathrm{SL}, \mathrm{ND}$ & $\mathrm{N}_{2}, \mathrm{HD}$ & $\mathrm{N}_{2}, \mathrm{ND}$ & Abb. \\
\hline 1P3N & $\mathrm{HCOOH}$ & + & ++++ & ++ & +++ & D.14b \\
& $\mathrm{C}_{2} \mathrm{H}_{4}$ & 0 & + & 0 & + & D.14b \\
& $\mathrm{H}_{2} \mathrm{CO}$ & ++ & ++ & + & + & D.14e \\
& Methylketen & + & ++ & + & ++ & D.14d \\
\hline 4PAL & $\mathrm{HCOOH}$ & + & +++ & + & ++ & D.15c \\
& $\mathrm{C}_{2} \mathrm{H}_{4}$ & ++ & +++ & ++ & ++ & D.15c \\
& $\mathrm{H}_{2} \mathrm{CO}$ & +++ & +++ & ++ & + & D.15e \\
\hline
\end{tabular}

Tab. 6.7.: Nachgewiesene Produkte für 1-Penten-3-on und 4-Pentenal.

Tabelle 6.7 zeigt neben einer unter Niederdruckbedingungen erhöhten Ameisensäure- und Ethenausbeute auch, dass beide vermehrt in Synthetischer Luft gebildet werden. Dies wird durch die Reaktionsspektren in Abb. D.14bund D.15C bestätigt.

Wie bei 1-Penten-3-on zeigen sich auch für 4-Pentenal, z.T. im Bereich der C-O-Schwingungen, Signale bei 1116, 1074, 1004 und $998 \mathrm{~cm}^{-1}$. Letztere kann dem Ameisensäureanhydrid zugeordnet werden. Dieses wurde bereits in verschiedenen Studien zur Ethenozonolyse als ein Produkt identifiziert [38,60] und könnte, durch das Auftreten von Ameisensäure im Reaktionsgemisch ebenfalls bei der 4-Pentenalozonolyse gebildet werden. Da die Bande bei $1106 \mathrm{~cm}^{-1}$ von der der Ameisensäure überlagert ist, wird der Befund durch das Auftreten einer schwachen Bande bei $1830-1810 \mathrm{~cm}^{-1}$ gestützt, die sowohl im Hochdruckspektrum in Synthetischer Luft in Abb. D.15e zu erahnen und in den Druckdifferenzspekren in Abb. 6.16 deutlich zu erkennen ist.

Ethen kann sich durch den unimolekularen Zerfall der CRIEGEE-Intermediate bilden, wie WoLF et al. [17] bereits für Methylcyclohexen zeigen konnten. Dort zerfällt das syn-Cl über den Hydroperoxidkanal, nach Abspaltung von Kohlenstoffmonoxid stabilisiert sich das entstehende Alkylradikal schließlich unter Ethenabspaltung (vgl. Abb. 2.8 in Kap. 2.2.2.2). Dieser Bildungsweg ist durch die mögliche Stoßstabilisierung der Intermediate in Konkurrenz zum unimolekularen Zerfall abhängig vom Gesamtdruck. Die Ethenbildung ist für 4-Pentenal 


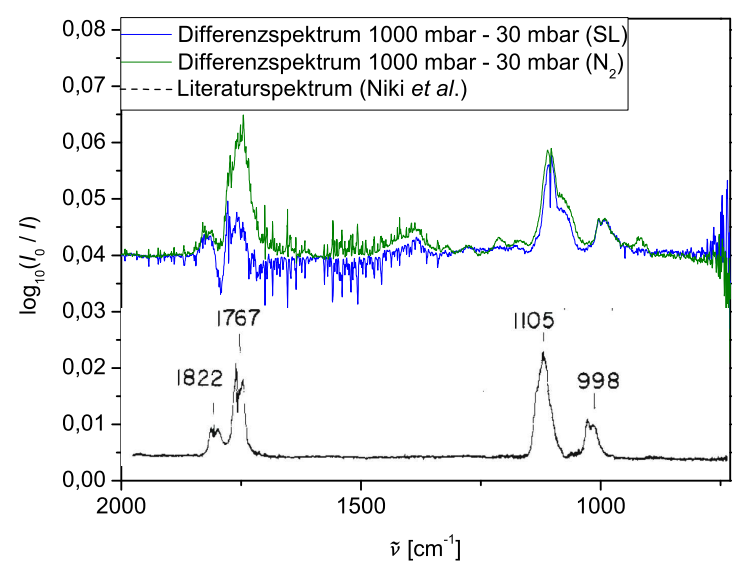

Abb. 6.16.: Vergleich der Druckdifferenzspektren der 4-Pentenalozonolyse mit einem Literaturspektrum von NIKI et al. [60].

in beiden Druckregimes recht ausgeprägt, wie die sichtbare rotationsaufgelöste Bande zwischen ca. $1050-900 \mathrm{~cm}^{-1}$ zeigt. Während WOLF diese Beobachtungen erst bei deutlich niedrigeren Drücken (ca. 150 mbar) machen konnte, ist für 4-Pentenal durch die destabilisierende Wirkung der Aldehydgruppe der C-C-Bindungsbruch offenbar schon bei Atmosphärendruck konkurrenzfähig zur Stoßstabilisierung und die gebildeten Intermediate nur in geringem Umfang stabilisierbar.

Zudem tauchen in Abb. D.14b der 1-Penten-3-onspektren drei recht intensive Banden mit Zentren bei 1091, 1030 und $988 \mathrm{~cm}^{-1}$ auf, die vor allem unter Hochdruckbedingungen hervortreten. WINTERHALTER et al. [126] konnten in diesem Wellenzahlbereich bei der Ozonolyse von $\beta$-Pinen Signale des entstehenden Sekundärozonids identifizieren. Unter Berücksichtigung möglicher Verschiebungen durch abweichende organische Reste am Trioxolanring könnten daher $\mathrm{C}$-O-Einfachbindungen und $\mathrm{O}-\mathrm{C}-\mathrm{O}-\mathrm{Schwingungen} \mathrm{für} \mathrm{einen} \mathrm{Teil} \mathrm{die-}$ ser Signale verantwortlich sein. Diese Strukturmotive tauchen allerdings auch in potenziell gebildeten Peroxiden auf, so dass diese ebenfalls in diesen Wellenzahlbereichen Signale zeigen können, wie bereits anhand der theoretisch berechneten Spektren in Abb. 6.14a gezeigt wurde. Daher können die Peaks an dieser Stelle nicht einer bestimmten Verbindung zugeordnet werden. Allerdings weist die Zunahme der Intensität unter Hochdruckbedingungen darauf hin, dass die Verbindung erst bei recht hohen Gesamtdrücken stabilisiert wird 
und ansonsten zerfällt, was allerdings für die Sekundärozonide ebenso wie das Propionylradikal als eines der Edukte der theoretisch berechneten Peroxide zutrifft 6 , wodurch auch die Bildung der gewählten Peroxide aus Propionylperoxylradikal und den entstehenden stabilisierten CRIEGEE-Intermediaten unter Hochdruckbedingungen begünstigt sein sollte. Die höhere Bandenintensität in Synthetischer Luft spricht für eine Beteiligung von Sauerstoff, was Peroxide als Verursacher ebenfalls nicht ausschließt.

Die Formaldehydbildung in der Ozonolyse des 1-Penten-3-on ist eher vom Druck als vom verwendeten Badgas abhängig, wobei auch jene nicht sehr stark ausgeprägt ist. 4-Pentenal zeigt in Synthetischer Luft in beiden Druckregimes eine höhere Ausbeute als in Stickstoff, wo wiederum unter Niederdruckbedingungen eine leicht reduzierte Ausbeute vorlag.

Der CO-Bereich in Abb. $\mathrm{D.14C}$ zeigt mit seiner Bandenintensität eher eine Druck- als eine Badgasabängigkeit (vgl. auch die berechneten CO-Ausbeuten in Tab. (B.2), Kohlenstoffmonoxid kann unter anderem aus dem Zerfall schwingungsangeregter CRIEGEE-Intermediate entstehen. Weiterhin zeigt die Differenzbildung der Reaktionsspektren durch die geschwungene Grundlinie, dass eine weitere Substanz in diesem Wellenzahlbereich absorbiert. Durch Korrektur dieses Bereiches um die CO-Signale kann in Abb. D.14d ein Signal zwischen $2165-2100 \mathrm{~cm}^{-1}$ (Zentrum bei ca. $2136 \mathrm{~cm}^{-1}$ ) sichtbar gemacht werden, dass wahrscheinlich durch die Bildung von Methylketen $\left(\mathrm{H}_{3} \mathrm{C}-\mathrm{CH}=\mathrm{C}=\mathrm{O}\right) \mathrm{zu}$ erklären ist. Diese Substanz absorbiert laut WINTHER et al. [127] mit der antisymmetrischen $\mathrm{C}=\mathrm{C}=\mathrm{O}$-Valenz bei $\nu_{4}=3136 \mathrm{~cm}^{-1}$, was anhand des Literaturspektrums dieses Bereiches in Abbildung D.14f gezeigt ist. Die Bildung des Methylketens ist potenziell über mehrere Wege denkbar, bildlich werden die im Folgenden beschriebenen Reaktionen in Abb. 6.20a in Abschnitt 6.2.3 dargestellt.

Zunächst könnte das $\mathrm{C}_{4}-\mathrm{Cl}$ nach Wasserstoffatomabstraktion an der $\mathrm{CH}_{2}-$ Gruppe zwischen Keto- und Carbonyloxid-Kohlenstoffatom gespalten werden, woraus neben Methylketen als weitere Zerfallsprodukte aus der abgespaltenen $\mathrm{CHOOH}$-Einheit schwingungsangeregte Ameisensäure, Wasser und Kohlenstoffmonoxid entstehen könnten.

${ }^{6} \mathrm{Hou}$ et al. [125] bestimmten für die Reaktion Propionylradikal $+\mathrm{O}_{2}$ eine Reaktionsentalpie von $-32.5 \mathrm{~kJ} / \mathrm{mol}$. 
Auch das Propionylperoxylradikal kommt als Ursprungsmolekül für Methylketen in Betracht. In einer theoretischen Studie untersuchten Hou et al. [125] die Reaktion von Propionylradikalen mit Sauerstoff, wobei Methylketen als eines der Produkte berechnet wurde. Allerdings wird es von den Autoren bei höheren Gesamtdrücken als lediglich in geringer Ausbeute gebildete Verbindung von untergeordneter Bedeutung angesehen.

Einen weiteren möglichen Reaktionsweg des Propionylradikals eröffnen die in der Ozonolysereaktion gebildeten $\mathrm{OH}$-Radikale. Diese könnten durch die Wasserstoffabspaltung in $\beta$-Position zur Carbonylgruppe direkt zur Methylketenbildung führen. Eine analoge Reaktion wurde bereits von HAYNES et al. [128] für die Photolyse von Diethylketon vorgestellt. Innerhalb dieses Mechanismus abstrahieren Ethylradikale ein $\mathrm{H}$-Atom an der $\mathrm{CH}_{2}$-Gruppe des Ketons, wodurch das entstehende Radikal zum Zerfall zu Methylketen und Ethylradikalen gebracht wird.

Insgesamt ist in Abbildung [D.14d eine deutliche Druckabhängigkeit der Methylketenbildung zu beobachten. Dies weist auf die Bildung aus einer unter Niederdruckbedingungen nur unzureichend stabilisierbaren Verbindung hin. Auch ist lediglich eine leichte Hemmung unter Sauerstoffabwesenheit zu erkennen. Wenn nur die oben genannten Bildungswege als Möglichkeiten in Betracht gezogen werden, kommt die erste Reaktion in Frage, da in ähnlichem Maße wie Methylketen auch $\mathrm{CO}$ gebildet werden sollte. Die CO-Bildung ist unter Hochdruckbedingungen nicht signifikant vom Badgas abhängig, wie es auch für die Intensität der Methylketenbande zutrifft. Lediglich im Niederdruckfall wird mehr $\mathrm{CO}$ wie auch Methylketen in Synthetischer Luft gebildet. Auch der Weg, der von HAYNES [128] vorgeschlagen wurde, ist in Betracht zu ziehen, da die $\mathrm{OH}$ Radikalkonzentration in Synthetischer Luft höher sein sollte als in Stickstoff [48] und damit die Reaktion zu Methylketen vermehrt ablaufen sollte und die Ausbeute erhöht ausfällt.

\subsubsection{Carbonsäuren}

Die Produktzusammensetzung in der Gasphase zeigt unter Hochdruckbedingungen keine ausgeprägte Abhängigkeit vom verwendeten Badgas (Abb. D.17a und (D.19a), während in den Druckdifferenzspektren einige Unterschiede er- 
kennbar sind (Bild D.17b bzw. D.19b). Diese betreffen für Methacrylsäure zum einen den Gerüstbereich mit einem Signal bei $1146-1094 \mathrm{~cm}^{-1}$ in Stickstoff, für Butensäure bei 1171-1057 $\mathrm{cm}^{-1}$ in Synthetischer Luft. Weiterhin ist ein deutlicher Unterschied im Carbonylbereich $\left(\mathrm{C}=\mathrm{O}-\mathrm{Sch}\right.$ wingung bei $1860-1660 \mathrm{~cm}^{-1}$ ) sichtbar.

Bemerkenswert ist, dass schon in den Hochdruckspektren für Butensäure in Abb. D.19a bei $2200-2100 \mathrm{~cm}^{-1}$ deutliche Ketenbanden zu erkennen sind.

In den Reaktionsspektren der Methacrylsäure tauchen erstmals Signale oberhalb von $3200 \mathrm{~cm}^{-1}$ auf, was auf die Bildung von Verbindungen mit $\mathrm{OH}$ Gruppen hinweist, da die freie O-H-Schwingung im Bereich um $3500 \mathrm{~cm}^{-1}$ absorbiert [129]. Die für diese Peaks verantwortlichen Verbindungen scheinen druckunabhängig gebildet zu werden, da sie in den Differenzspektren nicht mehr zu sehen sind. Der etwas unterschiedliche Verlauf der Grundlinien in den Differenzspektren oberhalb von $3000 \mathrm{~cm}^{-1}$ ist nicht aussagekräftig. Es könnte sich hier um Reste der Eduktsignale handeln, die aufgrund des nichtlinearen Verhaltens der gebundenen $\mathrm{OH}$... O-Schwingungen durch Dimerbildung kaum vollständig zu korrigieren sind. Allerdings absorbieren in dem Bereich zwischen 2850-2720 $\mathrm{cm}^{-1}$ auch aldehydische $\mathrm{CH}$-Schwingungen [129], so dass die schwachen positiven Signale in diesem Bereich im Niederdruckspektrum teilweise mit der Differenz bei $1710 \mathrm{~cm}^{-1} \mathrm{im} \mathrm{C}=\mathrm{O}-$ Bereich der Aldehyde zusammengehen und erklärt werden können.

Die Badgasdifferenzspektren der Methacrylsäure in Abb. 6.17a zeigen im Hoch- und Niederdruckfall ähnliche Differenzen, allerdings in unterschiedlich starken Intensitäten, wobei die meisten Banden positiv, d.h. vermehrte Produktbildung in Synthetischer Luft, sind $\left(1710,1436,1312,947 \mathrm{~cm}^{-1}\right)$. Ausnahmen bilden die Banden im Carbonyl- (1794-1739 $\left.\mathrm{cm}^{-1}\right)$ und eine im Gerüstbereich bei $1146-1094 \mathrm{~cm}^{-1}$. Letztere könnte aufgrund inrer P-Q-R-Struktur eventuell zu einer $\mathrm{C}-\mathrm{O}-\mathrm{C}-$ Streckschwingung eines Anhydrids gehören. Weiterhin sind in den Differenzspektren im Bereich 1054-994 cm-1 und 968-931 cm-1 insgesamt drei recht breite Banden zu erkennen, die im Bereich potenzieller SOZ-Signale liegen, allerdings wegen fehlender Referenzspektren nicht sicher identifiziert werden konnten. Beispielhaft wurden die berechneten IR-Spektren zwei verschiedener Hydroperoxidverbindungen, die nach Vorschlag von NEEB et al. [59] aus der Reaktion des stabilisierten $\mathrm{C}_{1}-\mathrm{Cl}$ mit der in der Reaktion gebildeten 
Ameisensäure bzw. Methacrylsäure selbst stammen könnten, theoretisch bestimmt (Korrekturfaktor 0.9658). Es zeigen sich recht gute Übereinstimmungen mit den höchsten Signalen der experimentellen Spektren, ohne sie allerdings einer bestimmten der getesteten Verbindungen zuordnen zu können.

Insgesamt kann wie bei den untersuchten Carbonylverbindungen durch die Ähnlichkeit der beiden Diffenzspektrenarten (Gesamtdruck vs. Badgas) darauf geschlossen werden, dass eine Änderung des Gesamtdruckes für die gebildeten Intermediate eine ähnliche Wirkung entfaltet wie die An- oder Abwesenheit von Sauerstoff als Reaktionspartner.

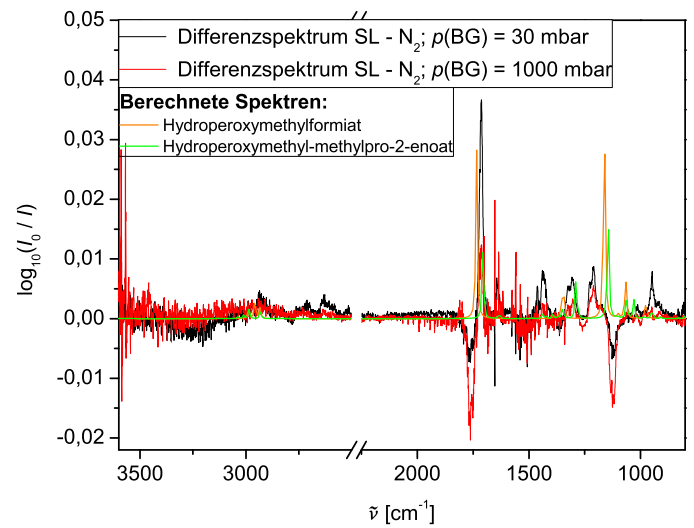

(a) Differenzspektren Synthetischer Luft und Stickstoff für Methacrylsäure, jeweils unter Hoch- und Niederdruckbedingungen, Vergleich mit Theoriespektren.

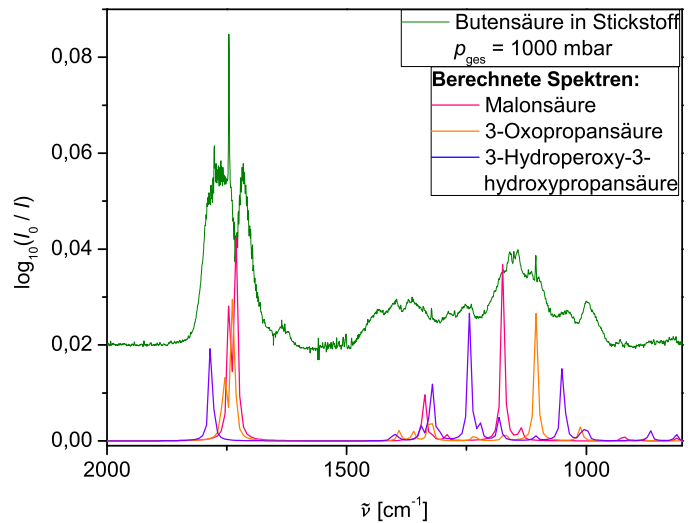

(b) Reaktionsspektren der Butensäure in Stickstoff (Hochdruckfall), Vergleich mit Theoriespektren.

Abb. 6.17.: Vergleich experimentell und theoretisch ermittelter Spektren $(B 3 L Y P / 6-311+G(d, p))$, Verschiebung letzterer jeweils um den Faktor 0.9658 .

Für Butensäure sind zwei breite Peaks um 997 und $1040 \mathrm{~cm}^{-1}$, die mit dem Gesamtdruck zunehmen, sowie eine starke Carbonylbande (1840-1650 cm$\left.{ }^{-1}\right)$ zu beobachten. Die erhöhte Intensität im Hochdruckfall sowie der Wellenzahlbereich könnten für die ersten beiden auf Sekundärozonide als Produkt hinweisen, eine sichere Zuordnung kann allerdings zunächst nicht erfolgen. Aus diesem Grund wurden theoretische Frequenzspektren (B3LYP/6-311+G(d,p)) für verschiedene Strukturen berechnet (vgl. Kap. 5.4), die während der Ozonolysereaktion der Butensäure entstehen können (s. Abbildung [.2). 3-Hydroperoxy3-hydroxypropansäure, Malonsäure und 3-Oxopropansäure entstehen bei der 
bimolekularen Reaktion des stabilisierten $\mathrm{C}_{3}-\mathrm{Cl}$ mit hydroxylgruppentragenden Verbindungen, z.B. Wasser, wie NEEB et al. [59] als möglichen Reaktionspfad vorschlagen. 3-Oxopropansäure wird zusätzlich direkt aus dem Zerfall des Primärozonids gebildet. Abbildung $6.17 \mathrm{~b}$ zeigt einen hohen Übereinstimmungsgrad aller drei möglichen Verbindungen (Korrekturfaktor 0.9658 ) bezüglich Peakposition und z.T. relativer Intensitäten mit den Hochdruckreaktionsspektrum in Stickstoff über den betrachteten Wellenzahlbereich, so dass eine Zuordnung einzelner Peaks durch die Restunsicherheit der Signalposition in den Theoriespektren kaum sinnvoll ist. Wahrscheinlich liegt ein Produktgemisch vor, das neben den in Betracht gezogenen möglichen Verbindungen weitere, oxidierte und in ähnlichen Bereichen absorbierende, Strukturen enthält. Weiterhin sei betont, dass die Hydroperoxyverbindung zwar mechanistisch möglich ist, allerdings nicht unbedingt in dieser Form gebildet werden muss und daher eher als Modellverbindung zu verstehen ist.

\begin{tabular}{|c|c|c|c|c|c|c|}
\hline Alken & Produkt & $\mathrm{SL}, \mathrm{HD}$ & $\mathrm{SL}, \mathrm{ND}$ & $\mathrm{N}_{2}, \mathrm{HD}$ & $\mathrm{N}_{2}, \mathrm{ND}$ & Abb. \\
\hline MACS & $\mathrm{HCOOH}$ & + & ++ & + & ++ & D.18b \\
& $\mathrm{H}_{2} \mathrm{CO}$ & ++ & ++ & ++ & ++ & D.18c \\
& Keten & + & ++ & + & +++ & D.18d \\
\hline \multirow{3}{*}{ BENS } & $\mathrm{HCOOH}$ & + & +++ & ++ & +++ & D.21b \\
& $\mathrm{H}_{2} \mathrm{CO}$ & ++ & ++ & ++ & ++ & D.21d \\
& Keten & ++ & ++++ & + & +++ & D.21d \\
\hline
\end{tabular}

Tab. 6.8.: Nachgewiesene Produkte für Methacrylsäure und Butensäure.

Die tabellarische Zusammenfassung zeigt, dass Ameisensäure wiederum unter Niederdruckbedingungen in größerer Ausbeute entsteht. In der Methacrylsäure- zeigt sich im Gegensatz zur Butensäureozonolyse keine Badgasabhängigkeit. Bei letzterer ist die Bandenintensität in Stickstoff leicht erhöht, was auf geringere Neigung der Intermediate zum Zerfall bei Stoßstabilisierung und gleichzeitiger Reaktionsmöglichkeit mit Sauerstoff hinweist.

Der $\mathrm{C}=\mathrm{O}-$ Carbonylbereich zeigt eine druck- und badgasunabhängige Bildung von Formaldehyd aus beiden Carbonsäuren, was auf den Zerfall des POZ als alleinige Quelle hindeutet.

Abbildung $\mathrm{D.18 \textrm {C }}$ zeigt für Methacrylsäure im Carbonylbereich ein weiteres Signal, dass in Stickstoff unter Hochdruckbedingungen am deutlichsten um 
$1757 \mathrm{~cm}^{-1}$ hervortritt. Unter anderem käme hier Ameisensäure in Frage, die um $1750 \mathrm{~cm}^{-1}$ ein starkes Signal zeigt (sehr ausgeprägter P-Zweig, Q-Zweig ist als scharfer Peak bei $1776 \mathrm{~cm}^{-1}$ erkennbar [130]). Desweiteren ist ein Dublettpeak bei $1837-1780 \mathrm{~cm}^{-1}$ erkennbar, der eventuell auf die Bildung einer Carbonsäure hinweist. Die Bildung einer Säure ist bei dieser Ozonolysereaktion wahrscheinlich, da der Peak um $1757 \mathrm{~cm}^{-1}$ in den Bereich der $\mathrm{C}=\mathrm{O}$ Schwingung eines Carbonsäuremonomers (1800-1740 cm-1 für Monomere, $1740-1650 \mathrm{~cm}^{-1}$ für Dimere [129]) fällt und auch im hohen Wellenzahlbereich bei $3465 \mathrm{~cm}^{-1}$ und $3386 \mathrm{~cm}^{-1}$ jeweils ein Signal erkennbar ist. Möglicherweise ist 2-Oxopropansäure, die zusammen mit dem unsubstituierten CRIEGEEIntermediat aus dem POZ-Zerfall ensteht, für diese Signale verantwortlich.

Auch für Butensäure ist bei $1776 \mathrm{~cm}^{-1}$ das Signal der Ameisensäure zu erkennen, dessen Intensität analog zu dem bei $1106 \mathrm{~cm}^{-1}$ mit steigendem Gesamtdruck geringer ausfällt, dies äußert sich auch in weniger ausgeprägten $P$ und R-Zweigen (ca. 1829-1730 $\mathrm{cm}^{-1}$ ). Zusätzlich ist auch mit der Überlagerung dieser Signale durch Peaks weiterer gebildeter Carbonyl- und Carbonsäureverbindungen zu rechnen (vgl. Abb. 6.17b).

Nach Korrektur der Spektren um Kohlenstoffmonoxid lässt sich in Abbildung D.18d] für Methacrylsäure unter allen Reaktionsbedingungen Keten erkennen, wobei die Ausbeute unter Niederdruckbedingungen im Vergleich zu den Hochdruckspektren erhöht ist und in Stickstoff vermehrt gebildet wird. Auch die Produktspektren der Butensäure zeigen im Bereich der Ketensignale (2200$2100 \mathrm{~cm}^{-1}$, Abb. (D.21d) die bereits bekannte Abhängigkeit vom Druck. Außerdem ist die Ausbeute in beiden Druckregimes jeweils in Synthetischer Luft leicht erhöht, was auf einen beschleunigten Zerfall der ketenbildenden Intermediate im Vergleich zur sauerstofffreien Umgebung hinweist.

Die Bildung von Ethen ließ sich in beiden Fällen nicht beobachten.

\subsubsection{Ketenbildung - Zusammenfassung}

Die Ketenausbeute ist bei der Butensäureozonolyse im Vergleich zu den übrigen vorher beschriebenen Edukten sehr hoch. Zur Veranschaulichung werden in Abb. 6.18 die Niederdruckspektren der Butensäure mit dem Strukturanalogon 1-Buten-3-ol und Methylcyclohexen als stärkstem nicht-voroxidierten Ketenbild- 
Abb. 6.18: Reaktionsspektren im Niederdruckbereich, Ketenabsorbanz: Vergleich von Methylcyclopenten, 3Buten-1-ol und Butensäure.

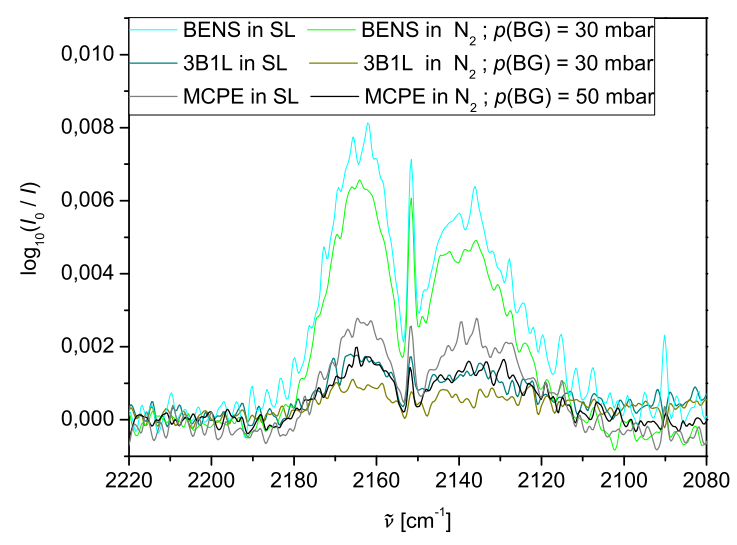

ner verglichen. Sie sind stets auf einen Ozonumsatz von 1 Pa skaliert.

Für alle Substanzen lässt sich feststellen, dass in Synthetischer Luft etwas mehr Keten als in Stickstoff gebildet wird. Dies lässt darauf schließen, dass die Reaktion eines Intermediates mit $\mathrm{O}_{2}$ aus dem Badgas das entstehende Produkt durch die Eintragung einer höheren Überschussenergie soweit destabilisiert, dass es bevorzugt zerfällt. Die verschiedenen Ausbeuten von Butensäure und dem analogen Alkohol weisen darauf hin, dass die in der Säureozonolyse gebildeten Zwischenprodukte durch den von vornherein höheren Oxidationsgrad eher zum Zerfall neigen und schon unter Atmosphärendruck nur unzureichend stoßstabilisiert werden können, wie sich schon in der deutlich sichtbaren Ketenbande unter Hochdruckbedingungen in Abb. D.19a zeigt.

In der Literatur wurden verschiedene Bildungswege für Keten bereits theoretisch und experimentell untersucht. An dieser Stelle werden exemplarisch drei dieser Arbeiten diskutiert, die sich mit der Ketenbildung aus verschiedenen radikalischen Vorläufern unter Reaktion mit Sauerstoff beschäftigen. Die Auswahl erfolgte aus den oben genannten Überlegungen, dass in Sauerstoffanwesenheit eine höhere Ketenausbeute zu beobachten war, dieser also als Reaktionspartner von Bedeutung sein könnte. Das Auftreten von sauerstoffhaltigen radikalischen Kohlenwasserstoffverbindungen ist in der Ozonolysereaktion bekannt. Auch OH-Radikale können für die Reaktionen von Bedeutung sein, wie in den im Folgenden vorgestellten Arbeiten gezeigt wird. Diese treten in sauerstoffhaltiger Umgebung in höherer Konzentration auf als in Stickstoffatmosphäre, allerdings sind diese Reaktionen in Anbetracht der wahrscheinlicheren Sauerstoffreaktion, durch die wesentlich höheren Partialdrücke, wohl von un- 
tergeordneter Bedeutung. Allerdings muss hier auch beachtet werden, dass die Radikal + Radikal-Reaktionen schneller ablaufen können und die Unterschiede in den Ketenausbeuten zwischen den beiden verwendeten Badgasen recht gering ausfällt.

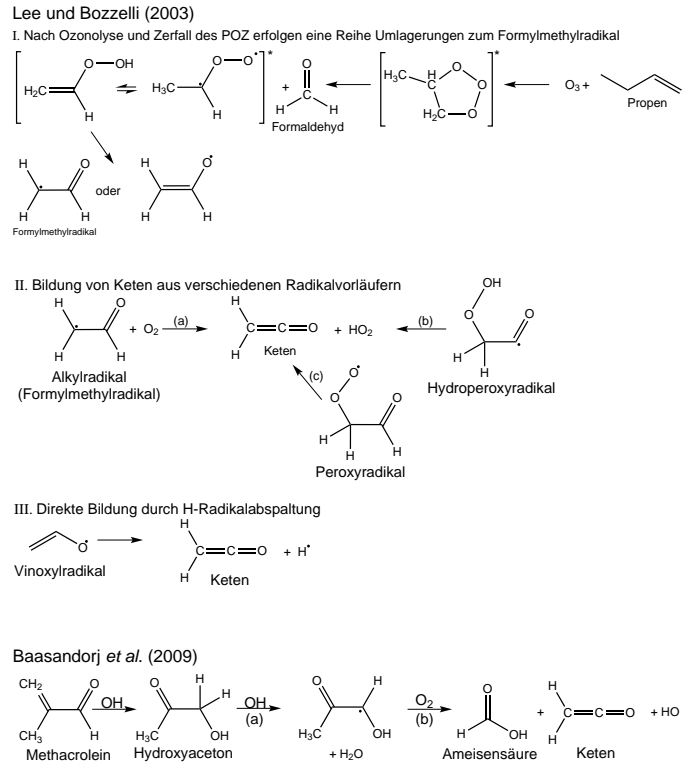

(a) Ketenbildung aus verschiedenen Radikalen nach LEE et al. [131] und BAASANDORJ et al. [132].

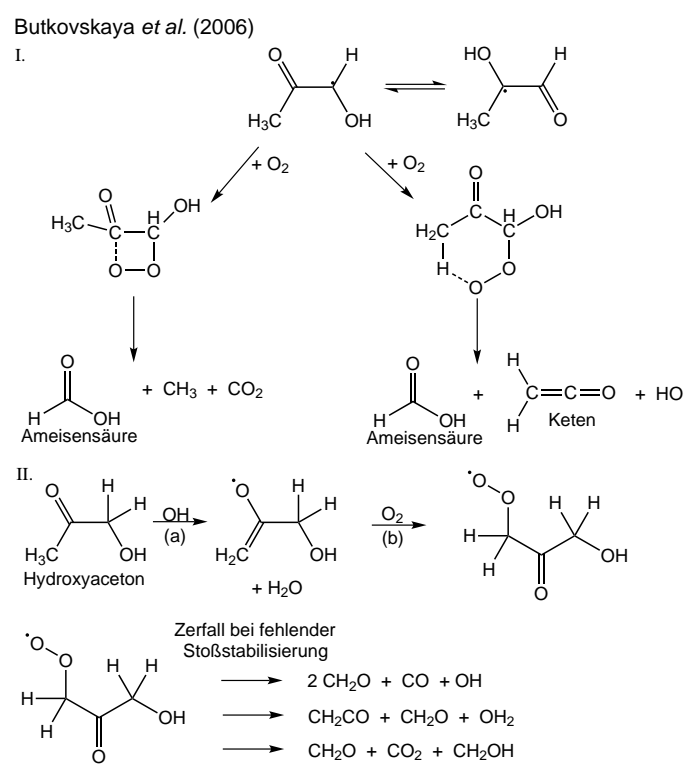

(b) Ketenbildung aus dem Zerfall verschiedener radikalischer Vorläufer nach BUTKOVSKAYA et al. [133].

Abb. 6.19.: Vorschläge zur Ketenbildung aus Reaktionen unter Sauerstoffbeteiligung in der Literatur.

Die theoretische Arbeit von LEE et al. [131] betrachtet unter anderem die Reaktion des Formylmethylradikals $\left(\mathrm{C}_{2} \mathrm{CHO}\right)$ mit Sauerstoff, aus der Keten und das $\mathrm{HO}_{2}$-Radikal entstehen. Ersteres wird z.B. in der Propenozonolyse gebildet, wie in Schritt (I.) der Abbildung 6.19a gezeigt ist. Wie auch unter anderem schon von OLZMANN et al. [113] berichtet, wird das Formylmethylradikal in der Ozonolyse ungesättigter Kohlenwasserstoffverbindungen aus dem CRIEGEEIntermediat gebildet, welches nach Wasserstoffwanderung und Bildung eines Vinylhydroperoxids schnell durch Bruch der schwachen $\mathrm{O}-\mathrm{OH}$-Bindung zerfällt. In Teil (II.) wird die nachfolgende Bildung von Keten durch unimolekularen Zerfall aus verschiedenen radikalischen Vorläufern gezeigt. Die genannten Peroxy- und Hydroperoxyradikale (Reaktionen b,c) können zunächst aus der vorangegangenen Reaktion des Alkylradikals mit Sauerstoff entstehen, statt 
dass nach bimolekularer Reaktion des Formylmethylradikals mit $\mathrm{O}_{2}$ direkt Keten und $\mathrm{HO}_{2}$ resultieren, wie in Reaktion (a) dargestellt. Weiterhin geben die Autoren als zusätzliche Reaktionsmöglichkeit den unimolekularen Zerfall des Vinoxylradikals $\mathrm{C}_{2} \mathrm{CHO}$ zu Keten und einem Wasserstoffradikal an, schränken allerdings ein, dass dieser nur bei niedrigen Drücken und hohen Temperaturen zur bimolekularen Reaktion konkurrenzfähig sei.

BAASANDORJ et al. [132] (Abb. 6.19a, unterer Teil) untersuchten experimentell und theoretisch die Kinetik der Hydroxyaceton $+\mathrm{OH}$-Reaktion bei Niederdruckbedingungen (2-5 Torr). Dabei wird das Hydroxyaceton in der Oxidationsreaktion von Methacrolein mit $\mathrm{OH}$-Radikalen gebildet und ist daher vor allem für die Ketenbildung aus der in dieser Arbeit betrachteten Methacrylsäure interessant, außerdem ist die Reaktion aufgrund des natürlichen Vorkommens der Carbonylverbindung auch atmosphärenrelevant. Das gebildete Hydroxyaceton kann anschließend, wie für methylsubstituierte Hydroperoxide bekannt (vgl. [17,48]), mit $\mathrm{OH}$-Radikalen unter Wasserabspaltung zu einem Alkylradikal reagieren, welches nach dem Vorbild von LEE et al. [131] mit Sauerstoff unter anderem zu Keten weiterreagieren könnte. Dieser Mechanismus ist allerdings wegen der zweimaligen Beteiligung von nur in geringer Konzentration vorkommenden $\mathrm{OH}$-Radikalen als eher unwahrscheinlich einzuschätzen.

Das zuvor betrachtete Hydroxyaceton und seine Oxidation durch $\mathrm{OH}$ Radikale wird auch in der Arbeit von BUTKOVSKAYA et al. [133] betrachtet, hier wird ebenfalls ein Mechanismus vorgestellt, der die auftretende Ketenbildung erklären soll (Abb. 6.19b). Nach der Bildung des mesomeriefähigen Alkylradikals (Teil I.), wie es etwa durch OH-Abspaltung aus einem Peroxyradikal entstehen kann, ist die sekundäre Reaktion mit einem Sauerstoffmolekül möglich. Dabei entsteht entweder ein vier- oder ein sechsgliedriger Übergangszustand, die beide aufgrund der enthaltenen Überschussenergie weiter zerfallen können, wobei im letzten Fall direkt Keten gebildet werden kann. Diese Reaktionen sind nach Ergebnissen der Autoren effektiver gegenüber anderen, je niedriger die Reaktionstemperatur gewählt wird, da die zyklischen Übergangszustände dann leichter ausgebildet werden. Eine alternative Reaktionsmöglichkeit stellen BUTKOVSKAYA et al. mit dem Zerfall des $\mathrm{O} \mathrm{OCH}_{2} \mathrm{C}(\mathrm{O}) \mathrm{CH}_{2} \mathrm{OH}$-Peroxyradikals vor. Dieses entsteht aus der $\mathrm{OH}$-Reaktion des Hydroxyacetons und anschließender $\mathrm{O}_{2}$-Addition (Reaktionen (a) und (b) in II.). Wenn dieses Peroxyradikal nicht 
stoßstabilisiert wird, stehen ihm verschiedene Zerfallskanäle offen, wobei der ketenbildende Schritt von den Autoren als eher unwahrscheinlich gegenüber den anderen genannten, exothermeren Zerfällen angesehen wird.

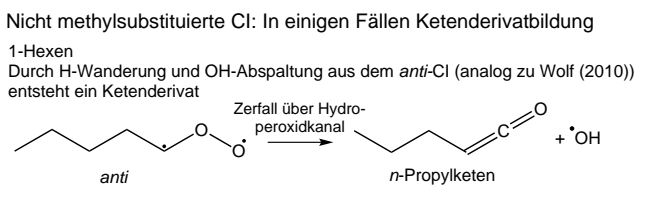

1-Penten-3-on: Mögliche Bildungswege für Methylketen

I. Direkter Zerfall des $\mathrm{C}_{4}-\mathrm{Cl}$ unter Abspaltung schwingungsangeregter Ameisensäure<smiles></smiles>

II. Direkter Zerfall des $\mathrm{C}_{4}-\mathrm{Cl}$ (Weiterführung des Vorschlags nach O'Dwyer (2010))

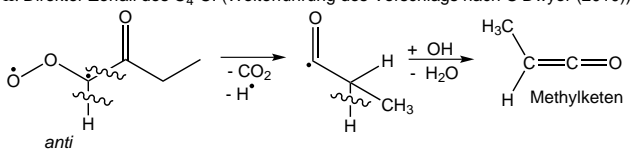

III. Zerfall des Propionylperoxylradikals nach Hou et al. (2007)<smiles>CC(=O)CC(=O)OOCCCO</smiles>
Propionylperoxylradikal

IV. Zerfall des Propionyradikals analog zu Haynes et al. (1951)

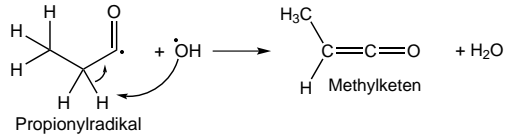

(a) Mögliche Wege zur Ketenderivatbildung einiger unsubstituierter Reaktanden, z.T. analog zU WOLF, O'DWYER, HOU, HAYNES [48,61, 125, 128].

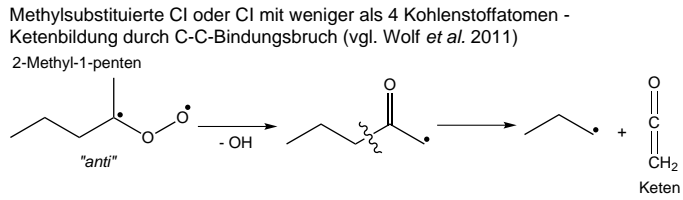<smiles>CC(=O)C=CCCC(C)=O</smiles>

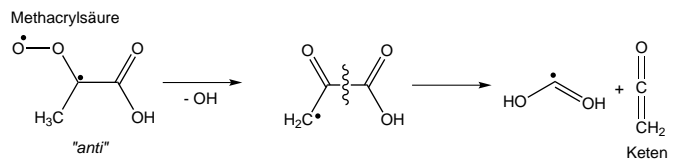

(b) Ketenbildung aus methylsubstituierten Ausgangsverbindungen, analog zu WOLF et al. [17].

Abb. 6.20.: Vorschläge zur Ketenbildung aus den verwendeten Edukten.

In den folgenden Ausführungen soll nun das unterschiedliche Reaktionsverhalten und die Neigung der in der vorliegenden Arbeit betrachteten Edukte zur Ketenbildung im Zusammenhang mit der reaktiven Struktur der Kohlenwasserstoffverbindungen interpretiert werden. In Abbildung 6.20a werden Reaktanden gezeigt, die keine Methylsubstituierung an der olefinischen Doppelbindung besitzen und infolgedessen in der Ozonolysreaktion kein Keten, sondern entsprechende Analoga bilden. Lediglich für 1-Hexen und 1-Penten-3-on konnten Signale im Wellenzahlbereich $2200-2100 \mathrm{~cm}^{-1}$ beobachtet werden, die aufgrund mechanistischer Überlegungen auf $n$-Propylketen bzw. Methylketen zu- 
rückzuführen sind. Im ersten Fall ist ein Zerfall über den Hydroperoxidkanal, wie bereits zuvor in Abbildung 2.11(S. 35) ausführlich dargestellt wurde, wahrscheinlich. Die Bildung von Methylketen in der Ozonolyse des 1-Penten-3-ons ist potenziell über viele verschiedene Wege denkbar, wobei die relativ hohe Signalstärke durchaus auf mehr als einen ketenbildenden Reaktionskanal hinweisen könnte. Neben dem direkten Zerfall des $\mathrm{C}_{4}$-CRIEGEE-Intermediates in Methylketen über den Hydroperoxidkanal (I.) oder Abspaltung verschiedener (radikalischer) Bruchstücke (II.), wie für die Bildung anderer Produkte in ähnlicher Art bereits von O'DWYER et al. [61] vorgeschlagen wurde, gibt es auch hier Vorschläge zur Ketenbildung unter reaktiver Beteiligung von Sauerstoff. Dieser wird zur Bildung des Propionylperoxylradikals (vgl. Abb 6.15a) aus dem Propionylradikal benötigt. Aus der Peroxylverbindung kann im Anschluss durch Abspaltung eines $\mathrm{HO}_{2}$-Radikals das Methylketen gebildet werden, wobei $\mathrm{Hou}$ et al. [125] diese Reaktion aufgrund ungünstiger Energetik als unwahrscheinlich gegenüber anderen Zerfallswegen bezeichnen.

HAYNES et al. [128] schlagen dagegen die direkte Bildung des Methylketens aus dem Propionylradikal aus der Reaktion mit einem OH-Radikal unter Wasserabspaltung vor, wobei aus der genannten Arbeit aufgrund unterschiedlicher Edukte und Reaktionsbedingungen (es wurde die Photodissoziation von Diethylketen untersucht) nicht ohne weiteres auf die Bedeutung für die vorliegende Methylketenbildung durch die Ozonolyse geschlossen werden. Unter anderem spricht die Reaktion durch die Abhängigkeit von einem in geringer Konzentration vorkommenden Radikal eher für eine geringere Bedeutung.

Abbildung 6.20bzeigt die bereits in den Arbeiten von WoLF et al. [17,48] vorgeschlagenen Reaktionswege für Edukte mit methylsubstituierter Doppelbindung. Bei diesen Verbindungen ist von einem Zerfall der CRIEGEE-Intermediate über $\mathrm{OH}$-Abspaltung und anschließendem $\mathrm{C}-\mathrm{C}$-Bindungsbruch auszugehen, wie für 2-Methyl-1-penten, Methylcyclopenten und auch die voroxidierte Methacrylsäure gezeigt wird.

Aus Butensäure resultiert trotz fehlender Methylsubstituierung, wie in Abb. 6.18 gezeigt, eine relativ hohe Ketenausbeute verglichen mit Methylcyclopenten als effektivstem nicht voroxidierten Ketenbildner und dem zur Säure analogen Alkohol. Prinzipiell sind verschiedene Wege, auf denen Keten gebildet werden kann, denkbar, die in der Diskussion in Abb. 7.1 (Seite 266) gezeigt 
und erörtert werden.

Für 4-Penten-1-ol, 4-Penten-1-al und 1-Penten-3-ol konnten keine Signale bei $2200-2100 \mathrm{~cm}^{-1}$ nachgewiesen werden. Für die ersten beiden Verbindungen scheint dies aufgrund der strukturellen Ähnlichkeit sinnvoll, da der Zerfall der Intermediate nicht zwangsläufig an den für die Ketenbildung entscheidenden Stellen im Molekül erfolgen muss. Allerdings weist das Fehlen eines Methylketensignals im Falle des zum 1-Penten-3-on bis auf den Oxidationsgrad identischen 1-Penten-3-ols darauf hin, dass eben dieser Unterschied entscheidend für das Reaktionsverhalten der in der Ozonolyse gebildeten Produkte sein kann. Die Beobachtungen weisen darauf hin, dass die Stabilität bestimmter, potenziell ketenbildender, Zwischenprodukte mit zunehmendem Oxidationsgrad des Eduktes abzunehmen scheint.

Diese These wird auch durch den Vergleich der Ausbeuten für Butensäure und 3-Buten-1-ol gestützt, da bei ersterer ungefähr die vierfache Intensität der Ketenbande im Vergleich zum Alkohol beobachtet werden kann.

Dass bei 3-Buten-1-ol jedoch im Gegensatz zu 1-Penten-3-ol Keten gebildet wird, weist darauf hin, dass die Länge der Kohlenstoffkette ein entscheidender Faktor für den Zerfall bestimmter Intermediate zu dem betrachteten Produkt sein kann, da bei größeren Molekülen mehr Freiheitsgrade zur Verfügung stehen, die eine Stabilisierung der Zwischenprodukte und eventuell nachfolgende Reaktionen gegenüber dem Zerfall bevorzugt ablaufen.

\subsubsection{Produktstudien unter $\mathrm{SO}_{2}$-Beteiligung}

In diesem Abschnitt werden Produktspektren gezeigt, die unter Anwesenheit von Schwefeldioxid aufgenommen wurden. Die Ergebnisse werden nach Strukturmotiv der Edukte geordnet präsentiert, wobei endozyklische Verbindungen eine Doppelbindung innerhalb, exozyklische eine außerhalb des Kohlenstoffringes besitzen. Generell birgt die Interpretation der gezeigten Infrarotspektren Probleme, da die Beteiligung von Schwefelsäure die Flüchtigkeit der entstehende Aggregate und damit die Aufteilung zwischen Gas- und Partikelphase stark beeinflusst. Aus diesem Grund sind Intensitätsunterschiede generell nicht nur auf eine vermehrte oder verminderte Bildung während der Reaktion, sondern potenziell auch durch eine unterschiedlich ausgeprägte Neigung zu Kondensa- 
tion aus der Gas- in die Partikelphase zurückzuführen.

\subsubsection{Endozyklische Alkene}

\section{Cyclohexen}

In Abbildung 6.21 sind Infrarotspektren von Cyclohexenozonolysen gezeigt. Dabei wurden bereits jeweils Differenzen zwischen unter $\mathrm{SO}_{2}$-Zugabe aufgenommenen Spektren bei verschiedenen Gesamtdrücken (in schwarz) bzw. $\mathrm{SO}_{2}$-haltigen und -freien Spektren bei identischem Gesamtdruck (in rot bei $p_{\text {ges }}=1000$ mbar, in grün bei $p_{\text {ges }}=50$ mbar) gebildet. Dies hat den Zweck, direkt den Einfluss des Schwefeldioxids auf die gebildeten Gasphasenprodukte zeigen zu können. Folglich zeigen positive Banden an, welche Produkte unter Hochdruckbedingungen oder $\mathrm{SO}_{2}$-Anwesenheit vermehrt gebildet werden bzw. in der Gasphase auftreten, negative Banden stehen für vermindert gebildete oder in der Gasphase vorliegende Verbindungen.

Die Spektren in Abb. 6.21a zeigen, dass Schwefeldioxid unabhängig von den herrschenden Druckbedingungen in der gewählten Reaktionszeit nicht vollständig umgesetzt wird $\left(\mathrm{SO}_{2}\right.$-Peak um $\left.1370 \mathrm{~cm}^{-1}\right)$. Auch zeigt sich druckabhängig kein deutlicher Unterschied im $\mathrm{SO}_{2}$-Umsatz, da das Differenzspektrum der beiden Produktspektren unter Schwefeldioxidzugabe bei 1000 bzw. 50 mbar keinen Peak mehr enthält.

Jedoch werden druckabhängig die Ausbeuten bestimmter Gasphasenprodukte beeinflusst. Das Differenzspektrum zwischen Hoch- und Niederdruckreaktion unter Zugabe von $\mathrm{SO}_{2}$ zeigt mit einem scharfen Q-Zweig bei $950 \mathrm{~cm}^{-1}$ eine vermehrte Bildung von Ethen unter Niederdruckbedingungen. Auch Ameisensäure mit scharfen Q-Zweigen bei 1106 und $1776 \mathrm{~cm}^{-1}$ ist hier zu erkennen. Auf die Bildung dieser Verbindungen scheint eine Schwefeldioxidzugabe bei Betrachtung der Diffenzspektren eine behindernde Wirkung auszuüben. Vor allem in dem in grün dargestellten Differenzspektrum bei Niederdruckbedingungen sind die Abnahmen bei den oben genannten Wellenzahlen zu beobachten, im Hochdruckfall sind sie im Rauschen dagegen gerade noch zu erahnen. Diese Beobachtungen könnten allerdings auch Artefakte von trotz Normierung leicht abweichenden Eduktumsätzen sein. 


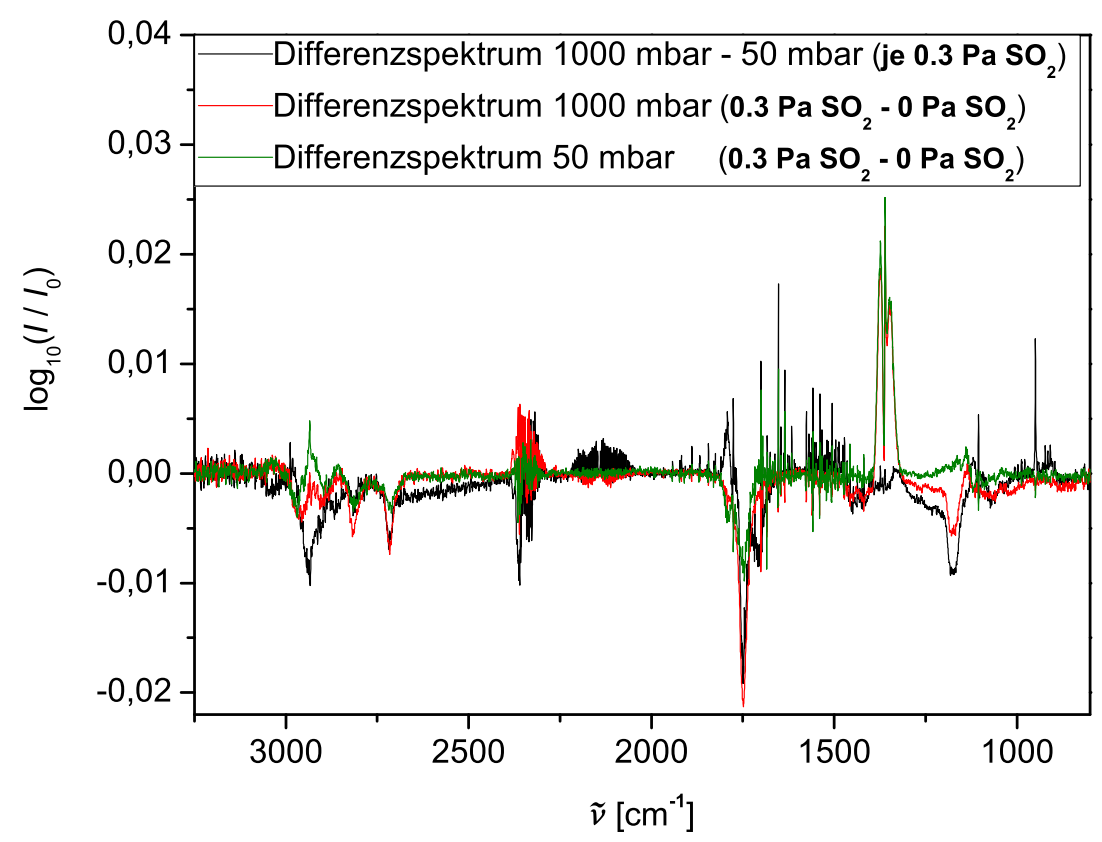

(a) Differenzspektren von Cyclohexenozonolysen bei Hoch- und Niederdruckbedingungen mit und ohne $\mathrm{SO}_{2}$-Zugabe. Die Referenzspektren ohne $\mathrm{SO}_{2}$ stammen aus bisher unveröffentlichten Daten von $\mathrm{L}$. WOLF.

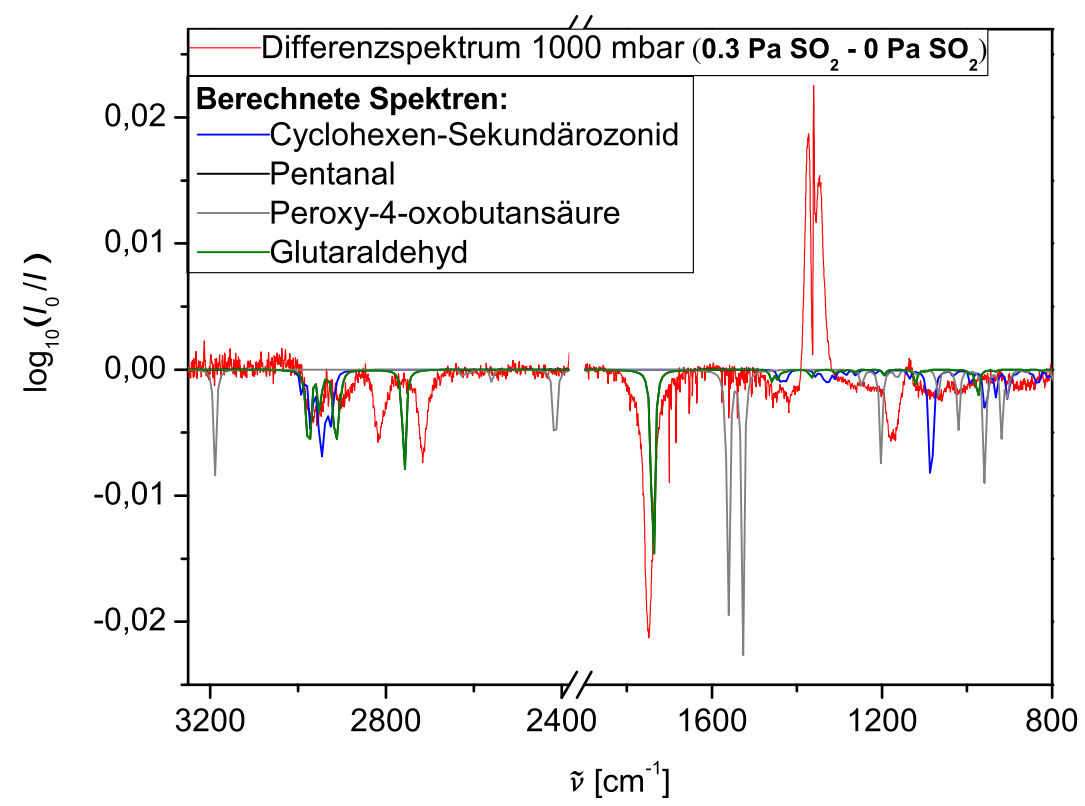

(b) Differenzspektrum von Cyclohexenozonolysen beim Gesamtdruck 1000 mbar mit und ohne $\mathrm{SO}_{2}$-Zugabe. Es wird verglichen mit Spektren aus quantenchemischen Rechnungen $((B 3 L Y P / 6-311+G(d, p))$, vgl. Kapitel[5.4]. Der Verschiebungsfaktor beträgt 0.965.

Abb. 6.21.: Ergebnisse der Produktbildungsexperimente des Cyclohexens unter $\mathrm{SO}_{2}$-Zugabe. 
Kohlenstoffmonoxid wird beispielsweise abhängig von den herrschenden Reaktionsdrücken, z.B. durch den Zerfall instabiler Intermediate, gebildet. Weiterhin unterliegt die rotationsaufgespaltene Bande einer ausgeprägten Druckverbreiterungsproblematik. Folglich ist $\mathrm{CO}$ bei Differenzbildung zwischen Hochund Niederdruckbedingungen zwischen $2500-2050 \mathrm{~cm}^{-1}$ deutlich zu erkennen. Weiterhin ist zu sehen, dass $\mathrm{SO}_{2}$-Zugabe die CO-Bildung im Hochdruckfall zu reduzieren scheint, während jedoch unter Niederdruckbedingungen kaum ein signifikanter Unterschied festzustellen ist.

Es sind einige negative Signale im Bereich von $\mathrm{C}=\mathrm{O}$-Carbonyl- (1750$1700 \mathrm{~cm}^{-1}$ ) und Gerüstschwingungen (um $1170 \mathrm{~cm}^{-1}$ ) zu erkennen, die unter Hochdruckbedingungen stärker ausgeprägt sind und damit das leicht verminderte Vorkommen einer Verbindung in der Gasphase in $\mathrm{SO}_{2}$-Anwesenheit anzeigen.

Unter Niederdruckbedingungen dagegen hat $\mathrm{SO}_{2}$ auf den Bereich der Gerüstschwingungen und auch auf den Carbonylbereich weniger Einfluss. Daraus lässt sich ein geringerer Einfluss auf die unimolekulare Chemie (bei Zuhilfenahme von $\mathrm{CO}$ und Ameisensäure als Referenzsubstanzen für den unimolekularen Zerfall angeregter Intermediate) unter Niederdruck- als unter Hochdruckbedingungen ableiten. Die Reaktion $\mathrm{SO}_{2}+$ CRIEGEE-Intermediat scheint also hauptsächlich unter Hochdruckbedingungen von Bedeutung zu sein.

Der Vergleich mit quantenchemisch berechneten Spektren in Abb. 6.21blegt nahe, dass durch Schwefeldioxidaddition im Reaktionsgemisch eine Substanz mit einem Aldehyd- oder Ketonstrukturmotiv nur reduziert in der Gasphase vorliegt. Dies könnte mit einer erhöhten Kondensation mittelflüchtiger oxidierter Verbindungen an sauren Partikelkeimen (vgl. Abschnitt 2.8) bzw. einer weiteren Reaktion der Carbonylverbindungen in $\mathrm{SO}_{2}$-Anwesenheit zu schwerflüchtigeren Verbindungen und daraus folgender erhöhter Kondensation zu erklären sein.

Der Vergleich mit verschiedenen möglichen Substanzen erlaubt allerdings keine genaue Zuordnung, da sowohl Pentanal als auch Glutaraldehyd in ähnlichen Wellenzahlbereichen ihre Signale zeigen und eine Unterscheidung, trotz Verwendung eines Korrekturfaktors (empirisch: 0.965) zum Ausgleich des harmonischen Ansatzes in den Rechnungen, daher nicht gesichert möglich ist. Im Übrigen ist aufgrund der Komplexität des Ozonolysemechanismus ohnehin zu 
erwarten, dass ein Gemisch mehrerer Substanzen höheren Oxidationsgrads entsteht. Lediglich die Peroxy-4-oxobutansäure und wahrscheinlich auch das mögliche Sekundärozonid sind aufgrund ihrer Signallage recht eindeutig als Teil des Produktgemisches auszuschließen, allerdings kann eine Vielzahl weiterer, nicht berechneter, Produkte ebenfalls zu den im Spektrum sichtbaren Peaks beitragen.

\section{$\alpha$-Pinen}

Zunächst werden in Abbildung 6.22a die Reaktionsspektren bei $p_{\text {ges }}=1000$ mbar mit (grün) und ohne $\mathrm{SO}_{2}$-Zugabe (blau) sowie das Differenzspektrum aus beiden (rot) gezeigt. Wie im Differenzspektrum zu erkennen, ist auch hier das zugegebene Schwefeldioxid nicht vollständig umgesetzt worden, zudem ist wie im Falle des Cyclohexens die Bildung von $\mathrm{CO}\left(2250-2050 \mathrm{~cm}^{-1}\right)$ in $\mathrm{SO}_{2}$-Anwesenheit reduziert.

Weiterhin ist zu beobachten, dass die Bildung von Ameisensäure unbeeinflusst zu bleiben scheint, da der scharfe Q-Zweig bei $1106 \mathrm{~cm}^{-1}$ nicht zu erkennen ist. Dagegen ist im Fingerprintbereich um $1500-800 \mathrm{~cm}^{-1}$ eine deutliche Intensitätszunahme zu beobachten, während im C=O-Bereich um $1730 \mathrm{~cm}^{-1}$ eine starke negative Bande auftritt. Letztere könnte, wie bereits bei Cyclohexen, mit einer erhöhten Kondensation mittelflüchtiger Verbindungen unter eventueller Beteiligung verschiedener Reaktionen mit $\mathrm{SO}_{2}$ oder $\mathrm{H}_{2} \mathrm{SO}_{4}$ zu erklären sein.

In Abbildung 6.22b zeigt der Vergleich des Differenzspektrums unter Hochdruckbedingungen mit einem berechneten Spektrum von Pinonaldehyd gewisse Übereinstimmungen. Vor allem im Bereich der Carbonyl-C=O-Absorbanz und der $\mathrm{CH}$-Schwingungen um $3000 \mathrm{~cm}^{-1}$ treten sowohl im experimentell als auch im theoretisch ermittelten Spektrum (wiederum nach Verschiebung des letzteren um einen Korrekturfaktor von 0.965) Signale in den gleichen Wellenzahlbereichen auf. Jedoch tritt im aufgenommenen Spektrum kein starkes Signal um $2800 \mathrm{~cm}^{-1}$ auf, das im theoretischen Spektrum als aldehydische $\mathrm{CH}$-Schwingung eine recht starke Intensität zeigt, stattdessen ist ein deutlicher Peak erst knapp unterhalb von $3000 \mathrm{~cm}^{-1}$ zu finden, was allerdings auch durch den empirisch bestimmten Verschiebungsfaktor des Theoriespektrums verursacht werden kann. 


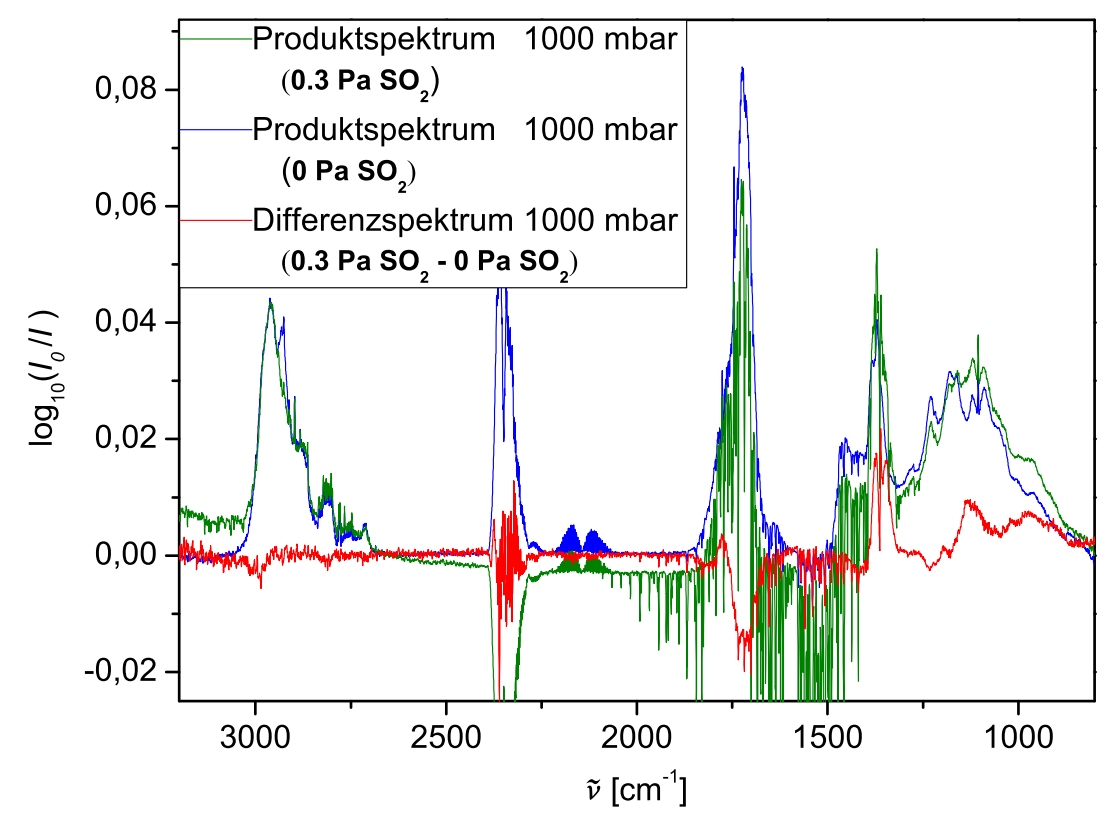

(a) Produkt- und Differenzspektrum von $\alpha$-Pinenozonolysen bei Hochdruckbedingungen mit und ohne $\mathrm{SO}_{2}$-Zugabe. Das Referenzspektrum ohne $\mathrm{SO}_{2}$ stammt von WOLF [48].

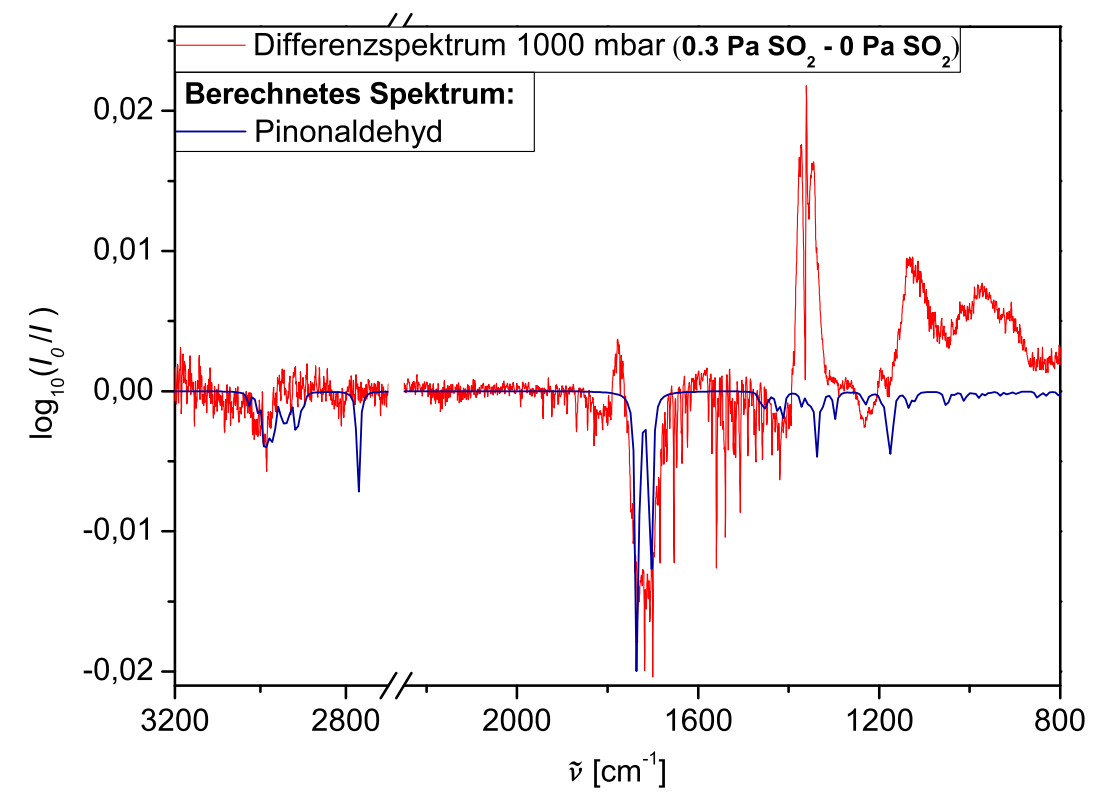

(b) Differenzspektrum von $\alpha$-Pinenozonolysen beim Gesamtdruck $p_{\text {ges }}=1000$ mbar mit und ohne $\mathrm{SO}_{2}$-Zugabe. Es wird verglichen mit einem Spektrum aus quantenchemischen Rechnungen (B3LYP/6$311+G(d, p)$, siehe Kapitel 5.4). Der Verschiebungsfaktor beträgt 0.965, zur besseren Erkennbarkeit wurde die Bandenintensität stärker gewählt, als es die Ausbeuten erwarten lassen. Der Vergleich relativer Intensitäten in beiden Spektren ist daher problematisch.

Abb. 6.22.: Ergebnisse der Produktbildungsexperimente des $\alpha$-Pinens unter $\mathrm{SO}_{2}$-Zugabe. 


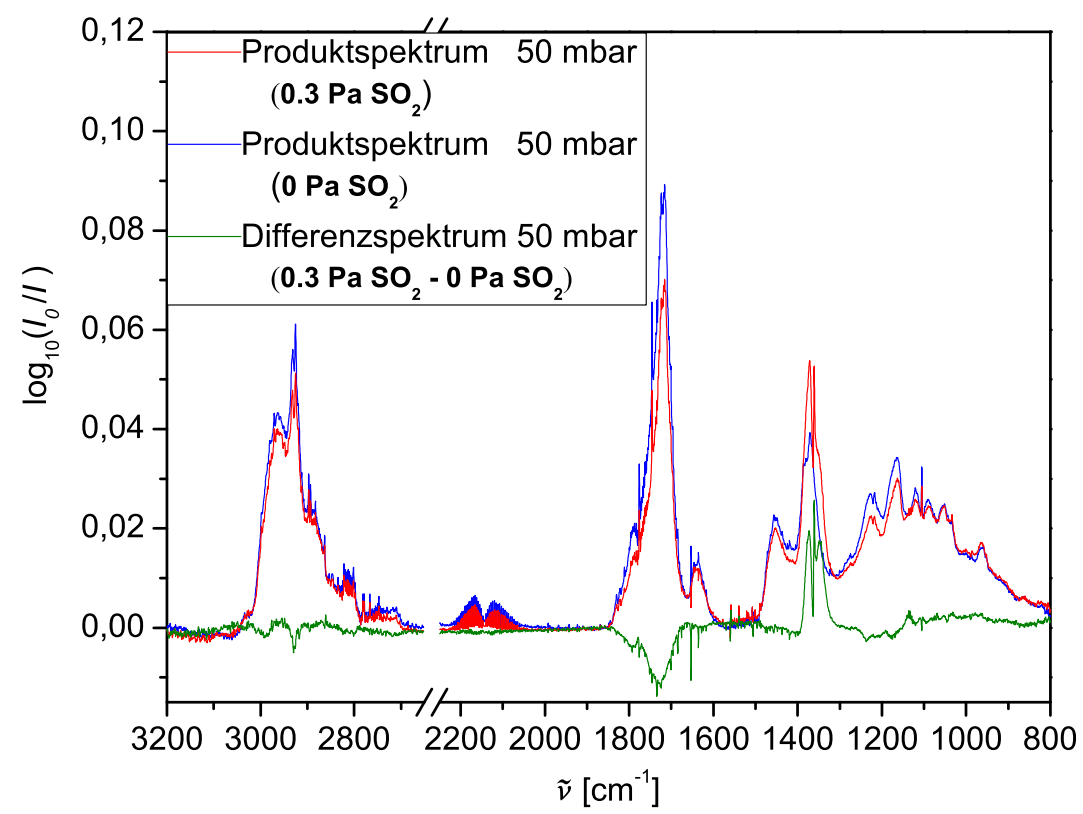

(a) Produkt- und Differenzspektrum von $\alpha$-Pinenozonolysen bei Niederdruckbedingungen mit und ohne $\mathrm{SO}_{2}$-Zugabe. Die Referenzspektren ohne $\mathrm{SO}_{2}$ stammen von WOLF [48].

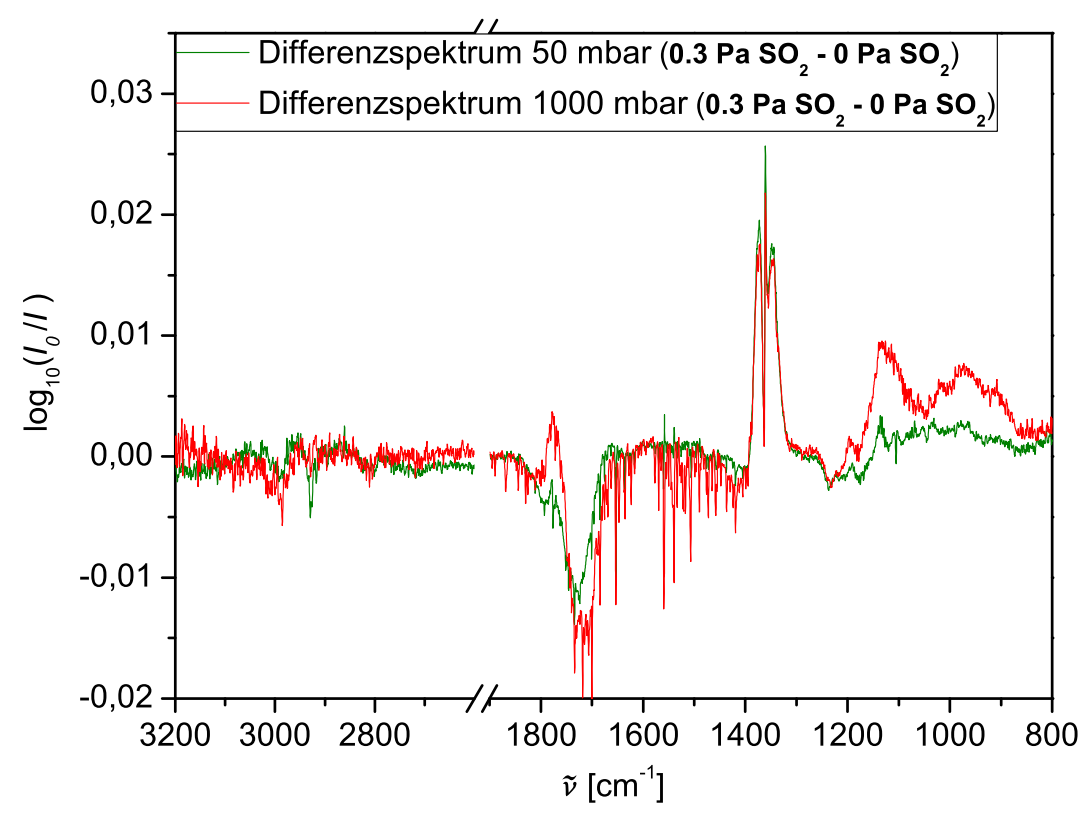

(b) Hoch- und Niederdruckdifferenzspektren von $\alpha$-Pinenozonolysen.

Abb. 6.23.: Vergleich der Produktbildungsexperimente des $\alpha$-Pinens unter $\mathrm{SO}_{2}{ }^{-}$ Zugabe bei verschiedenen Gesamtdrücken. 
Allerdings gilt auch hier wiederum die Anmerkung, dass wahrscheinlich ein Gemisch verschiedener Produkte zu den aufgenommenen Signalen beiträgt. Auch die Wahl des Korrekturfaktors zur Verschiebung des berechneten Spektrums könnte ungünstig gewählt sein, so dass die Bandenpositionen nicht ausreichend genau wiedergegeben werden. Die Bildung und damit auch die Detektion von Pinonaldehyd im IR-Spektrum ist also zwar wahrscheinlich, aber nicht unbedingt für die gesamte Signalsärke im $\mathrm{C}=\mathrm{O}$-Bereich verantwortlich.

Abbildung 6.23zeigt im oberen Teil (a) eine Darstellung der Produktspektren mit (rot) und ohne $\mathrm{SO}_{2}$-Zugabe (blau) sowie die Differenz dieser beiden IRMessungen im Niederdruckbereich bei $p_{\text {ges }}=50$ mbar. Das Differenzspektrum ähnelt dem der Hochdruckreaktion, wie auch der Vergleich der Differenzspektren im unteren Teil 6.23b verdeutlicht. Die CO-Ausbeute ist von der Schwefeldioxidzugabe unter Niederdruckbedingungen nahezu unbeeinflusst, während die Ameisensäureausbeute leicht reduziert scheint. Letzteres könnte allerdings auch durch Abweichungen im Alkenumsatz zustande kommen. Das Signal im $\mathrm{C}=\mathrm{O}$-Bereich um $1730 \mathrm{~cm}^{-1}$ ist bei dem geringeren Gesamtdruck weniger stark ausgeprägt, was entweder auf eine verstärkte Kondensation oder eine von vornherein geringere Produktion der beteiligten Carbonylkomponente schlieBen lässt. Auch im Fingerprintbereich unterhalb von $1200 \mathrm{~cm}^{-1}$ ist die Intensität im Vergleich zur Hochdruckaufnahme verringert. Im höheren Wellenzahlbreich tritt erst ab $2900 \mathrm{~cm}^{-1}$ ein Signal auf, welches sich unter Umständen auf $\mathrm{CH}$ Schwingungen der reduziert vorliegenden Carbonylkomponenten zurückführen lässt, da es ebenso wie der Peak um $1730 \mathrm{~cm}^{-1}$ eine negative Intensität aufweist.

\subsubsection{Exozyklische Alkene}

\section{$\beta$-Pinen}

Sowohl Produkt- als auch Differenzspektren (Abb. 6.24a) des exozyklischen $\beta$-Pinens zeigen einige systematische Unterschiede zu denen des endozyklischen $\alpha$-Pinen.

Zuerst fällt auf, dass das zugegebene $\mathrm{SO}_{2}$ komplett verbraucht wurde, da ein entsprechendes Signal um 1400-1300 $\mathrm{cm}^{-1}$ mit einem scharfen Q-Zweig 
bei $1361 \mathrm{~cm}^{-1}$ in den Reaktionsspektren nicht mehr zu sehen ist. Desweiteren ist eine starke Zunahme im Carbonyl-C=O-Schwingungsbereich um $1740 \mathrm{~cm}^{-1}$ wie auch im Fingerprintbereich (1440-1350, 1230-1150 und 975-850 $\left.\mathrm{cm}^{-1}\right)$ festzustellen.

In Abbildung 6.24b zeigt der Vergleich des Differenzspektrums zwischen $\mathrm{SO}_{2}$-An- und Abwesenheit eine hohe Übereinstimmung mit dem von WOLF [48] übernommenen Reinspektrum des Nopinons. Nopinon entsteht während der Ozonolyse von $\beta$-Pinen aus dem POZ-Zerfall ( $Y_{\mathrm{NPN}}=0.16 \pm 0.04$ [126]) und wird bei Schwefeldioxidzugabe vermehrt gebildet. Im Gegensatz dazu scheint sich die $\mathrm{SO}_{2}$-Zugabe ungünstig auf die Stabilität des Sekundärozonids auszuwirken, da der entsprechende Peak als negatives Signal im Differenzspektrum auftaucht. WINTERHALTER et al. berichten von Absorbanzen dieser Verbindung im Falle von Ameisensäurezugaben u.a. bei $976 \mathrm{~cm}^{-1}$ ( $Y_{\text {soz }}=0.16 \pm 0.05$ [126]). Dieser Peak ist in dem ohne $\mathrm{SO}_{2}$-Zugabe gemessenen (blauen) Spektrum bei $974 \mathrm{~cm}^{-1}$ anhand der Bandenform identifizierbar. Auch die weiteren Absorbanzen, die WINTERHALTER in seiner Arbeit angibt (um 1230, 1103 bzw. $1071 \mathrm{~cm}^{-1}$ ) entsprechen bis auf einige Wellenzahlen genau den Signalpositionen negativer Peaks im gemessenen Spektrum (um 1235, 1102 bzw. $1071 \mathrm{~cm}^{-1}$ ) und können daher dem Sekundärozonid des $\beta$-Pinens zweifelsfrei zugeordnet werden. Auch entprechen diese Ergebnisse denen von WoLF et al. [17] zur $\beta$ Pinenozonolyse. Schwefeldioxid scheint also die Stabilisierung des Sekundärozonids zu verhindern. 


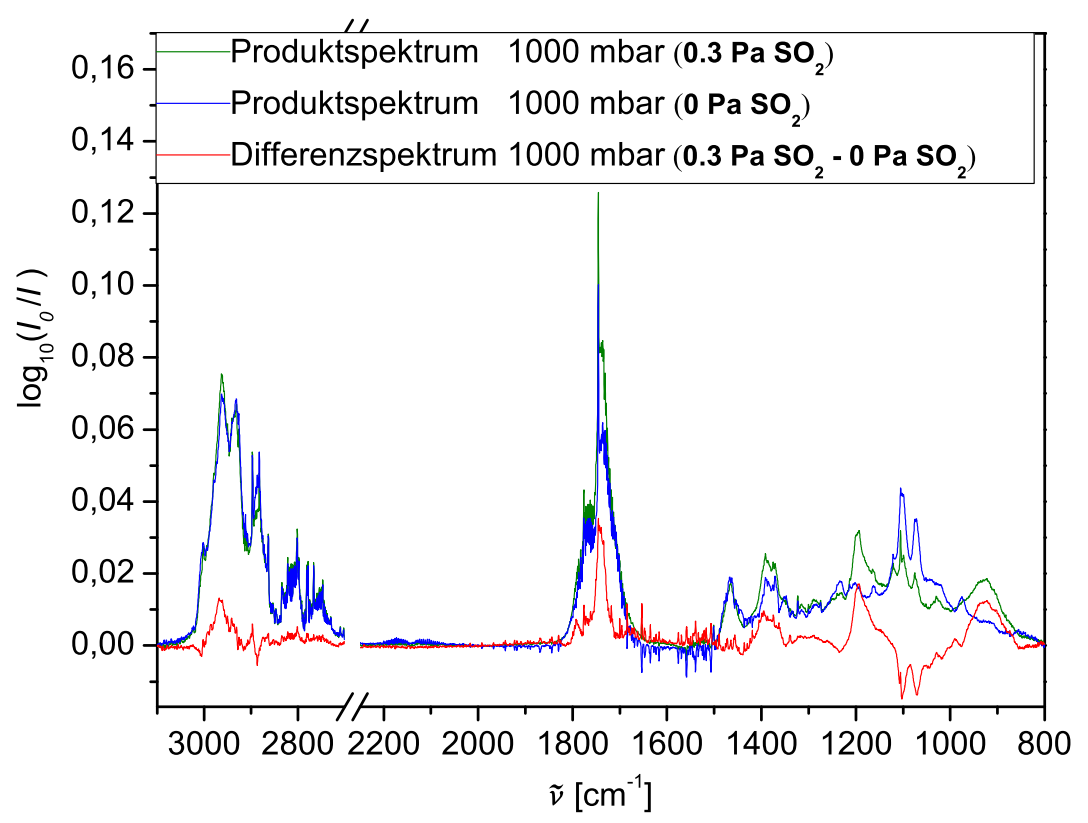

(a) Produkt- und Differenzspektrum von $\beta$-Pinenozonolysen bei Hochdruckbedingungen mit und ohne $\mathrm{SO}_{2}$-Zugabe. Das Referenzspektrum ohne $\mathrm{SO}_{2}$ stammt von WOLF [48].

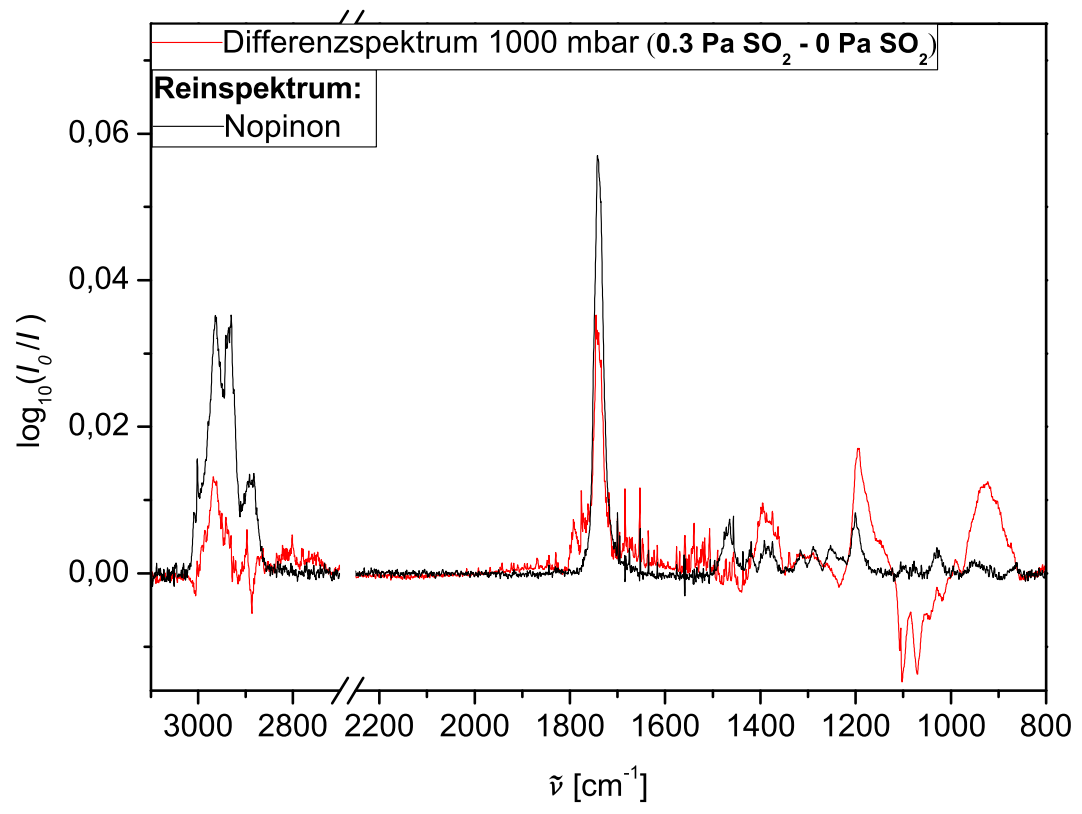

(b) Hoch- und Niederdruckdifferenzspektren von $\alpha$-Pinenozonolysen.

Abb. 6.24.: Vergleich des Hochdruckdifferenzspektrums des $\beta$-Pinens unter $\mathrm{SO}_{2}$-Zugabe mit Nopinon als möglichem Produkt. Das Reinspektrum des Nopinons stammt von WOLF [48]. 


\subsection{Produktstudien - Quantitative Auswertung}

In diesem Abschnitt erfolgen Zusammenstellungen der quantitativen Produktauswertungen für $\mathrm{OH}$-Radikale und Kohlenstoffmonoxid.

\subsubsection{OH-Ausbeute}

Die experimentelle Bestimmung der $\mathrm{OH}$-Ausbeuten erfolgt auf Grundlage der Kinetikmessungen. Wie in Kap. 2.5 dargestellt, kann die OH-Ausbeute direkt aus dem relativen Verbrauch von Alken und Ozon über GI. 2.28 ( $\left.Y_{\mathrm{OH}}=\alpha-1\right)$ bestimmt werden. Für die in Tabelle 6.9 angegebenen Werte der Ausbeute wurden ausschließlich Experimente mit Alkenüberschuss in Synthetischer Luft herangezogen, da bei diesen Bedingungen davon ausgegangen werden kann, dass die $\mathrm{OH}$-Radikale bevorzugt mit dem Alken reagieren. Eine zusätzliche Reaktion mit Ozon ist aufgrund der unterschiedlichen Reaktionsgeschwindigkeiten daher zu vernachlässigen und die fehlende Korrektur der letztgenannten Nebenreaktion sollte keinen großen Einfluss auf die erhaltenen Ergebnisse ausüben. Wo verfügbar, werden die durch Mittelwertbildung aus den in Tabelle A.6 im Anhang genannten Werten für $\alpha$ mit Literaturwerten für die Verbindung oder strukturell ähnliche Substanzen verglichen. Die angegebenen Fehler der relativen Verbräuche entsprechen Größtfehlern zum jeweiligen Mittelwert von $\alpha$.

Der Informationswert der $\mathrm{OH}$-Ausbeute liegt in der Möglichkeit, aus ihr auf den Anteil der gebildeten CRIEGEE-Intermediate rückschließen zu können. Wie bereits in der Theorie in Kap. 2.3.1 beschrieben, zerfallen syn- $\mathrm{Cl}$ über den Vinylhydroperoxidkanal und führen zur Bildung eines $\mathrm{OH}$-Radikals, während anti-Cl nach Dioxiranbildung und Umlagerung zu einer heißen Säure nur zu einem geringen Teil zur OH-Produktion beitragen können, da der Mechanismus an dieser Stelle auch auf andere Kanäle verzweigt. Nach Untersuchungen von ALAM et al. [40] resultiert aus der Ethenozonolyse, wo pro Eduktmolekül zwei $\mathrm{C}_{1}-\mathrm{Cl}$ gebildet werden und anschließend weiter zerfallen, lediglich eine $\mathrm{OH}$-Ausbeute von 0.17. Der Beitrag aus dem $\mathrm{C}_{1}$-Cl-Zerfall kann bei den untersuchten Verbindungen unter der gegebenen Bestimmungsgenauigkeit also vernachlässigt werden, da neben dem kleinen auch das große $\mathrm{Cl}$ gebildet wer- 


\begin{tabular}{|c|c|c|c|c|}
\hline Edukt & $\boldsymbol{\alpha}_{\exp }$ & $Y_{\mathrm{OH}, \exp }$ & $Y_{\mathrm{OH}, \mathrm{Lit}}$ & Referenz \\
\hline 1-Hexen & $1.4 \pm 0.1$ & 0.4 & 0.32 & RICKARD [37] \\
\hline $\begin{array}{l}\text { 2-Methyl-1- } \\
\text { penten }\end{array}$ & $1.7 \pm 0.1$ & 0.7 & 0.83 & $\begin{array}{c}\text { (Referenz: } \\
\text { 2-Methyl-1-buten) [37] }\end{array}$ \\
\hline Methylcyclopenten & $2.3 \pm 0.1$ & 1.3 & 0.9 & $\begin{array}{c}\text { (Referenz: } \\
\text { Methylcyclohexen) [37] }\end{array}$ \\
\hline 3-Buten-1-ol & $\begin{array}{l}1.4 \pm 0.1 \\
----\end{array}$ & $\begin{array}{r}0.4 \\
----\end{array}$ & $\begin{array}{c}0.56 \pm 0.15 \\
-\overline{0} \overline{4} \overline{1}--\end{array}$ & $\begin{array}{c}\text { (Referenz: 1-Buten) } \\
\text { ALAM [134] } \\
\text { (Referenz: } \overline{1-B u t e n)} \text { [37] }\end{array}$ \\
\hline 1-Penten-3-ol & $1.3 \pm 0.2$ & 0.3 & - & - \\
\hline 4-Penten-1-ol & $1.5 \pm 0.5$ & 0.5 & 0.37 & (Referenz: 1-Penten) [37] \\
\hline 1-Penten-3-on & $1.4 \pm 0.2$ & 0.4 & - & - \\
\hline 4-Pentenal & $1.2 \pm 0.2$ & 0.2 & 0.37 & (Referenz: 1-Penten) [37] \\
\hline Methacrylsäure & $2.4 \pm 0.5$ & 1.4 & $0.67 \pm 0.18$ & $\begin{array}{c}\text { (Referenz: } \\
\text { 2-Methylpropen) } \\
\text { ALAM [134] }\end{array}$ \\
\hline Butensäure & $1.6 \pm 0.1$ & 0.6 & $0.56 \pm 0.15$ & $\begin{array}{l}\text { (Referenz: 1-Buten) } \\
\text { ALAM [134] }\end{array}$ \\
\hline
\end{tabular}

Tab. 6.9.: Experimentell erhaltene $\mathrm{OH}$-Ausbeuten für die untersuchten Substanzen. 
den kann, wobei letzteres mit steigender Substitution der Doppelbindung mit höherer Wahrscheinlichkeit entsteht.

Der Vergleich der reinen $\mathrm{C}_{6}$-Kohlenwasserstoffverbindungen zeigt, dass 1 Hexen zur geringsten $\mathrm{OH}$-Ausbeute führt. Daraus kann auch auf die vergleichbar geringe Wahrscheinlichkeit der syn-Cl-Bildung geschlossen werden. Dies ist früher in diesem Kapitel schon im Zusammenhang mit Formaldehydbildung bei der 2-Methyl-1-pentenozonolyse (Abschnitt6.2.1.2) und der gebildeten Verhältnisse von großen und kleinen $\mathrm{Cl}$ dikutiert worden und würde den Beobachtungen entsprechen. Für Methylcyclopenten und 2-Methyl-1-penten dagegen entsteht mit größerer Wahrscheinlichkeit das disubstituierte $\mathrm{Cl}$ und damit resultiert für diese Verbindung folglich auch eine höhere $\mathrm{OH}$-Ausbeute. Der recht hohe Wert für den zyklischen Kohlenwasserstoff ist wahrscheinlich auf die relativ hohe interne Energie zurückzuführen. Da schon das endozyklische Eduktmolekül eine gewisse Ringspannung aufweist, die durch die Ozonanlagerung und Entstehung lediglich eines CI-Moleküls weiter erhöht und nicht teilweise durch eine Translationsmode abgebaut werden kann, ist die Stabilisierung der CRIEGEE-Intermediate nicht wahrscheinlich. So sollte ein höherer zerfallender Anteil der $\mathrm{Cl}$ zu einer höheren $\mathrm{OH}$-Ausbeute führen [44]. Da nach Untersuchungen von RICKARD et al. [37] der Hydroperoxidkanal die alleinige OH-Radikalquelle scheint, könnten bei Gültigkeit dieser Annahme anhand der $\mathrm{OH}$-Ausbeuten direkt der Anteil des gebildeten syn-Cl für die verschiedenen Verbindungen bestimmt werden. Allerdings weist der Wert von $Y_{\mathrm{OH}}>1$, der mechanistisch nicht unmittelbar zu erklären ist, entweder auf sekundäre $\mathrm{OH}$-Quellen oder eine Fehlbestimmung des Wertes hin.

Beim Vergleich der ungesättigten Alkohole zeigt sich eine mit dem relativen Abstand der beiden funktionellen Gruppen im Eduktmolekül leicht ansteigende $\mathrm{OH}$-Ausbeute, die sich allerdings innerhalb der Messgenauigkeit nicht signifikant unterscheidet. Zudem lassen die Literaturwerte der gewählten Vergleichssubstanzen eher einen gegenläufigen Effekt erwarten. Auch für die um eine Stufe höher oxidierten Carbonylverbindungen verringert sich die $\mathrm{OH}$-Ausbeute mit steigendem Abstand der Doppelbindung zum sauerstoffsubstituierten Kohlenstoffatom.

Eine ähnlich hohe Ausbeute wie der zyklische Kohnewasserstoff zeigt Methacrylsäure. Hier könnte der relative Verbrauch $\alpha$ durch zusätzlichen Säure- 
verbrauch durch die $\mathrm{Cl}$-Abfangreaktion beeinflusst sein, was sich auf die Berechnung der $\mathrm{OH}$-Ausbeute auswirkt.

\subsubsection{CO-Ausbeute}

\subsubsection{C6-Kohlenwasserstoffe}

Die CO-Ausbeuten $\left(Y_{\mathrm{CO}}\right)$ stellen unter anderem einen Marker für den Zerfall des $\mathrm{C}_{1}-\mathrm{Cl}$ dar und wurden über Zunahme des $\mathrm{CO}(\Delta(\mathrm{CO}))$ bezogen auf den Ozonverbrauch $\left(\Delta\left(\mathrm{O}_{3}\right)\right)$ während der Reaktion bestimmt.

$$
Y_{\mathrm{CO}}=\frac{\Delta\left(\mathrm{O}_{3}\right)}{\Delta(\mathrm{CO})}
$$

Kohlenstoffmonoxid kann innerhalb der Ozonolysereaktion über verschiede Reaktionskanäle gebildet werden, eine Zusammenstellung ist bei WoLF et al. zu finden [17]. Neben dem direkten Zerfall des unsubstituierten $\mathrm{C}_{1}-\mathrm{Cl}$ ( $\mathrm{vgl}$. Tabelle $2.1 \mathrm{~S}$. 25) kann es auch aus einem der möglichen Zerfallskanäle des anti- $\mathrm{Cl}$ entstehen, wo aus einer schwingungsangeregten Säure außer $\mathrm{CO}$ ein Alkoholmolekül entstehen kann (vgl. Abb. 2.4). Zudem ist auch die Bildung aus Folgereaktionen des Vinylhydroperoxidkanals möglich, da es während des Zerfalls des Radikals zu Ethen abgespalten wird (vgl. Abb. 6.10). Neben dem Zerfall des $\mathrm{C}_{1}$-Anteils zu $\mathrm{CO}$ ist allerdings zuvor die Ausbeute des $\mathrm{C}_{1}-\mathrm{Cl}$ aus dem Primärozonid zu berücksichtigen.

Die CO-Auswertungen der einzelnen Experimente für die azyklischen Kohlenwasserstoffe finden sich unter Angabe der Reaktionsbedingungen in Tabelle B.1 im Anhang. Es sind Experimente in Synthetischer Luft und Stickstoff, jeweils bei Hoch- und Niederdruckbedingungen, durchgeführt worden. In Tabelle 6.10 sind dagegen nur 1-Hexen und 2-Methyl-1-penten in Synthetischer Luft bei Atmosphärendruck gezeigt, die aufgrund verfügbarer vergleichbarer Literaturwerte an dieser Stelle eingehender betrachtet werden. Die Experimente wurden jeweils hinsichtlich der CO-Ausbeute bezogen auf den Ozonumsatz ausgewertet.

Die CO-Ausbeute ist bei 1-Hexen deutlich höher als bei 2-Methyl-1-penten. 


\begin{tabular}{|c|c|c|c|c|}
\hline Alken & $\begin{array}{c}\mathrm{C}_{1} \text {-Cl-Ausbeute } \\
\text { aus POZ-Zerfall }\end{array}$ & $\begin{array}{c}\text { Verzweigungs- } \\
\text { verhältnis } \\
\mathrm{C}_{1} \text {-Cl zu CO }\end{array}$ & $\begin{array}{c}\text { Berechnete } \\
\text { CO-Ausbeute }\end{array}$ & $\begin{array}{c}\text { Exp. bestimmte } \\
\text { CO-Ausbeute } \\
(1000 \text { mbar, SL) }\end{array}$ \\
\hline 1HEX & $0.53[37]$ & $0.44[39]$ & 0.23 & 0.26 \\
\hline 2M1P & $\begin{array}{c}0.28[37] \\
\text { (2-Methyl-1- } \\
\text { buten) }\end{array}$ & $0.44[39]$ & 0.12 & 0.11 \\
\hline
\end{tabular}

Tab. 6.10.: Abschätzung der CO-Ausbeuten der Ozonolysen von 1-Hexen und 2-Methyl-1-penten auf alleiniger Grundlage des $\mathrm{C}_{1}$-Cl-Zerfalls mit Literaturwerten von ATKINSON [39] und RICKARD [37], Vergleich mit experimentell bestimmten Ausbeuten bei $p_{\text {ges }}=1000$ mbar in Synthetischer Luft, normiert auf einen Ozonverbrauch von $1 \mathrm{~Pa}$.

Dies erklärt sich allein schon aus dem Bildungsverhältnissen der möglichen $\mathrm{Cl}$, da mit zunehmender Substitution der Doppelbindung im Verhältnis ein geringerer Anteil an unsubstituierten $\mathrm{C}_{1}-\mathrm{Cl}$ entsteht [37], wie die Werte in der Tabelle zeigen. Durch Multiplikation der beiden Verzweigungsverhältnisse lässt sich anschließend die sich aus den Literaturwerten ergebende CO-Ausbeute berechnen und mit der experimentell bestimmten vergleichen. Die erwarteten Werte stimmen mit $Y_{\mathrm{CO}}=0.23$ bzw. 0.26 für 1-Hexen und $Y_{\mathrm{CO}}=0.12$ bzw. 0.11 für 2-Methyl-1-penten (als Vergleichssubstanz wurde in Ermangelung eines Literaturwertes der für 2-Methyl-1-buten verwendet) sehr gut mit den experimentell bestimmten in Tabelle 6.10 überein. Daher stammt die ermittelte CO-Ausbeute wohl hauptsächlich aus dem Zerfall des $\mathrm{C}_{1}$-CRIEGEE-Intermediates, während die übrigen Zerfallswege zu CO nur eine geringere Bedeutung haben sollten.

Bei Betrachtung aller in Tabelle B.1 (S. XI) für die reinen Kohlenwasserstoffverbindungen aufgelisteten Werte fällt auf, dass unter allen Reaktionsbedingungen für 2-Methyl-1-penten die kleinsten und für Methylcyclopenten die größten CO-Ausbeuten resultieren. Die Werte weichen unter den verschiedenen experimentellen Bedingungen durch die Verwendung unterschiedlicher Badgase und Einstellung verschiedener Drücke nicht signifikant voneinander ab. Allerdings können die numerischen Ergebnisse auch durch die Verwendung verschiedener Kalibrierspektren beeinflusst sein. Kohlenstoffmonoxid zeigt mit seiner rotationsaufgelösten Bande zwischen 2250-2050 $\mathrm{cm}^{-1}$ im IR-Spektrum sowohl 
bei Konzentrations- als auch Druckänderungen nichtlineares Verhalten. Daher wurden zur Bestimmung der Ausbeuten jeweils Kalibrierspektren verwendet, die unter gleichen Druckbedingungen aufgenommen worden waren und ähnliche Signalstärken aufwiesen. Beim Vergleich der Werte muss daher eine Konzentrationsungenauigkeit von mindestens $0.02 \mathrm{~Pa}$ für den Ozonverbrauch und ebenso für die im Spektrum bestimmten CO-Partialdrücke mit dementsprechenden Auswirkungen auf die Genauigkeit der CO-Ausbeute ( [48], S. 77) berücksichtigt werden.

Der Zerfall von unsubstituierten $\mathrm{Cl}$ ist durch den Gesamtdruck nur eingeschränkt beeinflussbar, zusätzlich wird anhand der Literaturwerte für die Kanalaufteilung der größte Teil der CO-Ausbeute bereits erklärt. Aus diesem Grund können an dieser Stelle die $\mathrm{C}_{1}$-CRIEGEE-Intermediate für die azyklischen Alkene als Hauptquelle des gebildeten Kohlenstoffmonoxids angenommen werden, die druckunabhängig und ohne Beeinflussung vorhandenen Sauerstoffs im Badgas zerfallen. Vermutlich liegt bei 1-Hexen noch ein geringer Beitrag aus dem Zerfall des anti-Cl vor, der die Differenz zwischen Mess- und Literaturwert erklären könnte, allerdings weisen die Ergebnisse insgesamt auf einen geringen Zerfalls- und damit hohen Stabilisierungsgrad dieser Intermediate hin.

Für Methylcyclopenten dagegen lässt sich in Synthetischer Luft vor allem unter Niederdruckbedingungen ein deutlicher Anstieg der CO-Ausbeute im Vergleich zu Stickstoff als Badgas feststellen $\left(Y_{\mathrm{CO}_{\mathrm{N}} \mathrm{N}_{2}}=0.32\right.$ vs. $\left.Y_{\mathrm{CO}, \mathrm{SL}}=0.42\right)$. Methylcyclopenten kann aus der Ozonolyse keine unsubstituierten CRIEGEEIntermediate bilden. Es ist zu vermuten, dass die Bildung von Kohlenstoffmonoxid durch den Zerfall entstehender Intermediate im Vinylhydroperoxidkanal, wie WOLF et al. [17] inn für Methylcyclohexen vorgeschlagen haben, zustande kommt und der bereits für die Erklärung der Produktion von Ethen und Keten herangezogen wurde (vgl. Abb. 6.10).

\subsubsection{Voroxidierte Verbindungen}

Im Anhang findet sich in Tabelle B.2 (Seite XII) eine Zusammenstellung der CO-Ausbeuten in Experimenten mit Alkoholen, Carbonylverbindungen und Carbonsäuren. Bei den voroxidierten Verbindungen lässt sich im Gegensatz zu den azyklischen Kohlenwasserstoffverbindungen eine Druckabhängigkeit in der 
CO-Bildung feststellen. Generell zeigen sich unter Niederdruckbedingungen höhere CO-Ausbeuten als in Hochdruckexperimenten im gleichen Badgas. Weiterhin ist zu erkennen, dass die untersuchten Alkohole und Carbonylverbindungen in Synthetischer Luft höhere Ausbeuten zeigen als in Stickstoff, während die Carbonsäuren die gleiche Druckabhängigkeit, aber einen gegenteiligen Effekt bezüglich des Badgases zeigen.

Als Vorgriff zu den später dargestellten Ergebnissen der Partikelbildungsmessungen sei an dieser Stelle bereits angemerkt, dass die CO-Ausbeuten für die sauerstoffhaltigen Reaktanden ein ähnliches Verhalten bei Veränderungen des Druckes und des verwendeten Badgases wie die Partikelausbeuten zeigen.

\subsection{Partikelbildungsstudien}

In Tabelle A.4 in Anhang A.2.3 sind detaillierte Angaben zu Anfangskonzentrationen, Additiva und verwendeten Badgasen zu finden. Generell wurden die Anfangskonzentrationen der Alkene und des Ozons sowie die Reaktionszeiten in den jeweiligen Messreihen konstant gehalten, während Parameter wie der Gesamtdruck, die Beschaffenheit des Badgases oder die Zugabe von bestimmten Additiva variiert wurden. $\mathrm{Da}$ in den $\mathrm{SO}_{2}$-Zugabeexperimenten auch die Konzentration dieser Additiva einzeln variiert wurde, werden diese noch einmal unter Angabe eines Mittelwertes für die Anfangskonzentrationen des Alkens und Ozons wiederholt bzw. ausführlicher genannt.

Die vorgestellten Ergebnisse (ohne $\mathrm{SO}_{2}$-Beteiligung) wurden in der Regel nach einer Wartezeit von $t_{\mathrm{R}}=600 \mathrm{~s}$ zwischen Reaktionsbeginn und Aufnahmebeginn der SMPS-Messung erhalten. Ausnahmen bilden dabei die Substanzen Methylcyclopenten, 4-Penten-1-ol und 1-Penten-3-on, wo aufgrund höherer Reaktionsgeschwindigkeitskoeffizienten bereits nach $t_{R}=300 \mathrm{~s}$ mit der Aufnahme der Partikelmessungen begonnen werden konnte, da die Reaktion zu diesem Zeitpunkt vollständig abgelaufen war. Bei allen Experimenten herrschte in der Reaktionszelle eine Temperatur von $T=295 \pm 2 \mathrm{~K}$.

Vor den eigentlichen Partikelbildungsexperimenten wurden für alle Substanzen Messreihen mit variablen Ozonanfangskonzentrationen bei konstanter Al- 
kenkonzentration, die der in den durchzuführenden Messreihen entsprach, und gleich bleibendem Gesamtdruck durchgeführt. Auf diese Weise konnte sichergestellt werden, dass die Ozonanfangskonzentration jenseits der Nukleationsschwelle gewählt war. Dort sollten die Massenausbeuten linear mit der umgesetzten Alkenmenge zunehmen [21]. Beispielhaft ist dies in Abbildung 6.25 für die reinen $\mathrm{C}_{6}$-Kohlenwasserstoffe mit den sich ergebenden Masseausbeuten und gebildeten Anzahlen (jeweils Mittelwerte aus den drei partikelreichsten Verteilungen) gezeigt.

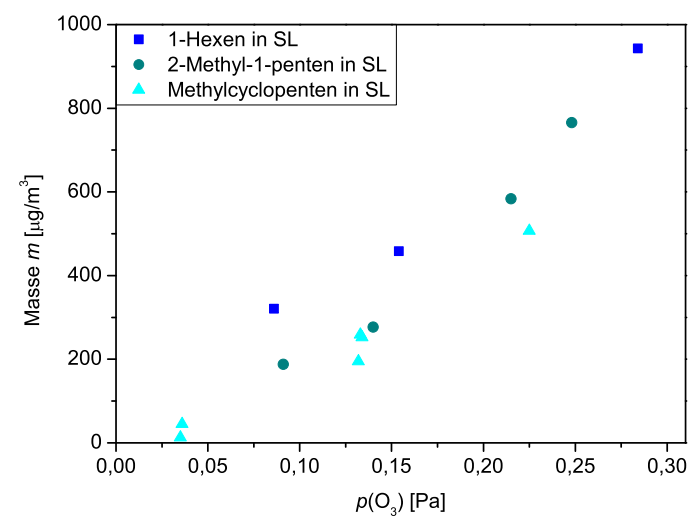

(a) Ozonpartialdruckreihe, Masse.

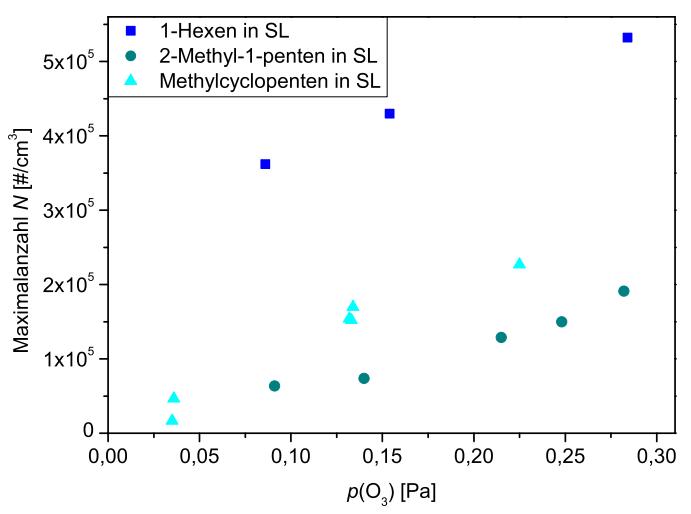

(b) Ozonpartialdruckreihe, Anzahl.

Abb. 6.25.: Ozonpartialdruckreihe zur Ermittlung der zu verwendenden Ozonanfangskonzentrationen bei $p_{\text {ges }}=460 \mathrm{mbar}$.

Wie an den Messpunkten sichtbar, verlaufen die Partikelausbeuten nicht durch den Ursprung, was ein Hinweis auf die zur Nukleation nötige Ozongrenzkonzentration ist. Für eine Vergleichbarkeit der Ergebnisse sollte ein einheitlicher Ozonpartialdruck verwendet werden. Daher wurde für die reinen Kohlenwasserstoffe mit einem Anfangspartialdruck von $p_{\mathrm{O}_{3}}=0.14 \mathrm{~Pa}$ ein Wert aus dem Bereich gewählt, in dem sich für alle Substanzen eine annähernd lineare Zunahme der Massenausbeute zeigte. Die Betrachtung der gebildeten Anzahlen diente an dieser Stelle nur als Zusatzinformation zum Ausschluss von Messfehlern. Für alle übrigen Substanzen wurde analog vorgegangen.

Die im weiteren Verlauf dieses Kapitels in den Abbildungen gezeigten Fehlerbalken ergeben sich aus den Abweichungen eines häufig gemessenen Punktes der jeweiligen Messreihe bei identischen Reaktionsbedingungen (meist Anzahlkonzentration und Massenausbeute bei $p_{\text {ges }}=460$ mbar). Für diesen Mess- 
wert wurde der prozentuale Fehler statistisch für ein Konfidenzintervall von $95.5 \%$ bestimmt und anschließend für die übrigen Ergebnisse übernommen. Der Student'sche Faktor wurde wie zuvor dem Fehlerrechnungsskript entnommen [118].

Bei Punkten oder Messreihen, für die keine oder nur wenige Wiederholungsmessungen durchgeführt wurden, wurden von der Mittelwertbildung und der Angabe von Fehlerintervallen abgesehen, um die Streuung nicht zu unterschätzen.

\subsection{1. $\mathrm{C}_{6}$-Kohlenwasserstoffe}

Zunächst werden in diesem Abschnitt die Ergebnisse zu Untersuchungen der druckabhängigen Partikelbildung gezeigt, bevor ein Vergleich des Badgaseinflusses auf die Ausbeuten bei den einzelnen Edukten erfolgt.

\subsubsection{Einfluss des Gesamtdruckes und des Badgases}

Wie in Abbildung 6.26 erkennbar, nehmen die Ausbeuten unabhängig vom verwendeten Badgas sowohl bezüglich Anzahl als auch Masse mit dem Gesamtdruck ab. Der Prozessdruck, unterhalb dessen eine vollständige Unterdrückung der Partikelbildung zu beobachten ist, ist abhängig vom verwendeten Alken, wie die Auftragungen 6.26a und 6.26b zeigen.

Während für 2-Methyl-1-penten und Methylcyclopenten schon bei einem Gesamtdruck von $p_{\text {ges }} \approx 30$ mbar keine definierte Verteilung mehr zu erkennen ist, sind die Kennzahlen für 1-Hexen $\left(N=1 \cdot 10^{5} \mathrm{~cm}^{-3}, m=100 \mu \mathrm{g} \cdot \mathrm{m}^{-3}\right)$ noch deutlich im messbaren Bereich, sogar bei 10 mbar werden für diese Substanz noch deutlich höhere Werte erreicht. Meist zeigen die Partikelausbeuten keine lineare Abhängigkeit vom Prozessdruck, sondern verlaufen oberhalb von 500 mbar etwas flacher als unterhalb, was auf eine Art „Sättigungsverhalten“ hinweist. Der Verlauf könnte etwa durch das Erreichen der höchstmöglichen Ausbeute an stabilisierbaren CRIEGEE-Intermediaten bei diesem Druck erklärt werden, weshalb sich bei weiterer Druckerhöhung keine starken Zunahmen mehr zeigen. Der in Stickstoff als Badgas auftretende lineare Verlauf für 1-Hexen und 2-Methyl-1-penten (Abb. 6.26C und 6.26d) kann aufgrund fehlender Wiederho- 


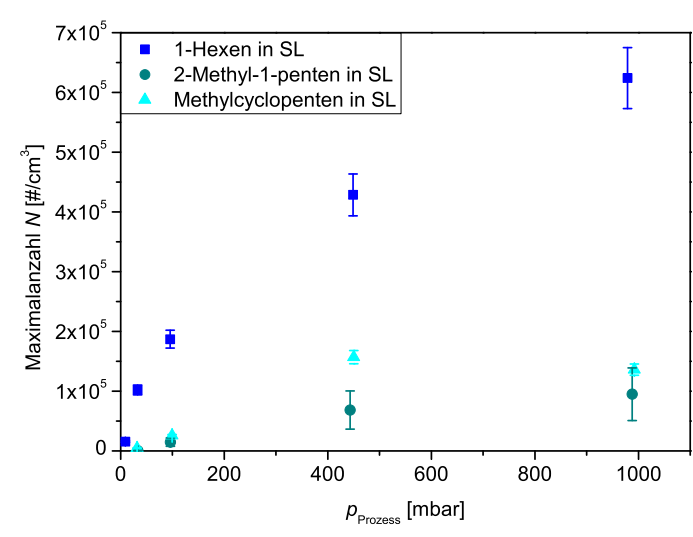

(a) Anzahlkonzentration.

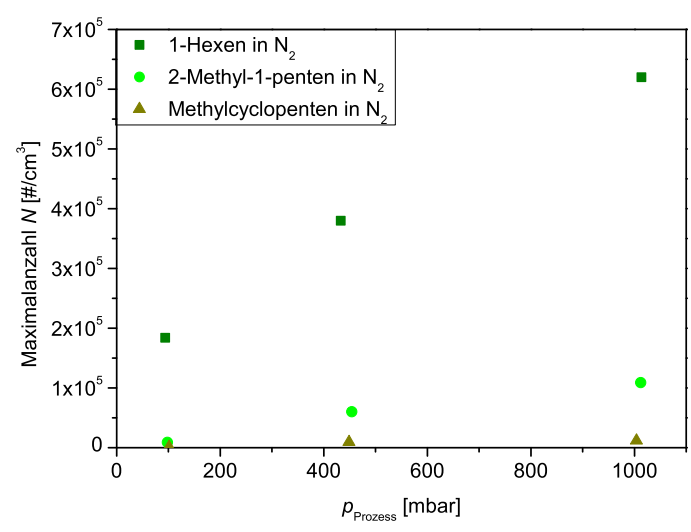

(c) Anzahlkonzentration.

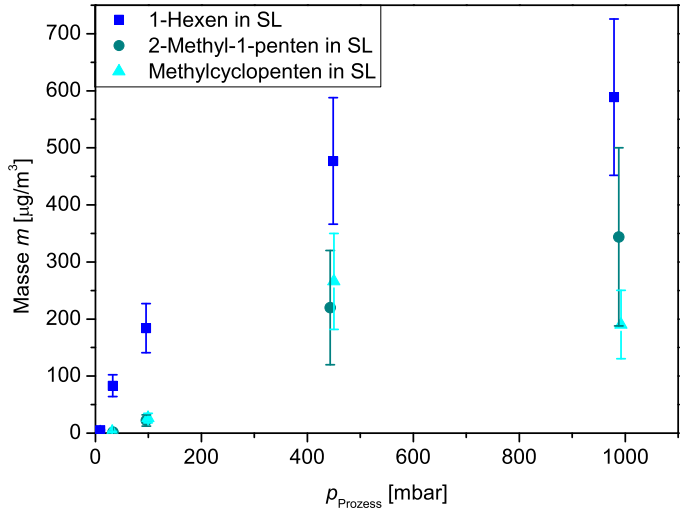

(b) Massenausbeute.

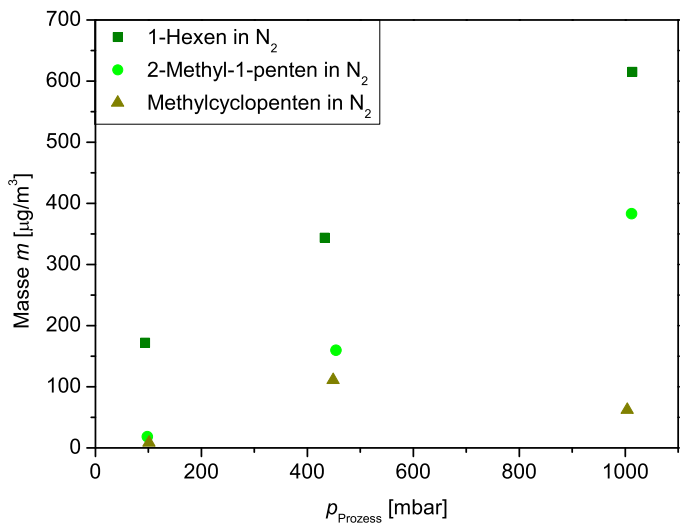

(d) Massenausbeute.

Abb. 6.26.: Untersuchung der druckabhängigen Partikelausbeute für 1-Hexen, 2-Methyl-1-penten und Methylcyclopenten in Synthetischer Luft bzw. Stickstoff.

lungsmessungen nicht als gesichert angesehen werden, könnte aber auf eine veränderte Chemie der Intermediate hinweisen. Auffällig ist, dass trotz der Ähnlichkeit in den Molekulargewichten der Ausgangsmoleküle und identischer Ozonumsätze 1-Hexen ein deutlich besserer Partikelbildner ist als 2-Methyl-1penten und Methylcyclopenten.

Für Methylcyclopenten scheinen Anzahlkonzentration und Masse oberhalb von 500 mbar unabhängig vom verwendeten Badgas wieder abzunehmen, allerdings überlappen sich die Fehlergrenzen, so dass der Effekt nicht aussagekräftig ist. Zudem unterliegen die Messwerte bei Drücken oberhalb von $p_{\text {ges }}=460$ mbar in der Regel stärkeren Schwankungen durch die etwas veränderte Einfüllprozedur durch zusätzliche Zugabe aus der Badgasflasche und 
daraus folgend der Durchmischungsverhältnisse der Reaktanden, die die Reproduzierbarkeit identischer Verhältnisse erschweren. Hieraus kann eine Veränderung in der Durchmischung der Reaktanden folgen, zudem benötigt dieses manuelle Auffüllen mit etwa $20 \mathrm{~s}$ bis auf den Enddruck deutlich länger als die reine Expansion (4s). Vor allem für das sehr schnell mit Ozon reagierende Methylcyclopenten kann dieser Unterschied in der Befüllung und damit verbundene Druckschwankungen große Auswirkungen auf die detektierten Ausbeuten haben.

Die Verringerung der Partikelausbeuten mit sinkendem Gesamtdruck lässt sich mit der unzureichenden Stoßstabilisierung bestimmter, zur Nukleation notwendiger Intermediate, wie CRIEGEE-Intermediate oder Alkoxy- und Peroxyverbindungen, erklären. Sie zerfallen unterhalb eines bestimmten Gesamtdruckes und können in der Folge nicht mehr zum Aufbau kondensierter Materie beitragen. Diese Ergebnisse stimmen mit den Beobachtungen von WOLF [48] überein. Bei den durch den Gesamtdruck beeinflussten Verbindungen kann es sich einerseits um Substanzen mit potenziell extrem niedrigem Dampfdruck handeln, die als Nukleationskeime (NIM) fungieren können und deren unzureichende Stabilisierung folglich vor allem durch eine reduzierte Anzahlkonzentration sichtbar wird. Falls andererseits eher mittelflüchtige Verbindungen vom Zerfall betroffen sind, reduziert sich die detektierte Gesamtmasse stärker.

Ausgehend von der Hypothese, dass vor allem oxidierte Zwischenprodukte wie $\mathrm{Cl}$ oder sauerstoffhaltige Radikale für die Nukleation verantwortlich sind, könnten erstere die im Substanzvergleich stark erhöhte Partikelausbeute des 1Hexens erklären. Bei der Ozonolyse dieses Alkens entsteht mit einem Verhältnis von $50: 50$ für $\mathrm{C}_{1}$ - und $\mathrm{C}_{5}-\mathrm{Cl}$ zwar verglichen mit methylsubstituierten Ausgangsverbindungen ein relativ geringer Anteil großer $\mathrm{Cl}$ (verglichen mit $28: 72$ für die Vergleichssubstanz 2-Methyl-1-buten) [37], allerdings scheinen diese Intermediate besonders gut stabilisierbar zu sein. DrozD et al. [44] stellten eine Studie vor, nach der die aus der trans-5-Decenozonolyse entstehenden $\mathrm{C}_{5}$-CRIEGEE-Intermediate recht langlebig sind, was auch mit ihrem Ursprung aus einem offenkettigen Alken, dessen Primärozonid im Unterschied zu denen endozyklischer Alkene wie Methylcyclopenten zu zwei Molekülen zerfällt, erklärt werden kann. Ein Teil der im Molekül enthaltenen Überschussenergie wird dabei in Translationsenergie umgewandelt, was zur Stabilisierung der bei- 
den Fragmente beiträgt. Diese Tatsache würde die erhöhte Ausbeute aus der 1-Hexenozonolyse unter Niederdruckbedingungen (10 bzw. 30 mbar) erklären. Auch der recht hohe Stabilisierungsgrad der $\mathrm{C}_{1}-\mathrm{Cl}$ von etwa $0.53 \mathrm{im}$ Vergleich zu etwa 0.28 für das Strukturanalogon zu 2-Methyl-1-penten nach RICKARD et al. (vgl. Tab 6.10, [37]) könnte für das hohe Partikelbildungspotential des linearen Alkens verantwortlich sein.

Zudem kann die Konformation der $\mathrm{Cl}$ von Bedeutung sein, denn es wird davon ausgegangen, dass das anti-Cl gegenüber syn- und unsubstituierten $\mathrm{Cl} z u$ einem größeren Prozentsatz stabilisierbar ist [48]. Während das $\mathrm{C}_{5}-\mathrm{Cl}$ des 1Hexens zumindest teilweise in anti-Konfiguration vorliegen sollte, ist die Bildung dieser Ausrichtung für Zwischenprodukte aus der 2-Methyl-1-pentenozonolyse nicht möglich, da hier neben dem unsubstituierten mit einer Ausbeute von etwa $72 \%$ [37] lediglich ein disubstituiertes $\mathrm{Cl}$ entsteht. Aus diesem Grund sollte ein Großteil der gebildeten $\mathrm{Cl}$ über den Hydroperoxidkanal zerfallen, da diese Reaktion sehr schnell abläuft.

Somit ist eine Weiterreaktion eines stabilisierten $\mathrm{C}_{1}$ - oder $\mathrm{C}_{5}$-CRIEGEEIntermediates für das 1-Hexen insgesamt wahrscheinlicher als für 2-Methyl-1penten und kann damit zumindest für einen Teil der erhöhten Ausbeute gegenüber den anderen Verbindungen verantwortlich sein.

Bei ausreichender Stabilisierbarkeit ist nach Untersuchungen von SADEZKY et al. [36, 53] die Bildung von längerkettigen Oligomeren aus unsubstituierten $\mathrm{C}_{1}$ - und längerkettigen $\mathrm{C}_{5}-\mathrm{Cl}$ grundsätzlich möglich. Da für 1-Hexen mit einem Anteil von etwa $50 \%$ der größte Anteil an $\mathrm{C}_{1}-\mathrm{Cl}$ und potenziell anti- $\mathrm{C}_{5^{-}}$ $\mathrm{Cl}$ entsteht, ist denkbar, dass Oligomerisierungsreaktionen zu einer erhöhten Partikelausbeute führen können. Diese Oligomere können aufgrund ihres hohen $\mathrm{O}$ : C-Verhältnisses und daraus folgender geringer Flüchtigkeit als Vorläufer von Partikelkeimen dienen. Bei Betrachtung von Abbildung 6.26 zeigt sich, dass sich die Anzahlkonzentrationen zwischen 1-Hexen und 2-Methyl-1-penten bzw. Methylcyclopenten deutlich stärker unterscheiden als die gebildeten Massen. Es ist daher davon auszugehen, dass die Entstehung von Nukleationskeimen bei 1-Hexen mit höherer Wahrscheinlichkeit möglich ist als für die anderen beiden Substanzen. Dieser Befund ließe sich mit der Rolle der potenziell gebildeten Oligomere erklären. Gestützt wird diese These durch die geringeren Anzahlkonzentrationen aus der 2-Methyl-1-penten- und Methylcyclopentenozo- 
nolyse. Da im ersten Fall nur zu etwa $28 \%$ das $\mathrm{C}_{1}-\mathrm{Cl}$ entsteht und die außerdem gebildeten $\mathrm{C}_{5}-\mathrm{Cl}$ weniger gut stabilisierbar sind als bei 1 -Hexen, ist eine Oligomer- / NIM-bildende Reaktion zwar auch für 2-Methyl-1-penten grundsätzlich möglich. Allerdings läuft sie in wesentlich geringerem Maße ab, was zu der beobachteten reduzierten Ausbeute führt. Die weitere Verringerung bei Methylcyclopenten ist wohl zumindest teilweise mit der Nichtbildung unsubstituierter $\mathrm{Cl}$ und den damit wohl reduziert auftretenden Oligomerisierungsreaktionen zu erklären. Aufgrund der potenziell vier möglichen $\mathrm{Cl}$ (vgl. Abb. 2.10 im Theorieteil) können höchstens mit einer $25 \%$-igen Wahrscheinlichkeit anti-Cl entstehen, die im Gegensatz zu den anderen drei Strukturen nicht bevorzugt über den Hydroperoxidkanal zerfallen.

Das verglichen mit den beiden anderen Alkenen recht geringe Partikelbildungspotential des Methylcyclopentens lässt sich neben den Überlegungen zu entstehenden Zwischenprodukten auch aus der Eduktstruktur erklären. Da der Fünfring von vornherein eine erhöhte Ringspannung besitzt (vgl. Abb. 6.34, S. 195), kann davon ausgegangen werden, dass auch die aus der Ozonolysereaktion entstehenden Intermediate eine erhöhte chemische Aktivierung aufweisen, was eine nur geringe Stabilisierbarkeit der Verbindungen bewirkt. Zusätzlich wirkt sich auch die im Vergleich zum Zerfall des Primärozonids in zwei CRIEGEE-Intermediate fehlende Translationsmode zur Energieabfuhr negativ aus.

Die zuvor im Substanzvergleich in identischen Badgasumgebungen gezeigten Messwerte werden in Abb. 6.27 noch einmal nach Edukten getrennt dargestellt, um leichter die Auswirkungen der Verwendung unterschiedlicher Badgase verdeutlichen zu können. Wie die einzelnen Auftragungen der Ausbeuten gegen den Gesamtdruck zeigen, weisen die erhaltenen Messwerte der azyklischen Alkene keine signifikante Abhängigkeit vom verwendeten Badgas, bzw. der An- oder Abwesenheit von Sauerstoff in der reaktiven Mischung auf. Lediglich das zyklische Methylcyclopenten zeigt eine stark reduzierte Ausbeute bei der Verwendung von Stickstoff als Badgas.

Diesem Befund könnten zwei verschiedene Effekte zugrunde liegen. Eine Möglichkeit ist, dass eine Alkoxy- / Peroxyradikalchemie für die Partikelbildung von 1-Hexen und 2-Methyl-1-penten im Gegensatz zum zyklischen Alken keinen entscheidenden Einfluss ausübt, da sich die detektierten Mengen in Sauerstoffan- und -abwesenheit nicht signifikant in Anzahl und Masse un- 


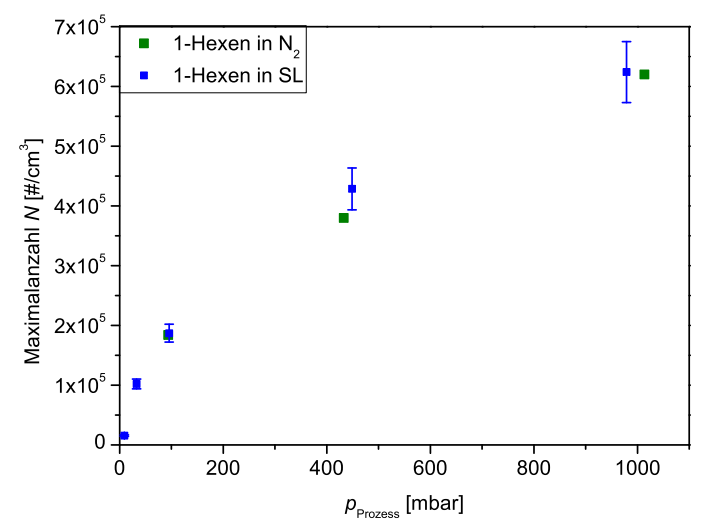

(a) Anzahlkonzentration.

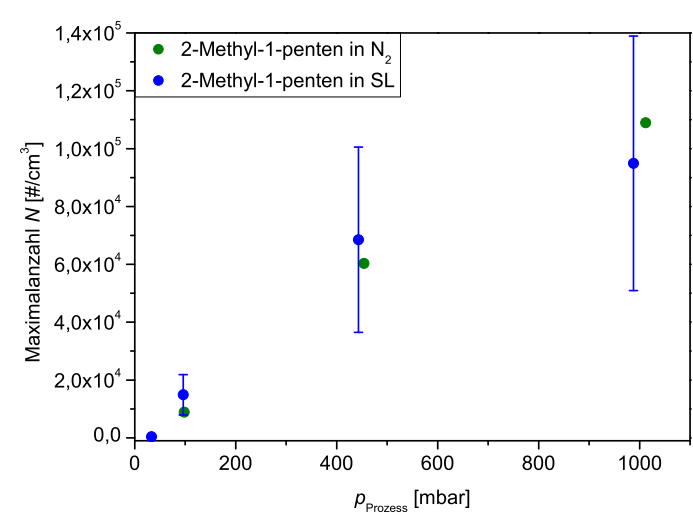

(c) Anzahlkonzentration.

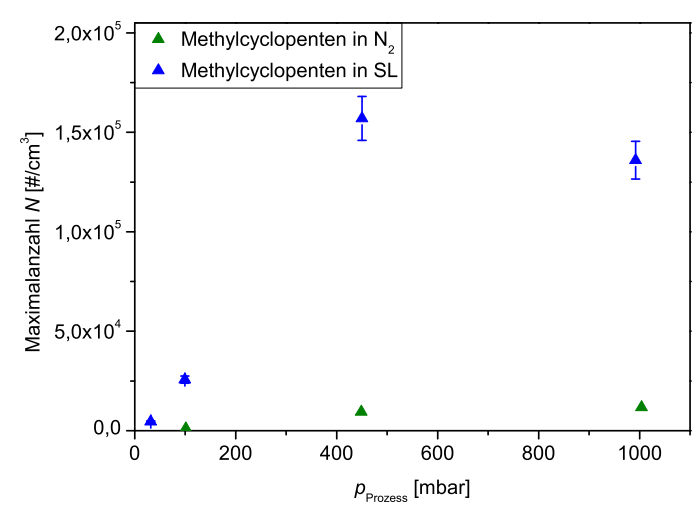

(e) Anzahlkonzentration.

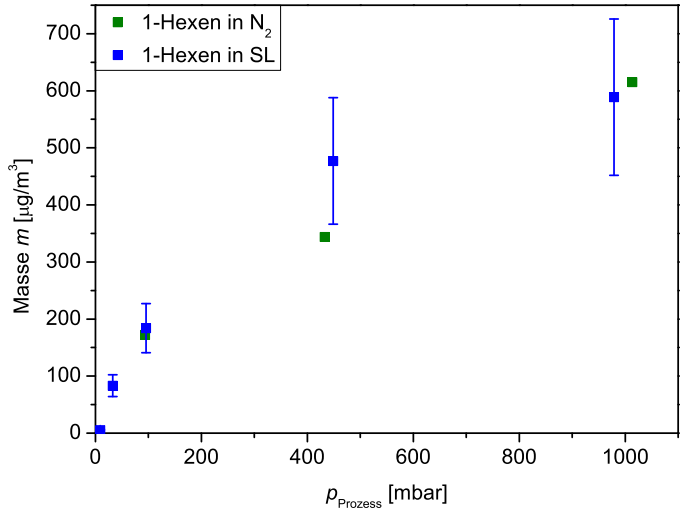

(b) Massenausbeute.

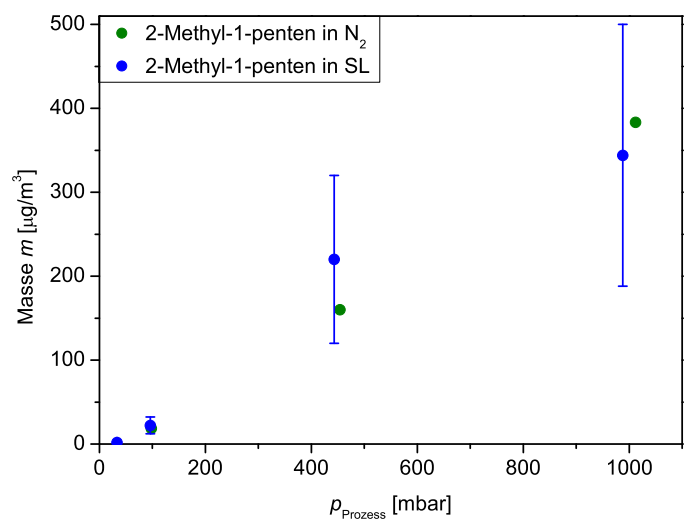

(d) Massenausbeute.

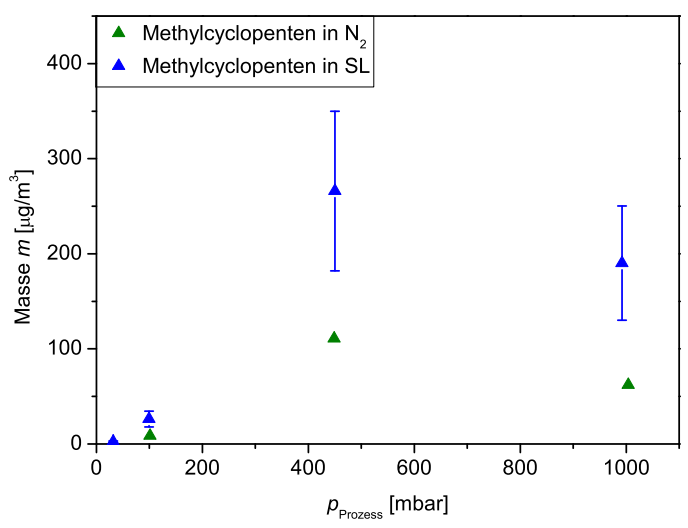

(f) Massenausbeute.

Abb. 6.27.: Untersuchung der badgasabhängigen Partikelausbeute für 1Hexen, 2-Methyl-1-penten und Methylcyclopenten, Vergleich der Ausbeuten in Synthetischer Luft und Stickstoff bei variiertem Druck. 
terscheiden. Im Gegensatz dazu sind die Ausbeuten für Methylcyclopenten in Stickstoff stark reduziert, was auf eine wichtige Rolle von $\mathrm{O}_{2}$ in einem effizienten Reaktionskanal für den Aufbau von Partikelkeimen und -masse, also schwer- und mittelflüchtigen Reaktionsprodukten, für dieses Alken hinweist [20,48].

Andererseits könnte auch hier die Stabilität der Intermediate wieder ein entscheidender Faktor sein. Auch bei der Verwendung von Stickstoff als Badgas kann nicht von einer völlig sauerstoffreien Reaktionsumgebung ausgegangen werden, aus Undichtigkeiten sowie der $\mathrm{He} / \mathrm{O}_{3}$-Mischung ist mit einem Anteil von ca. $p_{\mathrm{O}_{2}}=0.5$ Pa Sauerstoff im Gemisch zu rechnen. Wenn im Falle der azyklischen Verbindungen die entstehenden Intermediate schnell über den Vinylhydroperoxidkanal zerfallen oder auf andere Weise isomerisieren, können sich stabile Alkylradikale bilden. Diese können aufgrund der generell sehr schnell ablaufenden Reaktion mit Sauerstoff zu Peroxyradikalen, z.B.

$$
\mathrm{C}_{2} \mathrm{H}_{5}+\mathrm{O}_{2} \longrightarrow \mathrm{C}_{2} \mathrm{H}_{5} \mathrm{O}_{2}, \mathrm{~K}=8 \cdot 10^{-12} \frac{\mathrm{cm}^{3}}{\text { molecule } \mathrm{s}}
$$

auch mit dem geringen Sauerstoffpartialdruck unter Stickstoffatmosphäre zu den gleichen Produkten führen wie in Synthetischer Luft. Den Zwischenprodukten des Methylcyclopenten könnte dieser Reaktionsweg wegen ihrer hohen chemischen Aktivierung und damit höheren Neigung zum Zerfall verschlossen bleiben. Ein geringerer Anteil an Alkylradikalen bräuchte demnach eine höhere Sauerstoffkonzentration in der reaktiven Mischung, um mit einer ähnlichen Reaktionsgeschwindigkeit zu vergleichbaren Ausbeuten zu führen. Dies würde die deutlich ausgeprägtere Partikelbildung in Sauerstoffanwesenheit erklären.

\subsubsection{Einfluss von Radikalfängern}

Die Auftragungen der Ergebnisse gegen den Reaktionsdruck in Abbildung 6.28 veranschaulichen, dass die Zugabe von Radikalfängern abhängig vom verwendeten Alken sehr unterschiedliche Auswirkungen auf die gebildeten Ausbeuten haben kann.

Wie die Abbildungen 6.28a und 6.28b zeigen, hat die Zugabe von Cyclohexan in verschiedenen Mengen ( $p_{\mathrm{CHA}}=0.78$ mbar bzw. $p_{\mathrm{CHA}}=3.1 \mathrm{mbar}$ ) in der 1-Hexenozonolyse keinen signifikanten Einfluss auf die Partikelausbeuten 
bezüglich Anzahl und Masse. Dagegen bewirkt die Anwesenheit des Hydroxylgruppenträgers 1-Butanol bereits bei Addition von $p_{1 \mathrm{BUOH}}=0.78 \mathrm{mbar}$ zum Reaktionsgemisch eine komplette Unterdrückung der Nukleation. Diese Beobachtungen weisen darauf hin, dass $\mathrm{OH}$-Radikale für 1-Hexen keine entscheidende Rolle zu spielen scheinen, wobei einschränkend zu sagen ist, dass diese sogar durch die Zugabe sehr hoher Cyclohexanmengen nicht vollständig abzufangen sind, sondern ein kleiner Prozentsatz immer in der Reaktionsmischung verbleibt. Da Radikalreaktionen schnell ablaufen, könnte beispielsweise die im vorangegangenen Abschnitt beschriebene Oligomerisierungsreaktion im Sinne von SADEZKY et al. [36, 53], deren Kettenstart mit OH-Radikalen erfolgt (vgl.

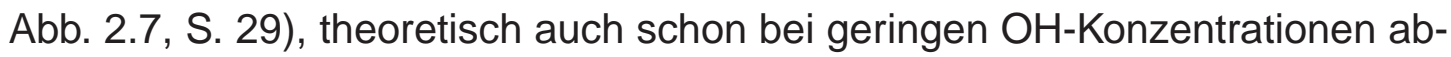
laufen. Zudem wäre auch ein Kettenstart durch andere in der Reaktion primär oder sekundär entstehende Radikale möglich, wie sie beispielsweise durch den Zerfall der syn-Cl über den Hydroperoxidkanal entstehen können.

Der Vergleich der Ergebnisse unter Cyclohexan- und 1-Butanolzugabe verdeutlicht, dass die beiden Fänger unterschiedlich in den Reaktionsmechanismus eingreifen. Allein durch die Abfangreaktion der $\mathrm{OH}$-Radikale ist die $\mathrm{Re}$ duktion der Ausbeuten kaum zu erklären, daher spielt wahrscheinlich das Vorhandensein einer Hydroxylgruppe im Fängermolekül eine Rolle. Wie unter anderem von NEEB et al. [59] und BONN et al. [18] berichtet, sind Moleküle mit $\mathrm{OH}$-Gruppe in der Lage, mit (stabilisierten) CRIEGEE-Intermediaten zu reagieren und so abzufangen. Soll diese Erklärung für den Effekt verantwortlich sein, müssten die entstehenden Reaktionsprodukte nicht partikelbildungsfähig sein, weil sie z.B. eine zu hohe Flüchtigkeit besitzen oder eher zerfallen als die Verbindungen, die durch bimolekulare Reaktionen der stabilisierten $\mathrm{Cl}$ mit anderen Molekülen entstehen.

Zusätzlich ist zu beachten, dass in der Reaktion 1-Butanol + $\mathrm{OH}$ potenziell $\mathrm{HO}_{2}$-Radikale entstehen, wie von KEYWOOD [55] für das strukturell ähnliche 2-Butanol berichtet wurde (vgl. Abb. 2.20 in Kap. 2.4.2 der Theorie). Diese wiederum induzieren nach den Ergebnissen von SADEZKY den Kettenabbruch in Oligomerisierungsreaktionen [36], so dass durch die vermehrt auftretende $\mathrm{HO}_{2}$-induzierte Abbruchreaktion die Bildung längerkettiger Oligomere unwahrscheinlicher und die Partikelausbeute verringert würde.

Bei der Methylcyclopentenozonolyse (Abb. 6.28c und 6.28d) bewirkt die Zu- 


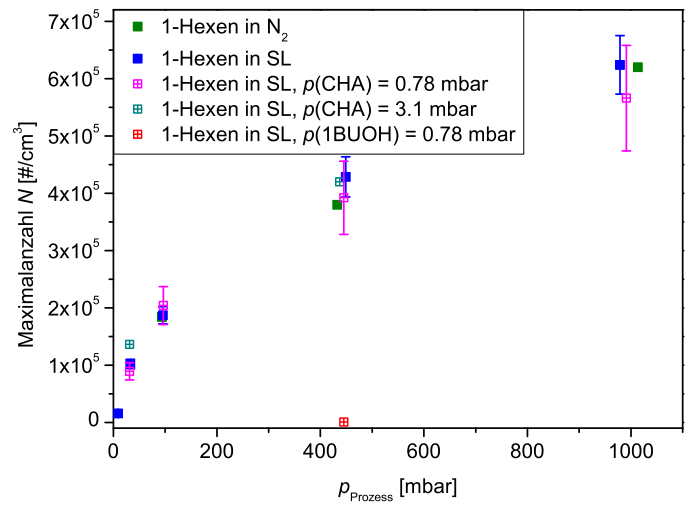

(a) Anzahlkonzentration.

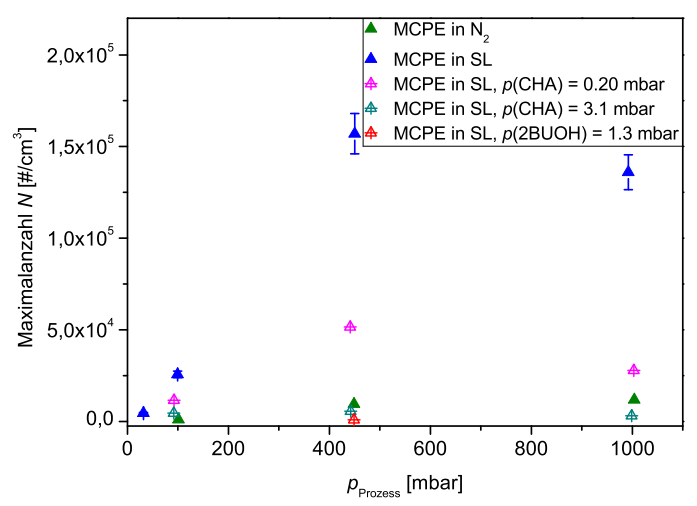

(c) Anzahlkonzentration.

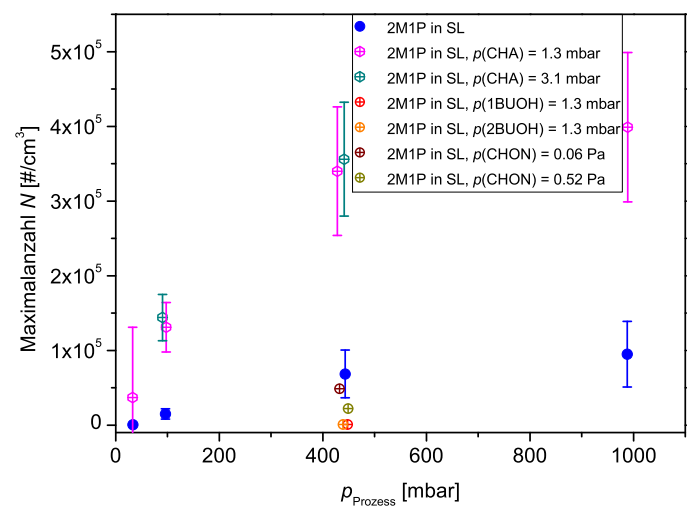

(e) Anzahlkonzentration.

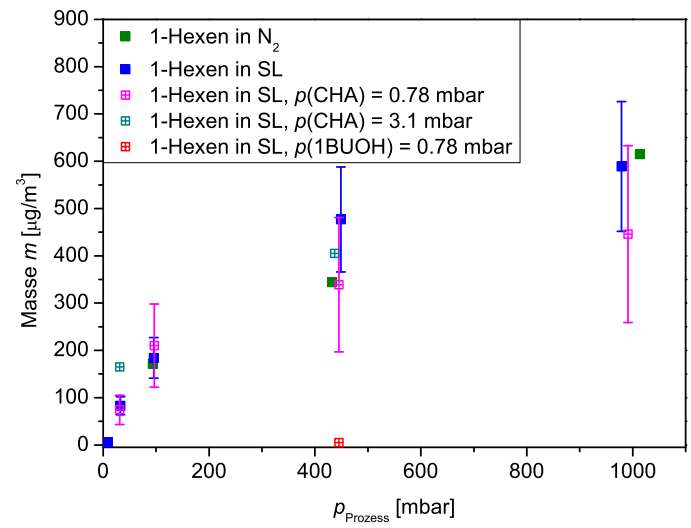

(b) Massenausbeute.

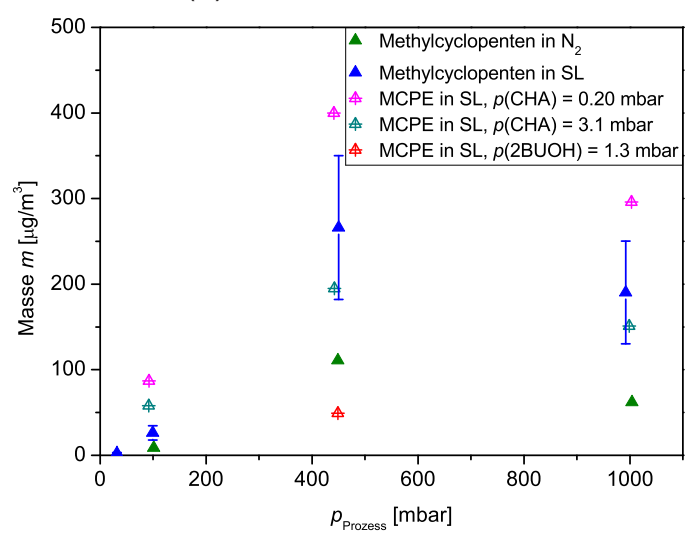

(d) Massenausbeute.

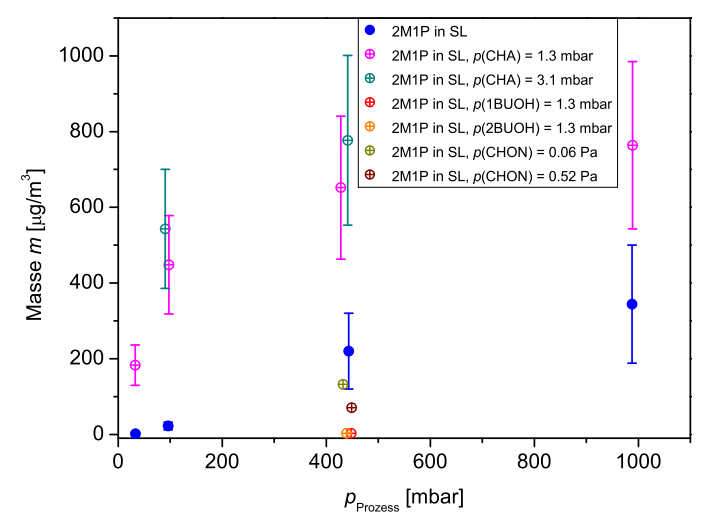

(f) Massenausbeute.

Abb. 6.28.: Untersuchung des Einflusses von Radikalfängern auf die Partikelausbeute für 1-Hexen, Methylcyclopenten und 2-Methyl-1-penten; Vergleich der Effekte durch Cyclohexan (CHA), 1-Butanol (1BUOH) und 2-Butanol (2BUOH). Für 2-Methyl-1-penten wurden zusätzliche Experimente unter Cyclohexanonzugabe (CHON, Oxidationsprodukt des Cyclohexans) durchgeführt. 
gabe von $p_{\mathrm{CHA}}=0.20$ mbar Cyclohexan einen Abfall der Anzahlkonzentration verglichen zur Ausbeute in Synthetischer Luft, während die gebildete Partikelmasse eine leichte Tendenz nach oben zeigt. Die Erhöhung auf $p_{\mathrm{CHA}}=3.1 \mathrm{mbar}$ führt zu einer Verringerung beider Kenngrößen, wobei die Anzahl nun der in Stickstoff gebildeten entspricht, während die Masse weiterhin im Bereich der Werte ohne Cyclohexanzugabe liegt. Wie bei 1-Hexen ist auch hier wiederum eine deutliche Reduktion der Ausbeuten bei Zugabe eines Hydroxylgruppenträgers die Folge, wobei in diesem Fall 2-Butanol zugegeben wurde $\left(p_{2 \mathrm{BUOH}}=1.3 \mathrm{mbar}\right)$. Da der Wert bei hoher Cyclohexanzugabe dem in Stickstoff gemessenen Ergebnis entspricht, könnte durch Zugabe von Radikalfängern an ähnlicher Stelle wie durch Abwesenheit von Sauerstoff in den Mechanismus eingegriffen werden. Dies würde für die Peroxyradikalchemie zutreffen, da die Bildung dieser Strukturen eher in Sauerstoffanwesenheit abläuft und die Bildung von Peroxyradikalen zumindest für Methylcyclohexen unter Einbindung von OH-Radikalen von HATAKEYAMA et al. [135] bereits berichtet wurde. Die fehlende Bildung von als NIM-Vorläufer fungierenden Peroxyverbindungen [12,48] würde zu einer starken Reduktion der Anzahlkonzentration führen, während die gebildeten Massen nicht zwangsläufig im gleichen Ausmaß betroffen sein müssen, da diese auch durch Aufkondensation an vorhandene Keime anderer, mittelflüchtiger, Verbindungen aufgebaut werden kann.

Die 2-Butanolzugabe kann ebenfalls die entstehenden Radikalverhältnisse beeinflussen. Nach KEYWOOD [55] wird beim Einsatz von Alkoholen zum Abfangen der $\mathrm{OH}$-Radikale die $\mathrm{HO}_{2}$-Konzentration erhöht. So können die $\mathrm{HO}_{2}{ }^{-}$ Radikale vermehrt Peroxyradikale abfangen und zu Reaktionsprodukten führen, die aufgrund ihres hohen Dampfdruckes nicht zur Partikelausbeute beitragen, wie es von DOCHERTY et al. [54] für $\beta$-Pinen vorgestellt wurde (Abb. 2.9 in Abschnitt 2.2.2.3). Allerdings kann auch hier die Abfangreaktion 2Butanol + CRIEGEE-Intermediat [59] einen entscheidenden Einfluss ausüben, indem sie die Oligomerisierungsreaktion behindert, was die NIM-Bildung nicht unbedingt in gleiche Maße wie die Produktion kondensierbarer Verbindungen beeinflussen muss. Beide Interpretationen stehen mit der beobachteten reduzierten Anzahlkonzentration und weniger betroffenen Masseausbeute im Einklang.

Die überraschendsten Effekte bei der Zugabe von Radikalfängern waren für 
2-Methyl-1-penten zu beobachten (Abb. 6.28e und 6.28f). Die Zugabe von Cyclohexan bewirkt eine starke Zunahme der gebildeten Anzahlkonzentrationen und Massenausbeuten über den gesamten Druckbereich. Die Erhöhung des Cyclohexanpartialdrucks von $p_{\mathrm{CHA}}=1.3 \mathrm{mbar}$ auf $p_{\mathrm{CHA}}=3.1 \mathrm{mbar}$ ändert die Ergebnisse nicht signifikant, es ist lediglich eine leichte Tendenz zu einer weiteren Zunahme zu erkennen.

Anscheinend tragen Produkte der Cyclohexan $+\mathrm{OH}$-Reaktion zum Aufbau von Partikelkeimen und -masse bei. Das Hauptprodukt dieser Reaktion ist Cyclohexanon $\left(Y_{\mathrm{CHON}}=0.5\right)$ [40], welches häufig für Aussagen über die Effektivität der Abfangreaktion herangezogen wird. Aus diesem Grund wurden zusätzlich zu den Radikalfängerexperimenten solche unter direkter Zugabe von Cyclohexanon durchgeführt. Die Abschätzung des nötigen Partialdruckes, erfolgte auf Grundlage der OH-Ausbeute bei der 2-Methyl-1-butenozonolyse, die von RICKARD et al. [37] mit 0.83 angegeben wird. Unter Einberechnung des verwendeten Ozonpartialdruckes von $p_{\mathrm{O}_{3}}=0.14 \mathrm{~Pa}$ und der erwarteten Cyclohexanonausbeute von ca. $50 \%$ ergibt sich eine potenziell entstehende Cyclohexanonmenge von $p_{\mathrm{CHON}}=0.05 \mathrm{~Pa}$. Da sich sowohl bei diesem Partialdruck als auch bei der stark überhöhten Zugabe von $p_{\mathrm{CHON}}=0.52$ mbar keine signifikante Beeinflussung der Ausbeuten verglichen zu denen in Synthetischer Luft ohne weitere Additiva beobachten ließ, kann Cyclohexanon als Verursacher ausgeschlossen werden.

Es kann davon ausgegangen werden, dass das Abfangen der $\mathrm{OH}$-Radikale in der Reaktionsmischung zu einer Erhöhung der Peroxyradikalmenge und Abnahme der $\mathrm{HO}_{2}$-Konzentration führt, da die Folgechemie der Abfangreaktion, wie in Abb. 2.19 [40] gezeigt, vielfache Reaktionen unter $\mathrm{RO}_{2}$ - oder $\mathrm{HO}_{2}$ Beteiligung beinhaltet, die daher nicht für andere Reaktionen zur Verfügung stehen. Auch hier kann es zu Oligomerisierungsreaktionen, wie von SADEZKY et al. [36] vorgestellt, kommen. Dabei bewirkt die durch Zugabe von Cyclohexan verringerte $\mathrm{HO}_{2}$-Konzentration eine Verringerung der Abbruchreaktion. Dies führt zu einer größeren Kettenlänge der gebildeten Produkte und damit zu einer erhöhten Partikelausbeute. Die Zugabe von 1- bzw. 2-Butanol bewirkt auch für 2-Methyl-1-penten, wie für die anderen Alkene beschrieben, eine Unterdrückung der Keimbildung und der Massenausbeute, die Interpretation ist erneut das direkte Abfangen der für die Partikelbildung bedeutsamen CRIE- 
GEE-Intermediate.

Eine Möglichkeit, den Effekt der Butanolzugabe näher zu untersuchen, wäre der Einsatz von CRIEGEE-Intermediatfängern. In der Literatur [57, 59] wird zu diesem Zweck häufig Ameisensäure (HFO) eingesetzt. Da nach Untersuchungen von NEEB et al. [57] aus der Reaktion des $\mathrm{C}_{1}$-Cl

$$
\mathrm{C} \mathrm{H}_{2} \mathrm{OO}+\mathrm{HCOOH} \longrightarrow \mathrm{CHO}-\mathrm{O}-\mathrm{CH}_{2}-\mathrm{OOH} \longrightarrow \mathrm{H}_{2} \mathrm{O}+\mathrm{HC}(\mathrm{O}) \mathrm{O}(\mathrm{O}) \mathrm{CH}
$$

lediglich Ameisensäureanhydrid und Wasser resultieren und in den Radikalpool daher weniger als beim Butanoleinsatz eingegriffen werden sollte, könnte auf diese Weise zugordnet werden, ob die Abfangreaktion des CRIEGEE-Intermediates oder die Änderung des $\mathrm{OH}-/ \mathrm{HO}_{2}$-Radikalverhältnisses für das Ausbleiben der Nukleationsereignisse verantwortlich ist. Allerdings könnten auch die genannten Folgeprodukte die Aerosolbildung in der Reaktionsmischung in unterwarteter Weise beeinflussen.

\subsubsection{Voroxidierte Verbindungen}

\subsubsection{Alkohole}

\section{3-Buten-1-ol}

Auch Messungen zur Partikelbildung bei der Ozonolyse von 3-Buten-1-ol zeigen eine deutliche Druckabhängigkeit der Ausbeuten sowie ein sehr hohes Partikelbildungspotential, obwohl mit $p_{\mathrm{O}_{3}}=0.15 \mathrm{~Pa}$ eine relativ geringe Ozonanfangskonzentration gewählt wurde. Außer Messungen in Synthetischer Luft und Stickstoff wurden auch solche in Synthetischer Luft unter Cyclohexanzugabe durchgeführt. Während die Partikelbildung in Stickstoff bei 30 mbar Reaktionsdruck bereits vollständig unterdrückt war, wurden in Synthetischer Luft deutliche Partikelverteilungen detektiert. Insgesamt ist in Synthetischer Luft eine Erhöhung der Ausbeuten bezüglich Anzahlkonzentration und Masse im Vergleich zu Stickstoff zu beobachten, vor allem ist dieser Unterschied mit einer fast verdoppelten entstehenden Aerosolmasse sehr ausgeprägt. Auch lässt sich im oberen Bereich kein Sättigungsverhalten feststellen, was auf eine potenziell höhere Ausbeute bei höherem Gesamtdruck und damit gut stabilisierbare zur Nukleation führende Intermediate hinweist. 
Die Experimente in Cyclohexananwesenheit zeigen, dass die Anzahl der gebildeten Partikel durch diesen Eingriff in den Mechanismus nicht so stark beeinflusst wird wie die detektierte Partikelmasse. Während die Anzahl weiterhin der in Synthetischer Luft entspricht, wird die Masse bis auf den in Stickstoff gemessenen Wert reduziert. Dies deutet darauf hin, dass die schwerflüchtigsten Reaktionsprodukte weiterhin gebildet werden und nach wie vor als Kondensationskeim wirken können, während mittelflüchtige kondensierbare Produkte in geringerem Maße entstehen.

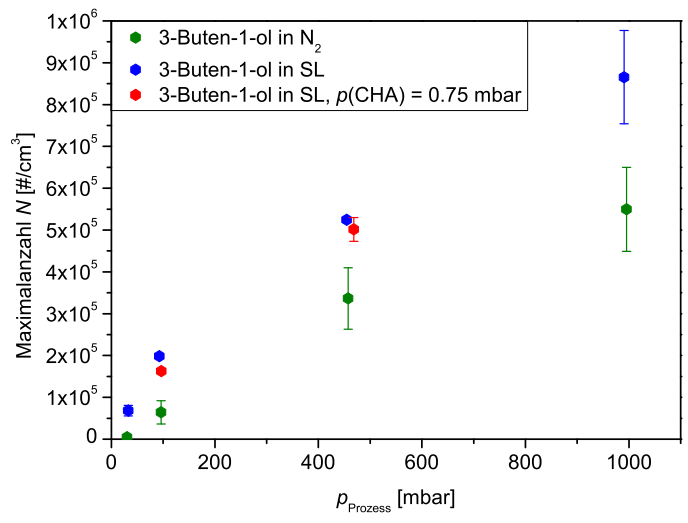

(a) Anzahlkonzentration.

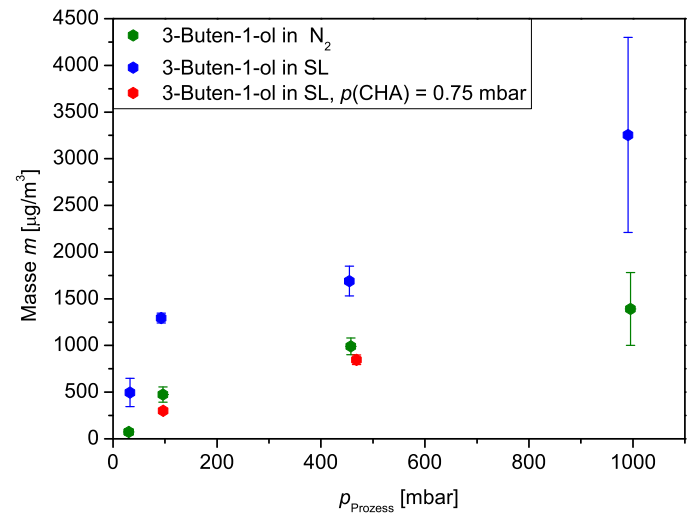

(b) Massenausbeute.

Abb. 6.29.: Untersuchung der druckabhängigen Partikelausbeute für 3-Buten1-ol in Stickstoff, Synthetischer Luft und Synthetischer Luft unter Zugabe von Cyclohexan (CHA).

Das druckabhängige Verhalten weist auf eine Beteiligung der CRIEGEEIntermediate hin, die mit steigendem Gesamtdruck zunehmend gut stabilisiert werden können. Die unterschiedlichen Ausbeuten in Sauerstoffanwesenheit und -abwesenheit unter Niederdruckbedingungen können wiederum ein Hinweis auf die Konkurrenz zwischen der Alkyl $+\mathrm{O}_{2}$-Reaktion mit Isomerisierungsvorgängen und anschließendem Zerfall sein. Während erstere zu sauerstoffreichen Verbindungen mit niedrigem Dampfdruck führen können, fragmentieren die Substanzen durch die verkürzte Lebensdauer durch unzureichende Stoßstabilisierung im zweiten Fall, bevor sie mit den Sauerstoffresten in der Mischung zu entsprechenden Produkten führen können.

Weiterhin sind die Beobachtungen bei Cyclohexanzugabe mit einer potenziellen Oligomerbildung zu erklären, da, vergleichbar mit den Ergebnissen bei 
Methylcyclopenten, ein Eingriff in die Radikalchemie vorgenommen wird. Wie die Reduktion zwischen den Messwerten in Synthetischer Luft und Stickstoff zeigt, führt die Reaktion von bestimmten Ozonolyseprodukten mit Sauerstoff zu einer Erhöhung der Partikelkeimzahlen und der Menge an kondensierter Materie. Die Messungen unter Radikalfängerzugabe macht dabei deutlich, dass die $\mathrm{OH}$-Chemie nicht unmittelbar Einfluss auf die schwerflüchtigsten, als NIM wirkenden Reaktionsprodukte besitzt.

Die hohen Partikelausbeuten für diese vergleichsweise kleine Molekülgröße sprechen für eine Beteiligung der $\mathrm{C}_{1}$ - und $\mathrm{C}_{3}$-CRIEGEE-Intermediate an einer Oligomerisierungsreaktion in der Gasphase, die effektiv zu einem Aufbau von Partikelkeimen und -masse führen.

\section{1-Penten-3-ol}

Generell sind auch für die Ozonolyse von 1-Penten-3-ol recht hohe Ausbeuten zu beobachten, obwohl jeweils lediglich $p_{\mathrm{O}_{3}}=0.10 \mathrm{~Pa}$ Ozon umgesetzt wurden. In diesem Fall konnte durch Badgaswechsel oder Cyclohexanzugabe kaum Einfluss auf die gebildeten Partikelanzahlkonzentrationen ausgeübt werden (Abb. 6.30a), erst ab einem Gesamtdruck von 500 mbar lässt sich eine leichte Reduktion in Stickstoff bzw. bei Zugabe eines Radikalfängers gegenüber den Punkten in Synthetischer Luft beobachten. Die detektierten Massenausbeuten werden stärker durch die Sauerstoffabwesenheit und Cyclohexanbeimischung beeinflusst, da die Ausbeuten in Stickstoff und noch ausgeprägter in Anwesenheit von Cyclohexan reduziert sind. Auch bei $p_{\text {ges }}=30 \mathrm{mbar}$ sind deutliche Aerosolausbeuten nachweisbar, allerdings lässt die starke Reduktion der Ausbeute mit abnehmendem Druck darauf schließen, dass cie Unterdrückung bei geringeren Gesamtdrücken durchaus eintreten kann. Die gemessenen Ausbeuten zeigen außerdem einen asymptotischen Verlauf, der für die detektierten Massen in Abb. 6.30b oberhalb von 500 mbar Reaktionsdruck ausgeprägter ist.

Die Messwerte lassen darauf schließen, dass $\mathrm{OH}$-Reaktionen für die Keimbildung keine übergeordnete Rolle spielen, während erstere in Verbindung mit Sauerstoff für die Bildung der kondensationsfähigen Masse entscheidender sind7. Bei Zugabe eines $\mathrm{OH}-F a ̈ n g e r s$ wird dagegen die Bildung mittel- und schwerflüchtiger Produkte eher gehemmt, was sich, wie bei den vorangegan-

\footnotetext{
${ }^{7}$ Eine weitergehende Interpretation findet sich im vergleichenden Fazit in Abschnitt 6.4.2.2.
} 


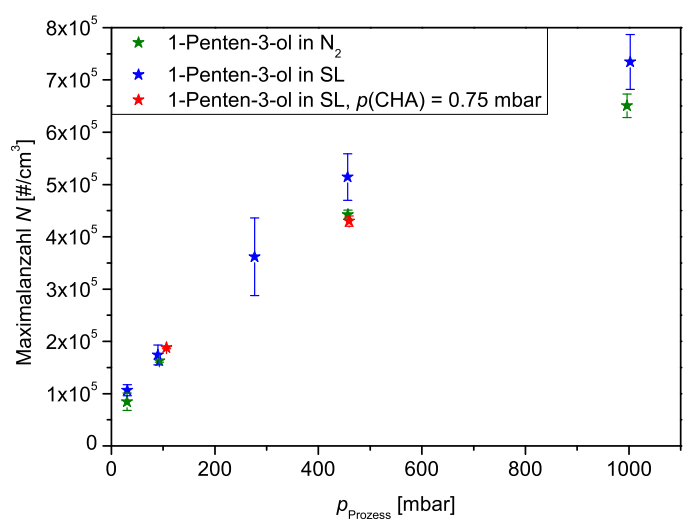

(a) Anzahlkonzentration.

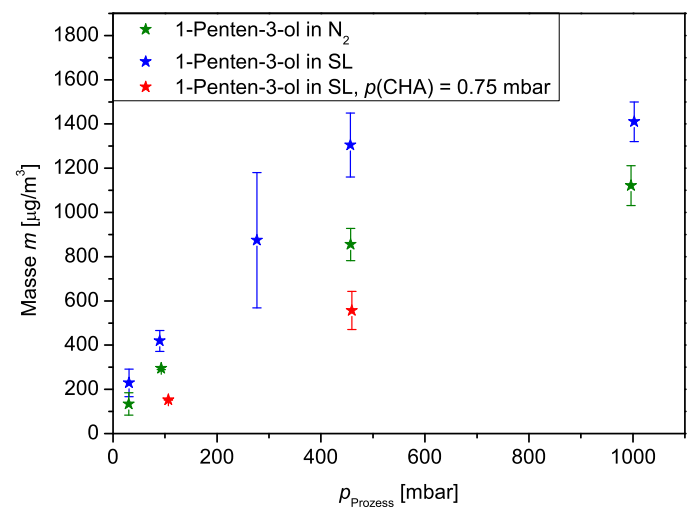

(b) Massenausbeute.

Abb. 6.30.: Untersuchung der druckabhängigen Partikelausbeute für 1-Penten3-ol in Stickstoff, Synthetischer Luft und Synthetischer Luft unter Zugabe von Cyclohexan (CHA).

genen Kohlenwasserstoffverbindungen, mit der Theorie der Oligomerbildung vereinbaren lässt.

\section{4-Penten-1-ol}

Bei der Ozonolyse von 4-Penten-1-ol wurden jeweils ca. $p_{\mathrm{O}_{3}}=0.20 \mathrm{~Pa}$ umgesetzt. Hier zeigt sich ein deutlich stärkerer Einfluss des in Synthetischer Luft enthaltenen Sauerstoffs als bei den übrigen Alkoholen. Wie in Abb. 6.31a und 6.31b erkennbar, ist die Ausbeute bezüglich beider Kenngrößen in Synthetischer Luft deutlich höher als in Stickstoff. Während die Anzahlkonzentration in Synthetischer Luft in der gleichen Größenordnung wie für die anderen Alkohole liegt, ist sie in Stickstoff um fast $80 \%$ reduziert. Auch der Rückgang der Massenausbeuten ist in Sauerstoffabwesenheit wesentlich stärker ausgeprägt.

Die Abhängigkeit vom Gesamtdruck zeigt mit einer Abnahme der Ausbeuten mit sinkendem Druck zwar generell die gleiche Tendenz wie bei den zuvor beschriebenen Verbindungen, allerdings bleiben die gebildeten Anzahlkonzentrationen in Stickstoff oberhalb von etwa 100 mbar konstant, während in Synthetischer Luft noch eine starke Zunahme über den gesamten Druckbereich zu verzeichnen ist. Ähnliches gilt für die Massenausbeute, wobei diese in Stickstoff ab etwa 200 mbar, in Synthetischer Luft oberhalb von etwa 500 mbar konstant bleiben.

Die Messergebnisse weisen darauf hin, dass Sauerstoff als Reaktionspartner 


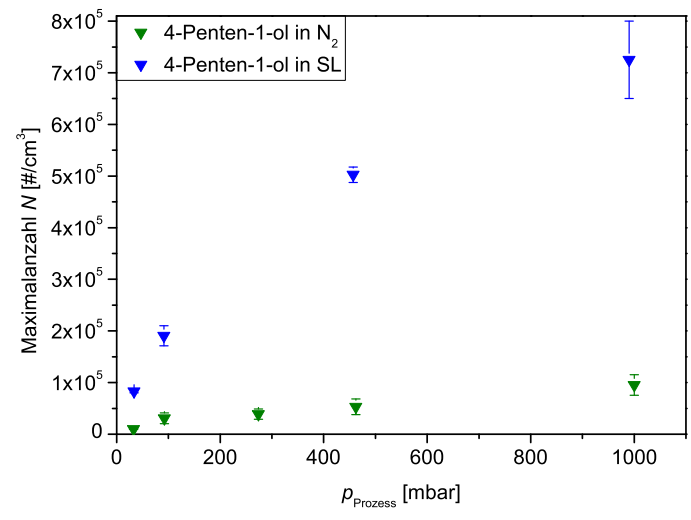

(a) Anzahlkonzentration.

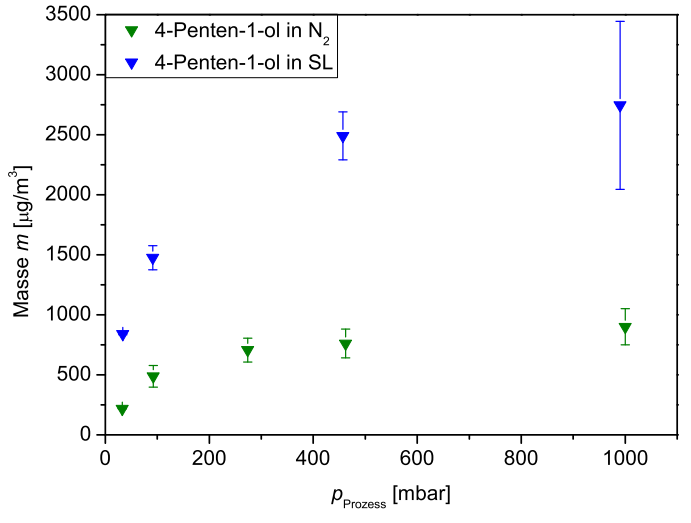

(b) Massenausbeute.

Abb. 6.31.: Untersuchung der druckabhängigen Partikelausbeute für 4-Penten1-ol in Stickstoff und Synthetischer Luft.

sowohl in die Reaktionen, die zur Ausbildung der als NIM wirkenden Kondensationskeime führen, als auch in denen zu mittel- und schwerflüchtigen Produkten, die anschließend auf den gebildeten Keimen kondensieren können, eine wichtige Rolle spielen. In Folge dessen scheint die Alkoxy- / Peroxychemie einen entscheidenden Teil innerhalb des Mechanismus darzustellen, wobei der vorliegende Restsauerstoffgehalt in Stickstoffatmosphäre nicht für konkurrenzfähige Reaktionen dieser Art auszureichen scheint. Auch sind hier unter Umständen sauerstoffinduzierte Oligomerisierungsreaktionen im Sinne des von SADEZKY et al. [36] vorgestellten Mechanismus denkbar. Allerdings müssten diese eher über die großen $\mathrm{Cl}$ ablaufen, da die unsubstituierten $\mathrm{C}_{1}-\mathrm{Cl}$ bei allen Alkoholen entstehen können und daher nicht für das unterschiedliche Reaktionsverhalten der Verbindungen gegenüber Sauerstoff verantwortlich sein sollten. Die erhöhte Ausbeute in Synthetischer Luft ist ein Hinweis darauf, dass durch den Einbau von $\mathrm{O}_{2}$ im Reaktionsverlauf für die Keimbildung und die Massenzunahme verantwortliche (Zwischen-) Produkte langlebiger sind und zu den Ausbeuten beitragen können, wahrscheinlich auch beschleunigt durch die Reduktion der Flüchtigkeit aus diesen zusätzlichen Funktionalitäten. Da sich jedoch, wie zuvor beschrieben, die Anzahl- / Massenzunahmen in Stickstoff ab einem Gesamtdruck von 100 / 200 mbar konstant verhalten, während sie in Synthetischer Luft über den gesamten Druckbereich bzw. zumindest bis 500 mbar zunehmen, ist von einer zusätzlichen chemischen Beeinflussung neben der 
Stoßstabilisierung auszugehen. Eine Betrachtung der Gasphasenprodukte bezüglich der Zerfallsprodukte zeigt eine erhöhte Bildung von CO in Synthetischer Luft bzw. Ameisensäure in Stickstoff, lässt also keine eindeutigen Schlussfolgerungen auf einen badgasabhängig erfolgenden beschleunigten Zerfall der Intermediate zu.

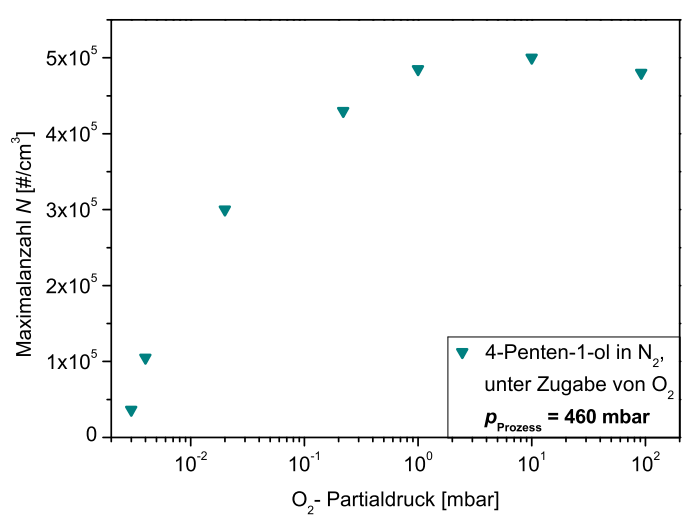

(a) Anzahlkonzentration.

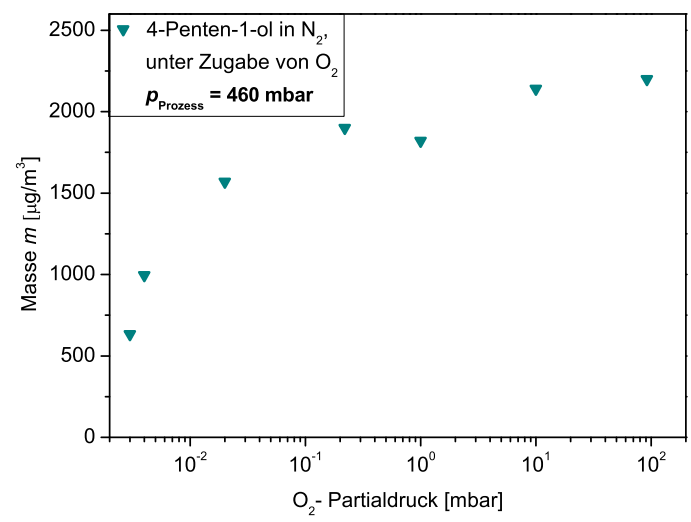

(b) Massenausbeute.

Abb. 6.32.: Untersuchung des Einflusses des Sauerstoffpartialdrucks für 4Penten-1-ol in Stickstoff unter variabler Zugabe von Sauerstoff.

Durch den deutlichen Unterschied in den Ausbeuten war es für 4-Penten-1ol interessant, eine Sauerstoffpartialdruckreihe, wie Abbildung 6.32 dargestellt, zu messen. Dies war der Versuch, durch gezielte Variation der Zugabe reinen Sauerstoffs herauszufinden, ob es eine "Grenzkonzentration“ gibt, ab der der in den Prozessdruckreihen beobachtete Badgaseffekt eintritt. Zu diesem Zweck wurde mit ca. $p_{\text {ges }}=460 \pm 10$ mbar der Gesamtdruck gewählt, bei dem sich die Ausbeuten am deutlichsten unterscheiden und der noch allein durch Expansion aus den Vormischkammern ohne zusätzliches Auffüllen aus der Druckgasflasche erreicht werden konnte. Ansonsten wurden die Anfangskonzentrationen wie bei den übrigen Partikelbildungsexperimenten gewählt.

Wie in Abb. 6.32a und Abb. 6.32b zu sehen ist, nimmt die Ausbeute von Anzahl und Masse mit steigendem Sauerstoffpartialdruck monoton zu. Es ist recht gut zu erkennen, dass die Graphen jedoch ab etwa $p_{\mathrm{O}_{2}}=\left(1 \cdot 10^{0}=1\right)$ mbar stark abflachen, zusätzlicher zur Verfügung stehender Sauerstoff also keinen großen Effekt mehr verursacht. 


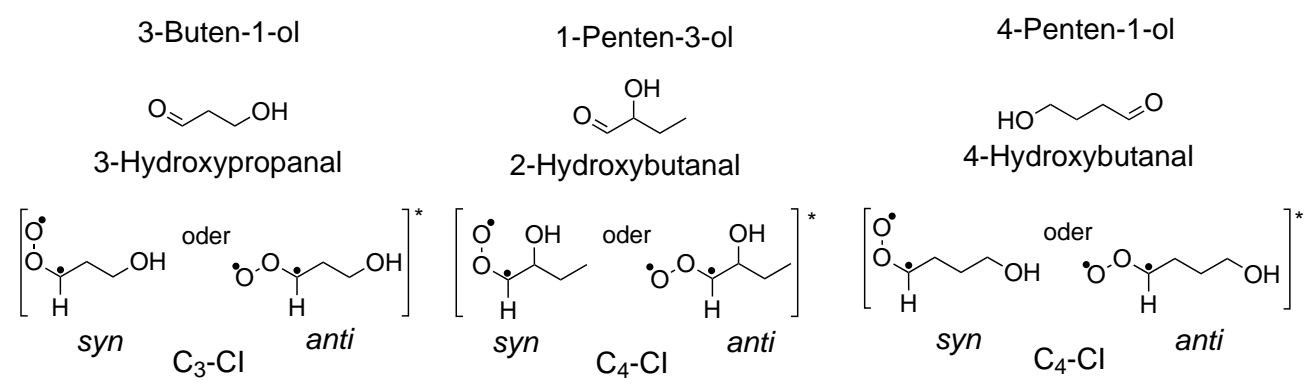

Abb. 6.33.: Bei der Ozonolyse von 3-Buten-1-ol, 1-Penten-3-ol und 4-Penten-1ol entstehende primäre Carbonylverbindungen und substituierte $\mathrm{Cl}$. Zusätzlich wird bei jeder Verbindung auch das $\mathrm{C}_{1}-\mathrm{Cl}$ gebildet.

\subsubsection{Fazit: Partikelbildung durch die Ozonolyse ungesättigter Alkohole}

Die im Vergleich zum 3-Buten-1-ol für die anderen beiden Alkohole im Niederdruckbereich etwas ausgeprägtere Partikelausbeute lässt sich vermutlich mit der um ein Kohlenstoffatom verlängerten Kette bei ansonsten ähnlicher Struktur erklären. Durch diese besitzen entstehende Intermediate mehr Freiheitsgrade und können einfacher, d.h. schon bei geringerem Badgasdruck, (stoß-) stabilisiert werden. Unter Umständen ist auch eine Mesomeriestabilisierung oder eine Stabilisierung durch eine Wasserstoffbrücke der radikalischen $\mathrm{Cl}$ im Falle des 1-Penten-3-ols leichter möglich, da die $\mathrm{OH}$-Gruppe in näherer Nachbarschaft liegt (vgl. Abb. 6.33).

Eventuell könnte sich für 1-Penten-3-ol ein sechsgliedriger Ring ausbilden, bei dem das am O-Atom positionierte Radikal und das Wasserstoffatom der $\mathrm{OH}$-Gruppe ähnlich wie bei einem tunneling-Prozess die Plätze tauschen und so stabilisiert werden. Generell ist die Anordnung in einem Sechsring energetisch deutlich günstiger als in einem potenziellen sieben- oder achtgliedrigen Ring, wie es bei analogem Verhalten für 3-Buten-1-ol bzw. 4-Penten-1-ol zu erwarten wäre und im Energiediagramm in Abbildung 6.34 veranschaulicht ist.

Insgesamt ist unerwartet, dass aus der Ozonolyse von 3-Buten-1-ol so hohe Partikelausbeuten resultieren, obwohl beispielsweise $0.05 \mathrm{~Pa}$ weniger Ozon als im Falle des 4-Penten-1-ol umgesetzt wird und die Kohlenstoffkette bei analoger Struktur um ein Atom verkürzt ist. Dies sollte eigentlich durch die Verfügbarkeit von weniger Freiheitsgraden die Stabilisierung von Ozonolyseprodukten 


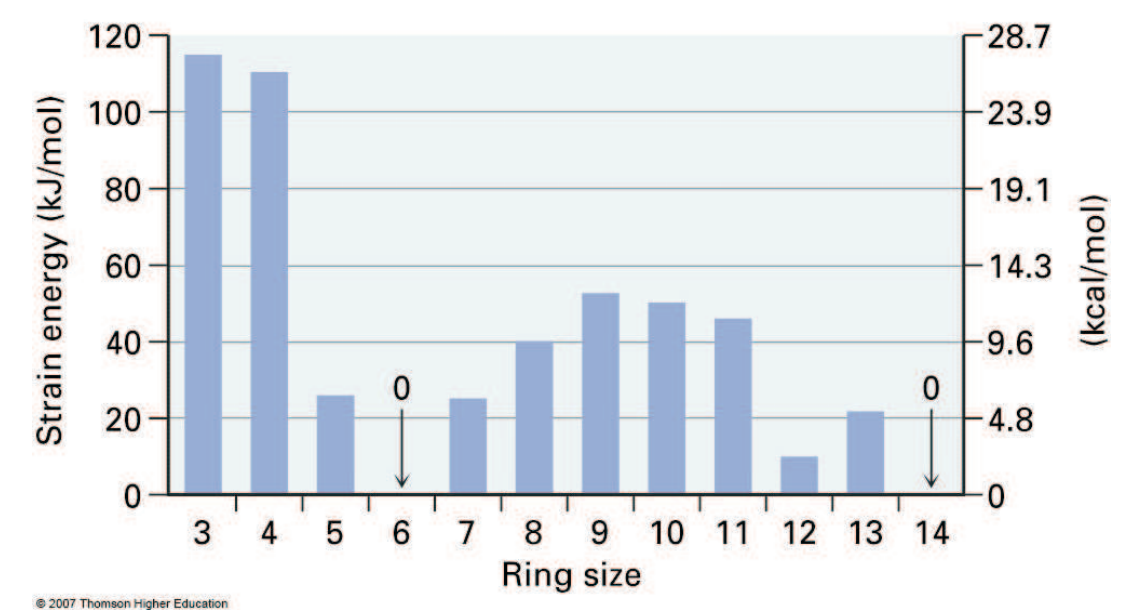

Abb. 6.34.: Ringspannungen für verschiedengliedrige Ringe, aus McMURRY [136], S. 113.

im Falle des 3-Buten-1-ol behindern. Andererseits zeigt dieses Ergebnis, wie schwer eine Voraussage des Nukleationsverhaltens während dieser Reaktionen allein aus der Grundstruktur des Eduktes heraus fällt.

Theoretisch könnten sich auch die unterschiedlichen aus dem POZ-Zerfall gebildeten Carbonylverbindungen (s. Abb. 6.33) auf die Partikelausbeuten auswirken, allerdings ähneln sie sich strukturell stark und sollten daher nicht für das stark unterschiedliche Verhalten der Substanzen verantwortlich sein. Lediglich 3-Hydroxypropanal besitzt durch die um ein Kohlenstoffatom verkürzte Kette ein etwas geringeres Molekulargewicht als die anderen beiden Substanzen, was sich jedoch auf die Partikelausbeute wegen eines potenziell höheren Dampfdruckes eher negativ auswirken sollte. Generell könnten die geringen Dampfdrücke für eine Kondensation auf vorhandenen Partikelkeimen ausreichend niedrig sein (für 3-Hydroxybutanal beträgt der Dampfdruck bei $20{ }^{\circ} \mathrm{C}$ z.B. $0.1 \mathrm{hPa}$ [137]; dazu zum Vergleich: $87 \mathrm{hPa}$ für Methacrylsäure, die nicht auskondensiert und so zur Partikelbildung beträgt). Allerdings würde die Bildung und der Einfluss der Carbonylverbindungen höchstens das generell recht hohe Partikelbildungspotential, nicht die unterschiedlichen Ausbeuten in beiden Badgasen für 4-Penten-1-ol im Gegensatz zu den ähnlichen für 1-Penten-3-ol erklären, da sie direkt aus dem POZ-Zerfall entstehen.

Der Vergleich der Ausbeuten in den verschiedenen Badgasen zeigt, dass die Struktur des Eduktes entscheidende Bedeutung für das Reaktionsverhal- 
ten hat, wenn Sauerstoff als Reaktionspartner zur Verfügung gestellt wird. Die Beobachtungen legen nahe, dass der relative Abstand des oxidierten und des ungesättigten Kohlenstoffatoms im Reaktanden berücksichtigt werden muss. So zeigen sich die größten Unterschiede in den Anzahlkonzentrationen bzw. Massenausbeuten zwischen Reaktionen in Synthetischer Luft und Stickstoff in 4-Penten-1-ol (drei $\mathrm{C}-\mathrm{C}$-Bindungen zwischen Doppelbindung und $\mathrm{OH}$-Gruppe) und die geringsten in 1-Penten-3-ol (eine $\mathrm{C}-\mathrm{C}$-Bindung), während 3-Buten-1-ol eher einen moderaten Effekt zeigt (zwei C-C-Bindungen).

Generell ist die erhöhte Massenausbeute in Sauerstoffanwesenheit für alle Alkohole ein Hinweis darauf, dass durch den Einbau von $\mathrm{O}_{2}$ in bestimmte Intermediate im Reaktionsverlauf für eine rasche Abnahme des Dampfdruckes durch die Bildung von Peroxyradikalen und folglich für eine größere Partikelausbeute gesorgt wird als durch die rein inerte Stoßstabilisierung durch Stickstoffmoleküle.

\subsubsection{Carbonylverbindungen}

\section{1-Penten-3-on}

Für 1-Penten-3-on zeigen sich ebenfalls recht deutliche Unterschiede in den Ausbeuten bei der Verwendung verschiedener Badgase, da diese in Synthetischer Luft deutlich höher ausfallen (Abb. 6.35).

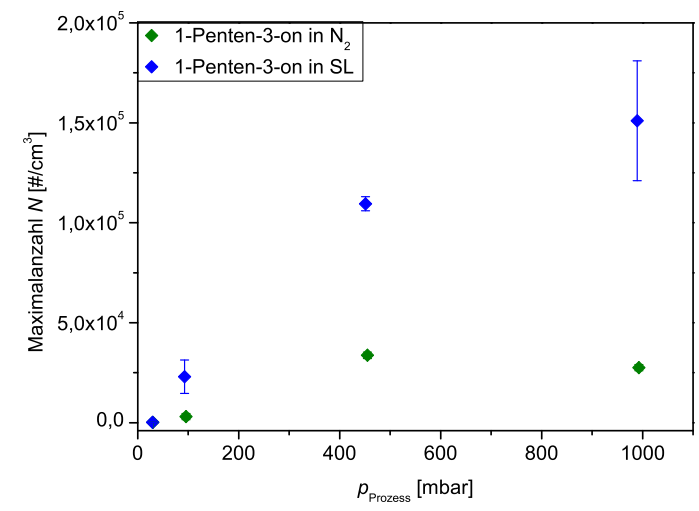

(a) Anzahlkonzentration.

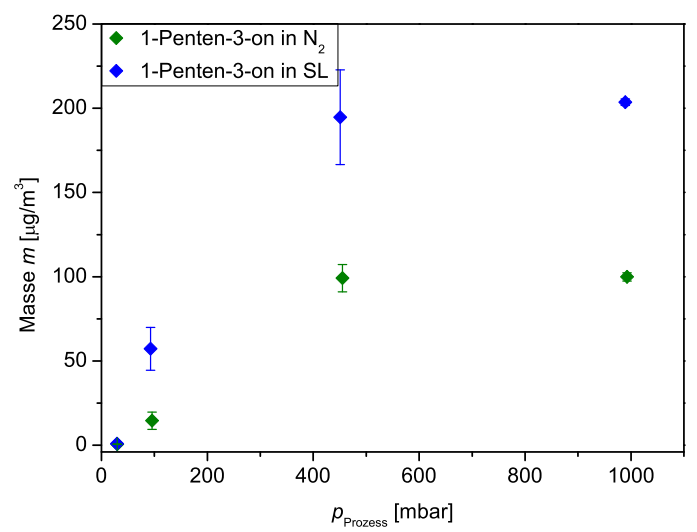

(b) Massenausbeute.

Abb. 6.35.: Untersuchung der druckabhängigen Partikelausbeute für 1-Penten3-on in Stickstoff und Synthetischer Luft. 
Die Nukleation konnte bei Niederdruckbedingungen in beiden Badgasen $\left(p_{\text {ges }}=30 \mathrm{mbar}\right)$ vollständig unterdrückt werden. Die Graphen in Abbildung 6.35a zeigen badgasabhängig unterschiedliche Verhalten, da die Anzahlkonzentration ab einem Gesamtdruck von etwa 460 mbar in Stickstoff konstant bleibt. In Synthetischer Luft nimmt sie noch bis zu dem Hochdruckmesswert bei $p_{\text {ges }}=1000$ mbar leicht zu, wobei sie allerdings gegen einen Grenzwert zu laufen scheint. Dagegen ist an Abb. 6.35b zu erkennen, dass die Massenzunahme ab einem Prozessdruck von $p_{\text {ges }}=460$ mbar in beiden Badgasen beendet ist.

Die sogar bei 100 mbar Prozessdruck noch recht geringe Partikelbildung weist darauf hin, dass die entstehenden, für die Nukleation verantwortlichen Intermediate nur sehr eingeschränkt aus sich selbst heraus durch interne Energieumverteilung stabilisiert werden können, sondern hochgradig von desaktivierenden Stößen mit Badgasmolekülen abhängig sind. Allerdings können auch diese im Hochdruckbereich nicht für vergleichbare Ausbeuten wie bei den untersuchten Alkoholen sorgen.

Die Änderung der Badgasumgebung und der Einfluss auf verschiedene Gasphasenprodukte lässt sich für 1-Penten-3-on besonders gut an dem in Abschnitt 6.2.2.2 (Abb. 6.14a, S. 143) beschriebenen Anwachsen der Signale im Frequenzbereich der potenziellen peroxidischen oder oligomerperoxidischen Banden in Synthetischer Luft gegenüber Stickstoff beobachten. Dieses Verhalten tritt auch beim Vergleich zwischen in Stickstoff aufgenommenen Hoch- und Niederdruckspektren auf. Somit kann festgehalten werden, das eine Erhöhung dieser Signale mit einer erhöhten Partikelbildung einhergeht. Die Klasse der Peroxyverbindungen stellt daher einen wahrscheinlichen Kandidaten für die Initiation eines Nukleationsereignisses für 1-Penten-3-on dar. Unter der Annahme, dass das Propionylperoxylradikal eines der wichtigsten dieser Art in der Ozonolyse des betrachteten Ketons darstellt, kann davon ausgegangen werden, dass sein beschleunigter Zerfall durch unzureichende Stabilisierung im Niederdruckbereich (zusammen mit dem der CRIEGEE-Intermediate) bzw. verminderte Bildung in Sauerstoffabwesenheit zu den beobachteten Unterschieden in Partikelausbeuten und Gasphasenprodukten führt.

Für 1-Penten-3-on wurden Sauerstoffpartialdruckreihen bei zwei verschiedenen Gesamtdrücken durchgeführt (Abb. 6.36). So sollte getestet werden, ob 


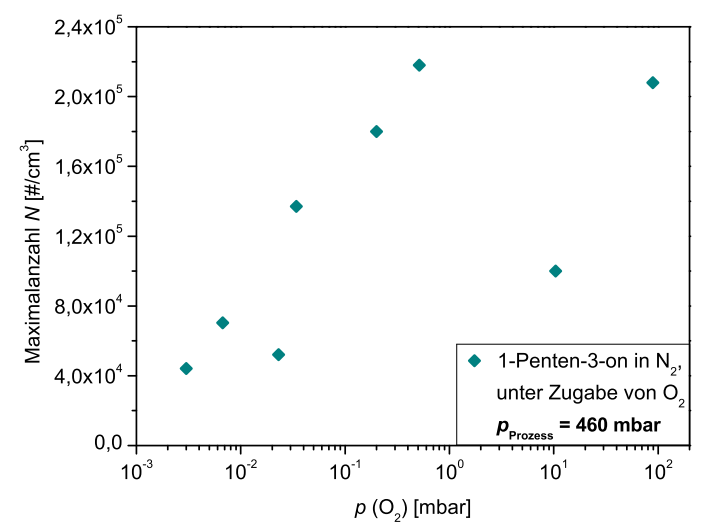

(a) Anzahlkonzentration.

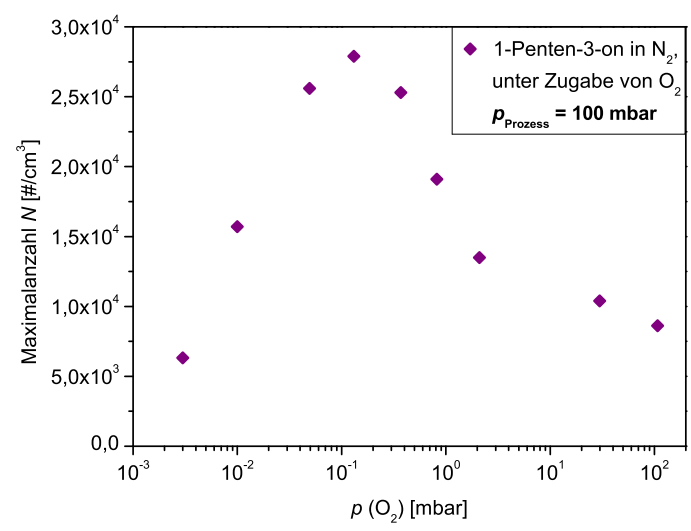

(c) Anzahlkonzentration.

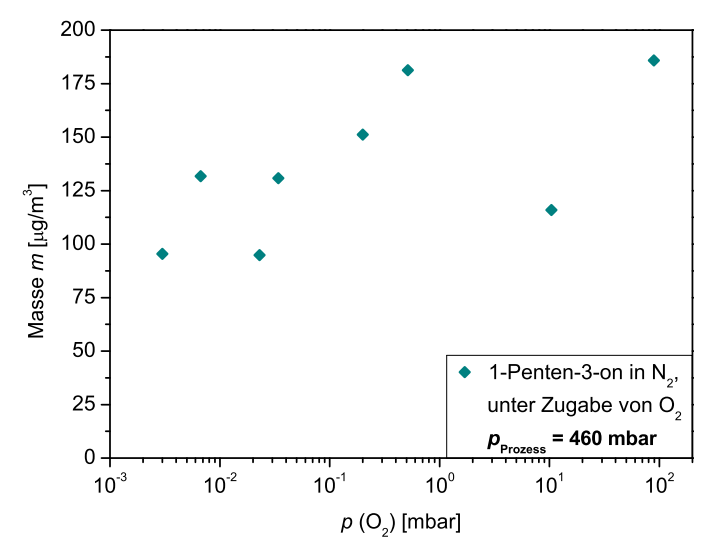

(b) Massenausbeute.

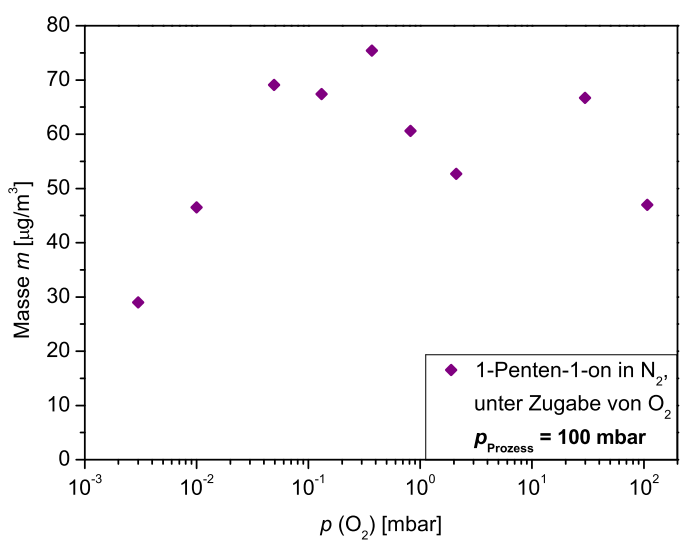

(d) Massenausbeute.

Abb. 6.36.: Untersuchung des Einflusses des Sauerstoffpartialdrucks für 1Penten-3-on in Stickstoff unter variabler Zugabe von Sauerstoff im höheren ( $p_{\text {ges }}=460 \pm 10$ mbar, oben) bzw. niedrigeren Druckbereich ( $p_{\text {ges }}=100 \pm 6$ mbar, unten).

sich das Reaktionsverhalten und damit auch der Einfluss der Sauerstoffanwesenheit unter verschiedenen Druckbedingungen unterscheidet.

Wie die Abbildungen 6.36a und 6.36c zeigen, steigen die Anzahlkonzentrationen in beiden Druckbereichen bis zu einem Sauerstoffpartialdruck von etwa $p_{\mathrm{O}_{2}, \mathrm{HD}}=\left(1 \cdot 10^{-1}=0.1\right)$ mbar bzw. $p_{\mathrm{O}_{2}, \mathrm{ND}}=\left(1 \cdot 10^{0}=1\right)$ mbar an, um dann bei letzterem bei höheren Zugaben stark abzunehmen. Für die Massenausbeuten gilt ähnliches, allerdings bleiben sie oberhalb der genannten Werte mehr oder weniger konstant.

Aufgrund der starken Schwankungsbreite der Messpunkte, wie sie in 6.36a 
für die Anzahl sowie auch für beide Abbildungen zur Massenausbeute oberhalb der genannten Grenzdrücke zu erkennen ist, erfolgt an dieser Stelle keine Interpretation des glockenförmigen Verlaufes in Abb. 6.36c. Da in diesen Messreihen nur wenige Wiederholungsmessungen durchgeführt wurden, kann keine Aussage über die tatsächlichen Fehlerbereiche getroffen werden, lediglich der starke Anstieg bei recht geringen Sauerstoffpartialdrücken, der aus abgesicherten Experimenten für 4-Penten-1-ol bereits bekannt ist (bzw. später beschriebenes ähnliches Verhalten für die Carbonsäuren), erscheint zuverlässig und auch sinnvoll.

Insgesamt kann durch den Anstieg der Ausbeuten bis zu einem die Eduktkonzentrationen bei weitem übersteigenden Partialdruck wie beim zuvor beschriebenen Alkohol davon ausgegangen werden, dass Sauerstoff auch hier ein wichtiger Reaktionspartner entstehender Intermediate in sekundären Prozessen darstellt.

\section{4-Pentenal}

Im Falle des 4-Pentenals wurde ein Anfangspartialdruck von $p_{\mathrm{O}_{3}}=0.20 \mathrm{~Pa}$ Ozon eingesetzt. Bei Betrachtung der gebildeten Anzahlkonzentrationen in Abb. 6.37a fällt auf, dass sich in Stickstoff und Synthetischer Luft identische Kuvenverläufe ergeben, die nicht gegen einen Grenzwert zu streben scheinen und für Stickstoff relativ konstant um etwa $N=8 \cdot 10^{4} \mathrm{~cm}^{-3}$ geringer gegenüber den Werten in Sauerstoffanwesenheit ausfallen.

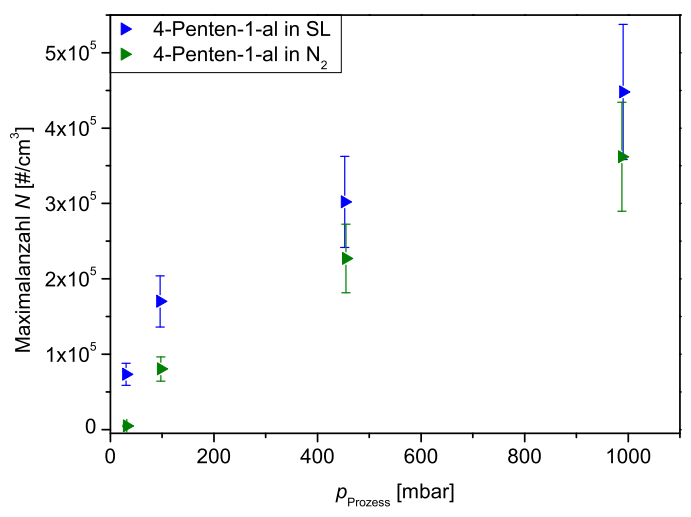

(a) Anzahlkonzentration.

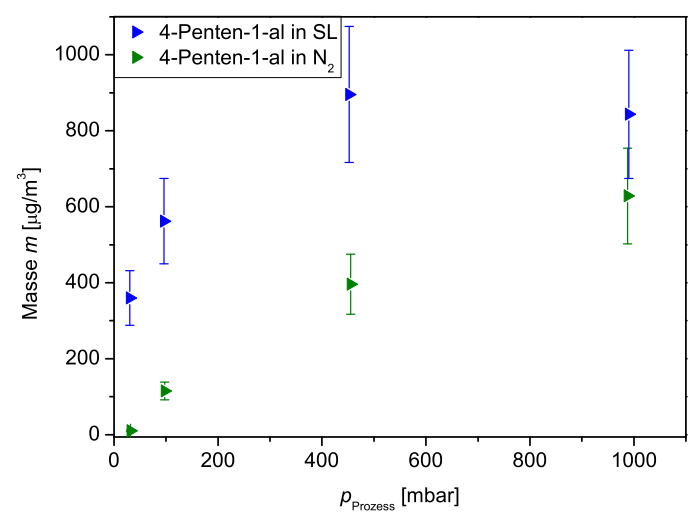

(b) Massenausbeute.

Abb. 6.37.: Untersuchung der druckabhängigen Partikelausbeute für 4-Penten1-al in Stickstoff und Synthetischer Luft. 
Die Massenausbeuten in Bild 6.37b zeigen badgasabhängiges Verhalten. Während die Zunahmen bis 500 mbar Gesamtdruck relativ parallel erfolgen, ändert sich der Wert in Synthetischer Luft jenseits dieses Druckes nicht mehr, während die Ausbeute in Stickstoff weiter zunimmt, ohne jedoch das Ergebnis des anderen Badgases zu erreichen.

Aus den Messwerten zur Anzahlkonzentration kann geschlossen werden, dass die Bildung der Nukleationskeime entweder größtenteils sauerstoffunabhängig erfolgen sollte oder aber so effektiv abläuft, dass sogar die geringe Sauerstoffrestkonzentrationen aus der Ozon / Heliummischung ausreichend für die Bildung der NIM ist. Die ähnlichen (extrapolierten) Kurvenverläufe der Messpunkte zeigen außerdem, dass Druckänderungen in beiden Badgasumgebungen ähnliche Folgen für die Stabilisierung nukleationsinduzierender Intermediate hat.

Der abflachende Verlauf der Massenausbeute im oberen Druckbereich weist darauf hin, dass der maximal mögliche Stabilisierungsgrad für nukleationsfördernde Intermediate erreicht ist, da im Gegensatz zur Stickstoffmessung kein Zuwachs mehr beobachtet werden kann.

Insgesamt ist die Sauerstoffbeeinflussung deutlich geringer ausgeprägt als es beim analogen Alkohol oder 3-Penten-1-on beobachtet werden konnte, was auf eine geringere Bedeutung der Alkoxy- / Peroxychemie für 4-Pentenal schlieBen lässt.

\subsubsection{Fazit: Partikelbildung durch die Ozonolyse ungesättigter Carbonylverbindungen}

1-Penten-3-on ist ein schlechter Partikelbildner, da hier mit einem Ozonpartialdruck von $p_{\mathrm{O}_{3}}=0.35 \mathrm{~Pa}$ gearbeitet werden musste, um zur Auswertung ausreichend definierte Partikelverteilungen zu erhalten. Sogar bei diesen Umsätzen sind die maximalen Ausbeuten mit einer Anzahlkonzentration von $N=1.5 \cdot 10^{5} \mathrm{~cm}^{-3}$ und einer Masse von $m=200 \mu \mathrm{g} \cdot \mathrm{m}^{-3}$, jeweils in Synthetischer Luft und Atmosphärendruck, viel geringer als bei dem analogen Alkohol $\left(N=8 \cdot 10^{5} \mathrm{~cm}^{-3}\right.$ und $m=1800 \mu \mathrm{g} \cdot \mathrm{m}^{-3}$ bei einem Umsatz von ca. $\left.\Delta\left(\mathrm{O}_{3}\right)=0.10 \mathrm{~Pa}\right)$.

Verglichen mit dem analogen Alkohol 4-Penten-1-ol ist auch 4-Pentenal der 

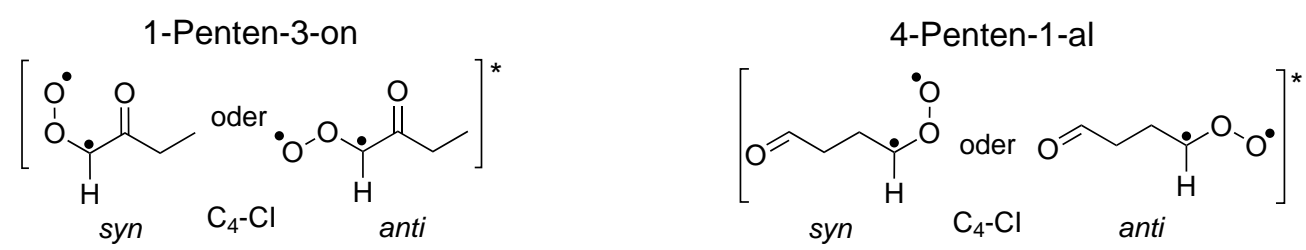

Abb. 6.38.: Bei der Ozonolyse von 1-Penten-3-on und 4-Penten-1-al entstehende substituierte $\mathrm{Cl}$. Zusätzlich wird bei jeder Verbindung auch das $\mathrm{C}_{1}$-Cl gebildet.

deutlich schlechtere Partikelbildner, da die Anzahlkonzentrationen und Masse in Stickstoff (bei identischem Ozonumsatz) jeweils um etwa die Hälfte, die Massenausbeute in Synthetischer Luft sogar um fast $75 \%$ reduziert sind. Allerdings werden für 4-Pentenal (in Vergleichsmessungen mit $p_{\mathrm{O}_{3}}=0.35 \mathrm{~Pa}$ ) fast doppelt so hohe Partikelausbeuten wie für 1-Penten-3-on detektiert. Dies könnte mit Unterschieden in der unimolekularen Chemie zusammenhängen.

Der Vergleich der entstehenden großen CRIEGEE-Intermediate (Abb. 6.38) zeigt, dass die syn-Konformation im Falle des 1-Penten-3-on-Cl durch die Nähe der Cabonyloxid- und des Keto-Sauerstoffatoms energetisch eher ungünstig wäre, dies tritt im Falle des terminalen 4-Penten-1-al nicht auf. Aufgrund weniger destabilisierender Wechselwirkungen könnten die $\mathrm{Cl}$ daher langlebiger sein als die des 1-Penten-3-on und die höheren Ausbeuten durch Folgereaktionen erklären.

Ein Unterschied zwischen Alkoholen und Carbonylverbindungen könnte durch die fehlende Möglichkeit zur Wasserstoffbrückenbindung, da statt der Hydroxylgruppe eine Ketogruppe vorhanden ist, verursacht sein. Eventuell können sich intramolekulare Wasserstoffbrücken zwischen den Alkoholmolekülen oder Alkohol und $\mathrm{Cl}$ ausbilden, die zu etwa einer Verdoppelung des Molekulargewichtes und damit verbunden zu erhöhter Kondensationsfähigkeit auf vorhandenen Keimen führen können, wodurch eine hohe Massenausbeute resultiert, während Carbonyle nicht auf diese Weise dimerisieren. Für eine Reduktion der Flüchtigkeit von Alkoholen im Vergleich zu analogen Carbonylverbindungen sprechen die im ersten Fall höher ausfallenden Siedepunkte [130]. Alkoholen steht durch die Hydroxylgruppe außerdem potenziell die direkte (Abfang-) Reaktion der $\mathrm{Cl}$ als Reaktionsweg offen, so dass sich unter Umständen aus dieser von NEEB et al. [59] für Carbonsäuren vorgeschlagenen Reaktion und daraus 
entstehenden Produkten mit hohem Molekulargewicht die erhöhten Ausbeuten im Vergleich zu den Carbonylverbindungen erklären lassen. Für diese These sprechen die Ergebnisse von LEE und KAMENS [52], nach denen Alkohole deutlich effektivere $\mathrm{Cl}$-Fänger als Carbonylverbindungen sind.

Insgesamt zeigt der Vergleich dieser, bis auf einen Oxidationsgrad identischen Verbindungen, welche Bedeutung die genaue Struktur des Eduktmoleküls und die daraus folgenden möglichen Wechselwirkungen auf das Partikelbildungsverhalten haben kann. Im Umkehrschluss ergibt sich die Erkenntnis, dass Vorhersagen des Reaktionsverhaltens aufgrund struktureller Ähnlichkeiten nur mit großer Unsicherheit möglich sind. Simulationen ohne experimentelle Vergleichswerte sollten also in Kenntnis der hier gezeigten Ergebnisse nur mit großer Vorsicht und unter Berücksichtigung dieser Tatsache zu Interpretationen herangezogen werden.

\subsubsection{Carbonsäuren}

Für die in diesem Kapitel beschriebenen Substanzen ergeben sich verglichen zu den zuvor gezeigten Ergebnissen recht große Schwankungen, die sich nicht nur in der Größe der Fehlerbereiche, sondern auch in einer recht ausgeprägten Streuung der aufgetragenen Punkte widerspiegelt. Die Probleme folgen unter anderem aus den komplizierten Wechselwirkungsverhältnissen der Carbonsäuren, die sich gesamtdruck- und konzentrationsabhängig verhalten können, zu einer Unsicherheit in der Bestimmung der Startkonzentration führen und erst nach ausführlichen Messungen über einen langen Zeitraum optimiert werden konnten. Sie führten zu der in Kap. 3.2.1 beschriebenen Experimentroutine.

Anders als bei allen zuvor beschriebenen Edukten ergeben sich bei den Carbonsäuren deutlich höhere Ausbeuten bei der Verwendung von Stickstoff als Badgas, was in den folgenden Abschnitten näher ausgeführt wird.

\section{Methacrylsäure}

In Partikelbildungsexperimenten mit Methacrylsäure wurden jeweils $p_{\mathrm{O}_{3}}=0.25 \mathrm{~Pa}$ Ozon umgesetzt. In den Abbildungen 6.39a und 6.39b werden die in verschiedenen Badgasen erhaltenen Ergebnisse, jeweils mit und ohne Cyclohexanzugaben, gezeigt. 
Zunächst ist auffällig, dass die Keimbildung in Sauerstoffanwesenheit stark reduziert ist. Auch in den Massenausbeuten zeigen sich Unterschiede, wobei diese relativ betrachtet geringer ausfallen. Die Partikelbildung wird unter Niederdruckbedingungen lediglich in Synthetischer Luft vollständig unterdrückt, in Stickstoff liegen die Ergebnisse deutlich höher. Daraus kann geschlossen werden, dass die Sauerstoffanwesenheit im Fall der Methacrylsäure für eine Destabilisierung der zur Nukleation notwendigen Intermediate sorgt, die in Stickstoff durch ausreichend effektive Stoßstabilisierung bereits bei einem Gesamtdruck von 30 mbar für eine sehr gut definierte Partikelverteilung sorgen.

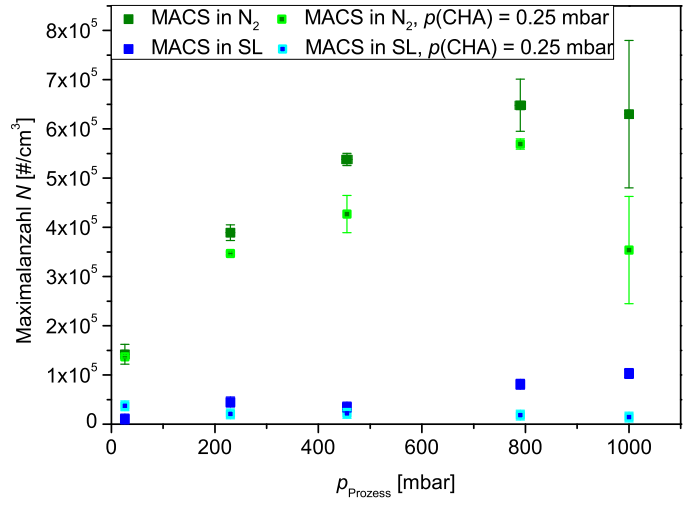

(a) Anzahlkonzentration.

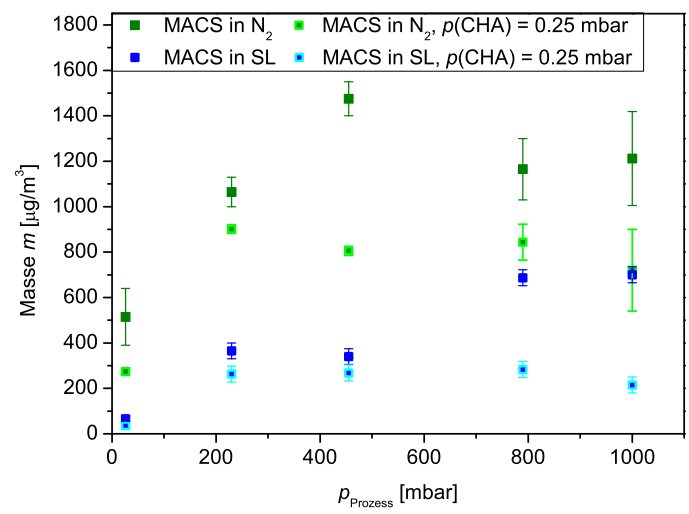

(b) Massenausbeute.

Abb. 6.39.: Untersuchung der druckabhängigen Partikelausbeute für Methacrylsäure in Stickstoff und Synthetischer Luft, jeweils auch unter Zugabe von Cyclohexan (CHA).

Die detektierten Anzahlkonzentrationen steigen in Stickstoff fast über den gesamten Bereich mit dem Gesamtdruck an, was der Stoßstabilisierung der für die Nukleation verantwortlichen Intermediate geschuldet sein dürfte. In Synthetischer Luft zeigen sich bis in einen relativ hohen Druckbereich von 800 mbar kaum nennenswerte Anzahlkonzentrationen. Bei Zugabe von Cyclohexan reduziert sich die Anzahl in beiden Badgasen etwas, wobei der generelle Trend erhalten bleibt. Die Reduktion bei Zugabe eines Radikalfängers gilt auch für die gebildeten Partikelmassen, wo die Messreihen für alle Messbedingungen einen ähnlichen Verlauf zeigen. Nach einem Anstieg im Niederdruckbereich bleiben die Massen in Stickstoff mit und ohne Cyclohexanzugabe (bis auf eine Ausnahme, bei der es sich wohl um einen Ausreißer handelt) ab einem Gesamtdruck 
von etwa 200 mbar recht konstant. Der kritische Bereich, in dem die Stoßstabilisierung wirksam wird, ist für die zur Masse beitragenden kondensationsfähigen Produkte damit für alle Messbedingungen (bezüglich Badgas / Radikalfänger) der Bereich ab einem Gesamtdruck von 200 mbar.

Die Ergebnisse zeigen, dass Sauerstoff über den gesamten Druckbereich die Bildung der schwerflüchtigsten, als Nukleationskeime dienenden, Produkte, behindert. Auch die mittel- und schwerflüchtigen, auf den Keimen kondensierbaren und auf diese Weise zur Massenausbeute beitragenden Verbindungen werden in geringerem Maße produziert.

Die Vergleichsexperimente unter Cyclohexanzugabe zeigen, dass der $\mathrm{OH}-$ Radikalchemie eher eine untergeordnete Rolle in der Partikelbildung bei der Methacrylsäureozonolyse zuzuordnen ist.

\section{Butensäure}

Bei der Butensäureozonolyse $\left(p_{\mathrm{O}_{3}}=0.10 \mathrm{~Pa}\right)$ zeigten sich bezüglich des Badgas- und Radikalfängereffektes ähnliche Beobachtungen wie für Methacrylsäure.

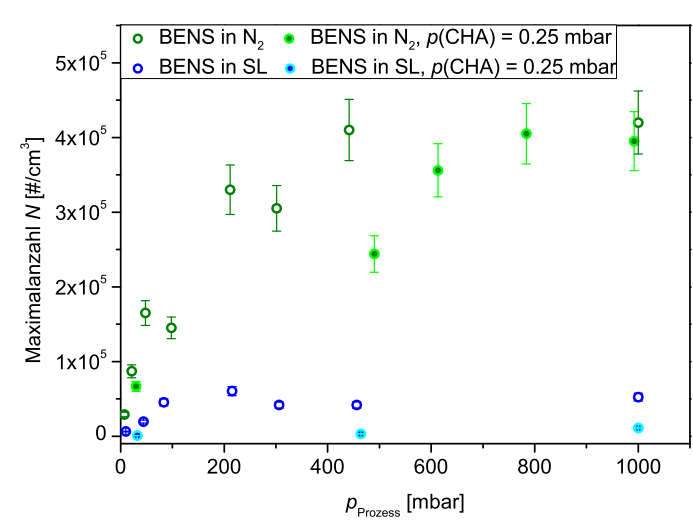

(a) Anzahlkonzentration.

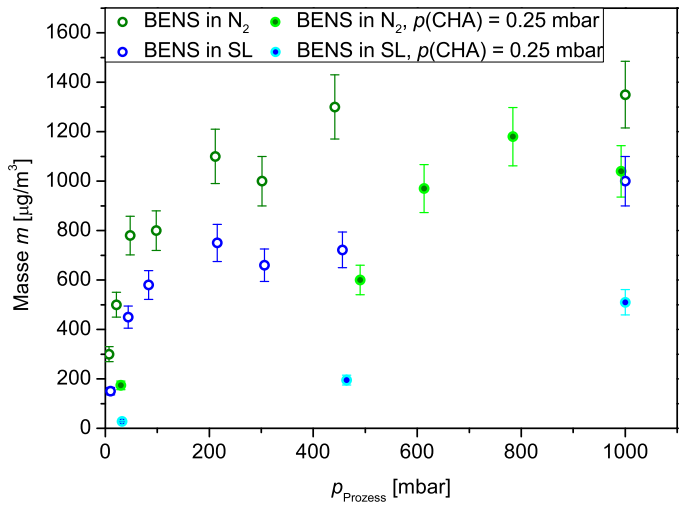

(b) Massenausbeute.

Abb. 6.40.: Untersuchung der druckabhängigen Partikelausbeute für Butensäure in Stickstoff und Synthetischer Luft, jeweils auch unter Zugabe von Cyclohexan (CHA).

Wie zuvor sind auch hier in Abb. 6.40 deutlich höhere Ausbeuten bei der Verwendung von Stickstoff zu verzeichnen, wobei die Unterschiede der gebildeten Anzahlkonzentrationen deutlich ausgeprägter sind als die der Massen. Die Zugabe von Cyclohexan als Radikalfänger hat in beiden Badgasen eine Tendenz 
zur Verringerung der detektierten Partikelmengen zur Folge, wobei dies in Synthetischer Luft bezüglich der Massen in Abb. $6.40 \mathrm{~b}$ besonders gut erkennbar ist.

Für die Experimente ohne Cyclohexanzugabe sind in den gemessenen Kenngrößen oberhalb eines Gesamtdruckes von etwa $p_{\text {ges }}=200-300$ mbar keine weiteren Zunahmen verzeichnet worden. Die Punkte scheinen gegen einen Grenzwert zu laufen, während in Synthetischer Luft unter Cyclohexanzugabe nur sehr geringe Anzahlen, die über den gesamten Druckbereich unter den Werten ohne Fängerzugabe liegen, beobachtet wurden (Abb. 6.40a).

Anhand der Ergebnisse lässt sich mutmaßen, dass die entstehenden Intermediate ab einem Gesamtdruck von etwa 200 mbar wirksam stoßstabilisiert sind. Die Stabilität einiger Zwischenprodukte scheint durch Sauerstoffanwesenheit bzw. Reaktionen mit $\mathrm{O}_{2}$ beeinträchtigt, wobei dies vor allem die keimbildenden Produkte betrifft, während kondensierbare mittel- und schwerflüchtige Substanzen nur leicht verringert vorliegen.

Die Unterdrückung der OH-Radikalreaktion zeigt in Synthetischer Luft deutlich größere Auswirkungen als in Stickstoff. Eine Erklärung für die unterschiedlichen Ausbeuten in der Anzahl in den beiden Badgasen wäre, dass die Reaktion mit $\mathrm{O}_{2}$ zur Peroxyradikalbildung führen, die aufgrund des hohen Sauerstoffanteils im Molekül durch den zuvor schon hohen Oxidationsgrad der Carbonsäure zu sehr destabilisiert werden und in der Folge zerfallen. Dagegen werden mittel- und schwerflüchtige kondensationsfähige Produkte weniger durch eine Änderung des Sauerstoffanteils in der Mischung beeinflusst, was auf eine von diesem Molekül unabhängige Reaktion hindeutet. Mögliche Wege zum Aufbau von Aerosolmasse wären hier eine Abfangreaktion der CRIEGEE-Intermediate durch Butensäure, zu der diese durch die $\mathrm{OH}$-Gruppe fähig sein sollte. Desweiteren kommt auch eine Oligomerisierungsreaktion nach SADEZKY in Frage [36, 53], da sowohl das $\mathrm{C}_{1}$ - als auch ein $\mathrm{C}_{3}$-Cl gebildet wird, die beide diese Reaktion eingehen könnten. Sauerstoff wird in diesem Mechanismus nur an einer Stelle zum Start der Reaktion benötigt (vgl. Abb. 2.7, S. 29), daher könnten geringe Spuren von $\mathrm{O}_{2}$, etwa aus der Ozon / Heliummischung oder Leckagen der Aerosolzelle (ca. 0.5 Pa), auch in Stickstoffexperimenten zum Start einer solchen Gasphasenpolymerisation ausreichend sein. In Synthetischer Luft sollte in jedem Falle die Anlagerung von Sauerstoff und dem damit verbundenen 
beschleunigten Zerfall durch zu hohe Überschussenergien der Produkte dominieren, da die niedrigen Ausbeuten in Sauerstoffanwesenheit allen beschriebenen Experimentvariationen gemein sind.

Einen Hinweis auf die Komplexizität der beschriebenen Vorgänge gibt die in Kap. 6.7 beschriebene Aerosolphasenanalyse.

\section{Sauerstoffpartialdruckreihen für die Carbonsäuren}

Für die beiden untersuchten Carbonsäuren wurden Sauerstoffpartialdruckreihen angefertigt, um das Verhalten der Nukleation bei variabler Sauerstoffkonzentration in der Reaktionsmischung genauer zu untersuchen (Abb. 6.41). Die Messungen wurden mehrmals wiederholt, daher wurden in diesem Fall Kurven durch die Messpunkte gelegt, die allerdings keine mathematische Aussage haben, sondern nur der Orientierung dienen sollen.

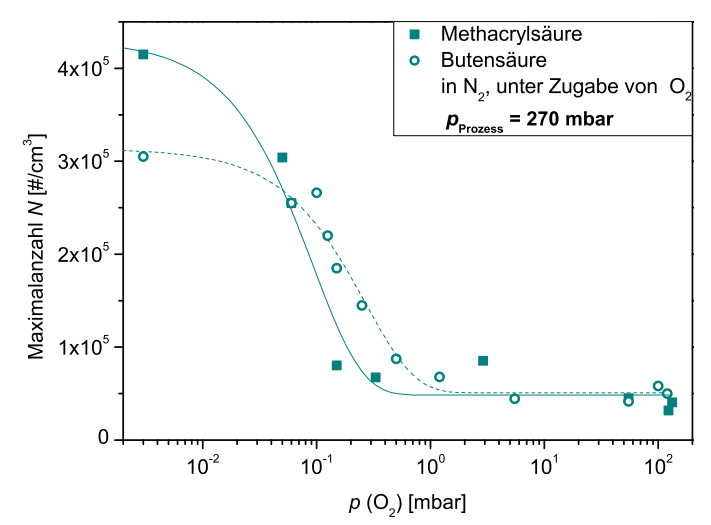

(a) Anzahlkonzentration.

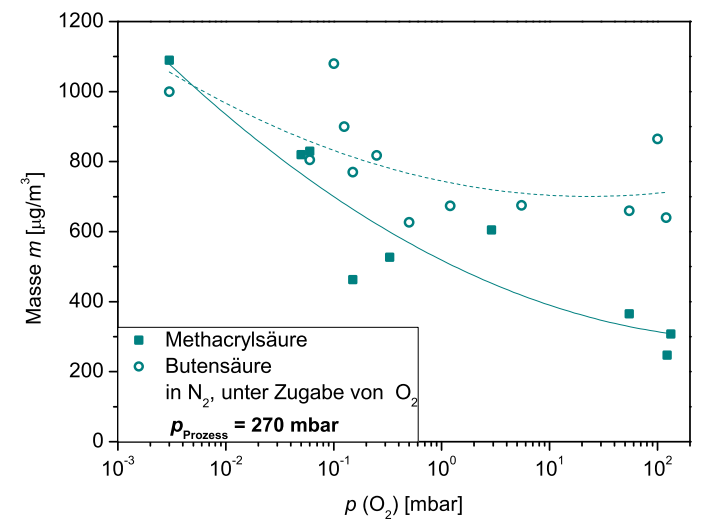

(b) Massenausbeute.

Abb. 6.41.: Untersuchung des Einflusses des Sauerstoffpartialdrucks für Methacrylsäure und Butensäure in Stickstoff unter variabler Zugabe von Sauerstoff im mittleren Druckbereich ( $p_{\text {ges }}=270 \pm 10 \mathrm{mbar}$ ).

Die Experimente wurden bei einem Gesamtdruck von $p_{\text {ges }}=270 \pm 10$ mbar durchgeführt, da die Messwerte in den zuvor untersuchten Prozessdruckreihen oberhalb dieses Druckes eine stärkere Streuung aufwiesen. Ozon- und Säurekonzentrationen entsprachen denen der zuvor beschriebenen Partikelbildungsexperimente.

Abbildung 6.41a zeigt, wie die Anzahlkonzentration mit steigendem Sauerstoffpartialdruck abnimmt, wobei dieser Effekt schon bei recht geringen Zugaben auftritt. Für Methacrylsäure ist bereits zwischen $p_{\mathrm{O}_{2}}=\left(1 \cdot 10^{-2}\right.$. 
$\left.1 \cdot 10^{-1}=0.01-0.1\right)$ mbar eine Reduktion der Anzahl zu beobachten, während dies für Butensäure erst bei $p_{\mathrm{O}_{2}}=\left(1 \cdot 10^{-1}-1 \cdot 10^{0}=0.1-1\right)$ mbar deutlich wird.

$\mathrm{Ab}$ einem Grenzdruck von etwa $p_{\mathrm{O}_{2}}=2$ mbar lässt sich die Anzahlkonzentration für beide Carbonsäuren nicht mehr durch weitere Erhöhung des Sauerstoffpartialdruckes beeinflussen.

Die gemessenen Massenausbeuten in Abb. 6.41b zeigen eine recht hohe Streuung. Trotzdem ist zu erkennen, dass die Zugabe von Sauerstoff einen größeren Effekt in der Methacrylsäureozonolyse entfaltet, was eventuell auch auf die unterschiedlichen Reaktivitäten zurückzuführen ist. Die Abnahme der Massen fällt relativ gesehen wesentlich geringer aus als die der Anzahlkonzentration und findet über den gesamten untersuchten Bereich statt.

\subsubsection{Untersuchung der Bedeutung sekundärer Reaktionen}

NeEB et al. [59] haben die Abfangreaktion von CRIEGEE-Intermediaten durch Hydroxylgruppenträger untersucht und festgestellt, dass die Zugabe von Ameisensäure in der Methacrylsäureozonolyse die Reaktion $\mathrm{Cl}+$ Carbonsäure verhindert. Durch die Zugabe dieses Fängers konnte die Kinetik der eigentlichen $\mathrm{O}_{3}+$ MACS-Reaktion untersucht werden, ohne dass zusätzlicher Säureverbrauch durch $\mathrm{Cl}$ die Bestimmung der Reaktionsgeschwindigkeit verfälschte. $\mathrm{OH}$-Radikale wurden in den genannten Studien gleichzeitig mit Cyclohexan abgefangen.

Dieser Effekt wurde in der gleichen Arbeitsgruppe auch mit anderen Hydroxyverbindungen, nämlich neben Ameisensäure auch Essigsäure, Methanol und Wasser [57], getestet und der Einfluss auf die Gasphasenprodukte IRspektroskopisch untersucht, wobei die Ameisensäure die höchste Reaktivität zeigte. Eine Analyse, wie sich diese Abfangreaktion auf den Partikelbildungsprozess auswirkt, erfolgte in dieser Studie nicht, allerdings ist aus anderen Arbeiten bekannt, dass beispielsweise die Zugabe von Wasser die Partikelausbeute verringert [18].

Zu näheren Untersuchung der Einflussmöglichkeiten auf sekundäre Reaktionen wurden zunächst Verhältnisreihen als Partikelbildungsexperimente bei konstantem Gesamtdruck durchgeführt, bei denen die Carbonsäurekonzentrationen variiert wurden, während die Ozonkonzentration unverändert blieb. Auf 
diese Weise sollte festgestellt werden, bis zu welchem Verhältnis die Konzentrationserhöhung der Carbonsäure Einfluss auf die Partikelbildung ausübt. Der Hintergrund ist der Folgende: Nimmt die Aerosolausbeute z.B. nur bis zu einem Verhältnis von $1: 1 \mathrm{zu}$, wird die Säure nur vom Ozon verbraucht. Wird zusätzlich das aus der Kinetikstudie bekannte Reaktionsverhältnis $\alpha$ einbezogen, wird gleichzeitig berücksichtigt, wieviel der Carbonsäure durch $\mathrm{OH}$-Radikale abgebaut werden. Sollte das Reaktionsverhältnis von Säure zu Ozon den Wert von $\alpha$ deutlich übersteigen, würde das für einen zusätzlichen Verbauch der Carbonsäure, etwa durch $\mathrm{Cl}$, sprechen.

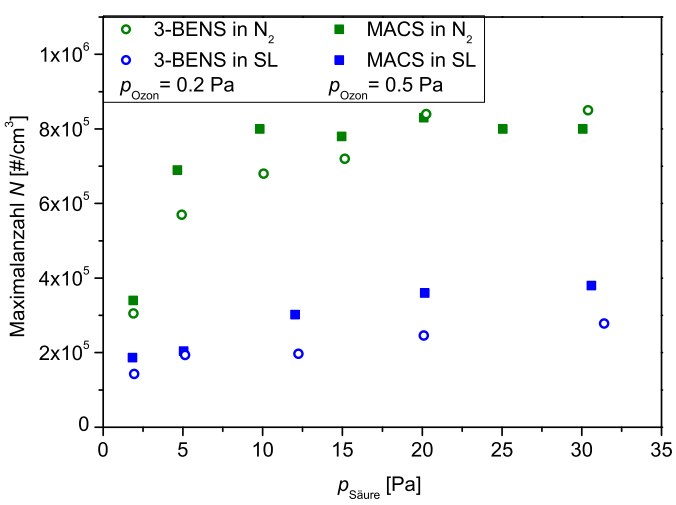

(a) Anzahlkonzentration.

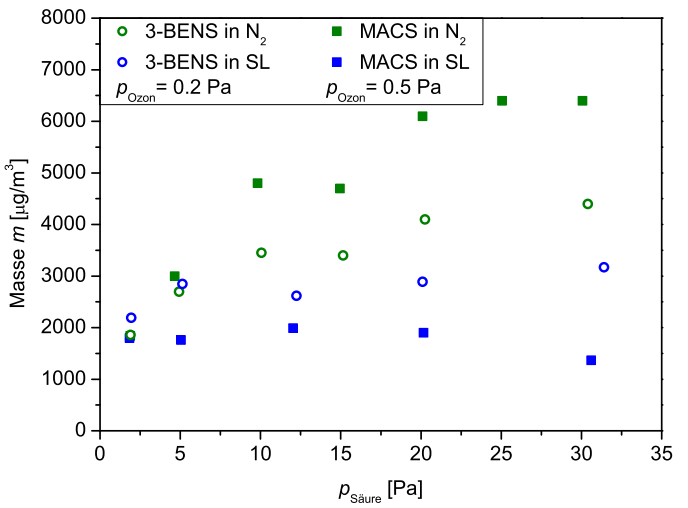

(b) Massenausbeute.

Abb. 6.42.: Untersuchung des Einflusses der Säurepartialdrücke für Methacrylsäure und Butensäure auf die Partikelausbeuten in beiden Badgasen bei konstantem Gesamtdruck ( $p_{\text {ges }}=460 \pm 10$ mbar).

Wie Abbildung 6.42 zeigt, hat die Säureanfangskonzentration eingeschränkt Einfluss auf die Ausbeuten. Die Messungen wurden mit, verglichen zu den übrigen Partikelbildungsexperimenten, verdoppelten Ozonanfangskonzentrationen

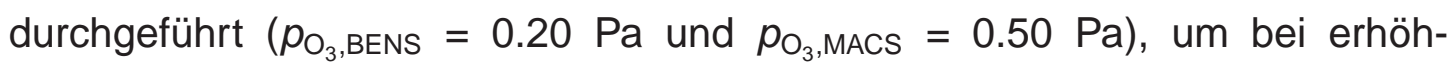
ten Umsätzen die Bedeutung der Säurekonzentration deutlicher beobachten zu können. Alle Messungen wurden bei einem konstanten Gesamtdruck von $p_{\text {ges }}=460 \pm 10$ mbar durchgeführt, da die Reproduzierbarkeit bis zu diesem allein aus der Vormischkammerexpansion erreichbaren Druck am höchsten war.

Die Anzahlkonzentrationen (Abb. 6.42a) zeigen, dass die starke Zunahme ab etwa einem Säurepartialdruck von $p_{\text {Säure }}=5 \mathrm{~Pa}$ größtenteils beendet ist. Während in Stickstoff zuvor für beide Säuren ein starker Anstieg zu beobachten ist, 
läuft die Anzahl jenseits dieser Konzentration gegen einen Grenzwert. In Synthetischer Luft ist dagegen über den gesamten Bereich ein geringer monotoner Anstieg der Anzahlkonzentration zu beobachten.

Die Massenausbeuten in Abb. 6.42b zeigen ein ähnliches Verhalten wie die Anzahl. Nur in Stickstoff sind auch jenseits der $p_{\text {Säure }}=5$ Pa noch Zunahmen zu beobachten, die für Methacrylsäure etwas ausgeprägter ausfallen als für Butensäure. In Synthetischer Luft sind für beide Carbonsäuren über den gesamten vermessenen Bereich recht konstante Werte das Ergebnis. Insgesamt muss jedoch beachtet werden, dass die Streuung in der Massenausbeute generell etwas ausgeprägter ist, so dass die erkennbare Sprung für die Stickstoffexperimente bei $p_{\text {Säure }}=15-20 \mathrm{~Pa}$ nur bedingt aussagekräftig ist.

In Stickstoff zeigt sich im Gegensatz zu Synthetischer Luft ein deutlicher Einfluss der Säurekonzentration, deshalb werden die Werte aus letzterem Badgas lediglich als Vergleichswerte herangezogen. Da zudem Butensäure deutlich intensiver untersucht wurde, wird sich die im Folgenden vorgestellte Überlegung vornehmlich auf diese Substanz beziehen, während die Methacrylsäure ebenfalls nur als Vergleich dient.

Aus den Kinetikexperimenten ergaben sich folgende Werte für den relativen Verbrauch $\alpha$ :

$$
\begin{array}{ll}
\text { - } \alpha_{\text {MACS }, \mathrm{N}_{2}}=2.70 & -\alpha_{\mathrm{BENS}, \mathrm{N}_{2}}=1.44 \\
\text { - } \alpha_{\mathrm{MACS}, \mathrm{SL}}=2.36 & -\alpha_{\mathrm{BENS}, \mathrm{SL}}=1.68
\end{array}
$$

Falls die Carbonsäure nur durch Ozon und $\mathrm{OH}$-Radikale abgebaut wird, sollte sich ein Einfluss auf die Partikelausbeuten also lediglich in einem Partialdruckbereich von $p_{\text {Säure }} \approx p_{\text {Ozon }} \cdot \alpha$ zeigen. Damit würden sich mit den Ozonanfangspartialdrücken von $p_{\mathrm{O}_{3} \text {, BENS }}=0.20 \mathrm{~Pa}$ und $p_{\mathrm{O}_{3}, \mathrm{MACS}}=0.50 \mathrm{~Pa}$ für die betrachteten Carbonsäuren folgende Zahlenwerte ergeben:

$$
\begin{gathered}
p_{\text {Säure,Badgas }} \approx p_{\text {Ozon }} \cdot \alpha_{\text {Säure,Badgas }} \\
p_{\mathrm{MACS}, \mathrm{N}_{2}} \approx 0.5 \mathrm{~Pa} \cdot 2.70=1.35 \mathrm{~Pa} \\
p_{\mathrm{BENS}, \mathrm{N}_{2}} \approx 0.2 \mathrm{~Pa} \cdot 1.44=0.29 \mathrm{~Pa}
\end{gathered}
$$


Durch den für Butensäure in Synthetischer Luft etwas höheren Wert für die relativen Verbräuche sollten die Partialdruckbereiche oberhalb des für Stickstoff berechneten Ergebnisses liegen, während der Wert für Methacrylsäure geringer ausfällt $\left(p_{\mathrm{BENS}, \mathrm{SL}}=0.34 \mathrm{~Pa}, p_{\mathrm{MACS}, \mathrm{SL}}=1.18 \mathrm{~Pa}\right)$.

Wie jedoch schon die nur anhand grober Abschätzung erhaltenen Ergebnisse für die Stickstoffwerte zeigen, scheinen die Carbonsäuren in sehr ausgeprägtem Maße in sekundäre Reaktionen eingebunden zu sein, da sich ein Einfluss der Konzentrationsvariation bis hin zu etwa $p_{\text {Säure }}=5 \mathrm{~Pa}$ zeigt. Neben den $\mathrm{OH}$ - und $\mathrm{O}_{3}$-induzierten und vermuteten $\mathrm{Cl}$-Reaktionen kann zwar noch eine Vielzahl weiterer Ozonolyseprodukte zum Abbau beitragen, allerdings sollte die $\mathrm{Cl}+$ Carbonsäurereaktion durch den hohen Überschuss der Säurekonzentration eindeutig dominieren.

Der Vergleich der theoretisch berechneten zu den experimentell erhaltenen Grenzkonzentrationen zeigt, dass bei der Butensäureozonolyse relativ gesehen eine größere Menge des Eduktes in sekundären Reaktionen verbraucht wird. Dieses Ergebnis zeigt sich in der Relation zur rechnerisch in der Ozonolysereaktion verbrauchten Säure, hier exemplarisch gezeigt für Experimente in Stickstoff.

$$
\begin{aligned}
& p_{\mathrm{MACS}, \mathrm{N}_{2}, \text { exp }}: p_{\mathrm{MACS}, \mathrm{N}_{2}, \text { theo }}=5 \mathrm{~Pa}: 1.35 \mathrm{~Pa} \approx 3.7 \\
& p_{\mathrm{BENS}, \mathrm{N}_{2}, \text { exp }}: p_{\mathrm{BENS}, \mathrm{N}_{2}, \text { theo }}=5 \mathrm{~Pa}: 0.29 \mathrm{~Pa} \approx 17.2
\end{aligned}
$$

Da sich die Konzentrationserhöhungen bis zu einem Partialdruck von $5 \mathrm{~Pa}$ in Stickstoff sehr stark auf die gebildeten Anzahlkonzentrationen auswirken, kann vermutet werden, dass diese sekundären Reaktionen unter Carbonsäurebeteiligung vor allem auf die Bildung der schwerflüchtigsten, als Keimvorläufer dienenden, Produkte auswirkt. Hier sind auch die kondensierbaren leichterflüchtigen, zur Massenausbeute beitragenden, Spezies etwas weniger betroffen. In Synthetischer Luft wird dagegen vermutlich die Reaktion bestimmter Intermediate mit dem in deutlichem Überschuss vorliegenden $\mathrm{O}_{2}$ diese Säurebeteiligung verhindern. Es könnte auch die destabilisierende Wirkung durch Sauerstoffanlagerung überwiegen, so dass die Konzentrationsvariation der Carbon- 
säure über den gesamten Bereich kaum einen sichtbaren Einfluss ausübt.

Einen weiteren Hinweis auf eine zusätzliche Reaktion von Säuremolekülen mit Verbindungen außer Ozon liefert eine genauere Betrachtung des relativen Verbrauchs $\alpha$ in der Butensäureozonolyse. Allerdings ist auch hier mit einer Restunsicherheit zu rechnen, da die zu diesem Wert beitragenden $\mathrm{OH}$ Radikale auch mit anderen Molekülen als dem Edukt reagieren könnten.

Zu diesem Zweck wurden die relativen Verbräuche in Synthetischer Luft und Stickstoff bei einer konstanten Ozonanfangskonzentration von $p_{\mathrm{O}_{3}} \approx 1 \mathrm{~Pa}$ und Butensäurekonzentrationen von $p_{\mathrm{BENS}}=5.0 \mathrm{~Pa}$ bzW. $p_{\mathrm{BENS}}=2.0 \mathrm{~Pa}$ und einem Gesamtdruck von $p_{\text {ges }}=460 \pm 10$ mbar durchgeführt (vgl. Tabelle A.6). Es ergaben sich folgende Werte:

$$
\begin{array}{ll}
\text { - } \alpha_{2.0 \mathrm{~Pa}, \mathrm{~N}_{2}}=1.23 \pm 0.10 & -\alpha_{2.0 \mathrm{~Pa}, \mathrm{SL}}=1.49 \pm 0.01 \\
\text { - } \alpha_{5.0 \mathrm{~Pa}, \mathrm{~N}_{2}}=1.65 \pm 0.01 & -\alpha_{5.0 \mathrm{~Pa}, \mathrm{SL}}=1.86 \pm 0.12
\end{array}
$$

Es zeigen sich in beiden Badgasen signifikant höhere Werte für den relativen Verbrauch bei höheren eingesetzten Säurepartialdrücken. Dies spricht für eine Beteiligung der Eduktmoleküle in der Sekundärchemie, die ausgeprägter bei der höheren Anfangskonzentration ausfällt, da eine sekundäre Reaktion dann mit höherer Wahrscheinlichkeit ablaufen kann.

Zunächst sollte festgestellt werden, ob sich bei nominell gleichem Reaktandenumsatz deutliche Unterschiede aus der Tatsache ergeben, dass nach Ablauf der primären Reaktion eine gewisse Restkonzentration an Ozon oder Butensäure im Produktgemisch vorliegt. Dazu wurde das Verhältnis der Edukte zueinander variiert.

Wie der direkte Vergleich der Reaktandenverhältnisse $1.0: 0.3$ sowie $0.5: 0.2$ (jeweils Butensäure : Ozon und in Pascal) und ihre Umkehrungen in Abb. 6.43 (gelb, orange / grau) zeigt, hat diese Variation keine signifikante Auswirkung auf die Ausbeuten in Anzahlkonzentration und Masse.

Bei einer leichten Veränderung des Verhältnisses von 1.0:0.3 zu 1.0:0.2 (gelb / gelbgrau) zeigt sich jedoch eine deutliche Verringerung der Ausbeuten, was auf eine recht starke Abhängigkeit der gebildeten Aerosolausbeute von der Ozonkonzentration schließen lässt. Allerdings zeigt der Vergleich der Ergebnisse mit Anfangspartialdrücken von 0.2:0.5 und deren Umkehrung 0.5:0.2 mit 


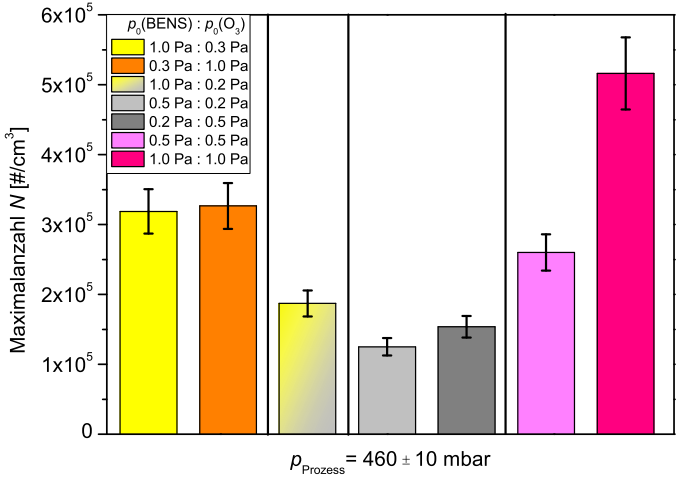

(a) Anzahlkonzentrationen.

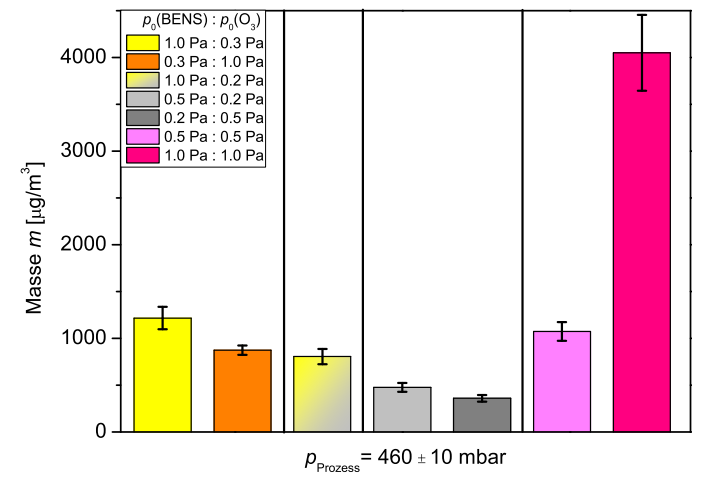

(b) Massenausbeuten.

Abb. 6.43.: Untersuchung des Einflusses verschiedener Eduktkonzentrationen auf die Partikelausbeuten bei der Butensäureozonolyse $\left(p_{\text {ges }}=460 \pm 10\right.$ mbar). Die Fehlerbalken wurden jeweils mit $\pm 10 \%$ des Mittelwertes abgeschätzt.

einem Reaktandenverhältnis von 0.5:0.5 (grau / magenta) bei letzterem eine deutlich höhere Anzahlkonzentration und Massenausbeute. Deutlich wirkt sich sie Anhebung beider Anfangskonzentrationen auf jeweils $1 \mathrm{~Pa}$ aus (pink), allerdings in verschiedenen Ausprägungen. Während sich die Anzahlkonzentration ungefähr verdoppelt, ist die Massenausbeute fast um den Faktor vier erhöht.

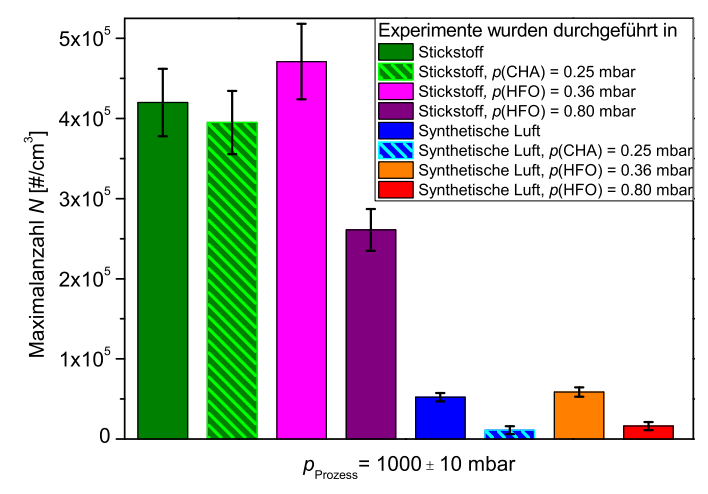

(a) Anzahlkonzentrationen.

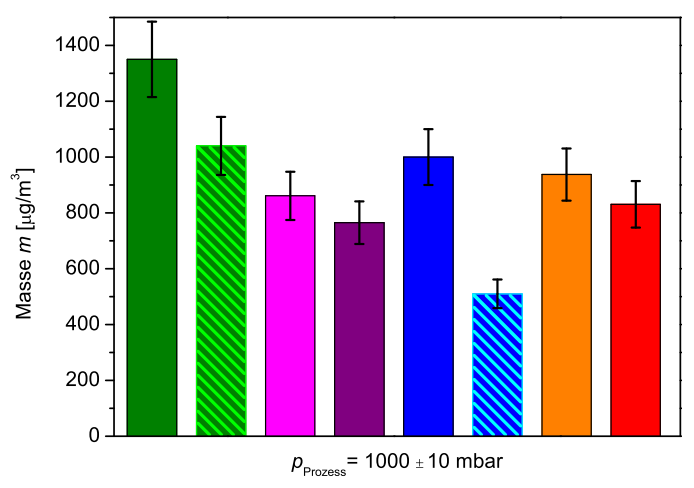

(b) Massenausbeuten.

Abb. 6.44.: Untersuchung des Einflusses der Badgase und verschiedener Additiva (CHA: Cyclohexan, HFO: Ameisensäure) auf die Partikelausbeuten bei der Butensäureozonolyse ( $p_{\text {ges }}=1000 \pm 10 \mathrm{mbar}$ ). Die Fehlerbalken wurden jeweils mit $\pm 10 \%$ des Mittelwertes abgeschätzt.

In Abbildung 6.44 sind die Partikelausbeuten verschiedener Butensäureozo- 
nolysen bei einem Gesamtdruck von $p_{\text {ges }}=1000 \pm 10$ mbar als Balkendiagramm dargestellt. Die einzelnen Messbedingungen unterscheiden sich dabei in der Wahl der Badgase und Additiva bzw. den Konzentrationen letzterer. Die Abbildungen der vollständigen Prozessdruckreihen sind aufgrund der zahlreichen miteinander zu vergleichenden Messreihen recht unübersichtlich und daher als Ergänzung im Anhang (Abschnitt $\mathrm{E}$ S. XXXIII) zu finden.

Auf den ersten Blick sind in Abb. 6.44a die durchgängig höheren Ausbeuten der Anzahlkonzentrationen in Stickstoff, unabhängig von sonstigen Beeinflussungen der Reaktionsumgebung durch Additiva, zu erkennen. Diese werden auch durch die Zugabe von Cyclohexan (CHA) oder Ameisensäure ( $H F O)$ mit der geringeren der gewählten Zugabemengen (entspricht dreifachem Überschuss bezüglich der Butensäure) zunächst nicht signifikant beeinflusst. Erst bei einer Erhöhung der Ameisensäurezugabe auf einen zwölffachen Überschuss ( $p_{\mathrm{HFO}}=0.80 \mathrm{mbar}$ ) zeigt sich eine deutliche Reduktion der Anzahlkonzentration.

Bei Verwendung von Synthetischer Luft entstehen generell recht geringe Anzahlkonzentrationen, die durch die Zugabe von Cyclohexan oder hohen Überschüssen von Ameisensäure noch einmal drastisch reduziert sind. Wie in Stickstoff hat die Zugabe von $p_{\mathrm{HFO}}=0.36$ mbar keine signifikanten Auswirkungen auf die Aerosolausbeute.

Die Massenausbeuten in Abb. 6.44bzeigen einen deutlich anderen Trend als die Anzahlkonzentrationen. Insgesamt unterscheiden sie sich untereinander bei verschiedenen Reaktionsbedingungen nicht so deutlich wie in der zuletzt betrachteten Auftragung. In Stickstoff reduzieren sich die Massenausbeuten bei Zugabe aller genannten Additiva, wobei diese Reduktion nur im Vergleich zum Experiment ohne Zugabe der Fängersubstanzen und nicht so sehr untereinander signifikant ist. In Synthetischer Luft scheint die Zugabe von Ameisensäure in beiden Fällen keine große Auswirkung zu haben, da nur unter Zugabe von Cyclohexan deutlich weniger Aerosolmasse als bei den übrigen drei Bedingungen detektiert werden konnte. 


\subsubsection{Fazit: Partikelbildung durch die Ozonolyse ungesättigter Carbonsäuren}

Die Untersuchung der Carbonsäuren zeigt, dass der Oxidationsgrad des Eduktmoleküls eine höhere Bedeutung für das Reaktionsverhalten zu haben scheint als die Struktur der Kohlenstoffkette. Dies lässt sich beispielsweise aus dem ähnlichen Reaktionsverhalten der beiden Carbonsäuren gegenüber den beiden verwendeten Badgasen oder Radikalfängerzugabe ableiten, wohingegen sich das der Butensäure stark von dem des 3-Buten-1-ols unterschiedet.

Die Säuren zeigen deutlich geringere Ausbeuten in Synthetischer Luft, dies kann mit destabilisierenden Effekten durch die Anlagerung von Sauerstoff an zur Nukleation notwendigen Ozonolyseprodukte erklärt werden.

Bei Betrachtung der Massenausbeuten wird deutlich, dass Butensäure der bessere Partikelbildner ist, da bei wesentlich niedrigerem Ozonumsatz $\left(p_{\mathrm{O}_{3}, \mathrm{BENS}}=0.10 \mathrm{~Pa}\right.$ gegenüber $\left.p_{\mathrm{O}_{3}, \mathrm{MACS}}=0.25 \mathrm{~Pa}\right)$ ähnliche Werte detektiert werden.

Das erhöhte Partikelbildungspotential für Butensäure im Vergleich zur Methacrylsäure muss auf die fehlende Methylsubstituierung zurückzuführen sein, da die Säurefunktionalität in beiden Verbindungen enthalten ist, beide eine terminale Doppelbindung und das gleiche Molekulargewicht $(M=86.09 \mathrm{~g} / \mathrm{mol})$ besitzen. Eine ähnliche Beobachtung zeigte sich bereits beim Vergleich von 1Hexen und 2-Methyl-1-penten, wo für das lineare Molekül ebenfalls signifikant höhere Ausbeuten resultierten.

Die Zugabe von $\mathrm{OH}$-Fängern führt zu einer Reduktion in Anzahlkonzentrationen und Massenausbeuten, wobei letztere nur in der Butensäureozonolyse in Synthetischer Luft deutlicher ausgeprägt ist.

Die Ergebnisse der im vorangegangenen Abschnitt dargestellten Experimente unter Zugabe verschiedener Fängermoleküle ergänzen das Bild hinsichtlich der Bedeutung der in der Ozonolysereaktion entstehenden CRIEGEE-Intermediate. Unter der Voraussetzung, dass diese eine wichtige Rolle in der betrachteten (eventuell substanzspezifischen) Aerosolbildung spielen, wird zunächst einmal deutlich, dass die Abfangreaktion der $\mathrm{Cl}$ mit Ameisensäure nicht sehr effektiv bzw. mit niedrigerer Geschwindigkeit als die potenzielle $\mathrm{Cl}+$ Butensäure-Reaktion abläuft. Dies lässt sich anhand der unter lediglich 
mit dreifachem Überschuss durchgeführten Partikelbildungsexperimenten (in magenta bzw. orange in Abb. 6.44 dargestellt) belegen, die in ihren Ausbeuten kaum einen Unterschied zu den reinen Badgasexperimenten erkennen lassen. Allerdings werden die Ausbeuten bei höheren Überschüssen durchaus beeinflusst, wobei sich die zusätzlich mögliche Abfangreaktion des $\mathrm{Cl}$ durch Ameisensäure unterschiedlich auf die einzelnen Größen in den beiden verwendeten Badgasen auszuwirken scheinen.

Während sich Anzahl und Massenausbeute bei Zugabe von $p_{\text {HFO }}=0.80$ mbar (zwölffacher Überschuss zu Butensäure) in Stickstoff verringern, lässt sich sogar bei diesen großen Überschüssen keine Veränderung für die Massenausbeuten in Synthetischer Luft im Vergleich zur reinen Ozonolyse feststellen, während die Anzahlkonzentration abnimmt. Dabei ist allerdings zu beachten, dass die Aerosolausbeute in Synthetischer Luft durch die reine Ozonolyse grundsätzlich verringert ist im Vergleich zu Stickstoff. Besonders ausgeprägt trifft dies auf die Anzahlkonzentration zu.

Die relative Betrachtung der Ergebnisse der Zugabeexperimente im Vergleich mit dem jeweiligen reinen Badgas zeigt nicht nur, dass die $\mathrm{OH}$-Radikalchemie in Stickstoff und Synthetischer Luft unterschiedlich starke Bedeutung besitzt, sondern auch, dass sich ebenfalls die Abfangreaktion der CRIEGEE-Intermediate mittels Ameisensäure in den verwendeten Badgasen unterschiedlich auswirkt, da sie die Ergebnisse in Stickstoff stärker beinflusst.

Insgesamt kann davon ausgegangen werden, dass die in der Ozonolysereaktion gebildeten $\mathrm{OH}$-Radikale in der Bildung von Partikelkeimen und ihren Vorläufern in einer Stickstoffatmosphäre nur eine geringe Bedeutung besitzen. Allerdings weisen die Ergebnisse darauf hin, dass die gebildeten CRIEGEEIntermediate eine hohe Bedeutung im Nukleationsprozess besitzen, was auch durch die Messungen in Ameisensäureanwesenheit bestätigt wurde. Da sich die Partikelbildung jedoch auch unter Ameisensäurezugabe nicht vollständig unterdrücken lässt, können diese nicht die einzige nukleationsinitiierende Spezies im Reaktionsgemisch sein.

Bei diesen Betrachtungen muss beachtet werden, das in Abb. 6.44 lediglich Messwerte bei Atmosphärendruck betrachtet wurden, wo durch Stoßstabilisierung der höchste Anteil an stablisierten CRIEGEE-Intermediaten vorliegen und der Einsatz eines $\mathrm{Cl}$-Fängers dementsprechend auch den größten Effekt rela- 
tiv zu Experimenten ohne Zugabe zeigen sollte. Demzufolge sollte sich anhand der Ameisensäurezugabeexperimente auch abschätzen lassen, ab welchem Gesamtdruck die in der Butensäureozonolyse entstehenden $\mathrm{Cl}$ effektiv stoßstabilisiert werden. Bei Betrachtung der Abbildung E.1 (Abschnitt E, S. XXXIII) ist ersichtlich, dass sich in der Auftragung der Anzahlkonzentrationen gegen den Prozessdruck (Abb. E.1a) ab einem Gesamtdruck von etwa 200 mbar Unterschiede in den Ausbeuten bei einer HFO-Zugabe von $p_{\mathrm{HFO}}=0.80 \mathrm{mbar}$ zeigen. Der gleiche Effekt ist für die Aerosolmassen schon ab 100 mbar Gesamtdruck sichtbar.

Somit kann davon ausgegangen werden, dass die CRIEGEE-Intermediate ab einem Druck von etwa $p_{\text {ges }}=100-200$ mbar effektiv stabilisiert werden können und durch sekundäre Reaktionen mit verschiedenen Verbindungen, wie beispielsweise dem Eduktmolekül, auch zur Aerosolausbeute beitragen. Die Ergebnisse unterhalb dieser Gesamtdrücke werden nicht durch HFO beeinflusst. Dies weist darauf hin, dass die Zunahme der Aerosolausbeuten im betrachteten Druckbereich vor allem auf die Stoßstabilisierung von nukleationsfördernden Ozonolyseprodukten zurückzuführen ist.

\subsubsection{Fazit: Partikelbildung aus der Alkenozonolyse}

Wie die dargestellten Abhängigkeiten zeigen, übt der Oxidationsgrad des ungesättigten Eduktmoleküls einen entscheidenden Einfluss auf das Partikelbildungsverhalten aus.

Sichtbar wird dies beispielsweise im direkten Vergleich der sich nur im Oxidationsgrad unterscheidenden Paare 1-Penten-3-ol / 1-Penten-3-on, 4-Penten-1ol / 4-Pentenal und 3-Buten-1-ol / Butensäure bei analoger Struktur des Kohlenstoffkörpers. Der generelle Trend, unter Sauerstoffanwesenheit höhere Partikelausbeuten zu produzieren, bleibt von den reinen Kohlenwasserstoffverbindungen bis zu den Carbonylverbindungen unverändert. Lediglich bei den hochoxidierten Säuren hat Sauerstoff eine behindernde Wirkung auf die Keimbildung und eingeschränkt auch auf den Massenaufbau.

Gerade bei den aus den untersuchten Säuren entstehenden, mit einem $\mathrm{C}_{3}{ }^{-}$ Grundkörper recht kleinen, CRIEGEE-Intermediaten, die mit der Säure- und der Carbonyloxidgruppe bereits zwei Substituenten mit ausgeprägtem -I-Effekt tra- 
gen, könnte durch die Anlagerung von weiterem Sauerstoff aus dem Badgas eventuell so viel Elektronendichte aus der Kohlenstoffkette abgezogen werden, dass es zum C-C-Bindungsbruch kommt. Die Folge ist der Zerfall dieser hochoxidierten Verbindungen zu kleineren Bruchstücken, deren Dampfdruck zu hoch für die Kondensation ist. In Kapitel 6.2.3 konnte die höhere Neigung zum Zerfall der stärker voroxidierten Butensäure gegenüber dem 3-Buten-1ol anhand der stark erhöhten Ketenbildung bereits gezeigt werden, was diese Annahme bestätigt.

Auch der Abstand der reaktiven Zentren zueinander scheint eine Bedeutung für das Verhalten bei der Partikelbildung zu besitzen. Scheinbar reagieren die Edukte mit deutlicheren Unterschieden in den Ausbeuten in verschiedenen Badgasen, je weiter die Doppelbindung und die voroxidierte Gruppe auseinanderliegen. Dies ergibt sich aus den Ergebnissen der Alkohole, wo beim größten Abstand der funktionellen Gruppen die größten Differenzen auftreten.

Die Partikelbildung ist auch in der Ozonolyse von Edukten mit relativ kurzen Kohlenstoffketten möglich. Wie die Ergebnisse von 3-Buten-1-ol und Butensäure zeigt, ist durch Voroxidierung eine ausreichende Reduktion des Dampfdruckes realisierbar, um große Ausbeuten zu erhalten.

Lineare Grundstrukturen scheinen für höhere Ausbeuten zu sorgen als Edukte mit Methylsubstitution an der Doppelbindung. Dies wird im Vergleich der Ausbeuten von 1-Hexen und 2-Methyl-1-penten bzw. Butensäure und Methacrylsäure deutlich, beide Paare besitzen jeweils die gleiche Summenformel und damit identisches Molekulargewicht. Bei den reinen Kohlenwasserstoffverbindungen zeigen sich mit 1-Hexen bei gleichem Ozonumsatz deutlich höhere Ausbeuten in Anzahlkonzentration und Massenausbeute. Das Verhalten gegenüber dem Badgaswechsel ist jedoch ähnlich, in beiden Fällen verringern sich die Kenngrößen in Stickstoff nur geringfügig. In Butensäure ist ebenfalls die höhere Ausbeute zu beobachten, vor allem unter Berücksichtigung der deutlich geringeren Ozonumsätze $\left(p_{\mathrm{O}_{3}, \mathrm{BENS}}=0.10 \mathrm{~Pa}\right.$ gegenüber $\left.p_{\mathrm{O}_{3}, \mathrm{MACS}}=0.25 \mathrm{~Pa}\right)$. Der Wechsel von Synthetischer Luft zu Stickstoff sorgt bei beiden Carbonsäuren gleichermaßen für einen starken Zuwachs in Anzahl und Masse.

Die Untersuchungen haben außerdem gezeigt, dass der Einsatz von Fängermolekülen, die bestimmte Zwischenprodukte der Ozonolysereaktion, wie $\mathrm{OH}$ Radikale oder CRIEGEE-Intermediate, abfangen sollen, teilweise zu unerwarte- 
ten Ergebnissen führen. So wurde in Abschnitt 6.4.1.2 dargelegt, dass der Einsatz von Cyclohexan als $\mathrm{OH}$-Radikalfänger abhängig vom verwendeten Ausgangsalken entweder keine (1-Hexen), eine leicht reduzierende (Methylcyclopenten) oder eine stark anregende (2-Methyl-1-penten) Wirkung auf die Aerosolbildung haben kann. Da mit 1- und 2-Butanol zwei weitere $\mathrm{OH}$-Radikalfänger Verwendung fanden, die unter Nichtberücksichtigung der Folgereaktionen und -produkte die gleiche Wirkung auf die $\mathrm{OH}$-Chemie haben sollten, allerdings mit der eduktunabhängigen vollständigen Unterdrückung jeglicher Aerosolbildung zu deutlich abweichenden Ergebnissen führten, zeigt dies, dass die Sekundärchemie der Fängersubstanzen nicht uneingeschränkt zu vernachlässigen ist.

Beispielsweise lassen die Ergebnisse im Falle des Cyclohexans darauf schließen, dass die Verwendung des Fängers nicht nur zur Verhinderung $\mathrm{OH}$ radikalinduzierter Reaktionskanäle und damit einer Vereinfachung des Partikelbildungsmechanismus führt. Stattdessen werden durch bestimmte Folgereaktionen Nebenprodukte gebildet, die zum Aufbau partikulärer Materie führen können, was eine Interpretation der eigentlich betrachteten Ozonolysevorgänge wiederum erschwert. Zusätzlich wird durch die hohen auftretenden Absorbanzen des $\mathrm{C}_{6} \mathrm{H}_{12}$ mit damit verbundenen Nichtlinearitäten und der Überlagerung der Signale der Ozonolyseprodukte allerdings auch die Untersuchung der Gasphasenchemie verkompliziert. Unter der Annahme, dass die Aerosolbildung eine Folge der in der Gasphase ablaufenden Prozesse ist, ist der Radikalfängereinsatz bei den gegebenen apparativen Möglichkeiten für Produktanalysen nur eingeschränkt sinnvoll. Vielmehr lohnt sich der Einsatz, um aus Vergleichsexperimenten zu Partikelmessungen für Ozonolysen im reinen Badgas erste Hinweise auf bedeutende Stellen im Mechanismus zu erhalten. Damit wird dem Ansatz der Kinetik gefolgt, der davon ausgeht, dass die Produktanalysen nicht durch die Alken $+\mathrm{OH}$-Reaktion verfälscht wird.

Eventuell können auch die Kohlenstoffmonoxidausbeuten teilweise als Marker dafür genutzt werden, in welchem Badgas eine effektivere Partikelbildung erfolgt. Zumindest ein stichprobenartiger Vergleich der Ausbeuten aus Ozonolysen voroxidierter Edukte zeigt, dass die gebildeten Menge CO die gleiche Tendenz wie die Partikelausbeuten zeigen. So entsteht in 3-Buten-1-ol in Synthetischer Luft mehr CO, während die Bildung bei Butensäure in Stickstoff ausgeprägter ist (vgl Tab. B.1 und B.2). Aufgrund der unter Niederdruckbedin- 
gungen jedoch recht hohen Unsicherheit in der Konzentrationsbestimmung des Kohlenstoffmonoxids und der Tatsache, dass dieses Verhalten bei 1-Hexen und 1-Penten-3-on nicht auftritt, bedarf diese These allerdings weiterer und ausführlicherer Experimente sowie einer deutlich intensiveren Beschäftigung mit den eduktspezifischen CO-Bildung, bevor hier gesicherte Aussagen getroffen werden können.

\subsection{Experimente unter Schwefeldioxidzugabe}

Sämtliche Experimente wurden in Synthetischer Luft als Badgas und bei Alkenüberschuss durchgeführt, so dass die Ozonkonzentration den Gesamtumsatz limitierte. In den Modellsubstanzen mit sechsgliedrigen Ringen wurde jeweils $p_{\mathrm{O}_{3}}=0.10 \mathrm{~Pa}$ Ozon umgesetzt (für Methylcyclopenten $p_{\mathrm{O}_{3}}=0.14 \mathrm{~Pa}$ ), während diese bei den Terpenen wegen des höheren Partikelbildungspotentials auf $p_{\mathrm{O}_{3}}=0.05 \mathrm{~Pa}$ reduziert werden musste. Trotz dieses geringen Umsatzes konnten die großen Anzahlkonzentrationen Koagulationseffekte und Nichtlinearitäten des CPC durch Verwendung verschiedener Zählmodi hervorrufen. Die Anfangskonzentrationen der bereits von WoLF et al. [17, 48] untersuchten Alkene wurden wie in den genannten Arbeiten gewählt, um eine Vergleichbarkeit der erhaltenen Ergebnisse zu gewährleisten. Trotzdem ergaben sich Differenzen in den tatsächlich verwendeten Ozonkonzentrationen, die auf den Einbau der neuen UV-Optik (Abb. 4.2 in Kap. 4.2.1.2, AUFBAU 2) und die damit verbundene genauere Konzentrationsbestimmung zurückzuführen ist. Diese Differenz führte in den aktuellen Messungen zu im Vergleich etwas verringerten Ausbeuten, wobei das generelle Verhalten jedoch erhalten blieb.

In den nachfolgend dargestellten Ergebnissen der Partikelbildungsexperimente mit endozyklischen Edukten werden erst Modellsubstanzen und anschließend atmosphärenrelevante Terpene präsentiert. Gleiches gilt für die Edukte mit exozyklischer Struktur. Die Modellsubstanzen bieten durch ihre einfachere Struktur leichter die Möglichkeit, Abhängikeiten der Partikelausbeute mit bestimmten Strukturmotiven oder Intermediaten in Verbindung zu bringen, die dann auf die Übertragbarkeit auf die biogenen Verbindungen überprüft werden können. 


\begin{tabular}{|c|c|c|c|c|}
\hline Alken & $p_{0, \text { Alken }}[\mathrm{Pa}]$ & $p_{0, \text { Ozon }}[\mathrm{Pa}]$ & $t_{\mathrm{R}}[\mathbf{s}]$ & $p_{\mathrm{SO}_{2}}[\mathrm{~Pa}]$ \\
\hline Cyclohexen & 1.2 & 0.09 & 300 & $0-0.313$ \\
\hline Methylcyclopenten & 0.5 & 0.14 & 300 & $0-0.100$ \\
\hline Methylcyclohexen & 1.2 & 0.09 & 300 & $0-0.100$ \\
\hline$\alpha$-Pinen & 1.0 & 0.04 & 300 & $0-0.100$ \\
\hline Me'thylencyclohexan & 3.0 & $0 . \overline{0} \overline{8}$ & $3 \overline{0} \overline{0}$ & $\overline{0}-0 . \overline{100}$ \\
\hline$\beta$-Pinen & 1.0 & 0.04 & 600 & $0-0.100$ \\
\hline
\end{tabular}

Tab. 6.11.: Auflistung der Anfangspartialdrücke der verwendeten Edukte für Partikelbildungsexperimente bei variablem Gesamtdruck unter $\mathrm{SO}_{2}-$ Zugabe, generell kann hier von einer einfüllbedingten Unsicherheit von $10-20 \%$ für $\mathrm{O}_{3}$ und $\mathrm{SO}_{2}$ ausgegangen werden.

Es wurden prinzipiell vier verschiedene Variationen der $\mathrm{SO}_{2}$-Experimente durchgeführt, wobei allerdings nur mit Cyclohexen sämtliche Messreihen und mit den übrigen Verbindungen nur bestimmte Versuche zum Vergleich einzelner Aspekte innerhalb des Mechanismus durchgeführt wurden. Eine Zusammenfassung der Reaktionsbedingungen findet sich in den angegebenen Tabellen. Die Untersuchungen umfassten die Variation der folgenden Parameter:

- der Gesamtdruck (Tabelle 6.11),

- die Schwefeldioxidkonzentration (Tabelle 6.11),

- die Auswirkungen der im Gemisch vorherrschenden relativen Luftfeuchtigkeit (Tabelle 6.12)

- und den Einfluss der Zugabe bestimmter Carbonylverbindungen auf die Partikelausbeuten (Tabelle 6.13).

\begin{tabular}{|c|c|c|c|}
\hline Alken & $\boldsymbol{p}_{\mathrm{SO}_{2}}[\mathrm{~Pa}]$ & $\boldsymbol{p}_{\mathrm{H}_{2} \mathrm{O}}[\mathrm{Pa}]$ & relative Luftfeuchte $\phi[\%]$ \\
\hline Cyclohexen & $0.0002-0.1$ & $0-700$ & $0-26.5$ \\
\hline$\alpha$-Pinen & 0.030 & $0-700$ & $0-26.5$ \\
\hline
\end{tabular}

Tab. 6.12.: Anfangspartialdrücke der verwendeten Additiva für Partikelbildungsexperimente zum Wassereinfluss unter $\mathrm{SO}_{2}$-Zugabe, Alken- und Ozonkonzentrationen sowie Reaktionszeit wie in Tab. 6.11. 


\begin{tabular}{|c|c|c|c|}
\hline Alken & $\boldsymbol{p}_{\mathrm{SO}_{2}}[\mathrm{~Pa}]$ & $\boldsymbol{p}_{\text {Propionaldehyd }}[\mathrm{Pa}]$ & $\boldsymbol{p}_{\text {Nopinon }}[\mathrm{Pa}]$ \\
\hline$\alpha$-Pinen & 0.030 & 0.3 & 0 \\
$\alpha$-Pinen & 0.030 & 0 & 0.3 \\
\hline
\end{tabular}

Tab. 6.13.: Anfangspartialdrücke der verwendeten Additiva für Partikelbildungsexperimente zum Carbonyleinfluss unter $\mathrm{SO}_{2}$-Zugabe, Alken- und Ozonkonzentrationen sowie Reaktionszeit wie in Tab. 6.11.

Eine Änderung in den Partikelausbeuten bei Zugabe von Schwefeldioxid lässt sich mit seiner Oxidation durch in der Ozonolysereaktion gebildeten $\mathrm{OH}$ Radikalen zu Schwefelsäure erklären [87] (vgl. Kap. 2.8). Auf welche Art die gebildete Schwefelsäure genau wirksam ist, wird jedoch seit längerer Zeit von verschiedenen Arbeitsgruppen kontrovers diskutiert. Während ZHANG et al. [22] aus ihren Untersuchungen auf die Bildung stabiler Verbindungen aus organischen Säuren und $\mathrm{H}_{2} \mathrm{SO}_{4}$ schließen, die in die Partikelphase übergehen, gehen BERNDT et al. [93] davon aus, dass die Alkenozonolyse und aus ihr stammende Produkte keine Bedeutung für den Partikelbildungsprozess besitzt, außer als $\mathrm{OH}$-Radikalquelle für die Schwefelsäureproduktion zu dienen.

BonN et al. [12] postulieren dagegen einen Mechanismus, in dem die organischen und anorganischen Komponenten interagieren. Ihre These besagt, dass die Produkte aus der Reaktion von Schwefelsäure und stabilisierten CRIEGEEIntermediaten, sogenannte Organosulfate, als Nukleationskeime im Partikelbildungsprozess wirken können.

Die durchgeführten Experimente hatten das Ziel, herauszufinden, ob sich eine der Theorien durch die gewählten Bedingungen bestätigen oder widerlegen lässt. Die Wirkung des Gesamtdruckes auf die durch $\mathrm{SO}_{2}$ beeinflusste Partikelbildung wurde im Rahmen dieser Arbeit erstmals untersucht.

Die Fehlerangaben der gezeigten Ergebnisse ergeben sich wie zuvor aus der relativen Abweichung eines häufig gemessenen Punktes unter Berücksichtigung des Student'schen Faktors für ein Konfidenzintervall von $95.5 \%$ [118]. Dabei wurden die Messungen bei einem Gesamtdruck von ca. 450 mbar und einem mitteleren $\mathrm{SO}_{2}$-Partialdruck von $0.01 \mathrm{~Pa}$ (Cyclohexen, Methylcyclopenten) bzw. 0.03 Pa ( $\alpha$-Pinen) herangezogen. Die relativen Abweichungen, die für $\alpha$ Pinen bestimmt wurden, wurden auch für die Verbindungen mit mehr als sechs 
Kohlenstoffatomen herangezogen, da die Ausbeuten für diese Verbindungen in einer ähnlichen Größenordnung liegen und vorausgesetzt wurde, dass die relativen Abweichungen stärker durch die Ausbeuten als durch die Struktur des Eduktes bestimmt sind.

\begin{tabular}{|c|c|c|}
\hline Alken & Rel. Fehler der Anzahl [\%] & Rel. Fehler der Masse [\%] \\
\hline \hline$\alpha$-Pinen & 11.0 & 32.0 \\
\hline Cyclohexen & 18.2 & 27.0 \\
\hline
\end{tabular}

Tab. 6.14.: Relative Fehler der Partikelausbeuten in $\mathrm{SO}_{2}$-Anwesenheit.

Zum Vergleich sind in Tabelle 6.14 die relativen Fehler angegeben. Zu beachten ist, dass bei kleineren Ausbeuten die relative Schwankung höher ausfallen kann und der Fehler unterschätzt werden könnte.

Die $\mathrm{SO}_{2}$-Experimente wurden bis zu Gesamtdrücken von höchstens $p_{\text {ges }}=450 \pm 10$ mbar durchgeführt, da dies der höchste aus der VMKExpansion erreichbare Druck ist. Die gleichbleibenden Expansions- und schnellen Durchmischungsvorgänge in der kürzestmöglichen Zeit ermöglichen die beste Reproduzierbarkeit.

\subsubsection{Cyclohexen}

\section{Abhängigkeit vom $\mathrm{SO}_{2}$-Partialdruck}

In den Abbildungen 6.45a und 6.45b sind in schwarz Anzahlkonzentrationen und Massenausbeuten unter Variation der $\mathrm{SO}_{2}$-Konzentration und der relativen Luftfeuchte dargestellt. Alle Werte wurden bei einem Gesamtdruck von $p_{\text {ges }}=450 \pm 10$ mbar erhalten. Es ist deutlich zu sehen, dass die Ausbeuten beider Kenngrößen mit dem $\mathrm{SO}_{2}$-Partialdruck stark zunehmen. Die Ergebnisse reinen Ozonolysereaktion lassen erkennen $\left(p_{\mathrm{SO}_{2}}=0 \mathrm{~Pa}: N \approx 4.7 \cdot 10^{3} \mathrm{~cm}^{-3}\right.$, $\left.m \approx 130 \mu \mathrm{g} \cdot \mathrm{m}^{-3}\right)$, dass Cyclohexen ein recht geringes Partikelbildungspotenzial zeigt. Beide Größen zeigen sich im geringeren Konzentrationsbereich sehr empfindlich gegenüber der $\mathrm{SO}_{2}$-Partialdruckerhöhung, während die Massenausbeuten ab etwa $p_{\mathrm{SO}_{2}}=0.05 \mathrm{~Pa}$ konstant bleiben. Die Anzahlkonzentrationen nehmen dagegen oberhalb dieses Wertes weiterhin monoton zu. 


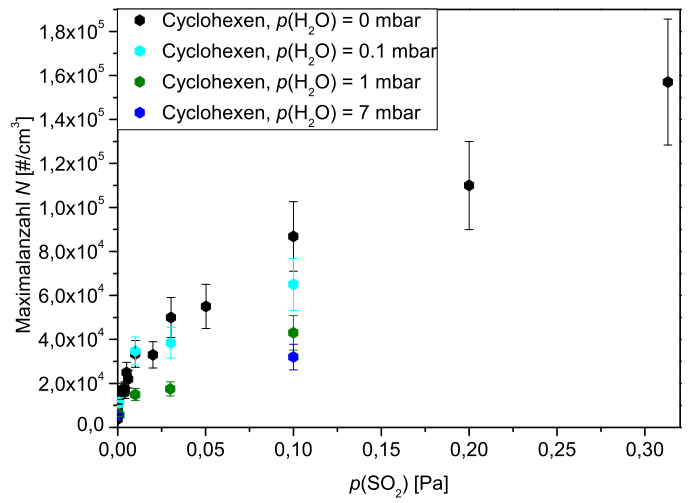

(a) Anzahlkonzentration.

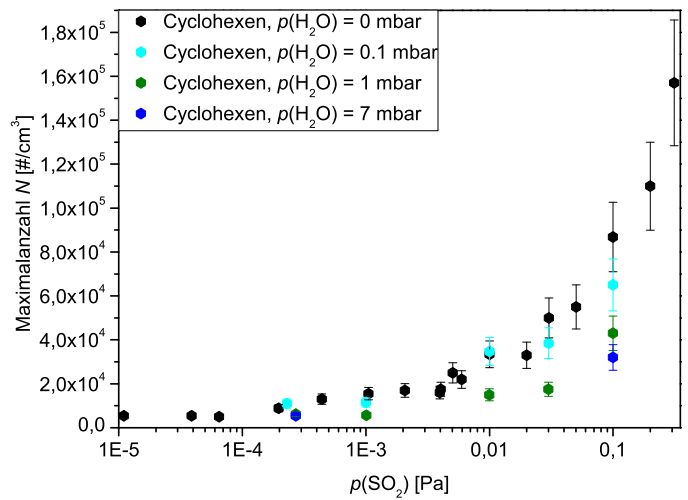

(c) Anzahlkonzentration, logarithmische Auftragung.

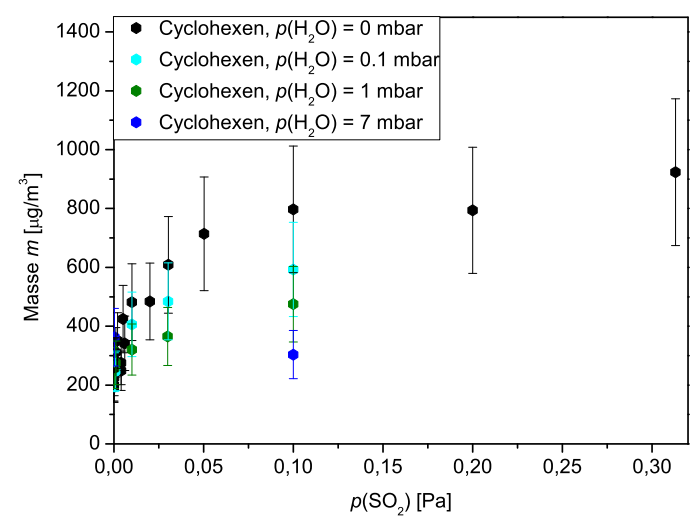

(b) Massenausbeute.

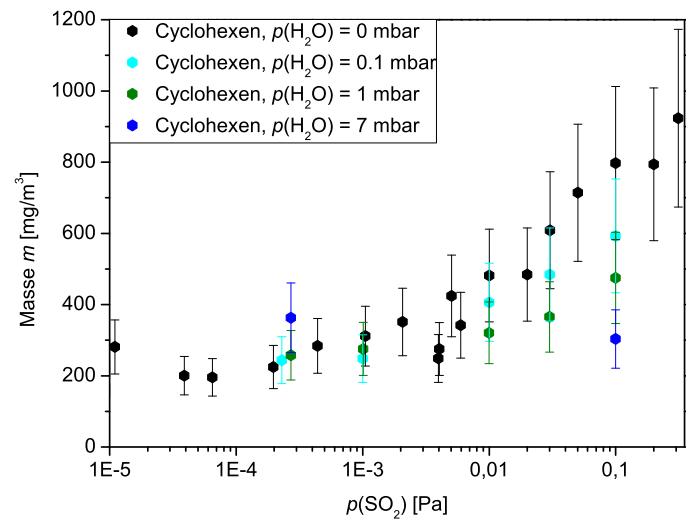

(d) Massenausbeute, logarithmische Auftragung.

Abb. 6.45.: Untersuchung des $\mathrm{SO}_{2}$-Einflusses auf die Partikelausbeute für Cyclohexen in Synthetischer Luft unter Zugabe verschiedener Wasserpartialdrücke bei $p_{\text {ges }}=460 \pm 10$ mbar. Neben den linearen werden auch logarithmische Auftragungen gezeigt.

Zur besseren Veranschaulichung ist der Bereich unterhalb von $p_{\mathrm{SO}_{2}}=0.12 \mathrm{~Pa}$ in den Abb. 6.45c und 6.45d noch einmal in logarithmischer Auftragung dargestellt. In der Auftragung der Anzahlkonzentration scheint bei etwa $p_{\mathrm{SO}_{2}}=0.01 \mathrm{~Pa}$ eine Art Grenzkonzentration erreicht, da der Anstieg zuvor recht moderat war, aber oberhalb dieses Wertes stark zunimmt. In der Massenausbeute ist dieses Verhalten nicht so ausgeprägt zu beobachten.

Das Verhalten der Anzahlkonzentrationen könnte durch die theoretische Betrachtung der Kinetik der Reaktion $\mathrm{SCl}+\mathrm{H}_{2} \mathrm{SO}_{4} \longrightarrow$ Partikelkeim mit dem 

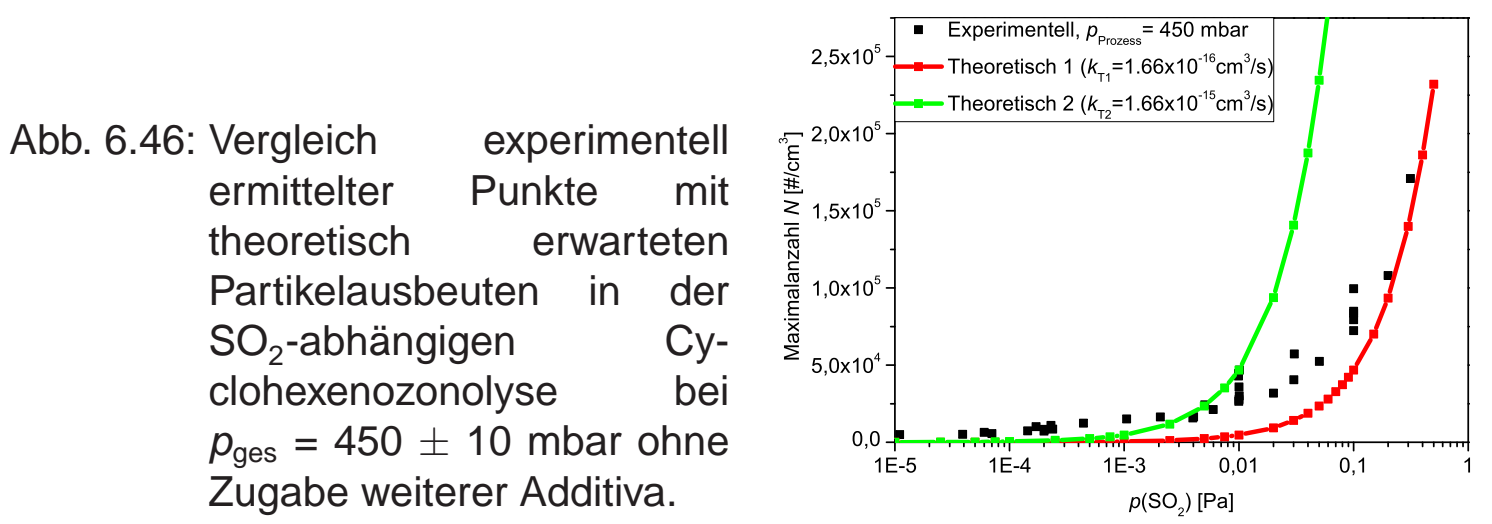

DARS-Programmpaket [138] erklärt werden8. Hier wurde nach Vorbild von BonN et al. [12] vorausgesetzt, dass sich die Anzahlkonzentration aus der zuvor genannten NIM-bildenden Reaktion ergibt, der Geschwindigkeitskoeffizient dieser Umsetzung wurde in den Untersuchungen anschließend variiert. In den Simulationen wurden der von WOLF [139] bestimmte Geschwindigkeits-

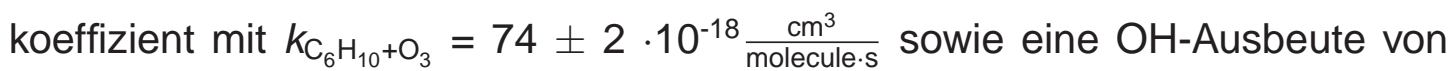
$Y_{\mathrm{OH}}=0.5$ vorausgesetzt. Unter trockenen Bedingungen (laut Badgasspezifikation für Synthetische Luft: $x_{\mathrm{H}_{2} \mathrm{O}}<2$ ppm) zeigte sich, dass die Schwefelsäurebildung auf den gleichen Zeitskalen wie die Ozonolyseraktion abläuft, wobei sich erstere weitgehend unabhängig von der zugegebenen Wassermenge zeigte, was sich aus der $\mathrm{SO}_{2}+\mathrm{OH}$-Reaktion als geschwindigkeitsbestimmendem Schritt erklärt [139]. Die mögliche Bildung zusätzlicher Schwefelsäure aus der Reaktion stabilisierter CRIEGEE-Intermediate wurde aufgrund des geringen Anteils gebildeter SCI [140] als vernachlässigbar angenommen.

Abbildung 6.46 zeigt, dass die experimentell gemessenen Punkte in ihrem prinzipiellen Trend von den Kurven, die sich bei Verwendung eines Geschwindigkeitskoeffizienten von $k_{T 1}=1.66 \cdot 10^{-16} \frac{\mathrm{cm}^{3}}{\text { molecule.s }}$ bzW. $k_{\mathrm{T} 2}=1.66 \cdot 10^{-15} \frac{\mathrm{cm}^{3}}{\mathrm{molecule}^{-\mathrm{s}}}$ ergeben, recht gut widergegeben werden.

In den Auftragungen in Abb. 6.45 sind neben den reinen Schwefeldioxidzugabeexperimenten zusätzlich einige Vergleichsmessungen bei verschiedenen relativen Luftfeuchtigkeiten zu sehen. Die in der Legende angegebenen volumetrisch bestimmten Wasserzugaben entsprechen einer relativen Luftfeuchte von $R H_{0.1 / 1 / 7 \mathrm{mbar}} \approx 0.4 / 3.8 / 26 \%$ (s. Tabelle 4.3 in Abschnitt 4.3.2). Die oh-

\footnotetext{
${ }^{8}$ Das genaue Vorgehen ist in [139] beschrieben.
} 
ne zusätzliche Wasserzugabe durchgeführten Experimente besitzen nominell eine relative Luftfeuchte von $R H=0.011 \%$, was aus dem nach Herstellerangaben maximalen Wassergehalt des Badgases von $x_{\mathrm{H}_{2} \mathrm{O}}<2 \mathrm{ppm} \stackrel{\wedge}{=} 2.0 \cdot 10^{-3} \mathrm{mbar}$ resultiert. Sie werden aufgrund dieses sehr niedrigen und nicht genau quantifizierbaren, da weit unter der Einfüllgenauigkeit liegenden, Wertes jedoch als "Trockenexperimente" betrachtet.

Die Zugabe von Wasser hat eine Verringerung der Aerosolausbeute zur Folge. Während bei einer relativen Lufteuchte von $R H=0.4 \%$ in Abb. 6.45a und $6.45 \mathrm{~b}$ die Reduktion erst ab einem Partialdruck zwischen $p_{\mathrm{SO}_{2}}=0.05-0.1 \mathrm{~Pa}$ signifikant ist, fallen die Ausbeuten bei $R H=3.8 \%$ schon ab $p_{\mathrm{SO}_{2}}=0.01 \mathrm{~Pa}$ geringer aus. Die Ausprägung des Wassereinflusses scheint also auch von der zugesetzten $\mathrm{SO}_{2}$-Menge abzuhängen.

\section{Abhängigkeit vom Gesamtdruck}

Es wurden auch Messreihen zur Bedeutung des Gesamtdruckes durchgeführt (Abb. 6.47), um festzustellen, ob dieser Faktor bei verschiedenen $\mathrm{SO}_{2}$-Zugaben ebenso bedeutsam für die Nukleation sein kann wie bei den zuvor durchgeführten reinen Ozonolyseexperimenten.

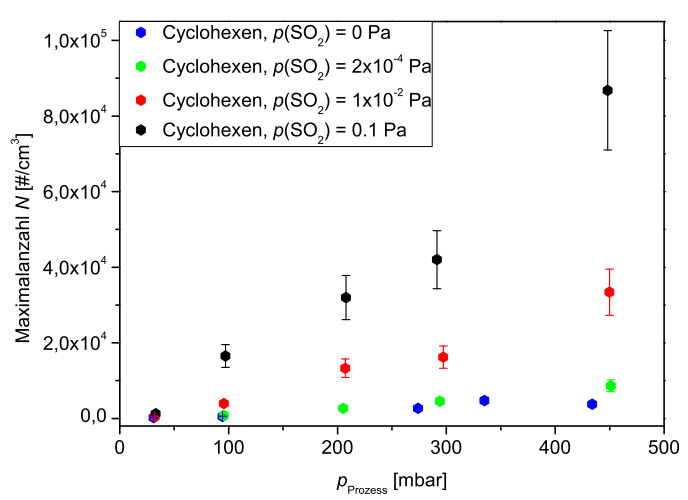

(a) Anzahlkonzentration.

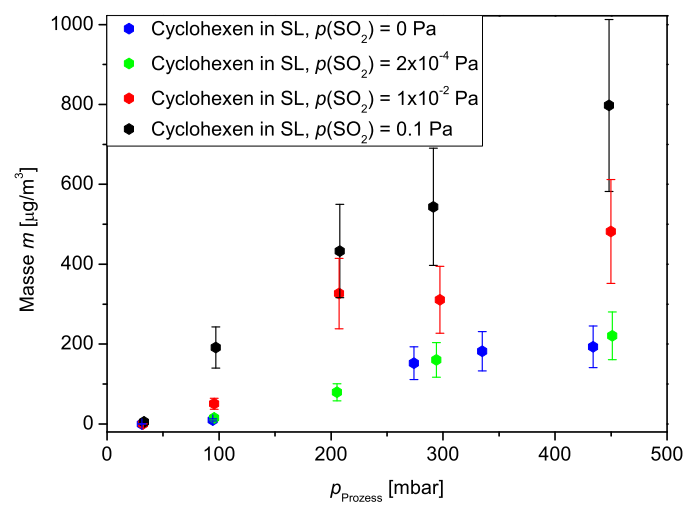

(b) Massenausbeute.

Abb. 6.47.: Untersuchung der druckabhängigen Partikelausbeute für Cyclohexen in Synthetischer Luft unter Zugabe verschiedener Mengen $\mathrm{SO}_{2}$.

Wie Abbildung 6.47a zeigt, ist die vollständige Unterdrückung der Nukleation bei jedem der gewählten Schwefeldioxidpartialdrücke im Niederdruckbereich möglich. Erst oberhalb eines Gesamtdruckes von etwa 80 mbar nehmen Anzahlkonzentration und Massenausbeute im Vergleich zur reinen Ozonolyse- 
reaktion zu, wobei dieser Effekt erst ab zugesetzten $\mathrm{SO}_{2}$-Partialdrücken von mindestens $p_{\mathrm{SO}_{2}}=0.01 \mathrm{~Pa}$ signifikant ist, während sich die Ausbeuten mit $p_{\mathrm{SO}_{2}}=2 \cdot 10^{-4} \mathrm{~Pa}$ nicht sehr stark von den $\mathrm{SO}_{2}$-freien Ergebnissen unterscheiden. Auch bei einem Gesamtdruck von 80 mbar zeigt nur der Messwert mit der höchsten $\mathrm{SO}_{2}$-Zugabe eine höhere Ausbeute als das reine Ozonolyseergebnis.

Insgesamt kann festgestellt werden, dass Schwefeldioxidzugaben die Nukleation unter Niederdruckbedingungen nicht effektiv erhöhen kann. Dafür spricht, dass die absolute Zunahme der Anzahlkonzentration bis etwa $p_{\text {ges }}=300$ mbar nicht so stark ausfällt wie die bei höherem Gesamtdruck. Dieser Effekt ist bei den Massenausbeuten nicht zu erkennen.

\subsubsection{Methylcyclopenten}

\section{Abhängigkeit vom Gesamtdruck}

Für Methylcyclopenten wurden einige Messungen unter Zugabe verschiedener $\mathrm{SO}_{2}$-Partialdrücke durchgeführt, um den Effekt auf die Partikelbildung bei Druckvariation vergleichend zu den Ergebnissen für Cyclohexen zu untersuchen. Dies bot sich an, da beide Verbindungen sich bei gleicher Kohlenstoffanzahl und endozyklischer Doppelbindung um die Methylsubstitution an dieser unterscheiden, wobei allerdings die unterschiedliche Ringgröße für eine höhere Ringspannung im Methylcyclopenten sorgt (vgl. Abb. 6.34).

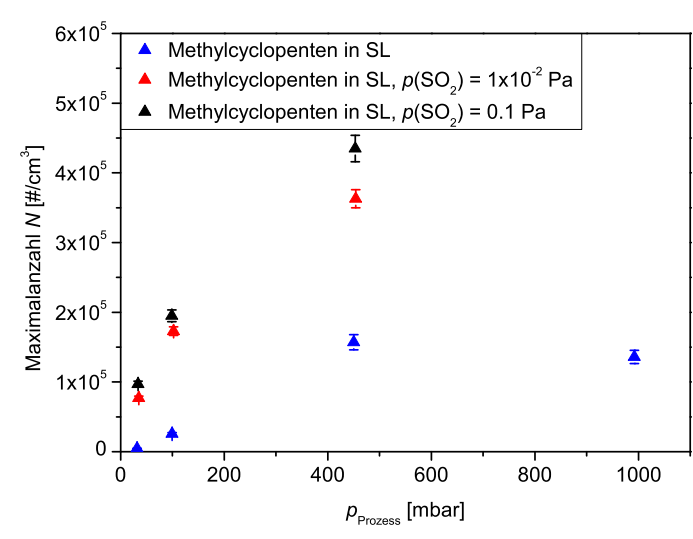

(a) Anzahlkonzentration.

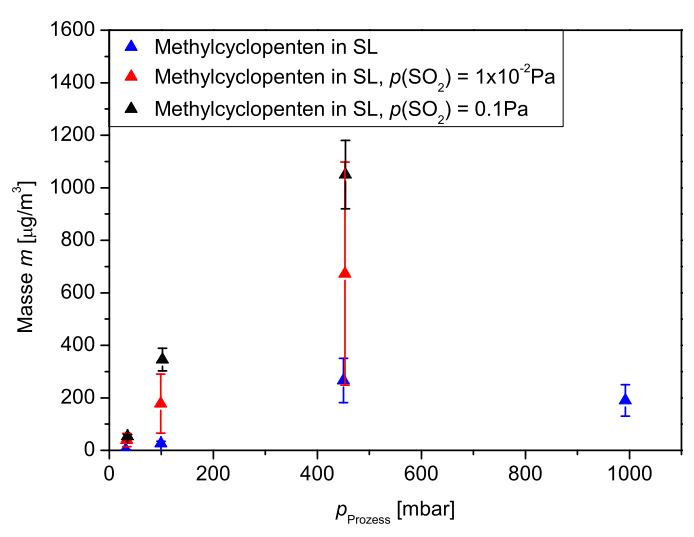

(b) Massenausbeute.

Abb. 6.48.: Untersuchung der druckabhängigen Partikelausbeute für Methylcyclopenten in Synthetischer Luft unter Zugabe von $\mathrm{SO}_{2}$. 
Wie Abbildung 6.48 zeigt, kann durch die Zugabe von $p_{\mathrm{SO}_{2}}=0.01 \mathrm{~Pa}$ die Ausbeute beider Kenngrößen bei der Methylcyclopentenozonolyse in Synthetischer Luft deutlich erhöht werden. Bei einer Steigerung auf $p_{\mathrm{SO}_{2}}=0.1 \mathrm{~Pa}$ nimmt die Anzahlkonzentration weiter zu (6.48a), während sich die Massenausbeute verringert, wie in Bild 6.48D dargestellt. Allerdings erhöht sich für die letztgenannte Messreihe auch die Streuung drastisch, wie die Fehlerbalken andeuten. Die Zunahmen für die Anzahlkonzentrationen werden mit steigendem Gesamtdruck geringer.

\subsubsection{Methylcyclohexen}

\section{Abhängigkeit vom Gesamtdruck}

Abbildung 6.49 zeigt, dass sich durch die Zugabe von Schwefeldioxid die Ausbeuten in der Methylcyclohexenozonolyse erhöhen lassen.

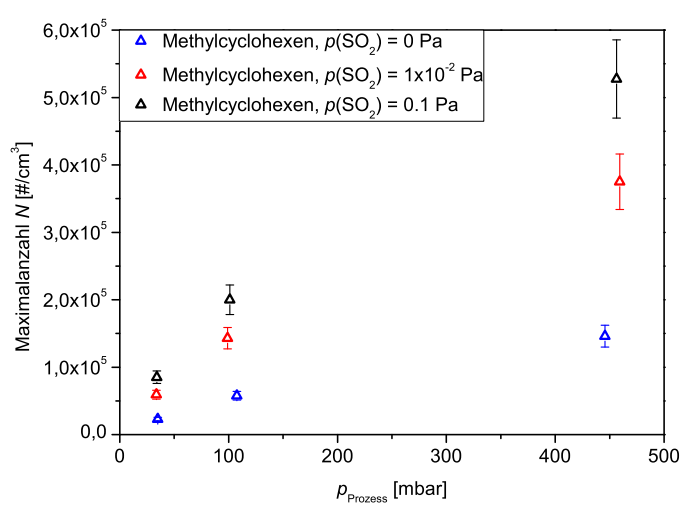

(a) Anzahlkonzentration.

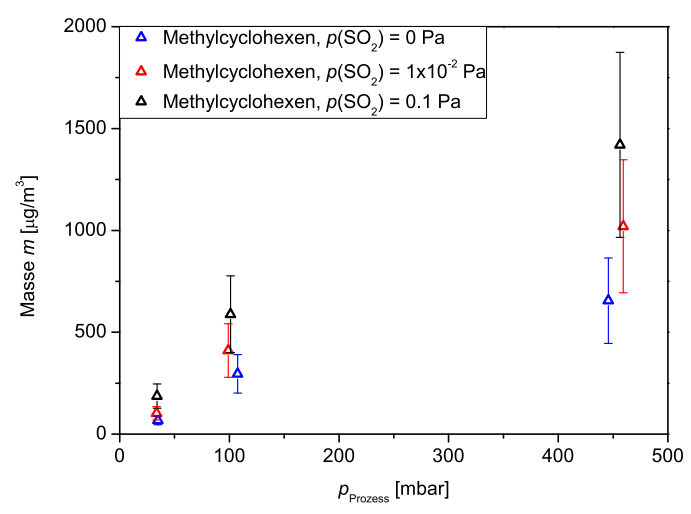

(b) Massenausbeute.

Abb. 6.49.: Untersuchung der druckabhängigen Partikelausbeute für Methylcyclohexen in Synthetischer Luft unter Zugabe verschiedener Mengen $\mathrm{SO}_{2}$.

Wie bei den zuvor beschriebenen Verbindungen verringern sich Anzahlkonzentration (Abb. 6.49a) und Massenausbeute (Abb. 6.49b) auch hier mit sinkendem Gesamtdruck, bei Niederdruckbedingungen lässt sich die Nukleation allerdings nicht vollständig unterdrücken. Letzteres lässt sich aus dem schon in $\mathrm{SO}_{2}$-Abwesenheit höheren Partikelbildungspotential des Methylcyclohexens verglichen zu Cyclohexen und Methylcyclopenten erklären. 
Auch unter Niederdruckbedingungen kann durch Schwefeldioxidzugabe die Anzahlkonzentration deutlich gesteigert werden, was über den gesamten Druckbereich zu beobachten ist. Dagegen unterscheiden sich die Massenausbeuten in allen Messpunkten nicht so klar, wenn auch in $\mathrm{SO}_{2}$-Anwesenheit eine Tendenz nach oben festgestellt werden kann. Die Zunahmen der Ausbeuten verhalten sich für beide Größen gleich, wobei die Punkte unter $\mathrm{SO}_{2}$-Zugabe mit steigendem Partialdruck verglichen zu den zu den $\mathrm{SO}_{2}$-freien Punkten lediglich nach oben parallelverschoben scheinen.

\subsection{4. $\alpha$-Pinen}

\section{Abhängigkeit vom $\mathrm{SO}_{2}$-Partialdruck}

Die Variation des Schwefeldioxidpartialdrucks scheint nur in einem Bereich ge-

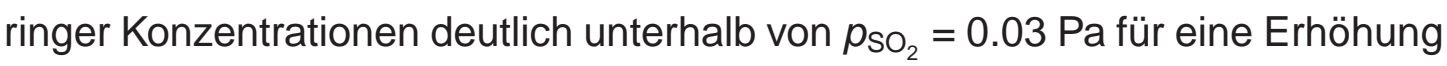
in den Anzahlkonzentrationen zu sorgen. Dies zeigen die Trockenexperimente (rot, Abbildung 6.50; $p_{\text {ges }}=450 \mathrm{mbar}$ ). Die Massenausbeuten nehmen dagegen über den gesamten Konzentrationsbereich zu, wobei der Anstieg unterhalb von $p_{\mathrm{SO}_{2}}=0.03 \mathrm{~Pa}$ jedoch, wie bei den Partikelanzahlen, deutlich steiler ausfällt.

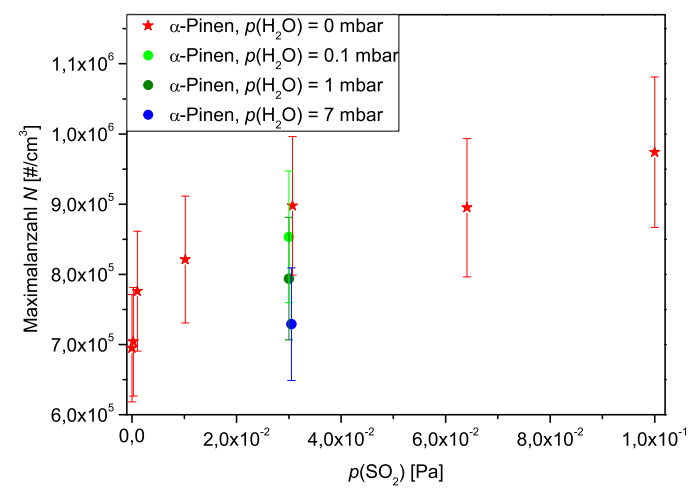

(a) Anzahlkonzentration.

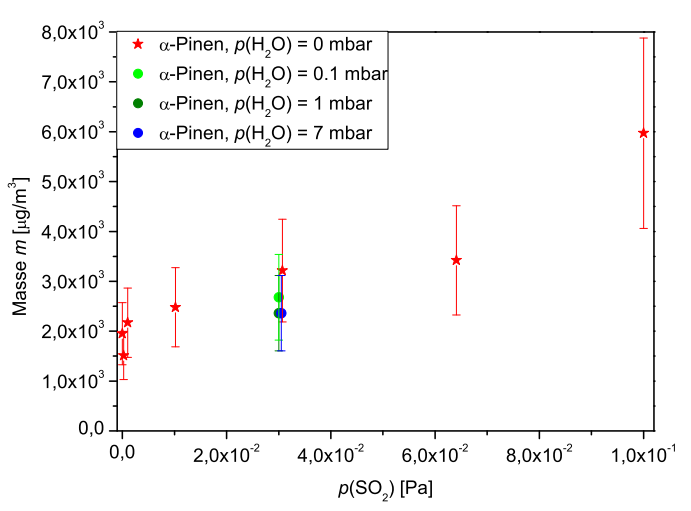

(b) Massenausbeute.

Abb. 6.50.: Untersuchung des $\mathrm{SO}_{2}$-Einflusses auf die Partikelausbeute für $\alpha$ Pinen in Synthetischer Luft unter Zugabe verschiedener Wasserpartialdrücke bei $p_{\text {ges }}=450 \pm 10$ mbar.

Die Zugabe verschiedener Wasserpartialdrücke zieht eine Verringerung der Ausbeuten nach sich, wobei sich unterschiedliche Auswirkungen auf Anzahl 
und Masse feststellen lassen. Während die Anzahlkonzentration mit steigender relativer Luftfeuchte über den gesamte vermessenen Bereich abnehmen, fällt die Beeinflussung der Massenausbeute geringer aus, da sich nur bei Zugaben von $p_{\mathrm{H}_{2} \mathrm{O}}=0.1$ bzw. 1 mbar eine Erniedrigung beobachten lässt, die erhöhte Addition von $p_{\mathrm{SO}_{2}}=7$ mbar allerdings keine weitere Reduktion hervorruft.

\section{Abhängigkeit vom Gesamtdruck}

Abbildung 6.50 zeigt die Abhängigkeit der Partikelausbeuten vom Gesamtdruck, wobei in diesem Fall Messungen bis hin zu Atmosphärendruck durchgeführt wurden. Die Ergebnisse zeigen, dass die Unterdrückung der Nukleation für $\alpha$-Pinen sogar unter extremen Niederdruckbedingungen bei ca. $p_{\text {ges }}=10$ mbar nicht möglich ist, da dieses Terpen ein sehr hohes Partikelbildungspotential besitzt. WOLF et al. [17] konnten zeigen, dass eine Unterdrückung erst unterhalb eines Gesamtdruckes von 3 mbar eintritt.

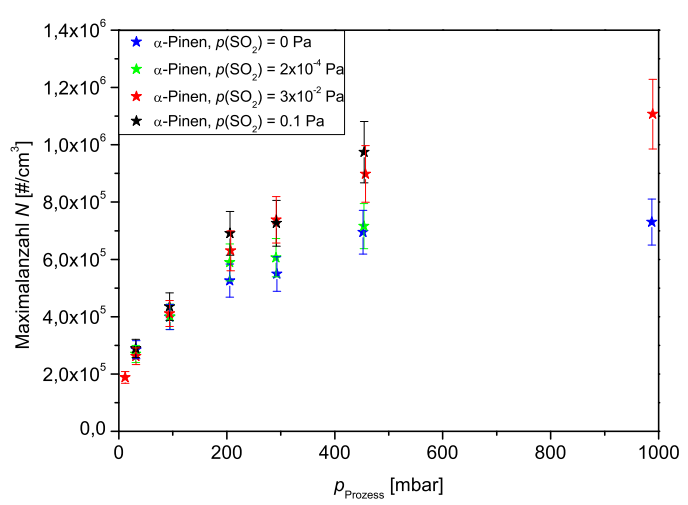

(a) Anzahlkonzentration.

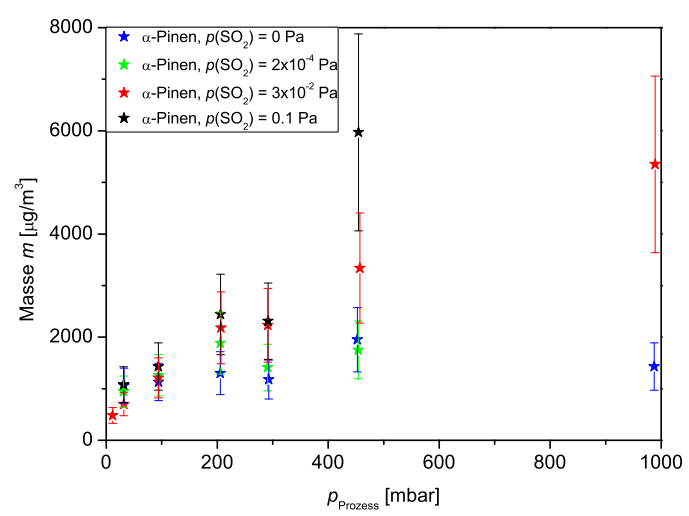

(b) Massenausbeute.

Abb. 6.51.: Untersuchung der Druckabhängigkeit bei der $\alpha$-Pinenozonolyse unter verschiedenen $\mathrm{SO}_{2}$-Zugaben.

Wie bereits im Falle des Cyclohexens ist auch hier eine unterschiedliche Auswirkung der $\mathrm{SO}_{2}$-Zugaben bei verschiedenen Prozessdrücken zu beobachten. Während sich die Ausbeuten bezüglich der Anzahlkonzentration bei niedrigeren Gesamtdrücken kaum unterscheiden (Abb. 6.51a), sind oberhalb von $p_{\text {ges }} \approx 200$ mbar mit steigendem Druck immer deutlichere Differenzen in den Partikelanzahlen zu verzeichnen. Für die gebildeten Aerosolmassen in Abb. $6.51 b$ gilt das gleiche, wobei die Ausbeuten relativ gesehen deutlich stärker durch die $\mathrm{SO}_{2}$-Zugabe erhöht werden als die Keimanzahlen. 


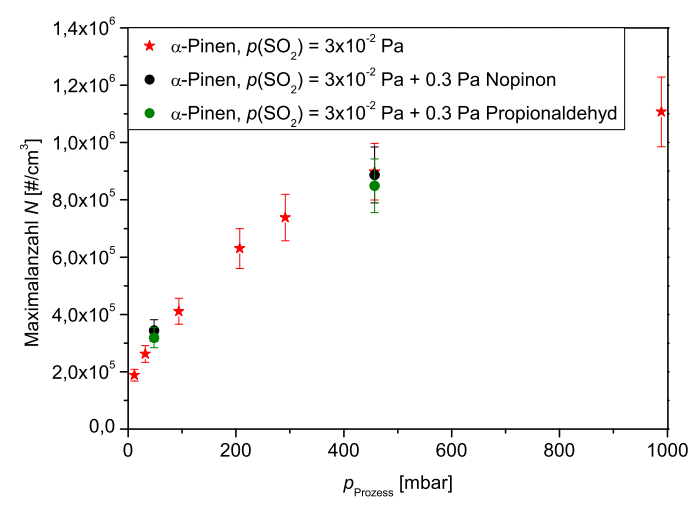

(a) Anzahlkonzentration.

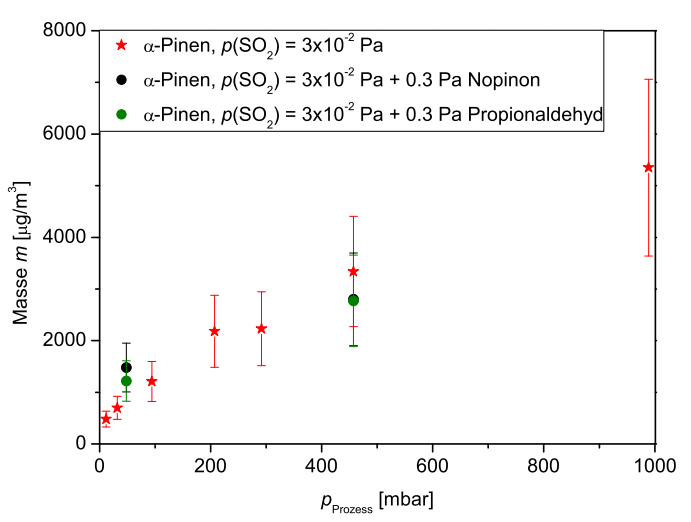

(b) Massenausbeute.

Abb. 6.52.: Untersuchung der Druckabhängigkeit bei der $\alpha$-Pinenozonolyse unter $\mathrm{SO}_{2}$-Zugaben in Anwesenheit von Nopinon und Propionaldehyd.

Ähnlich wie im Falle des 2-Methyl-1-penten, wo durch Zugabe von Cyclohexanon überprüft werden sollte, ob dieses Reaktionsprodukt für die starken Zunahmen der Aerosolausbeuten bei Cyclohexanzugabe verantwortlich ist (Abschnitt 6.4.1.2), wurden einige Vergleichsexperimente unter zusätzlicher Addition von Nopinon und Propionaldehyd durchgeführt.

Diese Substanzen sind keine direkten Reaktionsprodukte der $\alpha$ Pinenozonolyse und wurden lediglich als verfügbare Modellverbindungen für die tatsächlich enstehende Carbonylverbindung Pinonaldehyd (Ausbeute: $17.5 \%$ [115]) verwendet. Diese Additiva hatten jedoch auf die Ausbeuten einer Ozonolyse unter $\mathrm{SO}_{2}$-Anwesenheit keinen signifikanten Effekt. Wenn auch die Massenausbeuten im Niederdruckbereich leicht erhöhte und bei höherem Druck leicht erniedrigte Werte zeigen, sind diese zum einen meist noch innerhalb der Streubereiche. Zum anderen müssten sich bei einer tatsächlichen Beeinflussung durch die Anwesenheit von Carbonylkomponenten deutlich größere Unterschiede zeigen, da wesentlich höhere Partialdrücke verwendet wurden als bei Betrachtung der zu erwartenden Ausbeuten nötig gewesen wären. Die Reaktion von $\mathrm{SO}_{2}$ bzw. gebildeter Schwefelsäure mit aus der Ozonolyse stammenden Carbonylverbindungen kann also als Ursache für die Erhöhung der Partikelausbeuten bei $\mathrm{SO}_{2}$-Zugabe $\mathrm{zu} \alpha$-Pinenozonolysen ausgeschlossen werden. 


\subsubsection{Methylencyclohexan}

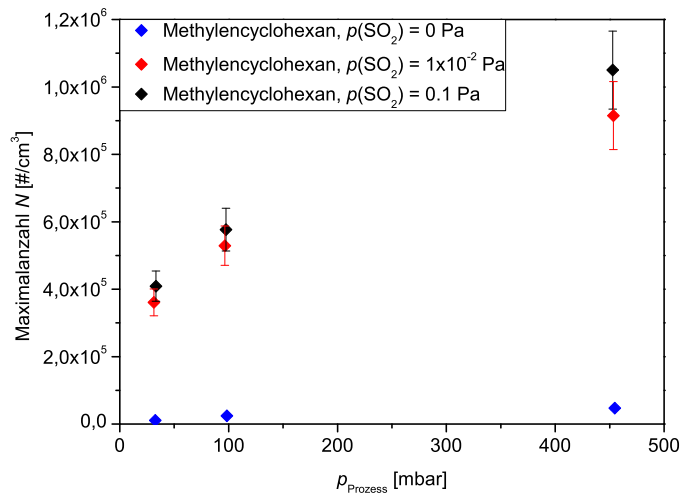

(a) Anzahlkonzentration.

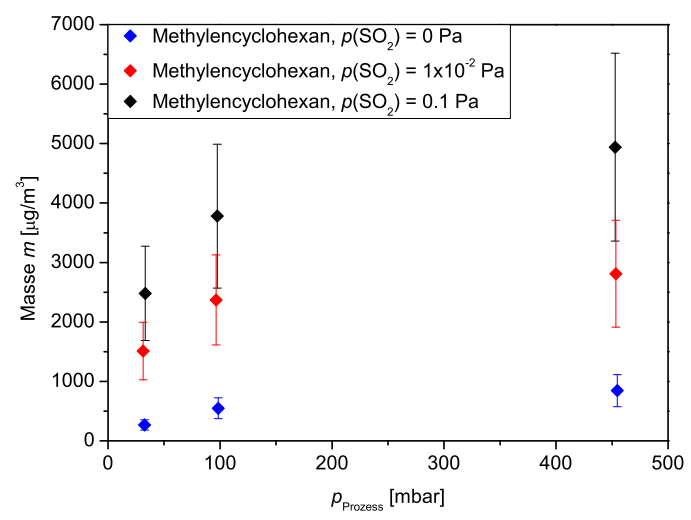

(b) Massenausbeute.

Abb. 6.53.: Untersuchung der druckabhängigen Partikelausbeute für Methylencyclohexan in Synthetischer Luft unter Zugabe verschiedener Mengen $\mathrm{SO}_{2}$.

Beim exozyklischen Methylencyclohexan wirkt sich die Schwefeldioxidanwesenheit deutlich stärker aus als bei Methylcyclohexen (Abschnitt 6.5.3). Auch schon bei einem relativ geringen Partialdruck von $p_{\mathrm{SO}_{2}}=0.01 \mathrm{~Pa}$ nimmt die Anzahlkonzentration stark zu (Abb. 6.53a), während die Zugabe der zehnfachen Menge $\mathrm{SO}_{2}$ im Vergleich zur zuvor genannten Messreihe nur noch zu geringen Zuwächsen führt. Diese Beobachtung gilt für den gesamten Druckbereich, so dass auch unter Niederdruckbedingungen deutlich höhere Ausbeuten als aus der reinen Ozonolyse zu verzeichnen sind.

Die Massenausbeuten nehmen in der $\mathrm{SO}_{2}$-Mischozonolyse ebenfalls $\mathrm{zu}$ (Abb. 6.53b), hier sind allerdings zwischen allen drei Messreihen ähnliche Trends zu beobachten und die Punkte erscheinen in ihrem Verlauf zu höheren Werten parallelverschoben.

Die Partikelbildung konnte auch ohne $\mathrm{SO}_{2}$-Zugaben unter Niederdruckbedingungen nicht vollständig unterdrückt werden. Methylencyclohexan zeigt im Niederdruckbereich die größte Beeinflussung durch die $\mathrm{SO}_{2} / \mathrm{H}_{2} \mathrm{SO}_{4}$-Chemie aller untersuchten Verbindungen. Lediglich für Methylcyclohexen konnte bei $p_{\text {ges }} \approx 30$ mbar ebenfalls ein Unterschied in den Ausbeuten festgestellt werden, wobei der Effekt dort allerdings stark abgeschwächt gegenüber den Beobachtungen für Methylencyclohexan ausfällt. 


\subsection{6. $\beta$-Pinen}

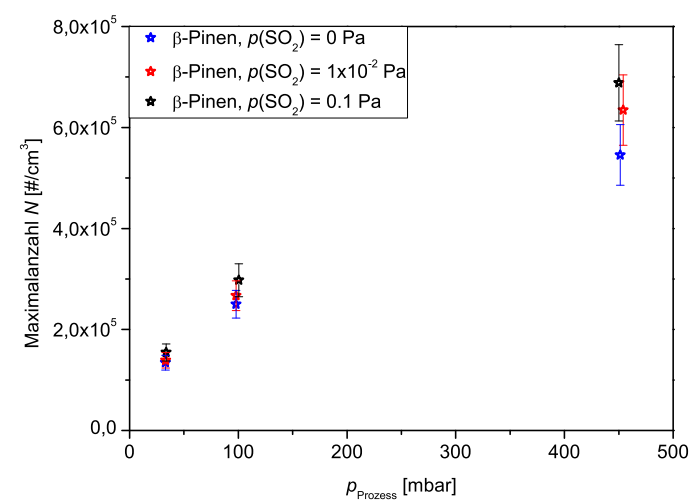

(a) Anzahlkonzentration.

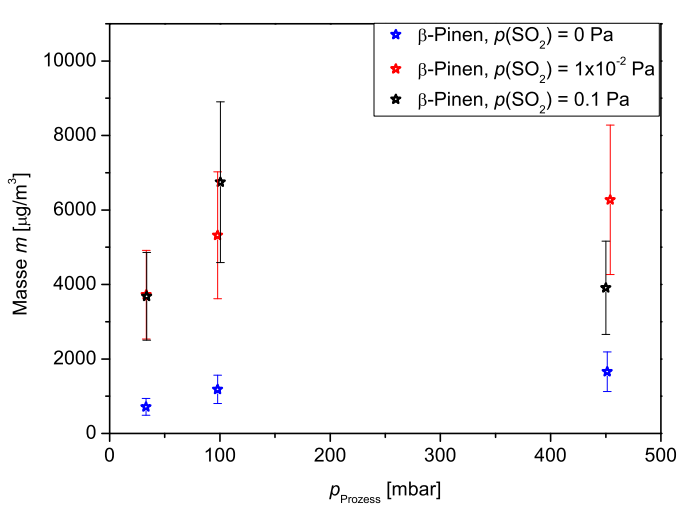

(b) Massenausbeute.

Abb. 6.54.: Untersuchung der Druckabhängigkeit bei der $\beta$-Pinenozonolyse unter verschiedenen $\mathrm{SO}_{2}$-Zugaben.

In der $\beta$-Pinenozonolyse ist die Nukleation unter den getesteten Niederdruckbedingungen nicht vollständig unterdrückt, auch hier ist dies erst unterhalb von $p_{\text {ges }} \approx 3$ mbar zu erwarten [17]. 6.54a zeigt, dass die Anzahlkonzentrationen durch $\mathrm{SO}_{2}$-Zugaben kaum beeinflusst werden, wie es auch schon für $\alpha$-Pinen zumindest bei niedrigen Gesamtdrücken zu beobachten war. Allerdings sind für das exozyklische Terpen auch im höheren Druckbereich um $p_{\text {ges }} \approx 450 \mathrm{mbar}$ kaum signifikante Unterschiede auszumachen, lediglich eine Tendenz zu höheren Anzahlen ist zu vermuten.

Abbildung 6.54b zeigt dagegen, dass sich die Massenausbeuten durchaus steigern, wobei sich keine deutlichen Unterschiede zwischen den beiden getesteten $\mathrm{SO}_{2}$-Partialdrücken feststellen lassen. Die vergleichsweise geringe Ausbeute bei $p_{\text {ges }} \approx 450$ mbar und hoher $\mathrm{SO}_{2}$-Zugabe ist im Rahmen der Messgenauigkeit nur bedingt aussagekräftig und unter Umständen eher auf ein Messartefakt zurückzuführen.

Im Vergleich zu $\alpha$-Pinen zeigt sich, dass $\beta$-Pinen aus der reinen Ozonolysereaktion wie unter $\mathrm{SO}_{2}$-Zugabe etwas weniger Partikelkeime bildet, während die Massenausbeuten im ersten Fall nahezu gleich sind. In $\mathrm{SO}_{2}$-Anwesenheit konnte dagegen eine höhere absolute Massenausbeute für $\beta$-Pinen detektiert werden. 


\subsubsection{Auswirkungen des Gesamtdruckes in $\mathrm{SO}_{2}$-Anwesenheit}

Die Druckabhängigkeit der Partikelbildung, wie sie bisher für Kohlenwasserstoffverbindungen jeglichen Oxidationsgrads nachgewiesen werden konnte, bleibt auch in Anwesenheit von Schwefeldioxid erhalten (vgl. auch [17, 48]). Dies kann, wie bei den reinen Ozonolyseexperimenten, unter anderem durch die fehlende Stoßstablisierung der CRIEGEE-Intermediate und anderer Zwischenprodukte unter Niederdruckbedingungen erklärt werden. Ein Marker für die Stabilität der CRIEGEE-Intermediate ist die Messung der Sekundärozonidkonzentration, da diese Produkte nur aus der bimolekularen Reaktion der $\mathrm{SCl}$ gebildet werden, wenn ihre Lebensdauern ausreichend lang und Konzentrationen hoch genug sind [140]. Wie bereits in Kap. 6.2.4.2 erwähnt, können die Signale dieser Verbindungen im Gerüstbereich um $1200-950 \mathrm{~cm}^{-1}$ IRspektroskopisch detektiert werden [17, 126].

WoLf et al. [17] konnten für exozyklische Verbindungen bereits nachweisen, dass sich die Ausbeuten der SOZ im Niederdruckbereich stark verringern, wobei nach Studien von PAULSON et al. [43] die Stabilisierbarkeit der Intermediate, zumindest für zyklische Kohlenwasserstoffe, ebenfalls stark durch die Alkengrundstruktur determiniert ist. Danach ergeben sich für die endozyklischen Alkene deutlich geringere Ausbeuten für das $\mathrm{SCl}$ als für exozyklische Verbindungen, die Zahlenwerte sind in Tabelle 6.15 angegeben. Die Abhängigkeit von der Alkenstruktur konnte in den neueren Studien von DROzD et al. [44] bestätigt werden, wobei ebenfalls eine ausgeprägte Druckabhängigkeit der SCIAusbeute nachgewiesen werden konnte.

Wie an den Werten für die SCl-Ausbeuten sichtbar wird, die sämtlich unter Atmosphärenbedingungen bestimmt wurden, erhöht sich der Anteil stabilisierter Intermediate stark mit der Zahl der verfügbaren Freiheitsgrade, also wachsender Atomanzahl des Moleküls (vgl. Cyclohexen $\mathrm{C}_{6} \mathrm{H}_{10}$, Methylcyclohexen $\mathrm{C}_{7} \mathrm{H}_{12}, \alpha$-Pinen $\mathrm{C}_{10} \mathrm{H}_{16}$ ). Weiterhin wird deutlich, dass Intermediate aus endozyklischen Alkenen wesentlich schlechter stabilisierbar sind als die der exozyklischen (vgl. Methylcyclohexen $\mathrm{C}_{7} \mathrm{H}_{12}$ und Methylencyclohexan $\mathrm{C}_{7} \mathrm{H}_{12}$ ). Letzteres lässt sich auf den unterschiedlichen Zerfall des Primärozonids dieser beiden Grundstrukturen zurückführen, da sich für endozyklische Verbindungen 


\begin{tabular}{|c|c|c|c|}
\hline Alken & $\boldsymbol{Y}_{\text {SCI }}[\%]$ & $\boldsymbol{Y}_{\text {OH }}[\%]$ & Referenz \\
\hline \hline Cyclohexen & 3.2 & 68 & ATKINSON [141] \\
Methylcyclohexen & 10.4 & 91 & PAULSON [43] \\
$\alpha$-Pinen & 12.5 & 82 & RICKARD [37] \\
\hline Methylencyclohexan & 21.6 & 75 & PAULSON [43] \\
$\beta$-Pinen & 24.9 & 24 & RICKARD [37] \\
\hline
\end{tabular}

Tab. 6.15.: Ausbeuten des stabilisierten großen CRIEGEE-Intermediates (SCI) für verschiedene zyklische Alkene. Sämtliche Werte stammen von PAULSON et al. [43]. Zusätzlich werden $\mathrm{OH}$-Ausbeuten angegeben, für die die Referenzen jeweils genannt werden. Alle Studien wurden unter Atmosphärendruck durchgeführt.

nur ein Molekül bildet, welches Carbonyl- und Carbonyloxidrest trägt und eine entsprechend hohe Schwingungsanregung besitzt. Demgegenüber zerfällt das aus einem exozyklischen Alken gebildete POZ in zwei getrennte Moleküle, was die Neubildung einer Translationsmode zum Abbau der Überschussenergie ermöglicht und die Stabilisierung des Cls vereinfacht (vgl. Abb. 2.10 auf S. 34). Günstig wirkt sich außerdem die Beibehaltung der Ringstruktur beim gebildeten CRIEGEE-Intermediat aus, da eine höhere Zahl an Schwingungsmoden verfügbar bleibt.

Allerdings können die konkreten Zahlenwerte nur als ein Anhaltspunkt für unterschiedliches Verhalten in der Aerosolbildung angesehen werden, da ihre Charakterisierung aufgrund der recht kurzen Lebensdauern problematisch ist und dementsprechend in der Literatur widersprüchliche Ergebnisse zu finden sind [37, 43, 44, 141, 142]. Die Ausbeuten von SCI werden in der Regel mit Hilfe einer Fängersubstanz bestimmt, ähnlich wie Cyclohexan für $\mathrm{OH}$-Radikale verwendet wird und aus der Cyclohexanonausbeute auf den $\mathrm{OH}$-Anteil zurückgeschlossen wird. Diese Methode führt jedoch für die noch immer sehr reaktiven $\mathrm{SCl}$ dazu, dass die Ergebnisse für die Ausbeuten des später ausgewerteten Produktes und der daraus extrapolierte Anteil der gebildeten Intermediate stark vom verwendeten Fängermolekül abhängt. Um dieses Problem zu umgehen, wurden die Ergebnisse einer einzigen Studie verwendet, in der die meisten der interessierenden Verbindungen unter gleichen experimentellen Bedingungen untersucht wurden und die Ergebnisse zumindest relativ zueinander aussagekräftig sein sollten. 
Die OH-Ausbeuten verhalten sich innerhalb der Substanzgruppen näherungsweise antiproportional zur SCl-Ausbeute. Diese Tatsache ist auf die unterschiedlichen Verhältnisse von syn- zu anti-Cl und den Zerfall nicht stabilisierter syn-Cl unter anderem in $\mathrm{OH}$-Radikale zurückzuführen.

Der Gesamtdruck könnte neben seinem Einfluss auf die Intermediatstabilität der Ozonolyseprodukte auch Bedeutung für die Schwefelsäurechemie bei $\mathrm{SO}_{2}{ }^{-}$ Zugabe haben. Dies könnte einerseits aus der Druckabhängigkeit der Reaktion $\mathrm{OH}+\mathrm{SO}_{2} \rightleftharpoons \mathrm{HOSO}_{2}$ resultieren, die von SOMNITZ [143] mittels quantenchemischer Rechnungen untersucht wurde. Nach seinen Ergebnissen verringert sich der Geschwindigkeitskoeffizient der Reaktion in Stickstoff und bei Zimmertemperatur bei einer Druckänderung von 500 mbar auf 30 mbar um den Faktor 2.5. Andererseits hätte das Badgas „ $M$ “ als Stoßpartner in den für die Oxidation von $\mathrm{SO}_{2}$ entscheidenden Schritten Bedeutung für die Bildung von $\mathrm{HSO}_{5}$ (Gl. 2.36, S. 54) [91], wobei die Bedeutung dieser Reaktion bisher ungeklärt ist und in den folgenden Betrachtungen daher zunächst vernachlässigt werden muss. LAAKSONEN et al. konstatieren in ihrer Arbeit lediglich, dass dieses Oxidationsprodukt durchaus ein besserer Keimvorläufer als Schwefelsäure sein kann, allerdings unterhalb von 80 mbar nicht stabilisierbar ist [91]. SIPILÄ et al. [92] schreiben $\mathrm{HSO}_{5}$ dagegen nur eine geringe Bedeutung zu, nachdem Messungen mit einer höheren Nachweisempfindlichkeit durchgeführt wurden, was diese Vernachlässigung gestattet. Dass sich außerdem in den IR-Spektren der endozyklischen Alkene kaum ein Unterschied im Schwefeldioxidumsatz bei verschiedenen Gesamtdrücken beobachten ließ, spricht ebenfalls gegen eine druckabhängige Schwefelsäurebildung (vgl. z.B. Abb. 6.23 und 6.21). Die übrigen Reaktionen im Schema auf S. [54/sollten dagegen druckunabhängig ablaufen, daher wird dies auch für die insgesamt vorliegende Schwefelsäurebildung angenommen.

Insgesamt kann also davon ausgegangen werden, dass bezogen auf die Bedeutung des Gesamtdruckes der Zerfall bzw. die Stabilisierung der aus der Ozonolyse gebildeten Intermediate für die ausgeprägte Druckabhängigkeit der gebildeten Aerosolausbeuten bedeutsamer als die Vorgänge im Schwefelsäurebildungsmechanismus sind.

In Tabelle 6.16 werden die Ergebnisse von Partikelbildungsexperimenten mit und ohne $\mathrm{SO}_{2}$-Zusatz miteinander verglichen, um die nukleationsfördernde 


\begin{tabular}{|c|c|c|c|c|}
\hline & \multicolumn{2}{|c|}{$\left.\left.\bar{N}_{(0.1 \mathrm{~Pa} \mathrm{SO}}\right)-\bar{N}_{(0 \mathrm{~Pa} \mathrm{SO}}\right)\left[\# \mathrm{~cm}^{-3}\right]$} & \multicolumn{2}{|c|}{$\frac{\left.\bar{N}_{(0.1 \mathrm{~Pa} \mathrm{SO}}\right)}{\left.\bar{N}_{(0 \mathrm{~Pa} \mathrm{SO}}\right)}$} \\
\hline Alken $\quad p_{\text {ges }}[\mathrm{mbar}]$ & 30 & 450 & 30 & 450 \\
\hline CHEX & $0.1 \cdot 10^{4}$ & $8.3 \cdot 10^{4}$ & 4.13 & 23.15 \\
\hline MCPE & $9.3 \cdot 10^{4}$ & $27.8 \cdot 10^{4}$ & 2.77 & 21.56 \\
\hline $\mathrm{MCHe}$ & $6.2 \cdot 10^{4}$ & $38.2 \cdot 10^{4}$ & 3.69 & 3.61 \\
\hline$\alpha$-Pinen & $0.4 \cdot 10^{4}$ & $27.9 \cdot 10^{4}$ & 1.01 & 1.33 \\
\hline $\mathrm{MCHa}$ & $39.8 \cdot 10^{4}$ & $100.3 \cdot 10^{4}$ & 38.40 & 22.22 \\
\hline$\beta$-Pinen & $2.0 \cdot 10^{4}$ & $14.3 \cdot 10^{4}$ & 1.15 & 1.26 \\
\hline
\end{tabular}

(a) Veränderung der Anzahlkonzentrationen.

\begin{tabular}{|c|c|c|c|c|}
\hline & \multicolumn{2}{|c|}{$\left.\left.\bar{m}_{(0.1 \mathrm{~Pa} \mathrm{SO}}\right)-\bar{m}_{(0 \mathrm{~Pa} \mathrm{SO}}\right)\left[\mu \mathrm{g} \mathrm{m}^{-3}\right]$} & \multicolumn{2}{|c|}{$\frac{\left.\bar{m}_{(0.1 \mathrm{~Pa} \mathrm{SO}}\right)}{\bar{m}_{\left(0 \mathrm{~Pa} \mathrm{SO} \mathrm{S}_{2}\right)}}$} \\
\hline${ }_{\text {Alken }} \quad p_{\text {ges }}[\mathrm{mbar}]$ & 30 & 450 & 30 & 450 \\
\hline CHEX & 5.1 & 604.3 & 26.5 & 4.88 \\
\hline MCPE & 49.6 & 601.0 & 13.40 & 2.34 \\
\hline $\mathrm{MCHe}$ & 118.4 & 765.0 & 2.75 & 2.16 \\
\hline$\alpha$-Pinen & 20.0 & 4020.0 & 1.02 & 3.06 \\
\hline $\mathrm{MCHa}$ & 2212.0 & 4095.0 & 9.27 & 5.85 \\
\hline$\beta$-Pinen & 2966.0 & $5562.0^{*}$ & 5.15 & $5.70^{*}$ \\
\hline
\end{tabular}

(b) Veränderung der Massenausbeuten.

Tab. 6.16.: Zusammenstellung der absoluten und relativen Veränderungen in den Ausbeuten bei $\mathrm{SO}_{2}$-Zugabe.

* Im Falle des $\beta$-Pinen wurden aufgrund der Abnahmen der Massenausbeute bei $p_{\text {ges }}=450$ mbar zum Vergleich der Wert bei 100 mbar verwendet. Die Ergebnisse bei $p_{\text {ges }}=450$ mbar betrugen $m=2253.3 \mu \mathrm{g} \mathrm{m}^{-3}$ bzw. 2.36. 
Wirkung des Schwefeldioxids respektive der gebildeten Schwefelsäure noch einmal zu verdeutlichen. Dazu werden jeweils Differenzen und Quotienten der gemessenen Anzahlkonzentrationen bzw. Massenausbeuten bei Niederdruckund Hochdruckbedingungen mit und ohne $\mathrm{SO}_{2}$-Zugabe gebildet, um absolute und relative Zuwächse zu erhalten. Um die Vergleichbarkeit bei der Betrachtung des Verhaltens zu gewährleisten, wurde als $\mathrm{SO}_{2}$-Konzentration $p_{\mathrm{SO}_{2}}=0.1 \mathrm{~Pa}$ gewählt, da mit dieser Konzentration bei allen verwendeten Alkenen Messungen durchgeführt wurden.

Die Ergebnisse zeigen, dass die Schwefeldioxidzugabe bei endozyklischen Eduktstrukturen einen sehr stark druckabhängigen Einfluss auf die Aerosolausbeute ausübt. $\alpha$-Pinen und Cyclohexen zeigen dabei eine ausgeprägtere Abhängigkeit vom Gesamtdruck als Methylcyclohexen. Bei letzterem war schon bei niedrigen Prozessdrücken eine hohe Aerosolausbeute zu beobachten, die Steigerung bei der Druckzunahme fiel relativ gesehen gering aus, wie die entsprechenden Zahlenwerte in Tab. 6.16zeigen.

Eine Möglichkeit der Erklärung für die Zunahme der Partikelanzahlen und -massen ist der mit dem bei höherem Gesamtdruck steigendem Anteil an stabilisierten CRIEGEE-Intermediaten. Darauf weisen die Ergebnisse der Simulationen hin, die zu diesem Thema durchgeführt wurden (vgl. Abb. 6.46][139]), in denen nur die Reaktion $\mathrm{SCl}+\mathrm{H}_{2} \mathrm{SO}_{4}$ als keimbildend vorausgesetzt wurde und deren Ergebnisse dem Verlauf der experimentell gemessenen Werte recht gut folgen. Auch die Betrachtung der Ausbeuten der endozyklischen Alkene für das stabilisierte $\mathrm{Cl}$ in Tabelle 6.15 zeigt eine zufriedenstellende Übereinstimmung mit den Steigerungen der Aerosolausbeuten bei Schwefeldioxidzugabe, was die Annahme der genannten Reaktion als entscheidenden Schritt im Mechanismus stützt. Die Auswertung der IR-Spektren zeigte für endozyklische Alkene bei allen untersuchten Druckbedingungen keinen vollständigen $\mathrm{SO}_{2}$-Verbrauch . Als einziger Unterschied zwischen Hoch- und Niederdruckbereich kann die Stabilisierung des $\mathrm{Cl}$ festgestellt werden, da die $\mathrm{OH}$-Radikalbildung sowie die Reaktion $\mathrm{OH}+\mathrm{SO}_{2} \rightleftharpoons \mathrm{HOSO}_{2}$ und daraus folgende Schwefelsäurebildung als druckunabhängig angenommen wird.

Die IR-Spektren von Reaktionen mit $\beta$-Pinen weisen dagegen auf einen vollständigen Umsatz der zugegebenen $\mathrm{SO}_{2}$-Partialdrücke hin, der mit einer Erhöhung gebildeter Carbonylstrukturen einhergeht. Nach den in Tabelle 6.15 
genannten Studien ergeben sich bei der Ozonolyse der exozyklischen Verbindungen geringere $\mathrm{OH}$-Ausbeuten als für die beiden analogen endozyklischen. Gleichzeitig werden zumindest unter Hochdruckbedingungen deutlich höhere Anteile des CRIEGEE-Intermediates stabilisiert, dies könnte ein Grund für den erhöhten $\mathrm{SO}_{2}$-Verbrauch in der reaktiven Mischung sein. Da der Vergleich des Produktspektrums mit einem Reinspektrum des Nopinons hohe Übereinstimmugen zeigt, wird davon ausgegangen, dass sich diese Verbindung, möglicherweise aus der Reaktion $\mathrm{Cl}+\mathrm{SO}_{2} \longrightarrow$ Nopinon $+\mathrm{SO}_{3}$, bildet. Das wiederum führt zu größeren Zunahmen in den Massenausbeuten durch eine höhere Rate in der $\mathrm{H}_{2} \mathrm{SO}_{4}$-Bildung, verglichen mit den Ergebnissen der endozyklischen Verbindungen.

Die Ergebnisse der Partikelmessungen zeigen für die Massenausbeuten beider Verbindungen und zumindest auch die Anzahlkonzentrationen der Modellverbindung Methylencyclohexan eine nur schwache Druckabhängigkeit. Schwefeldioxidzugaben sorgen im Niederdruckbereich bei letzterem relativ gesehen für eine stärkere Zunahme der Ausbeute als bei Atmosphärendruck, bei $\beta$-Pinen wirkt sich $\mathrm{SO}_{2}$ kaum auf die Anzahlkonzentration aus.

Schon bei $p_{\text {ges }}=30$ mbar kann durch $\mathrm{SO}_{2}$-Zugabe eine deutliche Steigerung der Ausbeuten erreicht werden, die sich anschließend bei Druckerhöhung kaum noch verändert (eine Ausnahme bildet hier die Anzahlkonzentration bei $\beta$-Pinen). Diese Beobachtung spricht für einen hohen Stabilisierungsgrad der $\mathrm{Cl}$ bereits bei Niederdruckbedingungen, wofür vor allem das anti-Cl in Frage kommt, da es nicht über den Vinylhydroperoxidkanal zerfallen kann und durch seine höhere Lebensdauer [33] leichter für bimolekulare Reaktionen zur Verfügung steht.

Im Falle des $\beta$-Pinens könnte also die bimolekulare Reaktion von $\mathrm{Cl}$ und $\mathrm{SO}_{2}$ für die Erhöhung der Ausbeuten, vor allem der Aerosolmasse, verantwortlich sein. Falls in der Reaktion mit Methylencyclohexan ebenfalls ein erhöhter Umsatz des Schwefeldioxids vorliegen würde, sollte dieser allerdings auf die recht hohe $\mathrm{OH}$-Ausbeute in Kombination mit Reaktionen der eher zum Zerfall neigenden syn-Cl zurückzuführen sein, da ihnen der Vinylhydroperoxidkanal offen steht (vgl. Abb. H.1]auf Seite XLim Anhang). Allein die OH-Ausbeute sollte nicht zu einer so hohen Steigerung der Ausbeuten bei $\mathrm{SO}_{2}$-Zugabe führen, dies zeigen die Ergebnisse der endozyklischen Alkene. 
Da die gemessenen Produktspektren keine Zunahme der Formaldehydausbeute zeigen, wie sie aus der Reaktion $\mathrm{C}_{1}-\mathrm{Cl}_{+} \mathrm{SO}_{2} \longrightarrow \mathrm{CH}_{2} \mathrm{O}+\mathrm{SO}_{3}$ zu erwarten wäre, scheint hauptsächlich das große $\mathrm{Cl}$ in Kombination mit $\mathrm{SO}_{2}$ den Einfluss auf die Aerosolbildung auszuüben, obwohl auch das $\mathrm{C}_{1}-\mathrm{Cl}$ zu $37 \%$ [39] bis $54 \%$ [40] stabilisiert wird und somit für bimolekulare Reaktionen zur Verfügung stünde.

Die vorangegangenen Ausführungen lassen darauf schließen, dass die Reaktion des $\mathrm{C}_{1}-\mathrm{Cl}$ wie auch des großen $\mathrm{SCl}$ aus endozyklischen Alkenen mit $\mathrm{SO}_{2}$ lagsamer abläuft als die entsprechende mit Intermediaten aus exozyklischen Verbindungen, während die Reaktion mit $\mathrm{H}_{2} \mathrm{SO}_{4}$ mit allen genannten Intermediaten recht schnell abläuft. Dies könnte zum einen an unterschiedlichen Geschwindigkeitskoeffizienten für die bimolekularen Reaktionen liegen oder zum anderen mit einem beschleunigten Zerfall der erstgenannten Intermediate und der daraus folgenden geringen Konzentration eines der Edukte und langsam ablaufenden Reaktion erklärt werden. Da sich auch die SOZ-Banden im Falle des $\beta$-Pinens nur unter $\mathrm{SO}_{2}$-freien Bedingungen beobachten lassen, kann eventuell auch die SOZ-bildende Reaktion zu den langsamen Reaktionen gezählt werden, die durch den Abfang der großen $\mathrm{Cl}$ mit Schwefelsäure verhindert wird. BonN et al. [12] schlägt als Alternative zu dieser Interpretation die SOZ-Aktivierung durch Schwefelsäure vor. Der Vorgang würde zu einer erhöhten Kondensation dieser Verbindung in die Partikelphase führen und die Beobachtungen ebenfalls erklären.

Neben den von den CRIEGEE-Intermediaten determinierten Reaktionen wurden in der Literatur auch andere Verbindungen als Schlüsselspezies in Betracht gezogen, die hier der Vollständigkeit halber in ihrer vermuteten Funktion und Bedeutung für die Aerosolbildung ebenfalls beschrieben werden sollen.

Schon genannt wurde die von SOMNITZ quantenchemisch untersuchte $\mathrm{HOSO}_{2}$-bildende Reaktion [143], für die eine gewisse Druckabhängigkeit festgestellt wurde, die aber im Rahmen der hier präsentierten Untersuchungen vernachlässigt wird. Außerdem sprechen die Ergebnisse der $\alpha$-Pinenozonolyse, bei der sich unter verschiedenen Druckregimes kein Unterschied im Schwefeldioxidumsatz zeigten, für dieses Vorgehen.

Dass organische Säuren eine fördernde Wirkung auf die Aerosolbildung in Schwefelsäureanwesenheit haben können, zeigt eine Studien von ZHANG et 
al. [22]. Der Effekt wurde auf irreversible Clusterbildung dieser beiden Säuren zurückgeführt und könnte auch unter den hier vorgestellten Ergebnisse eine Rolle spielen. Zur Abschätzung der Bedeutung dieser Reaktion wurde die Stabilität einzelner Dimere aus in der Ozonolyse gebildeten organischen Substanzen oder Wasser und Schwefelsäure quantenchemisch berechnet (Abschnitt 6.6).

Diese Berechnungen ergaben, dass beispielsweise die Wasserstoffbrückenbindungen in Dimeren zwischen Schwefelsäure und den untersuchten Carbonyl-, Carbonsäure- und Wassermolekülen in einer vergleichbaren GröBenordnung liegen. Dies lässt darauf schließen, dass Aldehyde durchaus als Dimere mit Schwefelsäure eine Rolle für die Partikelbildung spielen könnten, was sich mit den Beobachtungen in den IR-Produktspektren der endozyklischen Edukte decken würde. Da sich in diesen Spektren eine Reduktion im $\mathrm{C}=\mathrm{O}$-Bereich und im Bereich der aldehydischen $\mathrm{C}-\mathrm{H}$-Schwingung zeigt, könnte gefolgert werden, dass sich Dimere aus Carbonylverbindungen und $\mathrm{H}_{2} \mathrm{SO}_{4}$ bilden, die anschließend in die kondensierte Phase übergehen und daher im Gasphasenspektrum nicht mehr im gleichen Maße nachgewiesen werden können. In Falle des $\beta$-Pinen konnten keine analogen Beobachtungen gemacht werden, was allerdings mit dem in hohem Anteil gebildeten Nopinon zu tun haben könnte, welches im gleichen Wellenzahlbereich absorbiert.

Die Bildung von Carbonylverbindung-Schwefelsäure-Clustern ist nach den Ergebnissen der bisheringen theoretischen Betrachtungen thermodynamisch nicht begünstigt. So ist ein sich einstellendes Gleichgewicht zwischen kondensierter und gasförmiger Phase wahrscheinlicher, womit außerdem das Verbleiben von Carbonylsignalen im IR-Spektrum erklärt werden kann.

Diese Clusterformation sollte tendenziell bedeutsamer für exozyklische Alkene sein, da in der Ozonolysereaktion stets eine Carbonylverbindung aus dem SOZ-Zerfall resultiert [135], während in denen der endozyklischen zusätzliche radikalische Substituenten für eine Destabilisierung sorgen sollten. Insgesamt könnte die Clusterbildung druckabhängig sein, da die Bildung aus den Monomeren für eine Schwingungsanregung im Dimer sorgt.

Eine entscheidende Rolle einer säurekatalysierten Oligomerisierungsreaktion in der Partikelphase konnte durch Testexperimente unter Zugabe der Carbonylverbindungen Nopinon und Propionaldehyd in der $\alpha$-Pinenozonolyse aus- 
geschlossen werden, da sich auch bei großen Überschüssen keine signifikanten Auswirkungen auf die Aerosolausbeute zeigten. Eine ähnlich geringe Bedeutung sollte in $\mathrm{SO}_{2}$-Anwesenheit der Oligomerisierungsreaktion der stabilisierten CRIEGEE-Intermediate [36], die im Zusammenhang mit den linearen Alkenen diskutiert wurde, zukommen, da die $\mathrm{Cl}+\mathrm{SO}_{2}$-Reaktion in dieser Konkurrenzsituation schneller ablaufen sollte.

Allerdings ist auch die Bildung von reinen Schwefelsäureclustern eine Möglichkeit, wie Schwefeldioxidfolgeprodukte als NIM in den Partikelbildungsmechanismus eingreifen könnte [92]. Neben der Bildung von reinen anorganischen Säuredimeren sollte ebenfalls die ternäre Keimbildung mit Wasser oder organischen Ozonolyseprodukten möglich sein, wie KULMALA et al. [144] an einem anderen Beispiel (Wasser und Ammoniak) untersucht haben. In den durchgeführten Messungen konnte eine Druckabhängigkeit der Anzahlkonzentrationen beobachtet werden. Daher kann unter der Voraussetzung der druckunabhängigen Schwefelsäurebildung davon ausgegangen werden, dass eine entscheidende Kompontente zur Bildung der Partikelkeime aus dem anorganischen Teil des Mechanismus stammt und bei Niederdruckbedingungen "ausgeschaltet“ wird. Erst in Kombination mit einer organischen Komponente könnte sich folglich die ausbeuteerhöhende Wirkung auf die Aerosolbildung entfalten.

Die bisherigen Ausführungen bezogen sich hauptsächlich auf die betrachteten biogenen Terpene sowie ihre Modellsubstanzen. Obwohl aufgrund der strukturellen Analogie für Methylcyclopenten ein ähnlicher Mechanismus wie die für Cyclohexen angenomme Organosulfatbildung [12] vermutet werden könnte, kann aufgrund der nur in geringem Umfang als Vergleichsmessungen durchgeführten Experimente bisher nicht abgeschätzt werden, welche Intermediate durch die Schwefelsäureanwesenheit genau beeinflusst werden und damit für die höhere Ausbeuten sorgen. Auch für den Grund der Abnahme bei höheren $\mathrm{SO}_{2}$-Partialdrücken kann daher vor der Durchführung weiterer Messungen, wie für Cyclohexen geschehen, keine Aussage getroffen werden.

Allerdings ist $\mathrm{zu}$ beachten, dass der Anteil an stabilisierten CRIEGEEIntermediaten eher gering sein wird, da in der Ozonolyse nur ein $\mathrm{Cl}$ gebildet wird, welches durch das Fehlen einer Translationsmode zur Energieumverteilung und der schon vor der Reaktion recht hohen Ringspannung, die das Molekül zusätzlich destabilisiert. 


\subsubsection{Einfluss durch Wasserzugabe}

Wie bereits im Abschnitt2.8(S. 54) beschrieben, kann Schwefelsäure aus $\mathrm{SO}_{2}$ nur in Wasseranwesenheit gebildet werden. Allerdings ist im Rahmen der in dieser Arbeit vorgestellten Untersuchungen der hydroxylgruppenhaltigen Kohlenwasserstoffverbindungen bereits dargelegt worden, dass Substanzen mit $\mathrm{OH}$ Strukturmotiv in der Lage sind, stabilisierte CRIEGEE-Intermediate abzufangen und auf diese Weise in den Mechanismus einzugreifen [57]. Unter anderem wurde diese konkrete Reaktion auch von BonN et al. [18] untersucht. Sie sollte durch das Abfangen von reaktiven Intermediaten für eine Verringerung der Ausbeute sorgen. Aber auch andere Auswirkungen von Wasser in der Reaktionsmischung sind denkbar, die eher eine Erhöhung der Partikelanzahl oder -masse nach sich ziehen würden. Hier handelt es sich um die potenzielle Kondensation von Wasser auf den Partikelkeimen, die für eine höhere Massenausbeute sorgen sollte und von VIRKKULA et al. bearbeitet wurde [145]. Zudem wurde im vorherigen Abschnitt schon die essenzielle Rolle von Wasser in der ternären Nukleation diskutiert [144].

Es wird deutlich, dass durch Wasserzugabe nicht nur die Schwefelsäurebildung gesteuert wird, sondern auf vielfältige Weise in den Mechanismus eingegriffen wird, wobei nukleationsfördernde und -behindernde Vorgänge parallel ablaufen können und sich in ihren Effekten überlagern können. Dies verkompliziert eine Interpretation der Ergebnisse.

Die durchgeführten Experimente zeigten in allen Fällen eine Reduktion der Partikelanzahlen und der Gesamtmassen (vgl. Abb. 6.45a, 6.45b, 6.50a, 6.50b). In Tabelle 6.17 werden die Messergebnisse unter Wasserzugabe noch einmal zusammengefasst. Es werden die Veränderungen in Anzahl (Teil 6.17a) und Masse (Teil 6.17b) zwischen Experimenten mit maximaler relativer Luftfeuchte (ca. $26 \%$ ) und "Trockenexperimenten“ (Wasseranteil im Badgas $<3$ ppm laut Spezifikation) betrachtet.

Die Ergebnisse deuten darauf hin, dass schon der geringe Wasseranteil in den Trockenexperimenten ausreicht, um Schwefelsäure aus $\mathrm{SO}_{2} z$ z produzieren. Dies lässt sich aus den z.T. deutlich erhöhten Ausbeuten im Vergleich zu den reinen Alkenozonolysen ableiten. Da sich bei weiterer Wasserzugabe keine zusätzliche Erhöhung der Ausbeuten zeigt, ist davon auszugehen, dass ein 


\begin{tabular}{|c|c|c|c|c|c|c|}
\hline & \multicolumn{3}{|c|}{$\left.\bar{N}_{\left(700 \mathrm{~Pa} \mathrm{H}_{2} \mathrm{O}\right)}-\bar{N}_{(0 \mathrm{~Pa} \mathrm{H}} \mathrm{H}_{2} \mathrm{O}\right)\left[\mathrm{Hcm}^{-3}\right]$} & \multicolumn{3}{|c|}{$\frac{\overline{N_{\left(700 P^{2} H_{2} \mathrm{O}\right)}}}{\bar{N}_{\left(0 \mathrm{~Pa} \mathrm{H}_{2} \mathrm{O}\right)}}[\%]$} \\
\hline Alken $\quad p_{\mathrm{SO}_{2}}[\mathrm{~Pa}]$ & 0.0002 & 0.03 & 0.1 & 0.0002 & 0.03 & 0.1 \\
\hline $\begin{array}{l}\text { CHEX } \\
\alpha \text {-Pinen }\end{array}$ & $\begin{array}{c}-3417 \\
-\end{array}$ & $\begin{array}{c}- \\
-168600\end{array}$ & $\begin{array}{c}-54800 \\
-\end{array}$ & $\begin{array}{l}62 \\
-\end{array}$ & $\overline{81}$ & $\begin{array}{l}37 \\
-\end{array}$ \\
\hline
\end{tabular}

(a) Partikelanzahlen.

\begin{tabular}{|c|c|c|c|c|c|c|}
\hline & \multicolumn{3}{|c|}{$\bar{m}_{\left(700 \mathrm{~Pa} \mathrm{H}_{2} \mathrm{O}\right)}-\bar{m}_{\left(\mathrm{OPa} \mathrm{H} \mathrm{H}_{2} \mathrm{O}\right)}\left[\mu \mathrm{g} \mathrm{m}^{-3}\right]$} & \multicolumn{3}{|c|}{$\frac{\bar{m}\left(700 \mathrm{~Pa} \mathrm{H}_{2} \mathrm{O}\right)}{\left.\bar{m}_{(\mathrm{OPaH}} \mathrm{O}\right)}[\%]$} \\
\hline Alken $\quad p_{\mathrm{SO}_{2}}[\mathrm{~Pa}]$ & 0.0002 & 0.03 & 0.1 & 0.0002 & 0.03 & 0.1 \\
\hline $\begin{array}{c}\text { CHEX } \\
\alpha \text {-Pinen }\end{array}$ & $\begin{array}{c}137.9 \\
-\end{array}$ & $\begin{array}{c}- \\
-856\end{array}$ & $\begin{array}{c}-493.9 \\
-\end{array}$ & $\begin{array}{c}161 \\
-\end{array}$ & $\overline{73}$ & $\begin{array}{l}38 \\
-\end{array}$ \\
\hline
\end{tabular}

(b) Partikelmassen.

Tab. 6.17.: Absolute und relative Veränderungen der Partikelausbeuten durch Wasserzugabe in Experimenten mit verschiedenen $\mathrm{SO}_{2}$-Zugaben.

Wasserpartialdruck von etwa 0.5 $\mathrm{Pa}$ in der reaktiven Mischung vorliegt, da ab dieser Konzentration keine Verzögerung der Schwefelsäurebildung mehr eintritt [139].

Durch die beobachtete Abnahme der Ausbeuten kann davon ausgegangen werden, dass Cluster aus Wasser und Schwefelsäure (mit oder ohne organische Beteiligung) keine entscheidende Rolle für die Keimbildung besitzen. Dabei kann an dieser Stelle allerdings nicht ausgeschlossen werden, dass sich verschiedene Effekte überlagern.

Die beobachteten Reduktionen der Aerosolausbeuten können nach Untersuchungen von BoNN et al. [18] auf die Fängerwirkung der Wassermoleküle auf die stabilisierten $\mathrm{Cl}$ zurückzuführen sein. Dabei entstehen Wasserstoffperoxid und ein Keton, wobei die Auswirkungen aufgrund des höheren Anteils an $\mathrm{SCl}$ bei exozyklischen Alkenen deutlicher ausfallen sollten als bei endozyklischen (vgl. Tab. 6.15] und [45]). Die größere Beeinflussung der Ausbeuten des Cyclohexens im Vergleich zu denen des $\alpha$-Pinens ist mit der geringeren $\mathrm{OH}$ Ausbeute bei ersterem zu erklären, was auf einen geringeren Anteil des syn-Cl schließen lässt. Der unimolekulare Zerfall des anti-Konformers verläuft nach Studien von NGUYEN et al. langsamer als der des syn-Cl [146]. 


\subsection{Abschätzung der Clusterstabilitäten}

An dieser Stelle werden die Ergebnisse der quantenchemisch berechneten Clusterstabilitäten unter Beteiligung von Wasser, Schwefelsäure und verschiedenen organischen Ozonolyseprodukten dargestellt. Für die Durchführung und Interpretation dieser Rechnungen sei an dieser Stelle nochmals Philip Carlsson und Jonas Altnöder gedankt.

In Tabelle F.1. (s. S. XXXV]im Anhang) werden zunächst die Ergebnisse für die in den betrachteten Clustern enthaltenen berechneten Energien unter Angabe der Vorläuferverbindungen aufgelistet. Einen Überblick über die Monomere und Dimere sind in den angegebenen Abbildungen auf den Folgeseiten zu finden. Die aufgelisteten Bildungsenthalpien sind ohne vorheringe Nullpunktsenergiekorrektur angegeben. Aus diesen Werten und den ebenfalls berechneten Standardbildungs-GIBBS-Energien wurden anschließend unter Verwendung der Formeln 5.7 - 5.9 (S. 115) die in Tabelle gezeigten Bindungs- und Standard-Reaktions-GIBBS-Energien berechnet.

Dieses Vorgehen lässt nur eine grobe Abschätzung der für die Clusterbildung geltenden Abhängigkeiten sowie eine relative Betrachtung der Ergebnisse zu. Die numerischen Ergebnisse in Tabelle 6.18 können dahingehend gedeutet werden, dass negative GIBBS-Energien die Bildungswahrscheinlichkeit der betreffenden Verbindung erhöhen. Zumindest der stichprobenartige Literaturwertvergleich, der für einzelne Werte mit einer Arbeit von $\mathrm{RE}$ et al. [147] möglich war, zeigt im Rahmen der Unsicherheiten durch unterschiedliche verwendete Basissätze eine recht gute Übereinstimmung mit den im Rahmen dieser Arbeit berechneten Ergebnissen (vgl. Bildunterschrift der betreffenden Tabelle).

Anhand der erhaltenen Bindungsabstände $r$ sowie der Dissoziationsenergien $D_{0}$ der getesteten Oligomerstrukturen kann abgeschätzt werden, dass Wasser / Schwefelsäurecluster eine ähnliche Stabilität aufweisen sollten wie solche aus Carbonylverbindungen und Schwefelsäure, während die Cluster aus $\mathrm{H}_{2} \mathrm{SO}_{4}$ und Carbonsäuren durch Ausbildung stabilerer Wasserstoffbrückenbindungen eine höhere Stabilität besitzen. Letztere Cluster sollten daher auch irreversibel gebildet werden, während die übrigen durchaus in einem reversiblen Gleichgewicht mit den Monomeren vorliegen könnten. 


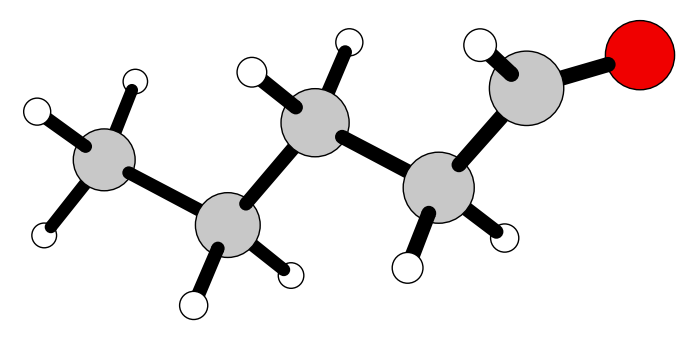

(a) Pentanal.

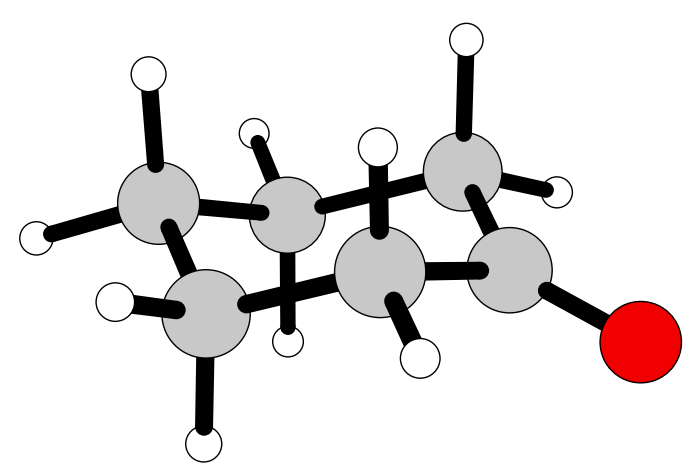

(c) Cyclohexanon.

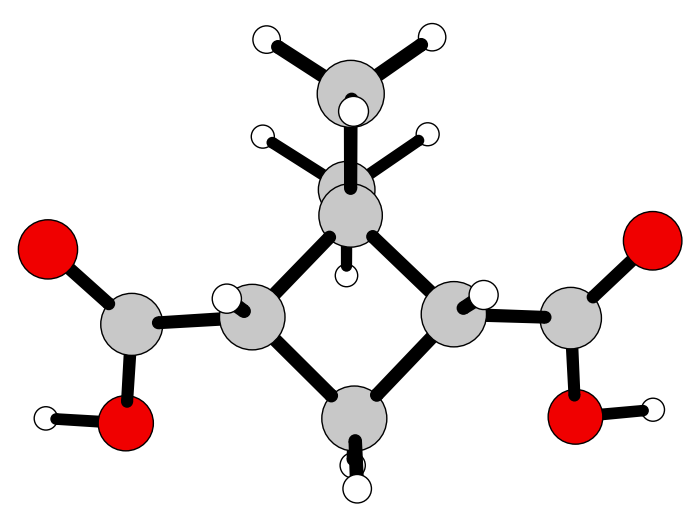

(e) Nopinsäure.

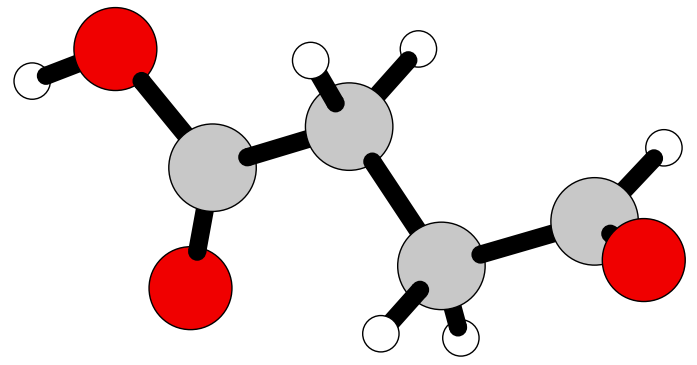

(b) 4-Oxobutansäure.

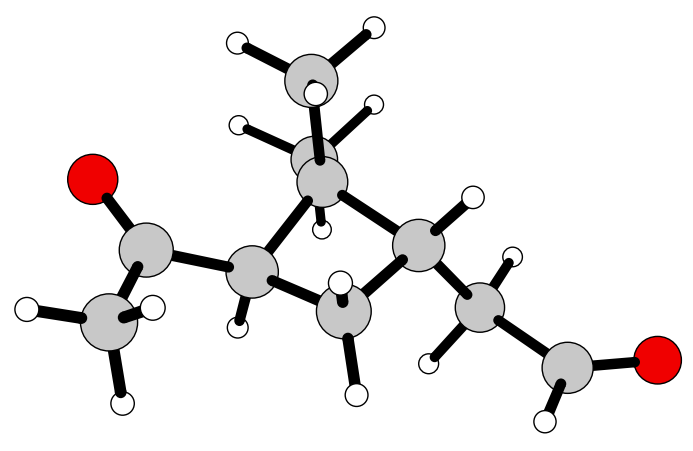

(d) Pinonaldehyd.

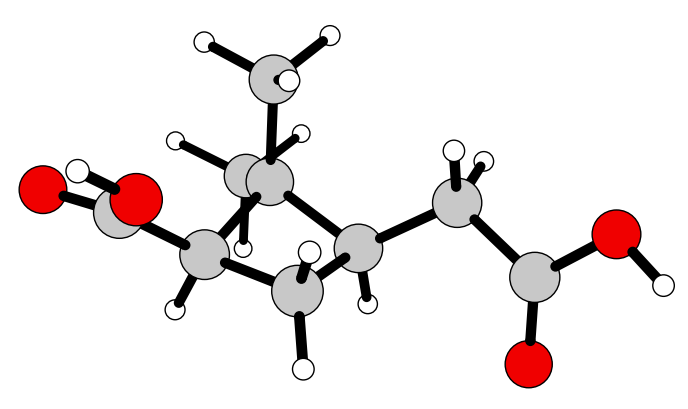

(f) Pininsäure.

Abb. 6.55.: Strukturen der Monomere aus den quantenchemischen Rechnungen, weiß: Wasserstoff; grau: Kohlenstoff; rot: Sauerstoff. 


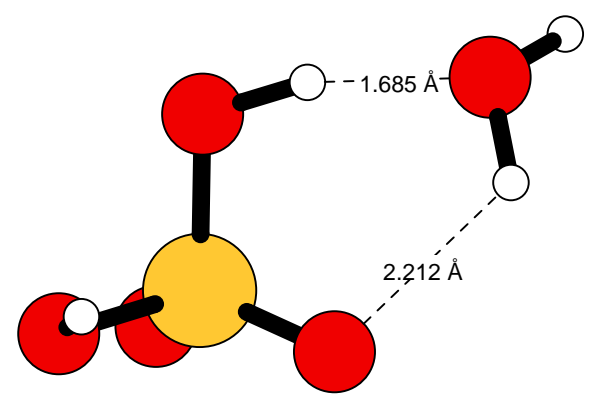

(a) Wasser- $\mathrm{H}_{2} \mathrm{SO}_{4}$-Cluster.

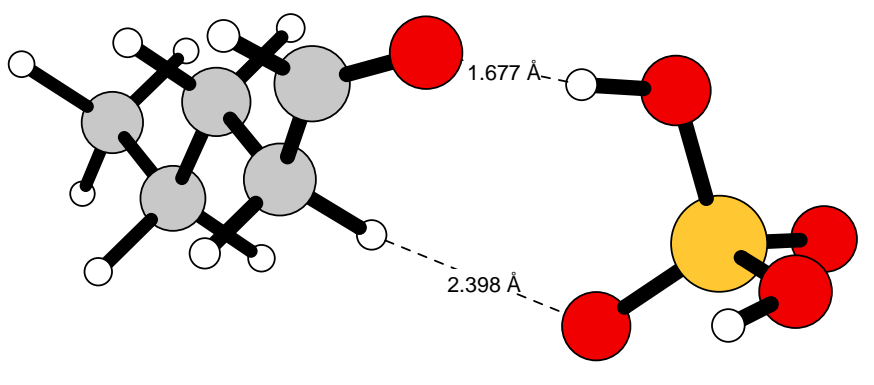

(b) Pentanal- $\mathrm{H}_{2} \mathrm{SO}_{4}$-Cluster.

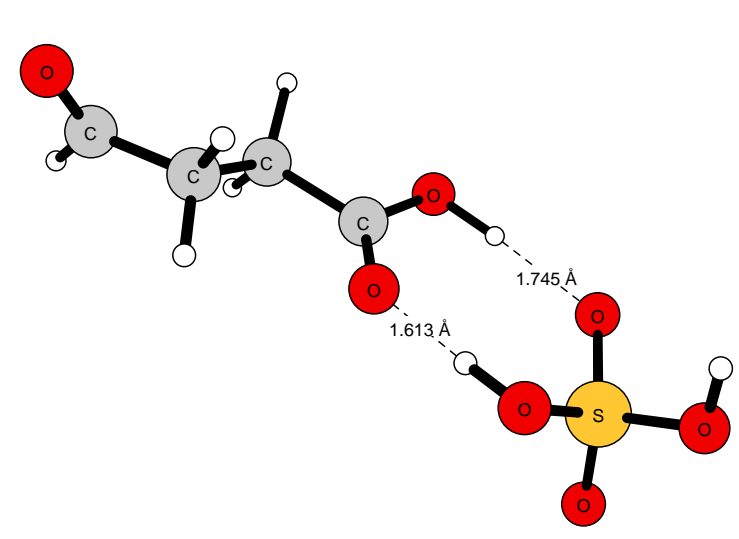

(c) 4-Oxobutansäure- $\mathrm{H}_{2} \mathrm{SO}_{4}$-Cluster (I).

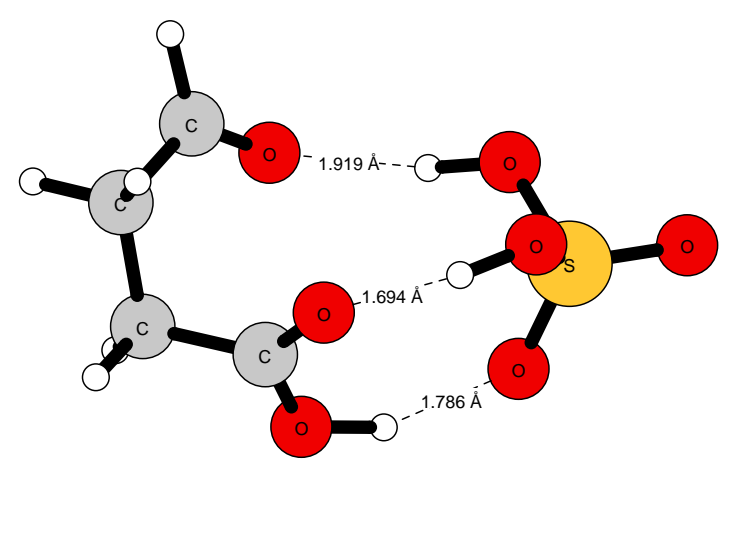

(d) 4-Oxobutansäure- $\mathrm{H}_{2} \mathrm{SO}_{4}$-Cluster (II).

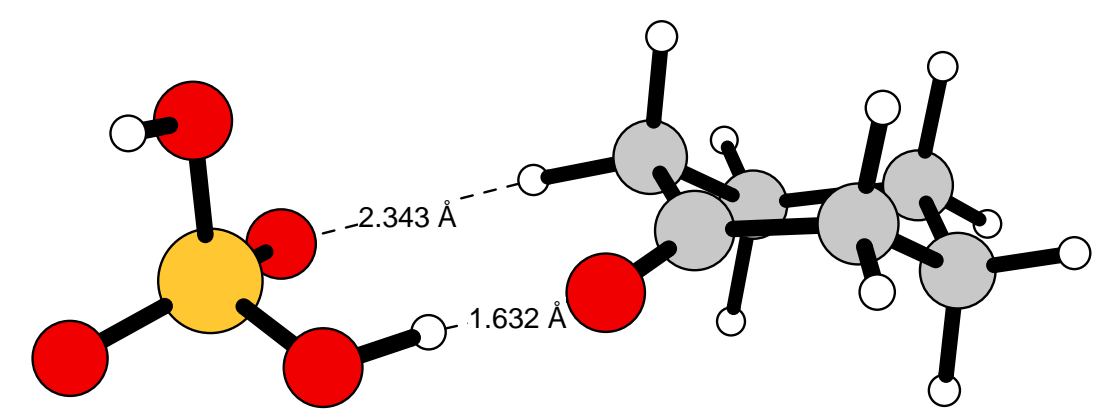

(e) Cyclohexanon- $\mathrm{H}_{2} \mathrm{SO}_{4}$-Cluster.

Abb. 6.56.: Strukturen der Oligomere aus den quantenchemischen Rechnungen, weiß: Wasserstoff; grau: Kohlenstoff; rot: Sauerstoff; gelb: Schwefel, Fortsetzung auf der nächsten Seite. 


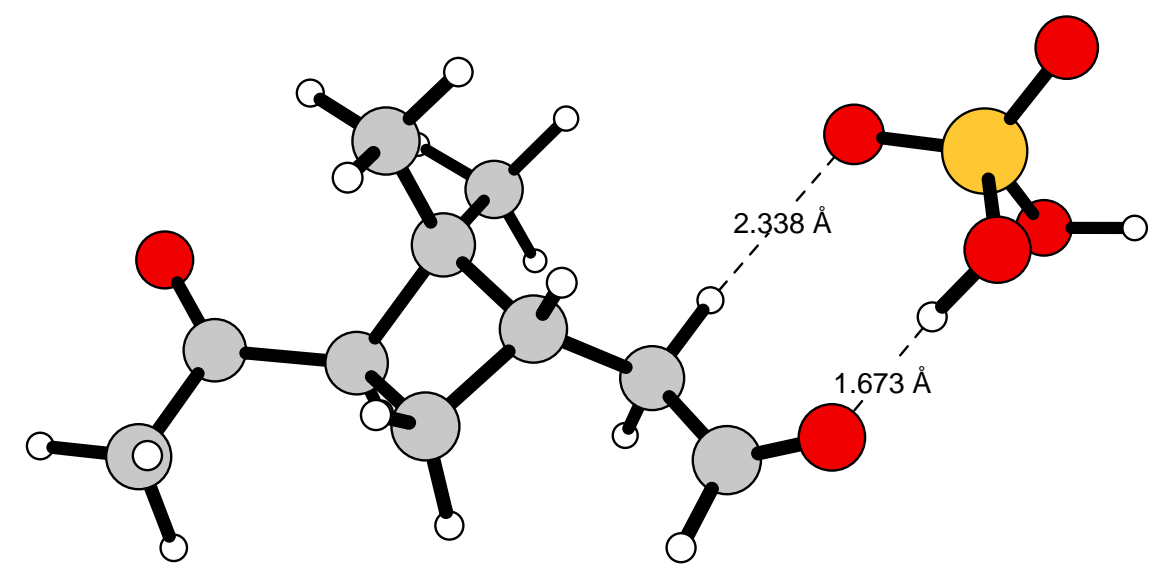

(f) Pinonaldehyd- $\mathrm{H}_{2} \mathrm{SO}_{4}$-Cluster.

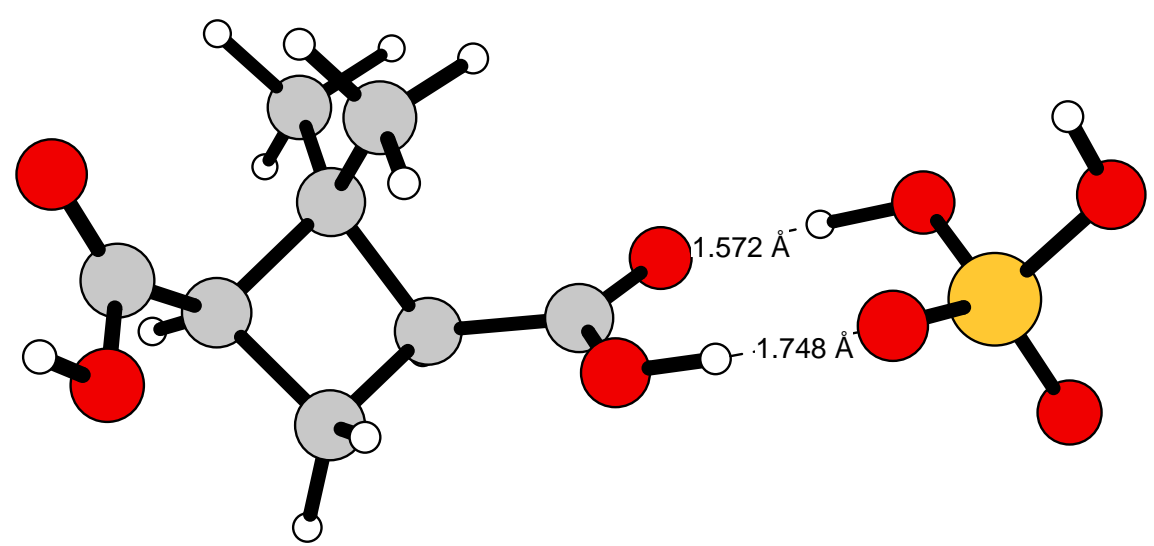

(g) Nopinsäure- $\mathrm{H}_{2} \mathrm{SO}_{4}$-Cluster.

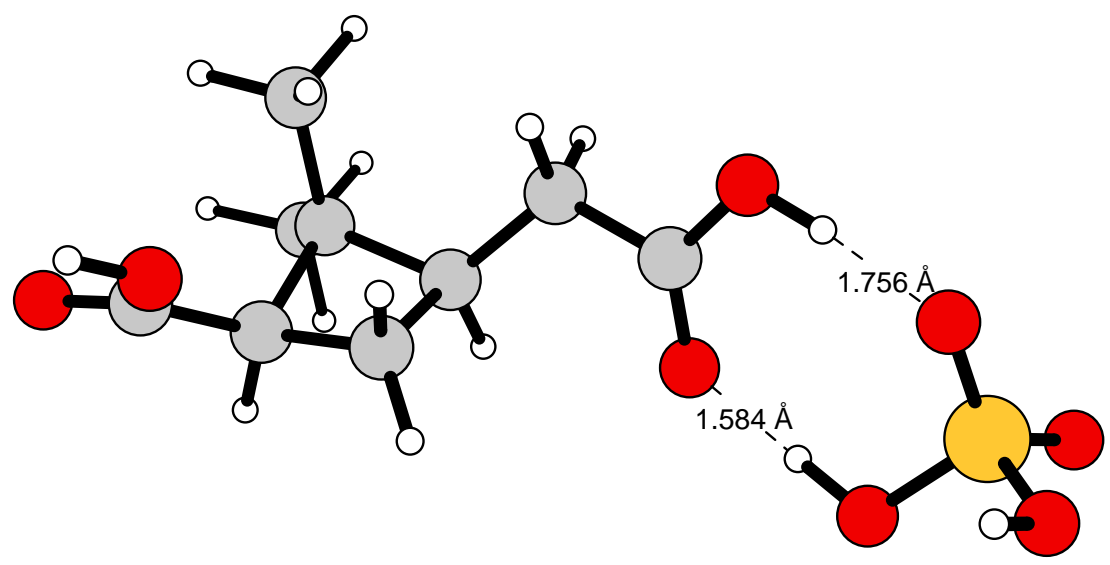

(h) Pininsäure- $\mathrm{H}_{2} \mathrm{SO}_{4}$-Cluster.

Abb. 6.56.: Strukturen der Oligomere aus den quantenchemischen Rechnungen, weiß: Wasserstoff; grau: Kohlenstoff; rot: Sauerstoff; gelb: Schwefel. 


\begin{tabular}{|l|c|c|c|}
\hline Cluster mit $\mathrm{H}_{2} \mathrm{SO}_{4}$ & $r[\AA]$ & $D_{0}\left[\mathrm{~kJ} \mathrm{~mol}^{-1}\right]$ & $\Delta_{\mathrm{R}} G^{0}\left[\mathrm{~kJ} \mathrm{~mol}^{-1}\right]$ \\
\hline Wasser & $1.685,2.212$ & 46.5 & 6.3 \\
Wasser & $1.669,2.153$ & $44.0^{* *}$ & $9.9^{* \star *}$ \\
Pentanal & $1.677,2.398$ & 46.9 & 2.7 \\
Cyclohexanon & $1.632,2.343$ & 56.4 & -4.0 \\
4-Oxobutansäure (I) & $1.613,1.745$ & 64.0 & -9.0 \\
4-Oxobutansäure (II) & $1.697,1.786,1.918$ & 79.8 & -9.1 \\
Pinonaldehyd & $1.673,2.338$ & 50.6 & -1.1 \\
Norpinsäure & $1.572,1.748$ & 68.5 & -145.2 \\
Pininsäure & $1.584,1.756$ & 66.6 & -122.5 \\
\hline
\end{tabular}

Tab. 6.18.: *Mit 6-311++G(3df,3pd) statt 6-311+G(d,p) als Basissatz.

** Zum Vergleich: $D_{0}=68.8 \mathrm{~kJ} \mathrm{~mol}^{-1}$ mit dem Basissatz $D 95(d, p)$ [147].

*** Zum Vergleich: $\Delta_{R} G^{0}=-4.3 \mathrm{~kJ} \mathrm{~mol}^{-1}$ [147], Basissatz wie oben.

Wasserstoffbrückenbindungslängen $r$, Dissoziationssenergie $D_{0}$ und Reaktionsenergie $\Delta_{R} G^{0}$ der Oligomere, Berechnungen siehe Kapitel 5.4. 


\subsection{Partikelphasenanalyse}

\subsubsection{Methode}

Im Rahmen einiger Untersuchungen wurden in Kooperation mit der Arbeitsgruppe von Prof. Dr. Thorsten Hoffmann in Mainz Partikelproben gesammelt und von dem dortigen Mitarbeiter Martin Beck analysiert. Dieser hat auch die im Folgenden gezeigten Ergebnisse tabellarisch zusammengestellt. Bei der verwendeten Methode handelt es sich um HPLC/ESI-MS (high performance liquid chromatography / electrospray ionization - mass spectrometry), die in Abb. 6.57 schematisch dargestellt ist und, ebenso wie die Hintergrundinformationen zu diesem Themenkomplex, der Dissertation von MARC-CHRISTOPHER REINNING [148] entnommen wurden.

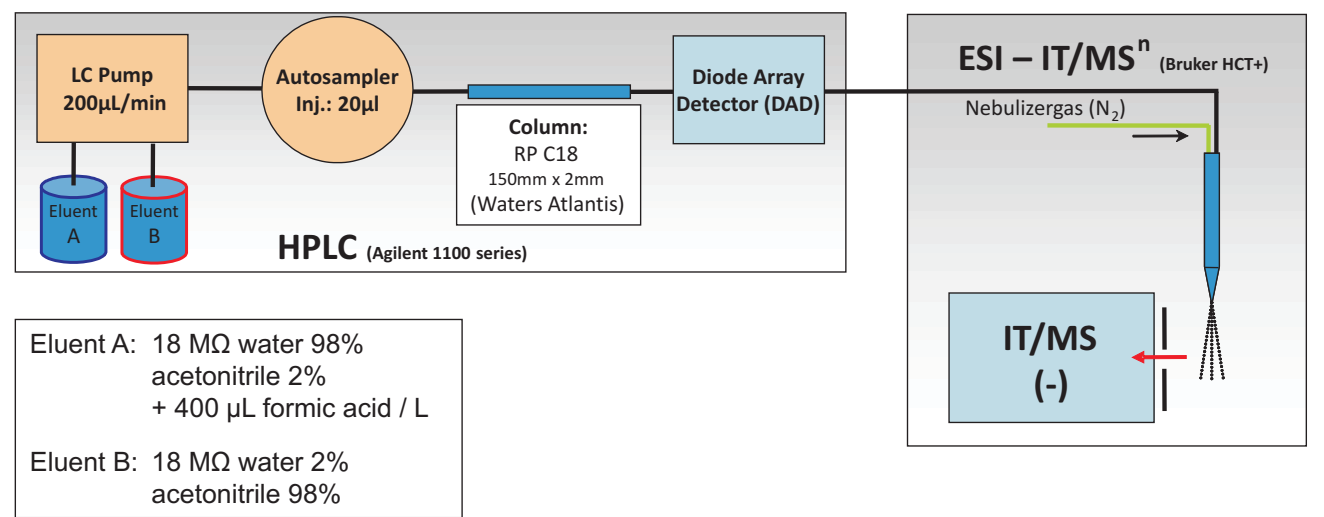

Abb. 6.57.: Experimenteller Aufbau zur Partikelanalyse, (HPLC/ESI-MS), entnommen aus M.-C. REINNING [148].

Die Methode ist insbesondere für die Analyse schwerflüchtiger Substanzen geeignet, da diese ohne große thermische Belastung in ihre Komponenten aufgetrennt werden können. Durch die Betrachtung der Retentionszeiten ergibt sich durch den Vergleich mit Standardsubstanzen eine bessere Identifizierbarkeit als bei der online-Massenspektrometrie. Zudem handelt es sich hier um eine sehr schonende lonisierungsmethode.

Zunächst wurden die Partikelmessungen wie in den vorangegangenen Messungen durchgeführt, lediglich der Ozonumsatz wurde verdoppelt, um mehr Aerosol zu produzieren. Es wurden Proben von den Reaktionsprodukten des 
$\alpha$-Pinens (Hochdruck- und Niederdruckbedingungen in Synthetischer Luft) und der Butensäure (stets Hochdruckbedingungen, in Synthetischer Luft und Stickstoff) genommen. Diese wurden in Filtern, die hinter eine Düse zwischen der Aerosolzelle und dem SMPS geschaltet wurden, aufgefangen, wobei mit einer zusätzlichen Pumpe ein definierter Durchfluss durch den Filter einreguliert wurde. Das Filtermaterial bestand aus PTFE-beschichteten Faserfiltern (PALLFLEX, T60A20, Pall Life Science, USA), welches eine besonders hydrophobe und inerte Oberfläche bereitstellt und so die genommene Partikelprobe in Beschaffenheit und chemischer Zusammensetzung möglichst wenig beeinflussen sollte [148]. Die Probennahme des Aerosols erfolgte über 20-30 Minuten, der Filter wurde anschließend in Schnappdeckelgläsern gelagert und bis zur weiteren Analyse mit Trockeneis gekühlt.

Die Partikelproben wurden anschließend mit einem Wasser / Methanolgemisch im Ultraschallbad extrahiert, filtriert und schließlich auf ein für die Analyse passendens Volumen eingeengt. Die mobile Phase besteht aus ELuent A (98 \% Wasser, $2 \%$, Acetonitril, $0.4 \mathrm{~mL}$ Ameisensäure) und Eluent B (98\% Acetonitril, $2 \%$ Wasser), die stationäre aus einer $\mathrm{C}_{18}$-Umkehrphase (Waters Atlantis T3 C18; $2.1 \times 15 \mathrm{~mm}$ ) [148].

Die Auswertung ergibt schließlich Peaks bei bestimmten Masse-zuLadungsverhältnissen $(\mathrm{m} / \mathrm{z})$, aus denen mit Hilfe der zugehörigen Retentionszeiten, die durch funktionelle Gruppen im Molekül beeinflusst werden, auf die Struktur des Moleküls rückgeschlossen werden kann.

\subsubsection{Analyseergebnisse}

Die Ergebnisse dieser Untersuchungen werden lediglich kurz dargestellt, da die Interpretation der Ergebnisse noch nicht abgeschlossen ist. Für die Durchführung der aufwendigen Auswertung möchte ich an dieser Stelle noch einmal Martin Beck aus Mainz danken.

\subsubsection{1. $\alpha$-Pinen}

Die relativen Ausbeuten einzelner Verbindungen aus den Aerosolproben des $\alpha$-Pinens sind in Abbildung 6.58 gezeigt. Die in der linken Spalte genannten 
$m / z$-Verhältnisse entsprechen den Massen der Produkte (bei einer nominellen Ladung von - 1), bei denen es sich meist um unterschiedlich verbrückte $\alpha$-Pinen-Dimere handelt. Bei den Brücken kann es sich um Ether- oder Estermotive handeln, wie sie bereits von MüLLER et al. [149] in Freiluftmessungen nachgewiesen werden konnten. Eine grüne Kennzeichnung steht für das Auftreten in Feldmessungen in der $\alpha$-Pinenozonolyse. Die Abkürzung „BP“ steht für ein bipolares Produkt.

\begin{tabular}{|c|c|c|c|c|c|c|c|}
\hline mbar & \multicolumn{2}{|c|}{10} & \multicolumn{2}{c|}{20} & \multicolumn{2}{c|}{465} & \\
\hline Produkte & Ausbeute & STABW & Ausbeute & STABW & Ausbeute & STABW & Gruppe \\
\hline $337-1$ & $0,0 \%$ & $0,0 \%$ & $0,4 \%$ & $0,1 \%$ & $100,0 \%$ & $9,5 \%$ & 1 \\
\hline $337-2 \mathrm{a}$ & $0,0 \%$ & $0,0 \%$ & $3,4 \%$ & $2,1 \%$ & $100,0 \%$ & $14,0 \%$ & 1 \\
\hline $337-4$ & $0,0 \%$ & $0,0 \%$ & $2,2 \%$ & $0,1 \%$ & $100,0 \%$ & $9,8 \%$ & 1 \\
\hline $371-1 \mathrm{~b}$ & $0,4 \%$ & $0,3 \%$ & $2,0 \%$ & $2,0 \%$ & $100,0 \%$ & $20,1 \%$ & 1 \\
\hline $371-1 \mathrm{a}$ & $0,5 \%$ & $0,2 \%$ & $2,5 \%$ & $1,1 \%$ & $100,0 \%$ & $23,0 \%$ & 1 \\
\hline 367 & $0,5 \%$ & $0,1 \%$ & $3,9 \%$ & $0,7 \%$ & $100,0 \%$ & $4,6 \%$ & 1 \\
\hline $337-2 \mathrm{~b}$ & $0,0 \%$ & $0,0 \%$ & $7,2 \%$ & $4,8 \%$ & $100,0 \%$ & $17,7 \%$ & $2 \mathrm{a}$ \\
\hline $329-3$ & $0,0 \%$ & $0,0 \%$ & $10,2 \%$ & $0,4 \%$ & $100,0 \%$ & $12,6 \%$ & $2 \mathrm{a}$ \\
\hline $329-2$ & $0,0 \%$ & $0,0 \%$ & $13,4 \%$ & $2,2 \%$ & $100,0 \%$ & $12,7 \%$ & $2 \mathrm{a}$ \\
\hline HPS & $1,7 \%$ & $0,4 \%$ & $8,2 \%$ & $2,7 \%$ & $100,0 \%$ & $14,3 \%$ & 2 \\
\hline 285 & $1,3 \%$ & $0,1 \%$ & $9,1 \%$ & $0,7 \%$ & $100,0 \%$ & $22,2 \%$ & 2 \\
\hline $329-1 \mathrm{~b}$ & $1,3 \%$ & $2,2 \%$ & $9,3 \%$ & $4,1 \%$ & $100,0 \%$ & $24,8 \%$ & 2 \\
\hline 355 & $2,1 \%$ & $0,2 \%$ & $12,8 \%$ & $4,0 \%$ & $100,0 \%$ & $10,1 \%$ & 2 \\
\hline $371-2$ & $2,2 \%$ & $0,7 \%$ & $14,2 \%$ & $1,8 \%$ & $100,0 \%$ & $11,9 \%$ & 2 \\
\hline $329-1 \mathrm{a}$ & $4,6 \%$ & $3,0 \%$ & $11,0 \%$ & $2,7 \%$ & $100,0 \%$ & $20,8 \%$ & $2 \mathrm{~b}$ \\
\hline 357 & $4,8 \%$ & $0,4 \%$ & $15,7 \%$ & $2,3 \%$ & $100,0 \%$ & $12,8 \%$ & $2 \mathrm{~b}$ \\
\hline 313 & $3,9 \%$ & $0,9 \%$ & $23,0 \%$ & $4,3 \%$ & $100,0 \%$ & $17,4 \%$ & 3 \\
\hline $369 \mathrm{BP}$ & $5,2 \%$ & $1,1 \%$ & $28,3 \%$ & $5,1 \%$ & $100,0 \%$ & $13,1 \%$ & 3 \\
\hline $\mathrm{m} / \mathrm{z} 353$ & $7,7 \%$ & $1,6 \%$ & $40,7 \%$ & $11,1 \%$ & $100,0 \%$ & $14,4 \%$ & 3 \\
\hline $331-\mathrm{D}$ & $11,9 \%$ & $9,0 \%$ & $48,9 \%$ & $21,6 \%$ & $100,0 \%$ & $60,6 \%$ & 3 \\
\hline $231-\mathrm{BP}$ & $20,8 \%$ & $2,9 \%$ & $43,1 \%$ & $5,9 \%$ & $100,0 \%$ & $8,4 \%$ & 3 \\
\hline $337-3$ & $203,0 \%$ & $66,2 \%$ & $574,8 \%$ & $68,0 \%$ & $100,0 \%$ & $55,2 \%$ & 4 \\
\hline
\end{tabular}

Abb. 6.58.: Auswertung der $\alpha$-Pinenergebnisse bei verschiedenen Gesamtdrücken $p_{\text {ges }}=(10,20,465)$ mbar.

Bei der Bestimmung der relativen Ausbeuten wurden die bei $p_{\text {ges }}=465 \pm 10$ mbar für die Vergleichbarkeit als $100 \%$ festgelegt, zudem sind die Standardabweichungen („STABW“) zu den jeweiligen Werten genannt. Die Zahlenwerte zeigen für fast alle gebildeten Produkte eine Abnahme mit dem Gesamtdruck, die sich sogar unter Niederdruckbedingungen zwischen $p_{\text {ges }}=20$ mbar und $p_{\text {ges }}=10$ mbar noch einmal recht stark bemerkbar macht. Zur besseren Unterscheidung werden die Verbindungen in mehrere Gruppen aufgeteilt (rechte Spalte). Gruppe 1 umfasst die Verbindungen, die in ihren relativen Ausbeuten sehr empfindlich auf eine Druckänderung reagieren und bei Hochdruckbedingungen verstärkt gebildet werden, in Gruppe 2 ist diese Änderung weniger stark, in Gruppe 3 nur noch schwach ausgeprägt. Gruppe 
4 liegt dagegen unter Niederdruckbedingungen verstärkt im Produktgemisch vor. Die farbigen Kennzeichnungen in den Spalten 2 und 4 sollen lediglich zur Orientierung dienen und ergeben sich aus den Gruppeneinteilungen.

Die Aufstellung verdeutlicht, dass in der Ozonolyse eine Vielzahl ähnlicher, aber durch bestimmte Analysemethoden dennoch unterscheidbarer Verbindungen in der Aerosolphase gebildet wird, die auch unterschiedlich auf eine Veränderunge der Reaktionsumgebung reagieren. Dies lässt sich auf Veränderungen der Gasphasenprozesse zurückführen, welche zur Bildung schwerflüchtiger und kondensierbarer Produkte führen.

Die starke Druckabhängigkeit der Gruppe 1 weist auf eine Beteiligung der CRIEGEE-Intermdiate hin, die unter Niederdruckbedingungen nur zu einem wesentlich geringeren Anteil stabilisiert und nur unter Hochdruckbedingungen für bimolekulare Reaktionen in ihrer Lebensdauer ausreichend werden. Nach Untersuchungen von DONAHUE et al. [140] wird bei 20 mbar nur 1-10\% des unter Hochdruckbedingungen stabilisierten Anteils an $\mathrm{SCl}$ gebildet.

Auch die Peroxy- / Alkoxychemie könnte bedeutsam sein, da diese Intermediate bei höherem Gesamtdruck ebenfalls besser stabilisiert werden können.

\begin{tabular}{|c|c|c|c|c|c|c|c|}
\hline mbar & \multicolumn{2}{|c|}{10} & \multicolumn{2}{c|}{20} & \multicolumn{2}{|c|}{465} & \\
\hline Produkte & Ausbeute & STABW & Ausbeute & STABW & Ausbeute & STABW & Gruppe \\
\hline HPS & $1,7 \%$ & $0,4 \%$ & $8,2 \%$ & $2,7 \%$ & $100,0 \%$ & $14,3 \%$ & 2 \\
\hline trans-Norpinsäure & $2,8 \%$ & $0,7 \%$ & $11,2 \%$ & $2,1 \%$ & $100,0 \%$ & $13,3 \%$ & 2 \\
\hline Pinsöure & $5,4 \%$ & $1,1 \%$ & $19,5 \%$ & $2,7 \%$ & $100,0 \%$ & $6,1 \%$ & $2 \mathrm{~b}$ \\
\hline $231-B P$ & $20,8 \%$ & $2,9 \%$ & $43,1 \%$ & $5,9 \%$ & $100,0 \%$ & $8,4 \%$ & 3 \\
\hline Terpenylic Acid & $29,0 \%$ & $7,5 \%$ & $42,5 \%$ & $0,9 \%$ & $100,0 \%$ & $9,3 \%$ & 3 \\
\hline
\end{tabular}

Abb. 6.59.: Ausbeuten der identifizierten Produkte der $\alpha$-Pinenozonolyse bei verschiedenen Gesamtdrücken $p_{\text {ges }}=(10 / 20 / 465)$ mbar.

Die Tabelle in Abbildung 6.59 gibt die identifizierten Produkte noch einmal namentlich an, welche bereits in Feldstudien nachgewiesen wurden und in der vorangegangenen Tabelle grün unterlegt sind.

\subsubsection{Butensäure}

Für Butensäure erschien der Badgaseffekt interessanter als die Druckabhängigkeit der Aerosolbildung. Aus diesem Grund wurden für diese Substanz Messungen bei gleichbleibend $p_{\text {ges }}=465 \pm 10$ mbar durchgeführt, wobei jedoch Synthetische Luft und Stickstoff als Badgase Verwendung fanden. 
In Tabelle 6.60 werden die identifizierten $\mathrm{m} / \mathrm{z}$-Verhältnisse der Produktverbindungen aufgelistet. In rot werden sich bei der Verwendung von Sticksoff statt Synthetischer Luft stark reduzierende Ausbeuten gekennzeichnet, in gelb leicht reduzierende, in grün gleichbleibende und in blau zunehmende Mengen.

\begin{tabular}{|c|c|}
\hline Produkt & Veränderung durch $\mathrm{O}_{2}$-Entzug \\
\hline 253 & -- \\
\hline 215 & -- \\
\hline $195-\mathrm{BP}$ & -- \\
\hline $333-1$ & -- \\
\hline $333-2$ & -- \\
\hline 291 & -- \\
\hline 439 & -- \\
\hline 251 & -- \\
\hline 365 & -- \\
\hline 335 & -- \\
\hline $221-1$ & - \\
\hline $221-2$ & - \\
\hline 223 & - \\
\hline 195 & 0 \\
\hline 207 & 0 \\
\hline 205 & + \\
\hline
\end{tabular}

Abb. 6.60.: Veränderungen in den Ausbeuten bei Verwendung von Stickstoff statt Synthetischer Luft, alle Experimente wurden bei $p_{\text {ges }}=465 \pm 10$ mbar durchgeführt.

Da bei den untersuchten Aerosolzusammensetzungen durch konstanten Gesamtdruck physikalische Druckeffekte keine Rolle spielen können, muss sich hier eine Beeinflussung der Intermediatstabilität durch chemische Reaktionen auswirken, die die Veränderungen nach sich ziehen.

Auch hier ist eine Vielzahl von Produkten zu beobachten und die Interpretationen noch nicht abgeschlossen, allerdings ist davon auszugehen, dass die CRIEGEE-Intermediat- sowie Peroxy- / Alkoxychemie im Aufbau der jeweiligen Verbindungen eine wichtige Rolle spielen. Wie bereits im Zusammenhang mit der Ketenbildung (Abschn. 6.2.3) und den reduzierten Aerosolausbeuten in Synthetischer Luft vermutet, scheint sich auch hier abzuzeichnen, dass die Reaktion von $\mathrm{O}_{2}$ mit bestimmten Zwischenprodukten letztere destabilisiert. Durch 
eine Fragmentation und die sich ergebende Dampfdruckerhöhung der Bruchstücke würden sie nicht mehr zur partikulären Materie beitragen können.

Auffallend ist vor allem die Abnahme hochmolekularer Produkte bei Sauerstoffentzug, die durch die eingeschränkte Partikelbildung in Synthetischer Luft kontraintuitiv erscheint. Hier scheinen massive Änderungen der Produkte in der Aerosolphase einzutreten, die unter Umständen durch die Ausbildung einer größeren Zahl an funktionellen Gruppen durch Reaktionen in der kondensierten Phase zu erklären sind. Vor allem das $\mathrm{m} / \mathrm{z}$-Verhältnis von 439 als Verbindung mit dem höchsten Molekulargewicht ist hier herauszustellen.

Ein Vorschlag zur Bildung bestimmter $m / z$-Verhältnisse auf Grundlage der $\mathrm{Cl}$ und verschiedenen Ozonolyseprodukten ist in Abb. 6.61 gezeigt, wobei diese Annahmen noch verifiziert werden müssen. 
Möglicher Reaktionsweg für die in der Partikelphase auftretenden Massepeaks Zunächst Zerfall über Vinylhydroperoxidkanal, anschließend Anlagerung des unsubstituierten $\mathrm{Cl}$<smiles>CC(C)COOC(C)C(=O)O</smiles>

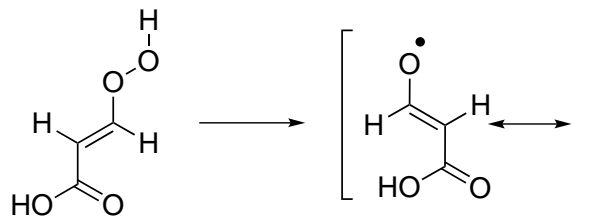<smiles>O=CC(=O)OCCO</smiles>

$\operatorname{syn}(\mathrm{m} / \mathrm{z} 104)$

Vinylhydroperoxid (m/z 104)

((E)-3-Hydroperoxyacrylsäure)

Vinoxylradikal (m/z 87)

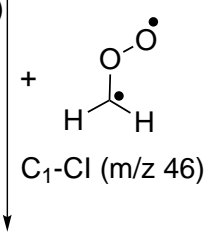<smiles>O=CC(C=O)(C(=O)O)C(O)OO</smiles>

$(\mathrm{m} / \mathrm{z} 179)$

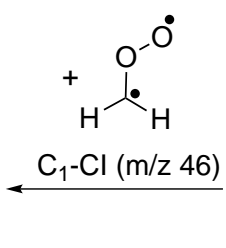

46)<smiles>O=CC(O)C(=O)OO</smiles>

$(\mathrm{m} / \mathrm{z} 133)$

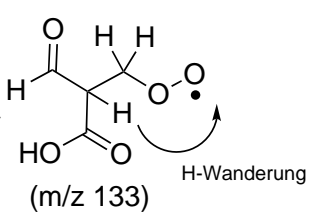

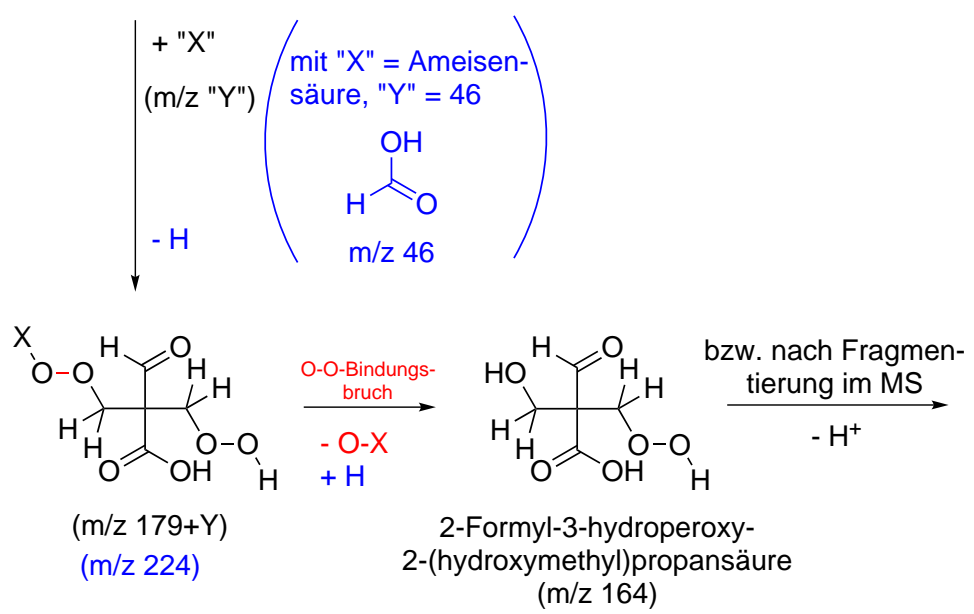

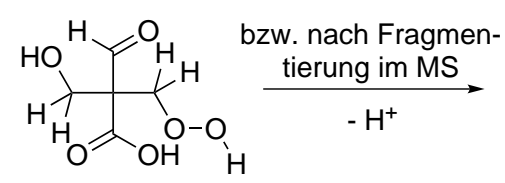

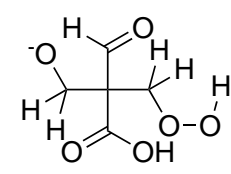

$(\mathrm{m} / \mathrm{z}$ 163)

Abb. 6.61.: Vorschlag für die Bildung von hochmolekularen Produkten in der Partikelphase, die Protonenabspaltung im letzten Schritt ist der experimentellen Methode geschuldet, da Anionen nachgewiesen werden. 


\section{Diskussion}

In diesem Kapitel sollen die in den vorangegangenen Abschnitten gezeigten Ergebnisse diskutiert werden. Interpretation und Literaturvergleich einiger Aspekte, wie die Bildung charakteristischer Produkte (Kap. 6.2) oder Spezifika der Partikelbildungsvorgänge verschiedener Substanzklassen (Kap. 6.4), wurde bereits an das Ende der jeweiligen (Unter-) Abschnitte vorgezogen. Dies war der Datenmenge, vor allem aber der Verschiedenartigkeit der einzelnen Verbindungsklassen und ihrem spezifischen Reaktionsverhalten in der Ozonolyse geschuldet. In diesem Kapitel werden die vielfältigen Gesichtspunkte nun zusammengeführt.

\subsection{Zentrale Rolle der $\mathrm{OH} / \mathrm{HO}_{2}$-Chemie? - Auswirkungen der Verwendung verschiedener Badgase}

\subsubsection{Kinetikstudien}

Allgemein ist es für die Bestimmung des Geschwindigkeitskoeffizienten der Ozonolysereaktion problematisch, dass innerhalb der reaktiven Mischung eine Vielzahl von Nebenreaktionen stattfinden, sobald die ersten Intermediate gebildet werden. Aus diesem Grund werden bei den meisten der in der Literatur zu findenden Studien bestimmte Fängermoleküle eingesetzt, um reaktive Spezies wie $\mathrm{OH}$-Radikale (meist $\mathrm{C}_{6} \mathrm{H}_{12}[9,59,68,69,81,117,119,150], \mathrm{CO}$ [61], 2-Butanol [81] oder 2-Propanol [151]) oder stabilisierte CRIEGEE-Intermediate (häufig Ameisensäure [59, 119]) abzufangen, da diese mit den Eduktmolekülen reagieren können und so zu einem zusätzlichen Verbrauch und tendenzi- 
ell zu hoch bestimmten Geschwindigkeitskoeffizienten führen. Nachteilig wirkt sich dieses Vorgehen bei der Verwendung der IR-Spektroskopie zur Konzentrationsbestimmung auf die Genauigkeit der Restkonzentrationen aus, da die Fängersubstanzen für eine effektive Wirkung in hohem Überschuss zugegeben werden müssen, was zu Überlagerungen der Edukt- und Fängerbanden im Spektrum führt. Die Absorbanzen, etwa für Cyclohexan und Ameisensäure, sind in einzelnen Bereichen außerdem so stark, dass die Signale in die Sättigung gehen. Dadurch können diese nicht immer vollständig aus dem Spektrum herauskorrigiert werden, was eine große Unsicherheit für die Bestimmung der Eduktrestkonzentrationen und damit der aus ihnen bestimmten Geschwindigkeitskoeffizienten bedeutet.

Für eine möglichst genaue Konzentrationsbestimmung wurde daher auf die Zugabe von Fängersubstanzen verzichtet. Stattdessen wurde aus den zu verschiedenen Zeitpunkten vorliegenden Restkonzentrationen die Umsätze der Edukte und damit der relative Verbrauch $\alpha=\frac{\Delta p(\text { Alken })}{\Delta p\left(\mathrm{O}_{3}\right)}$ (Gl. 2.19) bestimmt [35]. Da das Reaktionsverhältnis der Elementarreaktion $1: 1$ ist, kann unter Einbeziehung des relativen Verbrauches und seiner Abweichung von 1 die $\mathrm{OH}$ Ausbeute bestimmt werden ( $Y_{\mathrm{OH}}=\alpha-1$, Gl. 2.28). Dabei werden jedoch nicht nur der $\mathrm{OH}$-induzierte Ozonabbau, sondern auch der potenzielle Verbrauch beider Edukte durch $\mathrm{HO}_{2}$-Radikale sowie heterogene Prozesse, wie Wandverluste, vernachlässigt.

Die Ergebnisse (Kapitel 6.1 bzw. Tabelle A.3 sprechen allerdings dafür, dass die in der Auswertung getroffene Annahme, dass ausschließlich OH-Radikale zum zusätzlichen Alkenverbrauch beitragen, für eine Beschreibung der Ozonolysekinetik ausreicht. Dabei ist zu beachten, dass diese Aussage nur für die verwendeten experimentellen Bedingungen (z.B. Alkenüberschuss, moderate Drücke) und den beschriebenen Aufbau nachprüfbar gültig sind.

Es wurde ein Ansatz nach einem Vorschlag von NEEB et al. [35] verwendet, bei dem durch Berücksichtigung des relativen Verbrauchs statt eines effektiven Geschwindigkeitskoeffizienten $k_{\text {eff }}$, in dem außer der Ozonolysereaktion auch Nebenreaktionen durch zusätzlichen Verbrauch der Edukte beitragen, der um die $\mathrm{OH}$-Chemie korrigierte Koeffizient $k$ berechnet wird, der der eigentlich betrachteten Umsetzung zugeordnet wird: 


$$
\begin{array}{rlr}
-\frac{\mathrm{d}[\text { Alken }]}{\mathrm{d} t} & = & k_{\mathrm{eff}} \cdot[\text { Alken }] \cdot\left[\mathrm{O}_{3}\right] \\
& = & k \cdot\left(1+Y_{\mathrm{OH}}\right) \cdot[\text { Alken }] \cdot\left[\mathrm{O}_{3}\right] \\
& = & k \cdot \frac{\Delta p(\text { Alken })}{\Delta p\left(\mathrm{O}_{3}\right)} \cdot[\text { Alken }] \cdot\left[\mathrm{O}_{3}\right]
\end{array}
$$

Die Kinetikversuche der vorliegenden Arbeit (Kap. 6.1) zeigen, dass das experimentelle Verfahren für die unterschiedlichen Verbindungen, von reinen Kohlenwasserstoffverbindungen bis hin zu Carbonsäuren, sehr gut geeignet ist. Im Rahmen der bei solchen Untersuchungen erreichbaren Genauigkeit wurden für alle ungesättigten Verbindungen zu den verfügbaren Literaturwerten vergleichbare Ergebnisse erhalten (vgl. Tabelle A.3 auf Seite VII und dort zitierte Literatur). Die Abweichungen zwischen Mess- und Literaturwerten treten dabei in beide Richtungen auf, was eine systematische Fehlbestimmung unwahrscheinlich macht.

Dabei wurden die Experimente stets bei $p_{\text {ges }}=460 \pm 10$ mbar und bei Zimmertemperatur ( $T=295 \pm 2 \mathrm{~K}$ ) durchgeführt, um den Startzeitpunkt der infrarotspektroskopisch verfolgten Reaktion schnellstmöglich nach der Zusammenführung der Reaktanden durch simultane Expansion aus den Vormischkammern wählen zu können. In Experimenten von WOLF [48] waren zuvor keine Unterschiede zu den Geschwindigkeitskoeffizienten bei $p_{\text {ges }}=1000$ mbar festgestellt worden, die über die experimentbedingte Schwankungsbreite von etwa $10 \%$ hinausging 1 .

Der Einsatz des Ozonanalysators zur standardmäßigen Konzentrationsbestimmung ist für diese Experimente nur eingeschränkt geeignet, da seine Nachweisgrenze nur bei etwa $p_{\text {Ozon }} \approx 1$ Pa liegt [104]. Dies würde den verwendbaren Konzentrationsbereich sehr stark einschränken, da nach Expansion aus der Vormischkammer maximal $p_{\text {Ozon }} \approx 0.26 \mathrm{~Pa}$ in der Reaktionsmischung umgesetzt werden könnten. Da die Güte der angegebenen Geschwindigkeitskoeffizienten jedoch direkt von der Genauigkeit abhängt, mit der die Restkonzentra-

\footnotetext{
${ }^{1}$ Zu möglichen Gründen für die Abweichungen s. Anhang G
} 
tionen in der reaktiven Mischung bestimmt werden können, ist es von Vorteil, wenn über möglichst viele der gewählten Reaktionszeitpunkte Eduktbanden deutlich identifizierbar bleiben. Dies macht den Einsatz recht hoher Ozonanfangskonzentrationen (um $p_{\text {ozon }} \approx 1 \mathrm{~Pa}$ in der Aerosolzelle) wünschenswert und spricht gegen den standardmäßigen Betrieb des Ozonanalysators.

Generell liegt ein Vorteil des in den vorgestellten Messungen verwendeten Aufbau in der Möglichkeit, recht hohe Anfangskonzentrationen zu verwenden, da die Edukte erst durch die Expansion aus den Vorkammern zusammengeführt und auf einer deutlich kürzeren Zeitskala als der Reaktionszeit vermischt werden. Dies wiederum bedingt vergleichsweise kurze Messzeiten, da sich die Reaktion der Edukte durch die höheren Partialdrücke entsprechend beschleunigt. Die Kinetikmessungen anderer Arbeitsgruppen müssen wegen des, bedingt durch sehr kleine Konzentrationen eines der Edukte (meist unter $0.05 \mathrm{~Pa}$, vgl. [59, 72, 151]), langsamen Abbaus der Reaktanden oft über sehr lange Zeiträume aufgenommen werden. Durch die oft verwendeten sehr großen Volumina der Reaktoren muss zudem mit Inhomogenitäten durch lange Durchmischungszeiten gerechnet werden. Auch Wandverluste sind in allen Fällen in Betracht zu ziehen, wobei diese relativ gesehen bei größeren Volumina weniger ins Gewicht fallen sollten.

Die Ergebnisse von Kinetikstudien in verschiedenen Badgasen zeigen, dass sich der von JAPAR et al. [121] für Olefine gezeigte Effekt deutlich schnellerer Reaktionsgeschwindigkeiten in Stickstoff auch auf voroxidierte Verbindungen übertragen lässt. Für die verwendeten Carbonsäuren (Methacrylsäure und Butensäure) gilt dies ebenso wie für den zum Vergleich getesteten Alkohol 1-Penten-3-ol, was zeigt, dass dieser Effekt unabhängig vom Oxidationsgrad der Kohlenwasserstoffverbindung auftritt. Das lässt darauf schließen, dass zusätzliche Reaktanden in Sauerstoffabwesenheit außerhalb der betrachteten Ozonolysereaktion verbraucht werden. Nach Untersuchungen von TREACY et al. [72] kommen dafür hauptsächlich $\mathrm{OH}$ - und $\mathrm{HO}_{2}$-Radikale in Frage. Dabei geht aus einem Vergleich der Geschwindigkeitskoeffizienten hervor, dass Ozon wohl vor allem durch $\mathrm{HO}_{2}$ verbraucht wird $\left(\mathrm{O}_{3}+\mathrm{HO}_{2} \longrightarrow 2 \mathrm{O}_{2}+\mathrm{OH}\right)$, während $\mathrm{OH}$-Radikale deutlich schneller von den ungesättigten Kohlenwasserstoffverbindungen abgefangen werden und so zum zusätzlichen Alkenverbrauch beitragen. 
7.1. Zentrale Rolle der $\mathrm{OH} / \mathrm{HO}_{2}$-Chemie? - Auswirkungen der Verwendung verschiedener Badgase

Aus diesen Ergebnissen kann gefolgert werden, dass die Sauerstoffanwesenheit für den Abfang von zumindest $\mathrm{HO}_{2^{-}}$, und unter Umständen auch für den von $\mathrm{OH}$-Radikalen, sorgen muss. Eine mögliche Erklärung bietet die badgasabhängige Ausprägung der Peroxy- / Alkoxychemie. In Sauerstoffanwesenheit werden aus Alkylradikalen, die aus der Ozonolyse beim Zerfall der $\mathrm{Cl}$ gebildet werden, in einer Additionsreaktion mit $\mathrm{O}_{2}$ mit höherer Wahrscheinlichkeit Peroxyradikale $\left(\mathrm{RO}_{2}\right)$ gebildet [33] als in einer Stickstoffatmosphäre. Diese können zum einen durch die Selbstreaktion Alkoxyradikale ( $u$ nd $\mathrm{O}_{2}$ ) bilden, zum anderen durch das auf Seite 33 gezeigte Reaktionsschema aber auch $\mathrm{HO}_{2}$-Radikale abfangen.

Da diese Reaktion mit Peroxyradikalen deutlich schneller als die mit Ozon abläuft [130], können die $\mathrm{HO}_{2}$-Radikale in Synthetischer Luft auf effiziente Weise abgefangen und der zusätzliche Ozonverbrauch verhindert werden. Diese Reaktion hat auch für die Bildung schwerflüchtiger Verbindungen eine große Bedeutung [21], was im Abschnitt 7.2.1 näher beschrieben wird.

In Sauerstoffabwesenheit, durch die Verwendung von Stickstoff als Badgas, wird dagegen die Reaktion von Vinoxylradikal und Ozon wahrscheinlicher. Hier entsteht ein energetisch angeregtes Alkoxyradikal, das entweder weiter zerfallen oder stoßstabilisiert wird und im Anschluss Isomerisierungsreaktionen eingehen kann [20,48], wobei noch nicht abschließend bekannt ist, ob die $\mathrm{H}$ Abstraktion durch Sauerstoffatome in Alkoxyradikalen stattfindet und welche Bedeutung dieser zukäme.

Die Untersuchungen haben gezeigt, dass der recht einfache Ansatz von NEEB et al. [35] eine unabhängig von der Eduktstruktur universell einsetzbare Methode ist. Sogar für voroxidierte Verbindungen mit Hydroxylgruppen, wie ungesättigte Alkohole oder Carbonsäuren, konnten zufriedenstellende Übereinstimmungen mit Literaturwerten erhalten werden. Gerade für diese Verbindungsklassen, die für ihre Reaktion mit stabilisierten CRIEGEE-Intermediaten oder Dimerisierungseffekte bekannt sind und daher durch zusätzlichen Verbrauch der Kohlenwasserstoffverbindung die Bestimmung der Geschwindigkeitskoeffizienten verkomplizieren können, konnte damit gezeigt werden, dass der Einsatz von $\mathrm{Cl}$ - und $\mathrm{OH}$-Fängern und die Inkaufnahme der aus innen folgenden Problematik nicht immer notwendig ist. Beachtet werden muss, dass sich der relative Verbrauch $\alpha$ in diesen Fällen nicht zwangsläufig nur aus den 
$\mathrm{OH}-$ Reaktionen ergibt, sondern eventuell auch ein Beitrag aus Cl-Reaktionen stammt.

\subsubsection{Produktbildungsstudien - Abhängigkeit von Gesamtdruck und Badgas}

Die IR-spektroskopische Untersuchung der Ozonolyseprodukte in der Gasphase ergab, dass sich an einigen Stellen Unterschiede in der Produktzusammensetzung zeigen. Diese lassen sich auf die Verwendung unterschiedlicher Badgase zurückführen. Allerdings wurden die verschiedenen Reaktionskanäle bei sämtlichen verwendeten Substanzen durch die Änderung des Gesamtdruckes deutlich stärker beeinflusst.

Neben den in Kap. 5.2.2 genannten Produkten, die aus Fragmentierungen der in der Ozonolyse gebildeten Intermediate stammen können, konnten substanzabhängig für einige der untersuchten Edukte weitere Verbindungen anhand von Vergleichen mit Reinspektren, berechneten Frequenzspektren oder der Literatur identifiziert werden.

Die reinen Kohlenwasserstoffverbindungen Methylcyclopenten, 1-Hexen und 2-Methyl-1-penten zeigten bei einem Gesamtdruck von $p_{\text {ges }}=1000$ mbar keine ausgeprägte Abhängigkeit von der An- oder Abwesenheit von Sauerstoff. Dies gilt auch für die Mehrzahl der sauerstoffhaltigen ungesättigten Edukte, lediglich für 3-Buten-1-ol (Abschn. 6.2.2.1) und 1-Penten-3-on (Abschn. 6.2.2.2) ließen sich badgasabhängige Unterschiede in den Banden des Carbonyl- (um $1700 \mathrm{~cm}^{-1}$ ) und Gerüstbereiches (unterhalb von $1500 \mathrm{~cm}^{-1}$ ) feststellen. Deutlichere Unterschiede ließen sich dagegen bei der Betrachtung der Differenzen im gleichen Badgas bei unterschiedlichen Gesamtdrücken feststellen, wobei diese Unterschiede in ihrer Ausprägung teilweise von der Sauerstoffanwesenheit beeinflusst schienen. So war etwa die bei einigen Verbindungen auftretende Ketenbildung in Synthetischer Luft und unter Niederdruckbedingungen erhöht. Die Tabellen 7.1 und 7.2 geben einen Überblick über die für die jeweiligen Kohlenwasserstoffverbindungen qualitativ nachgewiesenen Produkte, unter welchen experimentellen Bedingungen dies am deutlichsten möglich war und auf welche Weise die Identifikation erfolgte. 
7.1. Zentrale Rolle der $\mathrm{OH} / \mathrm{HO}_{2}$-Chemie? - Auswirkungen der Verwendung verschiedener Badgase

Wie für die Alkene sowie die ungesättigten Alkohole (Tab. 7.1) zu erkennen ist, werden die betrachteten Produkte meist unter allen verwendeten Experimentbedingungen beobachtet. Bei den unter Niederdruckbedingungen (ND) vermehrt gebildeten Verbindungen handelt es sich um Zerfallsprodukte aus bestimmten Ozonolyseprodukten, die bei unzureichender Stoßdesaktivierung beschleunigt fragmentieren. Hier handelt es sich um Ameisensäure $(\mathrm{HCOOH}, \mathrm{Bil}-$ dungsmechanismus z.B. Abb. 2.5, S. 26), Ethen $\left(\mathrm{C}_{2} \mathrm{H}_{4}, \mathrm{~S}\right.$. 134), Keten $\left(\mathrm{H}_{2} \mathrm{CCO}\right.$, S. 153 f.) und seine Derivate. Die Bildung von Ameisensäure scheint für die Alkene eher druck- als badgasabhängig zu erfolgen, da sie unter Niederdruckbedingungen in Sauerstoffan- und - abwesenheit vermehrt gebildet wird. Bei den ungesättigten Alkoholen scheint die Bande in Stickstoff etwas intensiver auszufallen. Dies gilt auch für die Carbonsäuren (Tab. 7.2), während die Bildung bei der Ozonolyse der Carbonylverbindungen in Synthetischer Luft begünstigt ist.

Auch die Bildung von Ethen und Keten oder seinen Strukturanaloga ist in erster Linie durch fehlende Stoßstablisierung induziert, lediglich für die Ketenbildung ließen sich leichte Zunahmen bei der Verwendung von Synthetischer Luft (SL) beobachten.

Formaldehyd $\left(\mathrm{H}_{2} \mathrm{CO}\right.$, s. z.B. Abb. 2.2 S. 23) wird meist druckunabhängig aus dem Zerfall des Primärozonids gebildet. Allerdings ist in Einzelfällen auch eine Produktion aus sekundären Reaktionen unter Sauerstoffbeteiligung der CRIEGEE-Intermediate möglich, wie es z.B. in Abb. 6.12 (S. 136) für Methylcyclopenten dargestellt ist, die für eine stärkere Bande in Synthetischer Luft sorgen.

Zusätzlich wurden noch einige weitere in der Ozonolyse gebildete Verbindungen nachgewiesen. Sicher konnten 2-Oxobutanal für 1-Penten-3-on und Succinaldehyd für 4-Penten-1-al anhand des Vergleichs mit theoretisch berechneten Infrarotspektren $(B 3 L Y P / 6-311+G(d, p))$ identifiziert werden. Diese entstehen aus dem Zerfall der jeweiligen Primärozonide, der in erster Näherung druckunabhängig erfolgen sollte.

Für 1-Penten-3-on, Methacrylsäure und Butensäure wurden weiterhin die Spektren für einige Hydroperoxidverbindungen berechnet, die aus der Reaktion der jeweiligen CRIEGEE-Intermediate mit Peroxyradikalen (für 1-Penten-3-on nach [125]) bzw. mit in der Reaktion gebildeter Ameisensäure oder Eduktmolekülen (für Methacrylsäure, nach [59]) oder Wasser (für Butensäure, nach [59]) 


\begin{tabular}{|c|c|c|c|c|}
\hline Alken & Produkt & exp. Bedingung (max. bei) & Nachweisart & Abb. \\
\hline Methylcyclopenten & $\begin{array}{c}\mathrm{HCOOH} \\
\mathrm{C}_{2} \mathrm{H}_{4} \\
\mathrm{H}_{2} \mathrm{CCO} \\
\mathrm{H}_{2} \mathrm{CO}\end{array}$ & $\begin{array}{l}\text { alle }\left(\mathrm{N}_{2} / \mathrm{SL} N D\right) \\
\text { alle }(\mathrm{SL} N D) \\
\text { alle (SL ND) } \\
\quad \text { alle }\end{array}$ & $\begin{array}{l}\text { Reinspektrum } \\
\text { Reinspektrum } \\
\text { Literaturspektrum [123] } \\
\text { Reinspektrum }\end{array}$ & $\begin{array}{c}6.9 \mathrm{a} \\
6.9 \mathrm{c} \\
6.11 \mathrm{a}, 6.18 \\
6.11 \mathrm{c}\end{array}$ \\
\hline 1-Hexen & $\begin{array}{c}\mathrm{H}_{2} \mathrm{CO} \\
\mathrm{HCOOH} \\
\text { n-Propylketen (?) } \\
\mathrm{C}_{2} \mathrm{H}_{4}\end{array}$ & $\begin{array}{c}\text { alle }(\mathrm{SL} \mathrm{HD)} \\
\text { alle }(\mathrm{SL} N D) \\
\mathrm{N}_{2} / \mathrm{SL} N D \\
\mathrm{~N}_{2} \mathrm{ND} \text {, sehr schwach }\end{array}$ & $\begin{array}{c}\text { Reinspektrum } \\
\text { Reinspektrum } \\
\text { berechnetes Frequenzspektrum } \\
\text { Reinspektrum }\end{array}$ & \begin{tabular}{|l|}
$\mathrm{D.1c}$ \\
$\mathrm{D.1d}$ \\
$\mathrm{D.1a}$ \\
$\mathrm{D.1b}$ \\
\end{tabular} \\
\hline 2-Methyl-1-penten & $\begin{array}{c}\mathrm{HCOOH} \\
\mathrm{H}_{2} \mathrm{CCO} \\
\mathrm{H}_{2} \mathrm{CO}\end{array}$ & $\begin{array}{c}\text { alle }\left(\mathrm{N}_{2} / \mathrm{SL} \mathrm{ND}\right) \\
\mathrm{N}_{2} / \mathrm{SL} \mathrm{ND} \text {, sehr schwach } \\
\text { alle }\end{array}$ & $\begin{array}{l}\text { Reinspektrum } \\
\text { Literaturspektrum [123] } \\
\text { Reinspektrum }\end{array}$ & \begin{tabular}{|l|}
$D .2 b$ \\
$D .2 c$ \\
$D .2 d$ \\
\end{tabular} \\
\hline 3-Buten-1-ol & $\begin{array}{c}\mathrm{HCOOH} \\
\mathrm{C}_{2} \mathrm{H}_{4} \\
\mathrm{H}_{2} \mathrm{CO} \\
\mathrm{H}_{2} \mathrm{CCO}\end{array}$ & $\begin{array}{c}\text { alle }\left(\mathrm{N}_{2} \mathrm{ND}\right) \\
\text { alle }\left(\mathrm{N}_{2} / \mathrm{SL} N D\right) \\
\text { alle }\left(\mathrm{N}_{2} \mathrm{ND}\right) \\
\text { alle }\left(\mathrm{N}_{2} / \mathrm{SL} N D\right)\end{array}$ & $\begin{array}{c}\text { Reinspektrum } \\
\text { Reinspektrum } \\
\text { Reinspektrum } \\
\text { Literaturspektrum [123] }\end{array}$ & \begin{tabular}{|l|}
$\mathrm{D.5a}$ \\
$\mathrm{D.5a}$ \\
$\mathrm{D.5 \textrm {c }}$ \\
6.18 \\
\end{tabular} \\
\hline 1-Penten-3-ol & $\begin{array}{c}\mathrm{HCOOH} \\
\mathrm{H}_{2} \mathrm{CO}\end{array}$ & $\begin{array}{l}\text { alle }\left(\mathrm{N}_{2} \mathrm{ND}\right) \\
\text { alle }\left(\mathrm{N}_{2} \mathrm{ND}\right)\end{array}$ & $\begin{array}{l}\text { Reinspektrum } \\
\text { Reinspektrum }\end{array}$ & \begin{tabular}{|l|}
$\mathrm{D} .8 \mathrm{~b}$ \\
$\mathrm{D} .8 \mathrm{~d}$ \\
\end{tabular} \\
\hline 4-Penten-1-ol & $\begin{array}{c}\mathrm{HCOOH} \\
\mathrm{C}_{2} \mathrm{H}_{4} \\
\mathrm{H}_{2} \mathrm{CO}\end{array}$ & $\begin{array}{l}\text { alle }\left(\mathrm{N}_{2} \mathrm{ND}\right) \\
\text { alle }\left(\mathrm{N}_{2} / \mathrm{SL} \mathrm{ND}\right) \\
\text { alle }\end{array}$ & $\begin{array}{l}\text { Reinspektrum } \\
\text { Reinspektrum } \\
\text { Reinspektrum }\end{array}$ & $\begin{array}{l}\overline{D .11 b} \\
\mathrm{D.11b} \\
\mathrm{D.11c} \\
\end{array}$ \\
\hline
\end{tabular}

Tab. 7.1.: Zusammenfassung der qualitativ nachgewiesenen Gasphasenprodukte für Alkene und Alkenole. Angegeben wird, unter welchen Bedingungen sie (am ausgeprägtesten) auftraten, auf welche Weise der Nachweis erfolgte und wo die entsprechenden Spektren zu finden sind. Mit „(?)“ gekennzeichnete Verbindungen stehen für unsichere Nachweise bzw. berechnete Modellverbindungen für bestimmte Strukturmotive. 


\begin{tabular}{|c|c|c|c|c|}
\hline Alken & Produkt & exp. Bedingung (max. bei) & Nachweisart & Abb. \\
\hline 1-Penten-3-on & $\begin{array}{c}\text { 2-Oxobutanal } \\
\text { Hydroperoxide nach [125] (?) } \\
\text { HCOOH } \\
\text { Methylketen } \\
\mathrm{H}_{2} \mathrm{CO}\end{array}$ & $\begin{array}{l}\mathrm{SL} \mathrm{HD} \\
\mathrm{N}_{2}, \mathrm{SL} \mathrm{HD} \\
\text { alle (SL ND) } \\
\text { alle (SL ND) } \\
\quad \text { alle }\end{array}$ & $\begin{array}{l}\text { ber. Frequenzspektrum } \\
\text { ber. Frequenzspektren } \\
\text { Reinspektrum } \\
\text { Literaturspektrum [127] } \\
\text { Reinspektrum }\end{array}$ & \begin{tabular}{|l|}
6.15 \\
$6.14 \mathrm{a}$ \\
$\mathrm{D.14b}$ \\
$\mathrm{D.} .14 \mathrm{~d}$ \\
$\mathrm{D.14 \textrm {e }}$ \\
\end{tabular} \\
\hline 4-Penten-1-al & $\begin{array}{c}\text { Succinaldehyd } \\
\mathrm{HCOOH} \\
\mathrm{C}_{2} \mathrm{H}_{4} \\
\mathrm{H}_{2} \mathrm{CO}\end{array}$ & $\begin{array}{c}\mathrm{N}_{2} / \mathrm{SL} \mathrm{HD} \\
\text { alle (SL ND) } \\
\text { alle (SL ND) } \\
\text { alle (SL ND/HD) }\end{array}$ & $\begin{array}{l}\text { ber. Frequenzspektrum } \\
\text { Reinspektrum } \\
\text { Reinspektrum } \\
\text { Reinspektrum }\end{array}$ & \begin{tabular}{|l|}
$\mathrm{D.} 15 \mathrm{a}$ \\
$\mathrm{D.15 \textrm {c }}$ \\
$\mathrm{D.15 \textrm {c }}$ \\
$\mathrm{D.15a}$ \\
\end{tabular} \\
\hline Methacrylsäure & $\begin{array}{c}\text { Hydroperoxide nach [59] (?) } \\
\text { SOZ / Anhydrid (?) } \\
\mathrm{HCOOH} \\
\mathrm{H}_{2} \mathrm{CCO} \\
\mathrm{H}_{2} \mathrm{CO}\end{array}$ & $\begin{array}{c}\mathrm{SL} \mathrm{HD} \\
\mathrm{HD}\left(\mathrm{N}_{2}\right) \\
\text { alle }\left(\mathrm{N}_{2} / \mathrm{SL} N D\right) \\
\text { alle }\left(\mathrm{N}_{2} / \mathrm{SL} N D\right) \\
\text { alle }\left(\mathrm{N}_{2} \mathrm{ND}\right)\end{array}$ & $\begin{array}{l}\text { ber. Frequenzspektrum } \\
\text { Analogien [126, 130] } \\
\text { Reinspektrum } \\
\text { Literaturspektrum [123] } \\
\text { Reinspektrum }\end{array}$ & \begin{tabular}{|l|}
$6.17 \mathrm{a}$ \\
$\mathrm{D} .18 \mathrm{~b}$ \\
$\mathrm{D.} .18 \mathrm{~b}$ \\
$\mathrm{D.} .18 \mathrm{~d}$ \\
$\mathrm{D.18c}$ \\
\end{tabular} \\
\hline Butensäure & $\begin{array}{c}\mathrm{HCOOH} \\
\mathrm{SOZ}(?) \\
\mathrm{H}_{2} \mathrm{CO} \\
\mathrm{H}_{2} \mathrm{CCO} \\
\text { Malonsäure, } \\
\text { Oxopropansäure, } \\
\text { Hydroperoxid nach [59] (?) }\end{array}$ & $\begin{array}{l}\text { alle }\left(\mathrm{N}_{2} / \mathrm{SL} N \mathrm{ND}\right) \\
\text { alle }(\mathrm{SL} \mathrm{HD)} \\
\text { alle }\left(\mathrm{N}_{2} / \mathrm{SL} \mathrm{ND}\right) \\
\text { alle }\left(\mathrm{N}_{2} / \mathrm{SL} \mathrm{ND}\right)\end{array}$ & $\begin{array}{c}\text { Reinspektrum } \\
\text { Analogie [126] } \\
\text { Reinspektrum } \\
\text { Literaturspektrum [123] }\end{array}$ & $\begin{array}{l}\mathrm{D} .21 \mathrm{~b} \\
\mathrm{D} .21 \mathrm{~b} \\
\mathrm{D.21 \textrm {c }} \\
\mathrm{D.21d} \\
6.18 \\
6.17 \mathrm{~b}\end{array}$ \\
\hline
\end{tabular}

Tab. 7.2.: Zusammenfassung der qualitativ nachgewiesenen Gasphasenprodukte für höher oxidierte Verbindungen. Angegeben wird, unter welchen Bedingungen sie (am ausgeprägtesten) auftraten, auf welche Weise der Nachweis erfolgte und wo die entsprechenden Spektren zu finden sind. Mit „(?)“ gekennzeichnete Verbindungen stehen für unsichere Nachweise bzw. berechnete Modellverbindungen für bestimmte Strukturmotive. 
entstehen können. Diese Verbindungen konnten allerdings nicht zweifelsfrei identifiziert werden, da eine Vielzahl von funktionellen Gruppen (Ether-, Peroxystrukturen, Ozonide) in den betrachteten Wellenzahlbereichen absorbieren können. Daher sind die gewählten Substanzen auch ausdrücklich als Modellverbindungen für das gewählte Strukturmotiv zu verstehen, ohne dass eine gesicherte Aussage über die genaue Gestalt des Gesamtmoleküls zu treffen ist.

Gleiches gilt für die potenziellen Sekundärozonid- bzw. Anhydridsignale für die beiden Carbonsäuren, die nur unter Vorbehalt als mögliche Produkte anhand typischer Spektralbereiche und Literaturvergleich mit WINTERHALTER et al. und WOLF et al. [17, 126] bzW. NIKI et al. [60] zugeordnet werden konnten.
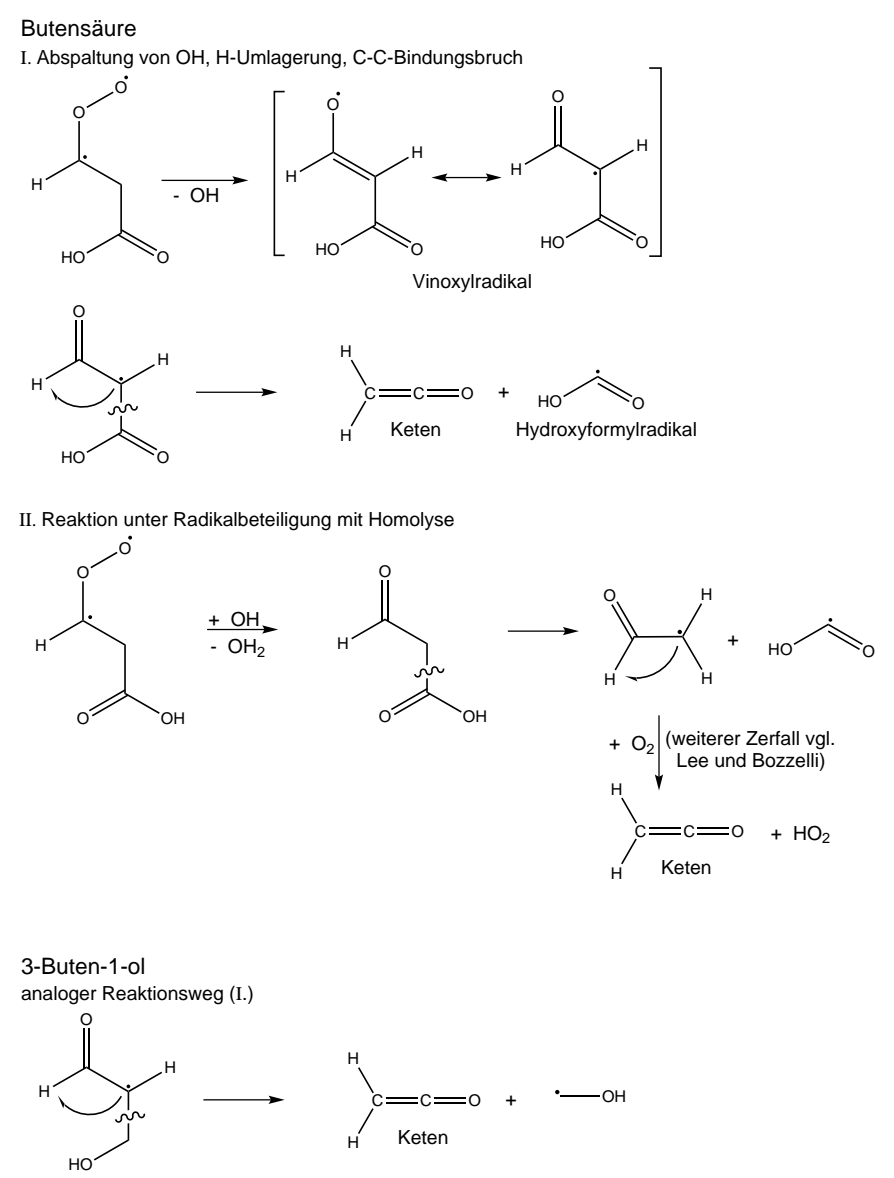

Abb. 7.1.: Postulierter ketenbildender Mechanismus für Butensäure und 3Buten-1-ol.

Besonderes Augenmerk lag auf der Ketenbildung, diese wurde bereits in Abschnitt 6.2.3 recht ausführlich behandelt. Das Hauptergebnis dieser Betrach- 
7.1. Zentrale Rolle der $\mathrm{OH} / \mathrm{HO}_{2}$-Chemie? - Auswirkungen der Verwendung verschiedener Badgase

tungen ist, dass die Ketenbildung einen Hinweis auf die Intermediatstabilität geben kann. In der vergleichenden Abbildung 6.18 werden die Ketenbanden von Butensäure, dem strukturell ähnlichen 3-Buten-1-ol und Methylcyclopenten als stärkstem Ketenbildner aller übrigen untersuchten Verbindungen gezeigt. Wie dort zu erkennen ist, verursacht die Änderung der Badgasumgebung bei allen Edukten eine weitere Zunahme bei Sauerstoffanwesenheit. Die schon für das zyklische Alken recht starke Ketenbildung kann mit der hohen Überschussenergie der in der Ozonolyse gebildeten Intermediate erklärt werden, da Methylcyclopenten durch die Spannung in seinem Fünfring und den zusätzlichen Energieeintrag durch die Ozonanlagerung und die ausbleibende Bildung einer Translationsmode beim Zerfall des Primärozonids chemisch aktiviert sein muss. Diese Instabilität beschleunigt den Zerfall in kleinere Fragmente, wie er auch in Abb. 6.10 auf Seite 134 gezeigt ist. Damit konnte der von WoLF et al. [17] für Methylcyclohexen postulierte Mechanismus bestätigt werden.

Der große Unterschied in der Bandenintensität der beiden voroxidierten Spezies weist darauf hin, dass auch die Intermediate aus der Butensäureozonolyse deutlich instabiler sein müssen als die des 3-Buten-1-ols. Wegen der hohen strukturellen Ähnlichkeit kommt als entscheidender Faktor vor allem der Oxidationsgrad in Frage. Die überproportional hohe Bildung im Fall der Butensäure spricht für eine Destabilisierung durch den hohen Sauerstoffanteil im Molekül.

Prinzipiell sind verschiedene Wege, auf denen Keten gebildet werden kann, denkbar, wie Abb. 7.1 zeigt. Ausgehend von dem $\mathrm{C}_{3}-\mathrm{Cl}$ kann, wie in Reaktion (I.) dargestellt, der Zerfall über den Hydroperoxidkanal erfolgen, wobei nach Abspaltung eines $\mathrm{OH}$-Radikals aus dem mesomeriestabilisierten Vinoxylradikal unter Bildung der eines Hydroxyformylradikals ( $\mathrm{HOC} \mathrm{O}$ ) direkt Keten entstehen kann. Eine weitere Möglichkeit stellt die Reaktion eines $\mathrm{OH}$-Radikals mit dem CRIEGEE-Intermediat dar (II.). Nach Abspaltung eines Sauerstoffradikals aus der Peroxygruppe wird nach $\mathrm{C}-\mathrm{C}$-Bindungsbruch neben einem Hydroxyformylradikal ein Alkylradikal ausgebildet, welches mit Sauerstoffmolekülen nach dem Vorschlag von LEE et al. [131] zu Keten weiterreagieren kann. Jedoch ist die von dem Angriff des $\mathrm{OH}$-Radikals abhängige Reaktion aufgrund der recht geringen Radikalkonzentration als eher unwahrscheinlich einzuschätzen. Für 3Buten-1-ol als Analogon zur Butensäure wird daher lediglich der Zerfall nach Reaktion (I.) in Abb. 7.1 gezeigt, der als wahrscheinlicherer Kanal anzusehen 
ist. Allerdings ist für diesen Bildungsweg entscheidend, welche Barriere für den H-Shift im zweiten Reaktionsschritt überwunden werden muss, was eventuell durch eine Modellierung der Reaktion geklärt werden kann.

Beiden Möglichkeiten ist gemeinsam, dass sie in sehr wenigen Schritten vom CRIEGEE-Intermediat zum Keten führen, was für ihre Wahrscheinlichkeit spricht. Allein aus der Ketenbildung kann allerdings nicht auf die Intermediatstabilität und damit universell auf das Partikelbildungvermögen rückgeschlossen werden, da für Butensäure auch bei experimentellen Bedingungen mit hoher Ketenausbeute ebensolche für das Aerosol nachgewiesen werden konnten. Weitere Untersuchungen könnten diesen Zusammenhang potenziell aufklären. Eventuell ist die Bildung von Ethen ein deutlicherer Hinweis auf eine niedrige Partikelausbeute oder die Ketenbildung bei hoher Aerosolbildung ist eine Besonderheit der Carbonsäuren.

Die leichten Zunahmen in der Ketenausbeute in Sauerstoffanwesenheit lassen darauf schließen, dass unter Umständen Sauerstoff direkt an der Reaktion beteiligt ist, wodurch das Intermediat durch die Einbringung von Überschussenergie zusätzlich destabilisiert wird und im Gegensatz zur reinen Stoßstabilisierung in Inertgasen bevorzugt zerfällt. Allerdings ist auch eine indirekte Beeinflussung durch Nebenreaktionen von in Sauerstoffanwesenheit in erhöhter Konzentrationen vorliegender Radikale oder Intermediate nicht auszuschlieBen. Diese Reaktionen machen offenbar auch nur einen kleinen Teil der gebildeten Ausbeute aus, da auch in Stickstoffatmosphäre Keten gebildet wird. Dieser Anteil kann, falls die Reaktion mit hohem Geschwindigkeitskoeffizienten abläuft, allerdings auch durch kleine Restkonzentrationen an Sauerstoff aus dem Badgas (<2 ppm laut Spezifikation), Undichtigkeiten der Apparatur und hauptsächlich dem zugegebenen $\mathrm{He} / \mathrm{O}_{3}$-Gemisch (abgeschätzt insgesamt etwa 0.3-0.5 Pa), effektiv zur Ketenbildung beitragen. Allerdings müssten zu diesem Komplex umfangreichere Messungen für die in beiden Badgasen vergleichbare Ausbeuten liefernden Edukte durchgeführt werden, bis gesicherte Aussagen möglich sind. Die Reduktion von Sauerstoffresten wäre beispielsweise durch die Verwendung von Stickstoff in einer höheren Reinheit, ein größeres Reaktionsvolumen, in dem die Sauerstoffverunreinigung aus der $\mathrm{He} / \mathrm{O}_{3}$-Zugabe einen geringeren Anteil ausmacht oder die Verwendung geringerer Ozonanfangskonzentrationen und damit verringerter Zugabe des Gemisches appara- 
7.1. Zentrale Rolle der $\mathrm{OH} / \mathrm{HO}_{2}$-Chemie? - Auswirkungen der Verwendung verschiedener Badgase

tiv problemlos realisierbar. Eventuell würde auch eine bessere Abdichtung der ozonzuführenden Leitungen einen Effekt zeigen. Eine in situ-Erzeugung des Ozons in der Reaktionszelle, wie einige Arbeitsgruppen sie praktizieren, wäre dagegen kontraproduktiv, da nur ein geringer Anteil des Sauerstoffs zu Ozon umgesetzt wird und jener im Reaktionsvolumen verbleiben würde.

Für die Substanzen mit großen Differenzen in den Ausbeuten in den verschiedenen Badgasen konnte dagegen eine Grenzkonzentration, ab der das $\mathrm{O}_{2}$ sich auf die Partikelbildung auswirkt, und damit auch auf das Produktgemisch in der Gasphase, nachgewiesen werden, die deutlich über den potenziellen Verunreinigungen liegt (vgl. Abb. 6.32, S. 193 für 4-Penten-1-ol, Abb. 6.41, S. 206 für die Carbonsäuren).

Die quantitativen Auswertungen beschränkten sich auf die Betrachtungen der Kohlenstoffmonoxid- und $\mathrm{OH}$-Ausbeuten, letztere ließen sich aus dem relativen Verbrauch der Edukte in den Kinetikmessungen berechnen.

Die voroxidierten Verbindungen zeigten im Gegensatz zu den reinen Kohlenwasserstoffverbindungen nicht nur eine recht deutliche Druckabhängigkeit in der CO-Bildung, die unter Niederdruckbedingungen stets ausgeprägter ist, sondern auch eine badgasabhängige Korrelation zu den gebildeten Partikelausbeuten. Nach den Ergebnissen in Tabelle B.2 und Kapitel 6.4.2 ergeben sich in dem Badgas, wo höhere Aerosolausbeuten beobachtet wurden, also Synthetische Luft mit Ausnahme der Carbonsäuren, tendenziell höhere COAusbeuten. Aufgrund der komplizierten CO-Konzentrationsbestimmung bedarf dieser Aspekt jedoch deutlich intensiverer Folgeuntersuchungen, zumal dieser Effekt für die Alkene nicht signifikant war.

Auch zwischen der OH-Ausbeute, die nach den Ergebnissen von JOHNSON und MARSTON [33] bei der Ozonolyse in erster Näherung allein auf den Zerfall der syn-Konformere der CRIEGEE-Intermediate zurückgeführt werden kann, und der gebildeten Aerosolausbeute lassen sich interessante Korrelationen beobachten, die in Abschnitt7.2.2.2 (S. 291) näher diskutiert werden. 


\subsection{Partikelbildungsmessungen}

Die Güte von klimarelevanten Vorhersagen allein auf Grundlage vergangegener Entwicklungen und Extrapolation auf die Zukunft birgt grundsätzlich große Unsicherheiten, wie die aktuell eher stagnierende Temperaturentwicklung statt einer vorhergesagten sich beschleunigten Erwärmung durch den Ausbau von Industrieanlagen in Schwellenländern zeigen. Auch die Verwendung verschiedener Modelle 2 trägt zur großen Schwankungsbreite der vorhergesagten Entwicklung bei, wie Abb. 7.2 zeigt.

The global climate of the 21st century

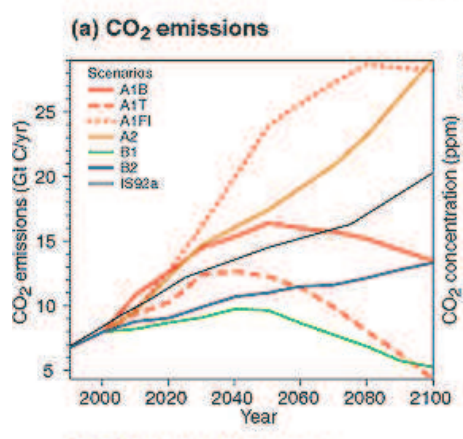

(b) $\mathrm{CO}_{2}$ concentrations

(c) $\mathrm{SO}_{2}$ emissions
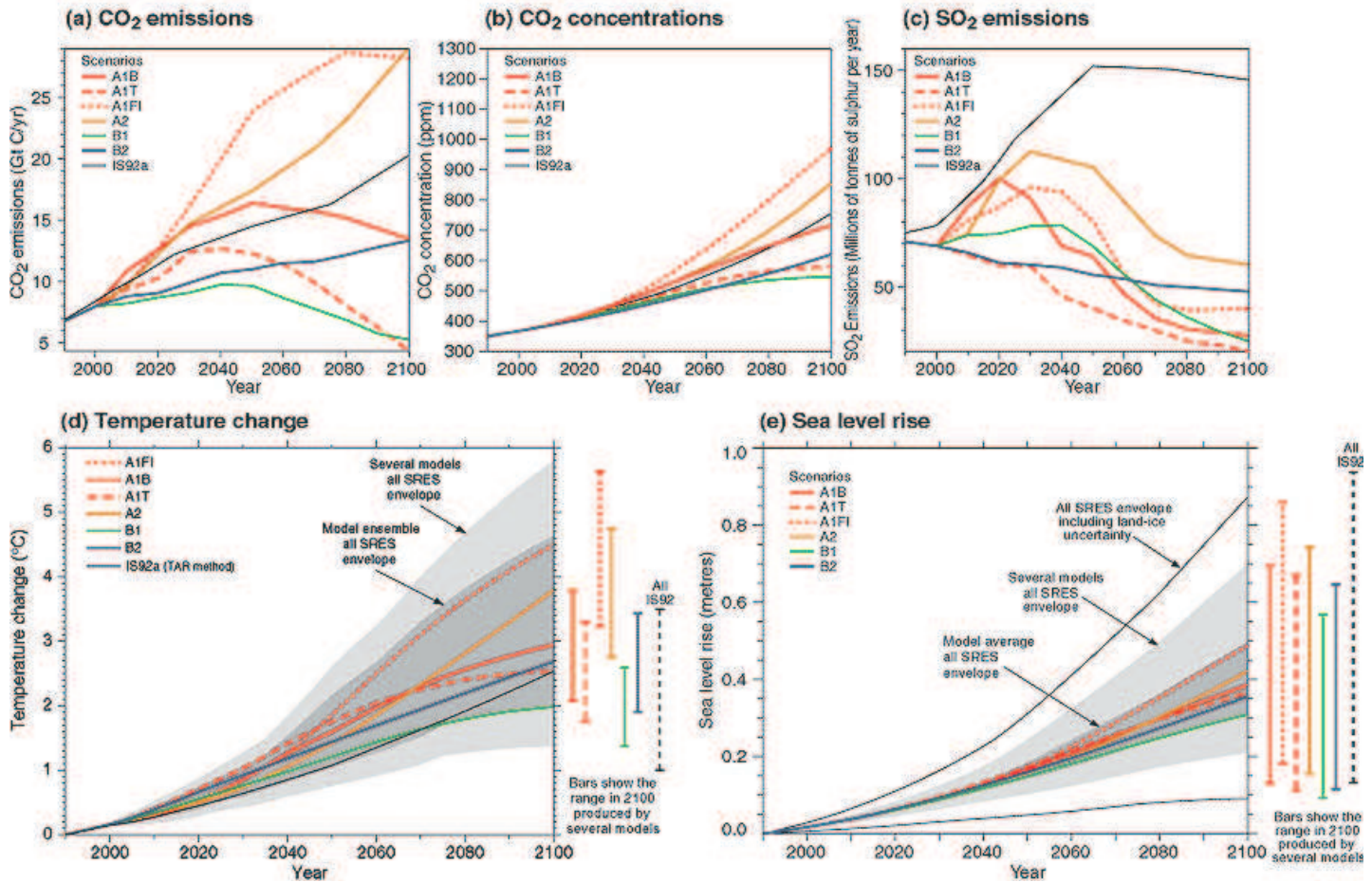

Abb. 7.2.: Vorhersage über die globale Klimaentwickung, entnommen aus dem Bericht des Weltklimarates von 2001 (IPCC - Intergovernmental Panel on Climate Change) [152].

Der Beitrag der Emission treibhausaktiver Gase aus anthropogenen Quellen ist zwar von hoher Bedeutung für die Gesamtentwicklung, allerdings können sich die Auswirkungen teilweise überlagern und kompensieren. Allein, dass

${ }^{2}$ Details zu den genannten verwendeten Modellen können abgerufen werden unter http://www.grida.no/publications/other/ipcc_tar/ (eingesehen am 24.03.2012). 
Stoffe mit potenziell erwärmender und abkühlender Konsequenz oft simultan freigesetzt werden, macht eine Abschätzung des Gesamteffektes extrem schwierig. Beispiele hierfür sind die aus Verbrennungsprozessen oft gemeinsam emittierten Verbindungen $\mathrm{CO}_{2}$, das durch Absorption von Strahlungsenergie aus dem Sonnenlicht die Atmosphäre aufheizen soll, und $\mathrm{SO}_{2}$, dem durch Bildung von Kondensationskeimen, verstärkter Wolkenbildung und Abschirmung von Sonnenstrahlung ein abkühlender Effekt zugesprochen wird [153]. Der letzte Punkt hätte auch für die Betrachtung der natürlichen Atmosphäre eine hohe Bedeutung, da es bereits seit den 1970er Jahren vielfältige Pläne für Geoengineering-Prozesse zur Reduktion der globalen Erwärmung gibt. Erstmals wurde dieser Gedanke 1977 von BUDYKo [153] unterbreitet. Die beobachteten abkühlenden Effekte großer Vulkanausbrüche durch in die Stratosphäre in großen Mengen freigesetzten Mengen Schwefeldioxid bilden dabei das Vorbild für Vorschläge, Schwefel oder Schwefeldioxid direkt in höhere atmosphärische Schichten einzubringen und damit die Abkühlung gezielt zu fördern. Nachteilige Effekte, wie etwa eine Versauerung des Regens, können jedoch bei diesem Vorgehen kaum abgeschätzt werden.

Der atmosphärische Partikelbildungsprozess hat durch die Funktion der Aerosole als Wolkenkondensationskeime eine große Bedeutung für das globale Klima und die Modellierung der Entwicklung der globalen Erwärmung [7]. Er ist zu einem hohen Anteil durch die Oxidation ungesättigter Kohlenwasserstoffverbindungen verursacht [1,5, 7, 10, 154].

Auch die Bedeutung von Aerosolen ist jedoch von großen Unsicherheiten behaftet (vgl. Abb. 1.1, insbesondere die Fehlerbereiche der Aerosoldaten), da der genaue Nukleationsmechanismus und vor allem die für den Übergang aus der Gas- in die Partikelphase entscheidenden Schritte unbekannt sind. Auch die intensive Forschung auf diesem Gebiet in Labor- und Feldstudien konnten zwar Anhaltspunkte, allerdings noch keinen geschlossenen und allgemeingültigen Mechanismus liefern. Schwierigkeiten ergeben sich aus dem statistischen Charakter der Freiluftmessungen. Dieser kann beispielsweise durch singuläre Ereignisse, die nicht als solche erkannt werden, wie nahegelegene Waldbrände oder Rodungen und daraus folgende Freisetzung der Reaktanden mit ungewöhnlich hoher Emissionsrate und eine Fehleinschätzung der realen Verhältnisse, verursacht werden. Messungen unter Laborbedingungen haben da- 
gegen den Nachteil, dass sich atmosphärische Vorgänge und Verhältnisse nur schwer simulieren lassen, wie die grundsätzliche Unterschätzung der Aerosolbildung bei Modellierungen auf alleiniger Grundlage von im Labor unter vereinfachten Bedingungen ohne Wechselwirkungen erhalteten Parametern zeigen [21].

KROLL und SEINFELD [21] schließen daraus, dass in der natürlichen Atmosphäre eine größere Zahl, bisher unbekannter, Partikelvorläufer und partikelbildender Prozesse existieren muss. Ein weiteres Problem, das HENRY und DONAHUE [19] identifizieren, ist das Verhältnis der sehr reaktiven Peroxyradikalverbindungen untereinander, welches für den Oxidationsprozess eine entscheidende Bedeutung besitzen kann.

Die Partikelbildungsstudien in Kapitel 6.4 haben gezeigt, dass die Verwendung unterschiedlicher Badgase, die Variation der Gesamtdrücke sowie die Zugabe bestimmter Additiva Auswirkungen auf die Aerosolausbeute haben kann, wobei nicht immer sicher ist, an welcher Stelle in den Mechanismus eingegriffen wird.

Dabei ist der jeweilige Effekt und auch seine Ausprägung stark von der Grundstruktur der ungesättigten Kohlenwasserstoffverbindung abhängig. In den folgenden Abschnitten sollen diese Auswirkungen diskutiert werden. Neben einem potenziellen Einfluss auf die Bestimmung der Geschwindigkeitskoeffizienten durch zusätzlichen Verbrauch der Edukte sind CRIEGEE-Intermediate auch für die Partikelbildung bedeutsame Strukturen, ebenso wie die aus innen gebildeten Folgeprodukte. Generell sind für die Partikelbildung Prozesse von zentraler Bedeutung, die für eine Verringerung des Dampfdruckes der entstehenden Produkte sorgen. Dies ist ein entscheidender Faktor, der auf die potenzielle Aerosolausbeute Einfluss nimmt, und kann durch verschiedene Prozesse realisiert werden.

\subsubsection{Beeinflussung der Intermediatchemie - Auswirkungen des Badgaswechsels}

Oxidationsreaktionen in der Gasphase bilden den entscheidenden ersten Schritt zur Ausbildung kondensierbarer Materie. KROLL und SEINFELD [21] 
identifizieren insgesamt drei Schlüsselstellen im Mechanismus, die die Flüchtigkeit der aus einem Alken entstehenden Produkte und damit auch ihr Potenzial in der Partikelbildung determinieren. Neben der initialen Oxidationsreaktion, die entweder durch eine $\mathrm{H}$-Abstraktion ohne Auswirkungen auf den Dampfdruck des Produktes oder die Addition des Oxidationsmittels an die Doppelbindung mit dampfdruckreduzierender Wirkung realisiert werden kann, sind auch die Folgereaktionen der entstehenden Peroxy- und Alkoxyverbindungen von entscheidender Bedeutung (vgl. Abb. 7.3). Zumindest die beiden letztgenannten Vorgänge können durch die Wahl des Badgases beeinflusst werden, wie an verschiedenen Stellen der Theorie und Auswertung bereits beschrieben wurde. Durch die Anwesenheit von Sauerstoff in der Reaktionsmischung wird unter anderem der Radikalpool beeinflusst. Wie in Abschnitt2.2.2.3.(S. 33) dargestellt, wird bei Verwendung von Synthetischer Luft die Peroxy- und Alkoxyradikalchemie bedeutsamer, da die Strukturen in größerer Ausbeute gebildet werden als unter sauerstoffarmen Bedingungen. Generell sind die Reaktionen und Produkte der Peroxy- / Alkoxychemie in Laborstudien höher einzuschätzen als bei Feldmessungen [54], da die verwendeten Eduktkonzentrationen meist höher sind, was auch eine größere Ausbeute für die oxidierten Strukturen verursacht. Allerdings ist in der natürlichen Atmosphäre zusätzlich generell von einem deutlich höheren $\mathrm{HO}_{2} / \mathrm{RO}_{2}$-Verhältnis als in den „sauberen“ Laborexperimenten auszugehen [19].

In einigen Studien wurden funktionelle Gruppen der Eduktverbindungen in ihrer Wirkung auf den Dampfdruck und damit den Partikelbildungsprozess untersucht. KROLL und SEINFELD [21] stellen in ihrer Arbeit die Bedeutung der Gasund Partikelphasenoxidation durch $\mathrm{OH}, \mathrm{O}_{3}$ und $\mathrm{NO}_{3}$, die durch die Addition polarer funktioneller Gruppen für eine Verringerung der Flüchtigkeit sorgt, heraus. Durch die Anlagerung sauerstoffhaltiger Substituenten wird ein um zwei GröBenordnungen stärkerer Effekt als durch die Verlängerung der Kohlenstoffkette um ein C-Atom verursacht. Die Oxidationsprozesse stehen dabei in Konkurrenz mit dem Bruch der Kohlenstoffkette, der durch die Bildung kleinerer Produkte für eine Erhöhung des Dampfdruckes der Komponenten sorgt und sich damit negativ auf die Partikelausbeute auswirkt. Dieser Vorgang ist in Abb. 7.3]in grün gekennzeichnet.

Die Volatilität der Produkte aus $\mathrm{RO}_{2}$-Kreuzreaktionen (rot markiert) wird von 


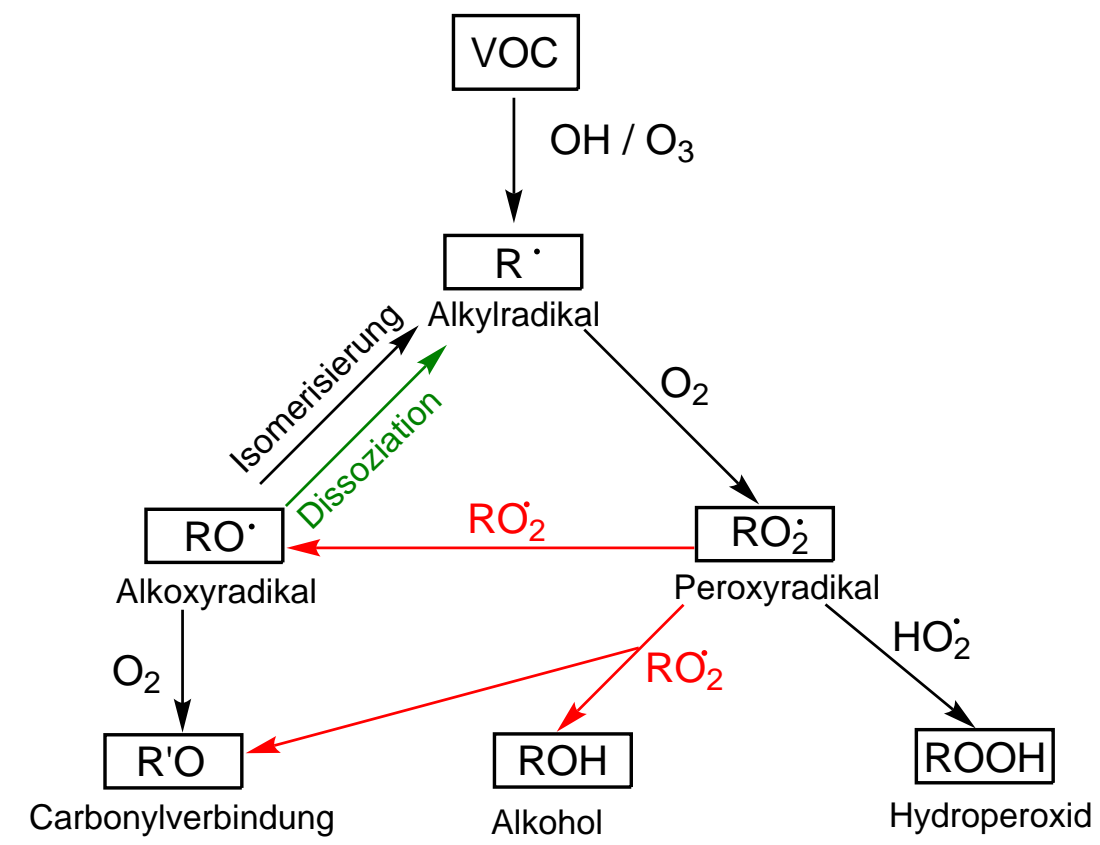

Abb. 7.3.: Vereinfachte Darstellung der atmosphärischen Oxidationsprozesse von volatilen organischen Substanzen (VOC) nach KROLL und SEINFELD [21] (ohne Nitratbeteiligung).

der Kanalverteilung bestimmt [54], die wiederum von der Struktur des Eduktes abhängig ist, sowie von den weiteren Reaktionen des Alkoxyradikals (vgl. Abschnitt 7.2.2) [54]. Hierbei können Alkoxyradikale (RO), Carbonylverbindungen $(\mathrm{RHO})$ oder Alkohole $(\mathrm{ROH})$ gebildet werden, die im Durchschnitt meist eine höhere Volatilität besitzen als die in der $\mathrm{RO}_{2}+\mathrm{HO}_{2}$-Reaktion gebildeten Hydroperoxide $(\mathrm{ROOH})$. Letztere sind als eine wichtige Komponente der Aerosolphase bekannt und konnten dort bereits von verschiedenen Arbeitsgruppen nachgewiesen werden [38, 57, 71, 155]. Sie sind auch in der Lage, in der Partikelphase weiter zu reagieren und dadurch in ihrer Flüchtigkeit noch weiter abgesenkt zu werden [71].

Durch die vermehrt ablaufende Abfangreaktion $\mathrm{RO}_{2}+\mathrm{HO}_{2}$ in Synthetischer Luft, wie sie auf S. 33 beschrieben ist [56], werden nicht nur schwerflüchtige Reaktionsprodukte gebildet, sondern potenziell auch andere Reaktionen unter $\mathrm{HO}_{2}$-Beteiligung beeinflusst. Beispielsweise sind die von SADEZKY [36, 53] vorgeschlagenen Oligomerisierungsreaktionen für unsubstituierte und für stabilisierte große CRIEGEE-Intermediate (in Gas- oder Partikelphase [21]) auch 
für einige der in dieser Arbeit verwendeten Edukte denkbar (vgl. Kap. 6.4.1f.). Diese Polymerisierungsvorgänge werden, wie in Abb. $2.7 \mathrm{~S}$. 29 dargestellt, durch Hydroperoxylradikale $\left(\mathrm{HO}_{2}\right)$ abgebrochen. Bei Vorliegen einer Oligomerisierungsreaktion als entscheidendem aerosolaufbauendem Vorgang für das betrachtete Edukt würde sich daher eine hohe $\mathrm{HO}_{2}$-Konzentration negativ auf die Ausbeute auswirken. Aus der Reaktion $\mathrm{OH}+\mathrm{O}_{3} \longrightarrow \mathrm{HO}_{2}+\mathrm{O}_{2}$ (Tabelle 2.3) ist vor allem in Sauerstoffabwesenheit eine höhere $\mathrm{HO}_{2}$-Reaktion zu erwarten, da die in Stickstoffatmosphäre verringerte $\mathrm{RO}_{2}$-Konzentration die Effektivität der Abfangreaktion $\mathrm{HO}_{2}+\mathrm{RO}_{2}$ (S. 33 ) vermindert.

Die Isomerisierungsreaktionen der Alkoxyradikale und der anschließende Zerfall in Produkte mit niedrigerer Volatilität tritt nur auf, falls eine $1,4-\mathrm{H}-$ Umlagerung möglich ist. Dieser Vorgang kann durch Substitution des $\mathrm{H}$ tragenden Kohlenstoffatoms beschleunigt werden, vor allem, wenn es sich um sauerstoffhaltige Substituenten handelt [21]. Dieser durch die Struktur des Edukts beeinflusste Aspekt wird im folgenden Abschnitt näher diskutiert. Im Allgemeinen ist die Reaktion von Alkoxyradikalen mit $\mathrm{O}_{2}$ aus dem Badgas dagegen bei Zimmertemperatur nicht konkurrenzfähig. Für größere organische Verbindungen spielt sie nur eine Rolle, wenn Isomerisierungen aufgrund struktureller Eigenschaften nicht möglich sind oder die Dissoziationsreaktionen lediglich primäre Alkylradikale bilden. Bei den herrschenden Temperaturen kann sie vernachlässigt werden [21].

Somit ist vor allem die Reaktion der Alkylradikale mit Sauerstoff und die entsprechende Folgechemie durch die Badgaswahl zu beeinflussen.

Die genannten $\mathrm{RO}_{2}$-abhängigen Vorgänge könnte die in einigen Fällen reduzierte Ausbeute unter Stickstoffatmosphäre erklären, wie sie etwa für Methylcyclopenten (Kap. 6.4.1.1), 4-Penten-1-ol (Kap. 6.4.2.1) und 1-Penten-3-on (Kap. 6.4.2.3) auftraten. Einerseits sind beschleunigte Abbruchreaktionen in der Oligomerbildung durch höhere $\mathrm{HO}_{2}$-Konzentrationen denkbar. Der Effekt des anwesenden Sauerstoffes könnte für die genannten Verbindungen allerdings auch mit einer vergleichsweise langsamen Reaktion von Alkyl- zu Peroyxradikalen erklärt werden, so dass die Partikelbildung über die Bildung von $\mathrm{RO}_{2}$-haltigen Produkten gegenüber Isomerisierungs- oder Dissoziationsreaktionen der Intermediate, die den Dampfdruck entweder nicht beeinflussen oder sogar erhöhen, bei geringen Sauerstoffkonzentrationen behindert ist. Zumindest für Methylcy- 
clopenten ist die hohe Überschussenergie der Ozonolyseprodukte nicht zu vernachlässigen, die für einen beschleunigten Zerfall der Intermediate [44] und damit eine geringe Aerosolausbeute sorgen könnte, falls die Reaktion mit Sauerstoff aus dem Badgas nicht durch sehr hohe vorliegende Konzentrationen begünstigt ist.

Bei den Edukten, die keine ausgeprägte Abhängigkeit vom Badgas aufweisen, also 1-Hexen, 2-Methyl-1-penten (vgl S. 182 ff.), 3-Buten-1-ol, 1-Penten3-ol (S. 188 f.) und 4-Pentenal (S. $199 \mathrm{f}$.), ist denkbar, dass geringe Verunreinigungen durch $\mathrm{O}_{2}$ im Stickstoff, aus dem Ozongemisch oder Undichtigkeiten der Aerosolzelle, bereits für eine effektiv ablaufende Peroxychemie ausreichend sind. Die aufgrund der hohen Bedeutung von oxidativen Schritten weniger wahrscheinliche Erklärung, dass Sauerstoff keine Bedeutung für die genannten Partikelbildungsprozesse hat, wären durch die Analyse der Aerosolphase und Bestimmung des $\mathrm{C}:$ O-Verhältnisses in den enthaltenen Molekülen zu belegen.

Da in den zuvor genannten Verbindungen, von den reinen Kohlenwasserstoffbis hin zu den Carbonylverbindungen, stets in Synthetischer Luft die höhere (oder zumindest gleich ausgeprägte) Neigung zur Aerosolbildung vorhanden war, scheint sich die Reaktionsfolge

$$
\begin{aligned}
\mathrm{R}+\mathrm{O}_{2} & \longrightarrow \mathrm{RO}_{2} \\
\mathrm{RO}_{2}+\mathrm{HO}_{2} & \longrightarrow \mathrm{ROOH}+\mathrm{O}_{2}
\end{aligned}
$$

eher förderlich auf die Aerosolbildung auszuwirken.

Auch an anderer Stelle lassen sich trotz relativer struktureller Ähnlichkeit der Eduktverbindungen teilweise große Diskrepanzen im Reaktionsverhalten feststellen. Hier handelt es sich um die Experimente unter Cyclohexanzugabe, die in der Methacrylsäureozonolyse für eine nur leichte Verringerung der Aerosolausbeuten sorgen, während die Ausbeuten in Anzahlkonzentration und Masse für 2-Methyl-1-penten dagegen stark zunehmen. Diese starke Zunahme ließ sich bisher noch nicht schlüssig erklären. Testexperimente unter Zugabe von Cyclohexanon, einem der Produkte der $\mathrm{C}_{6} \mathrm{H}_{12}+\mathrm{OH}$-Reaktion, konnten aber 
zumindest die Reaktionsprodukte der Abfangreaktion als Ursache ausschlieBen. Das Verhalten scheint sich auch nicht allein aus der Methylsubstitution der Doppelbindung zu ergeben, da Methacrylsäure kein vergleichbares Verhalten zeigt und auch in der Literatur bisher keine vergleichbare Beobachtung veröffentlicht wurde. Allerdings ist durch die ausgeprägten Differenzen im Molekül jenseits der Methylsubstitution die Interpretation allein aus dem Strukturvergleich heraus nicht unbedingt sinnvoll. Unter Umständen lässt sich die Beobachtung aus dem Eingriff in die Radikalverhältnisse erklären. Die Anwesenheit von Cyclohexan bewirkt eine Erhöhung der Peroxyradikalkonzentration in der Reaktionsmischung, wie Abb. 2.19)(S. 45) veranschaulicht. Durch den in der Folge effektiveren Abfang der $\mathrm{HO}_{2}$-Radikale durch Peroxyradikale aus der $\mathrm{OH}$-Abfangreaktion könnten Oligmerisierungsreaktionen zu größeren Molekülen führen und sich dementsprechend auch in einer höheren Aerosolausbeute äußern.

Um zu klären, warum dieser Effekt nur bei dem nicht voroxidierten Edukt mit der methylsubstituierten Doppelbindung auftrat, müssen allerdings weiterführende Experimente durchgeführt werden. Mögliche Ansätze wären hier die Variation der Kohlenstoffkettenlänge und des Oxidationsgrades der Eduktmoleküle.

Denkbar wäre als Grund für die starke Zunahme der Aerosolausbeute im Fall des 2-Methyl-1-penten etwa, dass ein spezifischer Typ der gebildeten Peroxyradikale aus der $\mathrm{C}_{6} \mathrm{H}_{12}+\mathrm{OH}$-Reaktion selektiv mit den Ozonolyseprodukten des Alkens eine Oligomerbildung eingeht. Prinzipiell wäre dies zwar aufgrund ähnlicher Strukturmotive (vgl. Abb. 7.4 in rot gekennzeichnet) denkbar, allerdings bliebe auch hier zu klären, warum dieser Effekt lediglich bei 2-Methyl-1-penten auftrat.

Allerdings zeigen diese Ergebnisse klar, dass jeder Eingriff in den komplizierten und weit verzweigten Mechanismus der Alkenozonolyse die Reaktionsfolgen und - produkte völlig anders beeinflussen kann als erwartet und jede Manipulation mehr Fragen aufwerfen kann als sie durch eine erwartete Vereinfachung des Systems beantwortet.

Eine auch wegen ihrer geringen Volatilität wichtige Verbindungsklasse innerhalb des Produktgemisches bei der Alkenozonolyse stellen die organischen Säuren dar. Das Vorkommen und die Bedeutung dieser ist vor allem unter $\mathrm{NO}_{\mathrm{x}^{-}}$ 


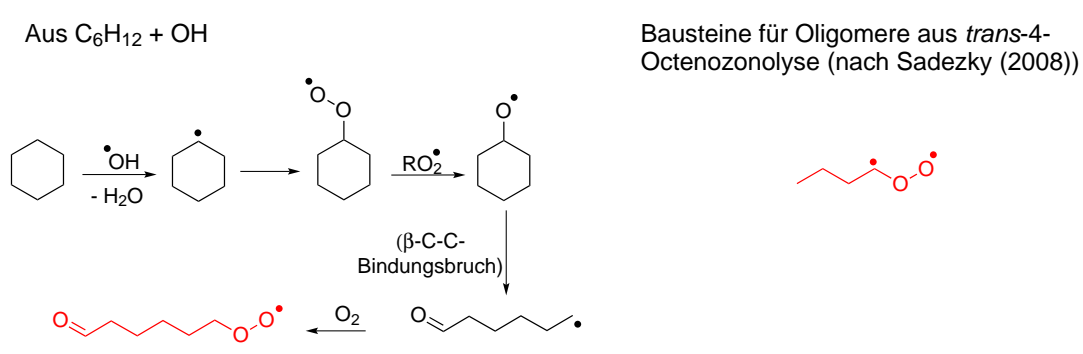

Abb. 7.4.: Vergleich auftretender struktureller Ähnlichkeiten in der $\mathrm{C}_{6} \mathrm{H}_{12}+\mathrm{OH}-$ Reaktion und der Oligomerisierungsreaktion nach SADEZKY et al. [36].

freien Bedingungen, wie sie in dieser Arbeit stets vorlagen, vielfach in der Literatur beschrieben [21,115, 126, 133], allerdings sind die Bildungswege noch immer nicht vollständig geklärt. Der direkt aus den CRIEGEE-Intermediaten stammende Teil scheint mit einer Ausbeute von weniger als $10 \%$ nur eine geringere Bedeutung zu besitzen. Wichtigere Bildungswege sind nach verschiedenen Studien wohl die Reaktionen $\mathrm{HO}_{2}+\mathrm{RC}(\mathrm{O}) \mathrm{O}_{2}$ (Acylperoxyradikal) [21], Isomerisierungen von Acyloxyradikalen $\left(\mathrm{R}_{1} \mathrm{C}(=\mathrm{O}) \mathrm{OR}_{2}\right)$ [115], der Zerfall von Persäuren $(\mathrm{RC}(=\mathrm{O}) \mathrm{OOH})$ [126] oder die Oxidation von 1,2-Hydroxycarbonylverbindungen [133]. Auch säurebildende Reaktionen in der Partikelphase sind nicht auszuschließen.

Ein großes Problem der Aerosolforschung ist, dass die aus Laborstudien gewonnenen Erkenntnisse über den Mechanismus der Partikelbildung bei ihrer Anwendung in der Simulation der natürlichen Atmosphäre die gebildeten Aerosolmengen grundsätzlich unterschätzen [21]. Dies deutet darauf hin, dass in der Realität eine deutlich höhere Vielfalt an potenziellen Partikelvorläufern und auch partikelbildenden Reaktionen ablaufen können, die in den Modellen bisher nicht berücksichtigt werden. Beispielsweise könnten Wechselwirkungen von Substanzen, die für sich gesehen eigentlich nicht zur Ausbildung von kondensationsfähigem Material geeignet sind, für solche Fehlbestimmungen verantwortlich sein. Einen Eindruck dafür geben etwa die Experimente unter Cyclohexanzugabe im Abschnitt 6.4.1.2. Aus diesem Grund wurden in der vorliegenden Arbeit eine große Bandbreite an verschiedenen Substanzen und Experimentvarianten untersucht, um einen Einblick in die Vorgänge zu erhalten. 


\subsubsection{Beeinflussung der Intermediatchemie durch die Struktur des Eduktes}

\subsubsection{Auswirkungen der Vorfunktionalisierung der Edukte}

Der Einbau polarer funktioneller Gruppen verringert, wie im vorherigen Abschnitt beschrieben, die Volatilität der Verbindungen. Allein die Betrachtung der Vorfunktionalisierungen der verwendeten Edukte erklärt daher einige der im Kapitel 6.4 erhaltenen Ergebnisse. Wie an verschiedenen Stellen der vorangegangenen Ausführungen bereits diskutiert wurde, sind die Aerosolausbeuten für die untersuchten Carbonylverbindungen vergleichsweise gering. Dieses geringe Partikelbildungspotential war trotz der hohen verwendeten Ozonkonzentrationen, die für einen erhöhten Umsatz im Vergleich zu den übrigen Untersuchungen sorgten, vor allem in Kenntnis der hohen Ausbeuten für das um ein Kohlenstoffatom verkürzte 3-Buten-1-ol unerwartet.

Die Betrachtung der Auswirkungen verschiedener sauerstoffhaltiger Substituenten auf die Flüchtigkeit der vorfunktionalisierten Kohlenwasserstoffverbindung bietet jedoch eine Erklärungsmöglichkeit. Da Hydroxyl- und Carboxylgruppen den Dampfdruck um etwa eine bzw. zwei Größenordnungen verglichen zu Aldehyd- oder Ketonfunktionalitäten absenken, ist bei der Alkenol- und Carbonsäureozonolyse schon die Eduktverbindung deutlich schwerflüchtiger als bei der Ozonolyse von Carbonylverbindungen. Dies lässt sich aus der vergleichsweise geringen Polarität letzterer erklären [21]. So kann, wenn nicht ein Bruch der Kohlenstoffkette für einen gegenläufigen Effekt sorgt, bei ungesättigten Alkoholen und Carbonsäuren durch weniger Oxidationsschritte als bei Carbonylverbindungen eine Absenkung des Dampfdrucks erreicht werden, die für einen Übergang aus der Gas- in die kondensierte Phase ausreichend ist. Auch die Produkte aus dem Zerfall über den Vinylhydroperoxidkanal oder bimolekularen Reaktionen der CRIEGEE-Intermediate können in ihrer Flüchtigkeit stark reduziert sein und wurden bereits in der Aerosolphase nachgewiesen [71]. Der Verlängerung der Kohlenstoffkette kommt im Gegensatz dazu nur eine geringere Bedeutung zu, da ein zusätzliches C-Atom nur für eine Reduktion der Flüchtigkeit um den Faktor 0.35 sorgt.

Auch die unterschiedlichen Auswirkungen der Sauerstoffanwesenheit lassen 
sich aus dieser Perspektive erklären. Während sich für Edukte mit einem niedrigerem Oxidationsgrad bis hin zum Keton die Sauerstoffanwesenheit durch die Verwendung von Synthetischer Luft positiv auf die Aerosolausbeuten auswirkt, hat sie auf die Partikelbildung aus der Carbonsäureozonolyse einen behindernden Effekt. Dies ließe sich mit einer steigenden Wahrscheinlichkeit des C-CBindungsbruchs bei der Anlagerung weiterer sauerstoffhaltiger Substituenten an das Kohlenstoffgerüst der Carbonsäure erklären.

Nach den Ausführungen von KROLL und SEINFELD [21] sind die Folgereaktionen der Peroxy- und Alkoxyradikale wichtig für die Entstehung partikulärer Materie. Auch in den vorliegenden Untersuchungen haben sich an verschiedenen Stellen Hinweise auf die Bedeutung in den untersuchten Aerosolbildungsstudien gezeigt. Dabei ist die Neigung der Peroxyradikale zum dampfdruckerhöhenden Zerfall durch ihre Struktur bestimmt, wobei verzweigte Verbindungen bevorzugter zerfallen als lineare [21]. Dies lässt sich mit den beobachteten Aubeuten von 1-Hexen vs. 2-Methy-1-penten und Butensäure vs. Methacrylsäure bestätigen, da jeweils für das erste Edukt der Oxidationsgrade die deutlich höheren Aerosolausbeuten resultierten.

Weiterhin sollte die Zerfallswahrscheinlichkeit mit dem Oxidationgrad zunehmen [21]. Dies ist auf den ersten Blick nicht zu bestätigen, da sich aus der Butensäureozonolyse in Abschnitt 6.4.2.5 bei einem Ozonumsatz von $p_{\mathrm{O}_{3}}=0.1 \mathrm{~Pa}$ und einem Gesamtdruck von $p_{\text {ges }}=460$ mbar in Stickstoff beispielsweise höhere Maximalkonzentrationen und Massenausbeuten zu ergeben scheinen als für die nicht voroxidierten Komponenten (s. Abb. 6.25, S. 176). Allerdings ist hier zu beachten, dass Partikelbildungsstudien meist unter atmosphärenähnlichen Bedingungen, das heißt in Synthetischer Luft, durchgeführt wurden. Gerade in Bezug auf die Peroxychemie ist die Verwendung von sauerstoffhaltigem Badgas für ihre Bildung entscheidend. Der Vergleich der Aerosolausbeuten zeigt folgerichtig deutlich geringere Ausbeuten der Carbonsäuren in Synthetischer Luft als für die verwendeten reinen Kohlenwasserstoffe, was die Aussagen von KROLL und SEINFELD [21] bestätigt. Dabei wird sogar der zuvor beschriebene Effekt auf dem Dampfdruck durch Voroxidation der Edukte überkompensiert, der im Vergleich zur Kettenverlängerung um zwei Kohlenstoffatome einen höheren Effekt haben sollte.

An dieser Stelle muss eingeschränkt werden, dass dieser Effekt lediglich im 
Vergleich der nicht voroxidierten Verbindungen mit den Edukten des höchsten Oxidationsgrads zutreffend erscheint. Faktoren wie die Dampfdruckerniedrigung durch funktionelle Gruppen werden dabei außer Acht gelassen. Aus der Alkenozonolyse ergeben sich deutlich höhere Ausbeuten in Anzahl und Masse bei der Verwendung von Synthetischer Luft und vergleichbaren Alkenumsätzen. Hier scheint die Auswirkung der funktionellen Gruppe die destabilisierenden Effekte durch die höhere Oxidationszahl also noch bei weitem zu übertreffen, da die untersuchten Alkohole ein um ein bis zwei Kohlenstoffatome verkürztes Gerüst im Vergleich zu den reinen Kohlenwasserstoffverbindungen besitzen.

Ebenfalls kann durch den Vergleich der hydroxylgruppentragenden Edukte geschlossen werden, dass sich die Addition von $\mathrm{OH}$-Gruppen im Vergleich zu Carboxylfunktionalitäten unter bestimmten Bedingungen positiver auf das Nuklationsvermögen auszuwirken scheint. Ausschlaggebend könnten hier die induktiven Effekte der beiden Funktionalitäten sein. Da die Carboxylgruppe zwei Sauerstoffatome am funktionalisierten C-Atom besitzt statt nur eines im Falle des Alkohols, könnte bei der Carbonsäure mehr Elektronendichte aus der Kohlenstoffkette abgezogen werden als beim Alkohol. So könnte die zusätzliche Destabilisierung bei Ausbildung einer Peroxygruppe an der radikalischen, zuvor die Doppelbindung enthaltenen Seite des Moleküls, bei Carbonsäuren zu einem beschleunigteren Bruch der Kohlenstoffkette sorgen als bei den Alkoholen.

Die Voroxidation kann die Partikelbildung aus der Ozonolysereaktion also dahingehend beeinflussen, dass Sauerstoff entweder nicht mehr, wie im Falle der Carbonsäure, oder nur noch in geringem $\mathrm{Maße}$, wie es die übrigen voroxidierten Spezies (außer 4-Penten-1-ol, s. S. 6.32) durch ihre recht hohen Ausbeuten auch in Stickstoff zeigten, für die Aerosolbildung benötigt wird.

Im Falle der Edukte mit recht kleinen Kohlenstoffgerüsten, wie den Carbonsäuren und 3-Buten-1-ol, aus denen lediglich $\mathrm{C}_{1}$ - und $\mathrm{C}_{3}$-Grundkörper aus der Ozonolysereaktion resultieren, sind Vorgänge auch jenseits der rein oxidativen Prozesse zu vermuten. Das zeigt die negative Wirkung des Sauerstoffes auf die Ausbeuten im Falle der Carbonsäuren. Hier könnten nicht-oxidative Vorgänge wie eine Art Gasphasenpolymerisation nach dem Vorbild von SADEZKY [36,53], die nach den Untersuchungen für unsubstituierte wie auch substituierte CRIEGEE-Intermediate möglich sind, eine denkbare Erklärung sein. Diese Reaktion 
verringert den Dampfdruck der Produkte pro zweier angelagerter Kohlenstoffatome um zwei Größenordnungen [21], wobei der Effekt durch die von vornherein im Molekül enthaltenen Sauerstoffatome noch drastischer ausfallen sollte. Auch andere bimolekulare Reaktionen könnten für voroxidierte Spezies eine höhere Bedeutung besitzen als für reine Kohlenwasserstoffverbindungen, was für die vergleichsweise hohen Ausbeuten sorgen könnte. Eine Möglichkeit ist die Ausbildung von Dimeren, etwa durch Wasserstoffbrückenbindungen, wobei das Molekulargewicht schlagartig verdoppelt und die Flüchtigkeit stark herabgesetzt wird. Vor allem für die Alkohole und Carbonsäuren sollte dies von Bedeutung sein, da sie jeweils eine $\mathrm{OH}$-Gruppe tragen. Alkohole und noch stärker die Carbonsäuren haben außerdem eine Neigung, CRIEGEE-Intermediate abzufangen, wie LEE und KAMENS [52] in ihren Studien nachweisen konnten. Diese agieren dabei deutlich effektiver als Carbonylverbindungen. Auch hier kann ein Unterschied in den deutlich geringeren Ausbeuten der Carbonylverbindungen im Vergleich zu den übrigen voroxidierten Gruppen liegen. Das Auftreten solcher Reaktionen ist wegen des stets hohen Überschusses der Kohlenwasserstoffverbindungen sehr wahrscheinlich.

Die prinzipielle Bedeutung dieser Reaktion für einen Teil der Aerosolausbeute konnte für die Butensäure bereits durch Experimente mit dem in großem Überschuss eingesetzten Cl-Fänger Ameisensäure sowie Experimenten unter Ozonüberschuss (Abschnitt 6.4.2.6) gezeigt werden, wie sie von NEEB et al. [59] für Carbonsäuren vorgeschlagen wurden.

Mit analogen Experimenten könnte dieses Reaktionsverhalten auch für die ungesättigten Alkohole verifiziert werden, wobei vor allem das Ausmaß der Beeinflussung im Vergleich zur Carbonsäure interessant wäre. Gleichzeitig könnten die Experimente auch Aufschluss über weitere sekundäre Reaktionen geben, da aus der alleinigen Abfangreaktion Alkenol $+\mathrm{Cl}$ die hohe Aerosolausbeute für 3-Buten-1-ol nicht erklärt werden kann. Darauf weisen die Experimente unter Butanolzugabe zum Abfangen der $\mathrm{OH}$-Radikale für die reinen Alkene hin (Kap. 6.4.1.2), da in diesen Experimenten prinzipiell die Aerosolausbeute bei Zugabe von 1- oder 2-Butanol stets fast vollständig unterdrückt wurde.

Die Molekulargewichte von Produkten aus der Reaktion 3-Buten-1-ol $+\mathrm{C}_{3}-\mathrm{Cl}$ (162.09 g/mol) und z.B. der Reaktion 1-Butanol $+\mathrm{C}_{5}-\mathrm{Cl}$ des 2-Methyl-1-pentens $(176.14 \mathrm{~g} / \mathrm{mol})$ unterscheiden sich nicht sehr stark und würden sogar für eine 
höhere Ausbeute im letzten Falle sprechen (s. Abb. 7.5).

In beiden Fällen werden Hydroperoxide gebildet. Als einzige strukturelle Unterschiede tauchen eine zusätzliche terminale Hydroxylgruppe und eine Doppelbindung bei der Reaktion des ungesättigten Alkohols auf. Dies resultiert nach KROLL und SEINFELD [21] für sich allein bereits in einer deutlichen Dampfdruckerniedrigung. Allerdings sind auch weitere oxidative Prozesse, die an der verbleibenden Doppelbindung in Anwesenheit der reaktiven Verbindungen Ozon und $\mathrm{OH}$-Radikalen unter Umständen effektiver ablaufen als an einem gesättigten Kohlenwasserstoffrest, denkbar.

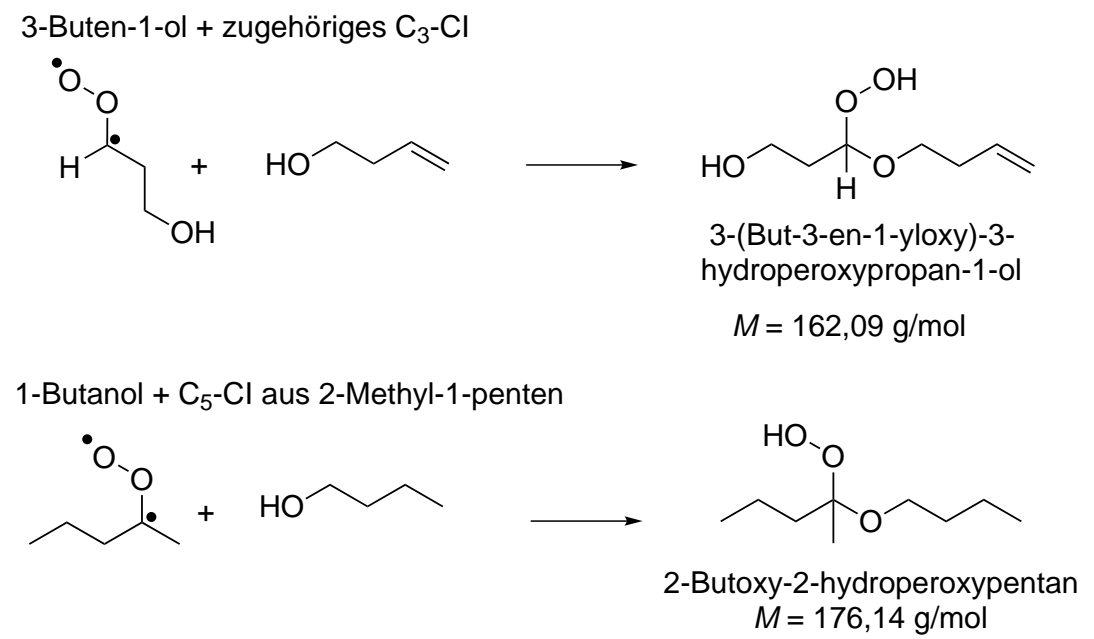

Abb. 7.5.: Darstellung der Abfangreaktion mit ungesättigtem und gesättigtem Alkohol.

Im vorangegangenen Abschnitt wurden bereits die Folgereaktionen der Alkoxyradikale als ein wichtiger Faktor für die Reduktion der Flüchtigkeit und damit der Aerosolausbeute genannt. Das Eintreten solcher Isomerisierungen ist nicht nur von der prinzipiellen Bildungsmöglichkeit solcher Strukturen in Anwesenheit von Sauerstoff in der reaktiven Mischung, sondern auch stark von der Struktur der Verbindung abhängig, da das Molekül ein an das Kohlenstoffatom gebundenes Wasserstoffatom in 1,4-Position zur Radikalposition besitzen muss. Dies trifft für alle untersuchten Edukte mit einem Gerüst mit mehr als vier Kohlenstoffatomen zu. Für die kleineren verwendeten Verbindungen, 3-Buten-1-ol, Butenund Methacrylsäure, befindet sich dagegen ein Wasserstoffatom in 1,4-Position an der jeweiligen Hydroxylgruppe. Ein Vergleich der Geschwindigkeitskoeffizi- 
enten für die Referenzreaktionen

$$
\begin{array}{ll}
\mathrm{C}_{2} \mathrm{H}_{2} \mathrm{OH}+\mathrm{OH} & \longrightarrow \mathrm{CH}_{3} \mathrm{CH}_{2} \mathrm{O}+\mathrm{H}_{2} \mathrm{Obzw} . \\
\mathrm{C}_{2} \mathrm{H}_{5} \mathrm{OH}+\mathrm{OH} & \longrightarrow \text { andere Produkte }+\mathrm{H}_{2} \mathrm{O} \text { [130] }
\end{array}
$$

ergibt, dass die letztgenannte Umsetzung einen um etwa einen Faktor zehn schnelleren Geschwindigkeitskoeffizienten besitzt. Unter Berücksichtigung des relativen Verhätnisses von an C-Atomen zu an O-Atomen gebundenen Wasserstoffatomen verringert sich die relative Wahrscheinlichkeit der Reaktion jedoch etwas. Dies erhöht die relative Reaktionswahrscheinlichkeit der Wasserstoffabstraktion in 1,4-Position von einer Hydroxylgruppe, zumal zusätzlich intermediär die energetisch günstige Ringstruktur ausgebildet wird [21], deren Auswirkungen an dieser Stelle aufgrund fehlender Literaturwerte nur schwer eingeschätzt werden kann. Vor allem eine Absenkung des Dampfdruckes durch weitere Folgereaktionen und eine höhere relative Stabilität der Folgeprodukte könnten die Bedeutung der Reaktion erhöhen.

Die Wasserstoffabstraktion in 1,4-Position bildet nach KROLL et al. [21] in relativ wenigen Reaktionsschritten ein Dihydrofuran aus, welches bereits eine geringe Flüchtigkeit besitzt und seinerseits wiederum durch geeignete Reaktanden ( $\mathrm{HO}, \mathrm{O}_{3}$ o.a.) schnell weiter oxidiert werden kann. Die Produkte dieser oxidativen Prozesse besitzen potenziell einen sehr niedrigen Dampfdruck und können damit zur Aerosolausbeute beitragen.

Eine Begünstigung dieser Umsetzung speziell bei Strukturen, die das betreffende Wasserstoffatom an der Hydroxylgruppe besitzen, könnte somit ein möglicher Erklärungsansatz für die sehr hohen Ausbeuten der kleinsten untersuchten Edukte sein.

Allerdings haben auch Reaktionen in der Partikelphase, die mit den vorhandenen experimentellen Mitteln bisher nicht aufgeklärt werden können, eine hohe Bedeutung für die erhaltenen Aerosolausbeuten. Eventuell können hier durch die Kooperation mit der Aerosolanalytik in Mainz ein tieferer Einblick gewonnen werden. Die Bedeutung der Partikelphasenreaktionen zeigten unter anderem Untersuchungen von TOBIAS et al. [71], in denen auch nach dem Abschluss der Gasphasenreaktionen noch deutliche Zunahmen des sekundären 
organischen Aerosols festgestellt wurden. Nach KROLL et al. [21] sind die Prozesse dabei mit denen in der Gasphase zu vergleichen, allerdings mit veränderter Kanalverteilung, wobei die Flüchtigkeit der Endprodukte stark von der Dissoziation der Alkoxyradikale bestimmt sei.

\subsubsection{Auswirkungen der Druckabhängigkeit - Die Rolle der CrIEgeE-Intermediate}

Neben der $\mathrm{RO}_{2}$ - / RO-Chemie sind auch die aus der Ozonolyse gebildeten CRIEGEE-Intermediate und ihre Stabilität von großer Bedeutung für das Partikelbildungpotential der untersuchten Verbindungen.

Wie die Untersuchungen der Druckabhängigkeit zeigen, sind die Partikelausbeuten substanzabhängig bei verschiedenen Gesamtdrücken unterdrückt. Aufgrund der hohen Bedeutung der CRIEGEE-Intermediate für die Aerosolbildung kann dieses $\mathrm{Maß}$ für die Stabilisierbarkeit der $\mathrm{Cl}$ auch als solches für das generelle Partikelbildungpotential der einzelnen Edukte angesehen werden. Beispiele sind die sehr guten Partikelbildner 1-Hexen ( $p_{\text {ges }}=10 \mathrm{mbar}$ ) und $\alpha$-Pinen ( $p_{\text {ges }}=2-3 \mathrm{mbar}$ ) und die unter den verwendeten experimentellen Bedingungen eher niedrige Ausbeuten liefernden Methylcyclopenten, 1-Penten-3-on, Cyclohexen oder 2-Methyl-1-penten ( $p_{\text {ges }}=30$ mbar).

Bei dieser Betrachtung muss beachtet werden, dass sich die unsubstituierten und die substituierten größeren CRIEGEE-Intermediate unterschiedlich leicht stabilisieren lassen. Die Kalkulationen können zwischen verschiedenen Studien jedoch variieren. So schätzen SADEZKY et al. [36, 156] ab, dass sich aus der Ozonolyse bestimmter Alkene oder Enolether mit 50-60 \% ein höherer Anteil der unsubstituierten $\mathrm{Cl}$ stabilisieren lässt als dies beispielsweise für $\mathrm{C}_{2}$ - oder $\mathrm{C}_{3}-\mathrm{Cl}$ mit etwa 20-40\% der Fall ist. OLzMANN et al. [113] sagen theoretisch, übereinstimmend mit experimentellen Werten von HATAKEYAMA et al. [46], einen Stabilisierungsgrad der unsubstituierten $\mathrm{Cl}$ von etwa $20 \%$ unter Niederdruckbedingungen (10 bzw. 13 mbar) voraus. Während HATAKEYAMA et al. [46] bei Atmosphärendruck eine Ausbeute von insgesamt 35-40 \% erhalten, unterschätzt die Simulation die Werte bei Atmosphärendruck allerdings um etwa den Faktor zwei [113]. Unter Atmosphärendruck konnten HATAKEYAMA et al. [46] weiterhin eine Ausbeute von etwa $30 \%$ für das $\mathrm{C}_{3}-\mathrm{Cl}$ aus der Ozo- 
nolyse des 2,3-Dimethyl-2-buten (TME) nachweisen. Für die gleiche Substanz können DROzD et al. [142] aus der TME-Ozonolyse bei einem Gesamtdruck von 50 Torr (ca. 67 mbar) für die SCl eine Ausbeute von etwa $15 \%$ bestimmen. Die gleiche Arbeitsgruppe konnte in einer anderen Studie [44] ebenfalls zeigen, dass für lineare Alkene unter Niederdruckbedingungen ein höherer Anteil an CRIEGEE-Intermediaten stabilisiert werden kann als bei zyklischen, bei denen diese Verbindungen kaum nachweisbar waren. Sie stellen weiterhin fest, dass die Bildung von SCI stark von der Anzahl der im Molekül enthaltenen Kohlenstoffatomen abhängig ist, wobei eine Kettenverlängerung um zwei bis drei C-Atome die Ausbeute stark erhöhen kann. Als einen Vergleichswert geben sie an, dass aus endozyklischen Alkenen erst ab einem Grundkörper von $\mathrm{C}_{10}$ bei Atmosphärendruck Cl stabilisiert werden können [44].

Genaue Aussagen zu den Zahlenwerten der tatsächlich vorliegenden stabilisierten Anteilen der $\mathrm{Cl}$ fallen aufgrund einer recht großen Schwankungsbreite, die aus den Eigenschaften der untersuchten Alkenen und den experimentellen Methoden resultiert, schwer. Trotzdem kann zumindest festgestellt werden, dass das $\mathrm{C}_{1}-\mathrm{Cl}$ relativ zur gebildeten Ausbeute der großen Intermediate gesehen etwas leichter stabilisiert werden kann. Dies kann vor allem durch den sehr schnell über den Vinylhydroperoxidkanal zerfallenden Anteil der synKonformere erklärt werden, während die anti-Cl durch die Blockade dieses Reaktionsweges eine längere Lebensdauer besitzen sollten [33]. Vor allem unter Niederdruckbedingungen und daher bei unzureichender Thermalisierung durch desaktivierende Stöße kann dies entscheidend das entstehende Produktgemisch beeinflussen.

Zumindest für einige der reinen Kohlenwasserstoffverbindungen sind im Gegensatz zu den voroxidierten Verbindungen direkte Literaturwerte oder indirekte Vergleichswerte durch Untersuchungen von Strukturanaloga verfügbar [37]. Durch einen Vergleich der allgemeinen Tendenzen können eventuell zumindest relative Aussagen über die Art und die Ausbeute der gebildeten stabilisierten $\mathrm{Cl}$ der sauerstoffhaltigen Edukte getroffen werden.

Für alle verwendeten Edukte jeden Oxidationsgrads konnte gezeigt werden, dass durch die Variation des Gesamtdruckes die Aerosolbildung reduziert wird. Die entscheidenden Intermediate im Produktgemisch, wo sich der Gesamtdruck über die Anzahl desaktivierender Stöße (ca $10^{10}$ pro Sekunde bei At- 
mosphärendruck [20]) direkt auf die Stabilität der Ozonolyseprodukte und damit die Aerosolausbeute auswirken kann, sind die aus dem Primärozonidzerfall in angeregtem Schwingungszustand entstehenden $\mathrm{Cl}$. Das Primärozonid kann dagegen kaum wirksam durch den Druck beeinflusst werden, was aus der chemischen Aktivierung des Moleküls durch die Ozonoanlagerung resultiert. Diese Verbindungen werden daher trotz ihrer relativ hohen Masse nicht zur Aerosolausbeute beitragen.

Die $\mathrm{Cl}$ dagegen, die eduktabhängig verschiedene Strukturen besitzen, sind für die Aerosolbildung durch verschiedene Arten bimolekularer Reaktionen für Laborexperimente zentral. Die Auswirkungen sollen, aufgrund der Vergleichbarkeit mit vorhandenen Literaturwerte exemplarisch für die nicht voroxidierten Verbindungen 1-Hexen, 2-Methyl-1-penten und Methylcyclopenten diskutiert werden. Zudem wird die Partikelbildung bei diesen Substanzen vorwiegend durch die Struktur des Kohlenstoffgerüstes und nicht durch zusätzliche, verschieden wirkende funktionelle Gruppen beeinflusst.

Zunächst entscheiden Edukt und Art der gebildeten $\mathrm{Cl}$ über die Stabilisierbarkeit der Intermediate. Die ist in der Methylcyclopentenozonolyse z.B. eher als gering einzuschätzen, da die Intermediate durch eine hohe chemische Aktivierung recht schnell zerfallen und nur sehr eingeschränkt stoßstabilisiert werden sollten [44]. Dazu kommt es durch die schon im Eduktmolekül enthaltene Ringspannung, den zusätzlichen Energieeintrag durch die Reaktion mit Ozon und die anschließende Bildung nur eines CRIEGEE-Intermediates. Dieses besitzt zwar ein hohes Molekulargewicht, allerdings neigt durch den fehlenden Energieabbau durch Ausbildung einer Translationsmode zur Fragmentierung. Die Auswirkungen dieser Instabilität sind in den geringen beobachteten Partikelausbeuten (s. S. $182 \mathrm{ff}$.) zu beobachten.

Wie in den Produktbildungsexperimenten und ihrer Diskussion dargelegt (Abschn. 6.2.3, 7.1.2), kann für Methylcyclopenten der von WOLF [17, 48] vorgeschlagene Mechanismus für Methylcyclopenten auch für das niedrigere Homologe bestätigt werden. Dies ließ sich an der Ethen- und Ketenbildung aus dem Zerfall von radikalischen Intermediaten (s. 6.10, S. 134) unter Niederdruckbedingungen belegen. Bei diesen Bedingungen ist die Partikelbildung unterdrückt.

Der Vergleich der sich um die Methylsubstitution der Doppelbindung und die Ringgröße in ihrer Struktur unterscheidenden Substanzen Cyclohexen und Me- 
thylcyclopenten zeigt, dass sich die Substitution an der Doppelbindung positiv auf das Nukleationsvermögen auszuwirken scheint, da bei einem Umsatz von etwa $p_{\mathrm{O}_{3}}=0.1 \mathrm{~Pa}$ die Anzahlkonzentration bei Methylcyclopenten um den Faktor 20 gegenüber Cyclohexen erhöht ist.

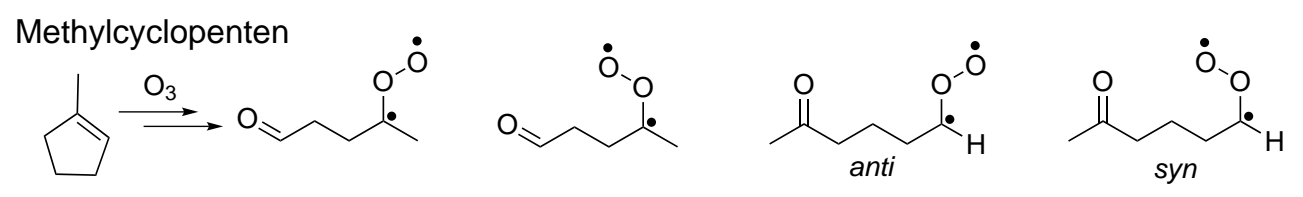

Cyclohexen
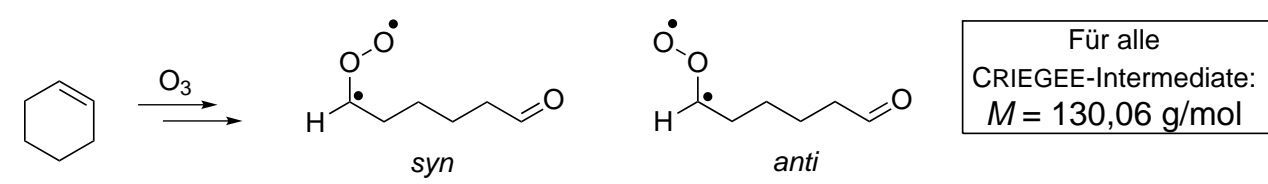

Abb. 7.6.: Sich in der Ozonolyse bildende CRIEGEE-Intermediate für Methylcyclopenten und Cyclohexen.

Das erhöhte Partikelbildungspotential für Methylcyclopenten ist in diesem Beispiel nicht allein aus der chemischen Aktivierung und einem dadurch induzierten beschleunigten Zerfall der $\mathrm{Cl}$ begründen, da diese für Cyclohexen aufgrund des energetisch günstigeren Sechsringes vergleichsweise gering ausfallen sollte. Da sie in ihrem Molekulargewicht identisch sind, wäre es naheliegend, die Struktur zur Erklärung heranzuziehen, doch auch die Betrachtung der gebildeten Konformere und die spezifischen Zerfallswege sind in diesem Fall nicht zielführend. Bei Cyclohexen liegt die Hälfte der gebildeten $\mathrm{Cl}$ in synKonformation vor und zerfält daher sehr schnell unter Bildung kleinerer Fragmente, womit sie nicht mehr zur Aerosolbildung beitragen können. Dagegen bilden sich in der Methylcyclopentenozonolyse neben syn- und anti-Konformation auch zwei disubstituierte $\mathrm{Cl}$ aus, die jedoch wie das syn-Cl über den Vinylhydroperoxidkanal zerfallen können [33]. Zur Interpretation dieses Effektes sind weitere Untersuchungen nötig, die für die MCPE-Cl eventuell effektiver ablaufende bimolekulare Reaktionen oder die Zerfallsgeschwindigkeit der disubstituierten $\mathrm{Cl}$ im Vergleich zu den syn-Konformeren behandeln.

Eine Methylsubstitution hat im Gegensatz zu zyklischen Alkenen auf azyklische Strukturen einen deutlich behindernden Effekt auf die Aerosolbildung, wie ein Vergleich der Ausbeuten von 1-Hexen und 2-Methyl-1-penten bzw. Buten- 
säure und Methacrylsäure zeigt (S. 178 bzw. Kap. 6.4.2.5).

Auch hier lässt sich mit den entstehenden Arten der CRIEGEE-Intermediaten argumentieren. Zunächst entstehen sie nach Untersuchungen von RICKARD et al. [37] für 1-Hexen im Verhältnis $C_{1}: C_{5}=50: 50$, für 2-Methyl-1-buten wird aufgrund der analogen Struktur der Wert für 2-Methyl-1-buten mit $\mathrm{C}_{1}: \mathrm{C}_{4}=28: 72$ zum Vergleich herangezogen. Der größere Anteil an entstehenden unsubstituierten $\mathrm{Cl}$ konnte durch Vergleich der Formaldehydausbeuten in Abbildung D.2e, S. XVII wie auch durch die CO-Ausbeuten (Tabelle 6.10) bestätigt werden, die für 2-Methyl-1-penten in größerer Menge anstelle des $\mathrm{C}_{1}$ - $\mathrm{Cl}$ aus dem POZZerfall resultieren.
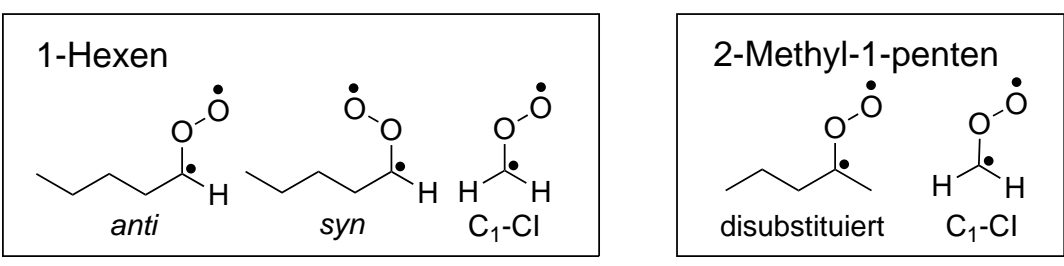

Abb. 7.7.: Sich in der Ozonolyse bildende CRIEGEE-Intermediate für 1-Hexen und 2-Methyl-1-penten.

Durch den größeren Anteil von unsubstituierten $\mathrm{Cl}$ und ihre leichtere Stabilisierbarkeit, die oben beschrieben wurde, sind für 1-Hexen Oligomerisierungsreaktionen nach dem Vorschlag von SADEZKY et al. [53] in größerem Umfang möglich, was die hohen Ausbeuten erklären kann.

Wenn von prinzipiell gleichen Bildungswahrscheinlichkeiten des unsubstituierten und substituierten [37] und weiterhin für die syn- und anti-Konformation des großen CRIEGEE-Intermediats ausgegangen wird, sollte für 1-Hexen etwa $25 \%$ als anti-Konformer vorliegen (vgl. Abb. 7.7). Diesem wird eine längere Lebensdauer und damit größere Neigung zu bimolekularen, die Flüchtigkeit potenziell verringernden Reaktionen zugesprochen [33, 89], was eine weitere Erklärung für die große Aerosolausbeute in der 1-Hexenozonolyse sein könnte.

Nach Untersuchungen von JOHNSON und MARSTON [33] führt die Stoßstabilisierung von schwingungsangeregten CRIEGEE-Intermediaten zur Bildung von syn- oder anti-konfigurierten $\mathrm{Cl}$, deren Verhältnis zueinander abhängig vom verwendeten Edukt recht stabil ist, da die Drehung um die Carbonyloxidbindung eine Barriere von etwa $200-250 \mathrm{~kJ} / \mathrm{mol}$ besitzt und daher nicht frei beweglich 
sein kann.

Das $\mathrm{OH}$-Radikal scheint vorwiegend aus dem Zerfall des syn-Cl über den Vinylhydroperoxidkanal zu stammen [157], während der Zerfall des anti-Konformers nach Bildung der "heißen“, schwingungsangeregten Säure vor allem zur Bildung von Verbindungen wie Methan, $\mathrm{CO}_{2}$, Keten, Wasser, Methanol und $\mathrm{CO}$ und wahrscheinlich nur zu einem kleinen Teil zu OH-Radikalen führt [33]. Dies ist in Abb. 2.4, S. 26 veranschaulicht, auch wenn Untersuchungen von KROLL et al. [156] für diesen Kanal eine Ausbeute von bis zu $30 \%$ vorhersagen. Aus den Untersuchungen einfacher Alkene folgern JOHNSON und MARSTON [33] jedoch, dass es trotz des wahrscheinlichen Eintretens mehrerer Bildungswege ausreichend ist, das syn- $\mathrm{Cl}$ als alleinige $\mathrm{OH}$-Quelle zu berücksichtigen. Jene zerfallen sehr schnell über den Vinylhydroperoxidkanal und bilden außer den genannten Radikalen weitere kleine Bruchstücke, die nicht ohne weiteres zur Partikelbildung beitragen können, da ihr Dampfdruck zu hoch ist (vgl. Abb. 2.5. S. 26). Die anti-Cl dagegen scheinen nach Ergebnissen von JOHNSON und MARSTON [33] leichter stabilisierbar zu sein und daher auch wahrscheinlicher für bimolekulare Reaktionen zur Verfügung zu stehen als syn-Konformer.

Diese Einschätzung kann durch die in Tabelle 7.3 zusammengefassten Ergebnisse bestätigt zu werden, da eine niedrige $\mathrm{OH}$-Ausbeute stets bei einer recht hohen Aerosolausbeute auftritt. Dies spricht für eine nukleationsfördernde Wirkung der in diesem Fall vermehrt gebildeten anti-Cl. Durch eine leichtere Stabilisierbarkeit und eintretende bimolekulare Reaktionen könnten in wenigen Schritten hochmolekulare und -oxidierte Verbindungen mit niedrigem Dampfdruck entstehen, die damit auch das Eintreten eines Nukleationsereignisses begünstigen können.

Beim Vergleich der in Tabelle 6.9, S. 170 aufgelisteten OH-Ausbeuten mit den angegebenen Literaturwerten wird deutlich, dass zumindest relativ gesehen die gleichen Tendenzen innerhalb der ungesättigten Substanzgruppen (Alken / Alkohol / Carbonylverbindung / Carbonsäure) resultierten. Weiterhin wird bei Betrachtung der Partikelauftragungen in Kap. 6.4 deutlich, dass innerhalb der untersuchten Stoffgruppen die Aerosolausbeute tendenziell mit zunehmender $\mathrm{OH}$-Ausbeute abnimmt. Dies ist in Tabelle 7.3 durch den Vergleich der experimentell in dieser Arbeit ermittelten $\mathrm{OH}$-Ausbeuten (von oben nach unten 
7.3. $\mathrm{SO}_{2}$-Experimente - Neue Ansatzpunkte durch Betrachtung des druckabhängigen Verhaltens?

\begin{tabular}{|c|c|c|c|}
\hline \hline Alken & $\boldsymbol{N}\left[\mathbf{c m}^{-3}\right]$ & $\boldsymbol{m}\left[\boldsymbol{\mu} \mathbf{g} \cdot \mathbf{m}^{-3}\right]$ & $\boldsymbol{Y}_{\text {OH,exp }}$ \\
\hline \hline 1-Hexen & $1.0 \cdot 10^{6}$ & 1400 & 0.4 \\
2-Methyl-1-penten & $3.3 \cdot 10^{5}$ & 1400 & 0.7 \\
Methylcyclopenten & $4.4 \cdot 10^{5}$ & 1200 & 1.3 \\
\hline 1-Penten-3-ol & $2.5 \cdot 10^{6}$ & 6500 & 0.3 \\
3-Buten-1-ol & $1.8 \cdot 10^{6}$ & 5700 & 0.4 \\
4.Penten-1-ol & $1.3 \cdot 10^{6}$ & 6300 & 0.5 \\
\hline 4-Pentenal & $7.5 \cdot 10^{5}$ & 2300 & 0.2 \\
1-Penten-3-on & $1.6 \cdot 10^{5}$ & 280 & 0.4 \\
\hline Butensäure & $2.1 \cdot 10^{6}$ & 6500 & 0.6 \\
Methacrylsäure & $1.1 \cdot 10^{6}$ & 3000 & 1.4 \\
\hline
\end{tabular}

Tab. 7.3.: Experimentell erhaltene $\mathrm{OH}$-Ausbeuten für die untersuchten Substanzen und Partikelbildungspotentiale. Sämtliche Werte wurden zur besseren Vergleichbakeit unter Annahme linearen Verhaltens jenseits der Nukleationsschwelle [33] auf einen Ozonumsatz von $\Delta\left(\mathrm{O}_{3}\right)=0.5 \mathrm{~Pa}$ skaliert. Bis auf die Carbonsäuren $\left(\mathrm{N}_{2}\right)$ wurden sämtliche Werte in Synthetischer Luft erhalten.

in den Stoffgruppen zunehmend) und den relativen Aerosolausbeuten veranschaulicht. Durch die Schwankungsbreite der einzelnen Messwerte, die hier nicht berücksichtigt wurde, und eine in Einzelfällen nicht streng gültige Linearität bei der Skalierung auf einen Ozonumsatz von $\Delta\left(\mathrm{O}_{3}\right)=0.5 \mathrm{~Pa}$ kann es zu Abweichungen kommen.

Zudem müsste dieser postulierte Zusammenhang durch potenzielle Unsicherheiten in der Bestimmung des relativen Verbrauches, wie sie etwa durch Nebenreaktionen der $\mathrm{Cl}$ mit Carbonsäuren auftreten können, in weiteren Messungen bestätigt werden.

\section{3. $\mathrm{SO}_{2}$-Experimente - Neue Ansatzpunkte durch Betrachtung des druckabhängigen Verhaltens?}

In den Experimenten wurden verschiedene zyklische Alkene auf ihr Partikelbildungsvermögen bei Zugabe von Schwefeldioxid untersucht (Kap. 6.5). Da 
diese Untersuchungen nur einen Nebenaspekt der insgesamt präsentierten Ergebnisse darstellen, sollen lediglich die Effekte diskutiert werden, die die Variation des Gesamtdruckes ausübt. Dieser Aspekt stellt im Zusammenhang mit der schwefelsäureinduzierten und - assistierten Nukleation einen neuen Ansatz dar. Auch unter Zugabe verschiedener $\mathrm{SO}_{2}$-Partialdrücke zog eine Verringerung des Gesamtdruckes die Reduktion der Aerosolausbeute nach sich, wie es auch für die zuvor beschriebenen Ozonolysen zu beobachten war.

Unter Niederdruckbedingungen zeigen die exozyklischen Alkene eine geringere Reduktion der Ausbeuten als endozyklische Strukturen. Dies könnte sich auf die leichtere Stabilisierbarkeit der CRIEGEE-Intermediate, was die Untersuchungen von PAULSON et al. [43] (s. Tab. 2.2, S. 27] für Atmosphärendruck) ergaben, erklären lassen. Auch unter Niederdruckbedingungen sollte die Ausbeute an $\mathrm{SCl}$ höher sein, was, unter Beteiligung der $\mathrm{Cl}$ am Partikelbildungsprozess geringere Zunahmen bei Druckerhöhung nach sich zieht. Zu erklären ist das durch die unterschiedlichen Zerfallswege der zyklischen Strukturen (Abb. 7.8), da beim Zerfall eines Exozyklus eine Fragmentierung in $\mathrm{Cl}$ und Carbonylverbindung auftritt, während für endozyklische Verbindungen nur ein Molekül mit beiden Funktionalitäten und damit verbunden höherer Neigung zur Fragmentierung entsteht [44]. Auch bleibt beim Zerfall exozyklischer Strukturen die Ringstruktur erhalten, was für eine höhere Zahl an Schwingungsmoden sorgt und damit eine bessere Umverteilung der Überschussenergie im Molekül ermöglicht.

Über den untersuchten Druckbereich war ein größerer relativer Effekt für die Edukte mit niedrigem Nukleationsvermögen zu beobachten, wie es die Abbildungen für Cyclohexen (Abb. 6.47, S. 225) und Methylcyclopenten (Abb. 6.48, S. 226) veranschaulichen, als für die Terpene als gute Partikelbildner (vgl Abb. 6.51 S. 229 für $\alpha$-Pinen, Abb. 6.54, S. 232 für $\beta$-Pinen). Die lässt sich auf die schwefelsäureinduzierte Bildung zusätzlicher Keime im Reaktionsgemisch, durch die beispielsweise mittelflüchtige organische Ozonolyseprodukte „aufgesammelt" werden können und damit eine größere Ausbeute für die Aerosolmasse detektierbar machen, zurückführen. Einen großen Einfluss durch die gebildete Schwefelsäure lässt sich somit nur auf die Anzahlkonzentration feststellen. Ein Beitrag zur Massenausbeute kann aufgrund von Kontrollmessungen, in denen lediglich Wasser und Schwefeldioxid bzw. Schwefeldioxid und Ozon in der 
Exozyklisch

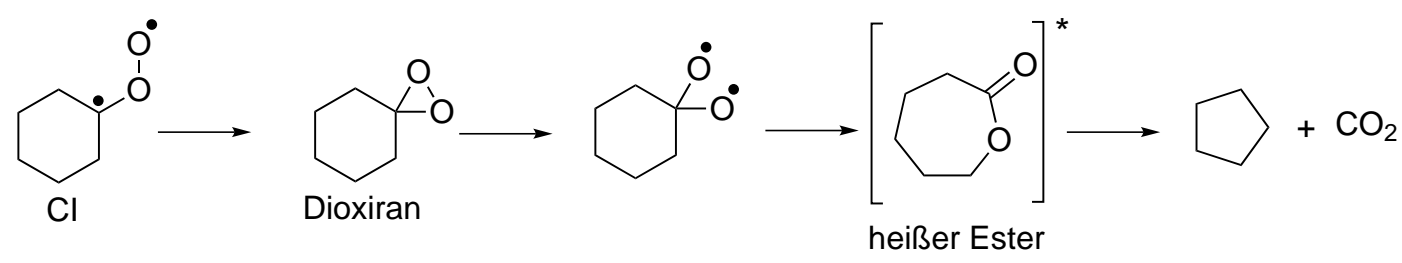

Endozyklisch<smiles>O=C1CCCCC1OCCO</smiles>

$\mathrm{Cl}$<smiles>CC1OOC1CCCCC=O</smiles>

Dioxiran<smiles>O=CC1CCCCCC1C(=O)O</smiles><smiles>CCCC[C+]C(=O)OCc1ccccc1</smiles>

Abb. 7.8.: Zerfall der CRIEGEE-Intermediate für endo- und exozyklische Alkene.

Reaktionszelle zusammengeführt und auf Nukleationsereignissse untersucht wurden, ausgeschlossen werden. Die abnehmende Tendenz der Aerosolausbeuten bei Wasserzugabe lässt den Schluss zu, dass schon unter trockenen Bedingungen genug Feuchtigkeit im Reaktionsgemsich vorhanden ist, um die Schwefelsäurebildung nicht zu verzögern [139].

Mit Hilfe der Produktstudien unter Schwefeldioxidzugabe (Kap. 6.2.4) konnte durch den Vergleich mit quantenchemisch berechneten IR-Spektren (Niveau: $B 3 L P Y / 6-311+G(d, p))$ für $\alpha$-Pinen und Cyclohexen nachgewiesen werden, dass in der Gasphase verschiedene Carbonylverbindungen reduziert vorliegen. Dies kann mit einem schwefelsäurekatalysierten Übergang mittelflüchtiger Substanzen in die Partikelphase, wie er bereits von GARLAND et al. [95] postuliert wurde, erklärt werden, wodurch in der Folge die detektierte Aerosolmasse erhöht wird. Alternativ könnte auch die Erniedrigung des Dampfdruckes dieser Carbonylverbindungen durch weitere Oxidation zu Carbonsäuren [94] für die verringerten Carbonylbanden in der Gasphase verantwortlich sein.

Die Betrachtung der Spektren des exozyklischen $\beta$-Pinens (Abb. 6.24, S. 168) zeigt im Gegensatz zu denen der endozyklischen Verbindungen einen vollständigen $\mathrm{SO}_{2}$-Umsatz sowie erhöhte Intensitäten der Carbonylbanden, welche anhand von Reinspektren als Nopinon identifiziert werden konnte. Diese Beobachtung lässt sich beispielsweise auf eine $\mathrm{SO}_{2}$-induzierte Reaktion der Form 
$\mathrm{SCI}+\mathrm{SO}_{2} \longrightarrow$ Carbonyl $+\mathrm{SO}_{3}$ zurückführen, bei der das CRIEGEE-Intermediat in eine stabilere Struktur reduziert wird. Dass dieser Effekt nur bei $\beta$-Pinen zu beobachten war, lässt sich ebenfalls mit der höheren Cl-Stabilität und damit der größeren Wahrscheinlichkeit bimolekularer Reaktionen erklären. Allerdings ist auch die mögliche Bildung von Organosulfaten durch eine Aktivierung der Sekundärozonide, wie BonN et al. [12] sie vorschlagen, in Betracht zu ziehen.

Zur genaueren Interpretation sind auch hier detailliertere Messungen nötig, die im folgenden Abschnitt 9 skizziert werden.

Insgesamt lassen sich die durchgeführten Experimente auf die in Abbildung 7.9 dargestellten Zusammenhänge und Abhängigkeiten zusammenfassen.

Einflussmöglichkeiten auf die Partikelbildung durch Veränderung der Reaktionsumgebung

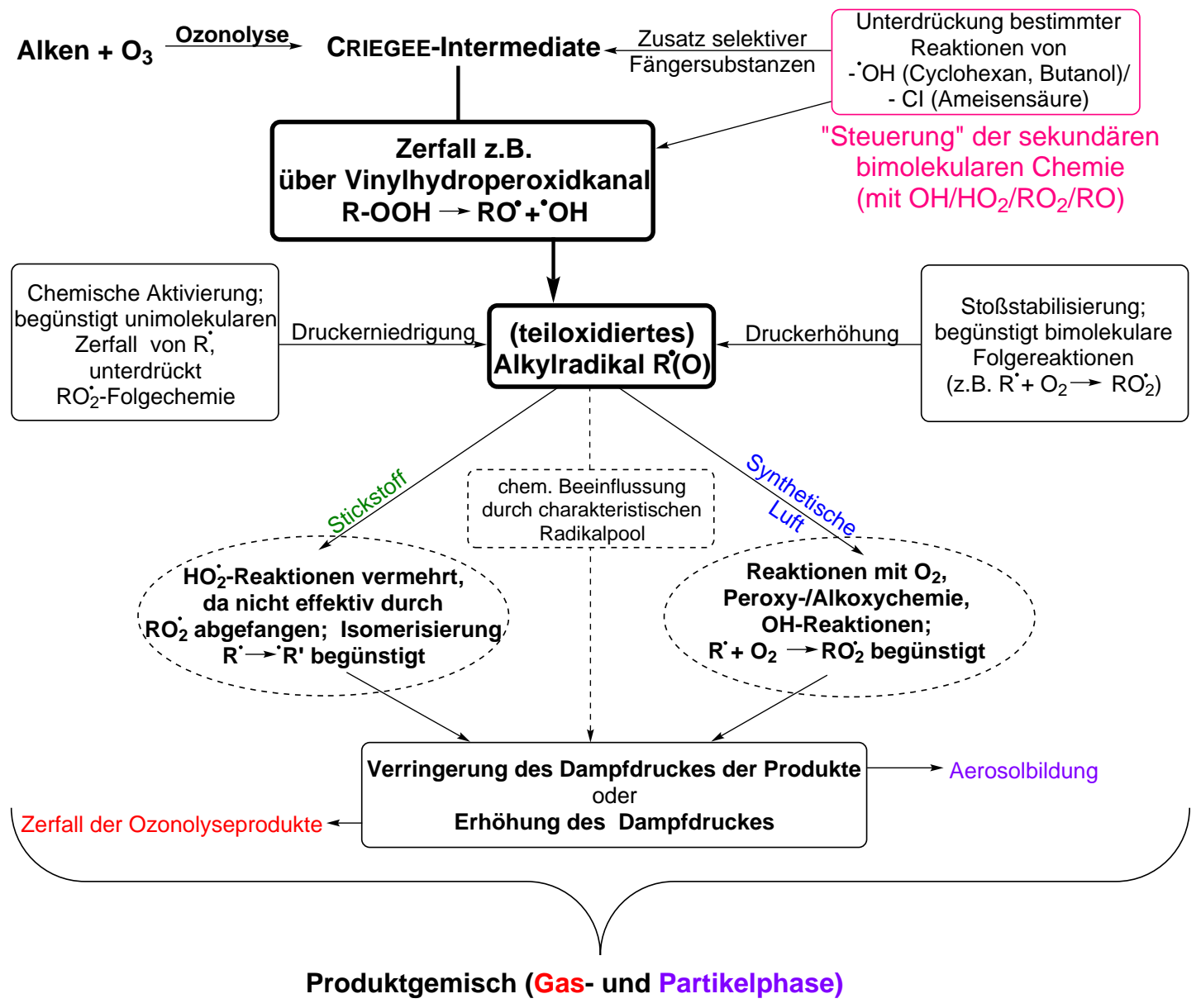

Abb. 7.9.: Schematische Darstellung der untersuchten Gesichtspunke bei der Aerosolbildung, Beeinflussung der Intermediatchemie. 
7.3. $\mathrm{SO}_{2}$-Experimente - Neue Ansatzpunkte durch Betrachtung des druckabhängigen Verhaltens?

Hier wird gezeigt, an welchen Stellen durch die jeweiligen Variationen der Experimentbedingungen in den Mechnismus eingegriffen wird. Es wird deutlich, dass durch die einzelnen Variationsmöglichkeiten vor allem auf die Chemie der Peroxyradikale und ihrer Folgeprodukten Einfluss ausgeübt wird. Auch das Verhältnis zwischen bimolekularer Reaktion und unimolekulrem Zerfall bzw. Isomerisierungsvorgängen ist vielfach betroffen. 


\section{Zusammenfassung}

In dieser Arbeit wurde eine große Bandbreite unterschiedlicher Verbindungen, aus deren Ozonolysereaktion potenziell Partikel entstehen können, untersucht. Dabei wurden verschiedene Substanzklassen untersucht, wobei nur einige der Edukte als biogen emittierte Verbindungen bekannt sind. Die Wahl der Substanzen erfolgte hauptsächlich auf Grundlage struktureller Analogien, die damit als Modellverbindungen für bestimmte Motive und biogene Substanzen Aussagen über das generelle Verhalten der Substanzklasse ermöglichen sollten.

Wie bereits vielfach nachgewiesen, können neben den untersuchten biogenen Terpenen auch ungesättigte voroxidierte Verbindungen biogen emittiert werden und die Ozonolysereaktion eingehen, Beispiele sind 1-Penten-3-ol und 1-Penten-3-on [62, 65, 158]. Zusätzlich wurden Methacrylsäure, Butensäure, 3Buten-1-ol, 4-Penten-1-ol und 4-Penten-1-al hinsichtlich ihres Reaktionsverhaltens betrachtet ( $\mathrm{vgl}$. Abb. 1.2). Bei Methacrylsäure handelt es sich um ein mögliches Oxidationsprodukt des in großen Mengen emittierten Terpens Isopren. Zudem wird organischen Säuren als Reaktionsprodukten in der Gas- [22] und in der Partikelphase [94] eine große Einflussmöglichkeit zugeordnet, weshalb die Ozonolyse dieser voroxidierten Verbindungen von großem Interesse war.

Zur Untersuchung der rein durch die chemische Struktur an der Doppelbindung verursachten Effekte wurden weiterhin 1-Hexen, 2-Methyl-1-penten und Methylcyclohexen (s. Abb. 1.3) gewählt.

Die Experimente wurden in einem $64 \mathrm{~L}$ fassenden kugelförmigen Reaktor durchgeführt (Kap. 3.1.4), in den die Reaktanden Ozon und Alken aus zwei Vormischkammern ( $V=40 \mathrm{~L}$, Kap. 3.1.5) nacheinander oder simultan eingegeben werden können. Durch die Einstellung des Vordrucks in den Vormischkammern kann die simultane Expansion innerhalb von etwa $4 \mathrm{~s}$ bis zu einem definierten Gesamtdruck von etwa 500 mbar unter schneller Durchmischung der Edukte erfolgen. Die Gasphasenanalyse wird durch FTIR-Spektroskopie er- 
möglicht. Zur Bestimmung der Partikelausbeuten wird nach Auffüllen der Reaktionszelle auf Atmosphärendruck ein SMPS-System verwendet, mit dem durch den Einsatz verschiedener Trennsäulen Aerosole im Größenbereich von etwa 5-770 nm nachgewiesen werden können.

In der vorliegenden Arbeit konnten durch Untersuchungen einer Vielzahl unterschiedlicher Strukturen mit stoffgruppentypischen Eigenschaften durch Variation der Reaktionsparameter einige Aspekte des Partikelbildungsmechanismus genauer beleuchtet werden.

Die die Gasphase betreffenden Experimente umfassten dabei ...

- die Bestimmung der bimolekularen Geschwindigkeitskoeffizienten für die Ozonolysereaktion, um die Vergleichbarkeit mit den in der Literatur zu findenden experimentellen Voraussetzungen zu gewährleisten (Kap. 6.1 und Tab. A.3. sowie die Betrachtung der Auswirkungen sauerstoffarmer Reaktionsbedingungen.

- die qualitative Untersuchung der Gasphasenspektren mittels Infrarotspektroskopie auf charakteristische Produkte bestimmter Reaktionen und aus dem Zerfall instabiler Intermediate (Kap. 6.2 und Abbildungen in Anhang D) unter Variation von Gesamtdruck und Badgas (Sauerstoffan- und Sauerstoffabwesenheit).

- die quantitative Auswertung der Kohlenstoffmonoxidausbeuten (Kap. 6.3.2 für Alkene, zusätzlich Tab. B.2 für voroxidierte Verbindungen).

- sowie die quantitative Auswertung der $\mathrm{OH}-A u s b e u t e n$ (Kap. 6.3.1) aus dem relativen Verbrauch der Edukte in den Kinetikmessungen.

Die Bestimmung der Geschwindigkeitskoeffizienten ergab eine zufriedenstellende Übereinstimmung mit den verfügbaren Literaturwerten, wie Tabelle A.3 zeigt. Es konnten erstmals Werte für die Ozonolyse von

- Butensäure: $k=(4.19 \pm 0.13) \cdot 10^{-18} \frac{\mathrm{cm}^{3}}{\text { molecule } \cdot \mathrm{s}}$,

- 4-Pentenal: $k=(7.84 \pm 0.22) \cdot 10^{-18} \frac{\mathrm{cm}^{3}}{\text { molecule.s }}$ und

- 4-Penten-1-ol: $k=(11.8 \pm 4.5) \cdot 10^{-18} \frac{\mathrm{cm}^{3}}{\text { molecule.s }}$ 
(jeweils in Synthetischer Luft) bestimmt werden.

Außerdem wurde gezeigt, dass durch die Berücksichtigung des relativen Verbrauchs beider Edukte der von NEEB et al. [35] vorgeschlagene Ansatz für sehr unterschiedliche Kohlenwasserstoffverbindungen universell einsetzbar ist. Gleichzeitig kann der relative Verbrauch $\alpha$ zur Bestimmung der OH-Ausbeute verwendet werden, was eine Einschätzung über das sich bildende syn / antiVerhältnis der CRIEGEE-Intermediate [33] und der Bedeutung sekundärer Radikalreaktionen der einzelnen Substanzen ermöglicht. Der aufgrund fehlender Literaturwerte oft nur anhand von Referenzsubstanzen mögliche Vergleich zeigt im Rahmen der üblichen Ungenauigkeiten solcher Bestimmungen (vgl. Tab. 6.9, S. 170) ebenfalls gute Übereinstimmungen. Es sind sowohl positive als auch negative Abweichungen festzustellen, die gegen eine systematische Fehlbestimmung sprechen. Der sehr hohe Wert für Methylcyclopenten bzw. Methacrylsäure $\left(Y_{\mathrm{OH}, \exp }>1\right)$ deutet auf sekundäre $\mathrm{OH}$-Quellen bzw. den zusätzlichen Verbrauch des Eduktes durch weitere sich bildende Intermediate neben dem $\mathrm{OH}$-Radikal hin.

Die quantitative Auswertung der CO-Ausbeuten konnte diese für die reinen Kohlenwasserstoffverbindungen allein auf den Zerfall des unsubstituierten CRIEGEE-Intermediates zurückführen und sekundäre Beiträge als von geringer Bedeutung identifizieren, wie in Tab. 6.10 S. 173 ersichtlich. Das überwiegend druck- und badgasunabhängige Verhalten lässt auf eine recht robuste Stabilisierbarkeit der Intermediate und ein stabiles Kanalverhältnis für den Zerfall schließen.

Aufgrund fehlender Literaturwerte für das Verzweigungsverhältnis von Primärozonid über $\mathrm{C}_{1}-\mathrm{Cl}$ zu $\mathrm{CO}$ konnte eine solche Zuordnung für die voroxidierten Spezies nicht erfolgen. Allerdings konnten unter den experimentellen Bedingungen mit hohen Partikelausbeuten auch höhere CO-Ausbeuten nachgewiesen werden. Hier ließ sich auch eine vermehrte Bildung unter Niederdruckbedingungen beobachten (Tab. B.2). Besonders im Niederdruckbereich sind jedoch für eine abgesicherte Interpretation ausführlichere Kalibrierungen nötig, um eine Korrelation, vor allem mit den Ausbeuten der $\mathrm{C}_{1}-\mathrm{Cl}$, feststellen zu können.

Durch die Analyse der Gasphasenprodukte konnten verschiedene charakteristische Produkte nachgewiesen werden, die verstärkt unter Niederdruckbe- 
dingungen aus dem Zerfall chemisch aktivierter Intermediate zu erwarten sind [17,20]. Hier handelt es sich neben Kohlenstoffmonoxid auch um Ameisensäure, Formaldehyd, Keten und Ethen ( $\mathrm{vgl}$. die zusammenfassenden Tabellen 7.1 und 7.2 sowie Kap. 6.2 und die Abbildungen in Anhang D). Weiterhin konnten im Fall der voroxidierten Spezies einige Carbonylverbindungen, die aus dem Zerfall des Primärozonids stammen, anhand des Vergleiches mit quantenchemisch berechneten IR-Spektren nachgewiesen werden $(B 3 L Y P / 6-311+G(d, p))$. Einige bei Atmosphärendruck mit höherer Intensität auftretende Banden konnten in Wellenzahlbereichen beobachtet werden, die von Druckabhängigkeit und Signalposition zu Anhydriden [157] oder Sekundärozoniden [48, 126] passen würden, allerdings noch einer genaueren Analyse bedürfen.

Bezüglich des Nukleationsverhaltens der Edukte in der Alkenozonolyse wurden als Schwerpunkte gesetzt...

- die Druckabhängigkeit der Partikelausbeuten (Kap. 6.4).

- die Auswirkungen von Sauerstoffanwesenheit in der Reaktionsmischung (Kap. 6.4 und darin besonders die Abbildungen 6.32, 6.36, 6.41).

- die Abhängigkeit des Partikelbildungspotentials von der reaktiven Struktur (azyklisch unverzweigt / verzweigt, zyklisch bei gleicher Kohlenstoffatomanzahl Kap. 6.4.1).

- der Einfluss der Vorfunktionalisierung in verschiedenen Oxidationsgraden (Kap. 6.4.2).

- der Einfluss von Radikalfängern (Kap. 6.4.1.2, 6.4.2.1, 6.4.2.5) sowie CRIEGEE-Intermediatfängern (Kap. 6.4.2.6).

- die prinzipelle Nachweisbarkeit sich verändernder Produktzusammensetzungen unter dem Einfluss verschiedener Gesamtdrücke ( $\alpha$-Pinen) oder Badgase (Butensäure) in Kap. 6.7.

Zusätzlich wurden die biogen emittierten Terpene [21,33,115] $\alpha$ - und $\beta$-Pinen (zugehörige Modellverbindungen: Methylencyclohexan, Methylcyclohexen, Cyclohexen, Methylcyclopenten, vgl. Abb. 1.5) unter dem Aspekt der schwefelsäureinduzierten Partikelbildung betrachtet. Da dieser in verschiedenen Studi- 
en [91-93] eine hohe Bedeutung auch für die atmosphärische Nukleation zugewiesen wird, wurden die genannten Verbindungen bei der Ozonolyse unter variabler $\mathrm{SO}_{2}$-Zugabe vor allem unter dem Gesichtspunkt der Druckabhängigkeit untersucht. Auch hier standen Gasphasenprodukte (Kap. 6.2.4) und die Partikelbildung (Kap. 6.5) im Fokus.

Für sämtliche verwendete Substanzen und Experimentvarianten konnte gezeigt werden, dass eine Reduktion des Gesamtdruckes die gleiche reduzierende Wirkung auf die Aerosolausbeute nach sich zieht. Dabei ist die für eine vollständige Unterdrückung nötige Verminderung abhängig vom Partikelbildungspotential des einzelnen Eduktes. Während sie für 2-Methyl-1-penten bereits bei $p_{\text {ges }}=30$ mbar eintrat, ist sie für $\alpha$-Pinen beispielsweise erst unterhalb von $p_{\text {ges }}=3$ mbar zu beobachten [17]. Dies lässt für alle Edukte auf eine Beteiligung der stoßstabilisierbaren CRIEGEE-Intermediate in der Aerosolbildung schließen, die unterhalb eines substanzspezifischen Grenzdrucks nicht mehr effektiv genug ihre Überschussenergie abführen können und zu leichtflüchtigen Produkten fragmentieren. Die Art der Beteiligung ist ebenfalls von der jeweiligen Kohlenwasserstoffverbindung und Faktoren wie der Art (substituiert vs. unsubstituiert) und Struktur (syn-vs. anti-Konformation) der gebildeten $\mathrm{Cl}$ sowie ihrer Verhältnisse untereinander determiniert. In Frage kommen hier oxidative Prozesse durch Peroxyradikale [33] oder nicht oxidative Prozesse wie eine Oligomerisierung [36,53], die jeweils in der Gas- oder Partikelphase auftreten können, den Dampfdruck der gebildeten Produkte absenken und damit für eine Kondensation sorgen. Diese Effekte konnten für die Carbonylverbindungen (Kap. 6.2.2.2) sowie die Experimente unter $\mathrm{SO}_{2}$-Zugabe (Kap. 6.2.4) durch die Betrachtung der Gasphasenspektren anhand von Intensitätsreduktionen von Banden potenziell mittelflüchtiger sauerstoffhaltiger Produkte beobachtet werden.

Die durchgeführten Experimente konnten zeigen, dass lineare Alkene unabhängig von ihrem Oxidationsgrad ein höheres Partikelbildungspotential besitzen als solche mit Verzweigungen an der Doppelbindung (1-Hexen vs. 2Methyl-1-penten, Butensäure vs. Methacrylsäure). Im Gegensatz dazu zeigte eine Methylsubstituierung der endozyklischen Doppelbindung eine nukleationsfördernde Beeinflussung (Methylcyclopenten vs. Cyclohexen), die offenbar sogar den energetisch ungünstigen Effekt eines fünf- statt sechsgliedrigen Ringes 
und die sich daraus ergebende Instabilität der Folgeprodukte durch chemische Aktivierung (vgl. Abb. 6.34) ausgleichen kann.

Eine Voroxidierung der zur Reaktion gebrachten Verbindungen senkt den Dampfdruck so weit [21], dass durch die Ozonolyse Produkte mit einem vergleichsweise kleinen $\mathrm{C}_{3}$-Grundkörper nukleationsfähig sind (3-Buten-1-ol, Abb. 6.29, Butensäure, Kap. 6.4.2.5). Dabei zeigen Verbindungen mit einer Hydroxylgruppe in beiden Badgasen deutlich höhere Aerosolausbeuten als Carbonylverbindungen. Dies kann mit der höheren Effektivität der Abfangreaktion des stabilisierten CRIEGEE-Intermediates für Alkohole und Säuren oder Dimerisierungseffekten im Gegensatz zu Aldehyden und Ketonen erklärt werden [52], aber auch eine Folge der von vornherein reduzierten Dampfdrücke bei Vorhandensein einer $\mathrm{OH}$-Gruppe im Molekül sein.

Die Ausprägung des Sauerstoffeinflusses, also die Differenz in den Ausbeuten in den verwendeten Badgasen Stickstoff oder Synthetischer Luft, nimmt allgemein bei den voroxidierten Edukten mit wachsendem Abstand zwischen den beiden funktionellen Gruppen zu.

Durch die Verwendung von Modellsubstanzen verschiedener Oxidationsgrade konnte außerdem gezeigt werden, dass sich im Reaktionsgemisch enthaltener Sauerstoff bei der Ozonolyse von Carbonsäuren negativ auf die Aerosolausbeuten auswirkt (Kap. 6.4.2.5). Bei allen anderen Verbindungen (Kap. 6.4.1f.) war eine Zunahme der Partikelausbeute in Synthetischer Luft zu beobachten. Dies lässt auf eine destabilisierende Wirkung der Anlagerung von sauerstoffhaltigen Gruppen bei übermäßig hohem O : C-Verhältnis auf das Kohlenstoffgerüst schließen. Eine mögliche Erklärung wäre die Verringerung der Elektronendichte in der Kohlenstoffkette durch Sauerstoffsubstituenten mit negativem induktivem Effekt und einem dadurch in Synthetischer Luft beschleunigten C-C-Bindungsbruch im Fall der Carbonsäureozonolyse. Dieser Effekt könnte durch Simulationen überprüft werden, indem Berechnungen zu Übergangszuständen und Elektronendichte im Kohlenstoffgerüst durchgeführt werden.

Untersuchungen unter Zugabe verschiedener $\mathrm{OH}$-Radikalfänger ergaben den Befund, dass diese Verbindungen nicht nur selektiv in die $\mathrm{OH}$-Chemie eingreifen, wie die unterschiedlichen Auswirkungen von Cyclohexan und 1- bzw. 2-Butanol auf die Partikelbildungsexperimente ergaben. Die hydroxylgruppenhaltigen Fängersubstanzen unterdrückten die Nukleation, während der Einsatz 
von Cyclohexan unabhängig vom Oxidationsgrad des Eduktes meist nur eine leichte Reduktion der Aerosolausbeuten bewirkte. Lediglich im Fall der 2Methyl-1-pentenozonolyse zog die Cyclohexanzugabe eine deutliche Steigerung der Ausbeute nach sich - ein Effekt, der in anderen Studien bisher nicht berichtet wurde.

Die unterschiedlichen Effekte lassen sich potenziell auf Nebenreaktionen zurückführen, da durch Cyclohexan das $\mathrm{HO}_{2} / \mathrm{RO}_{2}$-Verhältnis erniedrigt wird, was für einen später einsetzenden Abbruch von Oligomerisierungsreaktionen sorgen würde [36, 53]. Butanol kann durch seine $\mathrm{OH}-G r u p p e$ die für den Aufbau partikulärer Materie wichtigen CRIEGEE-Intermediate abfangen und damit negativ auf die Aerosolbildung einwirken. Beide Thesen bedürfen allerdings weitergehender Untersuchungen.

Der Einsatz von Ameisensäure zum Abfang von $\mathrm{Cl}$ konnte in Kombination mit der Untersuchung des Sauerstoffeinflusses und Experimenten mit variablen Eduktverhältnissen für Butensäure (Kap. 6.4.2.6) zeigen, dass die Butensäure + SCl-Reaktion zumindest für einen Teil der Aerosolausbeute verantwortlich ist. Geplante Untersuchungen dieser Art bilden ebenfalls für die untersuchten Alkenole einen vielversprechenden Ansatz für die Einschätzung der Bedeutung der $\mathrm{Cl}+$ Edukt-Reaktionen.

Durch die Analyse der Partikelphase konnte exemplarisch gezeigt werden, dass sich die Veränderungen in der Aerosolausbeute bei Variation der Reaktionsbedingungen in der Produktzusammensetzung nachweisen lassen. Für $\alpha$-Pinen wurden Proben bei verschiedenen Gesamtdrücken, für Butensäure unter Verwendung unterschiedlicher Badgase analysiert. Durch Einsatz eines HPLC/ESI-MS-Aufbaus [148] bei einem Kooperationspartner der Universität Mainz konnten massenspektrometrisch unterschiedliche Verbindungen sowie sich verändernde Anteile bestimmter auftretender Produkte nachgewiesen werden. Die Analyse der Ergebnisse ist bisher noch nicht abgeschlossen. Diese Methode ergänzt allerdings die bisher nur "von außen“ durch das SMPSSystem beobachteten Partikelausbeuten bezüglich Informationen zur Art der gebildeten Produkte, was potenziell Aufschluss über die keimbildenden Reaktionen geben kann.

Ozonolyseexperimente mit zyklischen Alkenen unter Zugabe von $\mathrm{SO}_{2}$ konnten ebenfalls eine Reduktion der Aerosolausbeuten bei abnehmendem Ge- 
samtdruck nachweisen (Abschn. 6.5). Da die Schwefelsäurebildung als druckunabhängig vorausgesetzt wird [139, 143], lässt dies auf eine Beteiligung der CRIEGEE-Intermediate schließen. Unterstützt wird diese These durch einen Vergleich der erwarteten Ausbeuten stabilisierter $\mathrm{Cl}$ (Tabelle 6.15, Werte nach PAULSON et al. [43]).

Da sich eine von der reaktiven Struktur des Zyklus abhängige Erhöhung der Partikelausbeuten bei Schwefeldioxidzugabe feststellen ließ, die mit steigendem Gesamtdruck und damit wachsendem Stabilisierungsgrad der $\mathrm{Cl}$ verstärkt beobachtet wurde, wird als Ursache die SCI + Schwefelsäure-Reaktion gesehen.

Die genannten Beobachtungen und zugrunde liegenden Effekte werden im Schema 8.1]abschließend zusammengefasst.

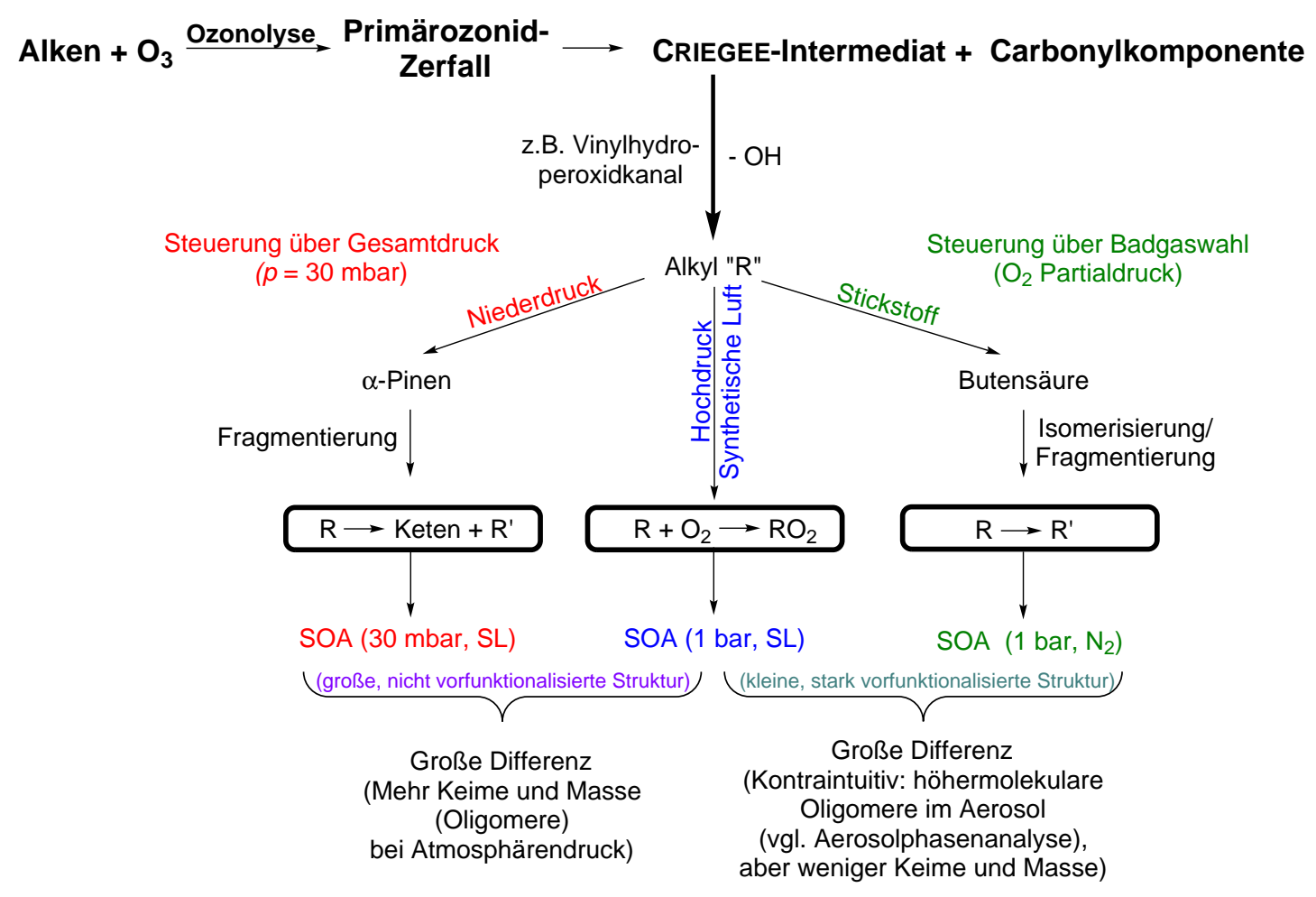

Abb. 8.1.: Zusammenfassung der Hauptergebnisse: Einflussfaktoren und Auswirkungen auf die Aerosolbildung am Beispiel der durch Aerosolphasenanalyse charakterisierten Substanzen. 


\section{Ausblick}

Durch die untersuchten Zusammenhänge zwischen der Variation der Reaktionsbedingungen und ihrer Auswirkungen auf die Nukleation haben sich einige Interpretationsansätze ergeben, deren zukünftige Untersuchung vielversprechend für die Aufklärung weiterer Details im aerosolbildenden Mechanismus erscheinen. Vor allem die Rolle der $\mathrm{OH}$ - oder $\mathrm{HO}_{2}-$ Nebenreaktionen sowie die Bedeutung der CRIEGEE-Intermediate für das Reaktionsverhalten der verschiedenen Verbindungstypen ist hier von zentralem Interesse.

Um den beobachteten Effekt der Radikalfänger Cyclohexan und 1- bzw. 2Butanol näher zu untersuchen, wäre die Verwendung alternativer Edukte bezüglich der reaktiven Struktur und dem Oxidationsgrad der Eduktverbindungen von Interesse. Vor allem die Homologen der untersuchten Verbindungen 1-Hexen und 2-Methyl-1-penten würden sich anbieten.

Im Zusammenhang mit der starken Zunahme der Aerosolausbeute bei der 2-Methyl-1-pentenozonolyse durch Cyclohexanzugabe, von der bisher in anderen Studien noch nicht berichtet wurde, wäre interessant zu untersuchen, ob sich der Effekt aus der Grundstruktur des Eduktes mit der Methylsubstitution an der Doppelbindung oder dem Eingriff in die Radikalchemie ergibt. Zur Überprüfung der Bedeutung der radikalinduzierten Folgereaktionen schlagen HENRY et al. [19] die Verwendung von Wasserstoffperoxid als OH-Fänger vor. Durch die OH-Abfangreaktion werden hier als radikalische Produkte lediglich $\mathrm{HO}_{2}$-Radikale gebildet [84], die jedoch in ihrer Relation zu Peroxyradikalen den Eigenschaften den atmosphärischen Verhältnissen entsprechen sollen. Auch diese Veränderung des Radikalpools kann wiederum für unerwartete Auswirkungen auf die Aerosolbildung sorgen, allerdings ließen sich hier die $\mathrm{HO}_{2}$ - oder $\mathrm{RO}_{2}$-Radikale als Verursacher identifizieren.

Durch den Einsatz von Butanolen als $\mathrm{OH}$-Fänger werden potenziell auch CRIEGEE-Intermediate abgefangen, wie in verschiedenen Studien nachgewie- 
sen werden konnte [18, 59,71]. Daher wären Untersuchungen, die die Bedeutung der $\mathrm{Cl}$ für den Aufbau partikulärer Materie in den Mittelpunkt stellen, von Interesse. Vor allem für die reinen Kohlenwasserstoffe, die mit der größten Bandbreite sich unterschiedlich auswirkenden $\mathrm{OH}$-Fängern untersucht wurden, und die ungesättigten Alkohole, deren hohe Ausbeuten potenziell auf die Reaktionen des Eduktmoleküls mit dem $\mathrm{Cl}$ zurückzuführen sind, wären dies vielversprechende Untersuchungen.

Denkbar wären in diesem Zusammenhang Experimente, in denen gezielt selektiv wirkende $\mathrm{Cl}$-Fänger, wie Ameisensäure, eingesetzt werden. Da nach den Veröffentlichungen von NEEB et al. [57] aus der Reaktion

$$
\mathrm{CH}_{2} \mathrm{OO}+\mathrm{HCOOH} \longrightarrow \mathrm{CHO}-\mathrm{O}-\mathrm{CH}_{2}-\mathrm{OOH} \longrightarrow \mathrm{H}_{2} \mathrm{O}+\mathrm{HC}(\mathrm{O}) \mathrm{O}(\mathrm{O}) \mathrm{CH}
$$

mit Wasser und Ameisensäureanhydrid potenziell stabile und nicht in den Partikelbildungsmechanismus oder die $\mathrm{OH} / \mathrm{HO}_{2}$-Chemie eingreifende Strukturen entstehen, könnte eine Zuordnung des unter Butanolzugabe beobachteten Effektes als Folge der $\mathrm{OH}$ - oder $\mathrm{Cl}$-Chemie erfolgen.

Auch Experimente unter Ozonüberschuss, wie sie exemplarisch für Butensäure durchgeführt wurden ( $\mathrm{vgl}$. Abschnitt 6.4.2.6) könnten bei geringeren resultierenden Ausbeuten Hinweise auf die Bedeutung der Reaktion Cl + Eduktmolekül für die Partikelbildung liefern.

Die in Abschnitt 7.2.2.2 diskutierte Bedeutung der stabilisierten $\mathrm{Cl}$ und ihrer relativen Anteile in der 1-Hexen- bzw. 2-Methyl-1-pentenozonolyse könnten durch Experimente unter Hexafluoracetonzugabe, wie sie von DROzD et al. [44] durchgeführt wurden, geklärt werden. Diese Verbindung fängt die stabilisierten $\mathrm{Cl}$ ab und könnte Informationen über die relativen Ausbeuten liefern. Denkbar wäre als Modellsystem die Ozonolysereaktion von 4,5-Dimethyl-4-octen, das durch seine Symmetrie lediglich CRIEGEE-Intermediate vom gleichen Typ wie die großen $\mathrm{Cl}$ in der 2-Methyl-1-pentenozonolyse bildet. Gleiches wurde bereits für trans-5-Decen unter Bildung analoger großer $\mathrm{Cl}$ zur 1-Hexenozonolyse durchgeführt [44] und könnte die relative Stabilität der Cl aufklären.

Interessant wäre eine Untersuchung, ob der nukleationshemmende Effekt der Sauerstoffanwesenheit allein auf die Carboxylgruppe oder auf eine Kombination der funktionellen Gruppe und der relativ kurzen Kohlenstoffkette zurückzuführen ist. Dies ließe sich anhand der Untersuchung längerkettiger Carbon- 
säuren, die eventell die destabilisierende Wirkung der Säuregruppe durch eine höhere Zahl von Schwingungsmoden kompensieren könnten, herausfinden, wobei allerdings die reduzierte Flüchtigkeit der Edukte für den Einfüllvorgang für das Erreichen ausreichender Konzentrationen oder durch Auskondensation in der Zelle zum Problem werden könnte.

Einer genaueren Untersuchung bedarf auch der Zusammenhang zwischen $\mathrm{OH}$ - und Aerosolausbeute, der von anderen Arbeitsgruppen bereits nachgewiesen werden konnte [21, 37]. Hier ließen sich Rückschlüsse auf die gebildeten Verhältnisse zwischen syn- und anti-Konformation der CRIEGEE-Intermediate ziehen. Nachzuprüfen wäre weiterhin, ob die erhöhte CO-Ausbeute bei höheren Aerosolausbeuten im Fall der voroxidierten Edukte auf einen erhöhten anti-Anteil hinweisen, der sich die Nukleation vorteilhaft auswirkt.

Auch aus den Untersuchungen der Ozonolysereaktion unter Schwefeldioxidzugabe ergeben sich potenziell weiterführende Experimente. Vor allem die Bedeutung der CRIEGEE-Intermediate in bimolekularen Reaktionen mit Schwefeldioxid oder Schwefelsäure sind hier von Interesse, da sie die Aufteilung von Gas- zu Partikelphase stark beeinflussen können.

Dazu sind Ergänzungen zu den Untersuchungen der Gasphasenprodukte, wie sie für Cyclohexen und $\alpha$-Pinen unter verschiedenen Gesamtdrücken durchgeführt wurden, denkbar. Die Ergänzung um Experimente für die Modellsubstanzen Methylencyclohexan und Methylcyclohexen in Hoch- und Niederdruckregime sowie $\beta$-Pinen im Niederdruckbereich könnte Aufschluss darüber geben, ob der berichtete Effekt der unterschiedlichen $\mathrm{SO}_{2}$-Umsätze (z.B. Kap. 6.2.4.2) zwischen endo- und exozyklischen Strukturen ein für die vorliegenden reaktiven Strukturen oder nur für die spezifischen Edukte Gültigkeit besitzt.

Die Bedeutung der CRIEGEE-Intermediate und Kreuzreaktionen mit $\mathrm{SO}_{2}$ und seinen Folgeprodukten könnten Modellexperimente mit den in dieser Arbeit verwendeten linearen oder einfacher zu interpretierenden symmetrischen Alkenen, die nur eine Art von $\mathrm{Cl}$ bilden, näher untersucht werden. Auch die Verwendung von Cyclopenten im Vergleich zu den Ergebnissen von Cyclohexen und Methylcyclopenten könnte von Interesse sein.

Auch wäre die Weiterführung von Simulationen zu erwarteten Partikelausbeuten, wie sie für Cyclohexen in guter Übereinstimmung durchgeführt werden konnte [139], für die biogenen Verbindungen von Interesse, da dieser sehr 
einfache Ansatz für die Partikelkeimbildung [12] durch seine zufriedenstellende Wiedergabe der Messergebnisse vielversprechend erscheint. Von theoretischer Seite kann zudem die Betrachtung der Schwefelsäurechemie und der Clusterbildung detaillierter erfolgen. Zentral ist hier etwa für das einen sehr starken Effekt zeigende Cyclohexen, inwieweit die Reaktion des stabilisierten CRIEGEE-Intermediates mit $\mathrm{SO}_{2}$ bzw. $\mathrm{H}_{2} \mathrm{SO}_{4}$ zu stabilen Produkten führt. Vor allem der Einfluss des Gesamtdruckes ist von Interesse.

Dies könnte auch einen Hinweis darauf liefern, ob sich die Reduktion der schwefelsäureassistierten Aerosolbildung nur auf die unzureichende Stabilisierbarkeit der $\mathrm{Cl}$ oder auch der Schwefelsäure und mit ihr gebildete Cluster zurückführen lässt. Da die $\mathrm{H}_{2} \mathrm{SO}_{4}+\mathrm{H}_{2} \mathrm{O}$-Reaktion makroskopisch sehr stark exotherm ist, könnte sich ein ähnliches Verhalten auch auf molekularer Ebene bemerkbar machen.

$\mathrm{Da}$ in zunehmender Höhe der atmosphärische Druck abnimmt, ist die Betrachtung der Stabilität sich bildender Cluster auch im Zusammenhang mit diskutierten Geoengineering-Prozessen [153] von zentralem Interesse.

Grundsätzlich ist der Ansatz der Untersuchung von kleinen Modellsubstanzen im Vergleich zu biogenen Verbindungen unter Betrachtung verschiedener Schlüsselstellen in Partikelbildungsmechanismus für seine weitere Aufklärung vielversprechend. Auch kann auf diese Art eine Einschätzung des Gesamtprozesses und ein erhöhtes Verständnis für atmosphärische Prozesse erlangt werden.

Durch die Variation der Reaktionsbedingungen, vor allem des Gesamtdruckes, der Zugabe selektiv in den Prozess eingreifender Additiva und der reaktiven Struktur der Edukte, ist die Indentifikation dieser Schlüsselstellen möglich. Dabei ist vor allem die Kontrollierbarkeit der Bedingungen als eine große Stärke des verwendeten Aufbaus anzusehen. Durch begleitende Simulationen können einzelne Aspekte der Nukleation näher betrachtet und eventuell aufgeklärt werden. Allerdings sollten diese immer im Zusammenhang mit dem Experiment interpretiert werden, da die Ergebnisse immer ein Stück weit von der Apparatur bestimmt sind. Dies zeigen die teils unerwarteten Ergebnisse, die sich nicht unmittelbar aus der Analogie mit anderen Untersuchungen erklären lassen. 


\section{A. Anhang}

\section{A.1. Verwendete Substanzen}

\begin{tabular}{|c|c|c|c|c|}
\hline Substanz & Summenformel & Abkürzung & Reinheit & Lieferant \\
\hline Methacrylsäure & $\mathrm{C}_{4} \mathrm{H}_{6} \mathrm{O}_{2}$ & MACS & $>98.0 \%$ & Fluka \\
\hline Butensäure & $\mathrm{C}_{4} \mathrm{H}_{6} \mathrm{O}_{2}$ & BENS & $97 \%$ & Fluka \\
\hline 1-Penten-3-ol & $\mathrm{C}_{5} \mathrm{H}_{10} \mathrm{O}$ & 1P3L & $98 \%$ & SAFC \\
\hline 4-Penten-1-ol & $\mathrm{C}_{5} \mathrm{H}_{10} \mathrm{O}$ & 4P1L & $\geq 99 \%$ & Fluka \\
\hline 3-Buten-1-ol & $\mathrm{C}_{4} \mathrm{H}_{8} \mathrm{O}$ & 3B1L & $96 \%$ & Fluka \\
\hline 1-Penten-3-on & $\mathrm{C}_{5} \mathrm{H}_{8} \mathrm{O}$ & $1 \mathrm{P} 3 \mathrm{~N}$ & $\geq 97 \%$ & Fluka \\
\hline 4-Penten-1-al & $\mathrm{C}_{5} \mathrm{H}_{8} \mathrm{O}$ & 4PAL & $\geq 97 \%$ & Fluka \\
\hline 1-Hexen & $\mathrm{C}_{6} \mathrm{H}_{12}$ & 1HEX & $\begin{array}{c}\text { zur } \\
\text { Synthese }\end{array}$ & VWR \\
\hline 2-Methyl-1-penten & $\mathrm{C}_{6} \mathrm{H}_{12}$ & 2M1P & $\geq 99 \%$ & Fluka \\
\hline 1-Methyl-cyclopenten & $\mathrm{C}_{6} \mathrm{H}_{10}$ & MCPE & $\geq 96 \%$ & Fluka \\
\hline Cyclohexen & $\mathrm{C}_{6} \mathrm{H}_{10}$ & cHEX & $>98.0 \%$ & Fluka \\
\hline 1-Methyl-1-cyclohexen & $\mathrm{C}_{7} \mathrm{H}_{12}$ & $\mathrm{MCHe}$ & $\geq 95 \%$ & Fluka \\
\hline Methylencyclohexan & $\mathrm{C}_{7} \mathrm{H}_{12}$ & $\mathrm{MCHa}$ & $\geq 98 \%$ & Fluka \\
\hline$\alpha$-Pinen & $\mathrm{C}_{10} \mathrm{H}_{16}$ & - & $\geq 99 \%$ & Aldrich \\
\hline$\beta$-Pinen & $\mathrm{C}_{10} \mathrm{H}_{16}$ & - & $\geq 99 \%$ & Aldrich \\
\hline Cyclohexan & $\mathrm{C}_{6} \mathrm{H}_{12}$ & $\mathrm{CHA}$ & $99.5 \%$ & Merck \\
\hline$n$-Butanol & $\mathrm{C}_{4} \mathrm{H}_{10} \mathrm{O}$ & $1 \mathrm{BuOH}$ & $\geq 99.5 \%$ & Aldrich \\
\hline 2-Butanol & $\mathrm{C}_{4} \mathrm{H}_{10} \mathrm{O}$ & $2 \mathrm{BuOH}$ & $\geq 99 \%$ & Aldrich \\
\hline Cyclohexanon & $\mathrm{C}_{6} \mathrm{H}_{10} \mathrm{O}$ & $\mathrm{CHON}$ & $\geq 99.5 \%$ & Fluka \\
\hline Schwefeldioxid & $\mathrm{SO}_{2}$ & $\mathrm{SO}_{2}$ & $\geq 99.98 \%$ & Messer \\
\hline Nopinon & $\mathrm{C}_{9} \mathrm{H}_{14} \mathrm{O}$ & NPN & $\geq 98 \%$ & Aldrich \\
\hline Propionaldehyd & $\mathrm{C}_{3} \mathrm{H}_{6} \mathrm{O}$ & PNA & $\geq 98 \%$ & Fluka \\
\hline
\end{tabular}


A. Anhang

\begin{tabular}{|c|c|c|c|c|}
\hline Substanz & Summenformel & Abkürzung & Reinheit & Lieferant \\
\hline \hline Helium & $\mathrm{He}$ & $\mathrm{He}$ & $\geq 99.996 \%$ & Air Liquide \\
\hline Sauerstoff & $\mathrm{O}_{2}$ & $\mathrm{O}_{2}$ & $\geq 99.995 \%$ & Air Liquide \\
\hline Stickstoff & $\mathrm{N}_{2}$ & $\mathrm{~N}_{2}$ & $\geq 99.9990 \%$ & Air Liquide \\
\hline $\begin{array}{c}\text { Synthetische Luft } \\
\left(20.5-\text { Vol\% } \mathrm{O}_{2} / \mathrm{N}_{2}\right. \\
(\text { Rest) })\end{array}$ & $\mathrm{O}_{2} / \mathrm{N}_{2}$ & $\mathrm{SL}$ & $\geq 99.9990 \%$ & Air Liquide \\
\hline
\end{tabular}

Tab. A.1.: Daten zu den verwendeten Substanzen.

\section{A.2. Experimentelle Bedingungen}

\section{A.2.1. Kinetikmessungen}

\begin{tabular}{|c|c|c|c|}
\hline Substanz & $\boldsymbol{p}_{\mathbf{O}_{3}}[\mathrm{~Pa}]$ & $\boldsymbol{p}_{\text {Alken }}[\mathrm{Pa}]$ & Badgas \\
\hline \hline Butensäure & $0.43-0.46$ & $3.02-5.10$ & $\mathrm{~N}_{2}, \mathrm{SL}$ \\
\hline Methacryl- säure & $0.41-0.97$ & $2.29-5.50$ & $\mathrm{~N}_{2}, \mathrm{SL}$ \\
\hline 4-Penten-1-ol & $0.14-0.58$ & $0.3-1.45$ & $\mathrm{SL}$ \\
\hline 1-Penten-3-ol & $0.18-0.95$ & $0.66-2.97$ & $\mathrm{~N}_{2}, \mathrm{SL}$ \\
\hline 3-Buten-1-ol & $0.19-0.50$ & $1.11-1.96$ & $\mathrm{SL}$ \\
\hline 4-Pentenal & $0.42-0.46$ & $1.55-4.95$ & $\mathrm{SL}$ \\
\hline 1-Penten-3-on & $0.18-0.28$ & $0.62-0.26$ & $\mathrm{SL}$ \\
\hline 2-Methyl-1-penten & $0.44-1.81$ & $0.99-2.20$ & $\mathrm{SL}$ \\
\hline $\begin{array}{c}\text { Methylcyclo- } \\
\text { penten }\end{array}$ & $0.25-0.43$ & $0.54-1.10$ & $\mathrm{SL}$ \\
\hline 1-Hexen & $0.43-1.86$ & $0.98-2.30$ & $\mathrm{SL}$ \\
\hline
\end{tabular}

Tab. A.2.: Zusammenfassung der experimentellen Bedingungen für Kinetikmessungen. Alle Messungen wurden bei $p_{\text {ges }}=460 \pm 10$ mbar und $T=295 \pm 2 \mathrm{~K}$ durchgeführt. 


\section{A.2.2. Produktbildung}

\begin{tabular}{|c|c|c|c|c|}
\hline Substanz & $p_{\mathrm{O}_{3}}[\mathrm{~Pa}]$ & $p_{\text {Alken }}[\mathrm{Pa}]$ & $\boldsymbol{p}_{\text {Additiv }}[\mathrm{Pa}]$ & $\begin{array}{c}\boldsymbol{p}_{\text {ges }} \\
{[\mathrm{mbar}]}\end{array}$ \\
\hline \hline Butensäure & $1.0 \pm 0.1$ & $5.0 \pm 0.1$ & - & $\begin{array}{c}30 / \\
1000\end{array}$ \\
\hline $\begin{array}{c}\text { Methacryl- } \\
\text { säure }\end{array}$ & $1.0 \pm 0.1$ & $5.0 \pm 0.1$ & - & $\begin{array}{c}30 / \\
1000\end{array}$ \\
\hline 4-Penten-1-ol & $0.99 \pm 0.01$ & $3.01 \pm 0.01$ & - & $\begin{array}{c}20 / 50 / \\
500\end{array}$ \\
\hline 1-Penten-3-ol & $0.98 \pm 0.08$ & $4.2 \pm 0.2$ & $63(\mathrm{CHA})$ & $\begin{array}{c}30 / \\
1000\end{array}$ \\
\hline 3-Buten-1-ol & $1.0 \pm 0.1$ & $5.0 \pm 0.3$ & $75(\mathrm{CHA})$ & $\begin{array}{c}30 / \\
1000\end{array}$ \\
\hline 4-Pentenal & $0.93 \pm 0.02$ & $4.2 \pm 0.2$ & $63(\mathrm{CHA})$ & $\begin{array}{c}30 / \\
1000\end{array}$ \\
\hline 1-Penten-3-on & $1.00 \pm 0.08$ & $4.2 \pm 0.2$ & $63(\mathrm{CHA})$ & $\begin{array}{c}30 / \\
1000\end{array}$ \\
\hline $\begin{array}{c}\text { 2-Methyl-1- } \\
\text { penten }\end{array}$ & $0.96 \pm 0.08$ & $5.0 \pm 0.1$ & - & $\begin{array}{c}30 / \\
1000\end{array}$ \\
\hline $\begin{array}{c}\text { Methylcyclo- } \\
\text { penten }\end{array}$ & $0.97 \pm 0.09$ & $3.0 \pm 0.1$ & - & $\begin{array}{c}50 / \\
1000\end{array}$ \\
\hline 1-Hexen & $0.99 \pm 0.04$ & $5.0 \pm 0.1$ & - & $\begin{array}{c}30 / \\
1000\end{array}$ \\
\hline Cyclohexen & $0.91 \pm 0.01$ & $2.11 \pm 0.02$ & $0.300\left(\mathrm{SO}_{2}\right)$ & $\begin{array}{c}30 / \\
1000 \\
(\mathrm{nur} \mathrm{SL})\end{array}$ \\
\hline$\alpha$ - Pinen & $0.86 \pm 0.01$ & $3.02 \pm 0.02$ & $0.3(\mathrm{NPN}, \mathrm{PNA})$ & $\begin{array}{c}30 / \\
1000 \\
(\mathrm{nur} \mathrm{SL})\end{array}$ \\
\hline$\beta$ - Pinen & 0.86 & 3.02 & $0.300\left(\mathrm{SO}_{2}\right)$ & $\begin{array}{c}30 / \\
1000 \\
(\mathrm{nur} \mathrm{SL})\end{array}$ \\
\hline & & & & \\
\hline
\end{tabular}

Tab. A.3.: Zusammenfassung der experimentellen Bedingungen für Produktbildungsmessungen bei $T=295 \pm 2 \mathrm{~K}$. Sofern nicht anders gekennzeichnet, wurden die Messungen sowohl in Stickstoff als auch in Synthetischer Luft durchgeführt. 


\section{A.2.3. Partikelbildungsexperimente}

\begin{tabular}{|c|c|c|c|c|}
\hline Substanz & $p_{\mathrm{O}_{3}}[\mathrm{~Pa}]$ & $p_{\text {Alken }}[\mathrm{Pa}]$ & $p_{\text {Additiv }}[\mathrm{Pa}]$ & Badgas \\
\hline Butensäure* & $0.1 \pm 0.01$ & $5.0 \pm 0.1$ & $25(\mathrm{CHa})$ & $\begin{array}{l}\mathrm{N}_{2}, \mathrm{SL}, \\
\mathrm{N}_{2} / \mathrm{O}_{2}\end{array}$ \\
\hline $\begin{array}{l}\text { Methacryl- } \\
\text { säure* }^{*}\end{array}$ & $0.25 \pm 0.01$ & $0 \pm 0.1$ & $25(\mathrm{CHA})$ & $\begin{array}{l}\mathrm{N}_{2}, \mathrm{SL}, \\
\mathrm{N}_{2} / \mathrm{O}_{2}\end{array}$ \\
\hline 4-Penten-1-ol* & $0.20 \pm 0.01$ & $3.0 \pm 0.1$ & - & $\begin{array}{l}\mathrm{N}_{2}, \mathrm{SL}, \\
\mathrm{N}_{2} / \mathrm{O}_{2}\end{array}$ \\
\hline 1-Penten-3-ol & $0.10 \pm 0.01$ & $3.0 \pm 0.1$ & $63(\mathrm{CHA})$ & $\mathrm{N}_{2}, \mathrm{SL}$ \\
\hline 3-Buten-1-ol & & & 75 (CHA) & $\mathrm{N}_{2}, \mathrm{SL}$ \\
\hline 4-Pentenal & & & 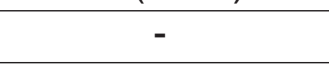 & $\mathrm{N}_{2}, \mathrm{SL}$ \\
\hline $\begin{array}{c}\text { 1-Penten-3- } \\
\text { on }^{* / *}\end{array}$ & $0.35 \pm 0.03$ & $3.0 \pm 0.1$ & - & $\begin{array}{l}\mathrm{N}_{2}, \mathrm{SL}, \\
\mathrm{N}_{2} / \mathrm{O}_{2}\end{array}$ \\
\hline $\begin{array}{l}\text { 2-Methyl-1- } \\
\text { penten }\end{array}$ & $0.140 \pm 0.015$ & $3.0 \pm 0.1$ & $\begin{array}{c}130(\mathrm{CHa}, \\
\text { 1-/2BuOH); } 310 \\
(\mathrm{CHa})\end{array}$ & $\mathrm{N}_{2}, \mathrm{SL}$ \\
\hline $\begin{array}{l}\text { Methylcyclo- } \\
\text { penten }\end{array}$ & $0.140 \pm 0.018$ & $0.50 \pm 0.01$ & $\begin{array}{c}20 / 310(\mathrm{CHa}) \\
500(2 \mathrm{BuOH}) ; \\
0.01 / 0.1 \mathrm{~Pa} \mathrm{SO}\end{array}$ & $\mathrm{N}_{2}, \mathrm{SL}$ \\
\hline 1-Hexen & $0.142 \pm 0.017$ & $3.0 \pm 0.1$ & $\begin{array}{c}78 / 310(\mathrm{CHa}) \\
78(1 \mathrm{BuOH})\end{array}$ & $\mathrm{N}_{2}, \mathrm{SL}$ \\
\hline Cyclohexen & $0.085 \pm 0.015$ & $1.28 \pm 0.05$ & $\begin{array}{c}0-0.313\left(\mathrm{SO}_{2}\right) \\
0-700\left(\mathrm{H}_{2} \mathrm{O}\right)\end{array}$ & SL \\
\hline$\alpha$ - Pinen & 0 & 0.05 & $\begin{array}{c}0-0.100\left(\mathrm{SO}_{2}\right) \\
0-700\left(\mathrm{H}_{2} \mathrm{O}\right)\end{array}$ & SL \\
\hline$\beta$ - Pinen & $0.040 \pm 0.010$ & $3.00 \pm 0.05$ & $0-0.100\left(\mathrm{SO}_{2}\right)$ & SL \\
\hline $\begin{array}{l}\text { Methylen - } \\
\text { cyclohexan }\end{array}$ & $0.085 \pm 0.015$ & $3.00 \pm 0.05$ & $0-0.100\left(\mathrm{SO}_{2}\right)$ & SL \\
\hline $\begin{array}{c}\text { Methylcyclo- } \\
\text { hexen }\end{array}$ & $0.085 \pm 0.015$ & $1.20 \pm 0.05$ & $0-0.100\left(\mathrm{SO}_{2}\right)$ & SL \\
\hline
\end{tabular}

Tab. A.4.: Experimentelle Bedingungen für Partikelbildungsmessungen bei $T=295 \pm 2 \mathrm{~K} ., \mathrm{N}_{2} / \mathrm{O}_{2}$ " zeigt dabei an, mit welchen Substanzen Sauerstoffpartialdruckreihen durchgeführt wurden.

$\mathrm{O}_{2}$-Reihe bei einem Gesamtdruck von ${ }^{*} 500 \mathrm{mbar} / \mathrm{bei}{ }^{* *} 100 \mathrm{mbar}$. 


\section{A.3. Geschwindigkeitskoeffizienten für die Ozonolysereaktion}

\begin{tabular}{|c|c|c|c|c|c|}
\hline Alken & $T[\mathrm{~K}]$ & $p_{\text {ges }}[\mathrm{mbar}]$ & $\begin{array}{c}\text { OH- } \\
\text { Fänger }\end{array}$ & $k\left[10^{-18} \frac{\mathrm{cm}^{3}}{\text { molecule.s }}\right]$ & Referenz \\
\hline \multirow[t]{4}{*}{ MACS } & $296 \pm 2$ & $973 \pm 7$ & $\begin{array}{l}\mathrm{C}_{6} \mathrm{H}_{12}, \\
\text { (HFO) }\end{array}$ & $4.1 \pm 0.4$ & NEEB [59] \\
\hline & $298 \pm 3$ & 1013 & $\begin{array}{l}\mathrm{C}_{6} \mathrm{H}_{12} \\
(\mathrm{HFO})\end{array}$ & $2.25 \pm 0.74$ & AL MULLA [119] \\
\hline & $295 \pm 2$ & $460 \pm 10$ & - & 3.74 & Exp.best. \\
\hline & $295 \pm 2$ & $460 \pm 10$ & - & $3.99, \mathbf{N}_{2}$ & Exp.best. \\
\hline & & & & & \\
\hline $\begin{array}{l}\text { Methyl- } \\
\text { propen }\end{array}$ & 298 & 1013 & - & $11.1 \pm 1.2$ & WEGENER [120] \\
\hline \multirow[t]{4}{*}{ BENS } & $295 \pm 2$ & $460 \pm 10$ & - & $3.58 \pm 0.13$ & Exp.best.,(UV1) \\
\hline & $295 \pm 2$ & $460 \pm 10$ & - & $4.19 \pm 0.13$ & Exp.best.,(UV2) \\
\hline & $295 \pm 2$ & $460 \pm 10$ & - & $4.49 \pm 0.04, \mathbf{N}_{2}$ & Exp.best.,(UV1) \\
\hline & $295 \pm 2$ & $460 \pm 10$ & - & $6.24 \pm 0.31, \mathbf{N}_{2}$ & Exp.best.,(UV2) \\
\hline 1-Buten & 298 & 1013 & - & $9.64 \pm 0.87$ & WEGENER [120] \\
\hline \multirow[t]{4}{*}{ 1P3L } & 295 & 1000 & - & 17.9 & MCGILLEN [80] \\
\hline & $293 \pm 2$ & 1000 & $\mathrm{CO}$ & $16.4 \pm 1.5$ & O'DWYER [61] \\
\hline & $290 \pm 2$ & 1000 & $\mathrm{C}_{6} \mathrm{H}_{12}$ & $17.9 \pm 1.8$ & GROSJEAN [68] \\
\hline & $295 \pm 2$ & $460 \pm 10$ & - & $21.5 \pm 3.2$ & Exp.best. \\
\hline 4P1L & $295 \pm 2$ & $460 \pm 10$ & - & $11.8 \pm 4.5$ & Exp.best. \\
\hline \multirow[t]{3}{*}{ 3B1L } & 295 & 1000 & - & 4.89 & MCGILLEN [80] \\
\hline & $295 \pm 4$ & 1000 & $\mathrm{C}_{6} \mathrm{H}_{12}$ & $4.9 \pm 0.4$ & GROSJEAN [69] \\
\hline & $295 \pm 2$ & $460 \pm 10$ & - & $8.64 \pm 1.02$ & Exp.best. \\
\hline \multicolumn{6}{|c|}{ Fortsetzung auf nächster Seite } \\
\hline
\end{tabular}


A. Anhang

\begin{tabular}{|c|c|c|c|c|c|}
\hline Alken & $T[\mathrm{~K}]$ & $p_{\mathrm{ges}}[\mathrm{mbar}]$ & $\begin{array}{c}\text { OH- } \\
\text { Fänger }\end{array}$ & $k\left[10^{-18} \frac{\mathrm{cm}^{3}}{\text { molecule } \cdot \mathrm{s}}\right]$ & Referenz \\
\hline 1P3N & $\begin{array}{c}295 \\
285 \pm 1 \\
293 \pm 2 \\
295 \pm 2\end{array}$ & $\begin{array}{c}1000 \\
- \\
1000 \\
460 \pm 10\end{array}$ & $\begin{array}{c}- \\
\mathrm{C}_{6} \mathrm{H}_{12} \\
- \\
-\end{array}$ & $\begin{array}{c}6.03 \\
6.04 \pm 0.40 \\
11.7 \pm 1.5 \\
6.97 \pm 0.46\end{array}$ & $\begin{array}{l}\text { MCGILLEN [80] } \\
\text { GROSJEAN [69] } \\
\text { O’DWYER [61] } \\
\text { Exp.best. }\end{array}$ \\
\hline 4PAL & $295 \pm 2$ & $460 \pm 10$ & - & $7.84 \pm 0.22$ & Exp.best. \\
\hline 1HEX & $\begin{array}{c}295 \\
287 \pm 1 \\
295 \pm 2 \\
295 \pm 2 \\
299 \pm 2 \\
295 \pm 2\end{array}$ & $\begin{array}{c}1000 \\
1000 \\
1000 \\
1000 \\
1000 \\
460 \pm 10\end{array}$ & $\begin{array}{c}- \\
\mathrm{C}_{6} \mathrm{H}_{12} \\
- \\
- \\
- \\
- \\
\end{array}$ & $\begin{array}{c}10.1 \\
9.4 \pm 0.4 \\
8.2 \\
13.6, \mathrm{~N}_{2} \\
11.1 \pm 0.3, \mathrm{~N}_{2} \\
11.6 \pm 1.5\end{array}$ & $\begin{array}{c}\text { McGILLEN [80] } \\
\text { GROSJEAN } \\
{[117]} \\
\text { Cox [159] } \\
\text { Cox [159] } \\
\text { JAPAR [121] } \\
\text { Exp.best. }\end{array}$ \\
\hline 2M1P & $\begin{array}{c}295 \\
289 \pm 4 \\
287 \pm 1 \\
295 \pm 2 \\
295 \pm 2\end{array}$ & $\begin{array}{c}1000 \\
1000 \\
1000 \\
1000 \\
460 \pm 10\end{array}$ & $\begin{array}{c}- \\
\mathrm{C}_{6} \mathrm{H}_{12} \\
\mathrm{C}_{6} \mathrm{H}_{12} \\
- \\
-\end{array}$ & $\begin{array}{c}13.1 \\
13.1 \pm 1.8 \\
12.5 \pm 1.1 \\
16.9 \\
12.0 \pm 0.6\end{array}$ & $\begin{array}{c}\text { MCGILLEN [80] } \\
\text { GROSJEAN [69] } \\
\text { GROSJEAN } \\
\text { [117] } \\
\text { Cox [159] } \\
\text { Exp.best. }\end{array}$ \\
\hline MCPE & $\begin{array}{l}296 \pm 2 \\
298 \pm 2 \\
295 \pm 2\end{array}$ & $\begin{array}{c}986 \\
1000 \\
460 \pm 10\end{array}$ & $\begin{array}{c}\mathrm{C}_{6} \mathrm{H}_{12} \\
\text { 2-Butanol } \\
- \\
-\end{array}$ & $\begin{array}{l}832 \pm 24 \\
670 \pm 100 \\
510 \pm 50\end{array}$ & $\begin{array}{l}\text { CUSICK [81] } \\
\text { TREACY [72] } \\
\text { Exp.best. }\end{array}$ \\
\hline cHEX & $\begin{array}{l}287 \pm 1 \\
298 \pm 2 \\
297 \pm 2 \\
295 \pm 1 \\
295 \pm 1 \\
299 \pm 2\end{array}$ & $\begin{array}{l}1000 \\
1000 \\
1000 \\
1000 \\
1000 \\
1000\end{array}$ & $\begin{array}{c}\mathrm{C}_{6} \mathrm{H}_{12} \\
- \\
- \\
- \\
- \\
-\end{array}$ & $\begin{array}{c}84.6 \pm 1.0 \\
85 \pm 8 \\
78 \pm 5 \\
151 \pm 10, \mathrm{~N}_{2} \\
151 \pm 10, \mathrm{~N}_{2} \\
169 \pm 15, \mathrm{~N}_{2}\end{array}$ & $\begin{array}{c}\text { GROSJEAN } \\
\text { [117] } \\
\text { TREACY [72] } \\
\text { NOLTING [160] } \\
\text { BENNETT [161] } \\
\text { BENNETT [161] } \\
\text { JAPAR [121] }\end{array}$ \\
\hline
\end{tabular}


A.3. Geschwindigkeitskoeffizienten für die Ozonolysereaktion

\begin{tabular}{|c|c|c|c|c|c|}
\hline Alken & $T[\mathrm{~K}]$ & $p_{\text {ges }}$ [mbar] & $\begin{array}{c}\text { OH- } \\
\text { Fänger }\end{array}$ & $k\left[10^{-18} \frac{\mathrm{cm}^{3}}{\text { molecule } \cdot \mathrm{s}}\right]$ & Referenz \\
\hline cHEX & $295 \pm 2$ & 500 & $\mathrm{C}_{6} \mathrm{H}_{12}$ & $73.9 \pm 2.4$ & WOLF [17] \\
\hline $\mathrm{MCHe}$ & $\begin{array}{l}296 \pm 2 \\
296 \pm 2 \\
295 \pm 2 \\
298 \pm 2 \\
298 \pm 1\end{array}$ & $\begin{array}{c}973 \\
986 \\
973 \\
1000 \\
500\end{array}$ & $\begin{array}{c}\mathrm{C}_{6} \mathrm{H}_{12} \\
\mathrm{C}_{6} \mathrm{H}_{12}, \\
\text { 2-Butanol } \\
\mathrm{C}_{6} \mathrm{H}_{12} \\
- \\
-\end{array}$ & $\begin{array}{c}74.6 \pm 0.5, \quad \mathbf{N}_{2} \\
146 \pm 10 \\
170 \\
166 \pm 12 \\
181 \pm 13\end{array}$ & $\begin{array}{c}\text { GREENE [150] } \\
\text { Cusick [81] } \\
\text { KOCH [9] } \\
\text { TREACY [72] } \\
\text { WOLF [48] }\end{array}$ \\
\hline $\mathrm{MCHa}$ & $\begin{array}{l}295 \pm 2 \\
293 \pm 2 \\
298 \pm 1\end{array}$ & $\begin{array}{c}973 \\
1000 \\
500 / 1000\end{array}$ & $\begin{array}{c}\mathrm{C}_{6} \mathrm{H}_{12} \\
\mathrm{C}_{6} \mathrm{H}_{12}, 2- \\
\text { Propanol } \\
-\end{array}$ & $\begin{array}{c}12 \\
10.6 \pm 1.9 \\
10 \pm 2\end{array}$ & $\begin{array}{c}\text { KOCH [9] } \\
\text { GROSJEAN } \\
{[151]} \\
\text { WOLF [48] }\end{array}$ \\
\hline$\alpha$-Pinen & $\begin{array}{l}297 \pm 2 \\
295 \pm 1 \\
295 \pm 2 \\
298 \pm 1\end{array}$ & $\begin{array}{c}1000 \\
1000 \\
973 \\
500\end{array}$ & $\begin{array}{c}- \\
- \\
\mathrm{C}_{6} \mathrm{H}_{12} \\
-\end{array}$ & $\begin{array}{c}86 \pm 13 \\
83 \pm 13 \\
87 \\
85 \pm 5\end{array}$ & $\begin{array}{c}\text { NOLTING [160] } \\
\text { ATKINSON [74] } \\
\text { Koch [9] } \\
\text { WolF [48] }\end{array}$ \\
\hline$\beta$-Pinen & $\begin{array}{l}297 \pm 2 \\
296 \pm 2 \\
295 \pm 2 \\
298 \pm 1\end{array}$ & $\begin{array}{l}1000 \\
1000 \\
973 \\
500\end{array}$ & $\begin{array}{c}- \\
- \\
\mathrm{C}_{6} \mathrm{H}_{12} \\
-\end{array}$ & $\begin{array}{c}14 \pm 2 \\
21 \pm 5 \\
15 \\
16 \pm 2\end{array}$ & $\begin{array}{c}\text { NOLTING [160] } \\
\text { AtKINSON [74] } \\
\text { Koch [9] } \\
\text { WolF [48] }\end{array}$ \\
\hline
\end{tabular}

Tab. A.5.: Geschwindigkeitskoeffizienten für die Ozonolysereaktion der verwendeten Substanzen mit Literaturangaben für den in den vorgestellten Experimenten entsprechendem Temperaturbereich. „Exp.best.“ kennzeichnet in der Aerosolzelle der eigenen Arbeitsgruppe bestimmte Werte. 


\section{A.3.1. Ergebnisse der einzelnen Kinetikmessungen}

\begin{tabular}{|c|c|c|c|c|}
\hline Kennung & $p_{0}$ (Alken) [Pa] & $p_{0}($ Ozon) $[\mathrm{Pa}]$ & rel. Verbrauch $\alpha$ & $k\left[10^{-18} \frac{\mathrm{cm}^{3}}{\text { molecule.s }}\right]$ \\
\hline 1HEXS020 & 2.10 & 0.442 & 1.46 & 9.74 \\
\hline 1HEXS021 & 0.93 & 0.433 & 1.42 & 11.78 \\
\hline 1HEXS022 & 1.40 & 0.461 & 1.43 & 11.31 \\
\hline 1HEXS023 & 2.30 & 0.892 & 1.37 & 12.52 \\
\hline 1HEXS024 & 0.98 & 1.860 & 1.18 & 12.90 \\
\hline 2M1PS024 & 2.00 & 0.456 & 1.71 & 11.49 \\
\hline 2M1PS025 & 0.99 & 0.671 & 1.63 & 12.06 \\
\hline 2M1PS026 & 1.70 & 0.442 & 1.82 & 11.68 \\
\hline 2M1PS027 & 2.20 & 0.887 & 1.74 & 12.29 \\
\hline 2M1PS028 & 0.99 & 1.810 & 1.39 & 12.44 \\
\hline MCPES028 & 0.54 & 0.258 & 2.26 & 463.12 \\
\hline MCPES030 & 1.10 & 0.425 & 2.30 & 553.12 \\
\hline 3B1LS016 & 1.96 & 0.435 & 1.49 & 8.64 \\
\hline 3B1LS017 & 1.67 & 0.245 & 1.33 & 9.05 \\
\hline 3B1LS018 & 1.11 & 0.185 & 1.36 & 8.23 \\
\hline 1P3LS010 & 1.88 & 0.380 & 1.64 & 21.0 \\
\hline 1P3LS011 & 0.70 & 0.945 & 1.02 & 20.6 \\
\hline 1P3LS013 & 2.88 & 0.467 & 1.32 & 23.0 \\
\hline 1P3LN013 & 0.71 & 0.289 & 0.76 & 29.2 \\
\hline 1P3LN014 & 2.97 & 0.495 & 1.04 & 28.8 \\
\hline 4P1LS007 & 0.95 & 0.188 & 2.16 & 12.35 \\
\hline 4P1LS008 & 1.50 & 0.195 & 1.98 & 9.14 \\
\hline 4P1LS009 & 0.50 & 0.144 & 2.06 & 22.63 \\
\hline 4P1LS010 & 0.30 & 0.583 & 1.52 & 8.64 \\
\hline 4P1LS011 & 1.20 & 0.292 & 1.07 & 11.93 \\
\hline 4P1LS016 & 1.30 & 0.294 & 1.60 & 8.64 \\
\hline 4P1LS017 & 0.50 & 0.473 & 0.93 & 9.05 \\
\hline
\end{tabular}

Fortsetzung auf nächster Seite. 
A.3. Geschwindigkeitskoeffizienten für die Ozonolysereaktion

\begin{tabular}{|c|c|c|c|c|}
\hline Kennung & $\boldsymbol{p}_{\mathbf{0}}$ (Alken) [Pa] & $\boldsymbol{p}_{\mathbf{0}}$ (Ozon) [Pa] & rel. Verbrauch $\boldsymbol{\alpha}$ & $\boldsymbol{k}\left[\mathbf{1 0}^{-18} \mathbf{c m}^{\mathbf{3}} \mathbf{\text { molecule.s }}\right]$ \\
\hline \hline 1P3NSKin1 & 0.62 & 0.195 & 3.24 & 5.06 \\
\hline 1P3NSKin2 & 1.09 & 0.201 & 1.34 & 6.28 \\
\hline 1P3NSKin3 & 2.63 & 0.263 & 1.64 & 7.18 \\
\hline 1P3NSKin4 & 1.44 & 0.277 & 1.21 & 6.91 \\
\hline \hline 4PALSKin1 & 4.13 & 0.484 & 1.57 & 7.98 \\
\hline 4PALSKin2 & 2.03 & 0.503 & 1.17 & 7.82 \\
\hline 4PALSKin3 & 1.55 & 0.507 & 1.09 & 7.53 \\
\hline 4PALSKin4 & 4.95 & 0.492 & 0.92 & 8.02 \\
\hline \hline MACSS026 & 5.10 & 0.421 & 2.36 & 3.74 \\
\hline MACSN028 & 5.70 & 0.411 & 2.70 & 3.99 \\
\hline \hline BENSS027 & 5.20 & 0.428 & 2.03 & 3.70 \\
\hline BENSS058 & 5.0 & 0.898 & 1.98 & 4.12 \\
\hline BENSS059 & 2.0 & 0.917 & 1.49 & 4.20 \\
\hline BENSS060 & 5.0 & 0.896 & 1.75 & 4.32 \\
\hline BENSS061 & 2.0 & 0.931 & 1.49 & 4.12 \\
\hline BENSN030 & 5.10 & 0.441 & 2.16 & 4.44 \\
\hline BENSN101 & 5.0 & 0.819 & 1.64 & 5.93 \\
\hline BENSN102 & 2.0 & 0.869 & 1.14 & 6.38 \\
\hline BENSN103 & 5.0 & 0.851 & 1.65 & 6.17 \\
\hline BENSN104 & 2.0 & 0.886 & 1.32 & 6.46 \\
\hline
\end{tabular}

Tab. A.6.: Angabe der Reaktionsbedingungen und Ergebnisse der in Kap. 6.1 präsentierten Kinetikmessungen. Zur Benennung der einzelnen Experimente: Nach einem vierstelligen Kürzel für die Substanz folgt „S“ oder „N“ für Synthetische Luft bzw. Stickstoff als Badgas. Die am Ende stehende laufende Nummer dient lediglich der Unterscheidung. Die Farben entsprechen denen der Auftragungen in der Auswertung, Abschnitt 6.1 



\section{B. Kohlenstoffmonoxidausbeuten der einzelnen Experimente}

\begin{tabular}{|c|c|c|c|c|}
\hline Alken & $\boldsymbol{t}_{\mathbf{R}}[\mathbf{s}]$ & $\boldsymbol{p}_{\text {ges }}$ (Badgas) [mbar] & $\boldsymbol{\Delta}\left(\mathbf{O}_{3}\right)[\mathrm{Pa}]$ & $\boldsymbol{Y}(\mathbf{C O})_{\exp }$ \\
\hline 1HEX & 345 & $1002(\mathrm{SL})$ & 0.94 & 0.26 \\
& & $33(\mathrm{SL})$ & 1.00 & 0.18 \\
& & $999\left(\mathrm{~N}_{2}\right)$ & 0.98 & 0.24 \\
& & $31\left(\mathrm{~N}_{2}\right)$ & 1.03 & 0.20 \\
\hline 2M1P & \multirow{2}{*}{345} & $1023(\mathrm{SL})$ & 0.87 & 0.11 \\
& & $33(\mathrm{SL})$ & 1.04 & 0.13 \\
& & $1006\left(\mathrm{~N}_{2}\right)$ & 0.89 & 0.11 \\
& & $32\left(\mathrm{~N}_{2}\right)$ & 1.04 & 0.14 \\
\hline MCPE & 223 & $973(\mathrm{SL})$ & 0.97 & 0.38 \\
& & $48(\mathrm{SL})$ & 1.06 & 0.44 \\
& & $1010\left(\mathrm{~N}_{2}\right)$ & 0.88 & 0.33 \\
& & $47\left(\mathrm{~N}_{2}\right)$ & 1.01 & 0.32 \\
\hline
\end{tabular}

Tab. B.1.: Auflistung der experimentell bestimmten CO-Ausbeuten für die $\mathrm{C}_{6}{ }^{-}$ Kohlenwasserstoffverbindungen. 


\begin{tabular}{|c|c|c|c|c|}
\hline Alken & $t_{\mathrm{R}}[\mathrm{s}]$ & $p_{\text {ges }}$ (Badgas) [mbar] & $\Delta\left(\mathrm{O}_{3}\right)[\mathrm{Pa}]$ & $Y(\mathrm{CO})_{\exp }$ \\
\hline \multirow[t]{4}{*}{ 3B1L } & \multirow[t]{4}{*}{702} & 998 (SL) & 0.89 & 0.14 \\
\hline & & 30 (SL) & 1.02 & 0.23 \\
\hline & & $1002\left(\mathrm{~N}_{2}\right)$ & 0.89 & 0.11 \\
\hline & & $31\left(\mathrm{~N}_{2}\right)^{2 \prime}$ & 0.95 & 0.14 \\
\hline \multirow[t]{4}{*}{ 1P3L } & \multirow[t]{4}{*}{702} & 1003 (SL) & 0.91 & 0.12 \\
\hline & & 33 (SL) & 1.01 & 0.21 \\
\hline & & $996\left(\mathrm{~N}_{2}\right)$ & 0.91 & 0.10 \\
\hline & & $28\left(\mathrm{~N}_{2}\right)$ & 1.06 & 0.16 \\
\hline \multirow[t]{6}{*}{ 4P1L } & \multirow[t]{6}{*}{462} & 502 (SL) & 0.86 & 0.09 \\
\hline & & 47 (SL) & 0.89 & 0.15 \\
\hline & & 21 (SL) & 1.00 & 0.16 \\
\hline & & $495\left(\mathrm{~N}_{2}\right)$ & 0.93 & 0.09 \\
\hline & & $51\left(\mathrm{~N}_{2}\right)$ & 0.88 & 0.12 \\
\hline & & $19\left(N_{2}\right)$ & 0.89 & 0.13 \\
\hline \multirow[t]{4}{*}{ 1P3N } & \multirow[t]{4}{*}{582} & 997 (SL) & 0.94 & 0.27 \\
\hline & & 32 (SL) & 1.01 & 0.44 \\
\hline & & $1001\left(\mathrm{~N}_{2}\right)$ & 0.92 & 0.27 \\
\hline & & $29\left(\mathrm{~N}_{2}\right)$ & 1.08 & 0.32 \\
\hline \multirow[t]{4}{*}{ 4PAL } & \multirow[t]{4}{*}{582} & 995 (SL) & 1.00 & 0.17 \\
\hline & & 33 (SL) & 1.01 & 0.34 \\
\hline & & $994\left(N_{2}\right)$ & 0.97 & 0.17 \\
\hline & & $32\left(\mathrm{~N}_{2}\right)$ & 0.98 & 0.29 \\
\hline \multirow[t]{4}{*}{ MACS } & \multirow[t]{4}{*}{1200} & 1001 (SL) & 1.00 & 0.04 \\
\hline & & 31 (SL) & 1.00 & 0.12 \\
\hline & & $997\left(\mathrm{~N}_{2}\right)$ & 0.99 & 0.07 \\
\hline & & $29\left(\mathrm{~N}_{2}\right)$ & 1.01 & 0.15 \\
\hline \multirow[t]{4}{*}{ BENS } & \multirow[t]{4}{*}{1200} & 1003 (SL) & 0.98 & 0.10 \\
\hline & & 30 (SL) & 0.97 & 0.15 \\
\hline & & $999\left(\mathrm{~N}_{2}\right)$ & 0.99 & 0.10 \\
\hline & & $34\left(\mathrm{~N}_{2}\right)$ & 1.01 & 0.17 \\
\hline
\end{tabular}

Tab. B.2.: Auflistung der experimentell bestimmten CO-Ausbeuten für die voroxidierten Kohlenwasserstoffverbindungen. 


\section{Reinspektren der verwendeten Substanzen (Auswahl)}

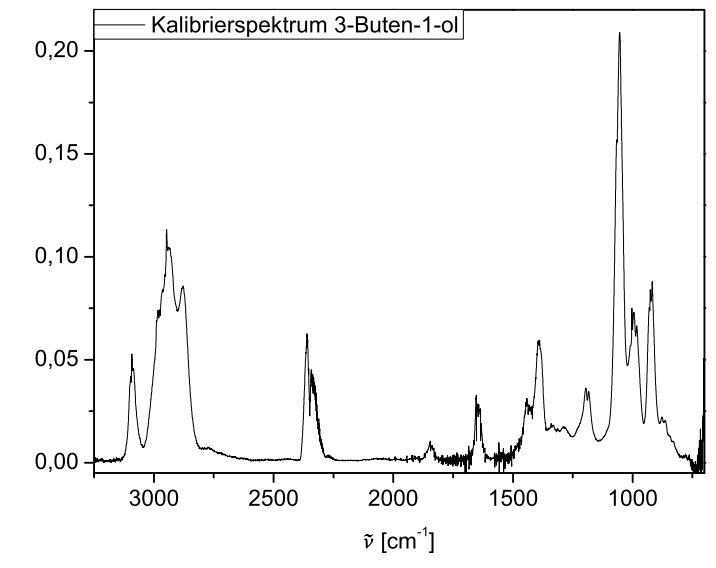

(a) $p_{3 \mathrm{~B} 1 \mathrm{~L}}=5.75 \mathrm{~Pa}, p_{\text {ges }}=1000 \mathrm{mbar} \mathrm{SL}$.

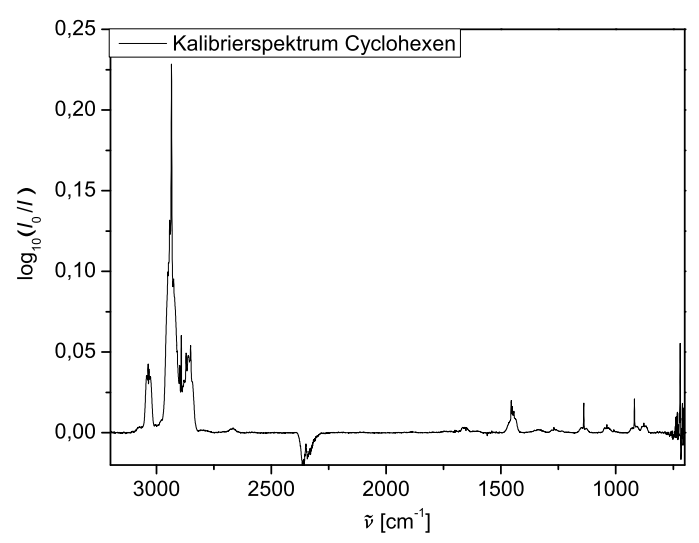

(c) $p_{\mathrm{CHEX}}=1.74 \mathrm{~Pa}, p_{\text {ges }}=500 \mathrm{mbar} \mathrm{SL}$.

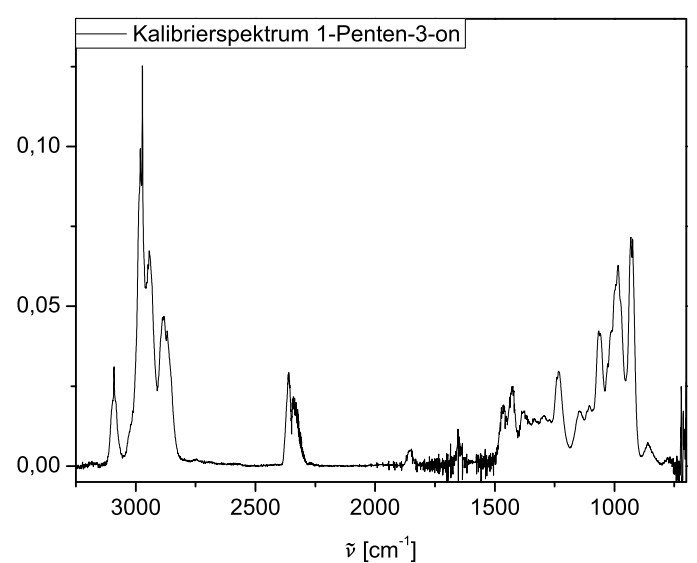

(b) $p_{1 \mathrm{P} 3 \mathrm{~L}}=3.14 \mathrm{~Pa}, p_{\text {ges }}=1000 \mathrm{mbar} \mathrm{SL}$.

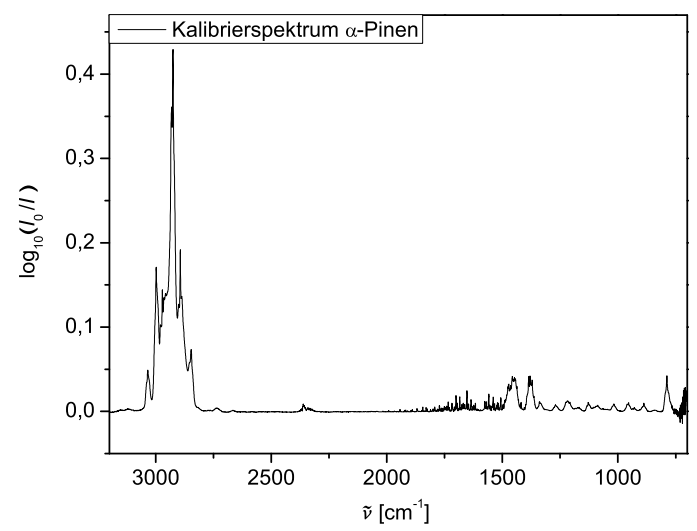

(d) $p_{\alpha-\text { Pinen }}=1.74 \mathrm{~Pa}, p_{\text {ges }}=500$ mbar SL.

Abb. C.1.: Kalibrierspektren aus [48] und [17] unter Angabe der verwendeten Partialdrücke, Gesamtdrücke und Badgase. 

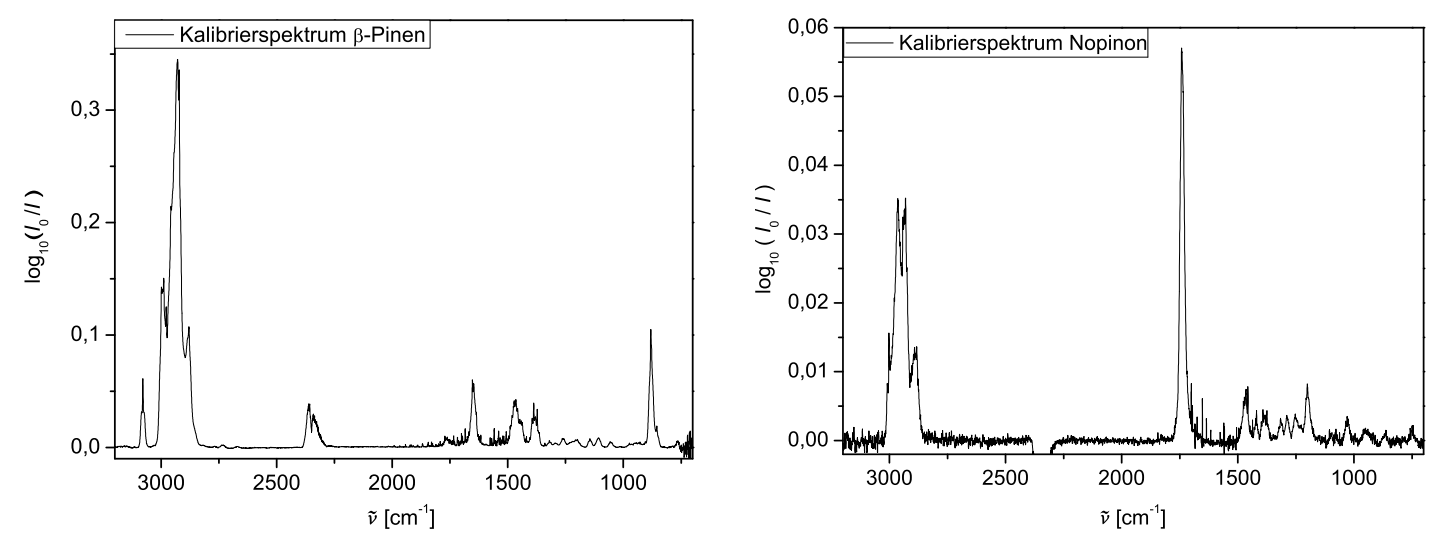

(a) $p_{\beta-\text { Pinen }}=5.26 \mathrm{~Pa}, p_{\text {ges }}=1000 \mathrm{mbar} \mathrm{SL}$.

(b) $p_{\text {Nopinon }}=1.30 \mathrm{~Pa}, p_{\text {ges }}=1000 \mathrm{mbar}_{2}$.
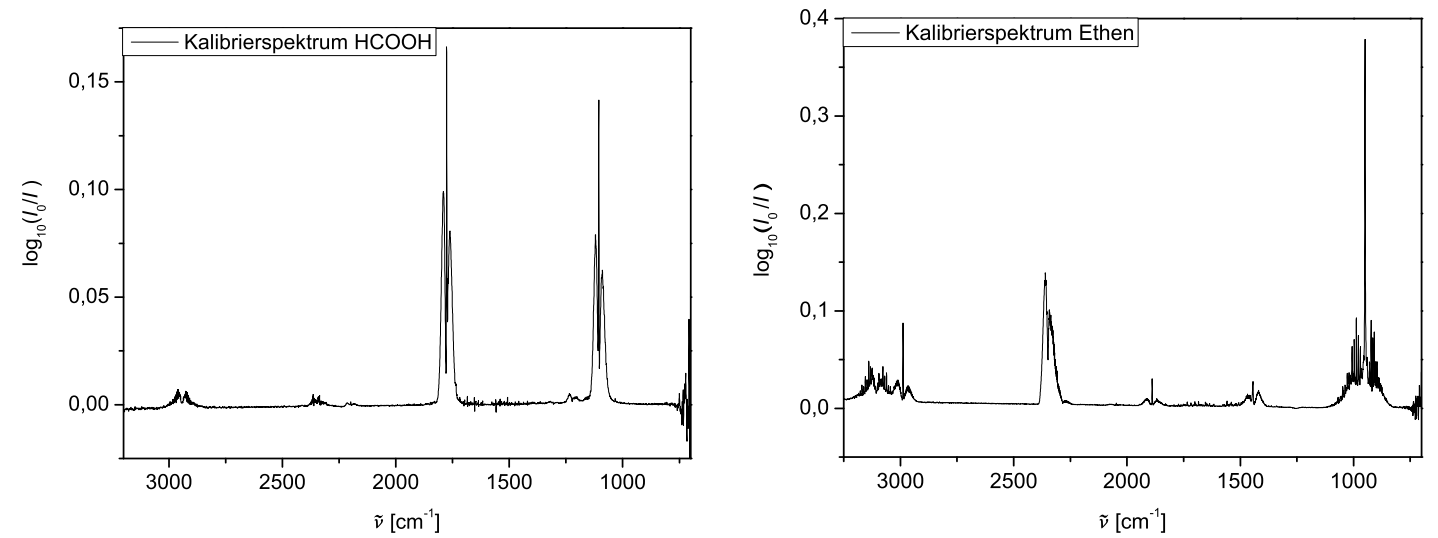

(c) $p_{\mathrm{HCOOH}}=0.80 \mathrm{~Pa}, p_{\text {ges }}=1000 \mathrm{mbar} \mathrm{SL}$.

(d) $p_{\text {Ethen }}=4.92 \mathrm{~Pa}, p_{\text {ges }}=1000 \mathrm{mbar} \mathrm{SL}$.

Abb. C.2.: Kalibrierspektren aus [48] und [17] unter Angabe der verwendeten Partialdrücke, Gesamtdrücke und Badgase. 


\section{Ergänzende Abbildungen zu Produktbildungsstudien}

\section{D.1. 1-Hexen}

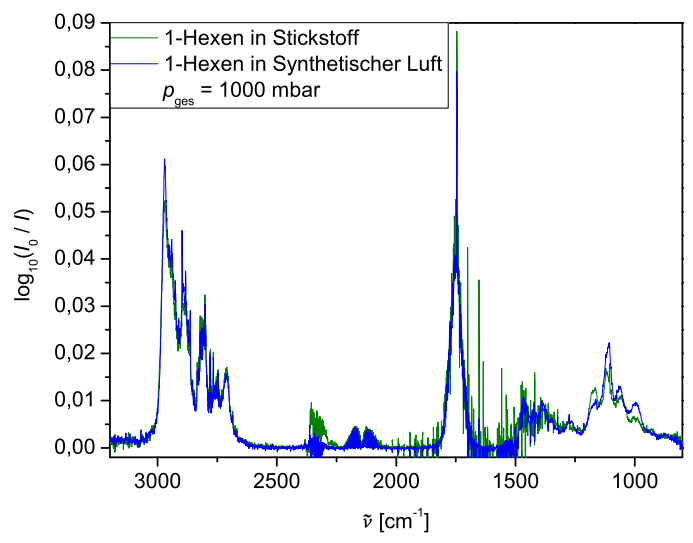

(a) Darstellung des Gesamtreaktionsspektrums des 1-Hexens in Synthetischer Luft und Stickstoff als Badgas bei einem Gesamtdruck von jeweils 1000 mbar.

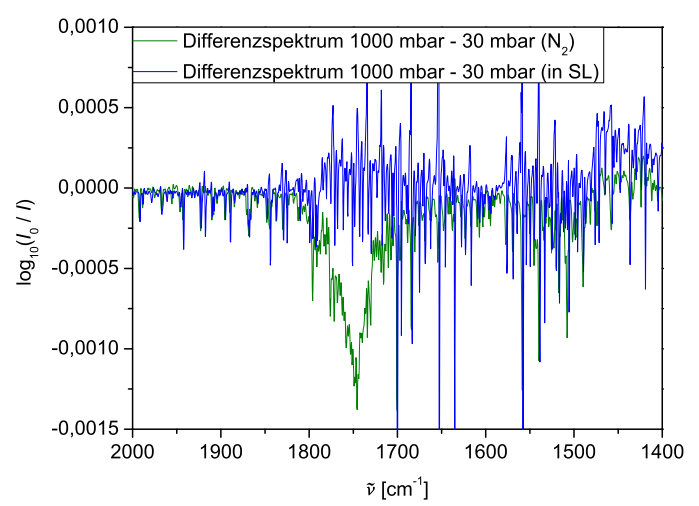

(c) Differenzspektren der Carbonylabsorbanz.

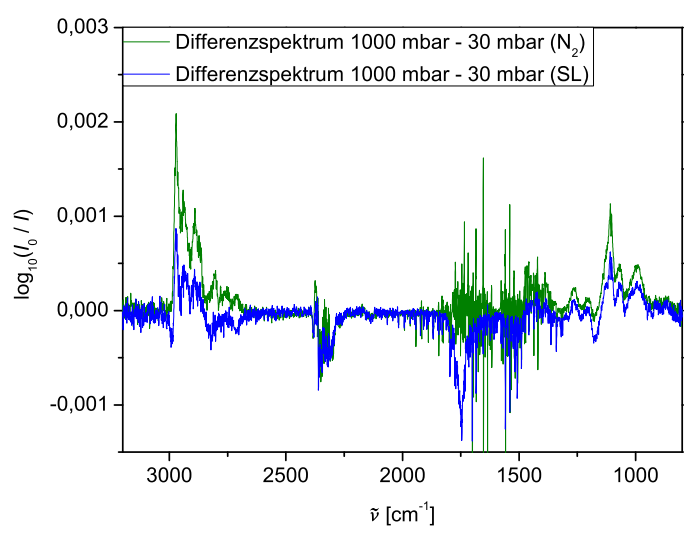

(b) Differenzspektren zwischen Hoch- und Niederdruckmessungen des 1-Hexens in Synthetischer Luft und Stickstoff als Badgas.

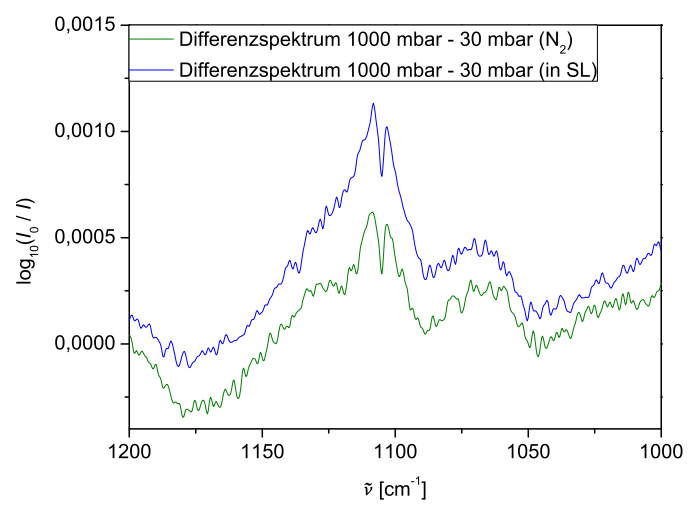

(d) Differenzspektren der Ameisensäureabsorbanz. 


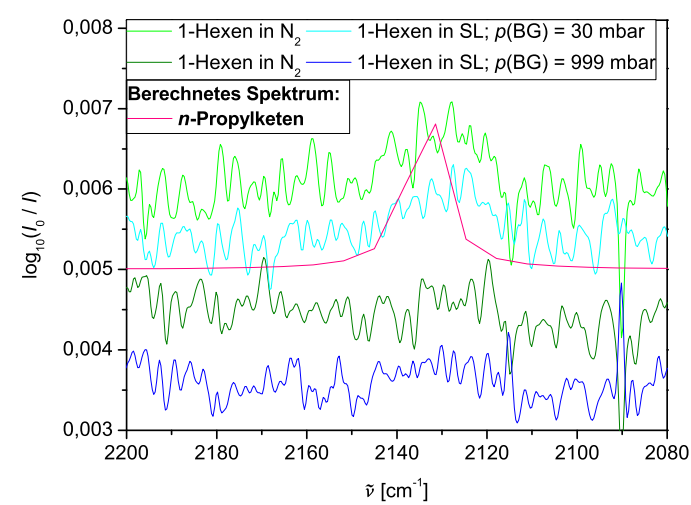

(a) Reaktionsspektren der Hoch- und Niederdruckmessungen des 1-Hexens im Detailbereich der Ketenabsorbanz. Sämtliche Spektren wurden CO-bereinigt.

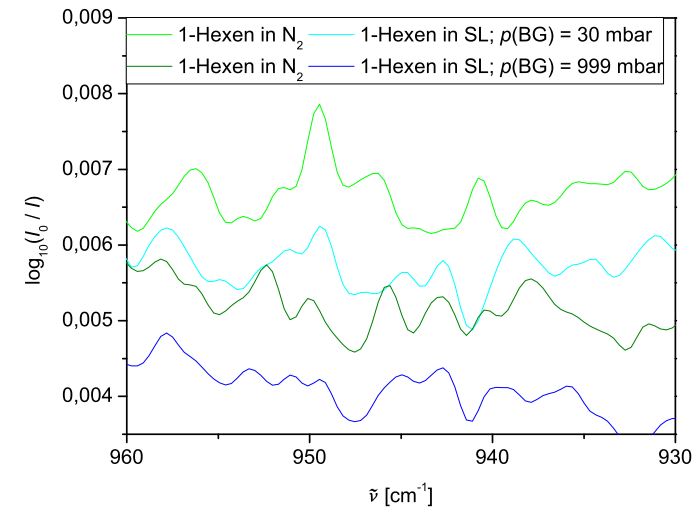

(b) Reaktionsspektren der Hoch- und Niederdruckmessungen des 1-Hexens, jeweils in Synthetischer Luft und Stickstoff als Badgas.

Abb. D.1.: Detailbereiche der 1-Hexen-Reaktionsspektren. 


\section{D.2. 2-Methyl-1-penten}

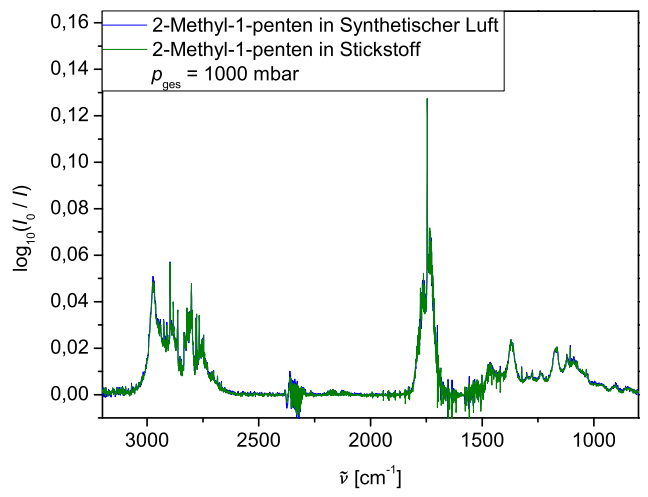

(a) Gesamtansicht der Reaktionsspektren.

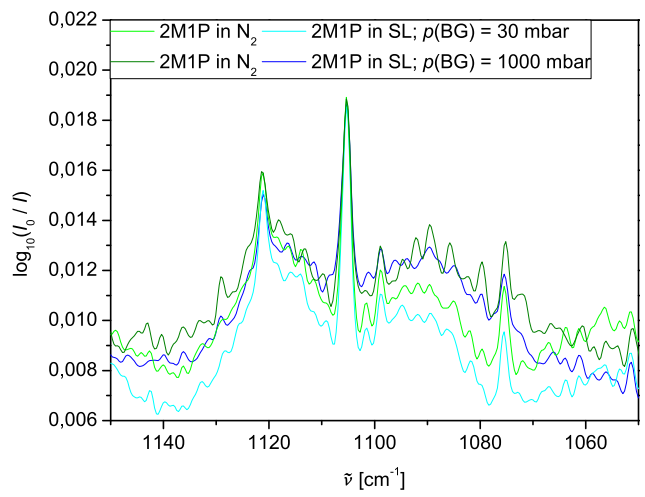

(b) Ameisensäure.

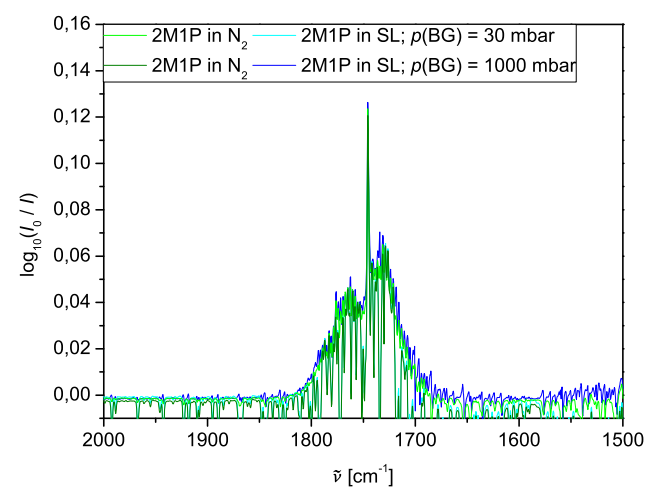

(d) Carbonylbereich.

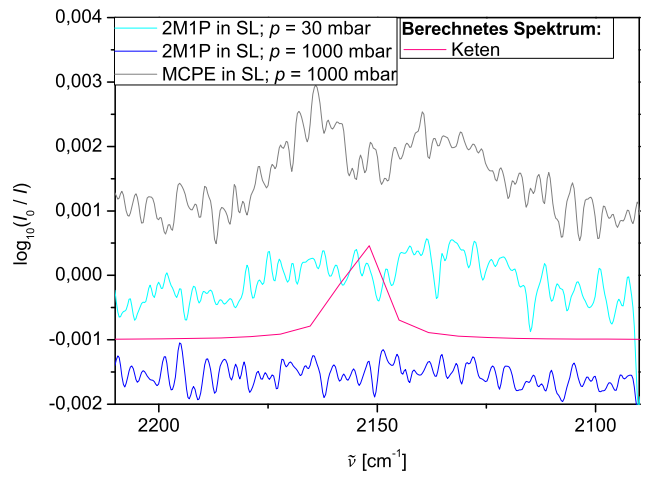

(c) Ketenabsorbanz nach CO-Bereinigung.

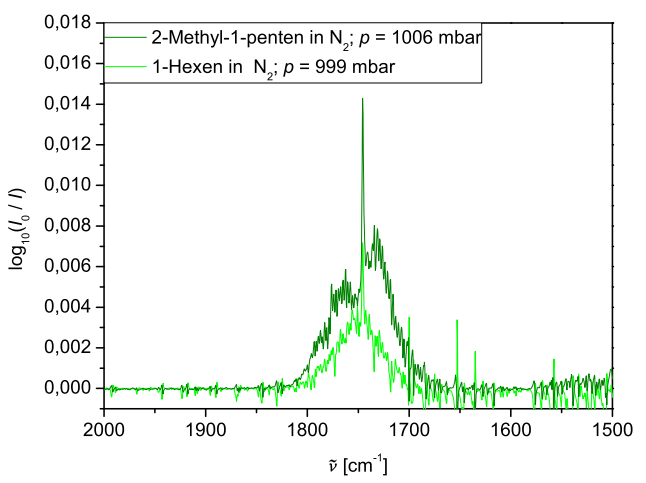

(e) Vergleich der Formaldehydausbeute (2M1P, 1HEX).

Abb. D.2.: Gesamtansicht und Detailbereiche der Reaktionsspektren des 2Methyl-1-pentens und Vergleich der Formaldehydausbeuten mit 1Hexen. 


\section{D.3. 3-Buten-1-ol}

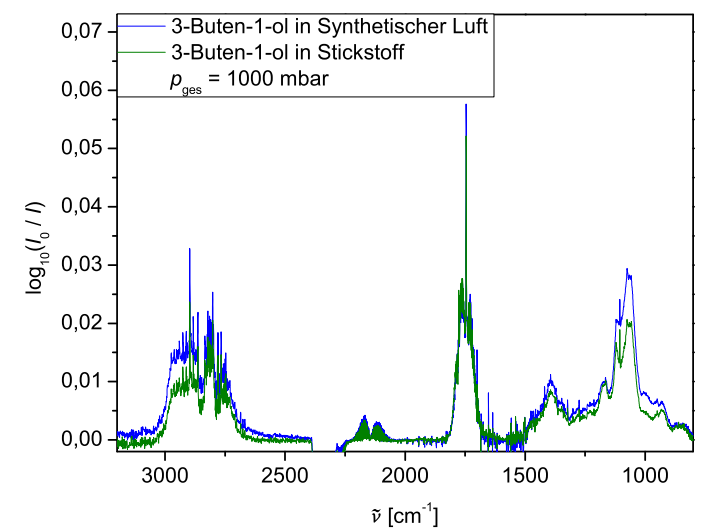

(a) Darstellung des Gesamtreaktionsspektrums des 3-Buten-1-ols in Synthetischer Luft und Stickstoff als Badgas bei einem Gesamtdruck von jeweils 1000 mbar.

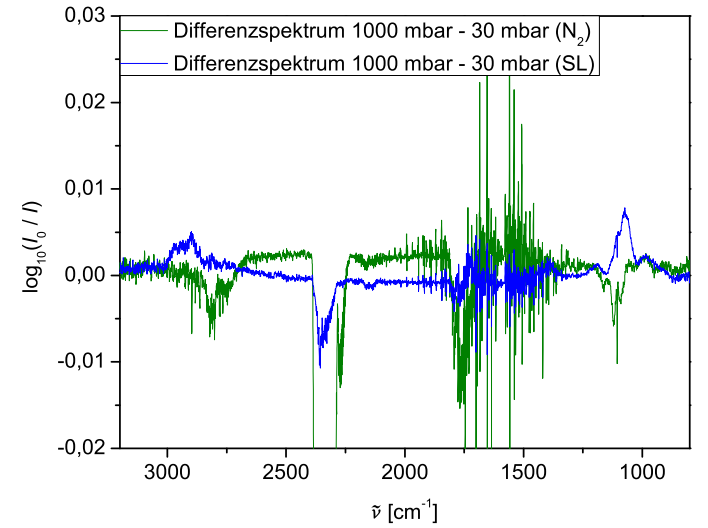

(b) Differenzspektren zwischen Hoch- und Niederdruckmessungen des 3-Buten-1-ols in Synthetischer Luft und Stickstoff als Badgas.

Abb. D.3.: Gesamtansicht der Reaktionsspektren bei Hochdruckbedingungen sowie der Differenzspektren unter verschiedenen Druckregimes für 3-Buten-1-ol.

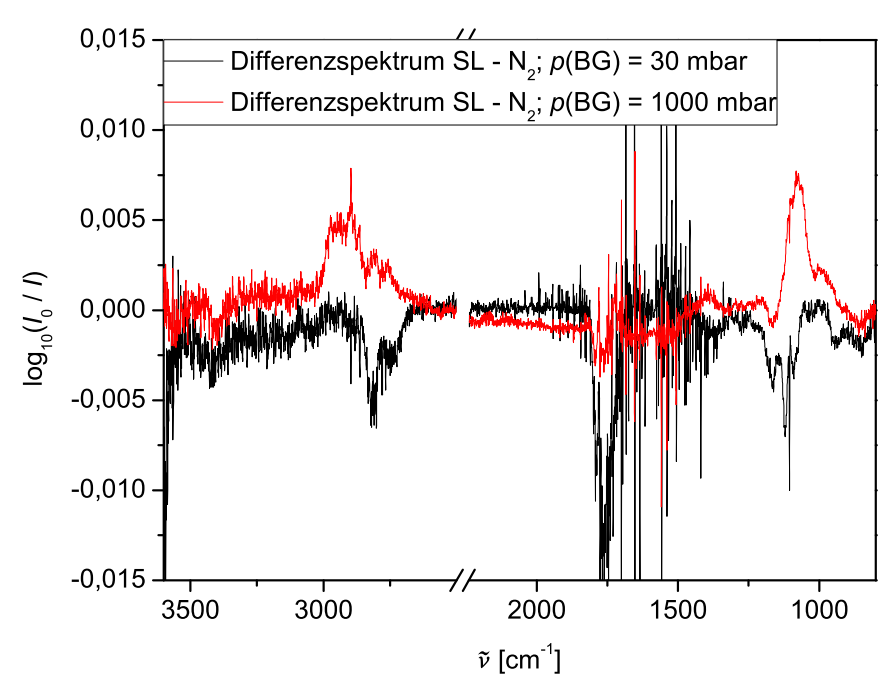

Abb. D.4.: Differenzspektren zwischen Reaktionen in Synthetischer Luft und Stickstoff für 3-Buten-1-ol, jeweils unter Hoch- und Niederdruckbedingungen, durch die Achsenunterbrechung wird lediglich das $\mathrm{CO}_{2}$ Signal ausgeblendet. 


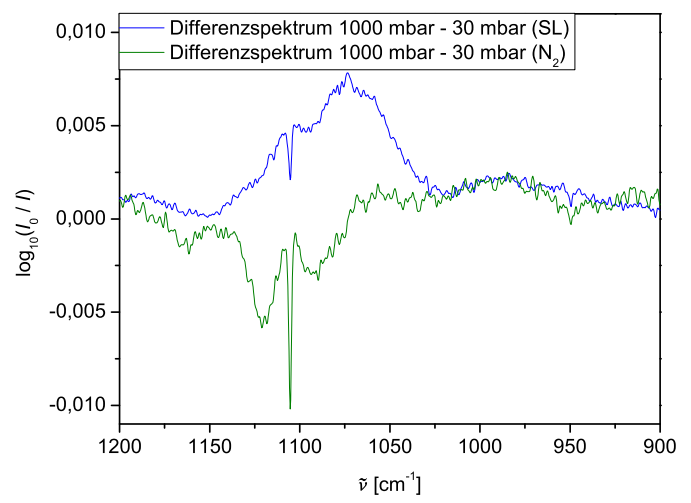

(a) Ameisensäure, Differenspektren.

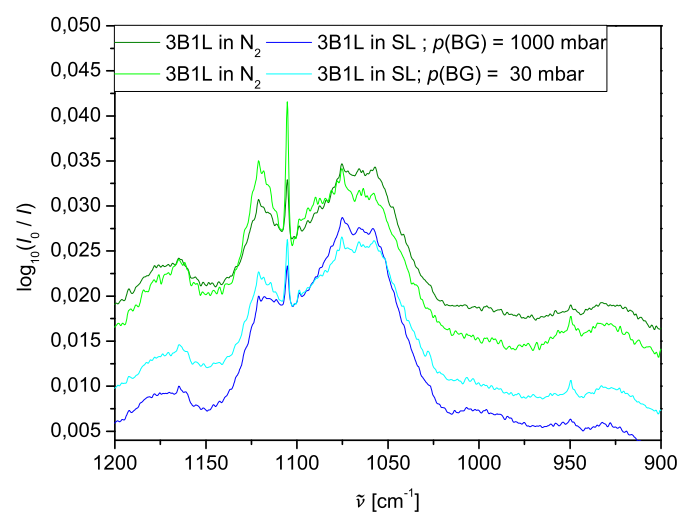

(b) Ameisensäure und Ethen, Reaktionsspektren.

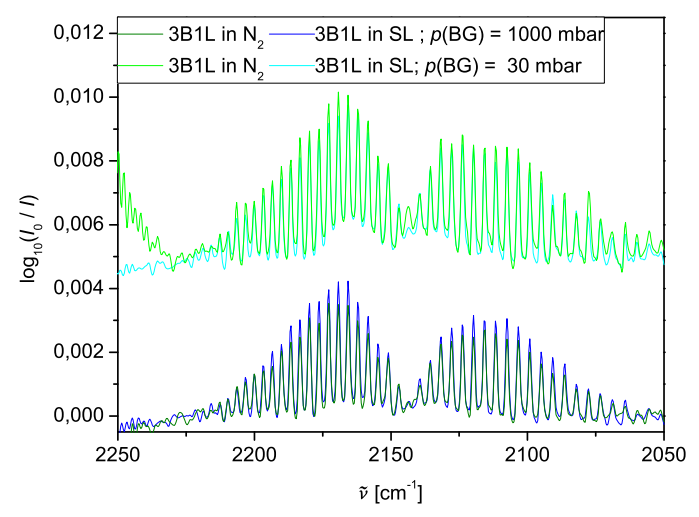

(d) CO- bzw. Ketenabsorbanz.

Abb. D.5.: Detailbereiche der Reaktionsspektren des 3-Buten-1-ols. 


\section{D.4. 1-Penten-3-ol}

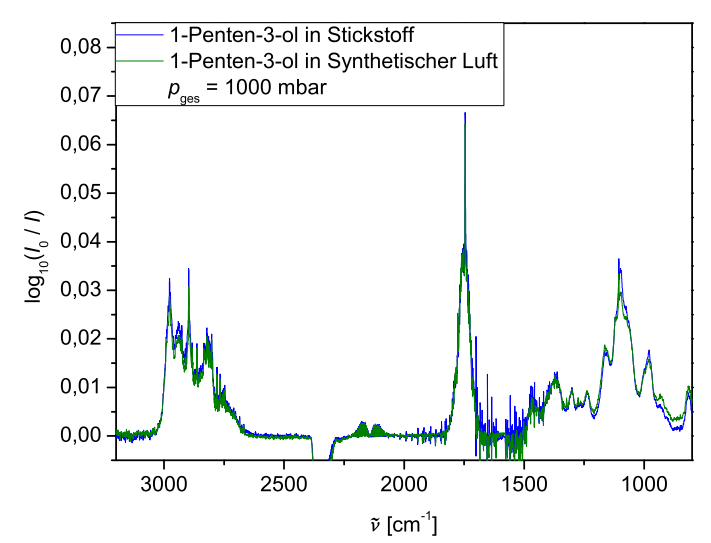

(a) Darstellung des Gesamtreaktionsspektrums des 1-Penten-3-ols in Synthetischer Luft und Stickstoff als Badgas bei einem Gesamtdruck von jeweils 1000 mbar.

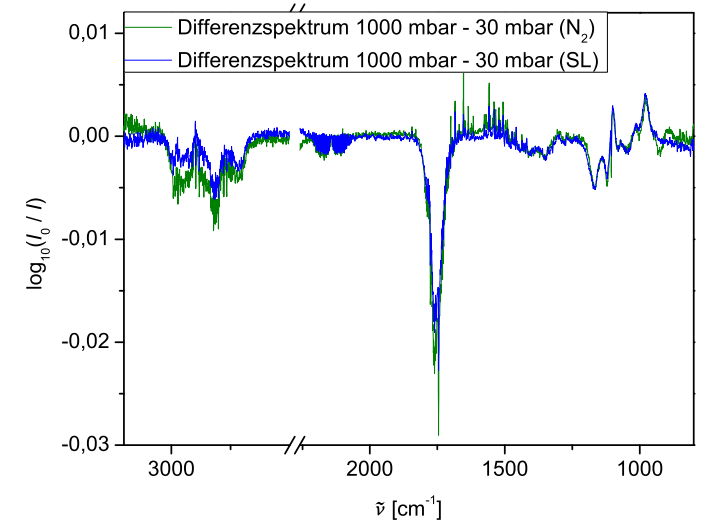

(b) Differenzspektren zwischen Hoch- und Niederdruckmessungen des 1-Penten-3ols in Synthetischer Luft und Stickstoff als Badgas.

Abb. D.6.: Gesamtansicht der Reaktionsspektren bei Hochdruckbedingungen sowie der Differenzspektren unter verschiedenen Druckregimes für 1-Penten-3-ol.

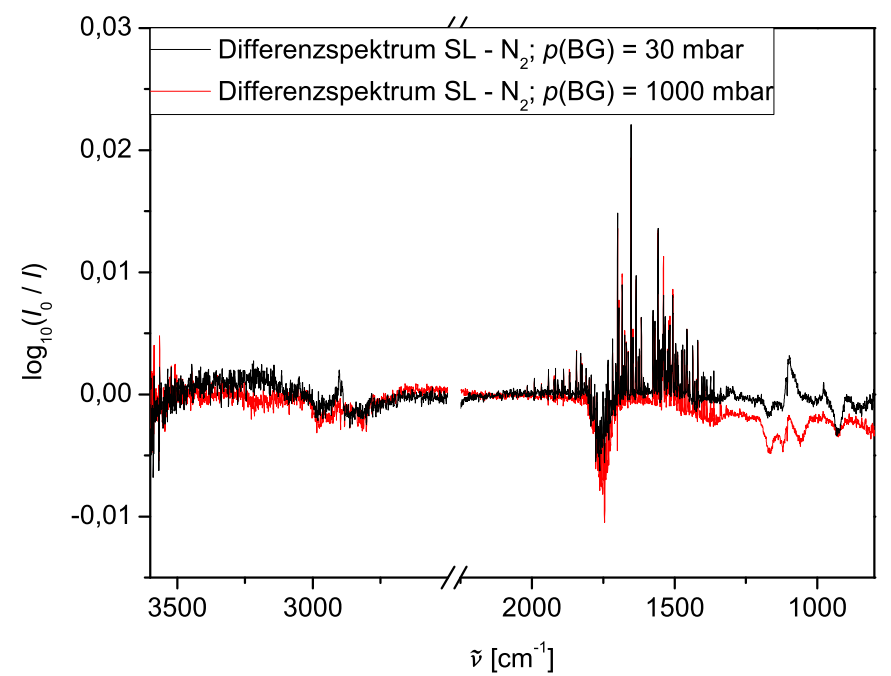

Abb. D.7.: Differenzspektren zwischen Reaktionen in Synthetischer Luft und Stickstoff für 1-Penten-3-ol, jeweils unter Hoch- und Niederdruckbedingungen, durch die Achsenunterbrechung wird lediglich das $\mathrm{CO}_{2}$ Signal ausgeblendet. 


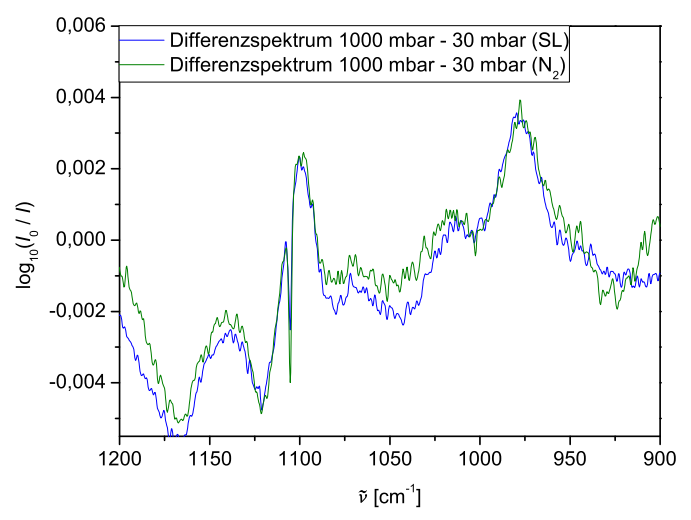

(a) Ameisensäure, Differenzspektren.

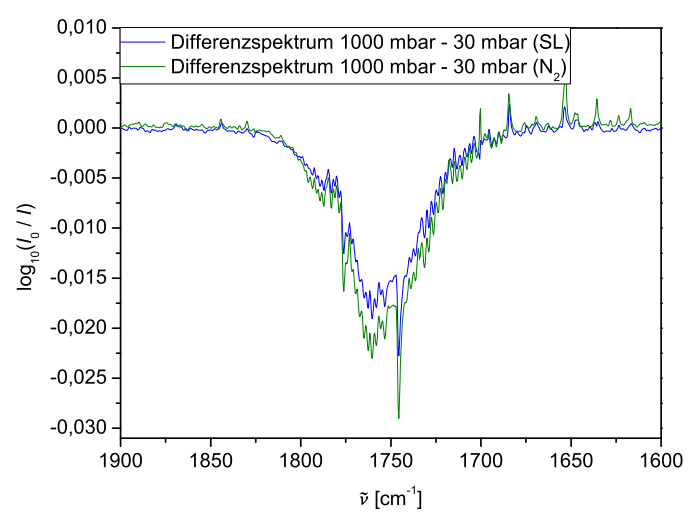

(c) Formaldehyd, Differenzspektren.

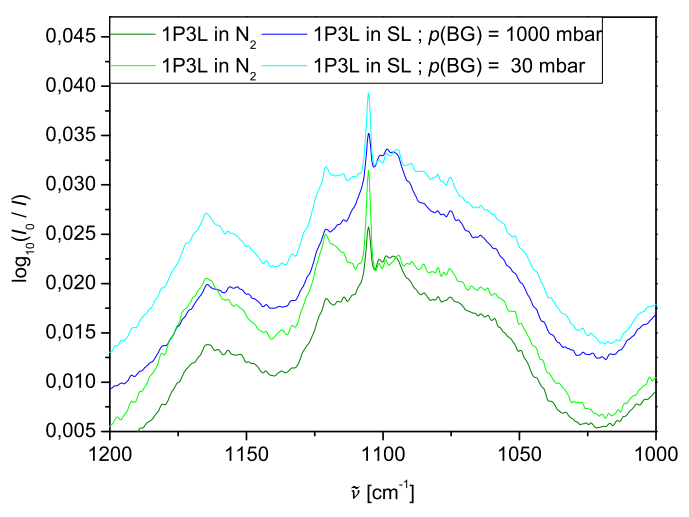

(b) Ameisensäure, Reaktionsspektren.

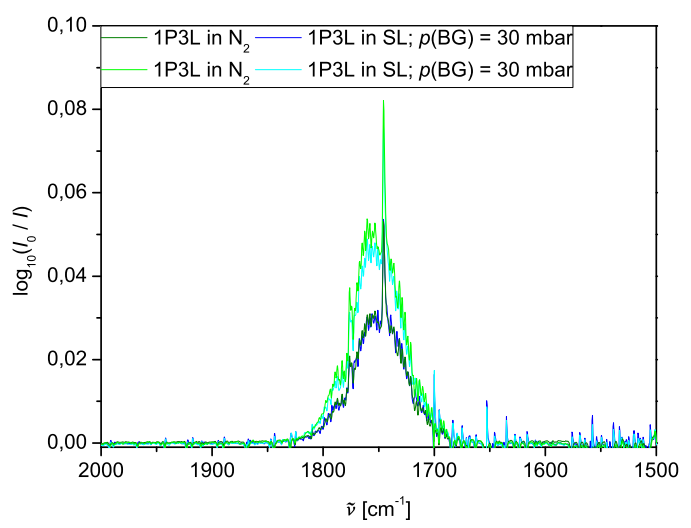

(d) Formaldehyd, Reaktionsspektren.

Abb. D.8.: Detailbereiche der Reaktionsspektren des 1-Penten-3-ols. 


\section{D.5. 4-Penten-1-ol}

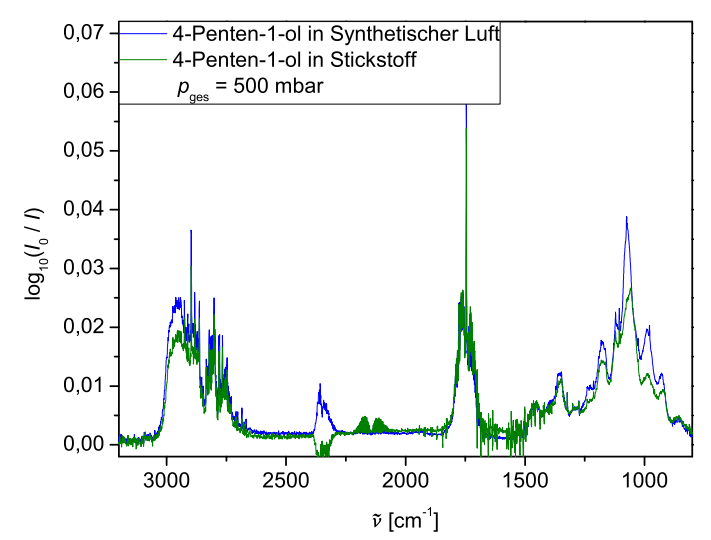

(a) Darstellung des Gesamtreaktionsspektrums des 4-Penten-1-ols in Synthetischer Luft und Stickstoff als Badgas bei einem Gesamtdruck von jeweils 500 mbar.

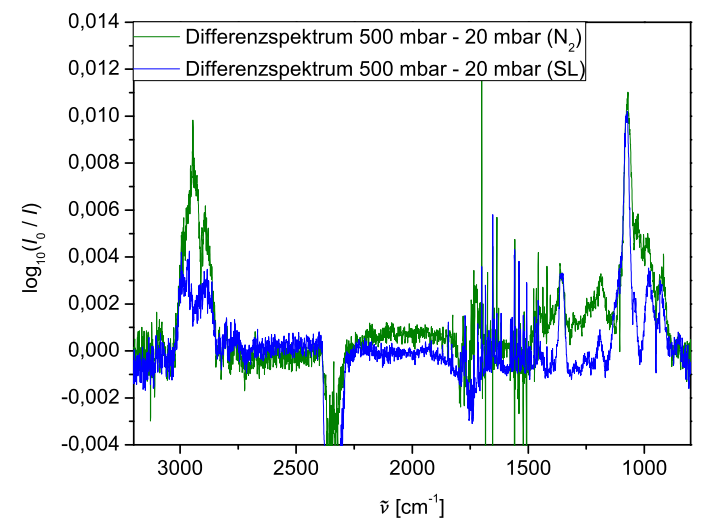

(b) Differenzspektren zwischen Hoch- und Niederdruckmessungen des 4-Penten-1ols in Synthetischer Luft und Stickstoff als Badgas.

Abb. D.9.: Gesamtansicht der Reaktionsspektren bei Hochdruckbedingungen sowie der Differenzspektren unter verschiedenen Druckregimes für 4-Penten-1-ol.

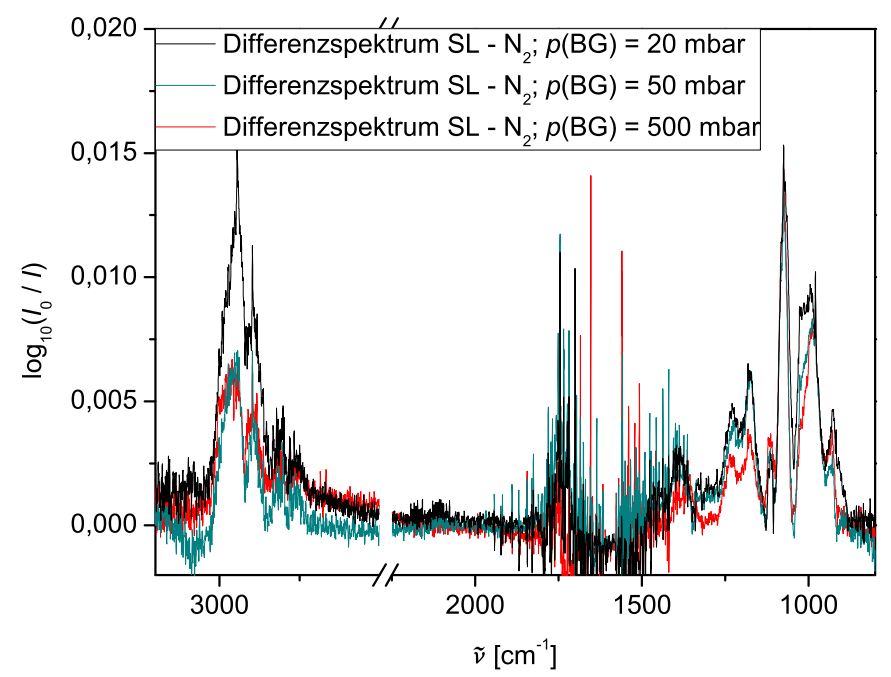

Abb. D.10.: Differenzspektren zwischen Reaktionen in Synthetischer Luft und Stickstoff des 4-Pentenols, jeweils unter Hoch- und Niederdruckbedingungen, durch die Achsenunterbrechung wird lediglich das $\mathrm{CO}_{2}$ Signal ausgeblendet. 


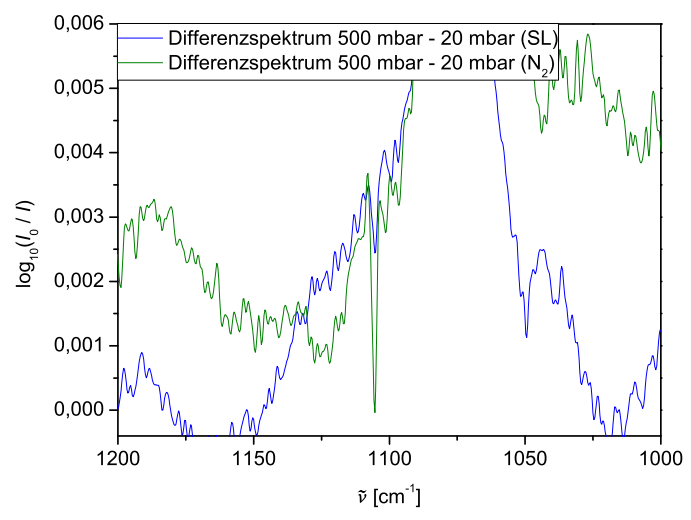

(a) Ameisensäure, Differenzspektren.

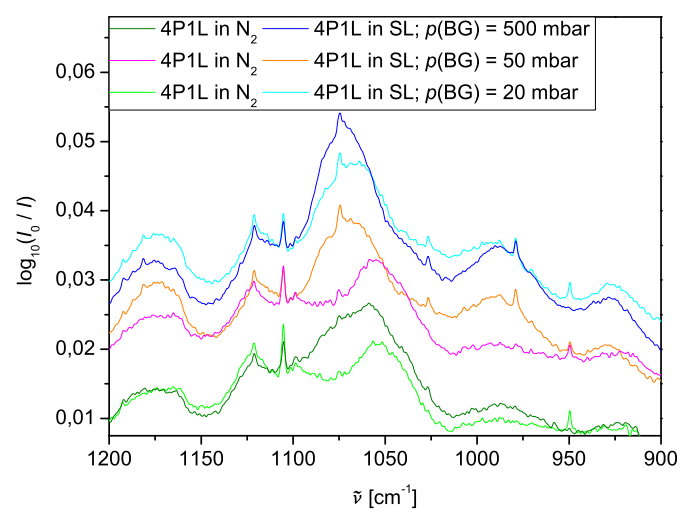

(b) Ameisensäure, Ethen, Gerüstschwingungen.

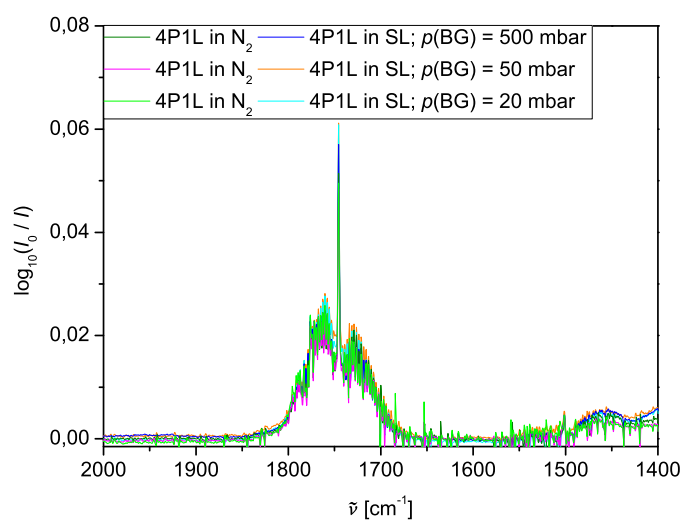

(c) Carbonylbereich.

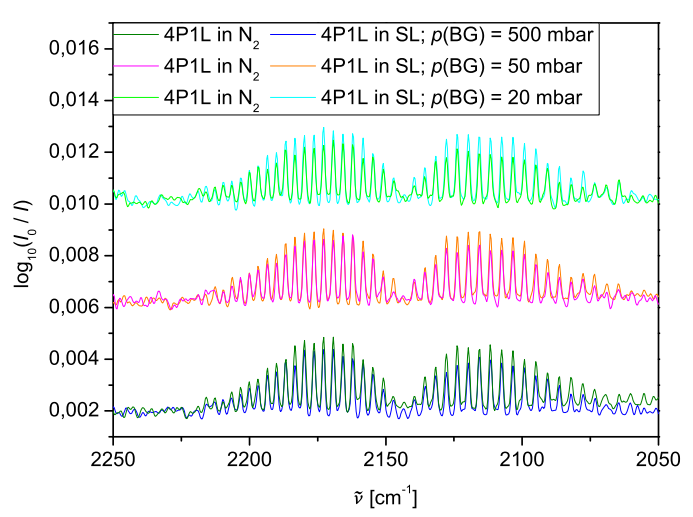

(d) Vergleich der CO-Banden.

Abb. D.11.: Detailbereiche der Reaktionsspektren des 4-Penten-1-ols. 


\section{D.6. 1-Penten-3-on}

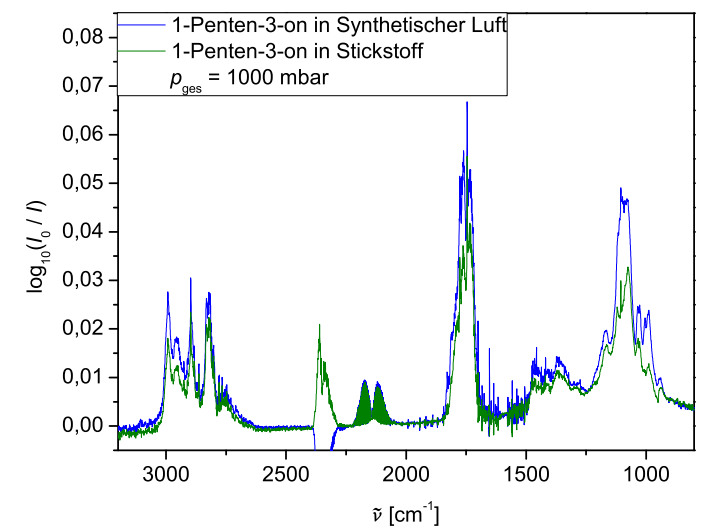

(a) Darstellung des Gesamtreaktionsspektrums des 1-Penten-3-ons in Synthetischer Luft und Stickstoff als Badgas bei einem Gesamtdruck von jeweils 1000 mbar.

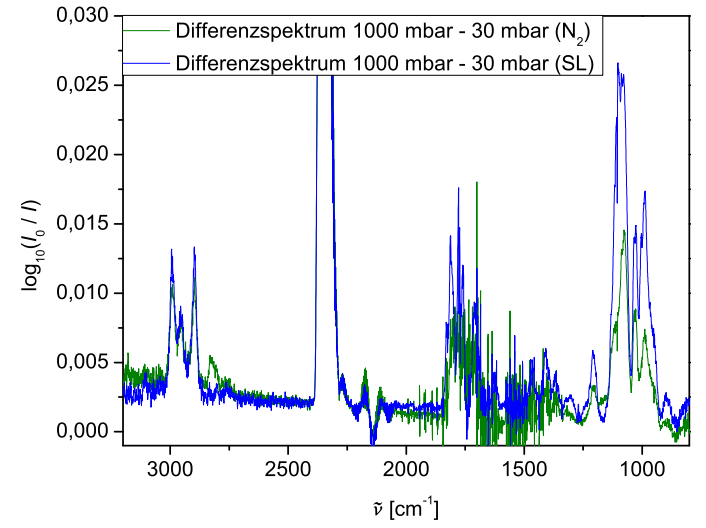

(b) Differenzspektren zwischen Hoch- und Niederdruckmessungen des 1-Penten-3ons in Synthetischer Luft und Stickstoff als Badgas.

Abb. D.12.: Gesamtansicht der Reaktionsspektren bei Hochdruckbedingungen sowie der Differenzspektren unter verschiedenen Druckregimes für 1-Penten-3-on.

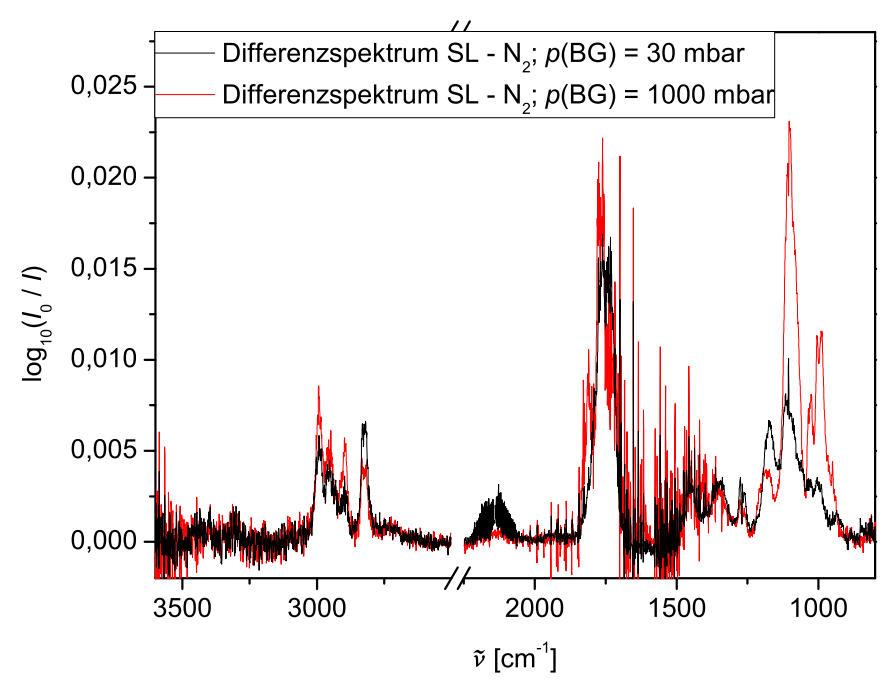

Abb. D.13.: Differenzspektren zwischen Reaktionen in Synthetischer Luft und Stickstoff für 1-Penten-3-on, jeweils unter Hoch- und Niederdruckbedingungen. 


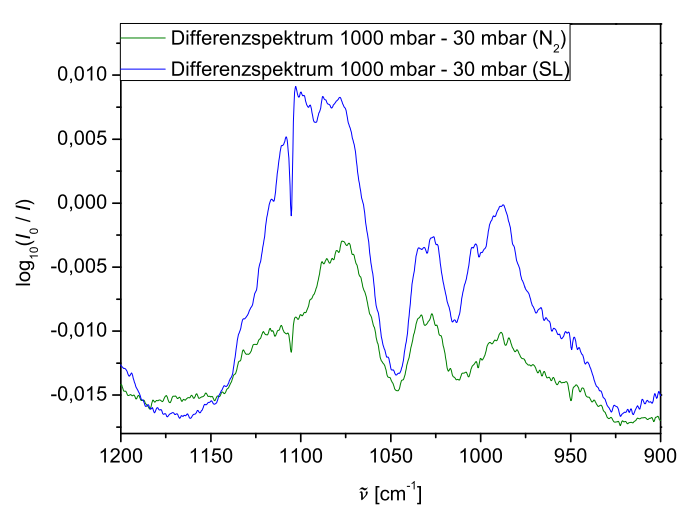

(a) Ameisensäure und Ethen, Differenzspektren.

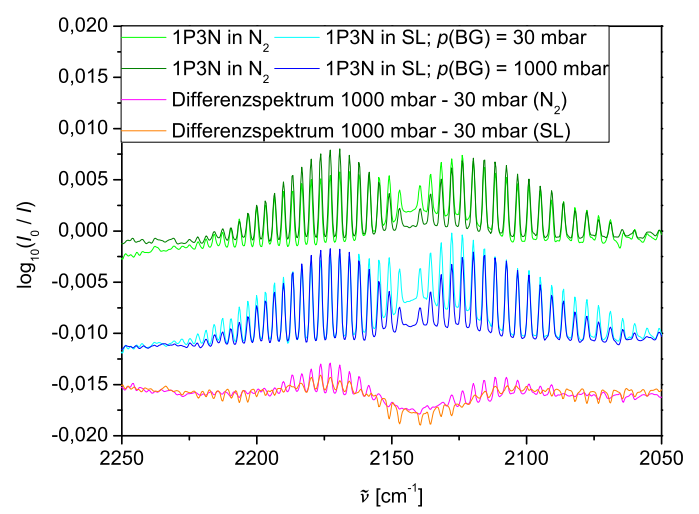

(c) CO-Bereich, Reaktions- und Differenzspektren.

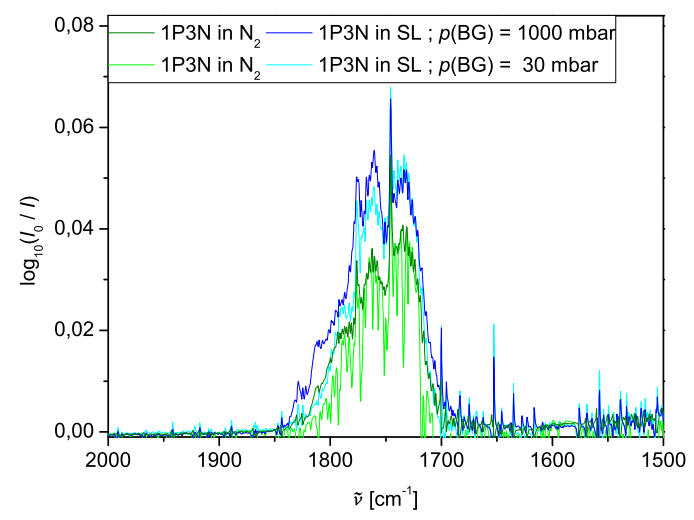

(e) Formaldehyd, Reaktionsspektren.

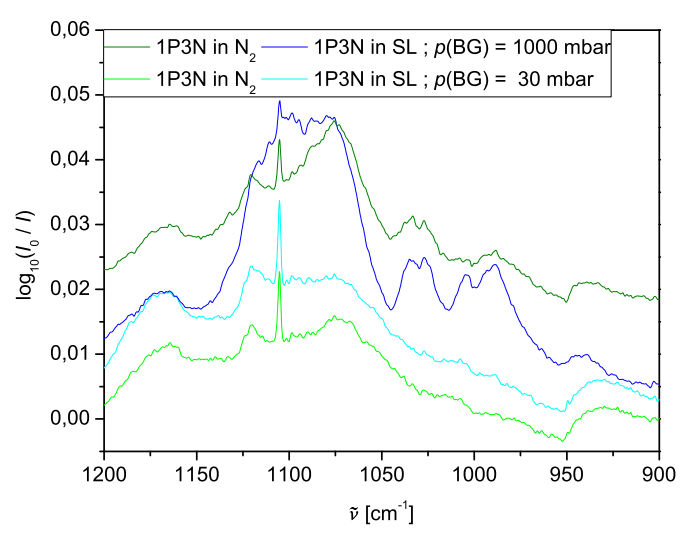

(b) Ameisensäure und Ethen, Reaktionsspektren.

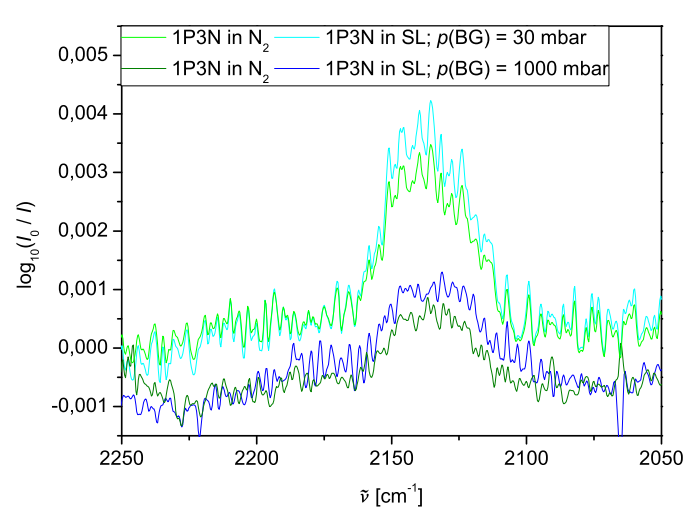

(d) Ketenabsorbanz 1-Penten-3-on.

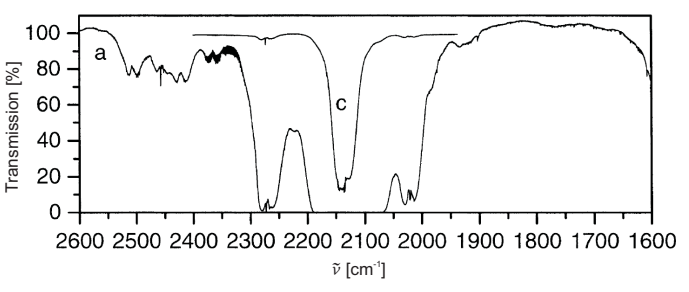

(f) Ausschnitt aus einem Literaturspekrum für Methylketen, entnommen aus WINTHER et al. [127].

Abb. D.14.: Detailbereiche der Reaktionsspektren des 1-Penten-3-ons. 


\section{D.7. 4-Penten-1-al}

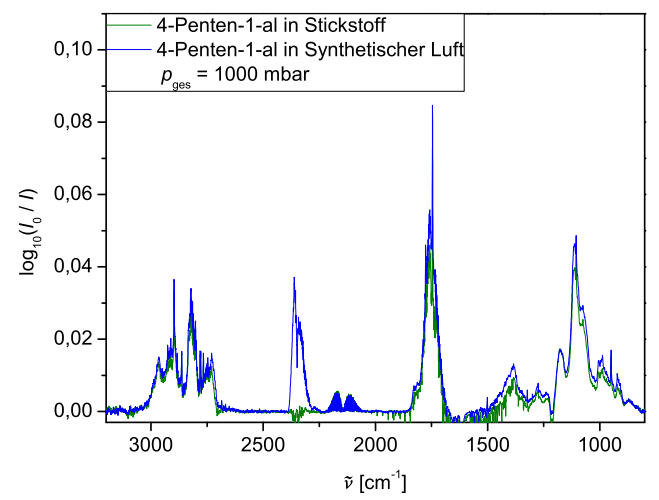

(a) Reaktionsspektren des 4-Penten-1-als.

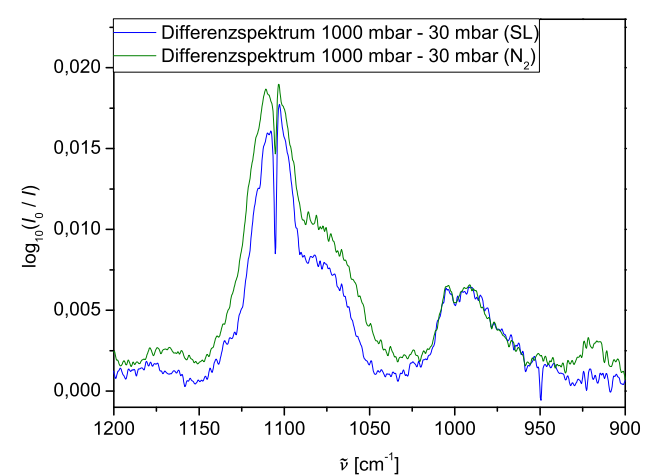

(b) Ameisensäure, Ethen, Differenzspektren.

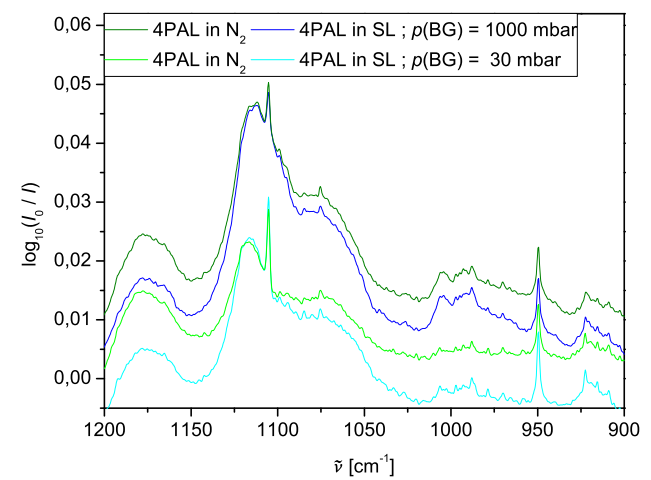

(c) Ameisensäure, Ethen, Gerüstschwingungen.

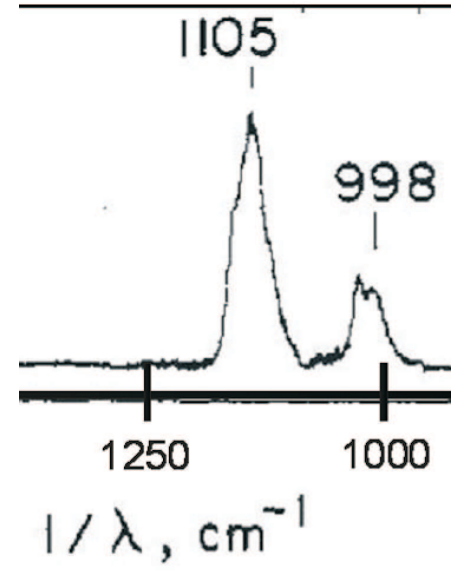

(d) Literaturspektrum Ameisensäureanhydrid nach NIKI et al. [60].

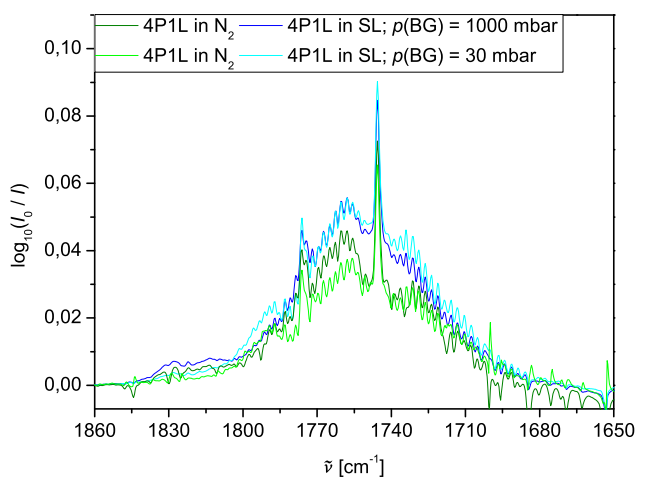

(e) Carbonylbereich.

Abb. D.15.: Gesamtansicht und Detailbereiche der Reaktionsspektren des 4Penten-1-als. 


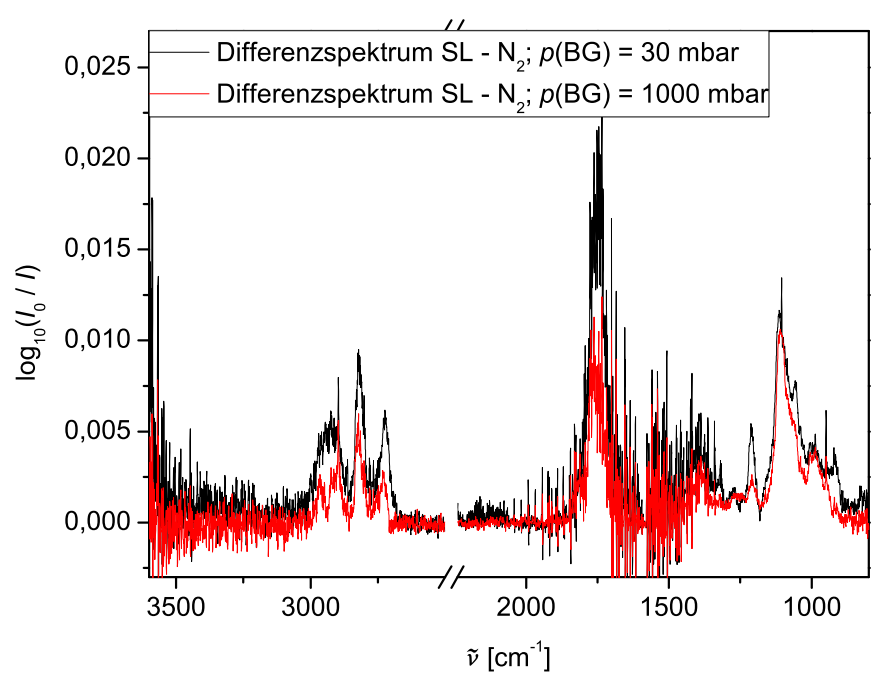

Abb. D.16.: Differenzspektren zwischen Reaktionen in Synthetischer Luft und Stickstoff für 4-Pentenal, jeweils unter Hoch- und Niederdruckbedingungen. 


\section{D.8. Methacrylsäure}

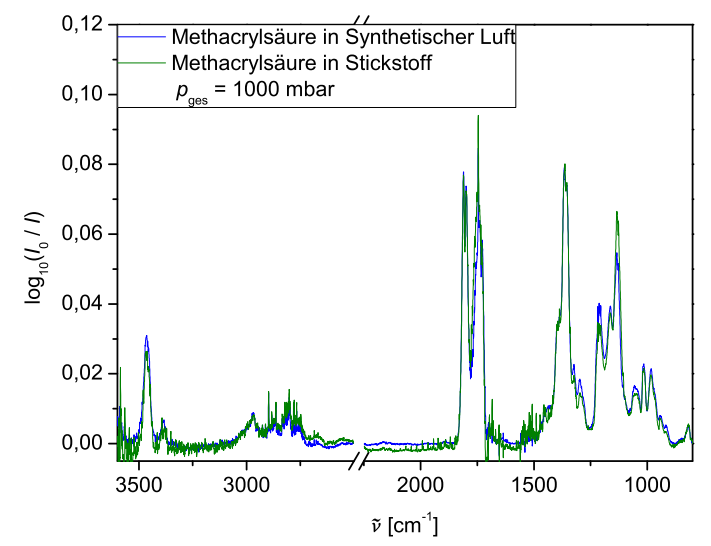

(a) Darstellung des Gesamtreaktionsspektrums der Methacrylsäure in Synthetischer Luft und Stickstoff als Badgas bei einem Gesamtdruck von jeweils 1000 mbar.

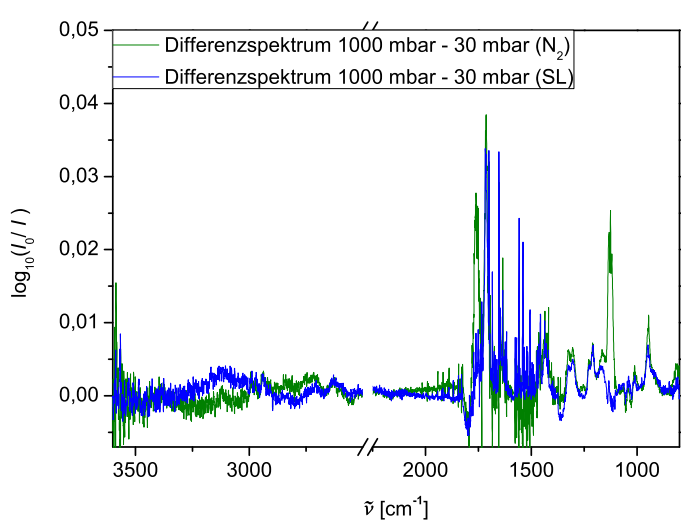

(b) Differenzspektren zwischen Hoch- und Niederdruckmessungen der Methacrylsäure in Synthetischer Luft und Stickstoff als Badgas.

Abb. D.17.: Gesamtansicht der Reaktionsspektren bei Hochdruckbedingungen sowie der Differenzspektren unter verschiedenen Druckregimes für Methacrylsäure. 


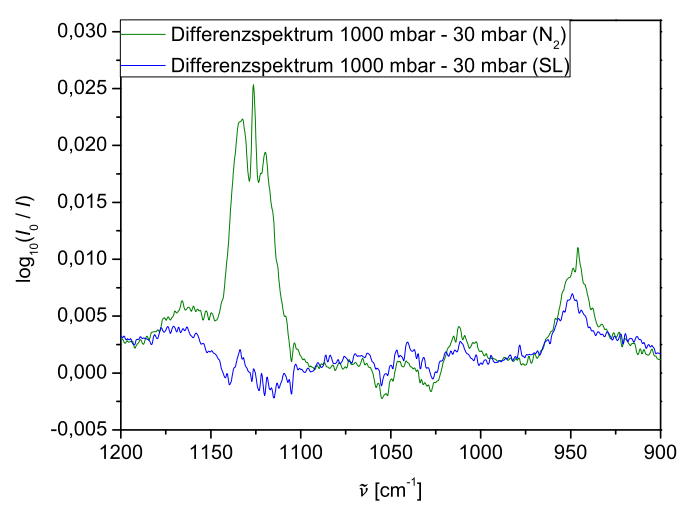

(a) Ameisensäure, Differenzspektren.

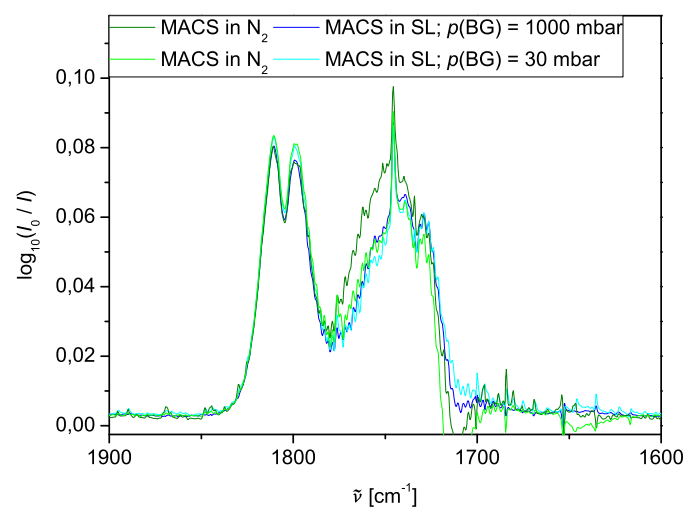

(c) Carbonylbereich.

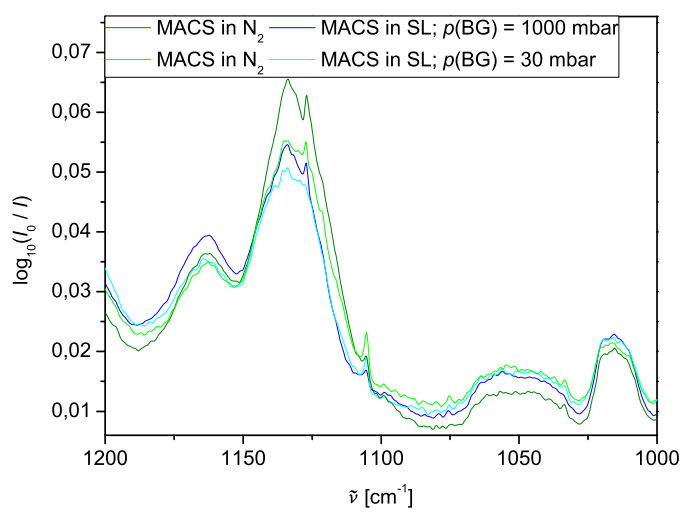

(b) Ameisensäure, Reaktionsspektren.

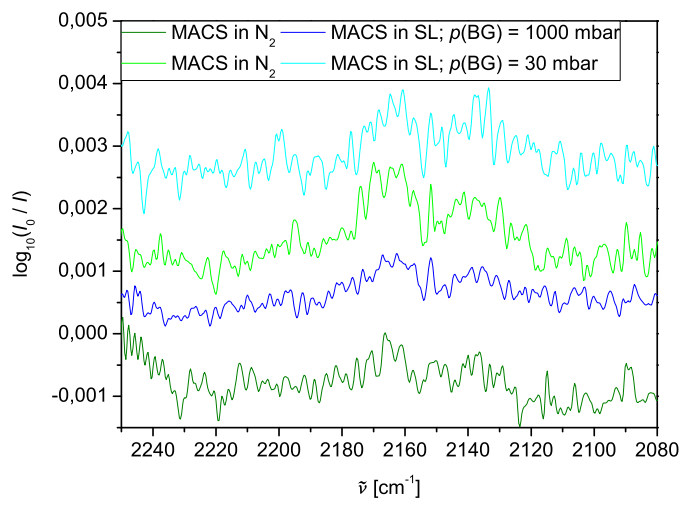

(d) Vergleich der Keten-Banden.

Abb. D.18.: Detailbereiche der Reaktionsspektren der Methacrylsäure. 


\section{D.9. Butensäure}

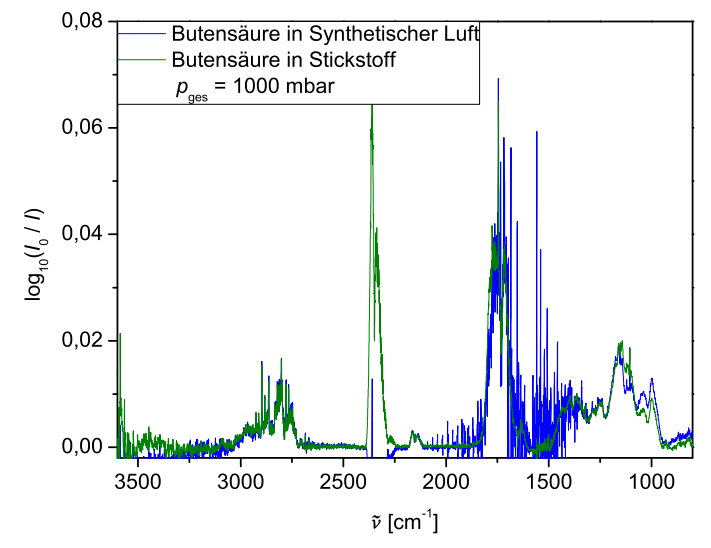

(a) Darstellung des Gesamtreaktionsspektrums der Butensäure in Synthetischer Luft und Stickstoff als Badgas bei einem Gesamtdruck von jeweils 1000 mbar.

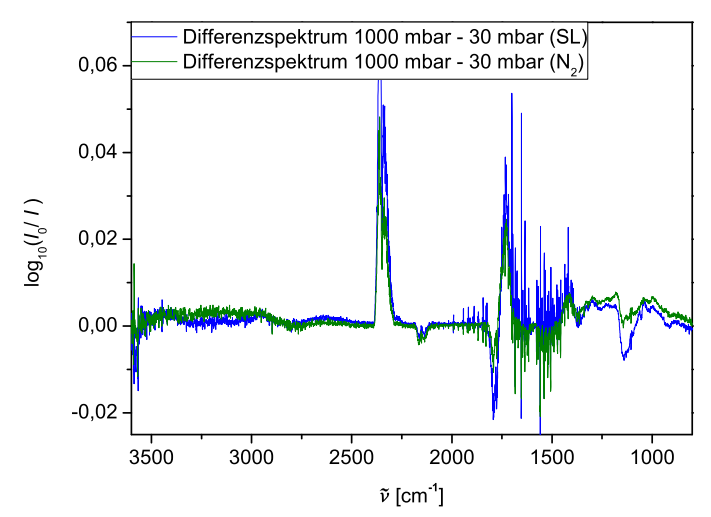

(b) Differenzspektren zwischen Hoch- und Niederdruckmessungen der Butensäure in Synthetischer Luft und Stickstoff als Badgas.

Abb. D.19.: Gesamtansicht der Reaktionsspektren bei Hochdruckbedingungen sowie der Differenzspektren unter verschiedenen Druckregimes für Butensäure.

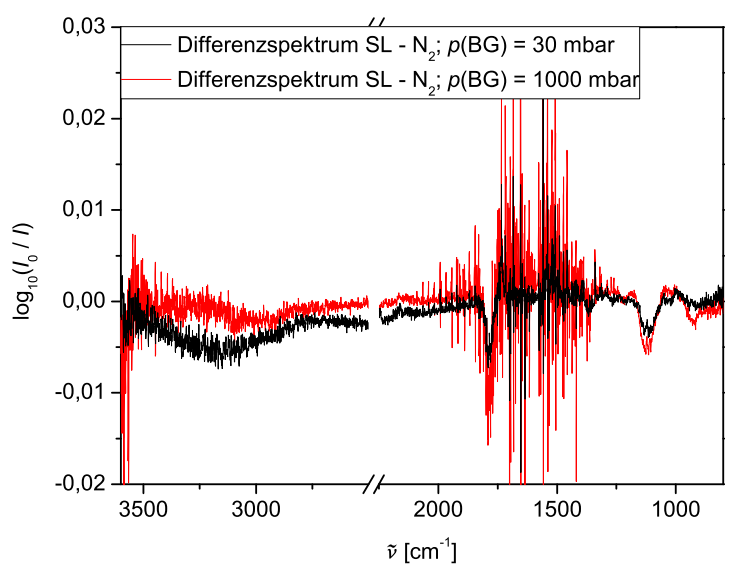

Abb. D.20.: Differenzspektren zwischen Reaktionen in Synthetischer Luft und Stickstoff für Butensäure, jeweils unter Hoch- und Niederdruckbedingungen, durch die Achsenunterbrechung wird lediglich das $\mathrm{CO}_{2}-$ Signal ausgeblendet. 


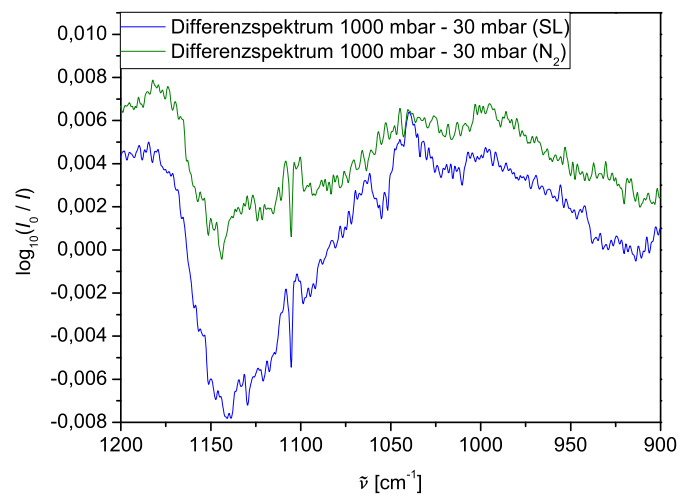

(a) Ameisensäure, Differenzspektren.

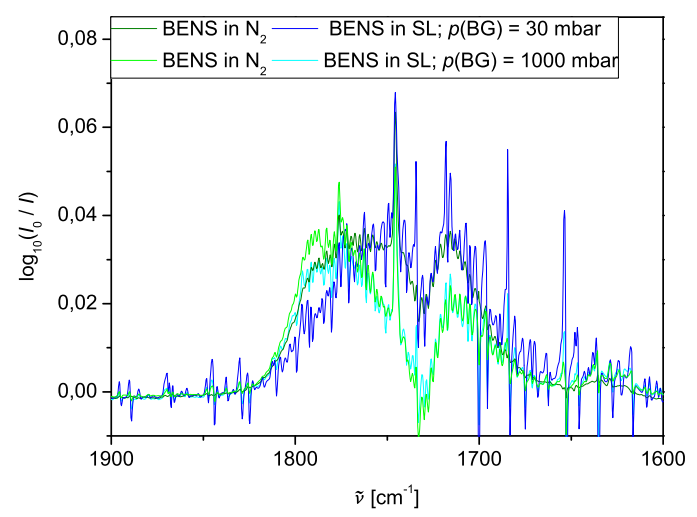

(c) Carbonylbereich.

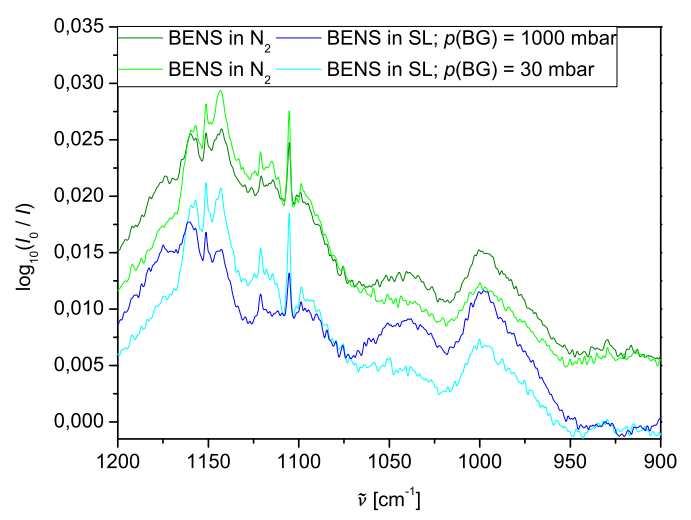

(b) Ameisensäure, Reaktionsspektren.

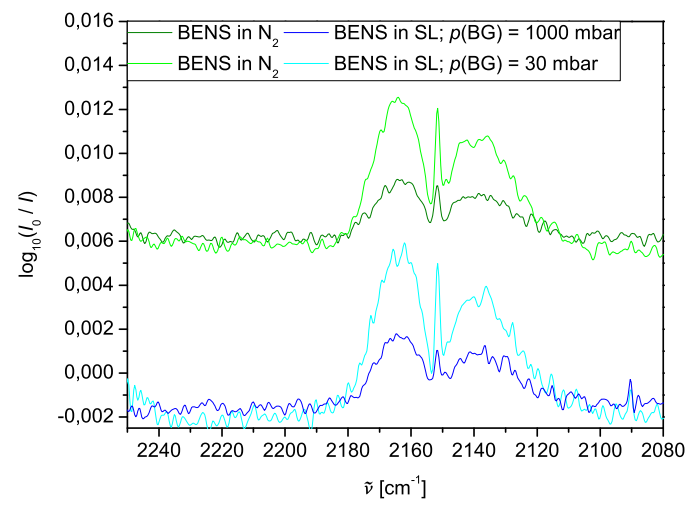

(d) Vergleich der Keten-Banden.

Abb. D.21.: Detailbereiche der Reaktionsspektren der Butensäure. 



\section{E. Ergänzende Abbildungen zur Partikelbildung - Butensäureozonolyse unter Zugabe verschiedener Additiva}

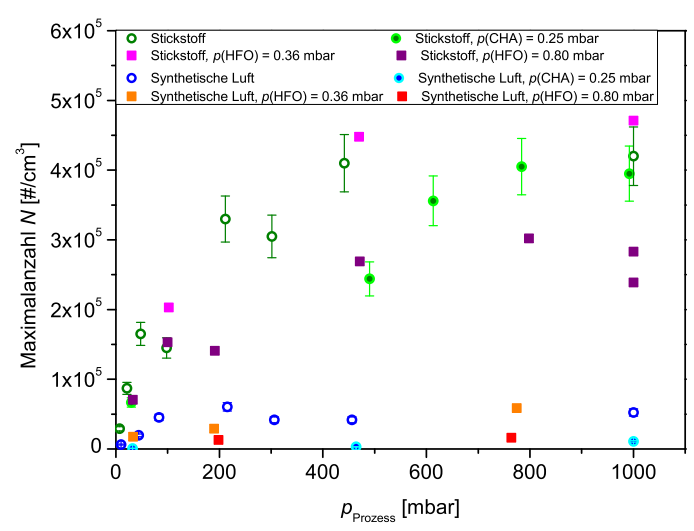

(a) Anzahlkonzentrationen.

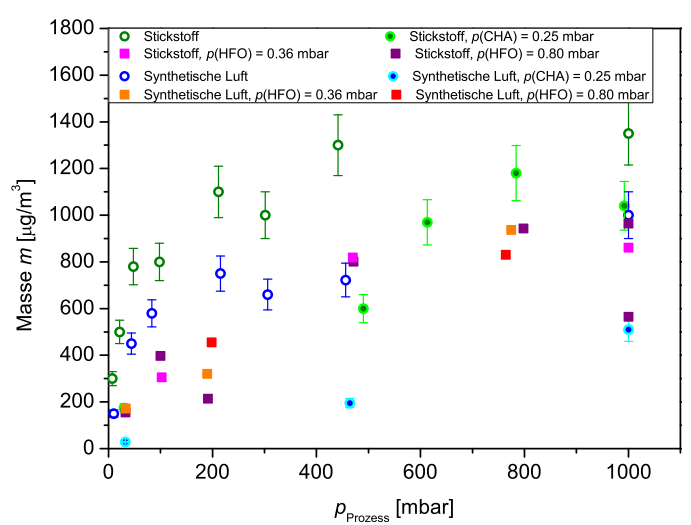

(b) Massenausbeuten.

Abb. E.1.: Untersuchung des Einflusses der Badgase und verschiedener Badgase und Additiva auf die Partikelausbeuten bei der Butensäureozonolyse $(T=295 \pm 2 \mathrm{~K})$. 

F. Ergänzende Werte zu Clusterstabilitäten unter $\mathrm{SO}_{2}$-Beteiligung 


\begin{tabular}{|c|c|c|c|c|}
\hline Verbindung / Cluster & Abb. & $\Delta_{\mathrm{f}} H(0 \mathrm{~K})^{*}\left[\mathrm{~kJ} \mathrm{~mol}^{-1}\right]$ & $\Delta_{\mathrm{f}} G^{0}(298.150 \mathrm{~K})\left[\mathrm{kJ} \mathrm{mol}^{-1}\right]$ & Vorläufer \\
\hline $\mathrm{H}_{2} \mathrm{SO}_{4}$ & ohne & -1838739.8 & -1838613.6 & $\mathrm{SO}_{2}$ \\
\hline $\mathrm{H}_{2} \mathrm{SO}_{4}^{* *}$ & ohne & -1838980.0 & -1838848.8 & \\
\hline Wasser & ohne & -200741.7 & -200676.130 & ohne \\
\hline Wasser $+\mathrm{H}_{2} \mathrm{SO}_{4}$ & $6.56 \mathrm{a}$ & -2039528.0 & -2039283.4 & \\
\hline Wasser** & ohne & -200756.4 & -200691.9 & \\
\hline Wasser $+\mathrm{H}_{2} \mathrm{SO}_{4}{ }^{* *}$ & ohne & -2039780.4 & -2039530.7 & \\
\hline Pentanal & $6.55 a$ & -713751.6 & -713096.3 & Cyclohexen \\
\hline Pentanal $+\mathrm{H}_{2} \mathrm{SO}_{4}$ & $6.56 \mathrm{~b}$ & -2552538.2 & -2551707.2 & \\
\hline 4-Oxobutansäure & $6.55 b$ & -1002512.3 & -1002077.4 & \\
\hline 4-Oxobutansäure $+\mathrm{H}_{2} \mathrm{SO}_{4}(\mathrm{I})$ & $6.56 \mathrm{c}$ & -2841316.1 & -2840700.0 & \\
\hline 4-Oxobutansäure $+\mathrm{H}_{2} \mathrm{SO}_{4}$ (II) & $6.56 \mathrm{~d}$ & -2841331.9 & -2840700.1 & \\
\hline Cyclohexanon & $6.55 \mathrm{c}$ & -813855.5 & -813146.2 & Methylencyclohexan \\
\hline Cyclohexanon $+\mathrm{H}_{2} \mathrm{SO}_{4}$ & $6.56 \mathrm{e}$ & -2652651.7 & -2651763.8 & \\
\hline Pinonaldehyd & $6.55 \mathrm{~d}$ & -1421099.5 & -1419946.0 & $\alpha$-Pinen \\
\hline Pinonaldehyd $+\mathrm{H}_{2} \mathrm{SO}_{4}$ & $6.56 f$ & -3259889.9 & -3258560.6 & \\
\hline Norpinsäure & $6.55 \mathrm{e}$ & -1609892.8 & -1608863.6 & $\alpha / \beta$-Pinen \\
\hline Norpinsäure $+\mathrm{H}_{2} \mathrm{SO}_{4}$ & $6.56 \mathrm{~g}$ & -3448701.0 & -3447622.3 & \\
\hline Pininsäure & $6.55 f$ & -1713141.8 & -1711964.7 & \\
\hline Pininsäure $+\mathrm{H}_{2} \mathrm{SO}_{4}$ & $6.56 \mathrm{~h}$ & -3551948.2 & -3550700.8 & \\
\hline
\end{tabular}

Tab. F.1.: * Ohne Nullpunktsenergiekorrektur.

${ }^{* *}$ Mit 6-311++G(3df,3pd) statt 6-311+G(d,p) als Basissatz.

Grundzustandsenergien der verschiedenen organischen Verbindungen, der Schwefelsäure und der Cluster. 


\section{G. Ergänzung zur UV-Analytik}

Die größte Fehlerquelle für die Bestimmung der Geschwindigkeitskoeffizienten bildet wahrscheinlich die Ozonkonzentrationsbestimmung über UV-Absorption (s. Kap. 4.2.1 und 4.2.1.2). Während sich die Konzentration der Kohlenwasserstoffverbindungen mit einer hohen Genauigkeit volumetrisch bestimmen lässt (nach Ergebnissen von WOLF 5 - $10 \%$ bei Eingabe über die Vormischkammer [48], bei direktem Einfüllen in die Aerosolzelle ist die Abweichung sogar noch eine Größenordnung kleiner), ist die Bestimmung des Ozonanfangspartialdrucks scheinbar fehlerbehafteter.

In Tabelle 4.2 (Kapitel 4.2.2, S. 94) wurde gezeigt, dass die Ergebnisse für die Ozonkonzentration nicht sehr stark von der verwendeten Messart abhängen, sondern unabhängig von verwendetem UV-Aufbau fast identische Ergebnisse lieferten. Allerdings stellte sich in den Partikelbildungsexperimenten mit den reinen Kohlenwasserstoffverbindungen heraus, dass die nachträglich ausgewertete Ozonkonzentration systematisch etwas unter der im Experimentverlauf mit Hilfe der aktuell angezeigten Spannung abgeschätzten lag. Der Grund muss im Umbau der UV-Optiken hin zu UV-AUFBAU 2 liegen, der direkt vor der Untersuchung dieser Verbindungen erfolgte, während die voroxidierten Spezies sämtlich mit UV-AUFBAU 1 bearbeitet wurden. Der neuere Aufbau besitzt eine leistungsfähigere UV-Lampe, die zudem weniger Leistungsschwankungen aufweist. Daher kann davon ausgegangen werden, dass mit diesem Werte mit einer höheren Verlässlichkeit erhalten werden. Dies hatte in den Partikelbildungsmessungen keine großen Auswirkungen, wie einige Nachmessungen mit Butensäure bestätigten.

Allerdings wurden in den Kinetikexperimenten signifikante und systematische vom UV-Aufbau abhängige Unterschiede zwischen den Geschwindigkeitskoeffizienten festgestellt. Die Werte mit UV-AUFBAU 2 fielen deutlich höher aus, wie ein Vergleich der Werte für Butensäure auf Seite VII zeigt. 
Vermutlich bietet die UV-Lampe des UV-AUFBAU 2 durch die höhere Strahlungsleistung eine verbesserte Nachweisempfindlichkeit, während die geringere Leistung und höhere Schwankungsanfälligkeit des älteren Modells zu einer tendenziellen Überschätzung der Ozonkonzentration geführt haben können, was den Geschwindigkeitskoeffizienten verringert (s. Gl. 2.17).

Allerdings kann davon ausgegangen werden, dass sich diese Unsicherheiten noch immer innerhalb der üblichen Schwankungsbreite von Kinetikmessungen, die immer auch ein Stück weit vom experimentellen Aufbau beeinflusst werden, bewegen. Unter Umständen ist trotzdem eine Überarbeitung der Auswertungsschemata sinnvoll, um genauere Aussagen über geringere Ozonkonzentrationsbereiche treffen zu können. 


\section{H. Ergänzende Reaktionsmechanismen}




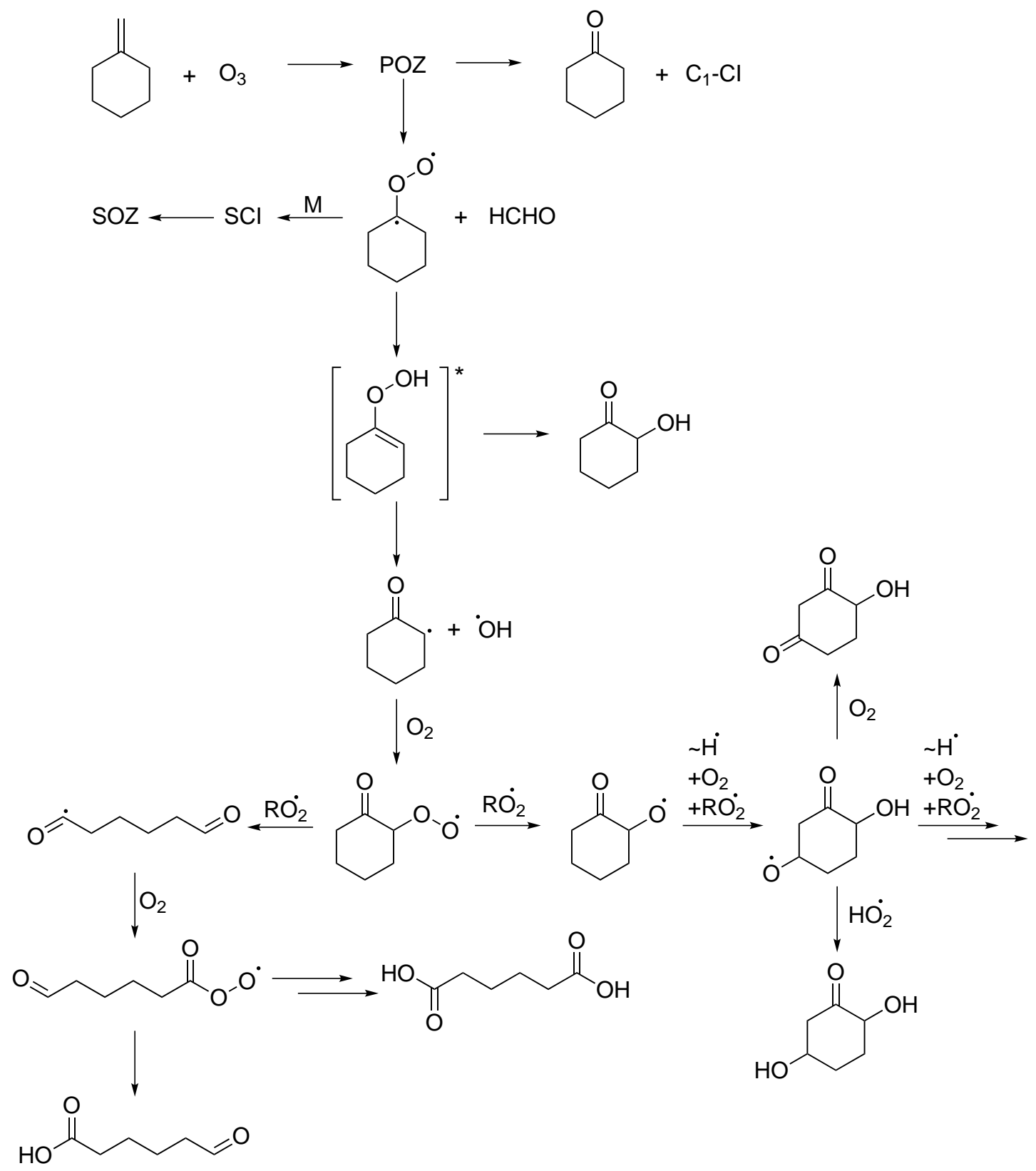

Abb. H.1.: Reaktionsmechanismus für die Ozonolyse von Methylencyclohexan nach HATAKEYAMA et al. [135] mit Ergänzungen nach WoLF [48], $\mathrm{KOCH}$ et al. [9] sowie WALSER et al. [162]. 


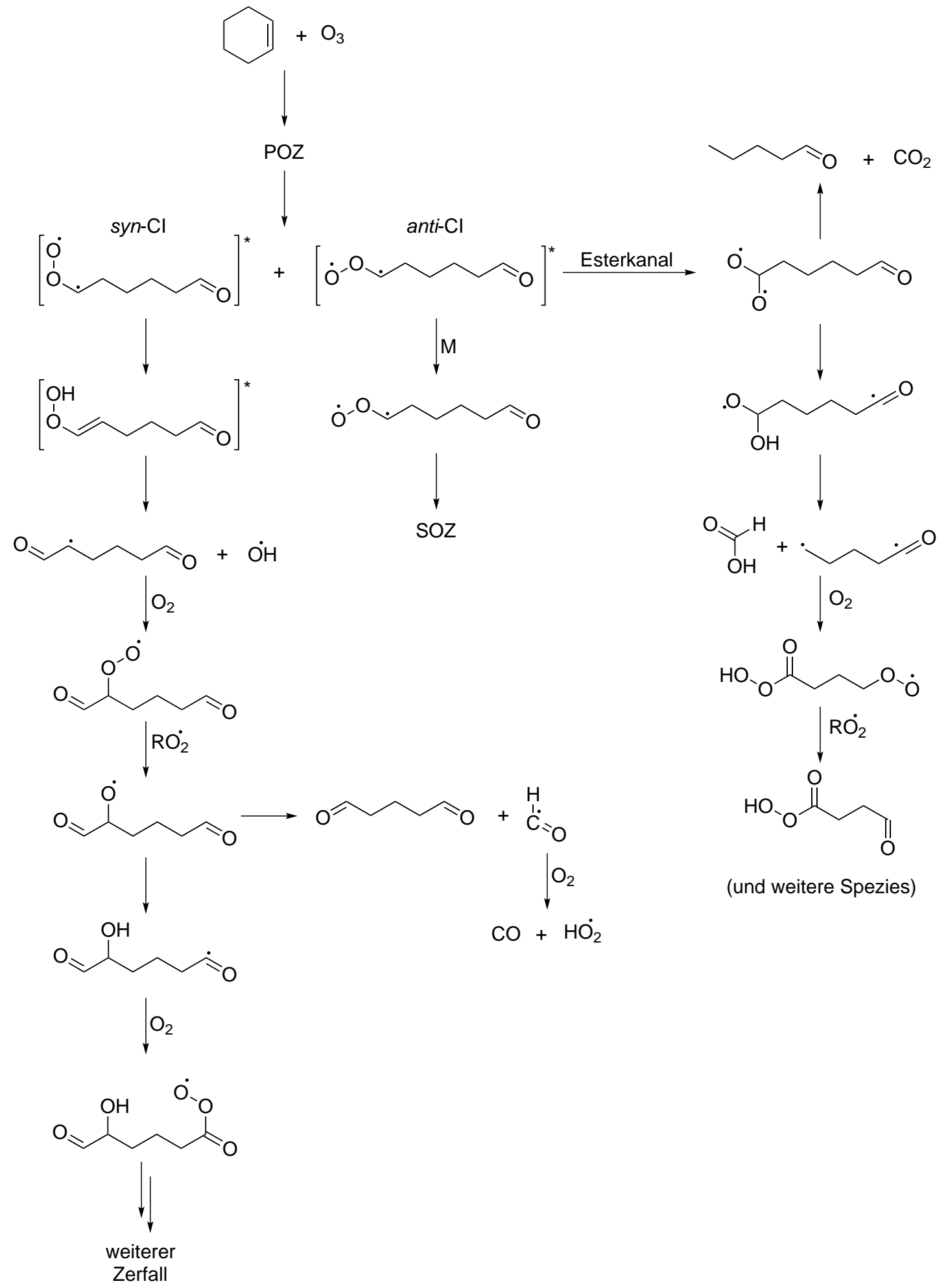

Abb. H.2.: Reaktionsmechanismus für die Ozonolyse von Cyclohexen nach ASCHMANN et al. [122] mit Ergänzungen nach JoHNSON et al. [33] sowie KEYWOOD et al. [55] . 


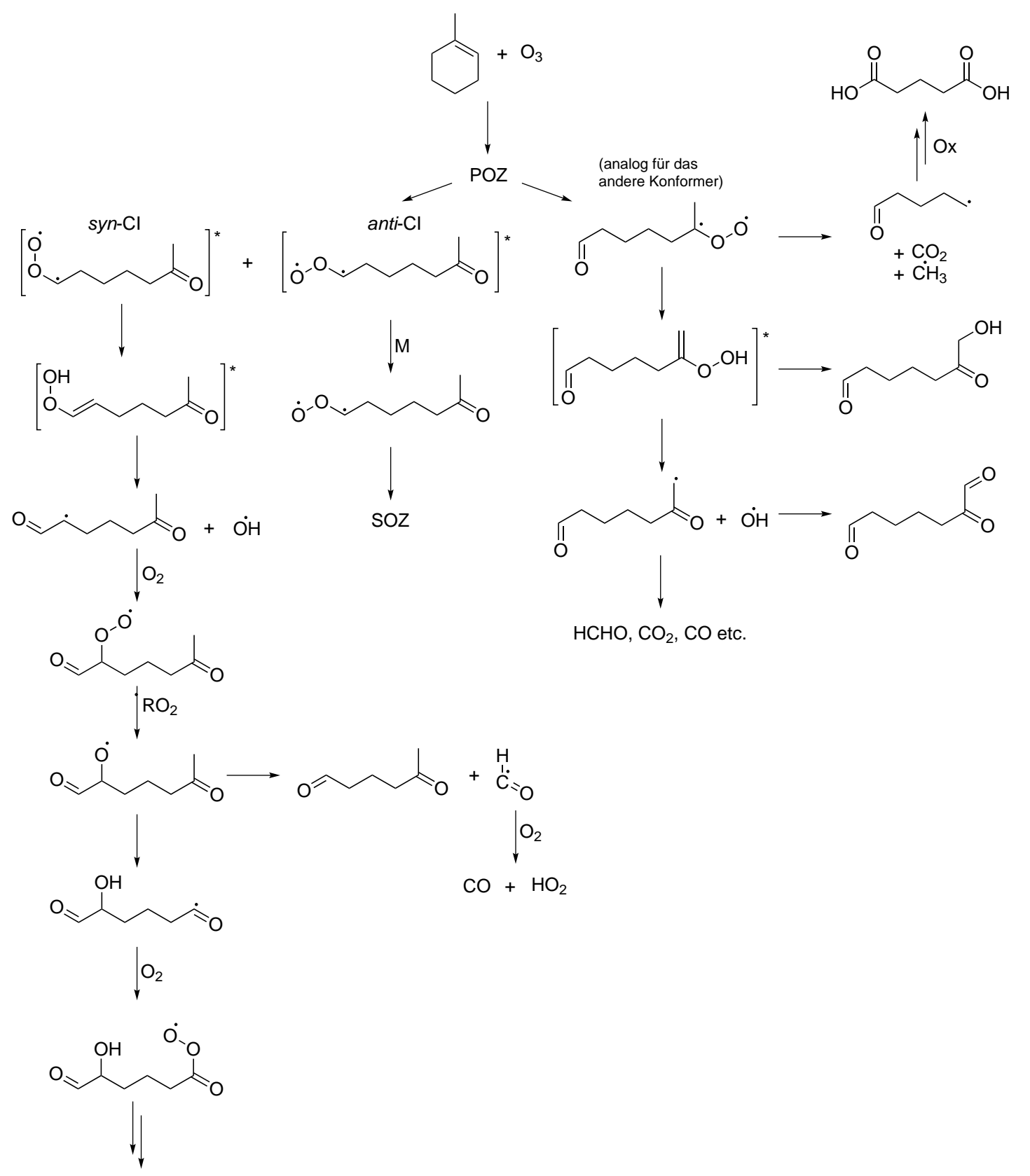

Abb. H.3.: Methylcyclohexenozonolyse, zu erwartende Zerfallsschritte. 


\section{Potenzielle Folgereaktionen voroxidierter Edukte}

\section{I.1. Abfangreaktionen des CRIEGEE-Intermediates und gebildete Produkte als Gegenstand zukünftiger Untersuchungen}

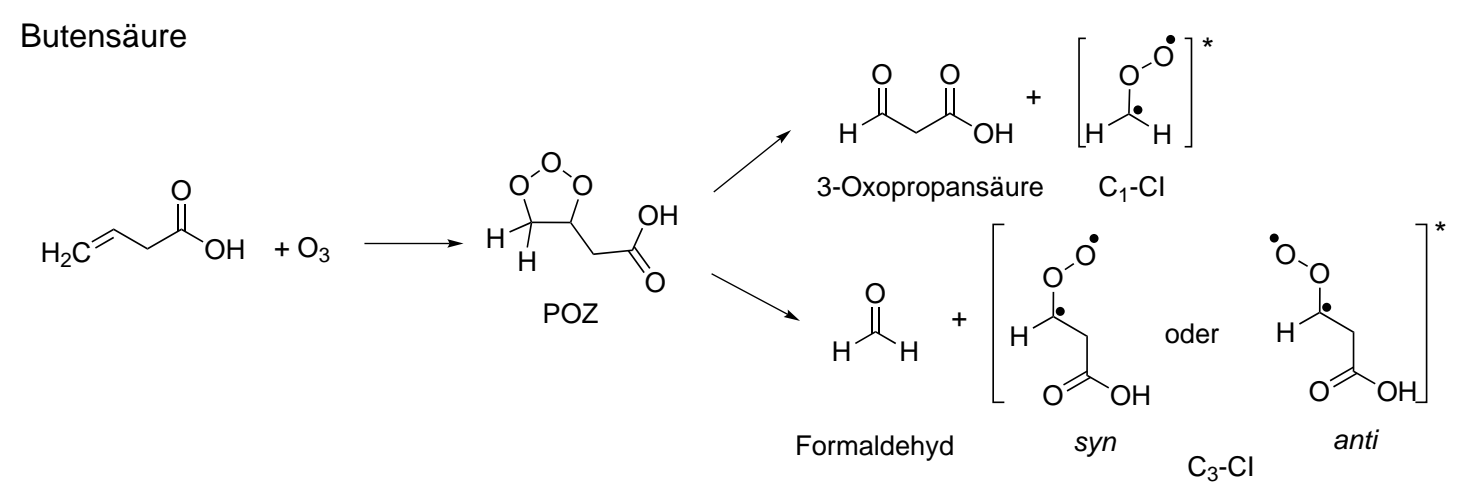

Abb. I.1.: Initialer Schritt der Ozonolysereaktion der Butensäure. 
Unimolekulare Folgereaktionen:

Zerfall des syn-Cl über den Hydroperoxidkanal<smiles>C[C@H]1C[C@@H](CC(=O)O)[C@H](C)[CH]O1</smiles>

syn<smiles>O=C(O)/C=C/OO</smiles>

Vinylhydroperoxid<smiles>CCC=C(O)CC</smiles><smiles>O=CCC(=O)O</smiles>

Vinoxylradikal

((E)-3-Hydroperoxyacrylsäure)

Zerfall von $\mathrm{C}_{1}-\mathrm{Cl}(\mathrm{R}=\mathrm{H})$ und anti- $\mathrm{Cl}\left(\mathrm{R}=\mathrm{C}_{2} \mathrm{H}_{3} \mathrm{O}_{2}\right)$

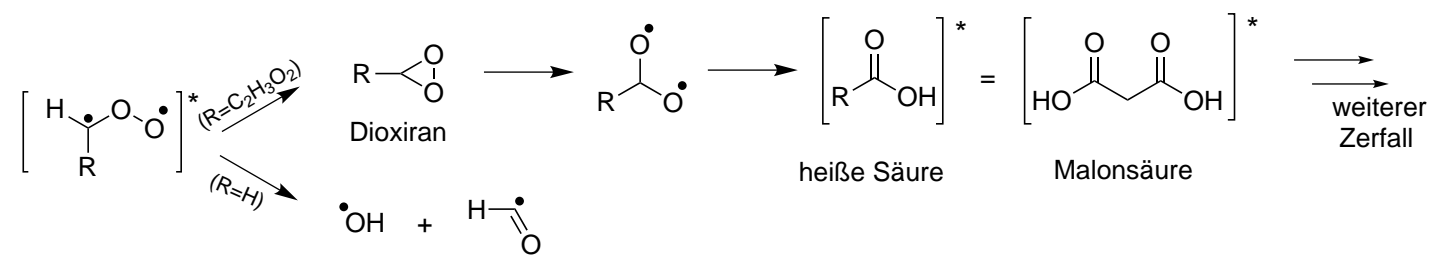

Vorschlag nach Neeb (1999)

Bimolekulare Folgereaktionen der stabilisierten $\mathrm{Cl}$ (am Beispiel Wasser und des verwendeten Edukts, weitere Reaktionen analog zu Abb. 2.7): unsubstituiert
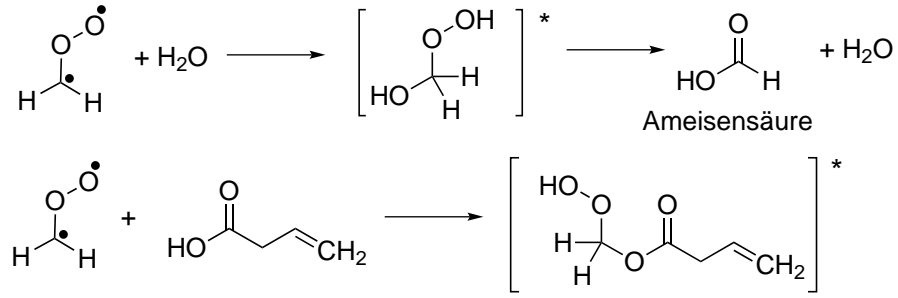<smiles>O=C(O)C[C@H](OO)O[C@@H](O)OO</smiles>

3-Hydroperoxy3-hydroxypropansäure
ODER<smiles>O=C(O)CC(=O)O</smiles>

Malonsäure
ODER<smiles>O=CCC(=O)O</smiles>
3-Oxopropansäure<smiles>COO[CH]CC(=O)O</smiles><smiles>C=CCC(=O)O</smiles><smiles>C1CCCCC1</smiles><smiles>C=CCC(=O)OC(CC(=O)O)OO</smiles>

3-(But-3-enoyloxy)3-hydroperoxypropansäure

Abb. I.2.: Folgechemie der Butensäure in Analogie zum Vorschlag von NEEB et al. [59] für Methacrylsäure. 
Vorschlag nach Neeb (1999)

Bimolekulare Folgereaktionen der stabilisierten $\mathrm{Cl}$ (am Beispiel Wasser und des verwendeten Edukts, weitere Reaktionen analog zu Abb. 2.7):

unsubstituiert
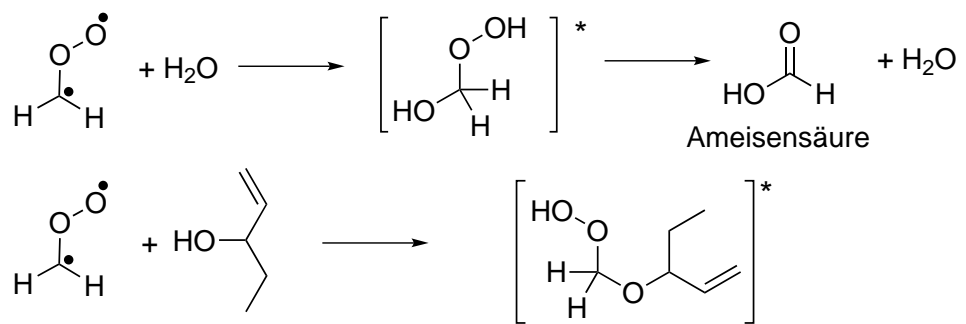

monosubstituiert

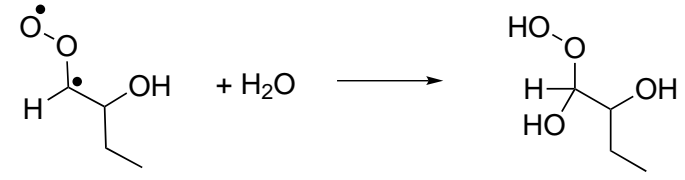

1-Hydroperoxybutan-1,2-diol<smiles>C=CC(O)CC</smiles>

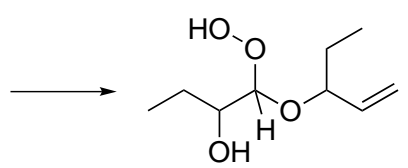

1-Hydroperoxy-1-(pent-1-en-3-yloxy)butan-2-ol

Abb. I.3.: Folgechemie des 1-Penten-3-ols in Analogie zum Vorschlag von NEEB et al. [59] für Methacrylsäure. 
Bimolekulare Folgereaktionen am Beispiel Wasser, der Produkte Formaldehyd und 2-Oxobutanal sowie des Eduktmoleküls unsubstituiert
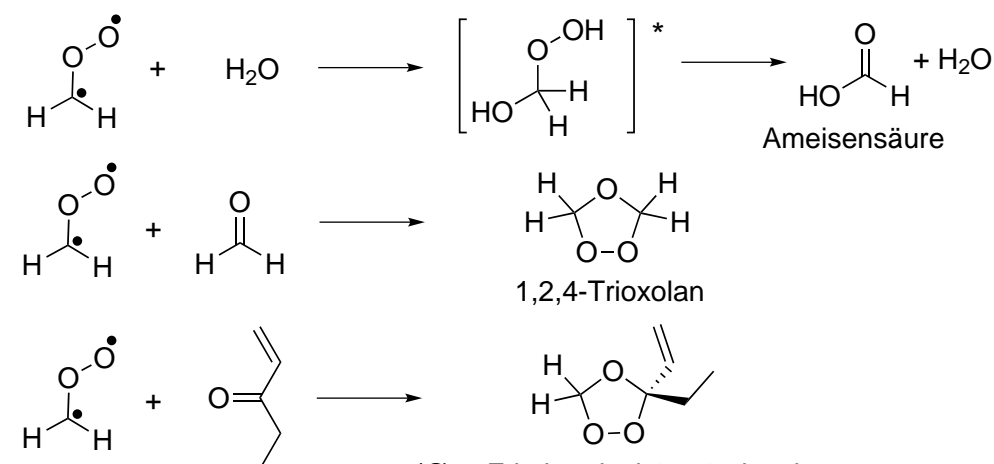

(S)-3-Ethyl-3-vinyl-1,2,4-trioxolan

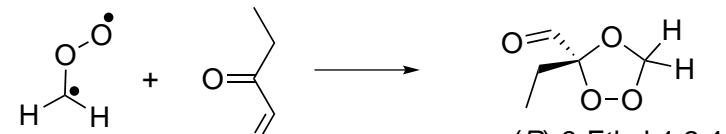

(R)-3-Ethyl-1,2,4trioxolane-3-carbaldehyd

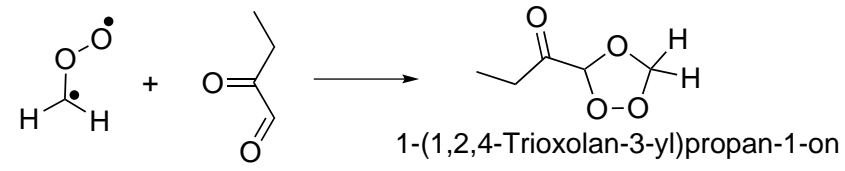

monosubstituiert

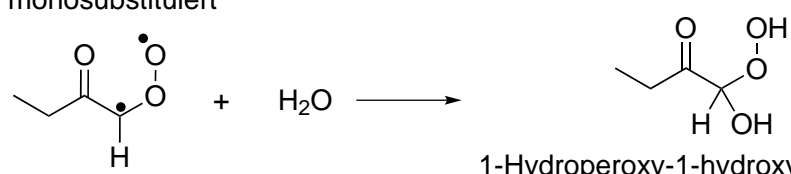

1-Hydroperoxy-1-hydroxybutan-2-on<smiles>CCC=O</smiles><smiles>CCC(=O)[C@@H]1[CH]COO1</smiles>

(R)-1-(1,2,4-Trioxolan-3-yl)propan-1-on<smiles>C=CC(=O)CC</smiles><smiles>C=CC1(CC)OOC(C(=O)CC)O1</smiles>

$1-((3 R, 5 S)-5$-Ethyl-5-vinyl1,2,4-trioxolan-3-yl)propan-1-on<smiles>CCC(=O)CC(=O)O[O+]</smiles><smiles>CCC(=O)C1OOC(C=O)(CC)O1</smiles>
3-Ethyl-5-propionyl-1,2,4trioxolane-3-carbaldehyde<smiles>CCC(=O)CC(=O)O[O-]</smiles>

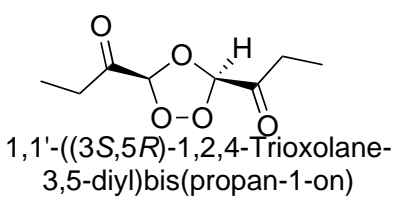

Abb. I.4.: Folgechemie des 1-Penten-3-ons in Analogie zum Vorschlag von NEEB et al. [59] für Methacrylsäure. 


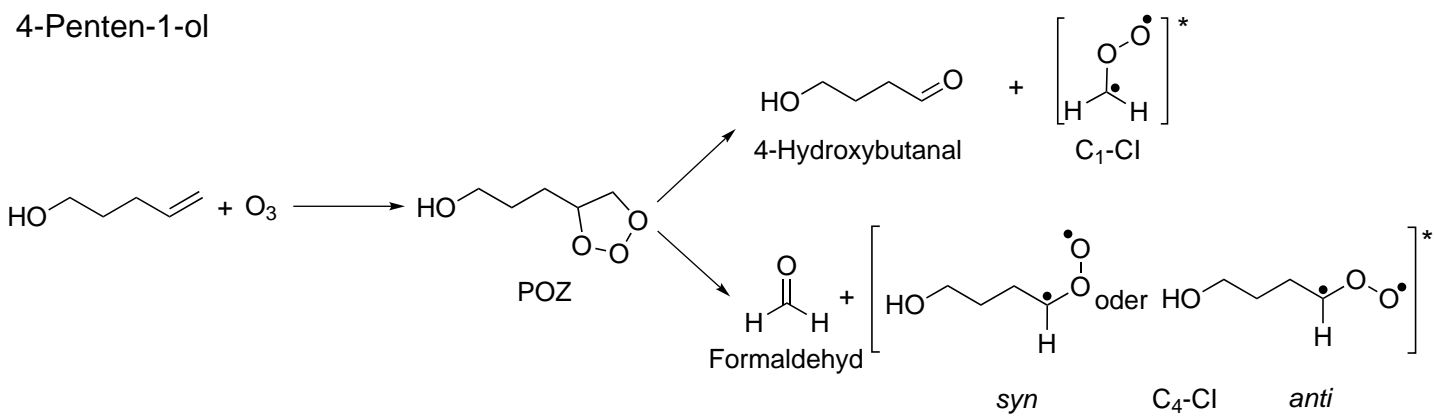

(Weitere Zerfallswege analog zu vorherigen Substanzen)

Abb. I.5.: Erste Schritte der Ozonolysereaktion des 4-Penten-1-ol.

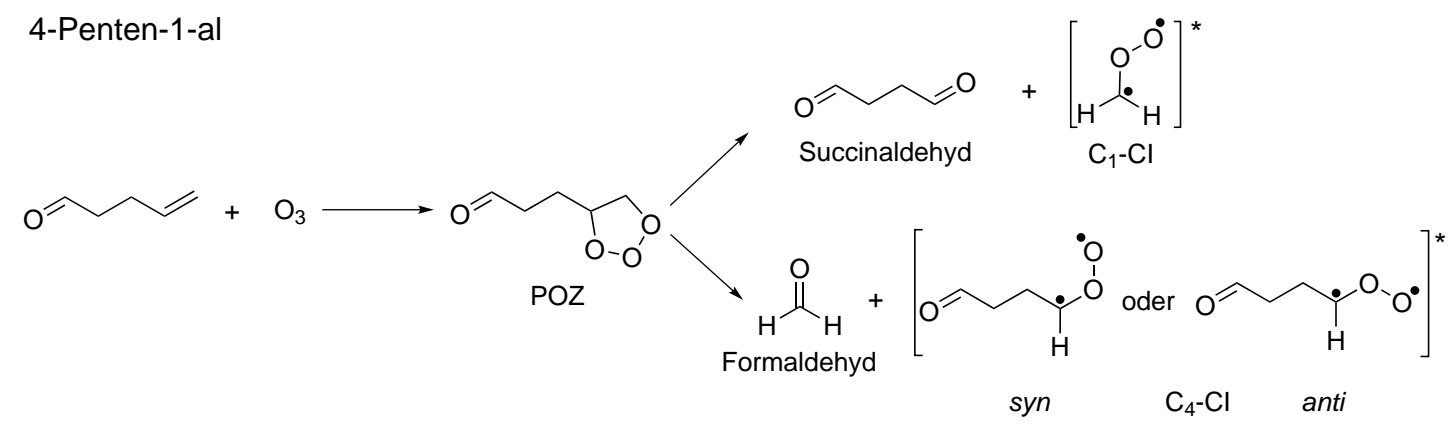

(Weitere Zerfallswege analog zu vorherigen Substanzen)

Abb. I.6.: Erste Schritte der Ozonolysereaktion des 4-Penten-1-al. 
Vorschlag nach Neeb (1999)

Bimolekulare Folgereaktionen der stabilisierten $\mathrm{Cl}$ (am Beispiel Wasser und des verwendeten Edukts, weitere Reaktionen analog zu Abb. 2.7):

unsubstituiert
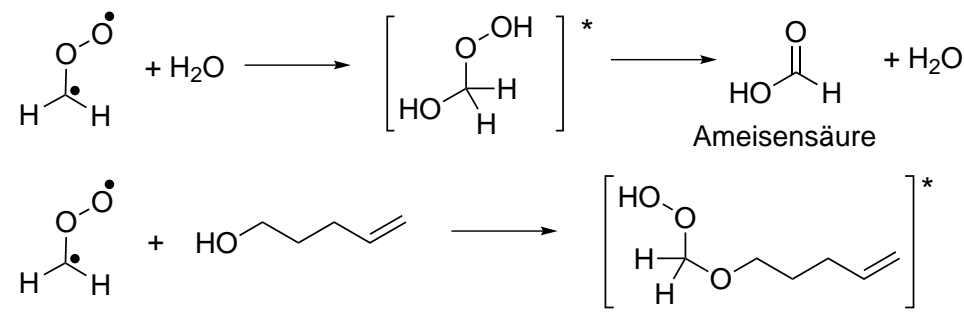

monosubstituiert<smiles>CO[C@H](O)CCCO</smiles><smiles>OCCCC(O)OO</smiles>

1-Hydroperoxybutan-1,4-diol<smiles>[O]OCCCCO</smiles>

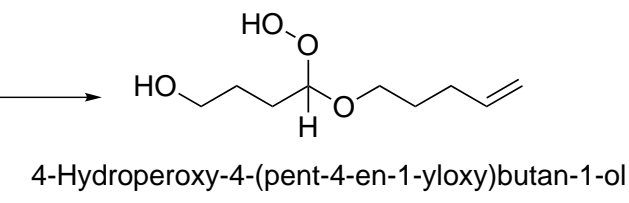

Abb. I.7.: Folgechemie des 4-Penten-1-ols in Analogie zum Vorschlag von NEEB et al. [59] für Methacrylsäure. 
Bimolekulare Folgereaktionen am Beispiel Wasser, der Produkte Formaldehyd und Succinaldehyd sowie des Eduktmoleküls

unsubstituiert
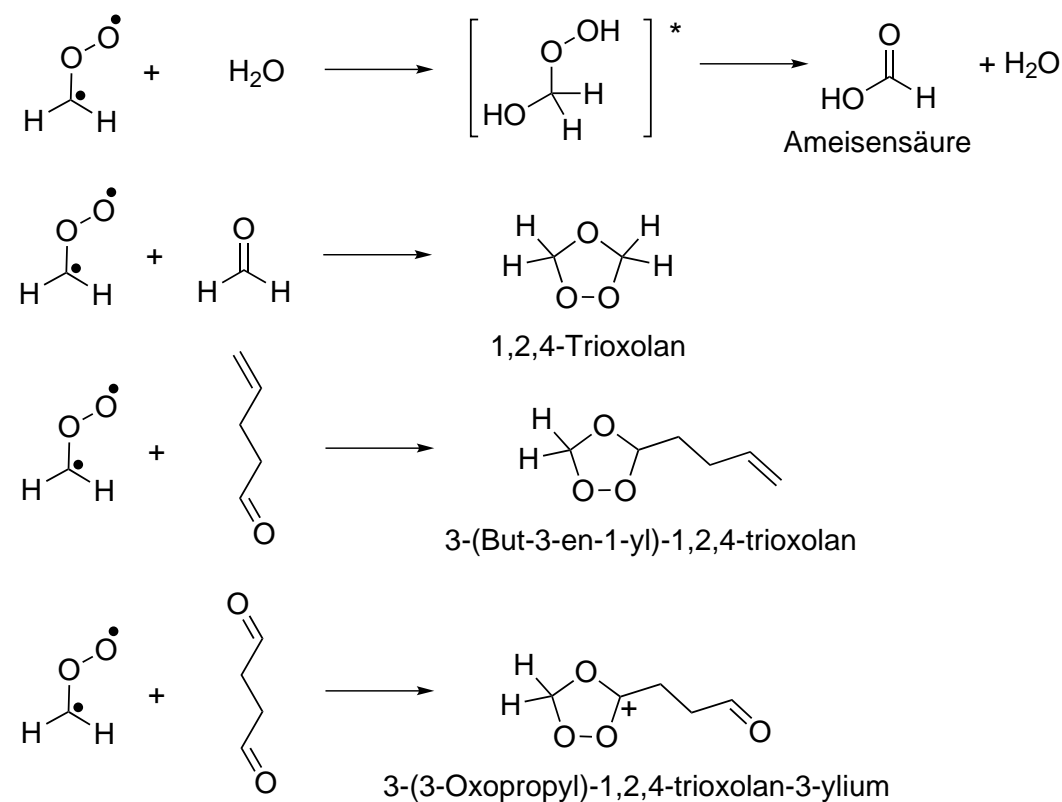

monosubstituiert<smiles>O=CCCCO[O+]</smiles><smiles>O=CCCC(O)OO</smiles>

4-Hydroperoxy-4-hydroxybutanal<smiles>CC=CCCCO[O+]</smiles><smiles>C[C@H]1OO[C@H](CCC=O)O1</smiles>

(R)-3-(1,2,4-Trioxolan-3-yl)propanal<smiles>C=CCCCC=O</smiles><smiles>C=CCC[C@H]1OO[C@@H](CCC=O)O1</smiles>

3-((3S,5S)-5-(But-3-en-1-yl)1,2,4-trioxolan-3-yl)propanal<smiles>CCCCC=O</smiles>

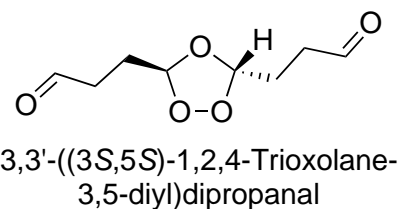

Abb. I.8.: Folgechemie des 4-Penten-1-als in Analogie zum Vorschlag von NEEB et al. [59] für Methacrylsäure. 
Vorschlag nach Neeb (1999)

Bimolekulare Folgereaktionen der stabilisierten $\mathrm{Cl}$, entsprechend des Mechanismus für Butensäure (am Beispiel Wasser und des verwendeten Edukts, weitere Reaktionen analog zu Abb. 2.7)

unsubstituiert
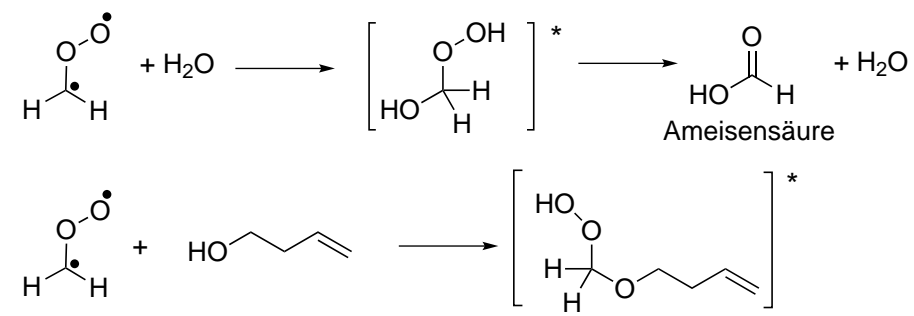

monosubstituiert

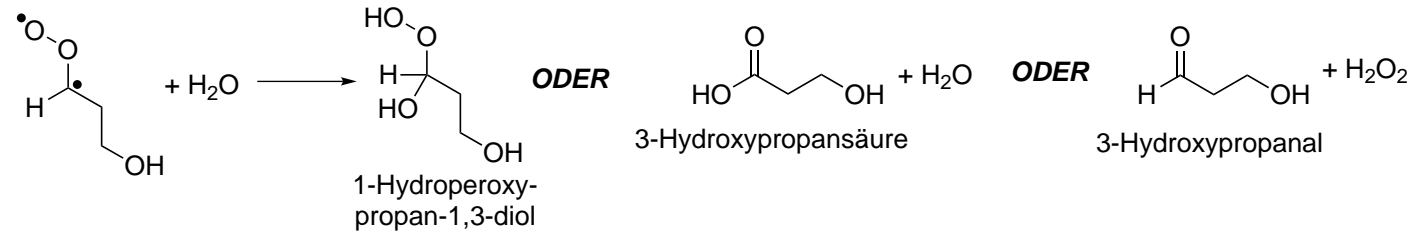<smiles>COOCCCO</smiles><smiles>C/C=C\CCOC(CCO)CCOO</smiles>

Abb. I.9.: Folgechemie des Butenols in Analogie zum Vorschlag von NeEB et al. [59] für Methacrylsäure. 


\title{
Verwendete Abkürzungen
}

\author{
AIM Aerosol Instrument Manager \\ AZ Aerosolzelle \\ BVOC biogenic volatile organic compound \\ CCN cloud condensatiion nuclei \\ CI CRIEGEE-Intermediat \\ CPC Condensation Particle Counter \\ DMA Differentieller Mobilitätsanalysator \\ FTIR Fourier-Transform-Infrarot \\ FWHM full width at half maximum \\ HD Hochdruck
}

HPLC/ESI-MS high performance liquid chromatography / electrospray ionization - mass spectrometry

HWHM half width at half maximum

IPCC Intergovernmental Panel on Climate Change

IR Infrarot

LDMA Long Differential Mobility Analyser

Ipm Liter pro Minute

MCT Quecksilber-Cadmiumtellurid 
Mischozonolyse Ozonolyse unter Zugabe von $\mathrm{SO}_{2}$

ND Niederdruck

NDMA Nano Differential Mobility Analyser

NIM Nukleationsinduzierendes Molekül

POZ Primärozonid

reine Kohlenwasserstoffverbindungen nicht vorfunktionalisierte Alkene

$\mathbf{R H}$ relative humidity

SCI stabilisiertes CRIEGEE-Intermediat

SL Synthetische Luft

slm Standard Liter pro Minute

SMPS Scanning Mobility Particle Sizer

SOA Sekundäres Organisches Aerosol

SOZ Sekundärozonid

UV Ultraviolett

VMK Vormischkammer

VOC volatile organic compound 


\section{Tabellenverzeichnis}

2.1. Ausbeuten der Zerfallsreaktionen des $\mathrm{C}_{1}-\mathrm{Cl} . \ldots . \ldots 25$

2.2. Ausbeuten des stabilisierten CRIEGEE-Intermediates (SCI) für verschiedene zyklische Alkene. Sämtliche Werte stammen von PAULSON et al. [43]. . . . . . . . . . . . . . . . . . . . 27

2.3. Exemplarischer Veraleich der Geschwindiakeitskoeffizienten für die Ozonolyse- und relevante Radikalreaktionen. . . . . . . . . . 49

2.4. Geschwindigkeitskoeffizienten der in Gl. 2.30 bis 2.36 gezeigten Reaktionen. . . . . . . . . . . . . . . . . 55

3.1. Einfüllschema der Edukte, abhängig von ungesättigter Kohlenwasserstoffverbindung und Experimentvariante. *Bei Radikalfängerexperimenten wurde das Alken in der Aerosolzelle vorgelegt.

4.1. Angabe der experimentell für den verwendeten Versuchsaufbau ermittelten Kalibrierkoeffizienten. Die Werte für Ozon, $\alpha$-, $\beta$ Pinen, Methylencyclohexan und 1-Methylcyclohexen wurden von WOLF [48] übernommen. . . . . . . . . . . . . . . . . . .

4.2. Vergleich der bestimmten Ozonkonzentrationen $x$ mit Hilfe verschiedener Messmethoden. Zum Vergleich der Werte aus VMK und $A Z$ müssen erstere Werte mit dem Faktor für das Volumenverhältnis $(f=0.26)$ multipliziert werden. Die Ergebnisse sind in Klammern angegeben. . . . . . . . . . . . . . . . . . . . . 94

4.3. Bestimmung der relativen Luftfeuchte in der Aerosolzelle. . . . . 101

5.1. Hauptprodukte der Ozonolysereaktion verschiedener ungesättiqter Verbindungen, die als Teil der Oligomere berücksichtigt wurden.116 
6.1. Ergebnisse der Kinetikauswertungen für reine $\mathrm{C}_{6}{ }^{-}$ Kohlenwasserstoffverbindungen. Bei den in den Referenzen verwendeten Fängersubstanzen handelt es sich um $\mathrm{CHA}$ : Cyclohexan, 2BuOH: 2-Butanol. . . . . . . . . . . . . . . . 121

6.2. Ergebnisse der Kinetikauswertungen für die ungesättigten Alkohole. . . . . . . . . . . . . . . . . . . . . . . . . . . 124

6.3. Ergebnisse der Kinetikauswertungen für die ungesättigten Carbonylverbindungen. . . . . . . . . . . . . . . . 126

6.4. Ergebnisse der Kinetikauswertungen für die ungesättigten Carbonsäuren. Bei experimentell ermittelten Werten wird angegeben, welcher Aufbau zur Ozonbestimmung (vgl. Abschnitt 4.2.1.2) verwendet wurde.

6.5. Nachgewiesene Produkte für 1-Hexen und 2-Methyl-1-penten. .137

6.6. Nachqewiesene Produkte für 3-Buten-1-ol, 1-Penten-3-ol und 1Penten-4-ol. . . . . . . . . . . . . . . . . . . . . . . . . . . . 141

6.7. Nachaewiesene Produkte für 1-Penten-3-on und 4-Pentenal. . . 146

6.8. Nachgewiesene Produkte für Methacrylsäure und Butensäure. . 152

6.9. Experimentell erhaltene $\mathrm{OH}$-Ausbeuten für die untersuchten Substanzen. . . . . . . . . . . . . . . . . . . . 170

6.10. Abschätzung der CO-Ausbeuten der Ozonolysen von 1-Hexen und 2-Methyl-1-penten auf alleiniger Grundlage des $\mathrm{C}_{1}$ - $\mathrm{Cl}$ Zerfalls mit Literaturwerten von ATKINSON [39] und RICKARD [37]. Vergleich mit experimentell bestimmten Ausbeuten bei $\mathrm{k}_{\text {ges }}=1000 \mathrm{mbar}$ in Synthetischer Luft, normiert auf einen Ozonverbrauch von $1 \mathrm{~Pa} . \ldots \ldots$. . . . . . . . . . . . . 173

6.11. Auflistung der Anfangspartialdrücke der verwendeten Edukte für Partikelbildungsexperimente bei variablem Gesamtdruck unter $\mathrm{SO}_{2}$-Zugabe, generell kann hier von einer einfüllbedingten Unsicherheit von $10-20 \%$ für $\mathrm{O}_{3}$ und $\mathrm{SO}_{2}$ ausgegangen werden. . 220

6.12. Anfangspartialdrücke der verwendeten Additiva für Partikelbildungsexperimente zum Wassereinfluss unter $\mathrm{SO}_{2}$-Zugabe, Alken- und Ozonkonzentrationen sowie Reaktionszeit wie in Tab. 6.11 . . . . . . . . . . . . . . . . . . . . . . 220 
6.13. Anfangspartialdrücke der verwendeten Additiva für Partikelbildungsexperimente zum Carbonyleinfluss unter $\mathrm{SO}_{2}$-Zugabe, Alken- und Ozonkonzentrationen sowie Reaktionszeit wie in Tab. 6.11. . . . . . . . . . . . . . . . . . . . . . . . . . 221

6.14. Relative Fehler der Partikelausbeuten in $\mathrm{SO}_{2}$-Anwesenheit. . . . 222

6.15. Ausbeuten des stabilisierten großen CRIEGEE-Intermediates (SCI) für verschiedene zyklische Alkene. Sämtliche Werte stammen von PAULSON et al. [43]. Zusätzlich werden $\mathrm{OH}$-Ausbeuten angegeben, für die die Referenzen jeweils genannt werden. Alle Studien wurden unter Atmosphärendruck durchgeführt. . . . . . . 234

6.16.Zusammenstellung der absoluten und relativen Veränderungen in den Ausbeuten bei $\mathrm{SO}_{2}$-Zugabe. ${ }^{*} \mathrm{Im}$ Falle des $\beta$ Pinen wurden aufgrund der Abnahmen der Massenausbeute bei $p_{\text {ges }}=450$ mbar zum Vergleich der Wert bei 100 mbar verwendet. Die Ergebhisse bei $p_{\text {ges }}=450$ mbar betrugen $m=2253.3 \mu \mathrm{g} \mathrm{m}^{-3}$ bzw. 2.36. . . . . . . . . . . . . . . . . . . . 236

6.17. Absolute und relative Veränderungen der Partikelausbeuten durch Wasserzugabe in Experimenten mit verschiedenen $\mathrm{SO}_{2}$ Zugaben. . . . . . . . . . . . . . . . . . . . . . . 243

6.18. *Mit 6-311++G(3df,3pd) statt 6-311+G(d,p) als Basissatz. ** Zum Vergleich: $D_{0}=68.8 \mathrm{~kJ} \mathrm{~mol}^{-1}$ mit dem Basissatz D95(d,p) [147]. ${ }^{* * *}$ Zum Vergleich: $\Delta_{\mathrm{R}} G^{0}=-4.3 \mathrm{~kJ} \mathrm{~mol}^{-1}$ [147], Basissatz wie oben. Wasserstoffbrückenbindungslängen $r$, Dissoziationssenergie $D_{0}$ und Reaktionsenergie $\Delta_{R} G^{0}$ der Oligomere, Berechnungen siehe Kapitel 5.4. . . . . 248

7.1. Zusammenfassung der qualitativ nachgewiesenen Gasphasenprodukte für Alkene und Alkenole. Angegeben wird, unter welchen Bedingungen sie (am ausgeprägtesten) auftraten, auf welche Weise der Nachweis erfolgte und wo die entsprechenden Spektren zu finden sind. Mit „(?)“ geke onzeichnete Verbindungen stehen für unsichere Nachweise bzw. berechnete Modellverbindungen für bestimmte Strukturmotive. 
7.2. Zusammenfassung der qualitativ nachgewiesenen Gasphasenprodukte für höher oxidierte Verbindungen. Angegeben wird, unter welchen Bedingungen sie (am ausgeprägtesten) auftraten, auf welche Weise der Nachweis erfolgte und wo die entsprechenden Spektren zu finden sind. Mit „(?)“ gekennzeichnete Verbindungen stehen für unsichere Nachweise bzw. berechnete Modellverbindungen für bestimmte Strukturmotive. . . . . . . . . . 265

7.3. Experimentell erhaltene $\mathrm{OH}$-Ausbeuten für die untersuchten Substanzen und Partikelbildungspotentiale. Sämtliche Werte wurden zur besseren Vergleichbakeit unter Annahme linearen Verhaltens jenseits der Nukleationsschwelle [33] auf einen Ozonumsatz von $\Delta\left(\mathrm{O}_{3}\right)=0.5 \mathrm{~Pa}$ skaliert. Bis auf die Carbonsäuren $\left(\mathrm{N}_{2}\right)$ wurden sämtliche Werte in Synthetischer Luft erhalten. . . . 291

A.1. Daten zu den verwendeten Substanzen. . . . . . . . . . . . . . . II

A.2. Zusammenfassung der experimentellen Bedingungen für Kinetikmessungen. Alle Messungen wurden bei $p_{\text {ges }}=460 \pm 10 \mathrm{mbar}$ und $T=295 \pm 2 \mathrm{~K}$ durchgeführt. . . . . . . . . . . . ॥

A.3. Zusammenfassung der experimentellen Bedingungen für Produktbildungsmessungen bei $T=295 \pm 2 \mathrm{~K}$. Sofern nicht anders gekennzeichnet, wurden die Messungen sowohl in Stickstoff als auch in Synthetischer Luft durchgeführt. . . . . . . . . . . . . . III

A.4. Experimentelle Bedingungen für Partikelbildungsmessungen bei $T=295 \pm 2 \mathrm{~K} ., \mathrm{N}_{2} / \mathrm{O}_{2}$ " zeigt dabei an, mit welchen Substanzen Sauerstoffpartialdruckreihen durchgeführt wurden. $\Theta_{2}$-Reihe bei einem Gesamtdruck von ${ }^{*} 500 \mathrm{mbar} /$ bei ${ }^{* *} 100$ mbar. . . . . . . .

A.5. Geschwindigkeitskoeffizienten für die Ozonolysereaktion der verwendeten Substanzen $\mathrm{m} t \mathrm{t}$ Literaturangaben für den in den vorgestellten Experimenten entsprechendem Temperaturbereich. „Exp.best.“" kennzeichnet in der Aerosolzelle der eigenen Arbeitsgruppe bestimmte Werte. 
A.6. Angabe der Reaktionsbedingungen und Ergebnisse der in Kap. 6.1präsentierten Kinetikmessungen. Zur Benennung der einzelnen Experimente: Nach einem vierstelligen Kürzel für die Substanz folgt "S“ oder „N“ für Synthetische Luft bzw. Stickstoff als Badgas. Die am Ende stehende laufende Nummer dient lediglich der Unterscheidung. Die Farben entsprechen denen der Auftragungen in der Auswertung, Abschnitt $6.1 \ldots \ldots \ldots$ IX

B.1. Auflistung der experimentell bestimmten CO-Ausbeuten für die $\mathrm{C}_{6}$-Kohlenwasserstoffverbindungen. . . . . . . . X XI

B.2. Auflistuna der experimentell bestimmten CO-Ausbeuten für die voroxidierten Kohlenwasserstoffverbindungen. . . . . . . . . XII

F.1. * Ohne Nullpunktsenergiekorrektur. **Mit 6-311++G(3df,3pd) statt 6$311+G(d \mid p)$ als Basissatz. Grundzustandsenergien der verschiedenen organischen Verbindungen, der Schwefelsäure und der Cluster. . . . . . . . . . . . . . . . . XXXVI 



\section{Abbildungsverzeichnis}

1.1. Verschiedene Effekte auf den vom IPCC eingeführten Strahlungsantrieb (radiative forcing) und ihre Unsicherheiten, entnommen aus dem aktuellen Bericht des Weltklimarates (IPCC - Intergovernmental Panel on Climate Change) [7]. . . . . . . . . . 6

1.2. Oxidierte Komponenten und die betrachteten Abhängigkeiten im Reaktionsverhalten. . . . . . . . . . . . . 8

1.3. Reine Kohlenwasserstoffverbindungen und Darstellung der untersuchten Abhängigkeiten. . . . . . . . . . . . . . . 9

1.4. Einflussmöalichkeiten auf (möalicherweise) entscheidende Schritte im Partikelbildungsprozess. . . . . . . . . . . . 10

1.5. Reine, biogen emittierte Kohlenwasserstoffverbindungen und zugehörige Modellsubstanzen. . . . . . . . . . . . . . . 11

2.1. Schematischer Aufbau eines MICHELSON-Interferometers, nach [27], S. 512. . . . . . . . . . . . . . . . . . . . . . . 19

2.2. Der CRIEGEe-Mechanismus, nach [32]. . . . . . . . . . . 23

2.3. Möaliche Strukturen der CRIEGEE-Intermediate in Gasphasenreaktionen. . . . . . . . . . . . . . . . . . . . . 24

2.4. Zerfall der schwingungsangeregten Ameisensäure aus einem anti-Cl, nach JOHNSON und MARSTON [33]. . . . . . . . . . . 26

2.5. Zerfall eines syn-Cl, nach JoHnson et al. [33]. . . . . . . . . . 26

2.6. Mögliche bimolekulare Reaktionen des CRIEGEE-Intermediats, nach [18,33,48-51]. . . . . . . . . . . . . . . . . . . . 28

2.7. Postulierte Oligomerisierungsreaktion bei der trans-3-HexenOzonolyse, nach SADEZKY et al. [36]. . . . . . . . . . . . . . 29

2.8. Postulierte Folgechemie des Vinylhydroperoxidradikals nach Hoyermann et al. sowie Wolf [20, 48]. . . . . . . . . . . 30 
2.9. Einfluss auf gebildete Produkte durch Radikale bei der $\beta$ Pinenozonolyse nach DOCHERTY et al. [54]. . . . . . . . . . . . 31

2.10. Möaliche Strukturen der CRIEGEE-Intermediate für die verwendeten Grundstrukturen der reinen Kohlenwasserstoffverbindungen. 34

2.11. Unimolekulare Folgechemie des 1-Hexens, adaptiert nach WOLF 481. . . . . . . . . . . . . . . . . . . . . . . . 35

2.12. Initialer Schritt der Methacrylsäureozonolyse. . . . . . . . . . . 36

2.13. Unimolekulare Folgechemie der Methacrylsäureozonolyse. . . . 37

2.14. Vorschlag zur $\mathrm{C}_{1}-\mathrm{Cl}+$ Aldehyd-Reaktion nach HATAKEYAMA et al. [58]. . . . . . . . . . . . . . . . . . . . . . . . . . . 37

2.15. Bimolekulare Folgechemie der stabilisierten $\mathrm{Cl}$ der Methacrylsäure, nach einem Vorschlag von NEEB et al. [59]. . . . . . . . . 38

2.16. Ozonolysemechanismus des 1-Penten-3-ol mit Folgereaktionen nach einem Vorschlag von O'DWYER et al. [61]. . . . . . . . . . 39

2.17. Ozonolysemechanismus des 1-Penten-3-on mit Folgeraktionen nach einem Vorschlag von O'DWYER et al. [61]. . . . . . . . . . 40

2.18. Ozonolysemechanismus des 3-Buten-1-ol mit unimolekularen Folgeraktionen nach einem Vorschlag von GROSJEAN et al. [65]. 41

2.19. Folgechemie der $\mathrm{C}_{6} \mathrm{H}_{12}+\mathrm{OH}$-Reaktion, nach ALAM et al. 40]. . 45

2.20. Folgechemie der 2-Butanol $+\mathrm{OH}$-Reaktion, entnommen aus KEYWOOD et al. [55]. . . . . . . . . . . . . . . . . . . . 46

2.21. Die säurekatalysierte Reaktion der Aldehyde, entnommen aus GARLAND et al. [95]. . . . . . . . . . . . . . . . . . . . . 57

2.22. Reaktion eines stabilisierten CRIEGEE-Intermediates mit $\mathrm{SO}_{2}$, nach HatAKeyama et al. [46, 58, 96]. . . . . . . . . . . . . . . 58

3.1. Schematischer Aufbau des Laborozonisators. . . . . . . . . . . 60

3.2. Schematischer Aufbau des Klassierers 3080L der Firma TSI (Diagramm @TSI Incorporated [98]), nach BRESCH [99], S. 60.] . 63

3.3. Funktionsweise des Impaktors, nach BRESCH [99]. S. 61. . . . 64

3.4. Schematischer Aufbau des 3022A CPC der Firma TSI (Diagramm @TSI Incorporated [102]), nach BRESCH [99], S. 67. . . . 66

3.5. Schematischer Aufbau der gesamten Apparatur, modifiziert nach WOLF [48], S. 47. . . . . . . . . . . . . . . . . . . . 68 
4.1. IR-Spektrum des Ozons - Darstellung der konzentrationsabhängigen Bandenform bei Änderung des Gesamtdruckes, entnommen aus [48]. . . . . . . . . . . . . . . . . . . . . . . . . 86

4.2. Schematischer Darstellung der UV-AufBaUten 1 bzw. 2 an Aerosolzelle bzw. Ozon-Vormischkammer zur Ozonkonzentrationsbestimmung. . . . . . . . . . . . . .

4.3. Absorbanzspektren des $\mathrm{SO}_{2}$ bei identischen Partialdrücken und verschiedenen Gesamtdrücken. . . . . . . . . . . . . . . . . . 97

4.4. Auftragungen der Absorbanz gegen den Partialdruck von $\mathrm{SO}_{2}$ für den Integrationsbereich $1405-1302 \mathrm{~cm}^{-1}$ und den Integrationsbereich 1240-1059 $\mathrm{cm}^{-1}$, es ergeben sich Korrelationskoeffizienten $>99 \%$ bei $p_{\text {ges }} \geq 500$ mbar. . . . . . . . . . . . . . . .

4.5. Auftragungen der Absorbanz gegen den Zelldruck für den Integrationsbereich 1405-1302 $\mathrm{cm}^{-1}$ und den Integrationsbereich $1240-1059 \mathrm{~cm}^{-1}$. . . . . . . . . . . . . . . . . 99

4.6. Reinspektrum des Cyclohexans. . . . . . . . . . . . . 102

5.1. Korrelation der Sianal- und Referenzspannuna vor (links) und nach (rechts) der Korrektur um die Ozonmesswerte. . . . . . . 105

5.2. Ausschnitt der Auftragung des Ozonpartialdrucks in der Vormischkammer, aufgetragen gegen die Aufnahmezeit des Voltmeters. . . . . . . . . . . . . . . . . . . . . . 106

5.3. Bestimmung des relativen Verbrauchs $\alpha$ (links) und Auftragung der Lösung des_Zeitqesetzes nach Gl. 2.27 gegen die Reaktionszeit (rechts). . . . . . . . . . . . . . . . . . . 108

5.4. Simulation einer Partikelverteilung. . . . . . . . . . . . . 113

5.5. Strukturen, für die mit Gaussian 03 Frequenzrechnungen angefertigt wurden. . . . . . . . . . . . . . . . 117

5.6. Strukturen, für die mit Gaussian 09 Frequenzrechnungen ange-

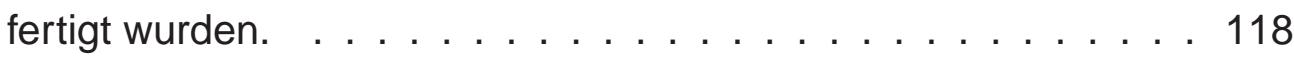


6.1. Darstellung der Ergebnisse der Kinetikmessungen für 1-Hexen (links) und 2-Methyl-1-penten (rechts). Alle Experimente wurden in Synthetischer Luft bei $p_{\text {ges }}=460 \pm 10$ mbar und $T=295 \pm 2 \mathrm{~K}$ durchgeführt. . . . . . . . . . . . . . . . . . . . 122

6.2. Darstellung der Ergebnisse der Kinetikmessungen für Methylcyclopenten. Alle Experimente wurden in Synthetischer Luft bei $p_{\text {ges }}=460 \pm 10$ mbar und $T=295 \pm 2 \mathrm{~K}$ durchgeführt.

6.3. Darstellung der Ergebnisse der Kinetikmessungen für 4-Penten1-ol (links) und 1-Penten-3-ol (rechts). Alle Experimente wurden bei $p_{\text {ges }}=460 \pm 10 \mathrm{mbar}$ und $T=295 \pm 2 \mathrm{~K}$ durchgeführt. Für 1-Penten-3-ol wurden Messungen sowohl in Stickstoff als auch in Synthetischer Luft durchgeführt.

6.4. Darstellung der Ergebnisse der Kinetikmessungen für 3-Buten1-ol. Alle Experimente wurden bei $p_{\text {ges }}=460 \pm 10 \mathrm{mbar}$ und $T=295 \pm 2 \mathrm{~K}$ in Synthetischer Luft durchgeführt. . . . . . . . . 125

6.5. Darstellung der Ergebnisse der Kinetikmessungen für 4Pentenal (links) und 1-Penten-3-on (rechts). Alle Experimente wurden in Synthetischer Luft bei $p_{\text {ges }}=460 \pm 10$ mbar und $T=295 \pm 2 \mathrm{~K}$ durchgeführt.

6.6. Darstellung der Ergebnisse der Kinetikmessungen für Methacrylsäure. Es wurden Experimente sowohl in Synthetischer Luft als auch in Stickstoff bei $p_{\text {ges }}=460 \pm 10 \mathrm{mbar}$ und $T=295 \pm 2 \mathrm{~K}$ durchgeführt.

6.7. Darstellung der Ergebnisse der Kinetikmessungen für Butensäure in Synthetischer Luft (links) und Stickstoff (rechts) bei $p_{\text {ges }}=460 \pm 10$ mbar und $T=295 \pm 2 \mathrm{~K}$.

6.8. Gesamtansicht der Reaktionsspektren bei Hochdruckbedingungen sowie der Differenzspektren unter verschiedenen Druckregimes für Methylcyclopenten.

6.9. Detailbereiche der Reaktions- und Differenzspektren bei verschiedenen Druckbedingungen für Methylcyclopenten (Ameisensäure, Ethen) 
6.10. Postulierter Bildungsweg für Ethen und Keten aus der Methylcyclopentenozonolyse, analog zum Vorschlad aus WoLF et al. [17] für Methylcyclohexen.

6.11. Detailbereiche der Reaktions- und Differenzspektren bei verschiedenen Druckbedingungen für Methylcyclopenten (Keten, Formaldehyd).

6.12. Postulierter Bildungsweg für Formaldehyd aus der Methylcyclopentenozonolyse, adaptiert nach TUAZON et al. [124]. . . . . . . 136

6.13. Möglicher Bildungsweg für Keten aus der 2-Methyl-1pentenozonolyse in Analogie zu einem von WoLF vorgeschlagenen Mechanismus [17]. . . . . . . . . . . . . . . . . . . . 139

6.14. Bildung der möalichen Peroxvprodukte, Veraleich der Differenzspektren mit Theoriepektren $(B 3 L Y P / 6-311+G(d, p))$. . . . . . . . 143

6.15. Bildung der möalichen Peroxyprodukte, Veraleich des Reaktionsspektrums mit 2-Oxobutanal $(B 3 L Y P / 6-311+G(d, p))$. . . . . 145

6.16. Vergleich der Druckdifferenzspektren der 4-Pentenalozonolyse mit einem Literaturspektrum von NIKI et al. [60]. . . . . . . . . . .

6.17. Vergleich experimentell und theoretisch ermittelter Spektren $(B 3 L Y P / 6-311+G(d, p))$, Verschiebung letzterer jeweils um den Faktor 0.9658.

6.18. Reaktionsspektren im Niederdruckbereich, Ketenabsorbanz: Vergleich von Methylcyclopenten, 3-Buten-1-ol und Butensäure.

6.19. Vorschläge zur Ketenbildung aus Reaktionen unter Sauerstoffbeteiligung in der Literatur. . . . . . . . . . . . . . . . . . . 155

6.20. Vorschläge zur Ketenbildung aus den verwendeten Edukten. . . 157

6.21. Eraebnisse der Produktbildungsexperimente des Cyclohexens unter $\mathrm{SO}_{2}$-Zugabe. . . . . . . . . . . . . . . . . . . . . . . . 161

6.22. Eraebnisse der Produktbildungsexperimente des $\alpha$-Pinens unter $\mathrm{SO}_{2}$-Zugabe. . . . . . . . . . . . . . . . . . . . . . . . 164

6.23. Veraleich der Produktbildunasexperimente des $\alpha$-Pinens unter $\mathrm{SO}_{2}$-Zugabe bei verschiedenen Gesamtdrücken. . . . . . . . . . 165

6.24. Vergleich des Hochdruckdifferenzspektrums des $\beta$-Pinens unter $\mathrm{SO}_{2}$-Zugabe mit Nopinon als möglichem Produkt. Das Reinspektrum des Nopinons stammt von WoLF [48]. . . . . . . . . . . . . 
6.25. Ozonpartialdruckreihe zur Ermittlung der zu verwendenden Ozonanfangskonzentrationen bei $p_{\text {ges }}=460$ mbar. . . . . . . 176

6.26. Untersuchung der druckabhängigen Partikelausbeute für 1Hexen, 2-Methyl-1-penteh und Methylcyclopenten in Synthetischer Luft bzw. Stickstoff.

6.27. Untersuchung der badgasabhängigen Partikelausbeute für 1 Hexen, 2-Methyl-1-penten und Methylcyclopenten, Vergleich der Ausbeuten in Synthetischer Luft und Stickstoff bei variiertem Druck.182 6.28. Untersuchung des Einflusses von Radikalfängern auf die Partikelausbeute für 1-Hexen, Methylcyclopenten und 2-Methyl1-penten; Vergleich der Effekte durch Cyclohexan (CHA), 1Butanol (1BUOH) und 2-Butanol (2BUOH). Für 2-Methyl-1penten wurden zusätzliche Experimente unter Cyclohexanonzugabe (CHON, Oxidationsprodukt des Cyclohexans) durchgeführt. 185 6.29. Untersuchung der druckabhängigen Partikelausbeute für 3Buten-1-ol in Stickstoff, Synthetischer Luft und Synthetischer Luft unter Zugabe von Cyclohexan (CHA) . . . . . . . . . . . . 189

6.30. Untersuchung der druckabhängigen Partikelausbeute für 1Penten-3-ol in Stickstoff, Synthetischer Luft und Synthetischer Luft unter Zugabe von Cyclohexan (CHA) . . . . . . . . . . . . 191

6.31. Untersuchung der druckabhänqigen Partikelausbeute für 4Penten-1-ol in Stickstoff und Synthetischer Luft. . . . . . . . . . . 192 6.32. Untersuchung des Einflusses des Sauerstoffpartialdrucks für 4-

Penten-1-ol in Stickstoff unter variabler Zugabe von Sauerstoff. . 193 6.33. Bei der Ozonolyse von 3-Buten-1-ol, 1-Penten-3-ol und 4Penten-1-ol entstehende primäre Carbonylverbindungen und substituierte $\mathrm{Cl}$. Zusätzlich wird bei jeder Verbindung auch das $\mathrm{C}_{1}$-Cl gebildet. . . . . . . . . . . . . . . . . . . . . . . 194

6.34. Ringspannungen für verschiedengliedrige Ringe, aus MCMURRY [136], S. 113. . . . . . . . . . . . . . . . . . . . 195

6.35. Untersuchung der druckabhänqigen Partikelausbeute für 1Penten-3-on in Stickstoff und Synthetischer Luft. . . . . . . . . . 196 
6.36. Untersuchung des Einflusses des Sauerstoffpartialdrucks für 1Penten-3-on in Stickstoff unter variabler Zugabe von Sauerstoff im höheren ( $p_{\text {ges }}=460 \pm 10$ mbar, oben) bzw. niedrigeren Druckbereich ( $p_{\text {ges }}=100 \pm 6$ mbar, unten). . . . . . . . . . . . . . . . 198

6.37. Untersuchung der druckabhänqigen Partikelausbeute für 4Penten-1-al in Stickstoff und Synthetischer Luft. . . . . . . . . . . 199

6.38. Bei der Ozonolyse von 1-Penten-3-on und 4-Penten-1-al entstehende substituierte Cl. Zusätzlich wird bei jeder Verbindung auch das $\mathrm{C}_{1}$-Cl gebildet. . . . . . . . . . . . . . . . . . . . . . . . 201

6.39. Untersuchung der druckabhängigen Partikelausbeute für Methacrylsäure in Stickstoff und Synthetischer Luft, jeweils auch unter Zugabe von Cyclohexan (CHA). . . . . . . . . . . . . . . . . . . 203

6.40. Untersuchung der druckabhängigen Partikelausbeute für Butensäure in Stickstoff und Synthetischer Luft, jeweils auch unter Zugabe von Cyclohexan (CHA). . . . . . . . . . . . . . . . . . . . . 204

6.41. Untersuchung des Einflusses des Sauerstoffpartialdrucks für Methacrylsäure und Butensäure in Stickstoff unter variabler Zugabe von Sauerstoff im mittleren Druckbereich $\left(p_{\text {ges }}=270 \pm 10\right.$ mbar $) . \ldots \ldots$. . . . . . . . . . . . . . . .

6.42. Untersuchung des Einflusses der Säurepartialdrücke für Methacrylsäure und Butensäure auf die Partikelausbeuten in beiden Badgasen bei konstantem Gesamtdruck ( $p_{\text {ges }}=460 \pm 10$ mbar). 208

6.43. Untersuchung des Einflusses verschiedener Eduktkonzentrationen auf die Partikelausbeuten bei der Butensäureozonolyse ( $p_{\text {ges }}=460 \pm 10$ mbar). Die Fehlerbalken wurden jeweils mit $\pm 10 \%$ des Mittelwertes abgeschätzt. . . . . . . . . . . . . . . . 212

6.44. Untersuchung des Einflusses der Badgase und verschiedener Additiva (CHA: Cyclohexan, HFO: Ameisensäure) auf die Partikelausbeuten bei der Butensäureozonolyse ( $p_{\text {ges }}=1000 \pm 10$ mbar). Die Fehlerbalken wurden jeweils mit $\pm 10 \%$ des Mittelwertes abgeschätzt. . . . . . . . . . . . . . 212 
6.45. Untersuchung des $\mathrm{SO}_{2}$-Einflusses auf die Partikelausbeute für Cyclohexen in Synthetischer Luft unter Zugabe verschiedener Wasserpartialdrücke bei $p_{\text {ges }}=460 \pm 10$ mbar. Neben den linearen werden auch logarithmische Auftragungen gezeigt. . . . 223

6.46. Vergleich experimentell ermittelter Punkte mit theoretisch erwarteten Partikelausbeuten in der $\mathrm{SO}_{2}$-abhängigen Cyclohexenozonolyse bei $p_{\text {ges }}=450 \pm 10$ mbar ohne Zugabe weiterer Additiva. 224

6.47. Untersuchung der druckabhängigen Partikelausbeute für Cyclohexen in Synthetischer Luft unter Zugabe verschiedener Mengen $\mathrm{SO}_{2}$. . . . . . . . . . . . . . . . . . . . . . . . . 225

6.48. Untersuchung der druckabhängigen Partikelausbeute für Methylcyclopenten in Synthetischer Luft unter Zugabe von $\mathrm{SO}_{2}$. . . . . 226

6.49. Untersuchung der druckabhängigen Partikelausbeute für Methylcyclohexen in Synthetischer Luft unter Zugabe verschiedener Mengen $\mathrm{SO}_{2}$. . . . . . . . . . . . . . . . . . . . . . . . . . . 227

6.50. Untersuchung des $\mathrm{SO}_{2}$-Einflusses auf die Partikelausbeute für $\alpha$-Pinen in Synthetischer Luft unter Zugabe verschiedener Wasserpartialdrücke bei $p_{\text {ges }}=450 \pm 10$ mbar.

6.51. Untersuchung der Druckabhängiakeit bei der $\alpha$-Pinenozonolyse unter verschiedenen $\mathrm{SO}_{2}$-Zugaben. . . . . . . . . . . . . . . . . 229

6.52. Untersuchung der Druckabhängigkeit bei der $\alpha$-Pinenozonolyse unter $\$ \mathrm{O}_{2}$-Zugaben in Anwesenheit von Nopinon und Propionaldehyd.

6.53. Untersuchung der druckabhängigen Partikelausbeute für Methylencyclohexam in Synthetischer Luft unter Zugabe verschiedener Mengen $\mathrm{SO}_{2}$. . . . . . . . . . . . . . . . . . . . . . . . . 231

6.54. Untersuchung der Druckabhänqiakeit bei der $\beta$-Pinenozonolyse unter verschiedenen $\mathrm{SO}_{2}$-Zugaben. . . . . . . . . . . . . . . . 232

6.55. Strukturen der Monomere aus den quantenchemischen Rechnungen, weiß: Wasserstoff; grau: Kohlenstoff; rot: Sauerstoff. . . 245

6.56. Strukturen der Oligomere aus den quantenchemischen Rechnungen, weiß: Wasserstoff; grau: Kohlenstoff; rot: Sauerstoff; gelb: Schwefel,

Fortsetzung auf der nächsten Seite. 
6.56. Strukturen der Oligomere aus den quantenchemischen Rechnungen, weiß: Wasserstoff; grau: Kohlenstoff; rot: Sauerstoff; gelb: Schwefel. . . . . . . . . . . . . . . . . . . . . . . 247

6.57. Experimenteller Aufbau zur Partikelanalyse, (HPLC/ESI-MS), entnommen aus M.-C. REINNING 148]. . . . . . . . . . . . . . 249

6.58. Auswertung der $\alpha$-Pinenergebnisse bei verschiedenen Gesamtdrücken $p_{\text {ges }}=(10,20,465)$ mbar.

6.59. Ausbeuten der identifizierten Produkte der $\alpha$-Pinenozonolyse bei verschiedenen Gesamtdrücken $p_{\text {ges }}=(10 / 20 / 465)$ mbar. . . . 252

6.60. Veränderungen in den Ausbeuten bei Verwendung von Stickstoff statt Synthetischer Luft, alle Experimente wurden bei $p_{\text {ges }}=465 \pm 10$ mbar durchgeführt.

6.61. Vorschlag für die Bildung von hochmolekularen Produkten in der Partikelphase, die Protonenabspaltung im letzten Schritt ist der experimentellen Methode geschuldet, da Anionen nachgewiesen werden.

7.1. Postulierter ketenbildender Mechanismus für Butensäure und 3Buten-1-ol. 266

7.2. Vorhersage über die globale Klimaentwickung, entnommen aus dem Bericht des Weltklimarates von 2001 (IPCC - Intergovernmental Panel on Climate Change) [152]. . . . . . . . . . . . . . . . 270

7.3. Vereinfachte Darstellung der atmosphärischen Oxidationsprozesse von volatilen organischen Substanzen (VOC) nach KROLL und SEINFELD [21] (ohne Nitratbeteiligung). . . . . . . . . . . . . . 274

7.4. Vergleich auftretender struktureller Ähnlichkeiten in der $\mathrm{C}_{6} \mathrm{H}_{12}+$ $\mathrm{OH}$-Reaktion und der Oligomerisierungsreaktion nach SADEZKY et al. [36]. . . . . . . . . . . . . . . . . . . . . . . . . . . . . . . 278

7.5. Darstellung der Abfangreaktion mit ungesättigtem und gesättigtem Alkohol. . . . . . . . . . . . . . . . . . . . . . . . 283

7.6. Sich in der Ozonolvse bildende CRIEGEE-Intermediate für Methylcyclopenten und Cyclohexen. . . . . . . . . . . . . . . . . 288

7.7. Sich in der Ozonolvse bildende CRIEGEE-Intermediate für 1Hexen und 2-Methyl-1-penten. . . . . . . . . . . . . . . . . . . . 289 
7.8. Zerfall der CRIEGEE-Intermediate für endo- und exozyklische Alkene. . . . . . . . . . . . . . . . . . . . . . . . . 293

7.9. Schematische Darstellung der untersuchten Gesichtspunke bei der Aerosolbildung, Beeinflussung der Intermediatchemie. . . . . 294

8.1. Zusammenfassung der Hauptergebnisse: Einflussfaktoren und Auswirkungen auf die Aerosolbildung am Beispiel der durch Aerosolphasenanalyse charakterisierten Substanzen. . . . . . . . 304

C.1. Kalibrierspektren aus [48] und [17] unter Anqabe der verwendeten Partialdrücke, Gesamtdrücke und Badgase. . . . . . . . . . . XIII

C.2. Kalibrierspektren aus [48] und [17] unter Angabe der verwendeten Partialdrücke, Gesamtdrücke und Badgase. . . . . . . . . . . XIV

D.1. Detailbereiche der 1-Hexen-Reaktionsspektren. . . . . . . . . XVI

D.2. Gesamtansicht und Detailbereiche der Reaktionsspektren des 2Methyl-1-pentens und Vergleich der Formaldehydausbeuten mit 1-Hexen. . . . . . . . . . . . . . . . XVII

D.3. Gesamtansicht der Reaktionsspektren bei Hochdruckbedingungen sowie der Differehzspektren unter verschiedenen Druckregimes für 3-Buten-1-ol. . . . . . . . . . . . . . . . . . . XVIII

D.4. Differenzspektren zwischen Reaktionen in Synthetischer Luft und Stickstoff für 3-Buten-1-ol, jeveeils unter Hoch- und Niederdruckbedingungen, durch die Achsenunterbrechung wird lediglich das $\mathrm{CO}_{2}$-Signal ausgeblendet. . . . . . . . . . . . . . . XVIII

D.5. Detailbereiche der Reaktionsspektren des 3-Buten-1-ols. . . . XIX

D.6. Gesamtansicht der Reaktionsspektren bei Hochdruckbedingungen sowie der Differenzspektren unter verschiedenen Druckregimes für 1-Penten-3-ol. . . . . . . . . . . . . . . . . . XXX

D.7. Differenzspektren zwischen Reaktionen in Synthetischer Luft und Stickstoff für 1-Penten-3-ol, jeweils unter Hoch- und Niederdruckbedingungen, durch die Achsenunterbrechung wird lediglich das $\mathrm{CO}_{2}$-Signal ausgeblendet. . . . . . . . . . . . . XXX

D.8. Detailbereiche der Reaktionsspektren des 1-Penten-3-ols. . . . . XXI 
D.9. Gesamtansicht der Reaktionsspektren bei Hochdruckbedingungen sowie der Differenzspektren unter verschiedenen Druckregimes für 4-Penten-1-ol. . . . . . . . . . . . . . . . . . . . . . . . XXII

D.10.Differenzspektren zwischen Reaktionen in Synthetischer Luft und Stickstoff des 4-Pentenols, jeweils unter Hoch- und Niederdruckbedingungen, durch die Achsenunterbrechung wird lediglich das $\mathrm{CO}_{2}$-Signal ausgeblendet. . . . . . . . . . . . . . . . XXII

D.11.Detailbereiche der Reaktionsspektren des 4-Penten-1-ols. . . . . XXIII

D.12.Gesamtansicht der Reaktionsspektren bei Hochdruckbedingungen sowie der Differenzspektren unter verschiedenen Druckregimes für 1-Penten-3-on. . . . . . . . . . . . . . . . . . . . . . XXIV

D.13.Differenzspektren zwischen Reaktionen in Synthetischer Luft und Stickstoff für 1-Penten-3-on, jeweils unter Hoch- und Niederdruckbedingungen. . . . . . . . . . . . . . . . . XXIV

D.14.Detailbereiche der Reaktionsspektren des 1-Penten-3-ons. . . . XXV

D.15.Gesamtansicht und Detailbereiche der Reaktionsspektren des 4Penten-1-als. . . . . . . . . . . . . . . . . . . XXVI

D.16.Differenzspektren zwischen Reaktionen in Synthetischer Luft und Stickstoff für 4-Pentenal, jeweils unter Hoch- und Niederdruckbedingungen. . . . . . . . . . . . . . . . . XXVII

D.17.Gesamtansicht der Reaktionsspektren bei Hochdruckbedingungen sowie der Differenzspektren unter verschiedenen Druckregimes für Methacrylsäure.

D.18.Detailbereiche der Reaktionsspektren der Methacrylsäure. . . . XXIX

D.19.Gesamtansicht der Reaktionsspektren bei Hochdruckbedingungen sowie der Differenzspektren unter verschiedenen Druckregimes für Butensäure. . . . . . . . . . . . . . . . . . . . . . XXXX

D.20.Differenzspektren zwischen Reaktionen in Synthetischer Luft und Stickstoff für Butensäure, jeweils unter Hoch- und Niederdruckbedingungen, durch die Achsenunterbrechung wird lediglich das $\mathrm{CO}_{2}$-Signal ausgeblendet. . . . . . . . . . . . . . . XXX

D.21.Detailbereiche der Reaktionsspektren der Butensäure. . . . . . . . XXXI 
E.1. Untersuchung des Einflusses der Badgase und verschiedener Badgase und Additiva auf die Partikelausbeuten bei der Butensäureozonolyse $(T=295 \pm 2 \mathrm{~K}) . \ldots \ldots \ldots \ldots \ldots$ XXXIII

H.1. Reaktionsmechanismus für die Ozonolyse von Methylencyclohexan nach HATAKEYAMA et al. [135] mit Ergänzungen nach WOLF [48], KOCH et al. [9] sowie WALSER et al. [162]. . . . . . . . . . X XL

H.2. Reaktionsmechanismus für die Ozonolyse von Cyclohexen nach ASCHMANN et al. [122] mit Ergänzungen nach JOHNSON et al. [33] sowie KEYWOOD et al. [55]. . . . . . . . . . . . . . . . . XLI

H.3. Methylcyclohexenozonolyse, zu erwartende Zerfallsschritte. . . . XLII

I.1. Initialer Schritt der Ozonolysereaktion der Butensäure. . . . . . . XLIII

I.2. Folgechemie der Butensäure in Analogie zum Vorschlag von NEEB et al. [59] für Methacrylsäure. . . . . . . . . . . . . . XLIV

I.3. Folgechemie des 1-Penten-3-ols in Analogie zum Vorschlag von NeEB et al. [59] für Methacrylsäure. . . . . . . . . . . . . . XLV

I.4. Folgechemie des 1-Penten-3-ons in Analogie zum Vorschlag von NEEB et al. [59] für Methacrylsäure. . . . . . . . . . . . . . XLVI

1.5. Erste Schritte der Ozonolysereaktion des 4-Penten-1-0l. . . . . . XLVII

1.6. Erste Schritte der Ozonolysereaktion des 4-Penten-1-al. . . . . . XLVII

I.7. Folgechemie des 4-Penten-1-ols in Analogie zum Vorschlag von NEEB et al. [59] für Methacrylsäure. . . . . . . . . . . . . . XLVIII

I.8. Folgechemie des 4-Penten-1-als in Analogie zum Vorschlag von NEEB et al. [59] für Methacrylsäure. . . . . . . . . . . . XLIX

1.9. Folgechemie des Butenols in Analogie zum Vorschlag von NEEB et al. [59] für Methacrylsäure. 


\section{Literaturverzeichnis}

[1] Lamb, B.; Grosjean, D.; Pun, B.; Seigneur, C. Review of the emissions, atmospheric chemistry, and gas/particle partition of biogenic volatile compounds and reaction products. Coordinating Research Council, Inc., 1999.

[2] Clean Air Act: National Ambient Air Quality Standards (NAAQS), 19902011. United States Environmental Protection Agency.

[3] Sydlik, U. Analyse der molekularen Mechanismen pathogener Effekte von ultrafeinen Partikeln: Induktion von Apoptose und Proliferation. Dissertation, Heinrich-Heine-Universität Düsseldorf, 2006.

[4] Haagen-Smit, A. Chemistry and Physiology of Los Angeles Smog. J. Ind. Eng. Chem., 44: 1342 - 1346, 1952.

[5] Guenther, A.; Karl, T.; Harley, P.; Wiedinmyer, C.; Palmer, P.I.; Geron, C. Estimates of global terrestial isoprene emissions using MEGAN (Model of Emissions of Gases and Aerosols from Nature). Atmos. Chem. Phys. Discuss., 6: 107 - 173, 2006.

[6] Sellegri, K.; Hanke, M.; Umann, B.; Arnold, F.; Kulmala, M.; Geron, C. Measurements of organic gases during aerosol formation events in the boreal forest atmosphere during QUEST). Atmos. Chem. Phys., 5: $373-$ 384, 2005.

[7] Forster, P.; Ramaswamy, V.; Artaxo, P.; Berntsen, T.; Betts, R.; Fahey, D. W.; Haywood, J.; Lean, J.; Lowe, D. C.; Myhre, G.; Nganga, J.; Prinn, R.; Raga, G.; Schulz, M.; Van Dorland, R. Changes in atmospheric constituents and in radiative forcing. In S. Solomon, D. Qin, M. Manning, 
Z. Chen, M. Marquis, K. B. Averyt, M. Tignor, and H.L. Miller, editors, Climate Change 2007: The Physical Science Basis. Contribution of Working Group I to the Fourth Assessment Report of the Intergovernmental Panel on Climate Change, Cambridge, United Kingdom and New York, NY, USA, 2007. Cambridge University Press.

[8] Ramanathan, V.; Carmichael, G. Global and regional climate changes due to black carbon. Nature. Geo., 1: 221 - 227, 2008.

[9] Koch, S.; Winterhalter, R.; Uherek, E.; Kolloff, A.; Neeb, P.; Moortgat, G. $\mathrm{K}$. Formation of new particles in the gas-phase ozonolysis of monoterpenes. Atmos. Environ., 34(23): 4031 - 4042, 2000.

[10] Seinfeld, J. H.; Pandis, S. N. Atmospheric Chemistry and Physics - From Air Pollution to Climate Change. WILEY-VCH, New York [u.a.], 1998.

[11] Kundel, M.; Thorenz, U.R.; Petersen, J.H.; Huang, R.-J.; Bings, N.H.; Hoffmann, T. Application of mass spectrometric techniques for the trace analysis of short-lived iodine-containing volatiles emitted by seaweed. Anal. Bioanal. Chem., 402: DOI 10.1007/s00216-011-5658-z, 2012.

[12] Bonn, B.; Kulmala, M.; Riipinen, I.; Sihto, S.-L.; Ruuskanen, T.M. How biogenic terpenes govern the correlation between sulfuric acid concentrations and new particle formation. J. Geophys. Res., 113: D12209, 2004.

[13] Donahue, N.M.; Trump, E.R.; Pierce, J.R.; Riipinen, I.; Kroll, J.H. Theoretical constraints on pure vapor-pressure driven condensation of organics to ultrafine particles. 38: doi:10.1029/2011GL048115, 2011.

[14] Sheldrick, G. Skript zur Vorlesung Umweltchemie. Georg-AugustUniversität Göttingen, 2006.

[15] Keunecke, C. Experimentelle Untersuchungen zur Produkt- und Partikelbildung bei der Ozonolyse von (Meth-)Acrylsäure in An- und Abwesenheit von molekularem Sauerstoff, 2008.

[16] Wolf, J.L.; Suhm, M.A.; Zeuch, T. Unterdrückte Partikelbildung durch kinetisch kontrollierten Ozonentzug:zur Bedeutung kurzlebiger Spezies bei der Alkenozonolyse. Angew. Chem., 121: 2265 - 2269, 2009. 
[17] Wolf, J.L.; Richter, S.; Pecher, J.; Zeuch, T. Pressure dependent mechanistic branching in the formation pathways of secondary organic aerosol from cyclic-alkene gas-phase ozonolysis. Phys. Chem. Chem. Phys., 13: 10952 - 10964, 2011.

[18] Bonn, B.; Schuster, G.; Moortgat, C. K. Influence of Water Vapor on the Process of New Particle Formation during Monoterpene Ozonolysis. J. Phys. Chem., 106(12): 2869 - 2881, 2002.

[19] Henry, K.M.; Donahue, N.M. Effect of the OH Radical Scavenger Hydrogen Peroxide on Secondary Organic Aerosol Formation from $\alpha$-Pinene Ozonolysis. 45: 696 - 700, 2011.

[20] Hoyermann, K.; Maarfeld, S.; Nacke, F.; Nothdurft, J.; Olzmann, M.; Wehmeyer, J.; Welz, O.; Zeuch, T. Rate coefficients for cycloalkyl + $O$ reactions and product branching in the decomposition of chemically activated cycloalkoxy radicals: an experimental and theoretical study. Phys. Chem. Chem. Phys., 12: 8953 - 8967, 2010.

[21] Kroll, J.H.; Seinfeld, J.H. Chemistry of secondary organic aerosol: Formation and evolution of low-volatility organics in the atmosphere. Atmos. Environ., 42: 3593 - 3624, 2008.

[22] Zhang, R.; Suh, I.; Zhao, J.; Zhang, D.; Fortner, E.C.; Tie, X.; Molina, L.T.; Molina, M.J. Atmospheric new particle formation enhanced by organic acids. Science, 304: 1487 - 1489, 2004.

[23] Falbe, J.; Regitz, M. [Hg.]. Römpp - Chemie Lexikon. Georg Thieme Verlag, Stuttgart [u.a.], 1992.

[24] Hollas, M. J. Moderne Methoden in der Spektroskopie. Vieweg, Braunschweig [u.a.], 1995.

[25] Banwell, C. N.; McCash, E. M. Molekülspektroskopie - Ein Grundkurs. Oldenbourg Verlag, München, 1999.

[26] Falbe, J.; Regitz, M. [Hg.]. Römpp Kompakt - Basislexikon Chemie. Georg Thieme Verlag, Stuttgart [u.a.], 1999. 
[27] Atkins, P.W. Physikalische Chemie. Wiley-VCH, Weinheim [u.a.], 2001.

[28] Signorell, R.; Hoyermann, K.; Hold, M.; Wehmeyer, J.; Zeuch, T. Skript zur Vorlesung Physikalische Chemie für Lehramtslandidaten I. GeorgAugust-Universität Göttingen, 2004/2005.

[29] Nothdurft, J. Die Oxidation des Cyclopentylradikals in der Gasphase. Examensarbeit, Georg-August-Universität Göttingen, 2002.

[30] Falbe, J.; Regitz, M. [Hg.]. Römpp - Chemie Lexikon. Georg Thieme Verlag, Stuttgart [u.a.], 1991.

[31] Reinhardt, C. . Christian Friedrich Schönbein und die Frühgeschichte der Katalyseforschung. Mitteilungen der Fachgruppe Geschichte der Chemie der GDCh, 6: 18 - 28, 1991.

[32] Criegee, R. Mechanismus der Ozonolyse. Angew. Chem., 21: 765 - 771, 1975.

[33] Johnson, D.; Marston, G. The gas-phase ozonolysis of unsaturated volatile organic compounds in the troposphere. Chem. Soc. Rev., 37: 699716, 2008.

[34] Uherek, E. Laboruntersuchungen der mechanistischen Bildung von Carbonsäuren in der Ozonolyse von Alkenen und ihrer Rolle bei der Aerosolbildung. Dissertation, Johannes-Gutenberg-Universität Mainz, 2003.

[35] Neeb, P.; Moortgat, G. K. Formation of $\mathrm{OH}$ Radicals in the Gas-Phase Reaction of Propene, Isobutene and Isoprene with $\mathrm{O}_{3}$ : Yields and Mechanistic Implications. J. Phys. Chem., 103: 9003 - 9012, 1999.

[36] Sadezky, A.; Winterhalter, R.; Kanawati, B.; Römpp, A.; Spengler, B.; Mellouki, A.; Le Bras, G.; Chaimbault, P.; Moortgat, G.K. Oligomer formation during gas-phase ozonolysis of small alkenes and enol ethers: new evidence for the central role of the Criegee Intermediate as oligomer chain unit. Atmos. Chem. Phys., 8: 2667 - 2699, 2008. 
[37] Rickard, A.R.; Johnson, D.; McGill, C.D.; Marston, G. OH Yields in the Gas-Phase Reactions of Ozone with Alkenes. J. Phys. Chem. A, 103: 7656 - 7664, 1999.

[38] Neeb, P.; Horie, O.; Moortgat, G. K. The Ethene-Ozone Reaction in the Gas-Phase. J. Phys. Chem. A, 102: 6778 - 6785, 1998.

[39] Atkinson, R. Gas-phase tropospheric chemistry of organic componds: a review. Atmos. Environ., 24A: 1 - 41, 1990.

[40] Alam, M.S.; Camredon, M.; Rickard, A.R.; Carr, T.; Wyche, K.P.; Hornsby, K.E.; Monks, P.S.; Bloss, W.J. Total radical yields from tropospheric ethene ozonolysis. Phys. Chem. Chem. Phys., 13: 11002 - 11015, 2011.

[41] Taatjes, C.A.; Meloni, G.; Selby, T.M.; Trevitt, A.J.; Osborn, D.L.; Percival, C.J.; Shallcross, D. Direct observation of the gas-phase Criegee intermediate $\left(\mathrm{CH}_{2} \mathrm{OO}\right)$. J. Am. Chem. Soc., 130: 11883 - 11885, 2008.

[42] Hasson, A.S.; Ho, A.W.; Kuwata, K.T.; Paulson, S.E. Production of stabilized Criegee intermediates and peroxides in the gas phase ozonolysis of alkenes, 2. Asymmetric and biogenic alkenes. J. Geophys. Res., 106: $34143-34153,2001$.

[43] Paulson, S.E.; Chung, M.Y.; Hasson, A.S. OH radical formation from the gas-phase reaction of ozone with terminal alkenes and the relationship between structure and mechanism. J. Phys. Chem. A, 103: 8125 - 8138, 1999.

[44] Drozd, G.T.; Donahue, N.M. Pressure dependence of stabilized Criegee intermediate formation from a sequence of alkenes. J. Phys. Chem. A, 115: 4381 - 4387, 2011.

[45] Chuong, B.; Zhang, J.; Donahue, N.M. Cycloalkene Ozonolysis: Collisionally Mediated Mechanistic Branching. J. Am. Chem. Soc., 126: 12363 12373, 2004.

[46] Hatakeyama, S.; Kobayashi, H.; Lin, Z.-Y.; Tagaki, H.; Akimoto, H. Mechanism for the reaction of $\mathrm{CH}_{2} \mathrm{O}_{2}$ withe $\mathrm{SO}_{2}$. J. Phys. Chem., 101: 4131 $-4135,1986$. 
[47] Kroll, J.H.; Sahay, S.R.; Anderson, J.G.; Demerjian, K.L.; Donahue, N.M. Mechanism of $\mathrm{HO}_{x}$ Formation in the Gas-Phase Ozone-Alkene Reaction. 2. Prompt versus Thermal Dissociation of Carbonyl Oxides to Form $\mathrm{OH}$. J. Phys. Chem. A, 105: 4446 - 4457, 2001.

[48] Wolf, J.L. Mechanismen der Partikelbildung bei der Ozonolyse von Alkenen. Dissertation, Georg-August-Universität Göttingen, 2010.

[49] Horie, O.; Neeb, P.; Moortgat, G. K. The reaction of the Criegee intermediate $\mathrm{CH}_{3} \mathrm{CHOO}$ in the gas-phase ozonolysis of 2-butene isomers. Int. J. Chem. Kinet., 29(6): 461 - 468, 1997.

[50] Hasson, A.S.; Orzechowska, G.; Paulson, S.E. Production of stabilized Criegee intermediates and peroxides in the gas phase ozonolysis of alkenes, 1. Ethene, trans-2-butene, and 2,3-dimethyl-2-butene. J. Geophys. Res., 106: 34131 - 34142, 2001.

[51] Jonsson, A.M.; Hallquist, M.; Ljungström, E. Impact of Humidity on the Ozone Initiated Oxidation of Limonene, $\Delta^{3}$-Carene, and $\alpha$-Pinene. Environ. Sci. Technol., 40: 188 - 194, 2006.

[52] Lee, S.; Kamens, R. M. Particle nucleation from the reaction of $\alpha$-pinene and $\mathrm{O}_{3}$. Atmos. Environ., 39(36): 6822 - 6832, 2005.

[53] Sadezky, A.; Chaimbault, P.; Mellouki, A.; Römpp, A.; Winterhalter, R.; Le Bras, G.; Moortgat, G.K. Formation of secondary organic aerosol and oligomers from the ozonolysis of enol ethers. Atmos. Chem. Phys., 6: $5009-5024,2006$.

[54] Docherty, K.; Ziemann, P. Effects of Stabilized Criegee Intermediate and $\mathrm{OH}$ Scavengers on Aerosol Formation from Reactions of $\beta$-Pinene with $\mathrm{O}_{3} .37: 877-891,2003$.

[55] Keywood, M.D.; Kroll, J.H.; Varutbangkul, V.; Bahreini, R.; Flagan, R.C.; Seinfeld, J.H. Effect of $\mathrm{OH}$ scavenger and the role of radical chemistry. Environ. Sci. Technol., 38: 3343 - 3350, 2004. 
[56] Alfassi, Z.B. The Chemistry of Free Radikals - Peroxyl Radicals. John Wiley \& Sons, 1997.

[57] Neeb, P.; Horie, O.; Moortgat, G. K. Gas-Phase Ozonolysis of Ethene in the Presence of Hydroxylic Compounds. Int. J. Chem. Kinet., 28: 721 730, 1996.

[58] Hatakeyama, S.; Akimoto, H. Reactions of Criegee intermediates in the gas phase. Res. Chem. Intermediat., 20: 503 - 524, 1994.

[59] Neeb, P.; Kolloff, A.; Koch, S.; Moortgat, G. K. Rate Constants for the Reactions of Methylvinyl Ketone, Methacrolein, Methacrylic Acid, and Acrylic Acid with Ozone. Int. J. Chem. Kinet., 30: 769 - 776, 1998.

[60] Niki, H.; Maker, P.D.; Savage, C.M.; Breitenbach, L.P. A FT IR Study of a Transitory Product in the Gas-Phase Ozone-Ethylene Reaction. J. Phys. Chem., 85: 1024 - 1027, 1981.

[61] O'Dwyer, M.A.; Carey, T.J.; Healy, R.M.; Wenger, J.C.; Picquet-Varrault, B.; Doussin, J.F. The Gas-phase Ozonolysis of 1-Penten-3-ol, (Z)-2Penten-1-ol and 1-Penten-3-one: Kinetics, Products and Secondary Organic Aerosol Formation. Z. Phys. Chem., 224: 1059 - 1080, 2010.

[62] Fall, R.; Karl, T.; Jordan, A.; Lindinger, W. Biogenic C5 VOCs: release from leaves after freeze-thaw wounding and occurrence in air at a high mountain observatory. Atmos. Environ., 35: 3905 - 3916, 2001.

[63] Kesselmeier, J.; Staudt, M. Biogenic Volatile Organic Compounds (VOC): An Overview on Emission, Physiology and Ecology. J. Atmos. Chem., 33: $28-88,1999$.

[64] Xu, Z.; Liu, Z.; Ge, M.; Wang, W. Uptake kinetics of 3-buten-1ol, 4-penten-1-ol and 3-methyl-3-buten-1-ol into sulfuric acid solution. Chin. Sci. Bull., 56: 1352 - 1356, 2011.

[65] Grosjean, D.; Grosjean, E.; Williams II., E.L. Atmospheric Chemistry of Unsaturated Alcohols. Environ. Sci. Technol., 27: 2478 - 2485, 1993. 
[66] Gutbrod, R.; Meyer, S.; Rahman, M.M.; Schindler, R.N. On the use of CO as Scavenger for $\mathrm{OH}$ Radicals in the Ozonolysis of Simple Alkenes and Isoprene. Int. J. Chem. Kinet., 29: 717 - 723, 1997.

[67] Malkin, T.L.; Goddard, A.; Heard, D.E.; Seakins, P.W. Measurements of $\mathrm{OH}$ and $\mathrm{OH}_{2}$ yields from the gas phase ozonolysis of isoprene. Atmos. Chem. Phys., 10: 1441 - 1459, 2010.

[68] Grosjean, E.; Grosjean, D. Rate Constants for the Gas-Phase Reactions of Ozone with Unsaturated Aliphatic Alcohols. Int. J. Chem. Kinet., 26: 1185 - 1191, 1994.

[69] Grosjean, D.; Grosjean, E.; Williams II., E.L. Rate Constants for the GasPhase Reactions of Ozone with Unsaturated Alcohols, Esters, and Carbonyls. Int. J. Chem. Kinet., 25: 783 - 794, 1993.

[70] Cavalli, F.; Geiger, H.; Barnes, I.; Becker, K.H. FTIR Kinetic, Product, and Modeling Study of the OH-Initiated Oxidation of 1-Butanol in Air. Environ. Sci. Technol., 36: 1263 - 1270, 2002.

[71] Tobias, H.J.; Ziemann, P.J. Thermal Desorption Mass Spectrometric Analysis of Organic Aerosol Formed from Reactions of 1-Tetradecene and $\mathrm{O}_{3}$ in the Presence of Alcohols and Carboxylic Acids. Environ. Sci. Technol., 34: $2105-2115,2000$.

[72] Treacy, J.; Curley, M.; Wenger, J.; Sidebottom, H. Determination of Arrhenius parameters for the reactions of ozone with cycloalkenes. J. Chem. Soc., Faraday Trans., 93: 2877 - 2881, 1997.

[73] G. Wedler. Lehrbuch der Physikalischen Chemie. WILEY-VCH, 2004.

[74] Atkinson, R.; Winer, A.M.; Pitts, J.N. Jr. Rate Constants for the Gas Phase Reactions of $\mathrm{O}_{3}$ with the Natural Hydrocarbons Isoprene and $\alpha$ - and ß-Pinene. Atmos. Environ., 16: 1017 - 1020, 1982.

[75] Atkinson, R.; Baulch, D.L.; Crowley, J.N.; Hampson, R.F.; Hynes, R.G.; Jenkin, M.E.; Rossi, M.J.; Troe, J. Evaluated kinetic and photochemical 
data for atmospheric chemistry: Volume II - gas phase reactions of organic species. Atmos. Chem. Phys., 6: 3625 - 4055, 2006.

[76] Atkinson, R.; Baulch, D.L.; Cox, R.A.; Crowley, J.N.; Hampson, R.F.; Hynes, R.G.; Jenkin, M.E.; Rossi, M.J.; Troe, J. Evaluated kinetic and photochemical data for atmospheric chemistry: Volume I - gas phase reactions of $\mathrm{O}_{x}, \mathrm{HO}_{x}, \mathrm{NO}_{x}$ and $\mathrm{SO}_{x}$ species. Atmos. Chem. Phys., 4: $1461-1738$, 2004.

[77] Atkinson, R.; Baulch, D.L.; Cox, R.A.; Crowley, J.N.; Hampson, R.F.; Kerr, J.A.; Troe, J. Evaluated Kinetic and Photochemical Data for Atmospheric Chemistry: Supplement III. J. Phys. Chem. Ref. Data, 18: 881 - 1097, 1989.

[78] Atkinson, R. Kinetics of the gas-phase reactions of $\mathrm{OH}$ radicals with alkanes and cycloalkanes. Atmos. Chem. Phys. Discuss., 3: 4183-4358, 2003.

[79] McGillen, M.R.; Carey, T.; Archibald, A.T.; Wenger, J.C.; Shallcross, D.E.; Percival, C.J. Structure-activity relationship (SAR) for the gas-phase ozonolysis of aliphatic alkenes and dialkenes. Phys. Chem. Chem. Phys., 10: 1757 - 1768, 2008.

[80] McGillen, M.R.; Archibald, A.T.; Carey, T.; Leather, K.E.; Shallcross, D.E.; Wenger, J.C.; Percival, C.J. Structure-activity relationship (SAR) for the prediction of gas-phase ozonolysis rate coefficients: an extension towards heteroatomic unsaturated species. Phys. Chem. Chem. Phys., 13: $2842-2849,2011$.

[81] Cusick, R.D.; Atkinson, R. Rate Constants for the Gas-Phase Reactions of $\mathrm{O}_{3}$ with a Series of Cycloalkenes at $296 \pm 2 \mathrm{~K}$. Int. J. Chem. Kinet., 37: $183-190,2005$.

[82] Bonn, B.; Korhonen, H.; Petäjä, T.; Boy, M.; Kulmala, M. Understanding the formation of biogenic secondary organic aerosol from $\alpha$ pinene in smog chamber studies: role of organic peroxy radicals. Atmos. Chem. Phys. Discuss., 7: 3901 - 3939, 2007. 
[83] DeMore, W.B.; Sander, S.P.; Golden, D.M.; Hampson, R.F.; Kurylo, M.J.; Howard, C.J; Ravishanskara, A.R.; Kolb, C.E.; Molina, M.J.

[84] Burrows, J.P.; Cliff, D.I.; Harris, G.W.; Thrush, B.A.; Wilkinson, J.P.T. Atmospheric reactions of the $\mathrm{HO}_{2}$ radical studied by laser magnetic resonance spectroscopy. Proc. R. Soc. A., 368: 463 - 481, 1980.

[85] Johnson, D.; Lewin, A.G.; Marston, G. The effect of Criegee-Intermediate scavengers on the $\mathrm{OH}$ yield from the reaction of ozone with 2-methylbut2-ene. J. Phys. Chem. A, 105: 2933 - 2935, 2001.

[86] Reiner, T.; Arnold, F. Laboratory investigations of gaseous sulfuric acid formation via $\mathrm{SO}_{3}+\mathrm{H}_{2} \mathrm{O}+\mathrm{M} \longrightarrow \mathrm{H}_{2} \mathrm{SO}_{4}+\mathrm{M}$ : measurement of the rate constant and product identification. J. Phys. Chem., 101: 7399 - 7407, 1994.

[87] Stockwell, W.R.; Calvert, J.G. The mechanism of the $\mathrm{HO}-\mathrm{SO}_{2}$ reaction. Atmos. Environ., 17: 2231 -2235, 1983.

[88] Milhelcic, D.; Heitlinger, M.; Kley, D.; Müsgen, P.; Volz-Thomas, A. Formation of hydroxyl and hydroperoxy radicals in gas-phase ozonolysis of ethene. Chem. Phys. Lett., 301: 559 - 564, 1999.

[89] Malkin, T.L.; Goddard, A.; Heard, D.E.; Seakins, P.W. Measurements of $\mathrm{OH}$ and $\mathrm{HO}_{2}$ yields from the gas phase ozonolysis of isoprene. Atmos. Chem. Phys., 10: 1441 - 1459, 2010.

[90] Fenske, J.D.; Hasson, A.S.; Ho, A.W.; Paulson, S.E. Measurement of Absolute Unimolecular and Bimolecular Rate Constants for $\mathrm{CH}_{3} \mathrm{CHOO}$ Generated by the trans-2-Butene Reaction with Ozone in the Gas Phase. J. Phys. Chem., 104: 9921 - 9932, 2010.

[91] Laaksonen, A.; Kulmala, M.; Berndt T.; Stratmann, F.; Mikkonen, S.; Ruuskanen, A.; Lehtinen, K.E.J.; Maso, M.D.; Aalto, P.; Petäjä, T.; Riipinen, I.; Sihto, S.-L.; Janson, R.; Arnold, F.; Hanke, M.; Ücker, J.; Umann, B.; Selegri, K.; O'Dowd, C.D.; Viisanen, Y. $\mathrm{SO}_{2}$ oxidation products other than 
$\mathrm{H}_{2} \mathrm{SO}_{4}$ as a trigger of new particle formation. Part 2: Comparison of ambient and laboratory measurements, and atmospheric implications. Atmos. Chem. Phys., 8: 7255 - 7264, 2008.

[92] Sipilä, M.; Berndt T.; Petäjä, T.; Brus, D.; Vanhanen, J.; Stratmann, F.; Patokoski, J.; Mauldin III, R.L.; Hyvärinen, A.-P.; Kulmala, M. The role of sulfuric acid in atmospheric nucleation. Science, 327: 1243 - 1246, 2010.

[93] Berndt, T.; Böge, O.; Stratmann, F.;. Atmospheric particle formation from the ozonolysis of alkenes in the presence of $\mathrm{SO}_{2}$. Atmos. Environ., 38: $2145-2153,2004$.

[94] Jang, M.; Czoschke, N. M.; Lee, S.; Kamens, R. M. Heterogeneous Atmospheric Aerosol Production by Acid-Catalyzed Particle-Phase Reactions. Science, 298(5594): 814 - 817, 2002.

[95] Garland, R.M.; Elrod, M.J.; Kincaid, K.; Beaver, M.R.; Jimenez, J.L.; Tolbert, M.A. Acid-catalyzed reactions of hexanal on sulfuric acid particles: Identification of reaction products. Atmos. Environ., 40: 6863 - 6878, 2006.

[96] Hatakeyama, S.; Kobayashi, H.; Akimoto, H. Oxidation of $\mathrm{SO}_{2}$ in the ozone-olefin reactions. J. Phys. Chem., 88: 4736 - 4739, 1984.

[97] Hermsdorf, D. Schnelle Expansion von überkritischen Lösungen zur Herstellung von organischen Nanopartikeln. Dissertation, Georg-AugustUniversität Göttingen, 2006.

[98] TSI Incorporated (Hrsg.). Series 3080 Electrostatic Classifiers., 2006. 1933792, Revision G. Shoreview, MN 55126 U.S.A.: TSI Incorporated.

[99] Bresch, H. Photoionisation von freien Aerosolpartikeln mit Synchrotronstrahlung. Dissertation, Freie Universität Berlin, 2007.

[100] Wiedensohler, A.; Fissan, H. J. Aerosol Charging in High Purity Gases. J. Aerosol Sci., 19: 867 - 870, 1988. 
[101] Wiedensohler, A. An Approximation of the Bipolar Charge Distribution for Particles in the Submicron Size Range. J. Aerosol Sci., 19: 387 - 389, 1988.

[102] TSI Incorporated (Hrsg.). Model 3022A Condensation Particle Counter., 2002. 1933763, Revision I. Shoreview, MN 55126 U.S.A.: TSI Incorporated.

[103] ÆE. Frisch, M. J. Frisch und G. W. Trucks. Gaussian 03, User's Reference, 2003. Benutzer-Handbuch.

[104] ansyco GmbH (Hrsg.). Bedienungsanleitung Ozon-Analysator Modell O341M., 2004. ansynco analytische Systeme und Componenten $\mathrm{GmbH}$, Karlsruhe.

[105] Griggs, M. Absorption coefficients of ozone in the ultraviolet and visible regions. J. Phys. Chem., 49: 857 - 859, 1968.

[106] Meschede, D. [Hg.]. Gerthsen Physik. Springer Verlag, 1956-2006.

[107] Barker, E.F. The Infra-Red Spectra of Triatomic Molecules. Rev. Mod. Phys., 149: 198 - 203, 1942.

[108] Lide, D.R. [Hg.]. Handbook of Chemistry and Physics, 76th Edition. CRC Press, 1995-1996.

[109] Atkinson, R.; Aschmann, S.M.; Carter, W.P. Effects of ring strain on gas-phase rate constants. 2. $\mathrm{OH}$ radical reactions with cycloalkenes. Int. J. Chem. Kinet., 15: 1161 - 1177, 1983.

[110] M. J. Frisch, G. W. Trucks, H. B. Schlegel, G. E. Scuseria, M. A. Robb, J. R. Cheeseman, J. A. Montgomery, Jr., T. Vreven, K. N. Kudin, J. C. Burant, J. M. Millam, S. S. lyengar, J. Tomasi, V. Barone, B. Mennucci, M. Cossi, G. Scalmani, N. Rega, G. A. Petersson, H. Nakatsuji, M. Hada, M. Ehara, K. Toyota, R. Fukuda, J. Hasegawa, M. Ishida, T. Nakajima, Y. Honda, O. Kitao, H. Nakai, M. Klene, X. Li, J. E. Knox, H. P. Hratchian, J. B. Cross, V. Bakken, C. Adamo, J. Jaramillo, R. Gomperts, R. E. Stratmann, O. Yazyev, A. J. Austin, R. Cammi, C. Pomelli, J. W. Ochterski, P. Y. 
Ayala, K. Morokuma, G. A. Voth, P. Salvador, J. J. Dannenberg, V. G. Zakrzewski, S. Dapprich, A. D. Daniels, M. C. Strain, O. Farkas, D. K. Malick, A. D. Rabuck, K. Raghavachari, J. B. Foresman, J. V. Ortiz, Q. Cui, A. G. Baboul, S. Clifford, J. Cioslowski, B. B. Stefanov, G. Liu, A. Liashenko, P. Piskorz, I. Komaromi, R. L. Martin, D. J. Fox, T. Keith, M. A. Al-Laham, C. Y. Peng, A. Nanayakkara, M. Challacombe, P. M. W. Gill, B. Johnson, W. Chen, M. W. Wong, C. Gonzalez, and J. A. Pople. Gaussian 03, Revision C. 02. Gaussian, Inc., Wallingford, CT, 2004.

[111] M. J. Frisch, G. W. Trucks, H. B. Schlegel, G. E. Scuseria, M. A. Robb, J. R. Cheeseman, G. Scalmani, V. Barone, B. Mennucci, G. A. Petersson, H. Nakatsuji, M. Caricato, X. Li, H. P. Hratchian, A. F. Izmaylov, J. Bloino, G. Zheng, J. L. Sonnenberg, M. Hada, M. Ehara, K. Toyota, R. Fukuda, J. Hasegawa, M. Ishida, T. Nakajima, Y. Honda, O. Kitao, H. Nakai, T. Vreven, J. A. Montgomery, Jr., J. E. Peralta, F. Ogliaro, M. Bearpark, J. J. Heyd, E. Brothers, K. N. Kudin, V. N. Staroverov, R. Kobayashi, J. Normand, K. Raghavachari, A. Rendell, J. C. Burant, S. S. Iyengar, J. Tomasi, M. Cossi, N. Rega, J. M. Millam, M. Klene, J. E. Knox, J. B. Cross, V. Bakken, C. Adamo, J. Jaramillo, R. Gomperts, R. E. Stratmann, O. Yazyev, A. J. Austin, R. Cammi, C. Pomelli, J. W. Ochterski, R. L. Martin, K. Morokuma, V. G. Zakrzewski, G. A. Voth, P. Salvador, J. J. Dannenberg, S. Dapprich, A. D. Daniels, Ö. Farkas, J. B. Foresman, J. V. Ortiz, J. Cioslowski, and D. J. Fox. Gaussian 09, Revision A.02. Gaussian, Inc., Wallingford, CT, 2009.

[112] Scott, A.P.; Radom, L. Harmonic Vibrational Frequencies: An Evaluation of Hartree-Fock, Møller-Plesset, Quadratic Configuration Interaction, Density Functional Theory, and Semiempirical Scale Factors. J. Phys. Chem., 100: 16502 - 16513, 1996.

[113] Olzmann, M.; Kraka, E.; Cremer, D.; Gutbrod, R.; Anderson, S. Energetics, Kinetics, and Product Distributions of the Reactions of Ozone with Ethene and 2,3-Dimethyl-2-butene. J. Phys. Chem. A, 101: 9421 - 9429, 1997.

[114] Alecu, I.M.; Zheng, J.; Zhao, Y.; Truhlar, D.G. Computational Thermo- 
chemistry: Scale Factor Databases and Scale Factors for Vibrational Frequencies Obtained from Electronic Model Chemistries. J. Chem. Theor. Comp., 6: 2872 - 2887, 2010.

[115] Jenkin, M.E.; Shallcross, D.E.; Harvey, J.N. Development and application of a possible mechanism for the generation of cis-pinic acid from the ozonolysis of $\alpha$-and $\beta$-pinene. Atmos. Environ., 34: 2837 - 2850, 2000.

[116] Kalberer, M.; Yu, J.; Cocker, D.R.; Flagan, R.C.; Seinfeld, J.H. Aerosol formation in the cyclohexene-ozone system. Environ. Sci. Technol., 34: 4894 - 4901, 2000.

[117] Grosjean, E.; Grosjean, D. Rate Constants for the Gas-Phase Reaction of C5-C9 Alkenes with Ozone. Int. J. Chem. Kinet., 27: 1045 - 1054, 1995.

[118] Eckold, G. Grundbegriffe der Fehleranalyse bei praktischen Messungen. Skript zum Fehlerrechnungsseminar. Georg-August-Universität, Göttingen, 2008.

[119] Al Mulla, I.; Viera, L.; Morris, R.; Sidebottom, H.; Treacy, J.; Mellouki, A. Kinetics and Mechanisms for the Reactions of Ozone with Unsaturated Oxygenated Compounds. Chem. Phys. Chem., 11: 4069 - 4078, 2010.

[120] Wegener, R.; Brauers, T.; Koppmann, R.; Bares, S.R.; Rohrer, F.; Tillmann, R.; Wahner, A.; Hansel, A.; Wisthaler, A., E. Simulation chamber investigation of the reactions of ozone with short-chained alkenes. J. Geophys. Res., 112: D13301, 2007.

[121] Japar, S.M.; Wu, C.H.; Niki, H. Rate Constants for the Reaction of Ozone with Olefins in the Gas Phase. J. Phys. Chem., 78: 1017 - 1020, 1974.

[122] Aschmann, S.M.; Tuazon, E.C.; Arey, J.; Atkinson, R. Products of the Gas-Phase Reaction of $\mathrm{O}_{3}$ with Cyclohexene. J. Phys. Chem., 107: 2247 - 2255, 2003.

[123] Duncan, J.L.; Ferguson, A.M.; Harper, J.; Tonge, K.H.; Hegelund, F. HighResolution Infrared Rovibrational Studies of the $A_{1}$ Species Fundamentals of Isotopic Ketenes. J. Mol. Spectrosc., 122: 73 - 93, 1987. 
[124] Tuazon, E.C.; Aschmann, S.M.; Arey, J.; Atkinson, R. Products of the Gas-Phase Reaction of $\mathrm{O}_{3}$ with a Series of Methyl-Substituted Ethenes. Environ. Sci. Technol., 31: 3004 - 3009, 1997.

[125] Hou, H.; Wang, B. Ab inition study of the reaction of propionyl $\left(\mathrm{CH}_{3} \mathrm{CH}_{2} \mathrm{O}\right)$ radical with oxygen $\left(\mathrm{O}_{2}\right)$. J. Chem. Phys., 127: 054306, 2007.

[126] Winterhalter, R.; Neeb, P.; Grossmann, D.; Kolloff A.; Horie, O.; Moortgat, G. K. Products and Mechanism of the Gas Phase Reaction of Ozone with $\beta$-Pinene. J. Atmos. Chem., 35: 165 - 197, 2000.

[127] Winther, F.; Meyer, S.; Nicolaisen, F.M. The infrared spectrum of methylketene. J. Mol. Spectrosc., 611: 9 - 223, 2002.

[128] Haynes, W.S.; Gardner, P.D. Formation of Methylketene in the Photolysis of Diethyl Ketone. J. Am. Chem. Soc., 73: 3065 - 3067, 1951.

[129] Hediger, H.J. Infrarotspektroskopie. Grundlagen, Anwendungen, Interpretation. Akademische Verlagsgesellschaft Frankfurt am Main, 1971.

[130] NIST Chemistry Webbook. (http://webbook.nist.gov/chemistry).

[131] Lee, J.; Bozzelli, J.W. Thermochemical and Kinetic Analysis of the Formyl Methyl Radical $+\mathrm{O}_{2}$ Reaction System. J. Phys. Chem. A, 107: $3778-$ 3791, 2003.

[132] Baasandorj, M.; Griffith, S.; Dusanter, S.; Stevens, P.S. Experimental and Theoretical Studies of the Kinetics of the $\mathrm{OH}+$ Hydroxyacetone Reaction As a Function of Temperature. J. Phys. Chem. A, 113: 10495 - 10502, 2009.

[133] Butkovskaya, N.I.; Pouvesle, N.; Kukui, A.; Mu, Y.; Le Bras, G. Mechanism of the $\mathrm{OH}$-Initiated Oxidation of Hydroxyacetone over the Temperature Range 236-298 K. J. Phys. Chem. A, 110: 6833 - 6843, 2006.

[134] Alam, M.S. Total Radical Production and Degradation Products from Alkene Ozonolysis. Dissertation, University of Birmingham, 2011. 
[135] Hatakeyama, S.; Akimoto, H. Reaction of ozone with 1-methylcyclohexene and methylenecyclohexane in air. Bull. Chem. Soc. Japan, 63: 2701 - 2703, 1990.

[136] McMurry, J. Organic chemistry. Pacific Grove, Calif. : Brooks/Cole, 2007, 2007.

[137] GESTIS-Stoffdatenbank, 2012. Institut für Arbeitsschutz der Deutschen Gesetzlichen Unfallversicherung.

[138] Digital Analysis of Reactive Systems (DARS), 2012. http://loge.se/Products/DARS-Basics.html.

[139] Carlsson, P.T.M.; Dege, J.E.; Keunecke, C.; Krüger, B.C.; Wolf, J.L.; Zeuch, T. Pressure dependent aerosol formation from the cyclohexene gas-phase ozonolysis in the presence and absence of sulfur dioxide: a new perspective on the stabilisation of the initial clusters. submitted, zzz: yyy, 2012.

[140] Donahue, N.M.; Drozd, G.T.; Epstein, S.A.; Presto, A.A.; Kroll, J.H. Adventures in ozoneland: down the rabbit-hole. Phys. Chem. Chem. Phys., 13: 10848 - 10857, 2011.

[141] Atkinson, R.; Aschmann, S.M. Radical production from the gas-phase reactions of $\mathrm{O}_{3}$ with a series of alkenes unter atmosperic conditions. Environ. Sci. Technol., 27: 1357 - 1363, 1993.

[142] Drozd, G.T.; Kroll, J.; Donahue, N.M. 2,3-Dimethyl-2-butene (TME)Ozonolysis: Pressure dependence of Stabilized Criegee Intermediates and Evidence of Stabilized Vinyl Hydroperoxides. J. Phys. Chem. A, 115: 161 - 166, 2011.

[143] Somnitz, H. Quantum chemical and dynamical characterization of the reaction $\mathrm{OH}+\mathrm{SO}_{2} \rightleftharpoons \mathrm{HOSO}_{2}$ over an extended range of temperature and pressure. Phys. Chem. Chem. Phys., 6: $3844-3851,2004$.

[144] Kulmala, M.; Hämeri, K.; Aalto, P.P.; Mäkelä, J.M.; Pirjpla, L.; Nilsson, E.D.; Buzorius, G.; Rannik, Ü.; Maso, M.D.; Seidl, W.; Hoffmann, T.; Jan- 
son, R.; Hansson, H.-C.; Viisanen, Y.; Laaksonen, A.; O'Dowd, C.D. Overview of the international project on biogenic aerosol formation in the boreal forest (BIOFOR). 53B: $324-343,2001$.

[145] Virkkula, A.; Dingenen, Y.; Raes, F.; Hjorth, J. Hygroscopic properties of aerosol formed by oxidation of limonene, $\alpha$-pinene, and $\beta$-pinene. J. Geophys. Res. Atmos., 104: 3569 - 3579, 1999.

[146] Nguyen, T.L.; Peeters, J.; Vereecken, L. Theoretical study of the gasphase ozonolysis of $\beta$-pinene $\left(C_{10} H_{16}\right)$. Phys. Chem. Chem. Phys., 11: $5643-5656,2009$.

[147] Re, S.; Osamura, Y.; Morokuma, K. Coexistence of neutral and ion-pair clusters of hydrated sulfuric acid $\mathrm{H}_{2} \mathrm{SO}_{4}\left(\mathrm{H}_{2} \mathrm{O}\right)_{n} n=1-5-\mathrm{A}$ molecular orbital study. J. Phys. Chem., 18: 3535 - 3547, 1999.

[148] Reinning, M.-C. Massenspektrometrische Untersuchungen und Nachweise von organischen Hydroperoxiden und höhermolekularen Verbindungen im biogenen sekundären organischen Aerosol. Dissertation, Johannes Gutenberg Universität Mainz, 2009.

[149] Müller, L.; Reinning, M.-C.; Warnke, J.; Hoffmann, Th. Unambiguous identification of esters as oligomers in secondary organic aerosol formed from cyclohexene/ $\alpha$-pinene ozonolysis. Atmos. Chem. Phys., 8: 1423 1433, 2008.

[150] Greene, C.R.; Behnke, W.; Zetzsch, C. Rate Constants for the Gas-Phase Reactions of $\mathrm{O}_{3}$ with a Series of alkenes at $296 \pm 2 \mathrm{~K}$. Int. J. Chem. Kinet., 24: $803-811,1992$.

[151] Grosjean, E.; Grosjean, D. A Kinetic and Product Study of the Gas-Phase Reaction of Ozone with Vinylcyclohexane and Methylene Cyclohexane. Int. J. Chem. Kinet., 29: 855 - 860, 1997.

[152] Forster, P.; Ramaswamy, V.; Artaxo, P.; Berntsen, T.; Betts, R.; Fahey, D. W.; Haywood, J.; Lean, J.; Lowe, D. C.; Myhre, G.; Nganga, J.; Prinn, R.; Raga, G.; Schulz, M.; Van Dorland, R. Changes in atmospheric constituents and in radiative forcing. In S. Solomon, D. Qin, M. Manning, 
Z. Chen, M. Marquis, K. B. Averyt, M. Tignor, and H.L. Miller, editors, Climate Change 2001: The Physical Science Basis. Contribution of Working Group I to the Third Assessment Report of the Intergovernmental Panel on Climate Change, Cambridge, United Kingdom and New York, NY, USA, 2001. Cambridge University Press.

[153] Budyko, M.I. Climatic Changes. American Geophysical Society, Washington D.C:, 1977.

[154] Kerminen, V.-M.; Virkkula, A.; Hillamo, R.; Wexler, A. S.; Kulmala, M. Secondary organics and atmospheric cloud condensation nuclei production. J. Geophys. Res., 105: 9255 - 9264, 2000.

[155] Docherty, K.S.; Wu, W.; Bin Lim, Y.; Ziemann, P.J. Contributions of Organic Peroxides to Secondary Aerosol Formed from Reactions of Monoterpenes with $\mathrm{O}_{3}$, volume $=39$, year $=2005$,. Environ. Sci. Technol., pages $4049-4059$.

[156] Kroll, J.H.; Donahue, N.M.; Cee, V.J.; Demerjian, K.L.; Anderson, J.G. Gas-Phase Ozonolysis of Alkenes: Formation of $\mathrm{OH}$ from Anti Carbonyl Oxides, volume $=124$, year $=2002$,. J. Am. Chem. Soc., pages $8518-$ 8519.

[157] Niki, H.; Maker, P.D.; Savage, C.M.; Breitenbach, L.P.; Hurley, M.D. FTIR Spectroscopic Study of the Mechanism for the Gas-Phase Reaction between Ozone and Tetramethylethylene. J. Phys. Chem., 91: 941 - 946, 1987.

[158] Grosjean, D.; Williams II., E.L.; Grosjean, E. Atmospheric Chemistry of Isoprene and of Its Carbonyl Products. Environ. Sci. Technol., 27: 830 840, 1993.

[159] Cox, R.A.; Penkett, S.A. Aerosol Formation from Sulphur Dioxide in the Presence of Ozone and Olefinic Hydrocarbons. J. Chem. Soc., 68: 1735 - 1753, 1972. 
[160] Nolting, F.; Behnke, W.; Atkinson, R. A Smog Chamber for Studies of the Reactions of Terpenes and Alkanes with Ozone and $\mathrm{OH}$. J. Atmos. Chem., 6: 47 - 59, 1988.

[161] Bennett, P.J.; Harris, S.J.; Kerr, J.A. A Reinvestigation of the Rate Constants for the Reactions of Ozone with Cyclopentene and Cyclohexene under Atmospheric Conditions. Int. J. Chem. Kinet., 19: 609 - 614, 1987.

[162] Walser, M.L.; Desyaterik, Y.; Laskin, J.; Laskin, A.; Nizkorodov, S.A. High resolution mass spectrometric analysis of secondary organic aerosol produced by ozonization of limonene. Phys. Chem. Chem. Phys., 10: 1009 - 1022, 2008. 

Ich versichere, dass ich die Arbeit selbstständig verfasst und keine anderen als die angegebenen Hilfsmittel verwendet habe.

Göttingen, den 10. August 2012

Claudia Keunecke 



\section{Lebenslauf}

- Geburtsdatum und -ort: 23.August 1983 in Bad Gandersheim, Niedersachsen - Staatsangehörigkeit: deutsch

Schulische Ausbildung:

- September 1990-Dezember 1991: Besuch der Grundschule Kreiensen/Greene

- Januar 1992-Juli 1994: Besuch der Bürgerschule Alfeld (Grundschule)

- September 1994-Juni 1996: Besuch der Orientierungsstufe Alfeld

- August 1996-Juni 2003: Besuch des Gymnasium Alfeld

- 26. Juni 2003: Zeugnis der allgemeinen Hochschulreife (Note 2.2)

Studium:

- 1. Oktober 2003: Beginn des Studiums „Lehramt am Gymnasium“ zum Wintersemester 2003/2004 an der Georg-August-Universität Göttingen mit der Fächerkombination Chemie und Geschichte

- 21. Oktober 2004: Bestehen der Zwischenprüfung im Fach Alte Geschichte (Teil Römische Geschichte; der Teil Griechische Geschichte wurde bestanden am 15. April 2004)

- 14. Juli 2005: Bestehen der Zwischenprüfung im Fach Chemie

- Oktober 2005: Immatrikulation im Fach Physik als drittes Unterrichtsfach (für 1 Semester)

- 4. Februar 2006: Bestehen der Zwischenprüfung im Fach Geschichte

- 16.12.2008: Bestehen der Ersten Staatsprüfung für das Lehramt an Gymnasien (Note 1.6)

- Seit 1.02.2009: Promotion im Fach Chemie (Physikalische Chemie im Rahmen des Mathematisch-Naturwissenschaftlichen Promotionskollegs GAUSS) 
- 1.02.2009: Kollegiatin des Graduiertenkollegs 782 (Spektroskopie und Dynamik molekularer Knäuel und Aggregate)

- 1.10.2009-29.08.2011: Weiterbildendes Fernstudium "Lasertechnik“ an der Friedrich-Schiller-Universität Jena (Abschlussnote „sehr gut“), Erwerb der Sachkunde als "Laserschutzbeauftragter"

- 1.02.2010-31.01.2012: Stipendiatin des Graduiertenkollegs 782 (Spektroskopie und Dynamik molekularer Knäuel und Aggregate)

- 1.02.2012-31.07.2012: Stipendiatin der Georg-August-Universität Göttingen im Rahmen des Stipendienprogrammes der Fakultät für Chemie 